Wolfgang Schindler

\title{
Untersuchungen zur Grammatik appositionsverdächtiger Einheiten im Deutschen
}

Max Niemeyer Verlag Tübingen 1990 


\section{Iniversitärs. \\ Biblicthek \\ $36740+64$ \\ München}

CIP-Titelaufnahme der Deutschen Bibliothek

Schindler, Wolfgang : Untersuchungen zur Grammatik appositionsverdächtiger Einheiten im Deutschen / Wolfgang Schindler. - Tübingen : Niemeyer, 1990

(Linguistische Arbeiten ; 246)

NE: GT

ISBN 3-484-30246-1 ISSN 0344-6727

(c) Max Niemeyer Verlag GmbH \& Co. KG, Tübingen 1990

Das Werk einschließlich aller seiner Teile ist urheberrechtlich geschützt. Jede Verwertung außerhalb der engen Grenzen des Urheberrechtsgesetzes ist ohne Zustimmung des Verlages unzulässig und strafbar. Das gilt insbesondere für Vervielfältigungen, Übersetzungen, Mikroverfilmungen und die Einspeicherung und Verarbeitung in elektronischen Systemen. Printed in Germany.

Druck: Weihert-Druck GmbH, Darmstadt 
INHALTSVERZEICHNIS

VORWORT

VIII

VERZEICHNIS DER ABKURZUNGEN

IX

HINWEISE ZUR KENNZEICHNUNG OBJEKTSPRACHLICHEN MATERIALS

1. EINLEITUNG

.1

2. DER PHANOMENBEREICH

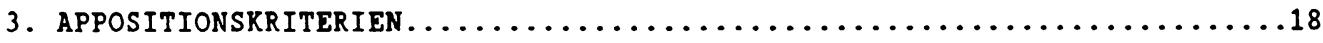

3.1. Kategoriale Füllung 19

3.2. Intonatorische/Graphematische Abtrennung des Appositivs 20

3.3. Stellungsverhalten von Basis und Appositiv 21

3.4. Funktion des Appositivs bzgl. der Basis 22

3.5. Paraphrasen (Umformungsbeziehungen) 24

3.6. Kasuskongruenz 29

3.7. Referenzidentität 32

3.8. Weglabbarkeit $\quad 35$

3.9. Appositionslexeme (Funktionslexeme) 35

3.10. Vertretung der Basis durch das Appositiv 37

3.11. Satzgrenzenbestimmtheit $\quad 37$

3.12. Attribution 38

3.13. Textnotwendigkeit 39

3.14. Sprecherdivergenz und sprecherpräsenz $\quad 40$

3.15. Adordinativităt $\quad 41$

3.16. Negationsskopus $\quad 42$

3.17. Wahrheitswertkonstanz $\quad 44$

3.18. Rekursivităt und Koordination $\quad 45$

3.19. Pronominalisierung und anaphorische Bezüge 46

3.20. Passivierung $\quad 46$

3.21. Dependenz 46

3.22. Konstituenz 46

4. ERSTE BEGRIFTSKLARUNGEN UND ABGRENZUNGSPROBLEME $\ldots \ldots \ldots \ldots \ldots \ldots \ldots \ldots \ldots$

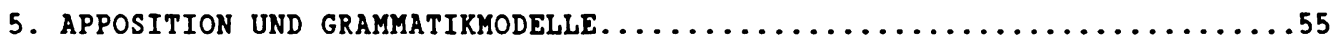

5.1. Apposition und Konstituentenstruktursyntax $\quad 56$

5.2. Apposition und X-Bar-Syntax $\quad 61$

5.3. Apposition und Dependenzgrammatik $\quad 65$ 
5.4. Apposition und kategoriale Grammatik 67

5.5. Modellvorstellung: Elementarsatz, Gastsatz und Hospitanten

6. DIE EIGENSCHAFTEN DES APPOSITIONS-PROTOTYPEN $\ldots \ldots \ldots \ldots \ldots \ldots \ldots \ldots \ldots$

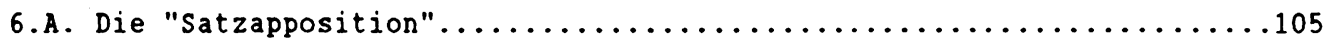

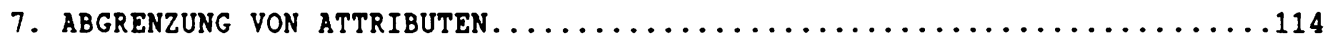

7.1. Die "engen Appositionen" 121

7.2. Das nicht-restriktive Adjektivattribut 135

7.3. Gefloatete NP-Elemente 138

7.4. Adverbial-Romplexe im Vorfeld 138

7.5. Translativphrasen mit wie 144

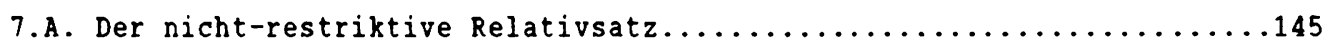

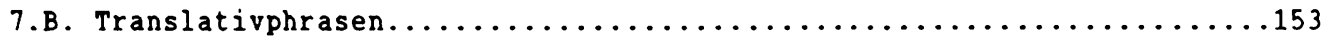

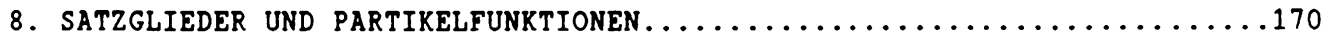

8.1. Satzglieder $\quad 170$

8.1.1. Die adverbialen Gliedsätze 171

8.1.2. Adverbiale als-Phrasen 172

8.1.3. Die prädikativen Attribute 174

8.2. Partikelfunktionen $\quad 175$

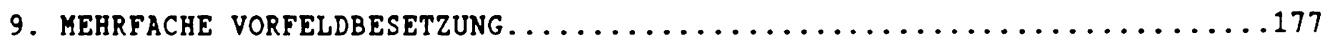

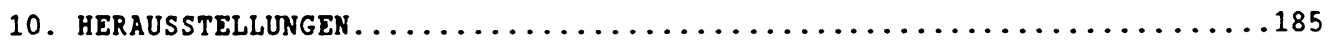

10.1. Linksversetzung 187

10.2. Rechtsversetzung 188

10.3. Nachtrag 189

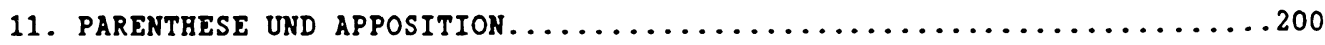

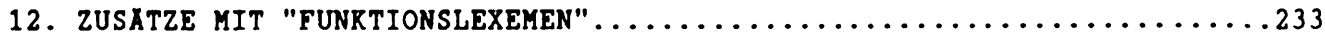

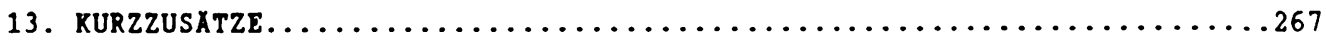

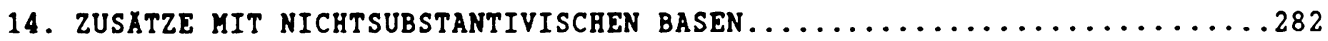

14.1. Basis: (Baupt)Satz, Zusatz: (Baupt)Satz 282

14.2. Basis: Verbend-Satz, Zusatz: Verbend-Satz 285

14.3. Basis: Infinitivgruppe, Zusatz: Infinitivgruppe 286

14.4. Basis: Verbalphrase, Zusatz: Verbalphrase 287

14.5. Basis: Verb, Zusatz: Verb 287

14.6. Basis: (attributives) Adjektiv, Zusatz: (attributives) Adj 287 
14.7. Basis: Prädikativ, Zusatz: Prädikativ

14.8. Basis: Determinans, Zusatz: Determinans (bzw. Genitiv-NP)

15. ZUSAMMENSCHAU DER NP-BEZOGENEN ZUSATZE.

15.1. Beteiligte Kategorien

15.1.1. Kategorien der Bezugseinheit

15.1.2. Rategoriale Füllung des Zusatzes

15.1.2.1. ohne Erweiterungen

15.1.2.2. Erweiterungsmöglichkeiten

15.2. Abtrennung

295

15.3. Stellung

298

15.4. Funktionen (auf semantischer und pragmatischer Ebene) 304

15.5. Umformungsbeziehungen

309

15.6. Kasuskongruenz

313

15.7. Referenzidentität

314

15.8. Weglabbarkeit

314

15.9. Appositionslexeme

315

15.10. Appositiv vertritt Basis 315

15.11. Satzgrenzenbestimmtheit 315

15.12. Attribution 315

15.13. Textnotwendigkeit 315

15.14. Sprecherdivergenz und sprecherpräsenz 316

15.15. Adordinativität 317

15.16. Tragweite von skopusbildenden Ausdrücken 317

15.17. Wahrheitswertkonstanz 320

15.18. Rekursivität und Koordination 320

15.19. Pronominalisierung und anaphorische Bezüge 322

15.20. Passivierung; syntaktische Operationen 322

15.21. Dependenz 323

15.22. Konstituenz 325

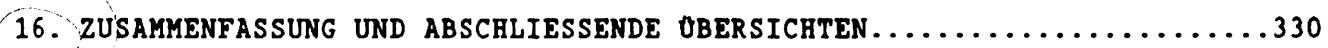

16.1. Zusammenfassung $\quad 330$

16.2. Die relevanten distinktiven Merkmale der Hospitanten 338

16.3. Der Bereich der Hospitanten $\quad 340$

16.4. Die Wortgruppenzusatze 342

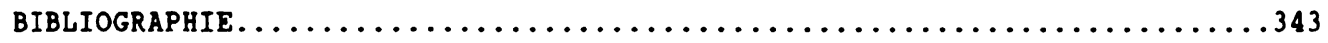


VORHORT

Die vorliegende Untersuchung ist eine überarbeitete und gestraffte Fassung meiner Arbeit "Untersuchungen zur Grammatik appositionsverdăchtiger Einheiten im Deutschen", die im Sommersemester 1989 von der Philosophischen Fakultät Sprach- und Literaturwissenschaft II der Ludwigs-Maximilians-Universităt München als Dissertation im Fach Germanistische Linguistik angenommen wurde.

An dieser Stelle möchte ich Herrn Prof. Dr. Elmar Seebold danken, der das Erstgutachten zu meiner Dissertation verfaste. Ebenfalls danken möchte ich Herrn Prof. Dr. Hans Altmann, der das Korreferat übernahm; ihm verdanke ich auch die Aufnahme der Arbeit in diese Reihe. Zudem möchte ich Herrn Prof. Dr. Theo Vennemann danken, der das dritte Gutachten verfabte.

Für zahlreiche Rorrekturvorschlăge danke ich Frau Marie LeBras. Ich danke auch Herrn Prof. Dr. E. Faucher für seine Bemūhungen, mir vergriffene Tagungsunterlagen zugänglich zu machen. 


\begin{tabular}{|c|c|c|c|}
\hline A & Apposition & DNSTR & instrumentales Adverbial \\
\hline Adj & Adjektiv & inf & infinit/nicht-finit \\
\hline AdjA & Adjektivattribut & InfGr & Infinitivgruppe \\
\hline AdjP & Adjektivphrase & KAUS & kausales Adverbial \\
\hline Adv & Adverb & KNeg & kontrastierende Negation \\
\hline AdvA & Adverbattribut & KOMIIT & komitatives Adverbial \\
\hline AdvP & Adverbphrase & KOND & konditionales Adverbial \\
\hline ADVB & Adverbial (e) & Kong & Kongruenz \\
\hline ADVBS & satzbezogene ADVB & Konj & Konjunktion \\
\hline ADVBV & verbbezogene ADVB & Konjadv & Konjunktionaladverb \\
\hline Akk/akk & Akkusativ & KONSTXK & konsekutives Adverbial \\
\hline AKKO & Akkusativobjekt & Kan & konzessives Adverbial \\
\hline ALRZ & Alternativen nennender Zusatz & koord & koordinierend \\
\hline AIZ & Alternative angebender Zusatz & KP & Kompositum \\
\hline APP & Apposition & KURZ & Kurzzusatz \\
\hline APREL & appositiver Relativsatz & KV & Kopulaverb \\
\hline ARG & Argument & las & lockeres appositionelles Syntagma \\
\hline Artdf & definiter (= bestimmter) Artikel & LOK & lokales Adverbial \\
\hline ATTR & Attribut & LV & Linksversetzung \\
\hline AZ & ausschließender Zusatz & MOD & modales Adverbial \\
\hline B & Basis/Bezugselement & MP & Modalpartikel \\
\hline BZ & beispielnennender Zusatz & IN & Modalverb \\
\hline aN & Gattungsname & MZ & mengeneinschließender Zusatz \\
\hline DAPP & Dativapposition & $N \sqrt{12}$ & Negation (swort) \\
\hline Dat/dat & Dativ & NegPt & Negationspartikel \\
\hline Det & Determinator & NN & Nachname \\
\hline DetP & Determinatorenphrase & $\mathrm{Nom} / \mathrm{nam}$ & Nominativ \\
\hline DIR & direktionales Adverbial & NP & Nominalphrase \\
\hline DUR & duratives Temporaladverbial & NS & Nebensatz \\
\hline eas & enges appositionelles syntagma & NSPYZ & nichtspezifizierender zusatz \\
\hline DN & Eigenname & $\mathrm{NT}$ & Nachtrag \\
\hline EXT & Dxtraposition & $\mathrm{Nmm}$ & Mmeral (e); Zahlwort \\
\hline EZ & einschränkender Zusatz & OBJ & Objekt \\
\hline fin & finit & obl & obligatorisch \\
\hline PN & Familienname & OPRAD & Objektsprädikativ \\
\hline RR2Q & frequentatives Termporaladverbial & PA & Präpositionalattribut \\
\hline FT & freies Thema & PAR & Parenthese \\
\hline GA & Genitivattribut & PATIR & prädikatives Attribut \\
\hline Gen/gen & Genitiv & Perspron & Personalpronomen \\
\hline $\cos 0$ & Genitivobjekt & PKT & punktuelles Temporaladverbial \\
\hline GIS & Gliedsatz & P1 & Plural \\
\hline GP & Gradpartikel & PO & Präpositionalobjekt \\
\hline GT & Gliedteil & Posspran & Possessivpronamen \\
\hline GtS & Gliedteilsatz & PostP & Postposition \\
\hline HFZ & hinzufügender Zusatz & $\mathrm{PP}$ & Präpositionalphrase \\
\hline $\mathrm{HZ}$ & hervorhebender Zusatz & PRAD & Prädikat \\
\hline inf & infinit/nicht-finit & Präp & Práposition \\
\hline InfGr & Infinitivgruppe & PRAZ & prädizierender Zusatz \\
\hline
\end{tabular}


Pronadv Proncminaladverb

PtGr Partizip(ial)gruppe

PITV

PZ

QN

RDrZ

Relpron

Rels

RTSTR

RV

S

Sadv

SADV (B)

$\mathrm{Sb}$

SbG

SBJ/SUBJ

$\mathrm{Sg}$

SN
Prädikativ

präzisierender Zusatz

quantifizierendes Nomen

referenzklärender Zusatz

Relativpronomen

Relativsatz

restriktives Adverbial

Rechtsversetzung

Satz

Satzadverb

Satzadverbial

Substantiv

Substantivgruppe

Subjekt

Singular

Stoffname

$\begin{array}{ll}\text { SPEZ } & \text { spezifizierender Zusatz } \\ \text { subord } & \text { subordinierend } \\ \text { TWPP } & \text { temporales Adverbial } \\ \text { Ugs } & \text { umgangssprachlich } \\ \text { UR } & \text { unmittelbare Rechtsadjazenz } \\ \text { UZ } & \text { umformulierender Zusatz } \\ \text { V/Vb } & \text { Verb } \\ \text { V-1 } & \text { Verb-Erst (-Stellung) } \\ \text { V-2 } & \text { Verb-Zweit (-Stellung) } \\ \text { V-E } & \text { Verb-End (-Stellung) } \\ \text { VF } & \text { Vorfeld } \\ \text { WN } & \text { Vorname } \\ \text { VokNP } & \text { vokativische Nominalphrase } \\ \text { VP } & \text { Verbalphrase } \\ \text { W } & \text { Vollverb } \\ \text { VZ } & \text { verallgemeinernder Zusatz } \\ \text { ZZ } & \text { zurücknehmender Zusatz }\end{array}$


Akzeptabilităt: Die Grade der Akzeptabilităt(sminderungs) werden markiert durch "*" ('inakzeptabel'), "??" ('kaum akzeptabel' bzw. 'vermutlich inakzeptabel'), "?" ('in seiner Akzeptabilität fragwürdig bzw. unklar'), "(?)" ('einigermaßen, jedoch evtl. nicht voll akzeptabel').

In Obersichten findet sich statt " $\star$ " das Zeichen "-", statt "??" findet sich z.T. "(-)", statt "(?)" dann "(+)" und für völlige Akzeptabilităt "+".

Eckige Klammern: Zusätze in "[...]" innerhalb von Sprachbeispielen (oder Zitaten) markieren meine Hinzufügungen.

Großschreibung: Sie dient im Text der Hervorhebung, vor allem von Begriffen für grammatische Funktionen (und Rategorien).

Rursivdruck: In den Text eingebrachte Sprachbeispiele werden kursiv markiert.

Numerierung: Durchzăhlung der Beispiele innerhalb eines Kapitels, Notation in Rundklammern: zuerst die Kapitelnummer, dann die Beispielnummer, ggf. eine Minuskel - z.B.: (5.23b). Die Numerierung der Fußnoten erfolgt in gleicher Weise, nur daß ein großes "F" vor die Zahlen gesetzt wird - z.B. (F5.23).

Pausen: Eine Pause wird mit "+" markiert, eine Satzpause mit "++". In Verbindung mit Tonmustermarkierungen folgt "+" stets nach (z.B. p t).

Runde Klammern: Runde Klammern innerhalb von Sprachbeispielen markieren die optionale Anwesenheit von Spracheinheiten (zur Verdeutlichung etc.). Manchmal füge ich in sprachbeispiele funktionale Termini mit "(..)" ein.

Schrăgstriche ("/../"): Je ein Schrägstrich links und rechts eines Beispielsatzes zeigt an, daB ein Beispiel auf die lautlichen, gesprochensprachlichen Eigenschaften Bezug nimmt; innerhalb der Schrägstriche erfolgt Kleinschreibung. Ein einfacher Schrägstrich innerhalb eines Sprachbeispiel trennt mögliche einsetzbare Alternativausdrũcke voneinander.

Sperrung: Sie zeigt die Lage von Hauptakzenten (z.B. Fokusakzent einer GP, Rhemaakzent etc.) an.

Tonmuster: Sie werden mit "p" ('progredient'), "s" ('steigend') und "f" ('fallend') angegeben.

Unterstreichung: Sie dient im Text und in Sprachbeispielen der Hervorhebung. 


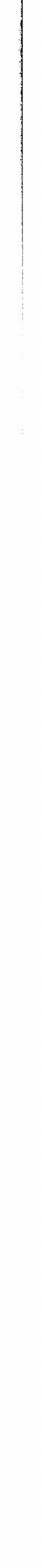




\section{BINLEITUNG}

Vorab ein "lesetechnischer" Hinweis für den Leser, der sich rasch über die Analysen und deren Ergebnisse informieren möchte: Das Kapitel 16 bietet dem Leser einen Oberblick über die "Ordnung" und die wesentlichen grammatischen Eigenschaften der in dieser Arbeit zentral diskutierten Konstruktionen. Neben Kapitel 16 seien zur schnellen Information noch Kap. 2 (Bereich appositionsverdăchtiger Muster), Kap. 5.5 (Modellvorstellung dieser Arbeit), Rap. 6 (Eigenschaften des Appositionsprototypen) und Kap. 15 (Zusammenschau der "appositionsverdächtigen" zusätze) empfohlen.

Bevor ich eigentlich beginne, möchte ich eine Vorklărung des Sprachgebrauchs anbieten. Sie besteht in der Annahme, das eine "appositionsverdăchtige Ronstruktion" sich in zwei Elemente zergliedern lassen muB: ein Bezugselement (eine Basis) und eine "Apposition" $z u$ diesem Element; beides zusammen bildet eine Appositionskonstruktion (1.1a). Die Terminologie verlăuft analog zu anderen syntaktischen Relationen. ${ }^{1}$ Man spricht $z$.B. bei einer Attributkonstruktion davon, daB Y Attribut relativ zur Basis X sei (1.1b); bei Satzgliedrelationen spricht man z.B. von objekten relativ zu einem Verb (1.1c). Damit wird nahegelegt, daß die "Apposition" - oder wie es am Schluß dieser Arbeit heiBen wird: der ZUSATZ - als eine syntaktische Relation zwischen zwei Einheiten aufgefast werden kann.

(1.1a) Der Baron, übrigens ein Knoblauchfan, betrat den Saal.

[ BASIS ] [ APPOSITION ]

[ APPOSITIONSKONSTRUKTION ]

(1.1b) Der Baron aus Gladbach betrat den Saal.

[ BASIS ] [ ATTRIBUT ]

[ATTRIBUTKONSTRUKTION]

(1.1c) Der Baron aus Gladbach betrat den Saal.

[VERB] [OBJEKT]

[SATZGLIEDRELATION]

Zur Beschreibung von Appositionsmustern existiert noch eine weitere Ausdrucksreise: die Termini Apposition, Basis und Appositiv von Raabe (1979: 225):

"Apposition" = Ronstruktion bestehend aus einer Basis und einem Appositiv, wobei die Basis Teil des Trăgersatzes, oder im Sonderfall mit dem Trăgersatz identisch ist".

Diese Festlegung sei an einem Beispiel demonstriert, das als "standardfall" bzw. "Prototyp" von Apposition gelten kann (cf. Raabe 1979: 223 f.):

1 Begriff nach Eisenberg (1986: $55 \mathrm{ff}$. ). 
[ TRAGERSATZ ] [ APPOSITIV ] [ TRAGERSATZ ]
(1.2) Kunibert Huber, mein bester Freund, hat mich betrogen.

[ BASIS ] [ APPOSITIV ]

[ APPOSITION ]

Raabes Sprachgebrauch ist insofern etwas ungewohnt, als üblicherweise mit "Apposition" das bezeichnet wird, was bei Raabe "Appositiv" genannt wird. In der vorliegenden Arbeit findet sich vor allem Raabes Sprachgebrauch, d.h. wenn ich sage, daB Y Appositiv zu/bzgl. X sei, dann ist das äquivalent mit der Aussage, $Y$ sei Apposition $z u / b z g l$. $X$. Ich verwende anstelle des Begriffs "Trägersatz" auch den Ausdruck "Gastsatz" (vgl. Kap. 5).

Die Terminologie wird sich im Verlauf dieser Arbeit verändern. Bis zum Ende der Abgrenzungserörterungen in Kap. 11 wird hauptsächlich von Bezugselement bzw. Basis und Appositiv bzw. Apposition (zu diesem Element) gesprochen. Beides zusammen ergibt eine Appositionskonstruktion, oder eigentlich genauer: eine "appositionsverdächtige Konstruktion". Man kann auch sagen, daß eine Appositionsrelation vorliegt, m.a.W.: Y (das Appositiv) fungiert bzgl. X (Basis) als Apposition. Später, wenn es darum geht, die i.e.s. "appositionsverdächtigen Muster" zu klassifizieren (Kap. 12-15), spreche ich mehr und mehr von Bezugselementen und unterschiedlichen (z.B. prädizierenden, spezifizierenden) ZUSATZEN zu diesen. Dabei sind die Bezugselemente Teile des Gastsatzes (Trägersatzes) und die zusätze "Gäste" bzw. "Hospitanten" in diesem. Nachstehend nochmals die zuerst verwendete Begriffichkeit (oberhalb des Beispiels) und die spater verwendete (unterhalb):

\section{APPOSITIONSVERDACHTIGE KONSTRUKTION}

[ APPOSITIONSKONSTRUKTION bZW. MUSTER ]

[BEZUGSELEMENT/BASIS] [APPOSITIV/APPOSITION]

(1.3) Kunibert Gschwendtner , übrigens ein Jurist, hat mich betrogen.

[BEZUGSELEMENT/BASIS] [ Z U S A T Z ]

[ GASTSATZ ] [ H.O S P I T A N T [ GASTSATZ ]

Als pauschale Vorwegnahme der Resultate dieser Arbeit sei bemerkt, daB der Bereich der Nicht-Basen der "appositionsverdăchtigen Ronstruktionen" - bislang wurde ja (s. Kap. 2) mehr als nur der "Prototyp" (s. Kap. 6) unter "Apposition" eingereiht - aufgelöst wird in mehrere Klassen von zusătzen zu Phrasen (des Gastsatzes). Es wird sich herausstellen, daB es eine eingrenzbare Menge von Einheiten $Y$ gibt, die als ZUSATZ zu ihrem Bezugselement $X$ fungieren, wobei es eine eingrenzbare Menge von Subklassen dieser Relation gibt, z.B. prádizierende, referenzklärende, spezifizierende zusătze etc.

Hausmann (1982: 437) schliebt seine Rezension der Appositionsmonographie von Raabe (1979) mit folgender Bemerkung ab:

"Das Thema Apposition ist lohnend und schwierig. Was die Grammatiker dazu bislang zusammengeschrieben haben, ist erstaunlich. Aber es war gewiB nicht fürsorglich, das Ausmisten dieses Augiasstalles statt einem linguistischen 
Herkules einem Doktoranden zu übertragen, der damit überfordert sein muBte."

Obwohl ich kein linguistischer Herkules bin, hat mich diese Bemerkung nicht von meinem für die Dissertation gewăhlten Thema abbringen können. Mir selbst erscheint der Bereich der "appositionsverdächtigen Konstruktionen" eher als Dschungel - vielfăltig, verworren, ohne sogleich erkennbare Zusammenhänge, unübersichtlich. Doch bin ich auch kein linguistischer Tarzan, der diesen Dschungel beneistert, sondern lediglich ein Neugieriger, den das Forschungsgebiet reizte.

DaB ich mir das Thema "Apposition" für meine Dissertation ausgewăhlt habe, obwohl z.B. mit Motsch (1965), Raabe (1974; 1979), Molitor (1979), Altmann (1981) oder Engel (1986) an Beispielmaterial, Differenzierungsaufwand und Begriffsdiskussion reiche Arbeiten vorliegen, hat vor allem folgende Gründe:

(a) Die in diesen Arbeiten (und zahlreichen weiteren; vgl. auch Rap. 2, 3) erzielten Definitionen von "Apposition" sovie die abgesteckten Appositionsphänomenbereiche sind nur teilweise miteinander verträglich. Eine Oberblendung der Resultate erbringt ein eher verwirrendes, relativ inhomogenes Bild vom Phănomen "Apposition", von der Intension und Extension dieses Begriffs. Um nur wenige Beispiele herauszugreifen: Die Explikationen von Altmann (1981) und Engel (1986) liegen recht nahe beieinander; sie sind z.B. insofern miteinander vereinbar, als nur "lockere" Strukturen (wie Dr. Mabuse, ein Spieler, wurde gefürchtet) akzeptiert werden. Molitor (1979) nimmt dagegen "enge strukturen" wie (der Spieler Dr. Mabuse wurde gefürchtet) hinzu. Raabe (1979) schliebt diese wiederum aus, läßt jedoch als Apposition grammatisch so verschiedene Strukturen zu wie:

(1.4a) Dr. Mabuse, er ist ein Spieler, wurde gefürchtet.

(1.4b) Dr. Mabuse - welche Oberraschung! - ist ein Spieler.

(1.4c) Er kommt, er kommt morgen.

(b) Obwohl man feststellen kann, das hinsichtlich eines Kernbereichs "prototypischer Appositionen" (1.5) eine relativ große Einigkeit besteht,

(1.5a) Dr. Mabuse, ein Spieler, war unersättich.

(1.5b) Dr. Mabuse, unersattlich wie immer, spielte die ganze Nacht.

(1.5c) Dr. Mabuse, hinter einew Vorhang, erteilte seine Anweisungen. ist es unbefriedigend, das bzgl. der weniger zentralen Muster bis hin zu den peripheren "appositionsverdăchtigen" Mustern kaum Obereinstimmung vorliegt. Die Vielzahl und Uneinheitlichkeit der von den verschiedenen Appositionsforschern angebotenen Abgrenzungsmerkmale gibt uns keine verbindliche Abgrenzungsstrategie zur Hand. Dies mag mit einer Eigenart "appositionsverdăchtiger" Konstruktionen zusammenhängen, die Matthews (1981: $235 \mathrm{f.}$ ) mit Anspielungen auf den Begriff der "Familienăhnlichkeit"2 und den Begriff des "Prototypen"3 so resũmiert:

2 s. Mittgenstein (1980). 
"(...) there is no positive property that is in common to all the constructions involved. (...) There is a tradition in linguistics which requires that terms should be defined with respect to our data, with necessary and sufficient conditions for their use. Apposition is a striking instance of a category that cannot be elucidated in that way. Instead we have a paradigm use, and other uses are linked to it by various forms of resemblance. Where the resemblances end is naturally indeterminate."

Zu klären ist also, welche Eigenschaften der "paradigm use" (1.5) aufweist, ob "Appositionskonstruktionen" tatsächlich keine Eigenschaft gemein haben (oder doch), und wann die khnlichkeiten zwischen einem "paradigm use" und einem peripheren Muster so gering sind, daB man letzteres einer anderen Kategorie bzw. Funktion zuweisen mus.

(c) Auf die vielen verschiedenen Ansätze zur Bestimmung von "Apposition" bezieht sich Schenk (1976: 9):

"Zusammenfassend läßt sich sagen, daß die Vorstellung, was Apposition bedeutet und in welchen Erscheinungsformen sie sich manifestiert, erheblichen Divergenzen in der Meinung der Forschung unterliegt. Die Vielfalt der Explikationsansătze (...) bewirkt demgemäß eine Vielfalt von Klassifikationsmethoden und -kriterien."

Dieser Zustand ist nicht zufriedenstellend; es sollte geprüf werden, welche Klassifikationskriterien sinnvoll und welche entbehrlich scheinen.

(d) Die Situierung von "Apposition" innerhalb einer Grammatik des Deutschen scheint auch noch nicht hinreichend geklärt. Gehört sie tatsächlich zu den attributiven Funktionen ${ }^{4}$ oder ist sie eine syntaktische Funktion eigener Art, die über adnominale Bezüge weit hinausreicht? ${ }^{5}$ Man könnte noch weitere Möglichkeiten ausführen, die die Literatur nahegelegt hat.

Es geht u.a. um die Frage, wie sich die "appositionsverdächtigen Erscheinungen" bzgl. ihrer Bezugselemente bzw. ihres "Bezugssatzes" verhalten. Mit welchen syntaktischen Erscheinungen'(z.B. Attribut, Satzglied, Herausstellung, Parenthese etc.) überschneiden sich "appositionsverdáchtige syntagmen", mit welchen könnten sie verwechselt werden?

(e) Es gibt "appositionsverdăchtige Muster" im Deutschen, deren grammatische Beschreibung entweder noch aussteht oder nur rudimentăr vorliegt, z.B.:

(1.6a) Ein Streichinstrument, genauer gesagt eine Geige, ertönte dort. Ein Mann, ein Mensch, krauchte aus dem Gebüsch.

(1.6b) Alle Vögel, ferner die Fledermäuse, erhoben sich in die Lüfte.

(1.6c) Helmut Kohl ( $\underline{C D U})$ ist der nächste Redner.

Aalen (Württemberg) ist eine Rreisstadt.

3 Matthews verwendet den Ausdruck "paradigm use". S. auch Putnam (1975), Rosch (1977).

4 Vgl. 2.B. Heidolph U.a. (1981: 290), Engel (1986: 189).

5 Z.B. Kolitor (1979: 222 f.), Raabe (1979: 329). 
Die erwăhnten Argumente zugunsten einer weiteren Arbeit über "Apposition" dürften genügen. Wie geht nun die vorliegende Arbeit methodisch vor?

In Kap. 2 werden sechzehn Arbeiten dahingehend ausgewertet, welche und wieviele Ronstruktionen bislang als "Appositionen" eingestuft worden sind. Dadurch erhălt man einen Bereich "appositionsverdăchtiger" Muster, der von mir grob vorsortiert wird. Dieser Appositionsbereich wird im Fortgang der Arbeit immer weiter aufgegliedert.

Rap. 3 bietet eine Obersicht über die Menge der verwendeten Appositionskriterien und deren Bündelung bei den einzelnen 16 ausgewerteten Arbeiten; die Kriterien werden jeweils kurz vorgestellt und hinsichtlich ihrer Leistungsfahigkeit diskutiert. Sofern ein Rriterium sich als heuristisch (einigermaBen) wertvoll erwiesen hat, wird es in späteren Kapiteln (6-15) verwendet. Zusătzlich werden vom Autor noch einige weitere Gesichtspunkte zur Analyse "appositionsverdăchtiger Muster" eingeführt.

Die Kriterien werden auf die spăter zur Diskussion stehenden Muster i.d.R. in der Reihenfolge angewendet, die sich aus Kap. 3 ergibt, wo sie der Băufigkeit nach sortiert sind. Es wäre evtl. nützlich gewesen, die Kriterien nach syntaktischen, semantischen und pragmatischen Gesichtspunkten zu sortieren, doch ich blieb bei der Băufigkeitsabfolge von Kap. 3. Dort kann der Leser stets nachschlagen. Die Appositionskriterien, m.a.W. die grammatischen Eigenschaften von Apposition, bündeln sich bei den einzelnen Mustern zu jeweils "individuellen" Merkmalsmengen, die das grammatische Verhalten der betreffenden Konstruktion charakterisieren.

Kap. 4 erőrtert einige Auffassungen von "Apposition" (als Ronstruktionsart, asyndetische Fügeweise etc.), die im Laufe der Appositionsforschung Erwăhnung fanden und trifft einige Sprachregelungen. Vor allem soll gezeigt verden, was die vorliegende Arbeit nicht unter Apposition versteht.

In Kap. 5 wird skizziert, welche Vorschläge zur Appositionsbeschreibung im Rahmen einzelner Grammatikmodelle (z.B. X-Bar-Syntax) angeboten werden. An Ende dieses Kapitels entrickelt der Verfasser selbst ein Modell, das von der nachstehenden Zweiteilung ausgeht: (a) den Elementarsatz, bestehend aus Valenztrăger bzw. Prădikat, Ergănzungen, Angaben sowie den Partikeln; in ihn wirken u.a. Rektion, Valenz, Negation, Erfragung; (b) den Maximalsatz; er besteht aus dem Elementarsatz und darin "eingeschalteten" GXSTEN resp. BOSPITANTEN (gemeint sind mit dem Einschaltungsmuster versehene EINSCHOBE und ZUSXT2E). Sie bleiben von im Gastsatz wirkenden Erscheinungen wie Rektion, Valenz etc. unbetroffen und sind syntaktisch wesentlich schwăcher in diesen integriert als die Elementarsatzelemente. Die "appositionsverdăchtigen" Muster werden grammatisch im Bereich der BOSPITANTEN verortet.

Das die vorliegende Arbeit weit unfassender als geplant ausfallen wird, wurde mir nach den Auswertungen von Kap. 2 und 3 sowie der Auffindung noch wenig behandelter Muster wie in $(1.6 a-c)$ bewust. Die ermittelten "appositionsverdāchtigen Ronstruktionen" waren zahlreicher und vielfaltiger, als ich es 
erwartete, und sie berührten sich offenbar mit einer vielzahl anderer grammatischer Erscheinungen wie z.B. Attribut, Satzglied, Herausstellung, Parenthese, Interjektion oder Korrektur etc., so daB all diese Begriffe (wenigstens knapp) gestreift werden musten, um die im engeren sinne "appositionsverdächtigen Muster" von eindeutig attributiven, satzgliedhaften u.a. grammatischen Erscheinungen abzutrennen (dazu v.a. Kap. 7-11).

Es erschien mir notwendig, den gesamten Bereich der "Einschaltungen" in einen Satz des Deutschen - darunter verstehe ich ein Syntagma aus Valenztrăger (Prădikat i.e.S.), Ergănzungen, möglichen Angaben und ggf. Partikeln (Negations-, Grad-, Modalpartikeln) - in Augenschein zu nehmen. "Einschaltung" bedeutet, das die betreffende spracheinheit durch das sog. "Einschaltungsmuster" markiert ist, d.h. links und rechts orthographisch durch Schreibzeichen resp. intonatorisch durch Pausen vom "Gastsatz" abgetrennt wird: ${ }^{6}$

(1.7a) Der Tiger, übrigens ein älteres Exemplar, maunzte Pia an.

(1.7b) Ein Tiger (Panthera tigris) war in dem Buch abgebildet.

(1.7c) Ein Geist, als es Mitternacht schlug, schwebte ins Zimmer.

(1.7d) Ein Tiger - wir haben sehr gestaunt! - spähte ins Zimmer. Ein Tiger spähte ins Zimmer. Wir haben sehr gestaunt!

(1.7e) Ein Tiger - o weh - sprang aus dem Busch. (O weh.) Ein Tiger sprang (o weh) aus dem Busch. (O weh.) Diese "Einschaltungen" unterteile ich (Rap. 5) in ZUSATZE (1.7a, b) und EINSCHOBE (1.7d, e). EINSCHUBE sind grammatisch betrachtet recht selbstandig, nicht auf einzelne Gastsatzelemente bezogen, sondern eher auf den gesamten Gastsatz; sie könnten keinesfalls eine syntaktische Funktion im Gastsatz ausüben; sie weisen i.d.R. eine große stellungsfreibeit auf und kommen sowohl zwischen den Gastsatz-Satzgliedern als auch meist links oder rechts des Gastsatzes vor. ZUSATZE sind auf ein Gastsatzelement (ein Bezugselement) bezogen und grammatisch weniger selbstandig; ihre stellungsmőglichkeiten sind begrenzter, die unmittelbare Adjazenz zum Bezugselement wird bevorzugt. EINSCHUBE sind auch insofern von ZUSATZEN zu unterscheiden, als man zwar sagen kann, $\underline{Y}$ sei (fungiere als) ZUSATZ $z u X$, nicht jedoch $Y$ sei (fungiere als) EINSCHUB zu $X$; man sprăche eher davon, daB $Y$ ein Einschub in $X$ sei. Es liegt nahe, ZUSATZ als funktionale Relation zwischen spracheinheiten anzusehen, EINSCHUB dagegen als konstruktionale Beziehung zwischen Gastsatz und Schaltsatz, Interjektion usw. (ebenfalls konstruktional: Satzkoordination, Satzverfügung etc.).

Dann gibt es Muster ohne Bezugselement unterhalb der Satzebene (1.7c), die einschubartig sind, jedoch im Gastsatz eine syntaktische Funktion erfüllen könnten (z.B. Temporaladverbial). Aufgrund ihrer potentiellen Integrierbarkeit verden solche Muster als SATZ-ZUSATZE eingestuft werden (Kap. 8.1.1, Kap. 11).

Im übrigen erscheint mir die Feststellung, daß man in Grammatiken des Deutschen (vgl. z.B. die in Kap. 2, 3 ausgewerteten) nicht allzu ausführlich uber

6 Zum Begriff "Schreibzeichen" s. Engel (1988: $819 \mathrm{ff.}$ ). 
den Bereich der Einschaltungen informiert wird, obwohl er eine Unzahl von Ronstruktionsmustern enthălt, die nicht pauschal mit "Apposition" und "Parenthese" abzudecken sind, als eine weitere Rechtfertigung, die Appositionsproblematik mitsamt ihrem Umfeld noch einmal aufzurollen.

Die vorliegende Untersuchung konzentriert sich im Bereich der ZUSATZE vor allem auf die zu Nominalphrasen möglichen, worunter nach der bisher entwickelten Begrifflichkeit mindestens die prototypische "lockere Apposition" (1.5) fảlt. Dennoch wird gezeigt (Kap. 12-16), das zusătze auch z.B. zu Adjektiven oder Verben möglich sind. Um der Gefahr zu entgehen, von der traditionellen Begrifflichkeit (und den mit ihnen verbundenen Assoziationen) irregeleitet zu werden, wird im Verlauf dieser Arbeit auf den Terminus "Apposition" verzichtet; überhaupt wird es vermieden, herkömmliche Begriffe wie "Parenthese", "Rechtsversetzung", "Nachtrag" U.a.m. zu verwenden. Die im engeren Sinn "appositionsverdăchtigen Konstruktionen" werden aufgelöst in einige Klassen von $\mathrm{ZU}-$ SATZEN (vor allem zu NPn). Dies bedeutet mitnichten, das die traditionellen Termini als obsolet anzusehen wăren. Nachfolgende Arbeiten kőnnten überlegen, wie die (Wieder)Einfuhrung jener Begriffe vorzunehmen wăre.

Die vorliegende Arbeit kann angesichts der Materialkomplexităt nur eine grobe Aufteilung der EINSCHOBE und ZUSATZE (ich nenne sie auch BOSPITANTEN eines Gastsatzes, im Ggs. zu den PARTIZIPANTEN des Elementarsatzes: Prádikat, Ergănzungen, Angaben, Partikeln) leisten. Sie vermag nur im Bereich vor allem der NP-bezogenen ZUSATZE detailliertere Analysen anzubieten (Rap. 6, 12-15).

Un die im engeren Sinne "appositionsverdăchtigen Muster" zu ermitteln, bot es sich an, zunăchst die Eigenschaften des "Appositionsprototypen" (1.5) in Kap. 6 genauer herauszuarbeiten, um mit deren Hilfe Vergleiche mit anderen "appositionsverdăchtigen Mustern" (Kap. 7-11; v.a. Kap. 12-15) anstellen zu können. Kap. 6.A enthălt zudem einen Exkurs zur sog. "Satzapposition", deren Status allerdings nicht befriedigend zu klăren var.

Die Kapitel 7-11 filtern aus den Phănomenbereich (Kap. 2) die Muster heraus, die sich eindeutig anderen syntaktischen Erscheinungen zuordnen lassen. Kap. 7 scheidet die Attribute aus. Dabei stellen sich zwei Muster als besonders problematisch heraus: die NP-adjazenten als-Phrasen (Rap. 7.B; s. 1.8a) und der nicht-restriktive Relativsatz (Rap. 7.A; Beispiel 1.8b). Es wird erörtert, ob sie als Attribute oder als ZUSXTZE zu gelten haben.

(1.8a) Pia als deine Anwaltin hât te das wissen müssen.

(1.8b) Pia, die dich ja anwaltlich berăt, häte das wissen müssen.

In Kap. 8 werden die Satzglieder und Partikelfunktionen ausgesondert. In Kap. 9 werden mehrfache Vorfeldbesetzungen und zwischen VF-Element und Finitum eingeschaltete Elemente ausgeschieden.

Das Rapitel 10 versucht, Berausstellungen (Altmann 1981) von "Appositionen" abzutrennen. Einfach erscheint dies bei der "Linksversetzung". Die "Rechtsversetzung" (1.9a) hingegen erweist sich als i.e.s. "appositionsverdachtig" und 
wird in Kap. 12, 15 genauer behandelt. Das Herausstellungsmuster "Nachtrag" (1.9b) stellt sich als intern vielfăltig heraus; untersucht werden auch die Mŏglichkeiten der Situierung des "Nachtrags" im Satzinneren (1.9b).

(1.9a) Diese Viecher, ich meine die Hammerhaie, wurden gefürchtet.

(1.9b) Die Haie, und zwar vor allem die Hammerhaie, wurden gefürchtet.

Der Geist ist in der Gruft verschwunden, und zwar um ein Uhr.

Der Geist ist, und zwar um ein Uhr, in der Gruft verschwunden.

Rapitel 11 problematisiert den Begriff Parenthese; es geht năher auf die EINSCBOBE (Parenthesen: traditionell v.a. Schaltsatz, Interjektion; aber auch Anreden etc.) ein und trennt diese von den ZUSATZEN (den i.e.s. "appositionsverdăchtigen Mustern") ab. Zudem werden als Einschaltung markierte Angaben (1.10a) behandelt. Die Grammatik solcher Muster kann allerdings nur rudimentär dargeboten werden. Als Einschaltungen markierte Gliedteile (1.10b) können potentiell (nach Wegfall der Einschaltungsmarkierungen) eine syntaktische Funktion im Gastsatz ausüben. Dazu wăren typische Zusătze (i.e.s. appositionsverdăchtige Einheiten) niemals imstande. Sie verden vorläufig als ZUSATZE behandelt, deren genauere grammatische Erfassung noch aussteht.

(1.10a) Der Tiger sprang - weil er Hunger hatte - aus dem Gebüsch. Der Tiger sprang - offensichtlich hungrig - aus dem Gebüsch.

(1.10b) Eine - notgedrungen einseitige - Weigerung war nicht sinnvoll. Kisten (mit Pulvertüten) standen dort herum.

Die Kapitel 12 mit 15 enthalten Analysen der i.e.S. "appositionsverdächtigen Muster". Die Kapitel 12, 13 und 15 gehen überwiegend auf die Eigenschaften NPbezogener ZUSATZE ein, wobei aber auch darauf hingewiesen wird, dab bestimmte Zusătze (übrigens nicht der "Appositionsprototyp") relativ viele Rategorien als Bezugselemente aufweisen können. Das Kap. 14 gibt einen Ausblick auf ZUSATZE zu nicht-substantivischen Basen.

In Kap. 12 verden ausfühlich die zusatztypen untersucht, die von sog. "Funktionslexemen" begleitet werden kőnnen (1.11a) oder mússen (1.11b):

(1.11a) Manche Haie, (vor allem) Blau- und Tigerhaie, werden gefürchtet.

(1.11b) Die Haie, obendrein die Muränen, waren ihnen unheimlich.

Dabei lassen sich vorlăufig fünf Zusatzklassen unterscheiden; der prădizierende Zusatz ("Prototyp") ist eine von diesen. Es bleibt eine Forschungsaufgabe, ob die Differenzierung nicht noch weiter hätte getrieben werden können.

In Kap. 13 verden die von mir als KURZzUSATZE bezeichneten Muster auf ihre grammatischen Eigenschaften hin geprüt. Es handelt sich um (zumeist) rundgeklammerte Einheiten (1.12), deren grammatische Behandlung bislang als stiefmüterlich gelten kann:

(1.12) Aalen (Kürttemberg) ist eine Kreisstadt.

Die Kosten (1,1 Millionen DH) übernahm der staat.

Die Orthographie (Rechtschreibung) făllt vielen schwer.

In Rap. 14 wird skizzenhaft auf zUSATZ-Möglichkeiten zu Phrasen ohne substantivischen Rern ( 1.13 zeigt Adj, V) eingegangen: 
(1.13) Bunt, vor allem rot und grün, waren die Hemden gevesen.

Vorbereitet, $\underline{d . h}$. gewaschen und geschnitten, wurde nun der salat.

Das 15. Rapitel bietet in einer Zusammenschau die grammatischen Eigenschaften vor allem der NP-bezogenen ZUSATZE. Die "prototypische Apposition" (Rap. 6) geht in einer dieser Klassen auf.

In der Bibliographie wird die zitierte Literatur, aber auch die nicht-zitierte, für die vorliegende Arbeit recherchierte Literatur aufgeführt, sofern sie thematisch auf die Appositionsproblematik bezogen ist. Die Literaturliste ist somit als Bibliographie zum Thema Apposition nutzbar.

Zum Abschluß noch eine Bemerkung: In dieser Arbeit werden zumeist selbstkonstruierte Sprachbeispiele diskutiert (was in den Gutachten kritisiert wurde). Bei erfundenen Beispielen besteht unter anderem die Verführung, die Belege so zu wăhlen, daß sie den eigenen theoretischen Aussagen nicht widersprechen, zudem besteht die Möglichkeit, daß die Leser den Akzeptabilitätsbeurteilungen nicht immer folgen können (und wollen). Folgende Gründe mögen den Beispielmißstand erklăren (nicht entschuldigen): Erstens sollte in dieser Arbeit die umfangreiche Literatur zum Thema Apposition verarbeitet werden, und zwar möglichst umfassend aus dem germanistischen und so viel wie möglich aus dem anglistischen und romanistischen Bereich. Allein dies hat viel zeit gekostet. Zweitens entwickelte sich die Arbeit so, daB eine Auseinandersetzung mit mehreren großen Themenbereichen aus der Grammatik (z.B. mit Parenthese, Attribut, Satzglied usf.) unumgănglich wurde - ein weiterer zeitverschlingender Faktor. Drittens erwiesen sich die Probleme, die aufgeworfen wurden, nicht nur als zahlreich, sondern auch als z.T. eminent widerborstig. Und schlieblich viertens: Mir stand für die Abfassung der Dissertation lediglich ein begrenzter Zeitraum zur verfügung ( $1 \mathrm{Jahr}$ ). Daher war es nicht möglich, auch noch ein umfassendes Beleg-Rorpus zu erstellen. Zukünftigen Arbeiten zum Thema sei allerdings angeraten, sich auf ein Belegkorpus zu stützen. Eine empirisch ausgerichtete Arbeit zur Appositionsproblematik bleibt ein Forschungsdesiderat. 
Da die vorliegende Arbeit einen neuerlichen Versuch macht, das Phănomen Apposition $z u$ beschreiben und von verwandten oder verwechselungsträchtigen Mustern abzugrenzen, erschien es methodisch sinnvoll, als Ausgangspunkt eine Sammlung derjenigen Sprachmuster anzulegen, die im Rahmen der grammatischen Beschreibung des Deutschen bereits einmal oder mehrmals als Beispiel für "Apposition" eingestuft worden sind. Die Menge der Beispiele dient als Eingabe in eine Abfolge von Abgrenzungserörterungen (v.a. Kap. 7-11). Nach "Ausfăllung" diverser Muster (als Attributskonstruktionen, Satzglieder etc.) bleibt am Ende eine Menge i.e.s. "appositionsverdächtiger Konstruktionen" übrig, die sich nicht in die bis dahin verwendete grammatische Beschreibung einfügen (Kap. 12-15). Diese Muster kōnnen dann nach ihren grammatischen Eigenschaften mithilfe einer eigens geprăgten Begrifflichkeit ("Zusătze") subklassifiziert werden.

Die Ermittlung des Appositionsbereichs erfolgt durch die Auswertung einer Auswahl von Arbeiten, die sich teilweise oder speziell mit dem Phänomen Apposition befaBt haben. Diese Auswahl besteht teils aus Grammatiken des Deutschen (A.-Liste), teils aus Monographien zur Apposition oder Arbeiten (B.-Liste), in denen die Apposition neben anderem eine ausführlichere Behandlung erfährt (in Rundklammern die hinfort verwendeten Abkürzungen):
A.1) Eisenberg (1986)
$=($ Ei 86)
A.2) Duden (1984)
$=(\mathrm{Du} 84)$
A.3) Engel (1982)
$=($ En 82)
A.4) Jung/Starke (1982)
$=$ (JS 82)
A.5) Schulz/Griesbach (1982)
$=($ SG 82)
A.6) Heidolph U.a. (1981)
$=($ He 81)
A.7) Helbig/Buscha (1980)
$=($ HB 80$)$
A.8) Erben (1972)
$=(\operatorname{Er} 72)$
A.9) Behaghel (1928)
$=(\operatorname{Be} 28)$

B.1) Engel (1986)

$=(\operatorname{En} 86)$

B.2) Altmann (1981) = (Al 81)

B.3) Molitor (1979) = (Mo 79)

B.4) Raabe (1979) = (Ra 79)

B.5) Motsch (1965)

$=$ (Mo 65)

B.6) Kusmin (1960)

$=(\mathrm{Ku} 60)$

B.7) Seiler (1960)

$=(\mathrm{Se} 60)$

Durch diese Auswahl dürften die für eine Appositionsdiskussion wichtigen Konstruktionen erfast sein. Der Leser wird im folgenden eine nicht unerhebliche Redundanz feststellen, selbst wenn man verschiedene ansătze zusammenführt. Natürlich ist nicht auszuschließen, daB weitere Konstruktionen existieren, für die zu prüfen wăre, ob sie nicht auch zum Appositionsbereich gehören könnten. ${ }^{1}$

Die folgende Obersicht ist noch nicht sehr ausdifferenziert, eher grob vorsortiert, und deutet die Mannigfaltigkeit der "Submuster" lediglich an. Sie bietet jedoch ein erstes Bild über die schier grenzenlose Extension des Be-

1 Man siehe z.B. den Aufsatz von Regula (1968), in dem eine Vielzahl "appositionsverdăchtiger" Konstruktionen (aus dem Französischen) behandelt wird. 
griffs Apposition, sobald man eine Zusammenschau verschiedener Forschungsergebnisse unternimmt. Die Zusammenstellung bietet:

- eine grobe Beschreibung der Muster (z.B. Kategorien, Fügungsart)

- illustrierende Beispiele

- eine Orientierung zur strukturierung in Basis (B) und Appositiv (A) 2

- die Angabe der Arbeiten, in denen der Typ behandelt wird ${ }^{3}$

- eine Hăufigkeitsangabe, die zeigt, in wievielen der sechzehn ausgewăhlten Arbeiten die jeweilige Ronstruktion behandelt wird

- die Angabe der wichtigsten Begriffe (mit Kap.nummer), unter denen das Muster wieder aufgegriffen wird (durch ">)" eingeleitet).

\section{Lockere Apposition zu MPs}

1.1. mit nominalem Appositiv (s. auch Kap. 6)

Kuno (B), mein bester Freund (A), / Sie, eine Linguistin, / Der $R$ 4, ein französisches Fabrikat, / Ein alter Mann, der Onkel von Frau Zingli,etc.

Ei 86, Du 84, En 82, JS 82, SG 82, He 81, HB 80, Er 72, Be 28, En 86, Al

81, Yo 79, Ra 79, Yo 65, Ku 60, Se 60 (= 16/16)

1) ZUSATZ (Rap. 12, 15), PARENThESE (Kap. 11).4

\section{2. mit nichtnominalem Appositiv}

1.2.1. $A=\operatorname{AdjP}$ (s. auch Kap. 6)

Pia (B), lustig und fidel (A), / Runo, des Neines überdrüssig,

Du 84, En 82 , He 81 , En 86 , Al 81 , Ra 79 , Ho $65(=7 / 16)$

\) ZUSATZ (Kap. 12, 15), PARENTHESE (Kap. 11).

1.2.2. A = PP (s. auch Kap. 6)

Jobst (B), in Katmandu (A), / Das Nirtshaus, im Nald vor der stadt,

Du 84, En 82, En 86, A1 $81 \quad(=4 / 16)$

\) ZUSATZ (Kap. 12, 15), PARENTHESE (Kap. 11).

1.2.3. $A=\operatorname{AdvP}($ s. auch Kap. 6)

Jobst (B), da unten (A), / Das lauschige Ortchen, dort drüben,

En 86, $\operatorname{Ra} 79(=2 / 16)$

\) ZUSATZ (Kap. 12, 15), PARENTHESE (Kap. 11).

2 Dazu murden die Analysevorschlăge (AB oder BA) der ausgewerteten Arbeiten gezăhlt; die hăfigere Einstufung wird angegeben.

3 Eine Arbeit erscheint in Rumdklammern, wenn der entsprechende TYP nicht uneingeschrănkt zur Apposition gerechnet, sondern als "Sconderfall" ("appositionsăhnlich, -artig" o.ă.) behandelt wird.

Z.B. in Kuno, jede Nacht, bört merkmirdige Achzer aus der Nuckucksuhr. 
1.2.4. $A=(\operatorname{Re} 1) \mathrm{S}$

Karl (B), den ich schon lange nicht mehr gesehen hatte (A),

Ra 79, Se $60(=2 / 16)$

\) ZUSATZ (Rap. 7A), ATTRIBUT (Kap. 7, 7A).

\section{Enge Apposition zu Monina/KPs}

) ATTRIBUTE (Kap. 7)

Ordnungsprinzipien: Hăufigkeit und Komplexität. Es gibt eine Reihe von subklassen, die hier (noch) nicht erscheinen - wie z.B. Der Rampf Clay-Frazier, Die Brigade Müller, Die Universitat Erfurt etc.

2.1. Gattungsname (CN) + Eigenname (EN)

Onkel (A) Fritz (B)/ Professor Huber / Ministerin Müller

Ei 86 , (Du 84), En 82 , JS 82, SG 82 , He 81 , ВB 80, Er 72, Be 28 , Мо 65 , $\mathrm{Ru} 60$, Se $60(=12 / 16)$.

2.2. Determinator $(D e t)+C N+E N$

Unser Onkel (B) Fritz (A)/ die Ministerin Müller

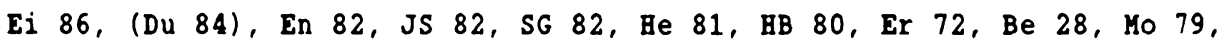
Mo $65, \mathrm{Ru} 60 \quad(=12 / 16)$.

2.3. Vorname/Eigenname + Det $+\mathrm{CN}$

Ludwig (B) das Kind (A)/ Katarina die GroBe

Ei 86 , Du 84, JS 82, SG 82 , HB 80, Er 72, Be $28,($ Ku 60$)(=8 / 16)$.

2.4. Vorname/EN + Familienname/EN

Nolfgang Peter Schindler / Pia (A) Nüller-Huber (B)

Ei 86, (Du 84), JS 82, SG 82, нB 80, (Er 72), Be 28, (Ku 60) (= 8/16).

2.5. Numerale + quantifizierendes Nomen + Nomen

ein Liter (B) herber Wein (A)/ zwei Sack Rartoffeln / eine Herde Kühe Ei 86, (Du 84), JS 82, SG 82, (Er 72), Be $28(=6 / 16)$.

2.6. Pron $+\mathrm{CN}$

Icb (B) Idiot (A)/ Wir Arbeiter / Euch ewigen Traumtänzern

(Du 84), He 81, HB 80, Er 72, Be 28, ( Ru 60) $(=6 / 16)$.

2.7. $\mathrm{EN}+\mathrm{CN}$

Hamburg (B) Bauptbahnhof (A)/Berlin Mitte

(Er 72), Be $28 \quad(=2 / 16)$. 
3. "Lockere" Typen ohne noninale Basen

3.1. $B$ = Adjektivphrase

Die Verben sind synonym (B), bedeutungsgleich (A).

Er ist ledig, d.h. ein Junggeselle.

Ho $79, \operatorname{Ra} 79(=2 / 16)$

) ZUSATZ (Kap. 12, 14, 15).

3.2. $B=$ Prăpositionalphrase

am anderen Ufer (B), hinter den Heiden (A), / am anderen Ufer, dort,

En 86, Mo $79(=2 / 16)$

) ZUSATZ (Kap. 12, 15), MEHRFACHE VORFELDBESETZUNG (Kap. 9), PARENTHESE (Kap. 11).

3.3. $B=$ Adverbphrase

dort hinten (B), unten (A), / dort, am anderen Ufer,

Du 84, En 86, Mo 79, Ra $79(=4 / 16)$

\) ZUSATZ (Kap. 12, 15), MEHRFACHE VORFELDBESETZUNG (Kap. 9), PARENTHESE (Kap. 11).

3.4. $B=\operatorname{Verb}($ alphrase)

Sie müssen verhungern (B), sterben (A).

DaB der Film "Lysistrata" uberhaupt nicht existiert (B), eine Erfindung ist (A), (...). 5

Mo 79, Ra $79(=2 / 16)$

\) ZUSATZ (Kap. 12, 14, 15).

3.5. $B=$ Determinator

Mit seiner (B), Müllers (A), Unterstützung / Mit dieser, meiner, Angel Mo 79 , Ku $60 \quad(=2 / 16)$

) ZUSATZ (Rap. 12, 14, 15).

3.6. BASIS $=$ Satz

3.6.1. APPOSITIV $=$ Satz

3.6.1.1. Wir kommen (B), wir kommen morgen (A).

Mo 79, $\operatorname{Ra} 79(=2 / 16)$

) SATZFOLGE (Kap. 14).

5 Beispiel aus Kolitor (1979: 188). 
3.6.1.2. Er wird diese Nacht in Paris angekommen sein (B), wenn die Umstände günstig sind (A). Auch:

Er wird diese Nacht, wenn die Umstände günstig sind (A), in Paris angekommen sein (B),

Ra $79(=1 / 16)$

) SATZGLIED (Kap. 8), PARENThESE (Kap. 11).

3.6.2. $A=N P$ (sog. "Satzapposition"; s. auch Kap. 6A)

sie hat gewonnen (B), ein Umstand, der sehr erfreulich ist (A).

Mo $79, \operatorname{Ra} 79(=2 / 16)$

\) (SATZ-)ZUSATZ (Rap. 15).

3.6.3. $A=P P$ (sog. "Nachtrag")

Sie lachten über ihn (B), aus SpaB (A).

Ra $79(=1 / 16)$

〉) HERAUSSTELLUNG (Rap. 10), PARENTHESE (Kap. 11).

\subsection{PARENTHESEN}

Sie werden wegen ihrer Bedeutung für einige Fragen - z.B. "Sind Appositionen reduzierte Parenthesen?" oder "Wo verlăuft die Grenze zwischen Parenthese und Apposition?" - als eigener Punkt aufgeführt.

Karl Müller (B), ich bewundere ihn sehr (A), hat eine Grammatik verfaBt.

Die Kerle haben, man glaubt es kaum, schon wieder einen Rausch.

Die Leute werden, wahrscheinlich, daraus ihre schlüsse ziehen.

Ra $79(=1 / 16)$

> PARENTHESE (Kap. 11)

4. "Bnge" TYpen (Rest)

4.1. $B=N / N P$

4.1.1. böse (A) Bunde (B) (nicht-restriktives ADJEKTIVATTRIBUT)

Se $60(=1 / 16)$

\) ATTRIBUT (Kap. 7).

4.1.2. (allein) (A) die Wissenschaft (B) (allein) (A) (GRADPARTIRELN)

Er $72(=1 / 16)$

) PARTIKELFUNKTION (Kap. 8).

4.1.3. Die straBen (B) sind alle (A) überfü11t. (GEFLOATETE QUANTOREN)

SG $82(=1 / 16)$

\) ATTRIBUT (Rap. 7). 
4.2. $B=$ nichtnominal

4.2.1. hier (B) am Knie (A) ((Adv) (PP) $)^{6}$

(Ei 86), (He 81), (= 2/16)

)) ATTRIBUT (Kap. 7), MEHRFACHE VORFELDBESETZUNG (Kap. 9).

\subsubsection{Im Stadion (B) auf der Tribüne (A) ((PP) (PP))}

(Mo 79; der Fall wird schlieblich als attributive Ronstruktion eingeordnet, sofern die zweite PP nicht durch Rommata abgetrennt wird. Da mit steinitz (1969) jedoch eine $z u$ diskutierende Arbeit u.a. zu "adverbialen Appositionen" vorliegt, wird dieser Typus hier erwăhnt). 7

) ATTRIBUT (Kap. 7), MEHRFACHE VORFELDBESETZUNG (Kap. 9).

\section{Strukturen nit "Translativ" (als, wie)}

5.1. Karl (B) als Arzt (A) hätte das wissen müssen.

(Ei 86; letztendlich aber bleibt die Frage der Einordnung offen), Du 84,

(JS 82), SG 82 , He 81, Er 72 , Mo $65(=6 / 16)$

\) ATTRIBUT (Rap. 7, 7B), SATZGLIED (Kap. 8).

5.2. Eine Chefin (B) wie Pia (A) wünscht sich jeder.

Du 84, (JS 82), SG 82, He 81, Er $72(=5 / 16)$

\) ATTRIBUT (Kap. 7).

\section{Adverbialadjektive nit Subjekt-/Objektbezug}

\subsection{Prădikatives Attribut zum Subjekt}

Kuno (B) kam krank (A) nach Bause.

Er 72, Be 28, (AI 81), Ho $65(=4 / 16)$

〉) SATZGLIED (Kap. 8).

\subsection{Prădikatives Attribut zum objekt}

Pia ab die Suppe (B) heiB (A).

Mo $65(=1 / 16)$

>) SATZGLIED (Kap. 8).

\section{Berausstellungen}

Sie finden sich bereits z.T. unter den lockeren nominalen Appositionen (z.B. Rechtsversetzung), werden aber hier wegen ihrer Bedeutung für Abgrenzungsfra-

6 Die Wertung als Apposition findet sich auch bei Droop (1977: $53 \mathrm{ff.}$.).

7 Auch Droop (1977: $40 \mathrm{ff.}$ ) wertet dieses Muster als Apposition. 
gen unter einem eigenen Punkt versammelt. Die Begrifflichkeit hat Altmann (1981) entwickelt.

\subsection{Linksversetzung}

DaB er sich getäuscht hat (A), das (B) glaube ich.

(Beispiel: Raabe 1979: 291; ins Deutsche ubertragen; $=1 / 16$ )

\) HERAUSSTELLUNG (Rap. 10).

\subsection{Rechtsversetzung}

(...) aber am Sonntag sei er eben nicht mit ihr (B), der Sekretärin (A), unterwegs (...)

(Beispiel Molitor 1979: 118); eine statistische Angabe wird nicht ausgewiesen, da die Problematik, Appositionen gegen Rechtsversetzung abzugrenzen, erst durch Altmann (1981) umfassend beschrieben vorden ist; davor wurden Rechtsversetzungen oft als Appositionen behandelt.

\) ZUSATZ (Kap. 12, 15), HERAUSSTELLUNG (Kap. 10).

\subsection{Nachtrag}

Ein Auto (B), und zwar ein großes (A), stand vor der Baustür.

(Beispiel Molitor 1979: 178); Ku 60 (S. 109), Ra $79(=3 / 16)$

) ZUSATZ (Kap. 12, 14, 15), HERAUSSTELLUNG (Kap. 10).

Somit ist die Menge der zu untersuchenden Konstruktionen vorgegeben. Bemerkenswert ist die Vielfalt der Ronstruktionen und die "statistische" Hăufigkeit. Die 10 an häufigsten bearbeiteten Typen sind:

1. Kuno, mein bester Freund,

(APPOSITIONSPROTOTYP; $16 / 16$ ).

2. Unser Onkel otto

3. Onkel otto

(ENGE NOMINALAPPOSITION mit Det; $12 / 16$ ).

4. Otto Huber (ENGE NOMINALAPPOSITION ohne Det; $12 / 16$ ).

5. Ratharina die Große (EIGENNAYENSEQUENZ; 8/16).

6. Pia, lustig und fidel,

7. Pia als Arztin

8. Ein Liter (herber) Wein

9. Ihr (ewigen) Traumtanzer

10. Eine Chefin wie Pia

(EIGENNAME + BEINAME histor. Personen; 8/16). (LOCKERES ADJP-APPOSITIV; 7/16). (NP + TRANSLATIVPHRASE; 6/16). (NP-QUANTIFIZIERTE NP; 6/16). (PRON + N/NP; 6/16). (NP + TRANSLATIVPHRASE; 5/16).

Hier făllt Folgendes auf: Nur bei 5 Konstruktionstypen (von 34 hier grob unterschiedenen) ist sich mindestens die Balfte der ausgewerteten Arbeiten einig, daß "Apposition" vorliege - eine nicht gerade große Einigkeit. Unter den 10 meistgenannten Konstruktionstypen gehört allein die Hălfte dem engen Appositionstyp an $(2-5,8)$, lediglich zwei Typen $(1,6)$ dem als "Prototyp" geltenden lockeren Appositionstyp. Viele yuster unter den 34 differenzierten (im 
Fortgang der Arbeit kommen noch einige Subklassen hinzu, z.B. Barry Birsch (SPD)) tauchen nur ein- oder zweimal auf.

Das Fazit kann folglich nur sein, das die obereinstimmung bzgl. der zum Phănomen Apposition gehörigen Muster eher uneinheitlich und gering ist. Es scheint zwischen allen aufgelisteten Typen eigentlich nur ein Zusammenhang durchgăngig $z$ bestehen: die angenommene strukturierbarkeit in Basis und Appositiv. Alle übrigen Merkmale (z.B. intonatorische/graphematische Abtrennung, Kasuskongruenz, Referenzidentităt etc.) treffen nur auf mehr oder weniger groBe Teilbereiche zu. Wenn man dies beispielsweise mit der Behandlung des Phănomens "Attribut" vergleicht, bei der die Yuster doch mit großer Obereinstimmung (z.B. Adjektiv-, Genitiv- und Prăpositionalattribut) aufgeführt werden, dann kann von einem relativ einheitlichen, überschaubaren (oder wenigstens im Kern einigermaßen geklărten) Phănomenbereich nicht die Rede sein. 


\section{APPOSITIONSRRITERIEN}

In diesem Absatz sollen die Rriterien beschrieben verden, die bislang zur Identifikation von Appositionskonstruktionen herangezogen worden sind. Wenn man die Apposition als grammatische Kategorie oder Funktion erfassen will, dann günstigerweise mithilfe eines reichhaltigen Bündels grammatischer Eigenschaften. Legt man ein relativ generelles Kriterium oder nur einige wenige Eigenschaften wie z.B. die "Referenzidentitat" oder die Definition "nominale Beifügung im gleichen Kasus" zugrunde, so erhăt man in der Folge sehr groBe Objektbereiche; ${ }^{1}$ man vereinigt dadurch eine vielfalt intern offensichtlich weiter aufgliederbarer Erscheinungen, die allesant "Apposition" geheißen werden. Doch welche Kriterien unter den vielen möglichen soll man zusammenfassen? Zunăchst einmal gar keine! Alle Kriterien, die sich nicht beim ersten Ansehen als unzweckmåßig erweisen, sollen zur Klassifikation herangezogen werden: Kongruenz, "Appositionslexeme", Stellungsverhalten, semantische/pragmatische Gesichtspunkte etc.

In diesem Rapitel werden die bislang verwendeten Rriterien gesammelt (nach der Hăufigkeit ihrer Anwendung), kurz beschrieben und einer ersten Prũfung ihrer Zweckmäbigkeit unterzogen. Eine oberprüfung oft verwendeter (und vielleicht zu selbstverstăndlich gewordener) Rriterien scheint angebracht, denn es wird sich herausstellen, das einige von ihnen in ihrem heuristischen bzw. definitorischen Wert wahrscheinlich uberschătzt worden sind; andere werden sich als entbehrlich erweisen; wiederum andere bislang weniger zentrale Merkmale werden ein größeres Gewicht erhalten.

Die vorzustellenden Kriterien stammen aus den in Kap. 2 ausgewerteten Arbeiten; dort kann man sie und ihre Abkürzungen nachsehen. Die Arbeit von Eisenberg (1986) bleibt hier unberücksichtigt, da er gar keine Begriffsklårung von "Apposition" anstrebt (ebd. 243).

Im folgenden ist aufgeschlüsselt, welche Rriterien in welchen Arbeiten erscheinen. Die Anordnung erfolgt nach der relativen Hafigkeit. Angegeben werden die wichtigsten Seiten, auf denen das Rriterium erwăhnt wird; sie stehen in eckigen Klammern, wenn das Kriteriun keine zentrale Rolle spielt:

1. Rategoriale Fülung: Du 84: 593; Eg 82: 156f.; JS 82: 110; SG 82: 362; He 81: 290; HB 80: 537; Er 72: 151; Be 28: 412; En 86: 192f.; Al 81: 57ff.; Mo 79: 20ff., 222f.; Ra 79: 285ff.; Ku 60: 123 .

2. Abtrennung: Du 84: 593; Eg 82: 158; JS 82: 110f.; SG 82: 362; He 81: 293; HB 80: 538; Be 28: 412ff.; En 86: 191; Al 81: 62; Mo 79: 21f.; Ra 79: 278ff.; Ru 60: 76 .

1 Die Referenzidentităt ist ganz (m.E. viel zu) zentral z.B. bei kolitor (1979: 2.B. 223) oder Jumg/Starke (1982: 110). Die Rasuskonoruenz ist bestimmend z.B. in DuDPN (1984: 593). 
3. Stellung: Du 84: 593; Eg 82: 157; JS 82: 110f.; SG 82: 362f .; HB 80: 538; Be 28: 412ff.; En 86: 189; Al 81: 62; Mo 79: 222; Ru 60: 77 .

4. Funktion A bzgl. B: Eg 82: 158f.; JS 82: 110; He 81: 292; Er 72: 151; En 86: 192; Al 81: 60; Mo 79: 222f.; Ra 79: 237ff.; Ku 60: 122f.; Se 60: 35ff. 5. Paraphrase: Eg 82: 158; JS 82: 110; He 81: 290ff.; Er 72: 151; En 86: 189; A1 81: 62; Mo 79: 222f.; Ra 79: 154ff.; Mo 65: 91ff.; Se 60: [37].

6. Kongruenz: Du 84: 593; Eg: 82 156f.; JS 82: 110; SG 82: 362; He 81: 290; HB 80: 537; Be 28: 412; Mo 79: 29f.; Ku 60: 123.

7. Referenzidentităt: JS 82: 110; HB 80: 537; En 86: [190f.]; Mo 79: 223; Ra 79: 255ff.; Ku 60: 123 .

8. A weglaßbar: JS 82: 110; En 86: 191; Mo 79: 181; Se 60: 11 .

9. Appositionslexeme: En 86: 191; Al 81: 62; Mo 79: 169ff.; Ra 79: $268 f f$.

10. Vertretung B durch A: JS 82: 110; HB 80: 537.

11. Satzgrenzenbestimmtheit: En 86: 189; Ra 79: $274 f$.

12. Attribution: Mo 79: 22ff., 222; Ku 60: [73].

13. Textnotwendigkeit: En 86: [187]; Ra 79: $252 \mathrm{ff}$.

14. Sprecherpräsenz: En 86: [188]; Ra 79: $264 f f$.

15. Adordinativităt: Ra 79: $226 \mathrm{ff}$.

16. Negationsskopus: Ra 79: 259ff.

17. Wahrheitswertkonstanz: Ra 79: $248 \mathrm{ff}$.

Man erhält aus den 15 ausgewerteten Arbeiten insgesamt $17 \mathrm{kriterien,} \mathrm{wobei} \mathrm{nur}$ die ersten 6 von mehr als der Halfte der Arbeiten verwendet werden. Die Kriterien 10 mit 17 werden nurmehr von maximal zwei Arbeiten herangezogen.

\subsection{Kategoriale Füllung}

Welche Elemente welcher Kategorie kŏnnen die Basis bzw. das Appositiv in einer Appositionskonstruktion reprăsentieren? Nicht selten wird davon ausgegangen, daß die Basis durch ein Nomen oder ein Pronomen (durch NPs) und das Appositiv durch ein Nomen (resp. durch eine NP) repräsentiert werden (z.B. Erben 1972: 151; Rusmin 1960: 123). Dies ist nicht verwunderlich, denn lange Zeit wurde der Ansicht gefolgt, eine Apposition sei "eine substantivische Beifügung im gleichen Rasus".

Manche Arbeiten lassen mehr Möglichkeiten der kategorialen Füllung der Appositive $z i_{i}{ }^{2}$ z.T. sind sie auch bereit, mehrere Rategorien beim Bezugselement zuzulassen, also z.B. PP, AdjP etc. ${ }^{3}$ Molitor (1979) last relativ viele Kategorien (beispielsweise auch Verben und VPn) zu. Bei Raabe (1979: 285 ff.) finden sich vielfăltigste Füllungsmöglichkeiten: Als Basen und als Appositive kónnen

2 Engel (1982: 158) läßt 2.B. NP, AdjP und PP als Appositive zu.

3 zu diesen Arbeiten gehoren u.a. Molitor (1979) und Bngel (1986). 
u.a. S, NS, N, Pron, Vb, Adj und Adv auftreten, wobei nicht alle Kombinationen untereinander als "Appositionskonstruktion" möglich sind, so z.B. nicht Nomina und Verben.

Die Möglichkeiten kategorialer Füllung von Basis und Appositiv müssen erfaßt werden. Sie stellen jedoch für sich genommen noch kein aussagekräftiges Kriterium dar; erst die Berücksichtigung der grammatischen Beziehungen zwischen zwei Rategorien ist heuristisch bzw. analytisch fruchtbar. Es gibt eine ganze Reihe möglicher Beziehungen zwischen Kategorien, z.B. subordinative (3.1a), koordinative (3.1b) oder auch "appositionsverdăchtige" (3.1c):

(3.1a) Die Mutter des Finsterlings kam herein.

(3.1b) Die Mutter und der Finsterling kamen berein.

(3.1c) Ihre Tante Eulalia Buber kam herein.

Ihre Tante, Eulalia Buber, kam herein.

Am Rande sei bemerkt, daß normalerweise kein appositives Verhăltnis zwischen Synsemantika (z.B. Präp, Abtönungspartikeln, Interjektionen etc.) angenommen wird. Somit lăgen im folgenden keine Appositionen vor:

(3.2a) Sie bat doch wohl nicht etwa Hermann Munster geehelicht?

(3.2b) Der Wagen rumpelte - rumps schepper ratatazong - in die Garage.

\subsection{Intonatorische/Graphenatische Abtrennung des Appositivs}

Diesem Kriterium kommt eine nicht unwesentliche Rolle in der Bestimmung von "Apposition" zu. Es handelt sich um die Abtrennung des Appositivs von der Basis, die entweder graphematisch durch Satzzeichen (Komma, Gedankenstrich, Rundklammer) erfolgt oder intonatorisch durch Sprechpausen, die sich links und rechts des "Appositivs" befinden (zudem erfolgt eine "zurückgenommene" Artikulation des Appositivs, abgestufte Akzentsetzung usw.). Es ist bemerkenswert, daß bei allen vom Verf. ausgewerteten Arbeiten uber Apposition die "lockere" (abgetrennte) Apposition als zentral angesehen wird:

(3.3) James Bond, ein moderner Mythos, schlagt wieder zu. Raabe (1979: 278) sieht dieses Kriterium als Merkmal mit hohem Wert an. Engel (1986: 191) fuhrt aus, "(...) das die Apposition immer formal gekennzeichnet ist, und zwar graphematisch oder prosodemisch (...)".

Dieses Merkmal hat die Eliminierung sămtlicher sog. "enger Appositionen" (3.4) sowie einiger weiterer, nicht selten zum Appositionsbereich gerechneter Ronstruktionen (3.5) zur Folge:

(3.4) Der Schauspieler Max Schreck spielte den Nosferatu. Bei einem Glas kühlem Wein kann man gut plaudern.

(3.5) Karl als Arzt bàtte nicht operieren dưrfen. Graf Drakula kam blutdürstig in die Blutbank.

4 Bierzu vor allem Altmann (1981: 62 f. u. 202 f.), auch Raabe (1979: 278 ff.). 
Der Appositionsbereich wird durch das Abtrennungskriterium ziemlich konsistent. Es erhebt sich allerdings die Frage, ob alle derartig "abgetrennten" Elemente als Appositionen anzusehen seien. Demzufolge müBten z.B. auch folgende unterstrichene Elemente Appositionen darstellen:

(3.6a) Sie haben dem Grafen (der bekanntlich Knoblauch verabscheut) Zaziki serviert.

(3.6b) Sie haben Graf Lambsdorf (FDP) nicht zum Fest eingeladen.

(3.6c) Sie haben den Grafen - das ist ja die Höhe - zum Fest eingeladen.

(3.6d) Sie haben den Grafen, liebe Freunde, zum Fest eingeladen.

Das Abtrennungskriterium wird sich als wesentliche Eigenschaft von "Gästen" (HOSPITANTEN) eines Satzes herausstellen, die man grob in EINSCHOBE (kein Satzglied als Bezugselement; $3.6 \mathrm{c}$, d) und ZUSATZE (mit Satzglied als Basis; 3.6a, b) einteilen kann. Alle "appositionsverdăchtigen Ronstruktionen" ohne Einschaltungsmuster scheiden in der Abgrenzungsdiskussion (Rap. 7-11) aus dem Appositionsbereich aus. Nicht alle Muster mit abtrennung sind jedoch i.e.s. appositionsverdăchtig (EINSCHOBE!).

\subsection{Stellungsverhalten von Basis und Appositiv}

Hierunter ist sowohl die relative topologische Beziehung von Basis und Appositiv zueinander $z u$ verstehen als auch das Verhalten von Appositionskonstruktionen in sog. "Herausstellungsstrukturen", wie es von Altmann (1981: 289-311) untersucht worden ist. Zudem kann man untersuchen, inwieweit Appositionskonstruktionen von stellungsverāndernden satzsyntaktischen Operationen wie SPALTSATZBILDUNG oder LINRSVERSCHACHTELUNG betroffen sind.

Bei der relativen stellungsbeziehung geht es vor allem darum, ob ein Appositiv auf den unmittelbaren Rontakt mit der Basis beschrankt oder inwieveit Distanzstellung möglich ist. Bei sog. "engen Appositionen" ist die Rontaktstellung (unmittelbare Adjazenz) obligatorisch:

(3.7) Der Schauspieler Max Schreck ist heute nahezu vergessen.

* Der Schauspieler ist Max Schreck heute nahezu vergessen.

Bei den "lockeren Appositionen" lassen sich z.T. auch relative Beziehungen zwischen Basis und Appositiv untersuchen; das Gleiche gilt bei einigen "weniger prototypischen" Syntagmen (3.8), wobei stets auf mögliche verănderungen der Ronstruktionsbedeutung $z u$ achten ist:

(3.8a) Pia als Zabnärztin arbeitet jetzt in Büro.

'Pia, die ja eigentlich Zahnărztin ist, arbeitet jetzt in Büro'.

(3.8b) Pia arbeitet jetzt als Zahnarztin in Buro.

'Pia arbeitet jetzt in der Funktion einer Zahnårztin in Büro'.

*'Pia, die ja eigentlich Zahnărztin ist, arbeitet jetzt in Büro'. 
Die meisten Darstellungen führen als Stellungseigenschaften entweder die strikte Nachstellung des Appositivs oder die Möglichkeit sowohl von Voran- wie auch Nachstellung an. 5

Das Stellungsverhalten ist wichtig und wird im einzelnen genauer zu untersuchen sein. Es wird sich herausstellen, daB der Unterschied zwischen EINSCHOBEN und ZUSATZEN grammatisch vor allem am stellungsverhalten festgemacht werden kann. Auch die am Ende der Arbeit ermittelten Zusatz-Klassen (die Muster, in denen die i.e.S. "appositionsverdächtigen" Konstruktionen aufgelöst werden) veisen ein unterschiedliches stellungsverhalten auf, das mit weiteren grammatischen Eigenschaften (z.B. Umformungsbeziehungen) korreliert.

\subsection{Funktion des Appositivs bzgl. der Basis}

Bei diesem Kriterium handelt es sich um die Feststellung der semantischen resp. der pragmatischen Konstruktionsbedeutung der Appositionskonstruktion. 6 Die inhaltlichen Beziehungen zwischen Basis und Appositiv stellen ein wichtiges Klassifikationskriterium dar, vor allem dann, wenn sie mit ausdrucksseitigen - d.h. syntaktischen - Unterschieden korrelieren; sie werden in den Kap. $6,12,13$ und 15 beschrieben. Das ofters verwendete semantische kriterium der "Referenzidentität von Basis und Appositiv" wird in Absatz 7 dieses Kapitels besprochen.

Zumeist finden sich Urteile der Art, daB Appositive "zusätzliche Erlăuterungen" zu einer Basiseinheit ${ }^{7}$ bzw. "zusätzliche Außerungen" (des Sprechers) ${ }^{8}$ darstellten. Eine sehr ausführliche Taxonomie möglicher pragmatischer Funktionen entfaltet Raabe (1979: $241 \mathrm{ff.}$ ). Dort werden zwei Basisfunktionen (ebd.

242) angesetzt:

- A. "Intentional objektive Information zur situierung und/oder Verbesserung der Primărtextrezeption des Hörers/Lesers";

- B. "Intentional subjektive Information zur zielgerichteten Beeinflussung des Hörers/Lesers".

Diese werden dann subkategorisiert. So gibt er u.a. für A. an: ${ }^{9}$

5 Mur Nachstellung z.B. in Duden (1984: 593), Engel (1986: 189); beides 2.B. in Engel (1982: 157), Helbig/Buscha (1980: 538).

6 Bine Diskussion, was genau semantische und pragmatische Funktionen unterscheide, muß unterbleiben. Generell zum Abgrenzungsproblem Semantik/Pragmatik: Vennemann/Jacobs (1982: 103ff., $139 \mathrm{ff}$. ), Grevendorf u.a. (1987: 298 ff., 374 ff.).

7 So z.B. Brben (1972: 151), Engel (1982: 158).

8 Hierzu Raabe (1979: 268 ff.), Engel (1986: 191 f.).

9 Ich übersetze Raabes Beispiele (frz.) ins Deutsche. 
- Retrospektive (Erinnerung) (ebd. 243): Die Schlacht von Hastings, in Jahre 1066, war ein großes Datum der Geschichte.

- Präzisierung (ebd. 244): Seine Frau, krank, kam uns eines Abends besuchen.

Zwei Beispiele gebe ich auch für B. wieder:

- Insinuation (ebd. $245 \mathrm{f}$. ): Es ist zu leicht, für unseren Dieb, diese Tür zu überklettern.

- Warnung (ebd. 246): Der R5, mit ziemlich zerbrechlichen Kardanwellen, findet sich auf Seite 5 unseres Kataloges.

Semantisch orientiert sind die Analysen von Seiler (1960: $19 \mathrm{ff.,} 35 \mathrm{ff.}$, nach denen die Apposition durch Charakterisierung und die Attribution durch Spezifikation gekennzeichnet seien: 10

(3.9a) b öse Bunde
Satellit Nukleus
Selektor Klasse
Spezifikation
ATTRIBUTION

$\begin{array}{ll}\text { (3.9b) böse } & \text { B u nde } \\ \text { Satellit } & \text { Nukleus } \\ \text { Rlasse } & \text { Selektor. } \\ \text { Charakterisierung }\end{array}$

Zu der Begrifflichkeit Seilers: Nukleus ist das Element eines Syntagnas, das zur gleichen Formklasse gehört wie das Gesamtsyntagma (also der Rern eines endozentrischen Syntagmas), der Rest ist Satellit (ebd. 9). Rlasse kann als (Individuen)Menge, Selektor als Untermengenbilder verstanden werden (ebd. 19 f.). Entscheidend ist, ob Nukleus oder Satellit selegieren, wobei in Seilers Beispiel der Intonation, insonderheit der Akzentsetzung, eine wesentliche Rolle zukommt: Die akzentuierte Konstituente des Syntagmas ist Selektor.

Die Rriterien Seilers dehnen den Appositionsbegriff auf Ronstruktionen aus, die traditionell den Attributen zugerechnet wurden, z.B.: Adjektivattribute (3.9b) und Relativsātze mit entsprechenden intonatorischen Markierungen (3.10a ist Charakterisierung/Apposition, 3.10b Spezifikation/Attribut; Seiler 1960:

$25 \mathrm{f} ., 35$ f.) :

(3.10a) $B$ u nde, die $b$ ö se sind, gehören in den Zwinger.

(3.10b) Hunde, die $b$ ö se sind, gehören in den Zwinger.

Seilers Verdienst besteht vor allem in der Einbeziehung intonatorischer Merkmale; doch sein Appositionsbegriff kann mit Raabe (1979: 127) kritisiert verden:

"Andererseits muß gesehen werden, daß Seilers Appositionsbegriff auf der Klasse-Selektor-Semantik gegründet und auf Nukleus-Satellit-Ronstruktionen begrenzt ist. Die Parenthesebetonung (...) wird (...) nicht berücksichtigt. Unter diesen Aspekten ist der Seilersche Appositionsbegriff enger als der traditionelle Appositionsbegriff. Seiler versteht unter Apposition weniger

10 Die Sperrung gibt die Lage des (Haupt) Akzents an. 
eine bestimmte Formklasse mit mehreren Eigenschaften als vielmehr eine Eigenschaft, nämlich das semantische Merkmal [t charakterisierend]". Dennoch, auch dies merkt Raabe (1979: 128) an, ist das Merkmal [+ charakterisierend] nicht unwichtig. Wenn man die "engen" Muster beiseite läßt ${ }^{11}$ und z.B. den lockeren Prototypen betrachtet, dann stieße Seilers Akzenttest zwar auf Probleme, weil die alleinige Akzentuierung des "Satelliten" (3.11b, vgl. 3.9a) inakzeptabel scheint; ${ }^{12}$ das Appositiv müste jedoch in jedem Fall semantisch als [+ charakterisierend] angesehen werden:

(3.11a) (?) Doktor Mab u se, ein Sp i eler, war ihm unheimlich.

(3.11b) ??Doktor Mabuse, ein Sp $i$ eler, war ihm unheimlich.

Anders ausgedrückt: Im engen Sinne appositionsverdächtige Muster (mit Abtrennung!) sind semantisch [- spezifizierend]. Nur in Ausnahmefallen kann eine nicht-charakterisierende Semantik zum Ausdruck kommen (Krifka 1983: 72, Fn. 2; in meinem Idiolekt sind Muster wie (3.12) zweifelhaft):

(3.12) ? Ich suche Emil Schmidt, den Pr e isträger (nicht den Juror). Andererseits sind beileibe nicht alle [+ charakterisierenden] Muster i.e.s. appositionsverdächtig:

(3.13) Doktor Mabuse, so vermuten wir, ist ein Spieler.

Doktor Mabuse, möglicherweise, ist ein Spieler.

Doktor Mabuse, lieber Inspektor, ist ein Spieler.

\subsection{Paraphrasen (Unfornungsbeziehungen)}

Der Begriff Paraphrase - eigentlich definiert als Synonymie-Relation zwischen Sätzen - sei hier weniger streng verstanden als Möglichkeit der Umschreibung bzw. alternativen Formulierung einer Sprachkonstruktion $X$ durch eine Sprachkonstruktion Y:

(3.14) Dr. Mabuse, ein Spieler, war ihm unheimlich $(X)$.

Dr. Mabuse, er war ein Spieler, war ihm unheimlich $(Y)$.

Ich spreche manchmal auch davon, daB ein Sprachmuster $X$ zu einem Sprachmuster $Y$ "in systematischen Beziehungen" stehe, was besagt, das zu allen (zumindest $z u$ vielen) Beispielen für $X$ ein analoges Beispiel in $Y$ gebildet werden kann.

Bereits bei Blümel (1910: 7) findet sich ein Hinweis auf die Ersetzbarkeit von ("lockeren") Appositionen durch Sătze (Relativsätze). Schwyzer (1947:9) verweist darauf, daß der Gedanke, "daß die Apposition auf einem Satz beruhe", bereits im 19. Jahrhundert geäuBert worden sei und hãlt "diese Auffassung für grundsătzlich einleuchtend". Und immer wieder findet sich in den oben aufgelisteten Arbeiten eine Bemerkung der Art, daB das Verhältnis zwischen Basis

11 Das charakterisierende Adjektiv wird in Kap. 7 der attributiven Relation zugeordnet.

12 Auch (3.11a) wirkt nicht völlig ummarkiert, weil der Akzent auf dem Appositiv i.d.R. etwas "zurückgenowmen" artikuliert wird (vgl. Altmann 1981: 63). 
und Appositiv innerhalb einer Apposition dem Verhälnis von Subjekt und Pradikativ in einem Kopulasatz entspreche. ${ }^{13}$ Vorgeschlagen wurden Beziehungen zwischen Appositionen und nicht-restriktiven Relativsätzen mit Kopulaverb (KV), Parenthesen mit KV oder einfach Vollsätzen mit $\mathrm{KV}$.

Die Blütezeit dieser Umformungsbeziehung wurde durch die Entwicklung der generativen Transformationsgrammatik (v.a. Chomsky 1957, 1965; einführend Bartsch/Lenerz/Ullmer-Ehrich 1977) eingeleitet. Einen guten Oberblick über die transformationelle Herleitung von Appositionskonstruktionen kann man sich bei Raabe (1979: $154 \mathrm{ff.}$ ) verschaffen, so daß eine ausführliche Diskussion hier unterbleiben kann. Beachtenswerte Arbeiten aus dem generativen Paradigma zur Ableitung von Appositionen sind $(a-f)$ :

(a) Motsch (1965), wo angesetzt wird, daB Appositionen als reduzierte Kopulasătze aufzufassen seien, wobei das Appositiv Prädikativ (3.15a) oder Subjekt (3.15b) sein kann:

(3.15a) Der Schwager, (der Schwager war) übrigens ein ruhiger, ordentlicher Mann, hörte sie erschrocken an.

(3.15b) Die Gefahrenstelle, ein entwurzelter Baum (ist die Gefahrenstelle), muB beseitigt werden.

(b) Rohrer (1968), worin angenommen wird, daB alle appositionellen Konstruktionen auf reduzierte Kopulasätze zurückgehen (ebd. 393). Methodisch wird so vorgegangen: In der Tiefenstruktur finden sich zunächst zwei koordinierte sätze (3.16a), von denen der zweite als "rechte Schwester" der NP Kuno per Einbettungstransformation angefügt wird (3.16b). Durch eine Tilgungstransformation entsteht dann die Apposition (3.16c):

(3.16a) Kuno spinnt und Kuno ist mein bester Freund.

(3.16b) Kuno, der mein bester Freund ist, spinnt.

(3.16c) Kuno, mein bester Freund, spinnt.

Uns interessieren allerdings weder die technischen Details der Umformungen noch die möglichen Schwierigkeiten, die man sich mit diesem Ansatz einfängt (hierzu Raabe 1979: $165 \mathrm{ff.}$ ). Von Interesse ist zunächst nur, daß eine Umformungs- bzw. Ableitungsbeziehung zwischen Kopulasatz und Apposition angenommen wird!

(c) Delorme/Dougherty (1972), die insofern interessant sind, als eine Hiderlegung der generellen Herleitung von Appositionen aus (Relativ)Satztiefenstrukturen versucht wird. Anhand des Typus Wir, die Polizisten, und Wir Polizisten wird zu zeigen versucht, dab eine Erzeugung durch eine der beiden folgenden Phrasenstrukturregeln weniger Probleme bereiten soll:

(3.17) NP > NP (NP)

$N P,($ Art $) N(S)$

13 Z.B. Erben (1972: 151), Altmann (1981: 62), Engel (1982: 158) und ein Teil der Arbeiten in Paradigma der generativen Transformationsgrammatik, z.B. Kotsch (1965) U.a.m. 
(d) Lange (1974) unternimmt den Versuch, zwei Quellen von Apposition nachzuweisen: nicht-restriktive Relativsätze und performative Sätze. Da das erste Verfahren bereits bekannt ist, sei nur das zweite vorgeführt. Appositionen wie (ebd. 132) :

(3.18) Icb werde dir Idiot docb nichts sagen! sind demnach herzuleiten aus zugrundeliegenden strukturen wie (ebd. 130; "PRAD" = "Prădikat", "ARG" = "Argument"):

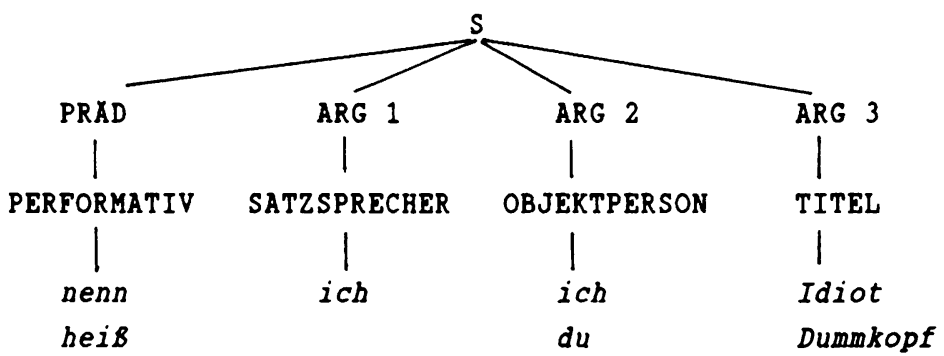

Hier erfolgt eine Ableitung aus einer Tiefenstruktur ohne KV unter Beteiligung sog. "Titulierungsverben". Ober eine parenthetische AnschlieBung an die Objektperson (3.20a) und Tilgungen entsteht die Appositionskonstruktion (3.20b):

(3.20a) Was hast du - ich nenne dich Dummkopf - angeste11t?

(3.20b) Was hast du Dummkopf angeste11t?

(e) Burton-Roberts (1975: $406 \mathrm{ff.}$ ), der eine Ableitung der Apposition (3.21a) aus (3.21b) vornimmt und nicht etwa aus (3.21c):

(3.21a) Manchester United, the champions, play today.

(3.21b) Manchester United play today. The champions play today.

(3.21c) Manchester United play today. Manchester United are the champions. Es seien kurz die Argumente vorgestellt: Wichtig ist die sog. Koreferenzbeziehung zwischen Basis und Appositiv, die impliziert, daB sowohl Basis als auch Appositiv allein auf die selbe Entităt referieren können. Gerade dies ist jedoch bei Prädikativen in Kopulasătzen nicht gegeben! In diesen kann lediglich dem Subjekt (also Manchester United) Referenzfähigkeit zugemessen werden. ${ }^{14}$ Prădikative NPs sind askriptiv, d.h. mit ihnen werden dem Subjekt Eigenschaften zugesprochen. ${ }^{15}$ Daher widersprechen sich Koreferenz und Ableitung der Appositive aus Prădikativen zugrundeliegender Sätze mit KV.

Die "Referenzidentităt" wird sich allerdings im weiteren (Punkt 7. dieses Kapitels, Rap. 6 Punkt 7) als renig zweckmäBiges Appositionskriterium erweisen. Somit liegt u.E. noch keine entscheidende Evidenz gegen eine Ropulasatzparaphrase vor.

14 Vgl. hierzu auch Strauson (1952: $145 \mathrm{ff}$.$) .$

15 Dies gilt allerdings nicht für alle Ropulasătze, nämlich mindestens nicht für die Identitätssătze vie der Kultusminister van Baden-Wurttemberg ist der Präsident des VFB Stuttgart. 
Ebenfalls verletzt wäre die von Burton-Roberts angenommene Bedingung, daB Basis und Appositiv "the same function with respect to the same constituent" (Burton-Roberts 1975: 407) aufweisen, da in (3.21c) zwar Manchester United Subjekt bzgl. play (today) ist, nicht jedoch the champions, die die Funktion Prädikativ haben. Nur durch eine Ableitung wie in (3.21b) ist gewährleistet, daB beide Appositionsteile hinsichtlich play (today) als subjekte fungieren.

Die Bedingung, Basis und Appositiv müBten beide in identischer funktionaler Beziehung zur gleichen Konstituente stehen, erscheint unnötig. Warum sollen in einer ungleichstufigen Konstruktion ( $Y$ ist Apposition $z U X$ !) beide Teile funktional identisch sein?

Burton-Roberts argumentiert mithin gegen eine Beziehung von Apposition und Kopulasatz, zumindest für den oben angeführten Fall. In einem anderen Fall, nämlich

(3.22) Mr Pontefract, an upholsterer, has big feet.

Mr Pontrefact/ *An upholsterer has big feet.

kann das Appositiv nicht die Subjektsfunktion übernehmen. Zu diesem Fall sagt Burton-Roberts (1975: 411):

"We conclude, then, that an upholsterer has its function in respect of $M r$

Pontrefact. This function must be attributive. [(3.22); W.S.] would thus be derived from a non-restrictive, copulative relativ clause and is not apposition".

Dann wird die überraschende Konsequenz gezogen (ebd. 412), daß "(..) an indefinite NP following a definite NP in subject position is clearly not apposition, (...)".

Hier sollen weder die technischen Implikationen des Vorgehens noch das kontraintuitive Fazit von Burton-Roberts näher diskutiert werden. Das Ansetzen der Appositionskriterien "Referenzidentität" und "gleiche Funktion bzgl. des Restsatzes" führt zu derartigen Ergebnissen. Auch hier kann ich keine ausreichende Evidenz gegen Kopulasatzparaphrasen wahrnehmen, sondern lediglich die Oberbetonung zweier Kriterien.

(f) Schapiro (1977) argumentiert wieder zugunsten einer Ableitung aus Relativsatztiefenstrukturen (z.B. ebd. $68 \mathrm{ff.}$ ). Dabei weist er auch auf sog. "Satzappositionen" hin:

(3.23) He smokes two packs a day, (which is) a desaster. Die Beziehungen zwischen Appositionen und satzfömigen Einheiten erstrecken sich allerdings nicht nur auf zugrundeliegende nicht-restriktive Relativsătze (als Kopulasätze) und Sätzen mit performativen Verben (cf. Lange 1974); auch Kopulasătze als Parenthesen (z.B. Hackel 1968: 110) sind als Möglichkeit genannt worden: 16

(3.24) Uns Pechvögeln ist der letzte Bus weggefahren. Uns - wir sind Pechvögel - ist der letzte Bus weggefahren.

16 Beispiel nach Heidolph u.a. (1981: 292 f.). 
Bei Engel (1986: 189; 202) findet sich der Vorschlag, bei bestimmten Appositionstypen eine Paraphrase durch einen Satz mit dem Verb meinen anzunehmen (Beispiel ebd. 189), womit er die Schwierigkeit in (3.26) umgeht:

(3.25) (..) drüben, in der Pfalz, (...).

Mit "drüben" meine ich die Pfalz.

(3.26) ?? (")drüben(") ist in der Pfalz.

In Molitor (1979: 90) und Heidolph u.a. (1981: 290 f.) wird eine Ableitung "enger" Appositionen von Sătzen mit heißen vorgeschlagen:

(3.27) Der Politiker Schmidt hat uns viel versprochen.

Der Politiker, der Schmidt heiBt, hat uns viel versprochen.

Um die sog. "engen appositionellen syntagmen" zu subklassifizieren, werden von Hackel (1968: v. a. $166 \mathrm{ff.;} 245 \mathrm{ff.;} 319 \mathrm{ff.;} 348 \mathrm{ff}$. et passim) diverse Umformungsmöglichkeiten verwendet. ${ }^{17}$ Im AnschluB ist nur ein kleiner Ausschnitt aus den angebotenen Möglichkeiten aufgeführt:

(3.28) die Arbeitsgruppe Müller : Müller führt die Arbeitsgruppe. das Pioniertheater Erfurt : Das Pioniertheater besteht in Erfurt. die Arbeitsgruppe Sport : Die Arbeitsgruppe befabt sich mit Sport. das Duo Köste-Dölling : Das Duo besteht aus Köste und Dölling. der Raum Halle-Leipzig : Der Raum liegt zwischen Halle und Leipzig. Hackel entwickelt aber auch Beziehungen zuischen Appositionen und nichtsatzformatigen Konstrukten, also z.B. die Beziehung zwischen "enger Apposition" und (Beispiele (Ausschnitt) aus Hackel 1968: 166, 245 f.))

- "lockerer Apposition": der Zahnarzt Schmidt/ Der Zahnarzt, Schmidt,/ Schmidt, der Zahnarzt.

- "als" + NP: Schmidt als Zahnarzt.

- "wie" + NP: Ein Zahnarzt wie Schmidt.

- "namens" + NP: Ein Zahnarzt namens Schmidt.

- Genitivattribut: Der Begriff Entfernung/ Der Begriff der Entfernung.

- Kompositum: Der Entfernungsbegriff.

Die Umformungsbeziehungen werden als Kriterium für das Vorliegen von Apposition für wichtig erachtet. Es ist zu erwarten, daB sich gleiche syntaktische Muster bzgl. Umformungsbeziehungen gleich oder zumindest sehr ähnlich verhalten. Oberdies werde ich (u.a. in Kap. 7) feststellen, daB gewisse "enge Appositionen" via "Reduktion" aus attributiven Syntagmen entstanden sind, so daB die Annahme einer Umformungsbeziehung durch die Sprachwirklichkeit Bestătigung findet. 18

$17 \mathrm{zu}$ den Unformungen Apposition/Satz vgl. auch Hackel (1972: 345 f.; 1973: $19 \mathrm{ff}$. ).

18 Vgl. Hackel (1972: 341 f.; 346 f.), Hackel (1975: 44). 


\subsection{Kasuskongruenz}

DaB das Appositiv mit dem Bezugselement in Kasus kongruiert, wird nicht selten als Merkmal einer Appositionskonstruktion angesetzt. Meist wird davon ausgegangen, daß der Normalfall die Kasuskongruenz ist, z.T. sogar davon, daß sie ausschließlich gilt. ${ }^{19}$ Teilweise wird angeführt (z.B. Jung/Starke 1982: 110; daher auch das Beispiel (3.29); Heidolph U.a. 1981: 290), daB das Appositiv unabhăngig vom Rasus der Basis im Nominativ auftreten könne:

(3.29) Am bedrücktesten fühlte ich mich des Abends, sonst meine liebste Tageszeit/ meiner liebsten Tageszeit.

Gerade wenn das Appositiv von Gradpartikeln, Satzadverbien u.a.m begleitet wird, z.T. auch bei der Anfügung von Relativsatzen, ist der Nominativ die Regel und die Kongruenz ungrammatisch (3.30a) oder zumindest weniger üblich $(3.30 b): 20$

(3.30a) Die Philosophie Kants, eines bedeutenden Denkers, ist schwierig. Die Philosophie Kants, auch ein bedeutender Denker/ *auch eines bedeutenden Denkers, ist schwierig.

(3.30b) ?Die Philosophie Kants, eines bedeutenden Denkers, der in Königsberg gelebt hat, ist schwierig.

Die Philosophie Kants, ein bedeutender Denker, der in Königsberg gelebt hat, ist schwierig.

Manchmal werden noch weitere Möglichkeiten erörtert, z.B. die sog. "Dativ-Apposition" (Gippert 1981; Engel 1982: 157). Es geht um die Frage, ob es eine Entwicklung gibt, den Dativ wie in (3.31) als "appositionsmarkierenden Kasus" zu verwenden (Beispiele z.T. nach Schönmann 1959: 159)

(3.31a) Sie waren geblendet von dem Auftreten des Anklagers, dieses Meisters des Bluffs/ diesem Meister des Bluffs.

(3.31b) Die Entscheidung liegt in den Bänden des Oberhauses als des obersten Gerichtshofes/ als dem obersten Gerichtshof.

19 So z.B. Schulz/Griesbach (1982: 362), Behaghel (1928: 412).

20 In übrigen gibt es durchaus "Warner", die das Kongruenzkriterium für "Apposition" kritisch betrachten, z.B. Zemb (1968: 277):

"Yême si le complément de dénomination direct [gemeint ist Rom in die Stadt Rom; H.S.] prenait le cas du nam qu'il complète, on ne pourrait en déduire qu'il s'agit d'une apposition, à moins de définir l'(〈apposition》) par l'accord des cas, ce qui conduirait à d'autres difficultés (épithètes et attributs du sujet et de l'objet) (...)".

Zudem liegen die Verhältnisse in anderen Sprachen durchaus so, dab das Appositiv in Nominativ erscheint, z.B. im Rumänischen; dort markiert nach Breyer/Bodmann/Bronsert (1987: 86) der Nominativ die Apposition "(...) wobei im Rumänischen in Gegensatz zim Deutschen der Noninativ auch dann die Apposition bestimnt, wenn das Bezugsnomen einen anderen Kasus aufweist (...)". Z.B.:

(F3.1) Vecinul - ni, lacatusul G.B., un spuse nimic despre accident. NACHBAR - Dat. SCHLOSSER G.B. (Nom.)

Seinem Nachbarn, (der/ dem) Schlosser G.B., sagte er nichts von dem Unfall. 
Als "falscher Dativ" wird diese Erscheinung von Schönmann (1959: 159-161) kritisiert, der "im Vermeiden des Genitivs", der "Unklarheit des Beugungsfalls des Bezugswortes" und der "Abhebung vom Restsatz" die Motive für das Abweichen von der Kongruenzregel vermutet. Hinter (1966: 25) konstatiert: "Ein Genitiv des Nomens kann in der Apposition durch einen Dativ aufgenommen werden" und kritisiert diese Erscheinung nicht.

Kolde (1971: 200) merkt bei der Behandlung von appositiven Phrasen der Art "als + NP" an, daß deren Verwendung mit Dativ "offenbar auf wenige Kontexte beschränkt" ist. Förster (1972: 81) zitiert den "Zweifelsfälle-DUDEN", 21 der anführt, daß sich bereits in der ersten Hălfte des 19. Jahrhunderts der inkongruente Dativ in reicher Zahl belegen lasse. Förster tendiert augenscheinlich (eine eindeutige stellung wird nicht bezogen) $z u$ folgender Erklărung: Die Kasuskongruenz schafft eine "fast nahtlose Verbindung zwischen Bezugswort und Apposition" (S. 82), wodurch diese "ihren Parenthesencharakter" verliert; die Markierung des Appositivs durch den Dativ "soll Emphase erzielen" (ebd.). Försters Fazit ist, daB der Dativ in dieser (appositionsmarkiernenden) Funktion "immer mehr an Bedeutung zu gewinnen scheint" (S. 83).

Leirbukt (1978) widmet sich der dativischen Apposition bei akkusativischem Bezugswort und wertet sie "als eine ernstzunehmende Regularitat und nicht als zufällige Entgleisung" (S. 5). Es wird eine "allgemeine Tendenz im heutigen Deutsch zur Verwendung des Dativs als einer Art Appositionskasus" konstatiert, wobei hinzugefügt wird, daß aufgrund des begrenzten Beispielmaterials dieses Resultat mit Vorbehalten behaftet bleibt (S. 14).

Einer der wichtigsten Aufsätze zum Thema Dativapposition (DAPP) ist Gippert (1981). Die darin diskutierten 256 Beispielsätze weisen folgende Verteilung auf (S. $33 \mathrm{ff.}$ ):

- 130 Fălle: adnominaler Genitiv (B) + DAPP

mit der Gesellschaft des Herrn Plessing (...), dem Sohne des Superintendenten

- 98 Fălle: Prăp + NP akk (B) + DAPP

Ich habe keine Entschuldigung für diese Niederlage, der höchsten, seit Mike Smith Manager ist.

- 11 Fálle: N nom (B) + DAPP

(...) fehlt die physiognomische Basis als einem Koordinatensystem, von dem aus die Laute erst ihren (...) sinn erhalten.

- 10 Fălle: Prăp + NP gen (B) + DAPP

Statt der üblichen Reigentänze, diesem Herumgehopse, (...).

- 3 Fålle: verbal regierte NP gen (B) + DAPP

(...) bediente man sich des Russischen (...) als schriftsprachlichem Medium.

21 DUDEN. 1965. Zweifelsfälle der deutschen Sprache. Mannheim u.a., Bibliograph. Institut. 
- 2 Fälle: verbregierte NP akk (B) + DAPP

(...) stattete (...) das PrivatschloB (...) als Luxushotel aus (...).

- 1 Fall: temporaler Genitiv (B) + DAPP

Freitags, als dem rubigsten Tage (...)

- 1 Fall: Zeitadverb (B) + DAPP

Heute als dem heiligen Pfingstfeste (...).

Als Resultat seiner Untersuchung urteilt er wie folgt ( $5.58 \mathrm{f}$. ):

"Die inkongruente Apposition im Dativ hat heute bereits einen weiten Anwendungsbereich: Sie tritt hăufig zu Bezugswörtern im Genitiv oder im Akkusativ nach Präpositionen, seltener auch zu solchen im Nominativ oder bloßen Akkusativ. (...) Ihre heutige Verbreitung führt zu dem Schlus, daß sie inzwischen bei einigen Sprechern zur Normalform der Apposition geworden ist". Um so überraschender wirkt nach diesen Arbeiten zur inkongruenten (Dativ)Apposition der Aufsatz von Bergenholtz (1985), in dem eine imposante Menge an Beispielmaterial zur Widerlegung der "Inkongruenzthese" aufgeboten wird mit dem Resultat (S. 33), daB von

- 4245 Appositionen mit deutlichem Kasusmorphem

- $4094(96,48)$ Kasuskongruenz und nur

- 151 (3,68) keine Kasuskongruenz

aufweisen. Hier erweist sich die Inkongruenz, ganz gleich, ob als Dativ- oder Nominativapposition realisiert, als verschwindend geringe Erscheinung. Was nun? Sowohl die empirischen als auch die theoretischen Befunde differieren bei der Bewertung der Frage "Kasuskongruenz oder -inkongruenz des Appositivs?" erheblich.

Ich werde bei der Untersuchung der kasusmorphologisch markierbaren Appositionskonstruktionen auf diese Problematik zurückkommen. Zunächst sei soviel festgehalten: Die Rasusbeziehungen zwischen Basis und Appositiv sind keineswegs unwesentlich, jedoch scheint die Formulierung einer generellen Regel wie z.B. "Bezugselement und Appositiv kongruieren in Kasus, wenn kasusmarkierbar" nicht möglich $z u$ sein. Entweder müssen zusatzregeln eingeführt verden, z.B. bei der lockeren Apposition, wenn dem Appositiv eine Gradpartikel oder ein Satzadverb vorausgeht (dann Nominativ!) oder die Regeln müssen umformuliert werden.

Obrigens tritt auch bei artikellosen nominalen Appositionen anstatt Kasuskongruenz der Nominativ auf - eine Regularităt, die auch Bergenholtz (1985: 38 f.) erwähnt (Beispiel aus DUDEN 1984: 660):

\section{(3.32) Das Virken dieses Mannes, Vorkämpfer/ *Vorkämpfers für die Rassen- gleichheit, (...).}

Die Kasuskongruenz muß als Appositionskriterium im Auge behalten werden, wenngleich sich bereits gezeigt hat, daB es etliche Ausnahmen zur Kongruenzregel (begleitende Partikeln, Satzadverbien etc.; artikellose Appositive) gibt. Zudem wăre es unbefriedigend, wenn Konstruktionen bei sonst gleichen (Umgebungs) Bedingungen und grammatisch sehr ähnlichem Verhalten (vgl. Kap. 6) nur 
wegen ihrer Kongruenzunfähigkeit nicht den Appositionen zugerechnet werden könnten:

(3.33) Pia, meine beste Freundin,/ , lustig und fidel,/, in Katmandu, erheiterte mal wieder die ganze Meute.

Im Verlauf dieser Arbeit wird deutlich, daß die Rongruenz des Appositivs im prototypischen Appositionsfall (später: prädizierender Zusatz) nur dann unbedingt durchzuführen ist, wenn sich das Appositiv auf mehr als eine Basis beziehen könnte (Desambiguierung):

(3.34) Der Bruder des Onkels, ein Pianist/ eines Pianisten, schnarcht. Sonst ist die Kongruenz entweder fakultativ oder sogar inakzeptabel. Andere appositionsverdăchtige Muster hingegen (die sich allerdings vom Prototypen unterscheiden und in Kap. 15 als referenzklärende, spezifizierende etc. Zusătze klassifiziert werden) müssen kongruieren:

(3.35) Man hat den Haien, (vor allem) den Hammerhaien/ *die Hammerhaie getrotzt.

Man nahm sich ihrer, der Singvögel/ *die singvögel, an.

\subsection{Referenzidentităt}

Dieses Kriterium spielte bei der Bestimmung von Appositionen bisher keine unerhebliche Rolle. Hier einige Fassungen dieses Merkmals: Demnach

- bezeichnet das Appositiv "die gleiche Erscheinung der objektiven Realität" wie die Basis (Jung 1982: 110);

- ist das Appositiv "referentiell mit [seinem] Bezugswort identisch" (Helbig/Buscha 1980: 537);

- verweisen "(..) die beiden NP des nebengeordneten as (..) in ein und demselben Zusammenhang auf ein und denselben Gegenstand (= Teilgedanken)" (Molitor 1979: 183).

- "Grundlegend für subordinierte ["enge"; W.S.] wie nebengeoranete ["lockere"; W.S.] as ist die Prădizierbarkeit der beiden appositiven Bestandteile über einen konstant gehaltenen Gegenstand bzw. Sachverhalt" (Molitor 1979: 213).

- "Das Appositiv oder ein Teil des Appositivs kann auch auf einen von der Basis ausgedrückten Sachverhalt (...), auf den mit der Basis verbundenen Modus des Hervorbringens $(.$.$) , auf einen von der Basis repräsentierten formalen As-$ pekt $(\ldots)$, auf einen mit der Basis verbundenen situationellen Aspekt (...) und auf eine durch die Basis ausgedrückte Bedeutung (...) zielen" (Raabe 1979: 257).

Raabes Aussagen - er hält die Referentenidentităt für ein zu enges Konzept seien durch folgende Beispiele erlăutert (Raabe 1979: 257): 
(3.36a) Il s'est tu, seul moyen de se faire pardonner.

Er hat geschwiegen, das einzige Mittel, um sich Verzeihung zu verschaffen.

(3.36b) Il est au lit, car il est malade.

Er ist im Bett - weil er krank ist.

(3.36c) Le soleil illume la mer: spectacle admirable!

Die Sonne erleuchtet das Meer: (welch ein) bewundernswertes Schauspiel!

In (3.36a) zielt das Appositiv auf den von der Basis (= S) ausgedrückten Sachverhalt; ${ }^{22}$ da man Sachverhalte gewönnlich nicht als Referenten auffast (wie Gegenstände bzw. Individuen), ist Referentenidentität hier ein unangebrachtes (zumindest ein mißverständlich formuliertes) Kriterium, wogegen Referenzidentität - je nach der Auffassung, was Referenz ist - zutreffend sein kann. Wenn die Referenz des Satzes Il s'est tu der Sachverhalt 'daß er geschwiegen hat' sein soll, dann könnte man davon sprechen, daB sich seul moyen de se faire pardonner als sprecherkommentar ebenfalls auf diesen Sachverhalt bezieht.

In (3.36b) zielt das Appositiv (laut Raabe) auf den mit der Basis verbundenen Modus des Hervorbringens. Dies mutet einigermaßen geheimnisvoll an; ich verstehe es jedenfalls nicht. In diesem Beispiel muB ein sehr allgemeiner Begriff von Referenzidentität angesetzt werden, damit behauptet werden kann, der Bezugssatz und die Kausalangabe (als Appositionskonstruktion!) bezögen sich auf den gleichen Sachverhalt o.a.

In (3.36c) zielt das Appositiv nach Raabe auf einen mit der Basis verbundenen situationellen Aspekt. Auch hier empfinde ich den Begriff der Referenzidentităt als sehr allgemein, wenn behauptet wird, daB sich der Satz (Basis) und der Sprecherkommentar (Apposition) auf den gleichen Sachverhalt o.ă. bezögen. Natürlich kann man das so fassen wollen, doch führt dies m.E. zu einer fast völligen Beliebigkeit des Referenzbegriffs.

Wie wenig es mit der Referentenidentität auf sich hat, demonstriert Raabe (1979: 258) an einem schlagenden Beispiel:

(3.37) Elle alluma une Bastos, cigarette très en vogue, et la porta à ses lèvres. (deutsch: Sie zündete sich eine Bastos an, eine Zigarette, die sehr in Mode war, und führte sie an ihre Lippen.)

Raabe bemerkt, daß die Basis auf eine konkrete Bastos, das Appositiv auf die Marke Bastos an sich referiere. Er schließt daraus (ebd. 258): "Der Begriff, der hier zum Tragen kommt, ist derjenige der (Bedeutungs-) Aquivalenz. In Sonderfăllen mag Bedeutungsidentităt vorliegen".

Besonders eindringlich tritt der Effekt, den Appositionsbereich insbesondere (allerdings nicht ausschlieblich) mittels der Referenzidentität konstituie-

22 Dieser Typ wird auch als sog. "Satzapposition" bezeichnet; hierzu v.a. Bichbaum (1978.). 
ren zu wollen, bei Molitor (1979) zutage, ${ }^{23}$ wenn er die Konstruktionen in (3.38a) einschliest, weil sich beide Teile "über einen konstant gehaltenen Gegenstand prädizieren lassen", und die in (3.38b) ausschliest, weil dies nicht der Fall ist:
(3.38a) (Der Politiker) (Schmidt). (Die Stadt) (Erfurt).
(3.38b) (Die Regierung) (Schmidt). (Der Bahnhof) (Erfurt).

Beide Muster werden in Kap. 7 der attributiven Relation zugewiesen, m.a.W.: Der Unterschied im Referenzverhalten macht nicht notwendigerweise einen Unterschied in der syntaktischen Konstruktion aus.

Folgendes sollte in diesem Abschnitt deutlich geworden sein: Die Referentenidentität von Basis oder Appositiv (möglicher Bezug auf den gleichen außersprachlichen Referenten; extensionale Identităt) ist als Appositionseigenschaft abzulehnen (vgl. 3.37!). Die Referenzidentität (Bezug auf den gleichen Sachverhalt o.ă.) ist als mögliches Kriterium zum einen nicht genau genug expliziert, zum anderen fragwürdig bei Beispielen wie z.B. (3.36). So allgemeine Feststellungen wie "Bezug auf die gleiche Erscheinung der objektiven Realität" dürten der Appositionsbestimmung wenig dienen.

Ein mehr versprechender Ansatz wurde in Raabe (1975) skizziert (in Raabe 1979 aber dann unzweckmäBig überdehnt), worin nicht nur extensionale Identität (3.39a), sondern auch z.B. Gruppe-Element-Beziehungen (3.39b) eingeschlossen werden:

(3.39a) Sepp Maier, die Katze von Anzing, flog durch den Strafraum.

(3.39b) Viele Vögel, vor allem Meisen, waren zu sehen.

Dadurch werden die möglichen Konstruktionsbedeutungen appositionsverdăchtiger Muster zutreffender erfast. Doch der Zusammenfassung der Muster in (3.39) widersprechen andere grammatische Eigenschaften; beispielsweise kann nur zu (3.39b) und zwar hinzugefügt werden, nur (3.39a) kann direkt in Beziehung zu einem Kopulasatz gesetzt werden (dazu in Kap. 15 mehr):

(3.40a) * Sepp Maier, und zwar die Katze von Anzing, (...). Sepp Maier ist die Katze von Anzing.

(3.40b) Viele Vögel, und zwar vor allem Meisen, (...).

*Viele Vógel sind vor allem Meisen.

Insgesamt scheint das semantische Kriterium Referenzidentitat (in allen angebotenen Fassungen) mehr Probleme als Klărungen nach sich zu ziehen. Ich werde im folgenden zwar versuchen, die Semantik der appositionsverdăchtigen Muster knapp zu umreiBen, aber nicht anstreben, die Referenzidentität als Appositionskriterium (als Kriterium für ZUSATZE) zu verwenden.

23 Vgl. auch Altmann (1981: 44): "Die Ursache für diese unhandliche Ausdehnung des Appositionsbegriffs sehe ich in einem unzureichenden Bedeutungskriterium". 


\subsection{Veglasbarkeit}

Daß das Appositiv ein ohne weiteres weglaßbares (ein rein zusatzliches, nicht primär zum Satz gehöriges) Element ist, spielt bei manchen Arbeiten eine Rolle. 24 Raabe (1979: $250 \mathrm{ff.}$ ) formuliert dies etwas genauer, indem er feststellt, daB die Eliminierung von Appositiven zwar nie den "semantischen Nert der Trägerentitäten" bzw. den Wahrheitswert berühre (3.41a), daß es aber durchaus zu "Störungen des formalen Wertes von Sătzen und Texten" (3.41b) kommen kann (Beispiele Raabe 1979: 250), die z.T. jedoch durch Permutation wieder aufhebbar sind $(3.41 \mathrm{c})$ :

(3.41a) Charly (, mein bester Freund,) kommt mich besuchen.

(3.41b) Ein Mann mit Vorsätzen, machte sich $M$. sofort an die Arbeit. * Machte sich $M$. sofort an die Arbeit.

(3.41c) M. machte sich sofort an die Arbeit.

In diesem Zusammenhang bemerkt Raabe (1979: $250 \mathrm{f.})$, daB

"(...) das Appositiv integraler Bestandteil von Texten, von komplexen Sätzen insofern ist, als es formal, durch Vorwärtspronominalisierungen (...) oder Rückwärtspronominalisierungen (...) in die Textkonstitution einbezogen wird $(. .)$.$" .$

Dies sei veranschaulicht durch das folgende Beispiel (Raabe 1979: 250; von mir ins Deutsche übertragen):

(3.42) Peter, Eigentümer zweier Bären, zeigt sie in der offentlichkeit. Appositive sind mithin zwar keine obligatorischen Satzkonstituenten (und somit "Angaben" im weitesten Sinne), immerhin aber textrelevante Bestandteile (Pronominalisierung!). Die "Weglabbarkeit" ist allerdings eine (syntaktische) Eigenschaft, die auf viele Bestandteile beliebiger Wortgruppen zutrifft, nămlich auf all jene, die valenztheoretisch unter "freie Angabe" (circonstant) fallen. DaB sich zwischen "Angabe" (insb. Adverbiale, fakultative Attribute) und den appositionsverdăchtigen Konstruktionen (wie 3.42) ein "grammatischer Graben" auftut, wird sich spăter zeigen (vgl. die Kap. 5, 6, 8, 11, 12, 15). Daher wird die Weglaßbarkeit im weiteren Verlauf meiner Arbeit keine nennenswerte Rolle spielen.

\subsection{Appositionslexene (Funktionslexeme)}

Die Möglichkeit, Appositive mithilfe von Appositionslexemen wie z.B. übrigens, bekanntlich, vor allem, z.B. u.a.m. zu markieren, stellt eine beachtenswerte Erkennungshilfe dar. Diese Ausdrücke indizieren die zwischen Basis und Appositiv bestehende semantische Relation (Präzisierung, Verallgemeinerung etc.),

24 Z.B. Seiler (1960: 11, hier ist Weglaßbarkeit ein wesentliches Kriterium zur Mukleus-/Satellit-Identifikation), Molitor (1979: 34 f., 181), Jung (1982: 110), Ingel (1986: 191). 
weshalb sie vor mir auch "Funktionslexeme" genannt werden. Raabe (1974: 329 ff.) war wohl für das Deutsche der erste, der "Zusatzlexeme" im Zusammenhang mit Appositionskonstruktionen eingehender erörtert hat. ${ }^{25}$ In Raabe (1979: 268 ff.) wird deren Funktion als "Markierung der Sprecherpräsenz" expliziert, d.h. der Sprecher kennzeichnet, was er als zusätzliche Information zu lancieren gedenkt (Beispiel ebd. 270): 26

(3.43) Der Arzt sagte, otto, übrigens ein Trinker, solle vortreten. In Altmann (1981; z.B. 80 f., 97-99) - wo sehr feine Differenzierungen zwischen Apposition (übrigens), Rechtsversetzung (ich meine) und Nachtrag (und zwar) vorgenommen werden - kommt den Appositionslexemen ein hoher heuristischer und distinktiver Wert $z u$, wenn es darum geht, appositionsverdächtige Muster einzuordnen. Darüber hinaus wird auch die Verbindbarkeit mit valenzfreien Adverbialen (v.a. temporale Adverbiale wie früher und Satzadverbiale wie vermutlich) und Gradpartikeln geprüf. Die folgenden drei Muster (3.44a-c) verhalten sich ungleich:

(3.44a) Der Hai, ein wahrer Riese, schwamm auf uns zu.

(3.44b) Er, der Blaubai, schwamm auf uns $z$.

(3.44c) Die Haie, vor allem die Blaubaie, schwammen auf uns $z u$.

(3.45a) Der Hai, übrigens/ *ich meinel *und zwar ein wahrer Riese, (...).

(3.45b) Er, *übrigens/ ich meinel *und zwar der Blaubai, (...).

(3.45c) Die Haie, (?) übrigens/ *ich meine/ und zwar vor allem die Blauhaie, schwammen auf uns $z u$.

(3.46a) Der Hai, bedauerlicherweise sogar ein wahrer Riese, (...).

(3.46b) ??Er, bedauerlicherweise sogar der Blauhai, (...).

(3.46c) Die Haie, bedauerlicherweise sogar vor allem die Blaubaie, (...). (3.44a) läßt sich mit übrigens (3.45a), Adverbialen und Gradpartikeln (3.46a) verbinden, (3.44b) mit ich meine (3.45b), wohl aber kaum mit ADVB und GPn (3.46b) und schlieblich (3.44c) am ehesten mit und zwar (3.45c) sowie mit ADVB und GPn (3.46c). Die unterschiedliche Hinzufügbarkeit stellt demnach ein klassifikatorisch wichtiges Merkmal dar und muB als Eigenschaft appositionsverdächtiger Muster untersucht werden.

Dennoch sollte nicht jede Einheit, die ein "Appositionslexem" enthălt, schlichtweg zur "Apposition" erklărt werden, selbst wenn sie appositionsverdăchtig aussieht ( 3.47 zeigt einen Schaltsatz und eine Satzfolge):

(3.47) Ein Hai - übrigens schien er hungrig - umkreiste uns.

Das machen wir; und zwar machen wir das alle zusammen.

Nur im Verband mit weiteren grammatischen Eigenschaften läßt sich eine ausreichend abgesicherte zuordnung vornehmen.

25 Für das Engl. 2.B. Norwood (1954: 269), Burtan-Roberts (1975: 17), Bitea (1977: 8). 26 Dgl. Engel (1986: 188, 191). 


\subsection{Vertretung der Basis durch das Appositiv}

Ein selten angewendetes Kriterium ist, daB die Apposition "im Satz ihr Bezugswort ersetzen kann" (Helbig/Buscha 1980: 537). Man betrachte hierzu einige Beispiele (3.48a aus Jung 1982: 110):

(3.48a) Der Direktor der Spinnerei, Förster, wurde prämiiert. Der Direktor wurde prämiiert.

Förster wurde prämilert.

(3.48b) Das Jahr 1989 hat 365 Tage.

? Das Jabr hat 365 Tage.

1989 bat 365 Tage.

(3.48c) Der Direktor, ideenreich und kreativ, wurde prämilert.

Der Direktor wurde prämiiert.

*Ideenreich und kreativ wurde prämilert.

(3.48a) zeigt einen akzeptablen "Vertretungsfall". (3.48b) führt vor Augen, daB die Vertretung keineswegs störungsfrei verlaufen muB; aufgrund der möglichen generischen Lesart des mittleren Satzes ist die Alleinrepräsentation des Konstrukts durch Das Jahr zumindest fragwürdig. Schlieblich demonstriert (3.48c) trotz aller "Appositionsverdächtigkeit", daß kategorial von der Basis differente Appositive nie die "Alleinvertretung" innehaben können.

All diese Befunde legen die Auffassung nahe, daB das "Vertretungskriterium" zur Analyse von Appositionen nicht viel beizutragen vermag, da zuviele Sonderfälle und störungen zu gewärtigen sind; überdies wäre es methodisch unzweckmäBig, von vorneherein auszuschlieBen, daB kategorial verschiedene Basis-Apposition-Konstellationen vorkommen. Es wird infolgedessen vernachlässigt werden.

\subsection{Satzgrenzenbestinntheit}

Dieses von Raabe (1979: $274 \mathrm{ff.}$ ) entwickelte (und von Engel 1986: $187 \mathrm{ff}$. aufgenommene) Merkmal besagt, daB Apposition ein Phänomen innerhalb eines Satzes ist (Zitat Raabe 1979: 278):

"Appositive überschreiten Satzgrenzen nicht. Unter Satzgrenze ist hier nicht die oberflächenstrukturelle graphematische Satzgrenze gemeint, obwohl sie in der Mehrzahl der Fälle mit dieser zusammenfallt. Appositive, die die oberflăchenstrukturelle Satzgrenze überschreiten, müssen ohne Bedeutungsveränderung im Trägersatz integrierbar sein. (...) Die Punktinsertion ist nur bei bestimmten Appositionstypen möglich".

Wichtig ist der Hinweis darauf, daB ein "orthographischer Satzbegriff" offensichtlich $z$ eng ist. An ein paar Beispielen sei das Gemeinte demonstriert:27

27 Z.T. an Raabe (1979: 275-277) angelehnt. 
(3.49a) Paul stürzte. Ein tragischer Vorfall.

Paul stürzte - ein tragischer Vorfall.

(3.49b) ??Ein tragischer Vorfall. Paul stürzte.

(3.49c) Pia, früher einmal meine beste Freundin, sab dort.

(?) Dort saB Pia. Früher einmal meine beste Freundin.

* Früher einmal meine beste Freundin. Dort sab Pia.

In (3.49a) würde man nach Raabe trotz des Punktes einen Satz mit stilistisch bedingter Punktinsertion annehmen, in dem eine sog. "Satzapposition" auftritt. Diese kann auch in den Satz vor den Punkt verschoben werden; sie kann aber nicht punktgetrennt diesem Satz voraufgehen (3.49b)! Ebenso verhält sich die "prototypische Apposition" in (3.49c). 28

Die Satzgrenzenbestimmtheit ist problematisch, weil kein "scharfer" Test vorliegt, um festzustellen, wo genau eine Satzgrenze verläuft, was Raabe (1979: 276) im übrigen zugibt. Der "Satzintegrationstest" (3.49), der nachweist, daB scheinbar auBerhalb eines Satzes befindliche Konstituenten innerhalb eines Satzes als "Appositive" fungieren, ist m.E. nicht klar genug.

Auch ich gehe davon aus, daB "Apposition" bzw. ZUSATZ (vgl. Kap. 12-15) eine grammatische Erscheinung ist, die innerhalb von (Gast)Sätzen zu lokalisieren ist. Beziehungen zwischen Sătzen bzw. satzwertigen Einheiten (wie z.B. Interjektionen, Anreden etc.) sind anders zu explizieren, nämlich als Satzfolgen oder als Einschaltungen (wenn z.B. ein Satz innerhalb eines anderen nicht-subordiniert erscheint; vgl. PARENTHESE, EINSCHUB in Kap. 11, 14). Da ich jedoch keine plausible Lösung der Bestimmung von Satzgrenzen kenne, vernachlässige ich im folgenden das satzgrenzenkriterium.

\subsection{Attribution}

Kusmin (1960: 73) weist bei der Untersuchung des "lockeren" nominalen Appositionstypus auf dessen "Fähigkeit zur Erweiterung durch verschiedene Arten von attributiven Bestimmungen, darunter auch durch Attributnebensätze (...)" hin. Molitor (1979: 22 ff., 222) operationalisiert diese Eigenschaft zur Scheidung von "engen appositionellen Syntagmen" (eas) und "lockeren appositionellen Syntagmen" (laS). Demnach kann bei las sowohl die erste als auch die zweite NP attributiv erweitert werden (3.50a), bei eas (3.50b) ist "nur die erste NP at-

28 Eine persönliche Beobachtung an Rande: "Zeitgeistzeitschriften" wie TFMPO bzw. WIFNER enthalten auffallend viele Beispiel für einen "abtrennenden", zergliedernden Stil wie in (3.49) vorgeführt; das betrifft nicht allein Appositionen, sondern auch andere syntaktische Funktionen. 3 exemplarische Beispiele:

(F3.2) Seltsame Situation. Eine Woche lang verbringen wir in Hamburger Studios und AuBenlocations, um eine Modegeschichte zu fotografieren. (HIDNJR 3/88, 16)

(F3.3) Alle wetzen die Messer. "Taz" und "Süddeutsche", "Stern" und "Spiegel", "FaZ" und "Enma". (TEMPO 2/88, 3)

(F3.4) Wer mitfliegen will, braucht ein Ticket. Für 3000 Mark. (WITNISR 11/87, 3) 
tributiv erweiterbar" (ebd. 23); bei postnominaler Attribution sind beim las wieder Basis und Appositiv frei attribuierbar (3.51a), das eas kann nur als Ganzes attribuiert werden (3.51b):

(3.50a) Der schlaue Bäcker, ein guter Geschätsmann, griff $z u$.

(3.50b) Der schlaue Bäcker Schmidt griff $z u$.

* Der Bäcker schlaue(r) Schmidt griff zu.

(3.51a) Der Bäcker an der Ecke, ein Mann mit Köpfchen, griff $z u$.

(3.51b) *Der Bäcker an der Ecke Schmidt griff $z u$.

Der Bäcker Schmidt an der Ecke griff $z u$.

Dieses Charakteristikum ist bei den Feinanalysen $z$ u beachten. Offensichtlich ist die Attributionsfähigkeit beider Konstruktionsteile eine Eigenschaft zumindest des "Appositionsprototyps" (3.50a, 3.51a). Ganz "scharf" ist das Merkmal jedoch nicht, denn es finden sich enge Appositionen mit beidteiliger Attributionsfähigkeit (3.52a) und appositionsverdächtige strukturen, deren Basis nicht attributionsfähig ist (3.52b; nach Altmann "Rechtsversetzung"; am Ende der Arbeit "referenzklärender Zusatz"); überdies finden sich appositionsverdächtige, jedoch vom "Prototyp" unterscheidbare Muster mit freier Attribution (3.52c; "Alternativen nennender Zusatz"):

(3.52a) Zwei große Schwärme hungrige Piranhas umkreisten die Anakonda.

(3.52b) Ich habe sie (*aus München), meine Traumfrau, getroffen.

(3.52c) Der Chef, m.a.W. der Mann mit der größten Schnauze, kam heraus. Wiederum bestätigt sich, wie sehr es auf die Kombinatorik der Eigenschaften ankommt, wenn man grammatisch relevante Appositions-bzw. "Zusatz"-Klassen aufstellen will.

\subsection{Textnotwendigkeit}

Dieses Merkmal stammt von Raabe (1979: 252 ff.). Es wird von Engel (1986: 187) aufgegriffen. Raabe (1979: 252) weist zunächst darauf hin, daß "oberflächenstrukturell" eine Apposition vereinzelt durchaus satz- oder textnotwendig sein kann, da sie z.B. den allgemeinen Pronominalisierungsregeln unterworfen ist (vgl. 3.42). Im ubrigen kann auch nichtpronominal auf in Appositiven gegebene Information verwiesen werden: ${ }^{29}$

(3.53) Legoff (, ein Geschaftsanwalt,) und Busquet (, ein ehemaliger General,) trafen sich bei Daniele. "Wie geht's?" fragte der Anwalt. "Es geht mir gut", antwortete der ehemalige General.

Ein Weglassen der Appositive hătte Interpretationsstörungen zur Folge (Wer ist der Anwalt? Legoff oder Busquet?). Für den inhaltlichen Bereich liegt hingegen keine Notwendigkeit der Appositive vor (s. auch Punkt 8. Neglabbarkeit). D.h., die Apposition (Raabe 1979: 252)

29 Beispiel nach Raabe (1979: 254 f.); von mir übersetzt. 
"(...) ist $\mathrm{n} i \mathrm{c}$ b $\mathrm{t}$ primărtextnotwendig in dem Sinn, daß sie von der Schreiber-, Sprecherperspektive her nicht als integraler Bestandteil des vorliegenden inhaltlichen Handlungs-, Zustandsbeschreibungsgerüsts angesehen wird. Sie ist nur sekundär für die stützung der primären Inhaltsstruktur nötig".

Raabe (1979: 255) schliebt seine Erörterungen über Textnotwendigkeit mit der Bemerkung $a b$, daB

"(..) das Appositiv im Kontext von Voraussetzungsbedingungen für nachfolgende Textteile einen unerläblichen Bezugspunkt darstellen kann, nicht aber

für vorausgehende Textteile oder seinen Trägersatz".

Die Text (nicht)notwendigkeit und die eben besprochenen Eigenschaften bei der Einbettung in Sătze/Texte sind vermutlich zutreffende Merkmale diverser appositionsverdächtiger Muster, die jedoch kaum in brauchbaren Tests zu operationalisieren sind. Zudem muß ich gestehen, das mir letztlich unklar blieb, was genau Raabe mit diesem Merkmal meinte. Daher wird die Textnotwendigkeit im folgenden vernachlăssigt.

\subsection{Sprecherdivergenz und Sprecherpråsenz}

Dieses auf viele der mit dem Einschaltungsmuster versehenen Konstruktionstypen zutreffende Merkmal stellt Raabe (1979: 264) auf:

"Das Merkmal beruht darauf, daß Komplementsătze, die in einen Autorsprechersatz mit "verbum dicendi" im Nukleus eingebettet sind, primär als von dem jeweilig eingeführten weiteren Sprecher 2 "gesprochen" anzusehen sind (...). Handelt es sich um im Komplementsatz eingebettete Appositive, so wird gemeinhin Sprecherdivergenz vorliegen, das heiBt, der Komplementsatz wird von Sprecher 2, das Appositiv vom Autorsprecher (in Einzelfăllen auch von Sprecher 2) "gesprochen"".

Dieses Merkmal demonstriert Raabe (1979: 269 f.) an folgendem Material:

(3.54a) Der Arzt sagte, otto, der ein Trinker sei, solle vortreten.

(3.54b) Der Arzt sagte, otto, der ein Trinker ist, solle vortreten.

(3.54c) *Der Arzt sagte, otto, der übrigens ein Trinker sei, solle vortreten.

(3.54d) Der Arzt sagte, otto, der übrigens ein Trinker ist, solle vortreten.

(3.54e) Der Arzt sagte, Otto, (übrigens) ein Trinker, solle vortreten. Es geht um die Frage, welcher Sprecher bei referierter Rede das Appositiv produziert habe, der referierende (aktuelle) sprecher oder der referierte? In (3.54b, d, e) stellen die Appositive AuBerungen des aktuellen Sprechers dar, wăhrend die Appositive in (3.54a) und (3.54c) nicht vom aktuellen sprecher stammen, sondern von diesem nur referiert werden; sie stammen von einem "Sprecher 2". Es zeigt sich, das Inakzeptabilităt entsteht, wenn nicht vom Autor- 
sprecher produzierte Appositive mit sog. "Appositionslexemen" verbunden werden (3.54c); diese kōnnen ausschließlich die Prãsenz des (Autor) Sprechers signalisieren, was sich an (3.54d) erkennen läßt.

Manchmal ist eine klare Entscheidung, von welchem Sprecher das Appositiv stammt, nicht leicht. Stammt in der Sowjetunion von Herrn Bauer oder vom aktuellen sprecher? ${ }^{30}$

(3.55) Herr Bauer hat gesagt, daB man, in der Sowjetunion, auch die Zweisprachigkeit behandelt.

Die Sprecherdivergenz manifestiert sich deutlich bei unmittelbarer Niedergabe direkter Rede. In (3.56a) äubert der aktuelle sprecher das Appositiv, in (3.56b) der referierte sprecher:

(3.56a) Der Arzt sagte: "Otto", ein Trinker, "soll vortreten".

(3.56b) Der Arzt sagte: "Otto, ein Trinker, soll hervortreten". Die Sprecherdivergenz stellt ein Merkmal dar, das auf appositionsverdăchtige Muster zutrifft. Anders als die in den Satz stark integrierten Erganzungen, Angaben und Partikelfunktionen stehen die gering integrierten ZUSATZE und EINSCHOBE (vgl. Kap. 5) bei Redewiedergaben zur Disposition sowohl des aktuellen als auch des referierten Sprechers:

(3.57a) Der Arzt sagte, "Otto", mir war das klar, "muß es aufgeben". Der Arzt sagte, "Otto, mir war das klar, mus es aufgeben".

(3.57b) Der Arzt sagte: "Otto", weil es zuviel wird, "muß es aufgeben". Der Arzt sagte: "Otto, weil es zuviel wird, muB es aufgeben".

Da dieses Merkmal jedoch auf eine große Menge von einschaltungsmarkierten Einheiten (Schaltsatze, Anreden, Interjektionen etc.) zutrifft, kann es zur Feingliederung des Bereichs der appositionsverdachtigen Muster nicht herangezogen werden; vielmehr beschreibt es eine generelle Eigenschaft von HOSPITANTEN (vgl. Kap. 5).

\subsection{Adordinativităt}

Raabe (1979: $229 \mathrm{ff}$. ) reiht die Apposition in die "adordinativen" Ronstruktionen ein. Zum besseren Verstăndnis eine Obersicht (ebd. 227):

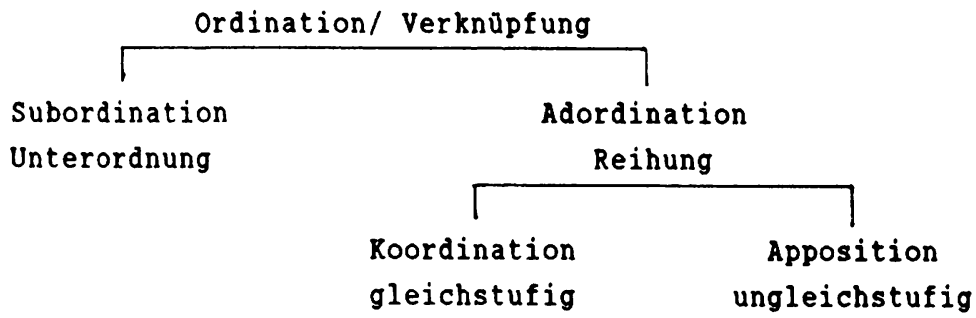

30 Beispiel nach Raabe (1979: 267); ubertragen. 
In gleichstufiger Adordination (= Koordination) sind beide Elemente (s. 3.59 "p, q") von gleicher Wichtigkeit; beide sind syntaktisch gleichermaßen in das übergeordnete syntagma integriert. Bei ungleichstufiger Adordination (= Apposition) ist dies nicht der Fall (ebd. 231):

(3.59) GLEICHSTUFIGREIT: $p q r$ s. UNGLEICHSTUFIGKEIT: $p \stackrel{q}{q}$.

Dieser Unterschied zeigt sich z.B. bei Anwendung von Negation:

(3.60a) Nicht Peter und sein bester Freund kamen, sondern Pia und Ute.

(3.60b) *Nicht Peter, sein bester Freund, kam, sondern Peter, sein ärgster Feind.

(3.60c) Nicht Peter, sein bester Freund, kam, sondern Kuno.

Die Negation kann in (3.60a) beide Konjunkte betreffen, in $(3.60 \mathrm{~b}, \mathrm{c})$ nur die Basis. "Das heiBt, daB explizites Reden eine gesonderte Bejahung bzw. Verneinung des Appositivs erfordert" (Raabe 1979: 231).

Das Appositiv steht also in relativ lockerer Beziehung zur Basis und zum Restsatz (zur Restaussage); es "stützt" den Wahrheitswert bzw. die Plausibilităt der Aussage, ohne für diese wesentlich zu sein. Damit steht es im Gegensatz $z u$ Koordination und Subordination, beide stets relevant für den Wahrheitswert der Aussage und in enger(er) Beziehung zur Restaussage.

Das Charakteristikum Adordinativität trägt zur Bestimmung von Apposition nicht allzu weit, weil es recht allgemein ist, d.h. man könnte alles, was in relativ lockerer Verbindung zur Aussage eines Satzes steht, als "adordiniert" ansehen. Das Merkmal trifft wiederum auf den weiten Bereich der HOSPITANTEN (Kap. 5) zu. (Zur "Adordination" s. auch Kap. 4 Abschnitt (a).)

\subsection{Negationsskopus}

Daß die Tragweite der Negation sich bei "lockeren" appositionsverdächtigen Konstruktionstypen nur auf die Basis, nicht aber auf das Appositiv erstreckt, führt Raabe (1979: 260; Beispiel von W.S. übersetzt) vor: ${ }^{31}$

(3.61a) Yves verneint, daß Charles, sein bester Freund, die Wahrheit gesagt habe.

(3.61b) Es ist nicht der Fall, daB Charles, sein bester Freund, die Wahrheit gesagt hat.

(3.61c) *Es ist nicht der Fall, daB Charles sein bester Freund ist. Eine Satznegation affiziert auf keinen Fall das Appositiv (3.61c); es kann nicht negiert werden, daB Charles sein bester Freund sei. Auch eine Sonderbzw. kontrastierende Negation betrifft ausschlieblich die Basis (3.62a):

31 Zum Negationsskopus s. Jacobs (1982). 
(3.62a) Nicht Pia, eine Köchin, kam herein, sondern Ute.

(3.62b) *Nicht Pia, eine Köchin, kam herein, sondern Pia, eine Malerin. Auch in Fragekontexten bleibt das Appositio unaffiziertimit (3.63) ist nicht "in Frage gestellt", daB Charles sein bester Freund ist: 32

(3.63) Hat Charles, sein bester Freund, die Wabrheit gesagt? Die "ungleichstufige Adordinativitat" der Apposition, ihr nichtnotwendiger, sekundărer charakter bedingt offensichtlich, daB diese abgeschirmt ist vor bereichsbildenden Operationen wie Negation und Frage. ${ }^{33}$ Formales Rorrelat der Abschirmung ist die graphematische resp. intonatorische Abtrennung. Natürlich kann das "Appositiv" selbst negiert oder von einer GP betroffen werden - nãm. lich dann, wenn die entsprechende Partikel innerhalb des "Appositivs" steht. 34

"Enge" und "lockere". Appositionsmuster verhalten sich hinsichtlich der Negation und Erfragung ubrigens völlig verschieden:

(3.64a) Nicht die Kóchin Pia trat ein, sondern die Malerin Ute. Nicht die Köchin Pia trat ein, sondern die Malerin Pia.

(3.64b) Trat die Kochin Pia ein? Nein, die Köchin Ute. Trat die Köchin Pia ein? Nein, die Malerin Pia.

(3.64c) Welche Róchin trat ein? Die Kóchin $P i a$. Welche Pia trat ein? Die $K \delta$ chin Pia.

(3.64d) Pia, die Köchin, trat ein.

? Welche Pia trat ein? ??Pia, die $K$ ö chin.

* Welche Köchin trat ein? *P $i$ a, die Köchin.

Bei den engen Mustern sind beide Konstruktionsteile einzeln kontrastierend negierbar und erfragbar (3.64a, b)! Frappierend ist der Unterschied bei der Anwendung sog. "Gliedteilfragen" (Fragen nach dem Attribut in einer Ronstruktion), die bei engen Typen unproblematisch ist (3.64c), bei den lockeren hingegen (3.64d) sind bereits die Fragestellungen merkwürdig, die Antworten aber deutlich inakzeptabel. Warum? Weil kein attributives Muster vorliegt!

Ein bislang nicht zur Appositionsanalyse angewandter Test besteht darin, die der Negation in einigen syntaktischen Aspekten verwandten Gradpartikeln auf appositionelle Konstruktionen anzuwenden: 35

(3.65a) Sogar Pia, die Kóchin, schnarchte, nicht nur Ute.

* Sogar Pia, die Köchin, schnarchte, nicht nur Pia, die Malerin.

(3.65b) Sogar die Kóchin Pia schnarchte, nicht nur die Malerin Pia.

32 Beispiel von Raabe (1979: 261); von H.S. ubersetzt.

33 Zur Negation als bereichsbildende Operation s. Jacobs (1982), zu illokutiven operationen bzw. Operatoren s. Jacobs (1984). Analoge Anwendung auf Gradpartikeln in Jacobs (1983).

34 Zum Beispiel: Pia, auch/ sogar Oberinspektorin, kam berein.

35 Vgl. Jacobs (1982) und Jacobs (1983). Der Bezugsbereich von Negation und Gradpartikel ist oft der ganze Satz, sie haben Stellungseigenschaften, die hinsichtlich ihrer Bezugselemente formuliert werden missen, sie sind nicht allein vorfeldfăhig u.a.m. 
Das Ergebnis ist, daB beim lockeren Typ (3.65a) nur die Basis, beim engen Muster (3.65b) beide Teile affizierbar sind.

Mit der Anwendung bereichsbildender Ausdrücke (Negation, Frage) hat Raabe ein sehr wichtiges Kriterium erarbeitet. Es ist eine grammatische Eigenschaft vieler HOSPITANTEN, von derartigen Operationen im Gastsatz abgeschirmt $2 \mathrm{u}$ sein. Auch z.B. der Schaltsatz in (3.66) ist von der Negation abgeschirmt:

(3.66) Es ist nicht der Fall, daB Pia - sie arbeitet als Köchin - die ganze Nacht schnarcht.

Andererseits läBt die Anwendung der erörterten Operationen Unterschiede im Bereich der i.e.S. "appositionsverdächtigen" Muster, der NP-ZUSATZE, hervortreten, z.B.:

(3.67a) Die Haie, schreckliche Meeresräuber, kamen nicht.

(3.67b) Daraus folgt: Die Haie kamen nicht.

* Schreckliche Meeresräuber kamen nicht.

Die Haie sind schreckliche Meeresräuber.

^Die Haie sind keine schrecklichen Meeresräuber.

(3.68a) Die Haie, vor allem die Hammerhaie, kamen nicht.

(3.68b) Daraus folgt: Die Haie kamen nicht.

(Vor allem) Die Hammerhaie kamen nicht.

* Die Haie sind (vor allem) Hammerhaie.

Wăhrend bei dem später als PRXDIZIERENDER ZUSATZ bezeichneten Muster (3.67a) der Zusatz unbetroffen bleibt und die Folgerung, das Haie schreckliche Meerestiere sind, zutreffend ist (3.67b), ist beim spāter als SPEZIFIZIERENDER ZUSATZ bezeichneten Muster (3.68a) der Zusatz affiziert und die sein-Folgerung unzutreffend.

Man kann noch weitere bereichsbildende, z.B. quantifizierende Ausdrücke prüfen, doch es zeigt sich stets (vgl. auch Rap. 6, 11, 15), daB sie nur Basiselemente, jedoch nur selten mit dem Einschaltungsmuster versehene Einheiten betreffen können. Diese Einschaltungen in einen "Gastsatz" leisten offensichtlich keinen (direkten) Beitrag zur Satzsemantik bzw. Satzproposition, so daB skopusbildende Operatoren (deren semantischer Beitrag zum Satz resp. zur Satzproposition offenkundig ist) meist nur auf den Gastsatz, nicht aber auf die Einschaltungen einzuwirken vermögen.

\subsection{Dahrheitswertronstanz}

Diese Eigenschaft wurde von Zemb (1968; 1972: 80; 1978: 706 ff.) eingeführt und ausfuhrlich untersucht. Nach Raabe (1979: 248 f.) gilt das Merkmal Vahrheitswertkonstanz

"(..) nicht nur für Appositionen, sondern auch für (tiefenstrukturell satzformatige) Roordinationen, für Sătze in asyndetischen Reihungen oder, noch allgemeiner, für Texte. Eine sichere Abgrenzung gestattet sie gegen- 
über subordinativ-determinativ-restriktiven strukturen. Sie besagt (...), daß weder das Hinzufügen noch das Tilgen des Appositivs den Wahrheitswert des Trăgersatzes verandert. (...). Wenn das Appositiv etwas beim Horer/Leser bewirken kann, dann eine stützung des jeweiligen Wahrheitswertes des Trăgersatzes".

Dieses Merkmal kann mittels des Heglabtests gepruft werden, wobei an die damit verbundenen Probleme (vgl. die Punkte 8 und 13 dieses Rapitels) erinnert sei.

Die Wahrheitswertkonstanz ist sicherlich ein auf "appositionsverdachtige" Ronstruktionen zutreffendes Merkmal, jedoch auch ein sehr allgemeines. Die "Appositionsbereiche" fallen u.a. deshalb bei Zemb (1968; 1978: 709) wie bei Raabe (1979: $285 \mathrm{ff}$.) sehr extensiv aus. Bei ihnen finden sich schaltsătze (3.69a), eingeschaltete Angaben (3.69b), eingeschaltete Verben (3.69c) etc., die ebensowenig den Trăgersatzwahrheitswert tangieren wie z.B. der "Appositionsprototyp" (3.69d). Daher wird das Merkmal im weiteren nicht zur Feinanalyse herangezogen. 36

(3.69a) Sie können natürlich [, wie sie ja wissen,] nicht alles wissen.

(3.69b) Die Letzten waren [, wie immer,] die letzten.

(3.69c) On avait négligé [, voire ignoré,] l'essence dynamique de la phrase.

(3.69d) Pia, eine Köchin, war nicht die letzte.

Es wurden gewiß nicht sämtliche möglichen grammatischen Eigenschaften zur Prüfung appositionsverdăchtiger Muster erörtert, doch läst die Menge der Kriterien, die sich als analytisch (heuristisch) interessant erwiesen haben, bereits auf eine ausreichende Differenzierung hoffen.

Abschließend möchte ich noch auf weitere Tests bzw. Rriterien hinweisen, die später in dieser Arbeit zur Anwendung kommen:

\subsection{Rekursivităt und Roordination}

Zu prüfen ist, inwiefern sich Appositive rekursiv erzeugen bzw. koordinieren lassen. Zwei Hypothesen stehen vor allem im Mittelpunkt: Gleiche (Sub-) Klassen von ZUSATZEN sind (in gewissem Umfang) rekursiv erzeugbar. Gleiche Zusătze sind koordinationsfăhig (z.B. 3.70a), ungleiche stören sich gegenseitig und widersetzen sich der Roordination (3.70c):

(3.70a) Pia, Rochin, 25 Jabre alt, erfolgreich, arbeitet gern.

(3.70b) Sie, Pia, Kochin, erfolgreich, arbeitet gern.

(3.70c) *Sie, Kochin, Pia, erfolgreich, arbeitet gern.

In (3.70a) treten drei gleichrangige (prădizierende) zusătze zu pia auf. In (3.70b) ist Pia (referenzklărender) zusatz zu sie, die letzten beiden (prădizierenden) zusătze beziehen sich auf Pia. In (3.70c) erweist sich die Abfolge von Kóchin und Pia als inakzeptabel. Die Zusătze gehören zwei verschiedenen Klassen an!

36 (3.69a, b) von Zemb (1978: 709); (3.69c) von Raabe (1979: 300). 


\subsection{Prononinalisierung und anaphorische Bezuge}

Hier soll untersucht werden, ob man sich auf Basis und/oder Appositiv anaphorisch beziehen kann und welche pro-Elemente zur verfügung stehen.

\subsection{Passivierung}

Appositionsverdăchtige Konstruktionen sollen daraufhin getestet werden, ob sie an satzsyntaktischen Operationen wie z.B. der PASSIVIERUNG teilnehmen (müssen), oder ob solche "Transformationen" nur die Basen betreffen. Satzsyntaktische operationen, die das stellungsverhalten betreffen (CLEFT, LINRSVERSCHACHTELUNG usf.) können z.T. bereits unter 3. "Stellung" abgehandelt verden. Die Durchführung dieses Tests kann man in Kap. 6 Abs. 20 nachlesen.

\subsection{Dependenz}

Es soll geklärt werden, ob in "Basis-Appositiv"-Konstruktionen ein Dependenzverhăltnis anzusetzen sei. Dependenz läBt sich mittels Rektion, Valenzforderungen, Weglasbarkeit sowie der kategorialgrammatischen Explikation (Operator-operand-Strukturen) von Vennemann (1977) nachweisen (s. Kap. 6 Punkt 21).

\subsection{Ronstituenz}

Ich halte das Problem, ob in appositionsverdăchtigen Konstruktionen Basis und Appositiv Ko-Konstituenten einer Konstruktion seien, für noch ungeklărt, obwohl dies in Konstituentenstruktursyntaxen (vgl. Kap. 5.1) of angenommen wird. Ronstituenz kann mithilfe von Konstituententests (Permutationstest etc.) expliziert werden. Wo es wichtig erscheint, werden Analysen hierzu vorgenommen (s. Kap. 6 Abs. 22). 


\section{BRSTE BEGRIFFSRLARUNGEN UND ABGRENZUNGSPROBLBME}

In diesem Kapitel werden einige generelle Bestimmungsversuche von "Apposition" erörtert, einige sprachregelungen getroffen und einige Abgrenzungsprobleme angedeutet. Manche "Vor-Urteile" über Apposition werden (terminologisch) ausscheiden, andere bleiben zu überprüfen. Vier Auffassungen (a-d) sollen zur Sprache kommen; nach diesen würde Apposition verstanden werden als

(a) grammatische Relation (neben Koordination und Subordination),

(b) asyndetische Fügeweise (neben der syndetischen Fügeweise),

(c) syntaktische Funktion (neben Attribut, Satzglied usw.),

(d) nicht-syntaktische "Funktion" (diverse Mőglichkeiten).1

(a) "Apposition" ist eine Relation zwischen zwei Einheiten, 2.B. zwischen zwei nominalen (4.1a), die weder subordinativ noch koordinativ ist, sondern "prádikativ". Expliziert wird diese Relation durch die Möglichkeit prădikativer Paraphrasen mit Kopulaverben (sein, heiBen; 4.1b): ${ }^{2}$

(4.1a) Der Fakir Krause/ Der Fakir, Krause, (spinnt).

(4.1b) Krause ist ein Fakir. Der Fakir heiBt Krause.

"Apposition" wäre dann innerhalb der folgenden Einteilung nichtfunktionaler Beziehungen bzw. innerhalb der folgenden konstruktionalen Aspekte der Verknüpfung von sprachlichen Einheiten anzusiedeln: ${ }^{3}$

- Roordination: Der Zabnarzt Krause und der Nervenarzt Huber. Weder ein- noch gegenseitige Bestimmung zweier Nuklei, symmetrische Relation.

- Subordination: Der Zahnarzt Krauses. Einseitige Bestimmung, in Nukleus und Satellit (GA) strukturierbar, asymmetrisch. Am Satelliten befindet sich u.U. ein formaler Subordinationsindikator (hier: Genitiv).

- Apposition: Herr Krause, ein Fakir, (săuft). Gegenseitige symmetrische "prädikative" Relation zwischen zwei Konstituenten.

Die "prădikative" Beziehung (explizierbar durch Umschreibungen mit Kopulasătzen) ist allerdings kein ausschliebliches Merkmal von "appositionsverdächtigen Mustern". Es gibt einige Fälle, die nicht appositionsverdachtig sind:

1 Syntaktische Funktionen (Objekt, Adverbial, Attribut etc.) werden von Einheiten ausgeulbt, die zum "Mementarsatz" (Kap. 5) gehören. Sie sind nicht z.B. durch das Einschaltumgsmuster vom Trägersatz abgetrennt. Andere Einheiten, vor allem solche, die in einen Satz eingeschaltet werden (Schaltsătze, Interjektionen, Anreden u.a.m.) bzw. einen Bezugssatz haben, ohne in diesen syntaktisch so fest integriert zu sein wie z.B. Satzglieder und Gliedteile, uben auch eine Funktion hinsichtlich des Satzes aus. So können Schaltsătze den "Bezugssatz" kommentieren, Herausstellungen die Informationsgliederung verändern etc. Man kann sich mit Altmann (1981: 46) z.B. so ausdrücken, daß Herausstellungen bzgl. des zugeordneten Satzes eine semanto-pragmatische Funktion erfullten. Mit dem Ausdruck "nicht-syntaktische Funktionen" ist derartiges gemeint.

2 vgl. zu dieser Auffassung Löbel (1986a: $103 \mathrm{ff.}$ ).

3 Funktionale Relationen wären z.B. "Objekt (zu)", "Attribut (zu)" etc. 
(4.2a) Das Laster der Trunksucht (Genitivattribut). Die Trunksucht ist ein Laster.

(4.2b) Der Onkel aus Amerika (Prăpositionalattribut). Der Onkel ist aus Amerika.

zudem können nicht alle "appositionsverdāchtigen", strukturell ähnlichen Konstruktionen prädikativ paraphrasiert werden:

(4.3a) Die Affen, übrigens possierliche Tiere, dösten. Die Affen sind übrigens possierliche Tiere.

(4.3b) Die Affen, vor allem die Gibbons, dösten. * Die Affen sind vor allem die Gibbons.

(4.3c) Die Affen, ferner die Löwen, dösten.

* Die affen sind ferner die Löwen.

Es erhebt sich die Frage, ob bei Appositionskonstruktionen eine symmetrische, wechselseitige Beziehung oder eine einseitige Relation vorliegt? Entweder ist. Apposition (hierin der Attribution vergleichbar) die Funktion einer Einheit $Y$ (= Apposition) $z u / b z g l$. einer Einheit X (= Basis), oder sie ist eine wechselseitige Funktion zwischen $X$ (Basis) und $Y$ (Apposition). Im ersten Fall wăre die Beziehung einseitig und jener beim Attribut ähnlich (z.B. "Y ist Genitivattribut $\left.z u X^{\prime \prime}\right)$. Der zweite Fall ähnelte der Koordination, ohne die Gleichwertigkeit beider Konjunkte aufzuweisen, da das "Appositiv" nicht in gleicher Weise wie ein Konjunkt in den Trägersatz integriert ist. Dieser Fall wird in der einschlägigen Literatur manchmal erwăhnt und bezieht sich of t auf sog. "enge Appositionen" wie der Fakir Krause, bei denen die Bestimmung, was Basis und was Appositiv sei, des öfteren schwerfält:

"Bei den appositionellen Ronstruktionen haben wir (...) von einem Verhăltnis wechselseitiger Bedingtheit auszugehen" (Hackel 1968: 88).

"Die Formulierung $>X$ ist Apposition $z u$ $Y<$ besagt, daB $X$ die Apposition und $Y$ der Rern ist, auf den $X$ bezogen ist. Sind Kern und Apposition nicht zu unterscheiden, dann ist diese Redeweise hinfallig und mus ersetzt werden durch $>X$ ist Apposition $z u Y$ und $Y$ ist Apposition $z u X($. Dieser Fall tritt häufig ein (...)" (Eisenberg 1986: 243).

"Ein >Umkippen< der Abhängigkeitsverhăltnisse ist aber gerade charakteristisch für die enge Apposition" (Eisenberg 1986: 244).

Es ist nach Ansicht mancher Darstellungen gerade diese "Schaukelsyntagmatik", 4 die "Kopflosigkeit" der Konstruktion bzw. die "Doppelköpigkeit", 5 welche die

4 Begriff von Mikus, R. F.: Die Koordination und die Syntagmatik. In: ZPSK 15 (1962), Heft 1, 137 ff. Zitiert nach Hackel (1970: 53).

5 Raabe (1979: 123 ff.) argumentiert, daß beim Muster "enge Apposition" (das er schließlich aus dem Appositionsbereich ausscheidet) eine generelle Kopfzuweisung unmöglich sei. In Kap. 7 Abs. 1 wird diese Auffassung korrigiert. Deutliche Ropf zuweisungen sind möglich. 
besondere Wesensart der Apposition ausmacht. 6 Dennoch ist die "wetterwendische" head-modifier-Verteilung kein sehr nützliches Definiens. 7 "Kopflosigkeit" ist erstens keine absolute Domäne der "Apposition" (4.4a) und zweitens keineswegs immer der Fall bei Mustern, die dem Phãnomen Apposition zugeordnet werden $(4.4 c)$ :

(4.4a) Pia und ihre Schwester (Roordination; kein head) traten ein. 8

(4.4b) Pia (Basis), Dozentin aus Köln (lockere APP), trat ein.

(4.4c) Wegen des Bahnhofs (head) Köln (modifier).

Beim lockeren appositionsverdăchtigen Muster (4.4b) ist durch die "Einschaltungsmarkierung" deutlich gemacht, welches Element bzgl. des anderen als "Apposition" (Zusatz) fungiert, wobei man kaum davon sprechen kann, das das Appositiv Dozentin aus Bonn den modifier zum head (zur Basis) Pia darstellt. Diese Relation fiele ja unter Subordination, die ofters durch Indikatoren wie Kasuszuweisung etc. markiert ist ${ }^{9}$ und gerade derartige Indikatoren fehlen hier, es sei denn, man rechnete das "Einschaltungsmuster" dazu, was zur Folge hätte, daß unzweckmäßigerweise alle mit diesem Muster versehenen "Einschaltungen" als modifier bzw. Satelliten zählten.

Bei den engen strukturen (4.4c) wird sich in Kap. 7 zeigen, daß man sie der attributiven Funktion zuordnen kann, z.B. weil durch Rektion bedingte Flexionsmerkmale wie bei anderen Attributkonstruktionen auch am Nukleus und nicht am Satelliten realisiert werden. Somit liegt eine einseitige Relation vor.

"Lockere Appositionen" wie (4.4b) stehen ebenfalls in einer einseitigen Relation zu ihrem Bezugselement, allerdings nicht im Verhăltnis der subordination, jedenfalls nicht im üblichen sinn (wie etwa bei Attributkonstruktionen).

Stellen alle grammatischen Relationen, die sich veder als Koordination noch als Subordination einstufen lassen, einen Fall von Apposition dar? Dies nimmt z.B. Raabe (1979: $226 \mathrm{ff}$, 329) an. Dadurch ergibt sich jedoch ein sehr umfas-

6 In diesem Sinne 2.B. Hockett (1955: $100 \mathrm{f}$ ) und Hackel (1970: $53 \mathrm{ff.,} \mathrm{88)} \mathrm{für} \mathrm{die} \mathrm{sog.} \mathrm{"enge}$ Apposition".

7 Als Kriterien für head vs. modifier gelten die bekannten Merkmale, das (a) der Kopf von der gleichen Kategorie ist wie die Cesamtkonstruktion, die Binzufügnng des Satelliten die Kategorie entweder gar nicht oder nur insoweit, als eine Valenzstelle abgebumden wird, verăndert, (b) der Ropf die Funktion der gesamten Guppe einnehmen könnte (der Satellit nicht), (c) der Satellit den Ropf semantisch năher bestimnt (eine Subklasse der Kopfreferenten bildet o.ä.). Neuere Beitrăge zur "Kopf-Diskussion" sind Zwicky (1985) und die Erwiderung von thdson (1987).

8 Vgl. Krifka (1983: 30): "Im Unterschied zu attributiven endozentrischen Konstruktionen gibt es aber bei der Koordination keinen Kopf". Anders Bockett (1955: 100): "John and Bill, for example, does not consist of attribute and head; it contains two heads, John and Bill (...). A (bipartite) construction of this sort may be called double-headed [im orig. kursiv; w.S.]".

9 Bein Attribut (als modifier) kann als Indikator z.B. der Genitiv realisiert sein. 
sender Appositionsbereich (vgl. Raabe ebd. $226 \mathrm{ff}$, , 329), der u.a. so verschiedenartige Konstruktionen wie die folgenden in sich vereinigt: 10

(4.5a) Der Wal, ein Säugetier, muß geschützt werden.

(4.5b) Der Wal - das sagt auch Pia - mus geschützt werden.

(4.5c) Er kommt, er kommt morgen.

(4.5d) Er will nur eines: Erfolg haben.

Es erscheint terminologisch unzweckmäBig, alle Konstruktionsmuster, die nicht durch Koordination oder subordination zu erfassen sind, pauschal als Appositionen zusammenzufassen. Einerseits führt dies zu einem sehr großen, inhomogenen Objektbereich (vgl. 4.5), andererseits ist der Begriff Apposition traditionell zu nahe bei den syntaktischen Funktionen verortet. (Man erinnere sich der ofters vertretenen Meinung, "Apposition" sei eine attributive subfunktion.) Allerdings wăre ein Begriff für die syntaktische Relation, die z.B. in (4.5a) zwischen der Wal und ein Săugetier besteht, wünschenswert.

Anstelle von "Apposition" wird vorgeschlagen, eine weder koordinative noch eindeutig subordinative Beziehung (Ronstruktionsart) zwischen zwei Einheiten wie in (4.5a) als ADORDINATION $z u$ bezeichnen. Ebenfalls denkbar wăre der Terminus HOSPITANZ (vgl. Kap. 5.5.). Dabei muB m.E. nicht notwendigerweise eine prădikative Paraphrase möglich sein; es scheint mir nicht einmal notwendig, daß überhaupt eine Paraphrase zur Verfügung stehen muß.11 Die "Apposition" selbst findet sich als submuster innerhalb der adordinativen Relation zUSATZ.

In einer Taxonomie der syntaktischen Relationen, die ich in Anlehnung an Lehmann (1983: v.a. 339-341) und Raabe (1979: $226 \mathrm{ff.}$ ) aufstelle, würden drei Hauptrelationen unterschieden: die Subordination (Dependenz; die beiden relationierten Konstituenten sind nicht gleichberechtigt, eine bestimmt die Auftretensmodalitaten der anderen), die Koordination enthălt nicht-subordinierte, gleichberechtigte Relata und die Adordination nicht-subordinierte Relata, die nicht gleichwertig sind: 12

syntaktische Relation
Subordination

10 Die beiden letzten Beispiele von Raabe (1979: 290, 292), vom Verf. ins Deutsche ubertragen.

$11 \mathrm{Vgl}$. (4.3b, c) wo man eher von einer "Satzkopiehypothese" ausginge:

(F4.1) Die Affen dösten. Ferner (dösten) die Lömen.

> Die Affen dösten, ferner die Löwen > Die Affen, ferner die Löwen, dösten.

$12 \mathrm{Vgl}$. auch Kap. 5.5. Modellvorstellung, wo die Termini ENNSCHUB und ZUSATZ deutlicher werden. 
Die nachstehenden Beispiele illustrieren die Unterteilungen:

(4.6a) Tarzan liebt Jane.

(4.6b) GroBe Affen knurrten.

(4.6c) Kuno und Pia gehen in die Kneipe.

(4.6d) Pia, sie hatte wieder Durst, ging in die Kneipe.

(4.6e) Pia, eine Künstlerin, geht in die Kneipe.

Die Haie, vor allem die Hammerhaie, umkreisten uns.

(b) "Apposition" ist eine besondere Fügeweise, nămlich die unvermittelte (asyndetische) Nebeneinanderstellung zweier (z.B. nominaler) Einheiten. ${ }^{13}$ Im Ggs. dazu gibt es die vermittelten (syndetischen) Fügeweisen, z.B. mittels Prăposition oder mittels des Kasus Genitiv:

(4.7a) Das Laster der [Genitiv] Trunksucht.

Das Laster Trunksucht ["Apposition"] .

(4.7b) Die Universität in [Präp] Köln.

Die Universität Köln ["Apposition"].

Hierbei wird "Apposition" als Fügeweise von Konstituenten aufgefaBt, etwa im Rahmen des folgenden Schemas:

- syndetische Fügung: durch Fügewörter (Ronjunktion, Prăposition) oder morphologische Fügemittel (Rasusaffix, z.B. "Genitiv").

- asyndetische Fügung: durch bloße "Apposition" (Juxtaposition). Dann wăren die Einheiten in (4.8) Appositionen, Einheiten wie in $(4.9,4.10)$ fielen jedoch nicht notwendigerweise darunter - es sei denn, man săhe beim ersten Typus von der graphematischen Abtrennung $a b$ und behandelte Mein Onkel Hugo und Mein Onkel, Hugo, gleich. Die Vernachlasssigung des Einschaltungsmusters erscheint jedoch unzweckmäBig; es ist kaum einzusehen, warum intonatorische resp. graphematische Mittel nicht als Fügungsmarkanten gelten sollten. In (4.10) liegt mit als ein explizites Fügemittel vor.

(4.8) Onkel Hugo. Das Laster Trunksucht. Die Poetin Anna Blume.

(4.9) Mein Onke1, Hugo, strickt. Das Laster, Faszinosum des Rleinbürgers, zog sie an.

(4.10) Pia als Arztin häte das wissen müssen.

"Apposition" mit unmarkierter bzw. unvermittelter Fũgung in eins zu setzen, erscheint definitorisch wenig sinnvoll. Wenn man den Appositionsbereich (vgl. Kap. 3) durchsieht, dann finden sich sowohl unvermittelte als auch vermittelte Muster diverser Art. Gerade der "Prototyp" (4.9) weist ein Markierungsmuster auf und ist somit kein Fall von Asyndese, wenigstens kein gewöhnlicher. Es liegt aber offenbar auch kein Fall von Syndese vor. Deshalb ergeht der Vorschlag, die unvermittelte Fügeweise nicht "Apposition", sondern JUXTAPOSITION zu nennen.

13 So z.B. Erben (1984: 103): "Die Neigung, (...) hypotaktische Retten zu vermeiden, wirkt sich auch in der Bevorzugung der Apposition oder appositionsăhnlicher Verbindungen als Brsatzform für genitivische Gefüge aus". Eines von Erbens Beispielen (ebd.) ist ein Stück Fleisch(es). 
(c) "Apposition" ist eine syntaktische Funktion (wie "Attribut", "Objekt" usf.). Zwei Sichtweisen sind möglich: (c1) die "Apposition" ist eine besondere Auspragung von Adnominalen (Substantivattributen) mit besonderen Merkmalen wie "Referenzidentităt von Basis und Appositiv", "Kasuskongruenz" u.a.m.:14

Adnominale: Das Laster der Trunksucht (Genitivattribut).

Die Universität in Köln (Präpositionalattribut).

Die Kólner Universität (Adjektivattribut).

Die Universität Köln (enge Apposition).

Die Universitat, ein Neubau, (lockere Apposition).

(c2) "Apposition" ist eine eigenständige syntaktische Funktion. 15 Tráfe (c1)

zu, dann wăre eine Benennung verfügbar, die alle Adnominale bezeichnete, die keine Fålle von GA, PA usf. darstellten, wobei ansonsten die "typischen" Adnominalmerkmale gegeben wăren (was nachzuweisen wăre!).

"Apposition" kann aber auch als eine der Attribution gleichberechtigte syntaktische Funktion aufgefast werden, wobei nicht unbedingt nur adnominale Funktionen inbegriffen sein dürfen (vgl. dort, am Ufer,). Dabei teilte die "Apposition" nicht unbedingt alle Attributscharakteristika, man vgl.:

Satzgliedfunktionen: Subjekt, objekt, Adverbial etc.

Partikelfunktionen: Gradpartikel, Negation etc.

Gliedteilfunktionen: Attribut, Apposition.

Man müßte folglich zeigen kōnnen, daß in $(4.11,4.12)$ zwar zwei nukleusbezügliche Funktionen vorlägen, aufgrund deutlicher Unterschiede aber zwischen Attribut (4.11) und "Apposition" (4.12) differenziert werden müBte:

(4.11) (Das Laster) (der Trunksucht). (Die Universität) (in Erfurt).

(4.12) (Das Laster) (Trunksucht). (Die Universitä) (Erfurt).

Zudem wăre u.a. nachzuweisen, daß diese Konstruktionen hinsichtlich Funktion und syntaktischem Verhalten "gleichwertig" sind mit solchen des lockeren appositionellen Typs, falls man beides unter Apposition zusammenfassen will:

(4.13) Der Tenor Olaf Kreisch singt wie Troubadix.

(4.14) Der Tenor, Olaf Kreisch, singt wie Troubadix.

In jedem Fall ist eine Abgrenzungserörterung Attribut versus Apposition erforderlich! In Kap. 7 wird sich herausstellen, daB das "enge Muster" (4.13) der attributiven Relation zuzuweisen ist; das "lockere Muster" (4.14) wird dagegen den HOSPITANTEN zugeordnet, genauer den ZUSATZEN (Kap. 12, 15). Somit wird eine gesonderte Benennung des engen, attributiven Musters notwendig.

Denkbar ist auch eine vier-Teilung syntaktischer Funktionen, die der "Apposition" einen selbstăndigen Rang zuerkennt:

14 In diesem Sinne z.B. Ingel (1988: 603 ff.), der sowohl den "engen" Typ (Tante Pulalia) als auch den lockeren (Bulalia, meine Tante,) zu den Nomensatelliten (-attributen) zăhlt.

15 Die "Adnominalansicht" vertreten u.a. einige Gramatiken des Deutschen (z.B. Duden 1984: 593, Engel 1988: 806). Molitor (1979) tendiert dazu, eine eigenstăndige Relation anzusetzen. 
- Satzgliedfunktionen

- Partikelfunktionen
- Gliedteilfunktionen

- Apposition(en).

In diesem Fall müssen zwei Abgrenzungsdebatten geführt werden: Apposition versus Satzglied(funktion) und Apposition versus Partikelfunktion (dazu Rap. 8 ). Im folgenden finden sich mögliche Problemfälle:

(4.15a) Pia, lustig und fidel, trat ins Zimmer ein.

(4.15b) Lustig und fidel trat Pia ins Zimmer ein.

(4.15c) Pia trat, Iustig und fidel, ins Zimmer ein.

(4.16a) Kuno als Maler arbeitet dort.

(4.16b) Kuno arbeitet dort als Maler.

Bei (4.15) geht es um die Einstufung der drei Vorkommnisse von Iustig und fidel. Möglich wären die Funktionen "Apposition" (v.a. in 4.15a), "modales Adverbial" (v.a. 4.15b; als variante "prádikatives Attribut", das sich wie ein Modaladverbial verhălt, vgl. Kap. 8), evtl. auch "Parenthese" (4.15c).16 In (4.16) wäre zu überprŭfen, ob "Attribut" oder "Apposition" (4.16a) bzw. ob ein Satzglied (Adverbial? 4.16b) oder eine "abgerückte Apposition" (ein abgerũcktes Attribut) vorliegt (dazu Kap. 7.B, 8).

Die Klärung der soeben angeschnittenen Fragen wird auf die speziellen Rapitel verschoben, doch erscheint bereits jetzt deutlich, das die "Apposition" (spăter: der Zusatz) sich mit den syntaktischen Funktionen berührt. Im Verlauf dieser Arbeit wird daher dafür argumentiert, daB Apposition resp. Zusatz als syntaktische Relation bzw. Funktion einer Spracheinheit $Y$ bzgl. einer Spracheinheit $X$ aufzufassen ist.

(d) "Apposition" ist eine Bezeichnung für "Anreicherungen" eines Satzes durch "Einschaltungen" unterschiedlichster Kategorien. Diese Einschaltungen weisen besondere Eigenschaften auf: graphematische bzw. prosodemische Markierung (Einschaltungsmuster), nur schwache grammatische Einbindung in den Gastsatz etc. Den Einschüben gemeinsam ist die (zugegeben: vage) pragmatische Funktion der "zusătzlichen, sekundaren Information". In etwa diesem Sinne - im Detail natürlich wesentlich differenzierter - entwickelt Raabe (1979: 329 et pass.) den Appositionsbegriff. Diese Appositionsauffassung ahnelt einer bestimmten Fassung des Parenthesebegriffs (z.B. Schwyzer 1939, Winkler 1969, Sommerfeldt 1985; mit jeweils etwas unterschiedlicher Fassung des Phănomenbereichs), der Einheiten wie Anreden, Schaltsătze, Interjektionen, eingeschaltete Satzglieder, nominale Appositionen etc. umfast:

16 Sofern man "Parenthese" iberhaupt als syntaktische Punktion auffassen will. Es liegt wohl naher, sie in Zusammenhang mit konstruktionalen Aspekten vie Satzfolge, -reihumg, -verknüpung etc. zu behandeln. 


\author{
$\mathrm{p}+$ meine damen und herren $\mathrm{p}+$ \\ $\mathrm{p}+$ das sei noch hinzugefügt $\mathrm{p}+$ \\ (4.17) /pia p+ hurra $\mathrm{p}+$ verlieb den kerl $\mathrm{ft+}$ /. \\ $\mathrm{p}+$ weil es so sein muste $\mathrm{p}+$ \\ $\mathrm{p}+$ eine Ornithologin $\mathrm{pt}$
}

Bei Fällen wie diesen zeigt sich die Notwendigkeit zu diskutieren, ob "Apposition" ein besonderer Fall von "pragmatischer Funktion" sei, also in eine GroBklasse mit Phänomenen wie KORREKTUR, ANREDE etc. gehörte, denn offensichtlich benutzen diese funktionell sehr verschiedenen Einheiten ähnliche grammatische Muster (Einschaltungsmarkierung usf.). 17 Es stellt sich die Frage, ob man nicht aufgrund des gleichen Musters eine einzige grammatische Funktion annimmt und diese dann nach pragmatischen Gesichtspunkten aufgliedert. (4.18) verdeutlicht die Problematik "Apposition" vs. "pragmatische Funktion" (Kap. 11-15):

(4.18a) Der Dickhäuter, (übrigens) ein Elefant, näherte sich.

(4.18b) Ein Pachyderm (ein Dickhäuter) năherte sich.

(4.18c) Der Dickhäuter, der groBe Dickhäuter, näherte sich.

(4.18d) Ein Elefant, (nein:) ein Nashorn(,) näherte sich.

(4.18a) zeigt den "Appositionsprototypen". Wie sehr ähneln ihm die folgenden Muster, in denen der ZUSATZ ein Fremdwort erlăutert (4.18b), eine Präzisierung (4.18c) bzw. eine Korrektur (4.18d) vornimmt? Es wird sich herausstellen, das eine rein pragmatische Gliederung nicht die einzig mögliche ist; zwischen den Einschaltungsmustern lassen sich auch deutliche grammatische Unterschiede herausarbeiten (v.a. Kap 12-15). Diese korrelieren bis zu einem gewissen Grad auch mit der jeweiligen pragmatischen Funktion des zusatzes.

Die Verwandtschaft von Appositionen und Parenthesen ist ofters erwăhnt worden (Schwyzer 1947: 9 f.; Altmann 1981: 63; Heidolph u.a. 1981: 292 f.); diverse Konstruktionen, die in einen "Gastsatz" eingefügt werden können, benutzen offenbar das gleiche "Einschaltungsmuster". Somit wird eine Abgrenzungsdiskussion "Apposition" vs. Parenthese bzw. "Apposition" vs. "Einschaltungen aller Art" notwendig (Kap. 9, 10; v.a. Kap. 11 und 12-15). Auch gegenüber den "Herausstellungen" sollten "Appositionen" abgegrenzt werden; Altmann (1981: 57 f., $289 \mathrm{ff.}$ ) hat auf die Verwechselungsmöglichkeiten von APPOSITION, NACHTRAG und RECHTSVERSETZUNG hingewiesen.

17 wăhrend syntaktische Funktionen als Relationen zwischen Spracheinheiten aufgefaßt werden, ist unter "pragmatischen Funktionen" die kommunikations- oder auch hörerbedingte Funktion einer Spracheinheit $\mathrm{zu}$ verstehen. So ist z.B. die Funktion von meine damen und herren in (4.17) die Aufnahme (bzw. Aufrechterhaltung) des Rontakts mit der Börerschaft. 


\section{APPOSITION UND GRAMMATIRMODELLE}

In diesem Kapitel sollen Vorschläge zur grammatischen Behandlung von appositionellen Konstruktionen skizziert werden, und zwar im Rahmen der folgenden Modelle: (a) Konstituentenstruktursyntax, (b) X-Bar-Syntax, (c) Dependenzgrammatik und (d) Rategorialgrammatik. Am Ende dieses Kapitels schlägt der Verfasser ein eigenes Modell vor, in dessen Rahmen man die "Apposition" bzw. die ganze Problematik der "Einschübe und Zusätze" im Deutschen behandeln kann.

Die erwähnten Modelle werden kurz erläutert, da im Folgenden manchmal auf sie Bezug genommen wird; zudem soll verdeutlicht werden, daB in den Grammatikmodellen selbst die Vorschläge zur Behandlung und Einordnung von "appositionsverdächtigen" Konstruktionen sich sowohl an Zahl als auch an Umfang eher gering ausnehmen; nicht selten werden Appositionen "en passant" abgehandelt. Die gröBte Aufmerksamkeit wird ihnen seitens der X-Bar-Theorie (Jackendoff 1977; zum Deutschen Vater 1986 b) geschenkt. Zudem soll gezeigt werden, daB die von den Modellen angebotenen Analysen nur teilweise miteinander vereinbar sind.

Die Diskussion der Vorschläge in den einzelnen Modellen wird knapp gehalten, denn es geht hier nicht um prinzipielle Modellkritik, sondern um die Frage, inwieweit die Lösungsangebote der Modelle verwertbar und fruchtbar sind für eine grünliche Durcharbeitung der Appositionsproblematik. Wo es geboten schien, habe ich (hoffentlich im Sinne der Modelle) über die referierten Analysevorschläge hinaus eigene Vorschläge unterbreitet.

Die Modellvorstellungen der generativen Transformationsgrammatik wurden ausführlich in Raabe (1979: $154 \mathrm{ff}$.; dort Angaben zur Spezialliteratur) diskutiert und brauchen hier nicht vorgestellt zu verden. Als (pauschales) Fazit kann im "gTG-Paradigma" folgende Vorgehensweise festgehalten werden:

Die Apposition als oberflächenstrukturelle syntaktische Erscheinung wird aus expliziteren, vornehmlich aus verbal-prådikativen satzförmigen Tiefenstrukturen durch Reduktions- bzw. Tilgungsoperationen erzeugt. Als Tiefenstrukturen werden häufig nichtrestriktive Relativsätze mit der Kopula sein bzw. parenthetische Kopulasătze angenommen, wobei das Appositiv im zugrundeliegenden Satz. meist (nicht immer, s. Motsch 1965, Raabe 1975) als Prädikativ fungiert. Getilgt verden dann Kopula und Subjekt und übrig bleibt das zur Apposition gewordene Prädikativ (vgl. 5.1a, b). Manchmal werden aber auch andere Verben als die "typischen" Kopulaverben zugelassen: ${ }^{1}$

(5.1a) Pia, (die) meine beste Freundin (ist), wird mich besuchen.

(5.1b) Kuno, (er war) früher ein Fan von de Sade, liebt heute simmel.

(5.1c) Ein Kerl, (er heist) Kuno Knall, hat den Schaden verursacht.

1 Vgl. Raabe (1975: 326), der annimnt, "(...) daß es falsch ist, nur parenthetische Ropulasatze als Basis für NPAP'-Ronstruktionen anzumehmen". Beispiel (5.1d) von Raabe (ebd.). 
(5.1d) Frau Grzyb, (Frau Grzyb besitzt (sitzt an)) Apparat 90, erwartet ihren Anruf.

Der Ansatz, aus einer gemeinsamen Tiefenstruktur (oder einigen wenigen gemeinsamen Tiefenstrukturen) die diversen oberflabchenstrukturellen Appositionskonstruktionen ableiten zu wollen, führt dazu, daß oberflächenstrukturell sehr verschiedene Konstruktionsmuster zu einer Funktion (Klasse) zusammengefaBt werden: zur Apposition. In dieser Arbeit wird davon ausgegangen, daB dies weder zweckmäBig noch notwendig ist, ohne daß damit der Wert transformationeller Erklărungsmodelle geschmălert werden soll. Beispielsweise kann man mit ihnen zeigen, daß oberflächenstrukturell verschiedenen Syntagmen die gleiche oder zumindest eine ăhnliche Konstruktionssemantik innewohnt. ${ }^{2}$ Eine Dominanz der Tiefenstruktur uber die oberflächenstruktur wird jedoch vom Verfasser abgelehnt. Zumindest kann m.E. eine gemeinschaftliche transformationelle Beziehung (eine gemeinschaftliche Tiefenstruktur) nicht allein schon eine grammatische Kategorie bzw. Funktion begründen. Ohne eine Bündelung dieser Gemeinsamkeit (bei den Appositionen die Kopulasatztiefenstruktur) mit weiteren Merkmalen bleiben die erreichten Generalisierungen und Einordnungen zu grob. Als Beispiel sei die gemeinsame Tiefenstruktur (5.2a) und die beiden Oberflächenstrukturen $(5.2 b, c)$ genannt:

(5.2a) Der Tiger schnurrt. Der Tiger ist jung und friedlich.

(5.2b) Der junge und friedliche Tiger schnurrt.

(5.2c) Der Tiger, jung und friedlich, schnurrt.

Die verschiedenen Oberflächenstrukturen, Attribut und "lockere Apposition", würde man aufgrund der gemeinsamen Tiefenstruktur wohl kaum zusammenfassen wollen, da deren grammatisches Verhalten weitgehend differiert (s. Kap. 6, 7).

In einem anderen Ansatz verden Appositionen (5.3b) mittels Phrasenstrukturregeln (5.3a) direkt (ohne Transformationen) erzeugt, z.B.:

(5.3a) NP, NP (NP)

(5.3b) Mir (NP) (Mănner (NP)); Giesbert (NP) (, ein Süffler, (NP)) etc.

\subsection{Apposition und Konstituentenstruktursyntax}

In einer Konstituentenstruktursyntax, welche "besteht-aus"- bzw. "Teil-Ganzes"-Relationen explizieren will, stellen sich hinsichtlich "appositionsverdachtiger" Konstruktionen folgende Fragen: Ist die Apposition Bestandteil ei-

2 so kann 2.B. durch die Rijckführung auf die Satz-Tiefenstruktur in (F5.1) die gemeinsame 'Besitzer-Besitz'-Semantik der Oberflächenstrukturen in (F5.2-F5.5) gezeigt resp. nachgerriesen werden:

(F5.1) Der Vater besitzt Bäuser.

(F5.2) die Báuser van Vater

(PRAPOSTTIONDLATTRIBUT') .

(55.3) die Bauser des Vaters

(GONTTVATIRIBUT).

(15.4) die väterlichen băuser

(ADNETIVATIRUBUT).

(F5.5) die Vaterhususer

(DETLRRMNATTVKCOMPOSTTUM) . 
ner Ronstruktion (einer Phrase) des Trägersatzes? Welches ist der Rnoten, der das Appositiv dominiert? Man betrachte einige Vorschläge (5.4 aus Raabe 1979: 123, leicht abgeändert; 5.5 aus Heidolph u.a. 1981: 296; 5.7 aus Lehmann 1984: 262, "NS" = "Nominalsyntagma" = NP):

$(5.4)$

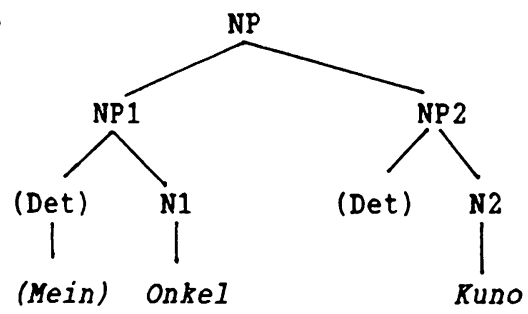

$(5.6)$

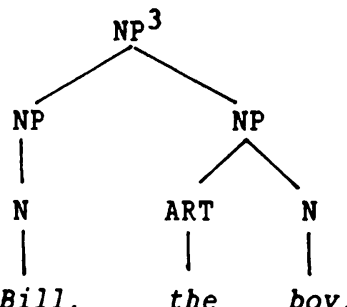

$(5.5)$

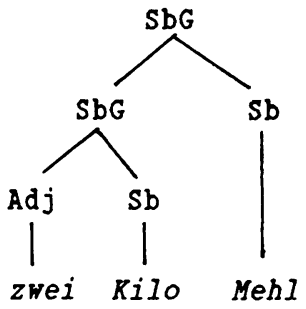

Auch in der "kontextfreien konstituentensyntax" von Clément/Thümmel (1975), in der Konstituenten vor allem als Distributionsklassen aufgefaBt werden (ebd. 11 f.), finden sich appositive Relativsätze und NP-Appositionen des "Prototyps" in die NP eingebettet. Der Knoten NP dominiert direkt zunăchst die Knoten Det (Determinantien) und den Knoten $\mathrm{N}_{6}$, das sind Nominale, die im Bezugsbereich von Determinantien liegen. $N_{6}$ wird in $N_{5}\left(N_{5}\right.$ ist dann weiter aufspaltbar und enthălt sowohl das Kernnomen wie auch 2 .B. restriktive Attribute etc.) und eine weitere Ronstituente " $\$$ " aufgespalten, die unter anderem durch einen appositiven Relativsatz bzw. eine lockere Apposition besetzt sein kann:

$(5.8)$

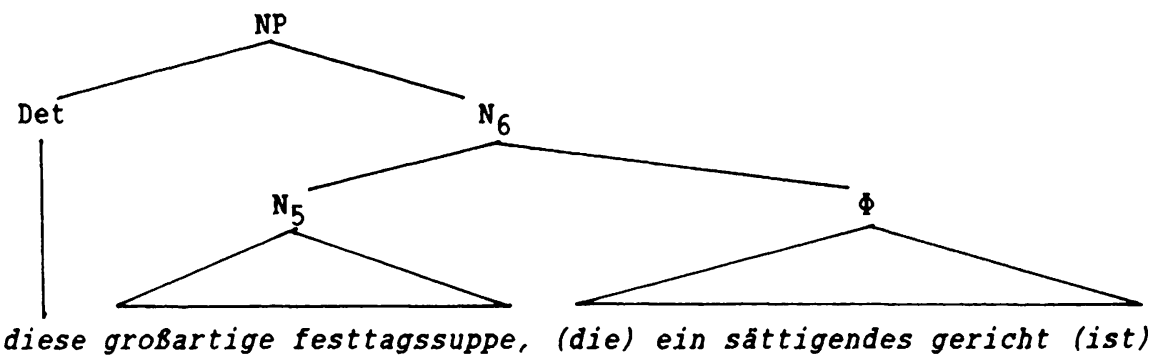

Interessant ist, daB die Einbettung der Parenthesen, die öfters mit Appositionen in Verbindung gebracht werden, hierarchisch höher erfolgt (also nicht un-

3 Delorme/Dougherty (1972: 9). Es ist anzunehmen, daß auch die "Akademie-Grammatik" (Heidolph u.a. 1981) das lockere Appositiv, ebenso wie den NP-bezogenen Schaltsatz, unter den NP-Knoten hắngen. Es wird davon gesprochen (ebd. 293), daß "(...) parenthetische Einschube einer SbG zugeordnet werden (...)", daß ein solcher Einschub jedoch "(...) der SbG zwar zugeordnet, aber nicht eigentlich untergeordnet ist (...)". 
ter NP). Unter dem in dieser Syntax ranghöchsten Rnoten "Phrasis ( $\Phi)$ " befinden sich die Interjektionsphrase (IP) und die Konjunktionsphrase (KjP); letztere kann expandiert werden $z$ Satzkomplex $2\left(S_{2}\right)$ und weiteren Konjunktionsphrasen. $S_{2}$ enthält fakultativ Parenthesen und obligatorisch Satzkomplex $1\left(\mathrm{SR}_{1}\right)$ : (5.9)

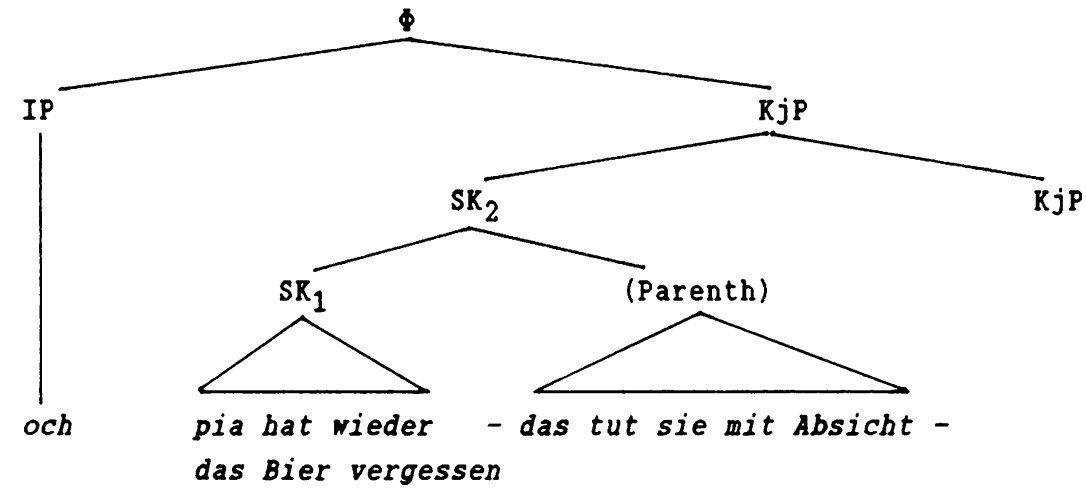

"Appositionsverdăchtige" Muster, die "enge" (5.4, 5.5) und die "lockere" (5.6, 5.7, 5.8) Apposition, werden als Konstruktionen mit zwei unmittelbaren Konstituenten (Basis und Appositiv) angesehen. Der Schaltsatz (als Parenthese) wird unterschiedlich eingestuft: In (5.9) ist er keine NP-, sondern Satz-Konstituente. Bei Heidolph u.a. (1981: 293) findet sich dagegen folgender strukturierungsvorschlag:

$(5.10)$

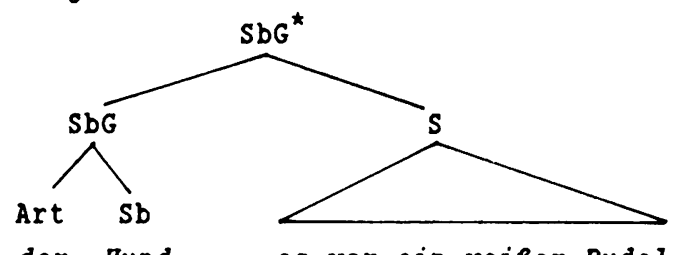

Rommentiert wird er folgenderweise (ebd.):

"Wenn solche parenthetischen Einschübe einer SbG zugeordnet werden, ergibt sich, ahnlich wie bei der Roordination, eine Erweiterung der Konstituentenstruktur (...). (...) Dadurch, das S der SbG zwar zugeordnet, aber nicht eigentlich untergeordnet ist, kann es seine intonatorische Selbstandigkeit erhalten".

Hier wird an kein Konstituenzverhăltnis im gewöhnlichen sinne gedacht, da von "Zuordnung" und nicht von "Unterordnung" die Rede ist! Das ist ein Hinweis darauf, daß sich möglicherweise neben Konstituenz (Teil-Ganzes-Beziehung mit Unterordnung) und Unabhăngigkeit noch ein drittes Verhăltnis zwischen Konstituenten annehmen labt, das man "Zuordnung" oder - wie ich spăter sage - "Assoziiertheit" nennen könte. In jedem Fall mus darauf geachtet werden, ob das herkömmliche Konzept der Ronstituenz gleichermaßen auf alle appositionsverdächtigen Muster mit Bezugselement zutrifft. 
Die oben angeführten Strukturierungen werden auch durch die Anwendung folgender sog. "Ronstituententests" nahegelegt: 4

- Topikalisierungstest (im Vorfeld steht eine Konstituente), z.B. Mein Onkel Kuno/ Jene Pia, die immer lacht, spinnt.

- Pro-Form-Test (gesamt ersetzbar ist eine Konstituente), z.B. Mein Onkel Kuno/ ER; Jene Pia, die immer lacht, / SIE

- Fragetest (erfragbar sind Konstituenten), z.B. Wer spinnt? Mein Onkel/ Mein Onkel Kuno/ Kuno.

Welcher Onkel spinnt? Mein onkel $K$ u no.

Welcher Kuno spinnt? Mein O nkel Kuno.

- Weglastest (weglaßbar sind Konstituenten), z.B. Mein Onkel Kuno/ Mein Onkel/ Kuno spinnt.

Die Appositive oben (5.4-5.7) reagieren überwiegend positiv auf die Tests (man prüfe es selbst nach); im folgenden finden sich nur die Resultate sowie Zweifelsfälle, die andeuten, daß jene Tests nicht immer unproblematisch sind:

$(5.4) \quad(5.5) \quad(5.6) \quad(5.7)$

$\begin{array}{llllc}\text { Topikalisierung B + A: } & \text { ja } & \text { ja } & \text { ja } & \text { ja } \\ \text { Pro-Form B + A: } & \text { er } & \text { die } & \text { he } & \text { die } \\ \text { Erfragung B + A: } & \text { wer? } & \text { was? } & \text { wer? } & \text { wer? } \\ \text { Erfragung B: } & \text { welch-? wieviel? wer? } & \text { wer? } \\ \text { Erfragung A: } & \text { welch-? was? } & \text { wer? } & \text { ? } \\ \text { weglasbar } & \text { A, B } & \text { A, B } & \text { A, B } & \text { nur B }\end{array}$

(5.11) Wer spinnt? Jene Pia/ ??Die immer lacht. Jene Pia/ ??Die immer lacht spinnt.

Die Testresultate legen es nahe, daß jeweils eine Konstruktion mit zwei unmittelbaren Konstituenten vorliegt. Dennoch ist ein genaueres Hinsehen notwendig, weil die Proben im Detail Probleme aufwerfen und z.T. widersprüchliche Ergebnisse erbringen. Die Konstituententests werden u.a. in Kap. 6, Abs. 22, Kap. 7 (!) und Kap. 15, Abs. 22 erörtert. Daher verweise ich nachstehend nur auf ein paar Problempunkte.

(a) Die Eindeutigkeit des Topikalisierungstests wird dadurch eingeschrănkt, daB nicht alles vor dem Finitum (im Verbzweit-Deklarativsatz) notwendigerweise zum Vorfeld (VF) zăhlt - es sind alternative, feinere strukturierungen denkbar (5.12). Z.T. dürfen sich "Basis" und "Appositiv" gar nicht gemeinsam im Vorfeld befinden (5.13-5.15).

(5.12) Apropos Haie - der Katzenhai, lieber Udo, schmeckt recht lecker. vor dem VF VORFEL D Parenthese Finitum MITTELFELD Apropos Fall - Eulalia Huber, Romanistin, erforscht den Genitiv.

4 Zu den Testverfahren z.B. Grevendorf u.a. (1987: $158 \mathrm{ff.}$ ). 
(5.13) Kuno (B) (*krank (A)) kam (krank) nach Hause.

(5.14) Pia spinnt (Satz = B), eine bedauerliche Tatsache (A). *Eine bedauerliche Tatsache spinnt Pia.

(5.15) Sie lachte über ihn, und zwar wegen seiner Machoallüren. ??Und zwar wegen seiner Machoallüren lachte sie über ihn.

(b) In (5.16) ist es nicht statthaft, mit der Pro-Form "Basis" und mutmaBliches "Appositiv" gesamt zu ersetzen; vielmehr können beide Einheiten nur getrennt ersetzt werden. Dieses Verhalten spricht dafür, zwei unabhängige Konstituenten bzw. Satzglieder anzusetzen, nämlich Subjekt/Objekt und prädikatives Attribut:

(5.16) Kuno (B?)/ Er kam krank (A?)/ so nach Bause. Pia aB die Suppe (B?)/ sie heiB (A?)/ so.

(c) Die Ziele von Fragetests lassen sich so formulieren: Was man zusammen erfragen kann, ist eine Konstituente. Was durch eine "Gliedteilfrage" (welch-, was für ein-) erfragt werden kann, ist eine Ko-Konstituente:

(5.17) Pia, meine beste Freundin, /, Juristin, kommt zu Besuch.

Wer kommt zu Besuch? Pia, meine beste Freundin/ Pia/ Meine beste Freundin/?Pia, Juristin/ *Juristin.

(5.18) Meine Freundin Pial Meine Freundin, Pia, kommt zu Besuch.

Welche Freundin kommt $z u$ Besuch? Meine Freundin $P i$ al «eine Freundin, $P$ i a.

Welche Pia kommt zu Besuch? Meine Fr e undin Pia/ *Meine Fr e undin, Pia.

Die gesamte Erfragung von Basis und Appositiv ist teils unproblematisch, teils jedoch fragwürdig (5.17). Eigenartig mutet es auch an, wenn auf die Frage nach dem ganzen Satzglied manchmal sogar nur eine Teilkonstituente antworten kann (und manchmal nicht; vgl. 5.17, 5.18).

Die Fragen nach einem Gliedteil (einer Teilkonstituente innerhalb einer Ronstruktion) führen dagegen $z$ unterschiedlichen Resultaten. Nur bei einigen engen Mustern können beide Teile der mutmaslichen Appositionskonstruktion erfragt werden (5.18). In Rap. 7 wird sich herausstellen, daB Gliedteilfragen nur auf Attribute, nicht aber auf "wirkliche" Appositionen - die ich spater Zusatze nenne - anwendbar sind.

(d) Der Weglastest spielt bei manchen Appositionsdarstellungen eine Rolle. So führen Helbig/Buscha (1980: 537) als Appositionsmerkmal an, daB das Appositiv die Basis im Satz syntaktisch zu vertreten vermag. Dadurch erhalt man zwar einen relativ engen Appositionsbereich (5.19 gehörte z.B. dazu), schliebt aber Konstruktionen aus (5.20), die offenkundig zum gleichen Typ gehören, nur veil sie minimale Unterschiede aufweisen: 5

(5.19) Seine beiden Söhne, Andreas und Michael, sind nett.

Seine beiden Söhne/ Andreas und Michael sind nett.

5 Beispiele 2.T. van Helbig/Buscha (1980: 538 ff.) inspiriert. 
(5.20) Sein Sohn, Modedesigner,/ Sein Sohn/ * Modedesigner ist nett.

Seine beiden Söhne, lustig und fidel, kamen herein.

Seine beiden Söhne/ *Lustig und fidel kamen herein.

Wenn man davon ausgeht, daß bei möglicher Weglasbarkeit eines Elements dies das Appositiv (der Satellit, das Dependens etc.) sein müste, hătte man einen Test zur Bestimmung von Nukleus-Satellit resp. Basis-Appositiv zur Hand. Relativiert wird der Eliminierungstest jedoch dadurch, daB außer den satzkonstituierenden valenztrăgern und deren notwendigen Ergänzungen so ziemlich alles weitere weglaßbar ist; somit ist er kein scharfes Analyseinstrument.

Zusammenfassend lăßt sich sagen, daß vor allem die gemeinsame Vorfeldfăhigkeit, die gesamte Ersetzung (durch eine Pro-Form) und die gesamte Erfragung bei vielen Mustern nahelegen, daß Basis und Appositiv (mindestens beim "engen" und wohl auch beim "lockeren" Typ - weniger dagegen z.B. beim Schaltsatz) Teile einer Konstruktion darstellen. Im Detail konnten allerdings auch problematische Effekte von Konstituententests entdeckt werden.

Der Verf. könnte sich angesichts der aufgebotenen Testevidenz mit der Konstituenz von Basis und Appositiv, zumal bei Mustern wie Tante Pia etc., durchaus zufriedengeben, wenn nicht ein gewisses Unbehagen z.B. gerade beim "Prototypen" (Pia, Modedesignerin, etc.) bestünde. Es speist sich vor allem aus folgender Quelle: der Anwesenheit des Einschaltungsmusters, das bewirkt, daB das "Appositiv" von Operationen (wie z.B. Negation) aus dem Gastsatz heraus abgeschirmt ist ("syntaktische Desintegration"); man beachte vor allem den Gegensatz zum engen Muster:

(5.21) *Nicht Pia, Modedesignerin, schnarcht, sondern Pia, Graphikerin.

(5.22) Nicht die Modedesignerin Pia schnarcht, sondern die Graphikerin Pia. In Kap. 6 wird der "Prototyp" hinsichtlich der Konstituenzfrage genauer geprüft. Auch die i.e.s. appositionsverdăchtigen Muster (spăter ZUSATZE genannt; s. Kap. 15), werden daraufhin zu prüfen sein.

\subsection{Apposition und X-Bar-Syntax}

Die $X$-Bar-Syntax ist eine Phrasenstrukturtheorie, die einen strukturell weitgehend analogen internen Aufbau von Phrasen wie NP, VP, PP und AdjP postuliert, und zwar dergestalt, daB ein lexikalischer Rern vorhanden ist, zu dem Komplemente dreier unterschiedlicher "Rangstufen" sowie spezifizierer (SPEC; das sind z.B. beim Nomen die Determinatoren und diverse Quantoren, beim Verb v.a. die Auxiliarverben) treten: ${ }^{6}$

6 Als Kerne (Röpfe, heads) kommen infrage: N, V, Adj, Präp; evtl. auch Adv. Ich entschließe mich hier für drei Bar-Stufen, ohne dies näher zu diskutieren und ohne damit andere Bar-Ronventionen als weniger beschreibungsadäquat zu werten. Statt der üblicheren Notation $X^{\prime}, X^{\prime}, X^{\prime}{ }^{\prime}, X^{\prime}{ }^{\prime}$ ' verwende ich $X_{0}, x_{1}, X_{2}, X_{3}$. 
(5.23)

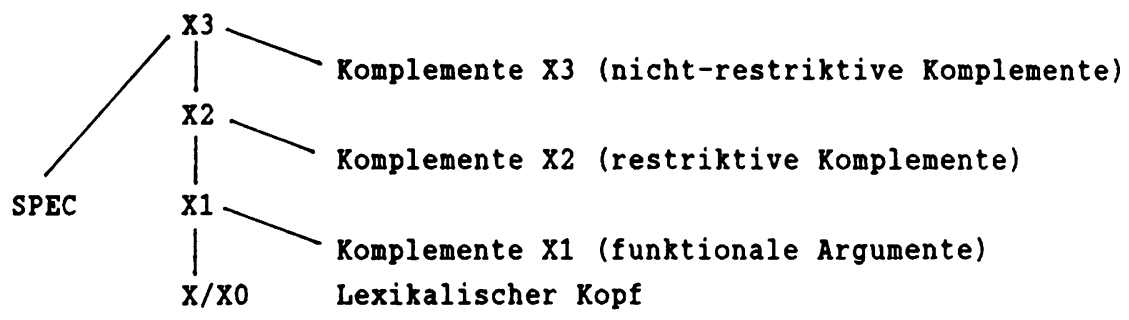

Die $\mathrm{X}$-Bar-Theorie bezieht in die strukturierung der Phrasen die Funktionen derjenigen Elemente mit ein, die als Komplemente zum Kern treten können; die nachstehende obersicht geht bereits spezieller auf die uns interessierenden NP-Strukturen ein (zur Veranschaulichung vgl. 5.24): 7

(a) Auf der Ebene $\mathrm{X1/N1}$ werden die "funktionalen Argumente" angesiedelt. Es handelt sich um die Komplemente, die das Kernlexem strikt subkategorisiert, m.a.H. valenzmåBig fordert bzw. bestimmt, wie z.B. die objekte des Verbs und obligatorische bzw. fakultative Nomenergänzungen.

(b) Auf der Ebene $\mathrm{X} 2 / \mathrm{N} 2$ befinden sich die "restriktiven Komplemente" (RK). Bei einer NP wăren das jene Attribute, welche die Denotatsmenge der Kern-NP einschrănken, so z.B. restriktive Relativsătze, Adverb(ial)attribute und PPn.

(c) Auf der $X 3 / N 3-E b e n e$ finden sich die "nicht-restriktiven bzw. appositiven (!) Komplemente", welche die Denotatsmenge unangetastet lassen, also z.B. appositive Relativsătze, NP-Appositionen und Parenthesen. Sie treten erst zur voll spezifizierten NP und beeinfluBen die Extension einer NP resp. den Wahrheitswert eines Satzes nicht.

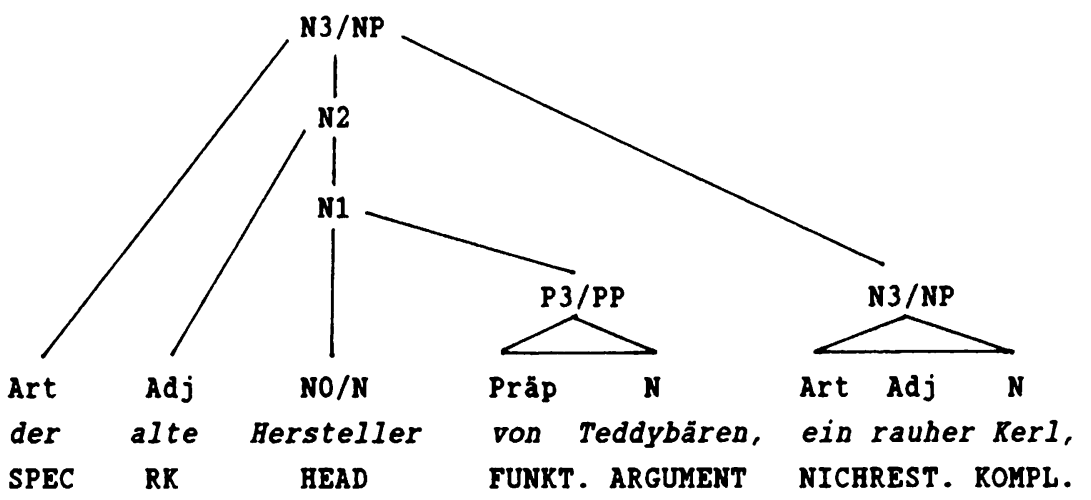

7 Ich folge hier in mesentlichen der Darstellung in Vater (1986b); vgl. auch Vater (1985). Zur Weiterentwicklung des X-Bar-Ansatzes bgzl. deutscher NPn und zur Kritik an Vater s. Haider (1988). 
Diese Ebenen sind nicht nur funktional unterscheidbar, sie kristallisieren sich auch im syntaktischen Verhalten heraus. So folgen z.B. rechts vom "head" einer NP (also NO) N3- auf N2-Komplemente und N2- auf N1-Komplemente. 8

Gerade wegen der in dieser Theorie getroffenen Unterscheidung zwischen einem Level der "Appositive" (N3) und den anderen beiden Levels eignet sich diese Darstellungsweise gut für unsere $\mathrm{Zwecke.} \mathrm{Zudem} \mathrm{werden} \mathrm{innerhalb} \mathrm{dieser}$ Theorie mehrere Tests zur Differenzierung der Ebenen angewendet, die hilfreich sein können: 9 (a) "comma intonation", (b) "appositives are immune to the scope of quantifiers and negation" und (c) N3-Komplemente können zu (artikellosen) Eigennamen treten (N2 nicht).

Ich möchte nun nicht die der $\mathrm{X}$-Bar-Syntax innewohnenden Probleme zur Sprache bringen, mus aber dennoch einige methodische Setzungen erlăutern. Es besteht z.B. Uneinigkeit darüber, auf welchem Level die NP-Spezifizierer, etwa Artikel und Quantoren (wie viele, alle etc.), anzusiedeln sind. Vater (1985: $35 \mathrm{ff} . ;$ 1986b: $135 \mathrm{ff.}$ ordnet sie der Ebene N1 zu. Es ist auch erwogen worden, den Determinator als Kern der NP (dann natürlich besser: der Determinatorphrase (DetP)) anzusehen - eine relativ neue Hypothese, der ich nicht weiter nachgehe. ${ }^{10}$ Ich beziehe mich in dieser Frage auf folgende Fakten: Appositive Komplemente wie z.B. nicht-restriktive Relativsătze oder "lockere Appositionen" treten an voll spezifizierte, voll determinierte NPn (vgl. Lehmann 1984: $259 \mathrm{ff.}, 270 \mathrm{ff.}$, denn sie restringieren die nominale Bezugseinheit nicht mehr - sie fügen der NP eine Zusatzinformation hinzu. Dies spräche dafür, die SPEC unterhalb des "Appositionslevels" anzuordnen. Determinatoren überführen (komplexe) CN-Phrasen in Voll-NPs; ${ }^{11}$ gemäB des semantischen Kompositionalitătsprinzips operieren restriktive Komplemente zuerst auf dem Kernnomen (Extensionseinschrănkung), dann folgen die Artikel. Dies spiegelt sich in folgender kategorialgrammatischer Reprăsentation wider:12

(5.25) ((Das) $\left.\mathrm{NP} / \mathrm{CN}((\text { Haus }) \mathrm{CN}((\text { des }) \mathrm{CN} / \mathrm{CN} / / \mathrm{CN}(\text { Vaters }) \mathrm{CN}) \mathrm{CN} / \mathrm{CN})_{\mathrm{CN}}\right)_{\mathrm{NP}}$

$8 \mathrm{Vgl}$. Vater (1986b: 132): "Je enger ein Ramplement an sein Regens gebunden ist, desto näher bei ihm steht es". Im Englischen ist dies wohl die Regel; ab sie im Deutschen gleichfalls gilt, wäre zu prüfen.

9 Hier die N3-relevanten Tests von Jackendoff (1977: 63, 171 ff.).

10 Siehe Rolde (1985: $250 \mathrm{f}$.) mit weiteren Hinweisen zu diesem Thema. Vgl. vor allem den Beitrag Haiders (1988), der folgendes vorschlägt (1988: 41):

"Die "NP" ist (...) eine Determinatorenphrase, d.h. eine Projektion des funktionalen Haupts D [= Determinator; K.S.], die als Kamplement eine NP enthält, d.h. die maximale Projektion der lexikalischen Kategorie $\mathbf{N}^{\prime \prime}$.

11 s. auch Krifka (1983: 31): "Der Artikel scheint also ein Derivationsmittel zur Oberführung von Appellativen in Nomina [gemeint ist: NP; W.S.] zu sein".

12 vgl. Haider (1988: 38 f.). 
Ich bleibe allerdings bei der in (5.23, 5.24) vorgestellten Reprăsentation, wenngleich man eigentlich einen $\mathrm{Zwischenknoten} \mathrm{zwischen} \mathrm{N} 2$ und $\mathrm{N} 3$ einzuführen hatte, um darzustellen, daB ein Determinator "nach N2 und vor N3" operiert.

Die zweite Uneinigkeit herrscht bzgl. der Anfügung von Adjektivphrasen als Attribute. Vater (1985: $49 \mathrm{ff}$; 1986: 140) schlägt aufgrund von Distributionsargumenten N1 vor. Jackendoff (1977: $72 \mathrm{f}$. ) setzt N2 an, weil dies die Ebene der restriktiven Komplemente ist. Ich entscheide mich auch hierfür.

Nun zu den Vorschlägen. 13 Jackendoff (1977:63 f., 169 ff.) siedelt sowohl die "lockere Apposition" als auch den nicht-restriktiven Relativsatz auf der Ebene N3 an; dazu seine Beispiele (ebd. Geklammerte Zusătze vom Verf.):

(5.26) I will sell you these bagpipes (N3), the finest in all Poland, for only 4000 zloty.

She presented Picasso (N3), then in his blue period, with a blue berry pie.

$(5.27)$

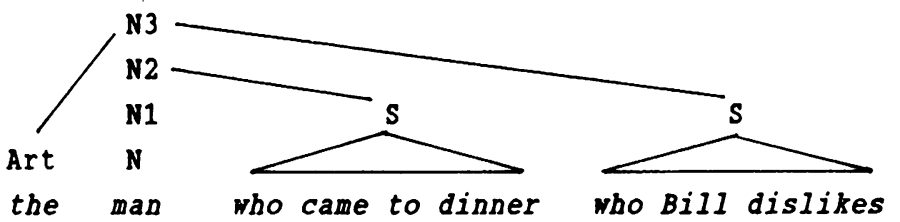

(restrictive)

(appositive)

Vater (1986b: $129 \mathrm{ff.}$ ) ordnet appositive Relativsätze, lockere Appositionen und Parenthesen (!) der N3-Ebene zu, enge Appositionen dagegen werden an N1 gefügt, da sie "(..) weder durch Valenzkomplemente noch durch restriktive oder appositive Komplemente erweitert werden können (...)" und im allgemeinen kein Determinans aufweisen (ebd. 131):14

(5.28)

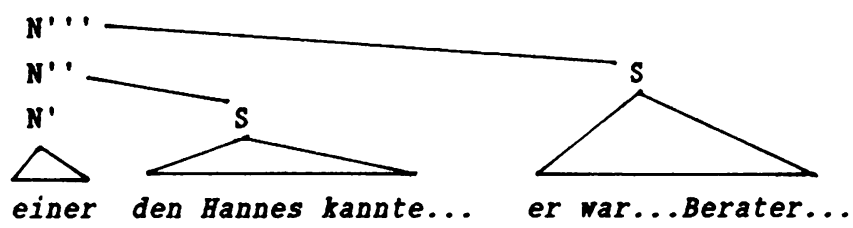

(5.29)

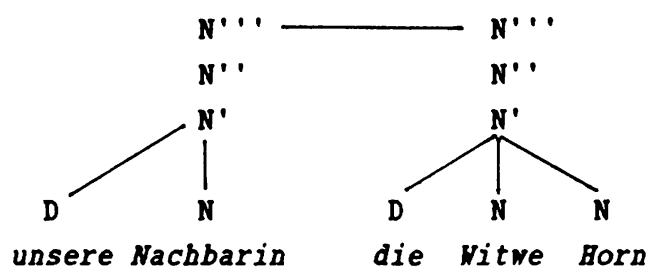

vaters unterschiedliche Zuordnung von engen und lockeren "Appositiven" ist insofern bemerkenswert, als ich in Kap. $7 \mathrm{zu}$ der Auffassung gelangen werde, daB

13 Eine hier nicht behandelte Arbeit mit $X$-bar-Vorschlăgen ist van Gestel (1986).

14 Vgl. auch Vater (1985: 27 f.). 
ein enges "Appositiv" tatsăchlich ein attributiver Modifikator ist, wăhrend lockere "Appositive" mit Recht als nicht-restriktive zusătze "ganz oben" angesiedelt werden. 15

Noch ein interessantes Detail: Kolde (1985) weist einschaltungsmarkierte Attribute ebenfalls der N3-Ebene $z u$ - ein nicht nur intuitiv überzeugender Vorschlag:

(5.30) Ich kann Ihnen noch dieses, übrigens voll unterkellerte, Haus anbieten.

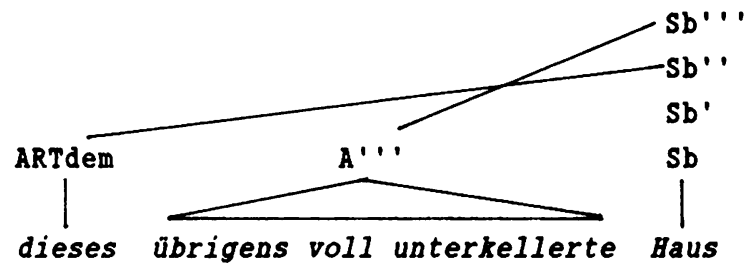

Im Rahmen der $X$-Bar-Syntax werden sămtliche nicht-restriktiven Zusătze, die auf eine NP bezogen sind - so auch NP-bezogene Parenthesen und sogar prănukleare Attribute mit Einschaltungsmarkierung - der N3-Ebene zugeordnet und somit als im Rahmen des Ansatzes hierarchisch höchstgelegene Teile (Ronstituenten) der NPn bestimmt; das scheint mir eine Darstellung zu sein, die sowohl von der formalen wie auch von der funktionalen Begründung her ansprechend ist und uberdies eine uberzeugende Parallelisierung von funktionalen (Nicht-Restriktivităt) und formalen (Nichtnegierbarkeit, Einschaltungsmarkierung, stellung ganz rechts etc.) Faktoren anbietet. Die Anordnung "enger Appositionen" auf einer niedrigeren $\mathrm{X}$-Bar-Stufe wird sich in Rap. 7 als gerechtfertigt erweisen, da sich dort ihr attributiver (restriktiver) Charakter zeigen wird.

\subsection{Apposition und Dependenzgrannatik}

Als Beispiel für eine dependenzielle Behandlung von Apposition sei Tesnière (1965; 1980; 5.32 aus Tesnière 1965: 163, ins Deutsche übertragen) herangezogen. Er ordnet die (lockere) Apposition dem "noeud substantival" zu, wenngleich die dependentielle Ronnexion (Beziehung) zwischen Bezugsnomen und Appositiv nicht vertikal, sondern horizontal reprasentiert wird:

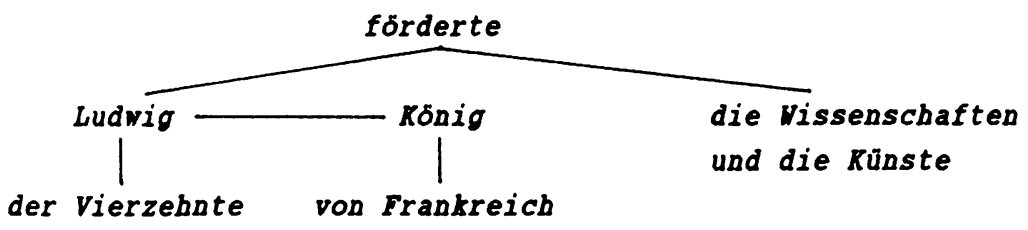

15 Jackendoff (1984) kammt zu dem Schlub, daB in Phrasen wie the actor John Gielgud die Dr-Phrase (die man als enges Appositiv bezeichnen kömte) wahrscheinlich auf der N2-Ebene anzusiedeln sei, wenngleich mancher Test auch für in spräche (ebd. $30 \mathrm{ff,} \mathrm{insb.} \mathrm{33).}$ 
Das Appositiv wird auf der gleichen Dependenzstufe angesiedelt wie das Bezugselement und kann somit nicht als "complément déterminatif" angesehen werden. 16 Eine Verbindung mit dem zentralen Regens (Verb) wird nicht angesetzt. Rlar geschieden wird die Apposition vom Attribut, bei dem eine vertikale Ronnexion besteht (vgl. von Frankreich zu König). Apposition wird von Tesnière weitgehend negativ bestimmt: weder Romplement noch Attribut noch Junktion etc. 17 Am ehesten zeige sie verwandtschaft mit koordinativen strukturen. 18

Der im Rahmen der Dependenzgrammatik arbeitende Tarvainen (1981: $98 \mathrm{f.}$ ) behandelt unter den "freien Angaben beim Substantiv" bzw. den "Angabeattributen" neben beispielsweise dem valenzunabhängigen Adjektiv- und dem Genitivattribut (z.B. genitivus possessivus) auch die Apposition, und zwar den lockeren Prototypen sowie die als-Phrase (Beispiele Tarvainen 1981: 99):

(5.33) Er kaufte einen grünen Teppich.

der Garten der Eltern.

Karl, mein bester Freund.

Die stadt als koniglicher Privatbesitz.

Tarvainen zufolge sind derartige Appositive valenzunabhangige Gliedteile, also ebenfalls Attribute wie das Adjektiv- oder Genitivattribut.

Eine interessante Bemerkung findet sich bei Engel (1982: 157; Unterstreichungen W.S.), der auch einen dependentiellen Ansatz verfolgt:

"Appositionen kommen nur bei Nominalphrasen oder Pronominalphrasen vor.

(...) Die gewăhlte Formulierung soll gleichzeitig zum Ausdruck bringen, daB Appositionen nicht Teile der Phrasen sind, denen sie zugeoranet werden".

In spăteren Arbeiten Engels $(1986 ; 1988)$ wird diese Ansicht, die leider nicht năher begründet wurde, nicht mehr vertreten; dort wird die Apposition vieder als besonderes NP-Attribut behandelt. "Enge Typen" wie Vetter stefan oder der Anwalt Rettig werden als fakultative Nomenergänzungen eingestuft, "lockere Appositionen" als Nomenangaben. 19

In einer Dependenzgrammatik geht es um die Abhăngigkeiten der satzteile untereinander, die sich vor allem in folgenden Phänomenen zeigt: (a) gerichtete Konkomitanz (wenn $X$ auftritt, kann auch $Y$ auftreten; wenn $X$ nicht auftritt, kann $Y$ nicht auftreten); anders formuliert: das Auftreten von $X$ ist Vorbedingung fưr das Auftreten von $Y$, weil es die Auftretensmodalităten von $Y$ (mit)

16 Vgl. Dupant (1985: 55).

17 Vgl. Dupant (1985: 57).

18 Tesnière (1965: 326): "Te trait de jonction est de forme identique au trait d'apposition (....) puisque tous deux sant horizontaux. Toutefois il est impossible de les confondre."

(F5.6)

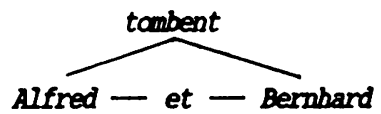

(jonction)
(55.7)

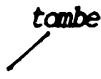

Alfred - man fils

(apposition)

19 Vgl. Drgel (1988: 610, $616 \mathrm{ff} ., 806 \mathrm{ff}$.$) .$ 
bestimmt; (b) Subkategorisierung von Rategorien (z.B. Vollverb bzgl. seiner Ergănzungen); (c) Zuweisung von Rektionsmerkmalen (Rasus, Genus); ${ }^{20}$ (d) $\underline{\text { sub- }}$ kategorisierung von Funktionen (Vollverb bzgl. seiner Ergånzungen); (e) $\underline{\mathrm{Zu}-}$ weisung von thematischen Rollen (z.B. Vollverb an seine Ergănzungen).

Dependenzverhăltnisse spielen in jedem Fall eine Rolle bei den engen Mustern des Typs onkel Kuno, mein onkel Kuno etc., 21 wohl aber nicht bei den "lockeren" Mustern (mein Onkel, ein Angestellter,), da offensichtlich keine Subkategorisierungsforderungen von seiten der Basis wirksam werden. Eine Zuweisung von Rektionsmerkmalen, Funktion und thematischer Rolle labt sich ebenfalls nicht ausmachen. Somit kann im Rahmen einer Dependenzgrammatik von den lockeren "Appositiven" tatsăchlich nicht mehr gesagt werden, als dab sie freie Angaben zum Nomen bzw. zur NP darstellen.

\subsection{Apposition und kategoriale Gramatik}

In kategorialen Grammatiken wird darauf abgezielt, syntaktische Operationen (z.B. Attribution) und semantische Operationen (z.B. Begriffsmodifikation) parallel zu repräsentieren. Einer syntaktischen operation soll eine semantische operation entsprechen. Wichtig ist dabei die sog. "Spezifikationsrelation": In einem Syntagma spezifiziert eine Ronstituente X (als Operator/Funktor/Spezifikator) eine Konstituente $Y$ (als Operand/Argument/Spezifikat), wobei sich zwei Moglichkeiten unterscheiden lassen: (a) wenn sich nach der Operation von $X$ auf $Y$ die Rategorie von $Y$ nicht verăndert hat, dann heist $X$ "Attribut" von $Y ;$ (b) wenn $X$ eine Valenzstelle von $Y$ abbindet, dann heiBt $X$ "Romplement" von $Y .{ }^{22}$ Folgende Beispiele mögen dies veranschaulichen: ${ }^{23}$
$(5.34)$
ATTRIBUT ROPF
(5.35) ROMPLEMENT ROPF
(eine) (fleiBige studentin)
Runibert schnarcht
$\stackrel{\mathrm{CN} / \mathrm{CN}}{L}=\mathrm{CN} \stackrel{\mathrm{CN}}{\mathrm{C}}$

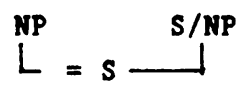

20 Nach Eisenberg (1986: 54 f.) liegt keine Cenuskongruenz zrischen Namina, Adjektiven und Determinantien vor; vielmehr regiert das Nomen - weil Genus eine Paradigmenkategorie desselben ist bei diesen mementen das cenus, das bei ihnen eine Binheitenkategorie darstellt.

21 Das betrifft mindestens Absatz 2. und 4.1.1. (evtl. auch 4.2.1. u. 4.2.2.) in Rapitel Phănomenbereich. Hierzu Engel (1988: 610, 616-618). Z.B.:

(15.8)

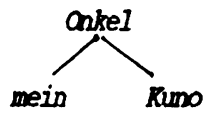

(F5.9) numo

ansel

22 Achtung Terminologiedschungel! "Romplement" in der X-Bar-Theorie bedeutet etwas ganz anderes.

23 "aN/ON" heißt 'ergibt mit einem aN ein neuerliches (kamplexeres) aN'; "S/NP" heißt 'ergibt mit einer NP einen Satz'. Die hier verwendete kategoriale Notation ist etwas krude, reicht für die Darstellungszwecke jedoch aus. 
Von Interesse ist nun, ob ein "Appositiv" in einer Spezifikationsrelation zu seinem Bezugselement steht. Krifka (1983) behandelt in seiner kategorialgrammatischen Darstellung des Swahili auch appositive Konstruktionen. Bei der "spezifizierenden Apposition" (entspricht "engen appositionellen Syntagmen") werden nach Krifka (1983: $28 \mathrm{f} ., 113 \mathrm{f}$.$) folgende Verhăltnisse angenommen:$

(5.36) Mister Jonston, jap. nakaso san; Herr Jonston.

(Der Eigenname spezifiziert den Titel.)

(5.37) Henry Jonston, jap. Nakaso Toshio; Pia Müller.

(Der Familienname spezifiziert den Taufnamen.)

(5.38) Die stadt München.

(Der Eigenname spezifiziert die stadt.)

Zur Verdeutlichung zwei Strukturrepråsentationen (Krifka 1983: 113, 114): 24

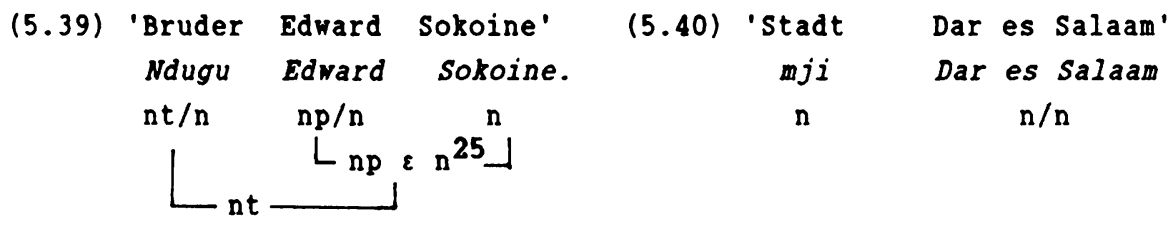

Sie zeigen, daß der Städtename als Attribut zu (die) stadt (bzw. Sokoine zu Edward und die Namensfügung $z u$ Ndugu) gewertet wird. Diese Annahmen verden (a) durch das Rriterium der Rategorienkonstanz (die Kategorie des Syntagmas ist syntaktisch entweder gleich derjenigen des operands oder zumindest insofern ăhnlich, als die Syntagmenkategorie eine Valenzstelle weniger aufweist als die Kopfkategorie) und (b) das Rriterium der universalen Relevanz gestützt; letzteres bezieht sich auf die Eigenschaft natürlicher Sprachen, Spezifikationsrichtungen topologisch relativ homogen $z u$ reprăsentieren, und zwar dergestalt, das in einer Sprache die Operatoren den Operanden (relativ) strikt voranstehen oder (relativ) strikt nachfolgen; man spricht dann von (relativ) konsistent pră- resp. postspezifizierenden Sprachen.

Des weiteren verden sog. "Klassifikatorkonstruktionen" in Augenschein genommen, die ja z.T. den (engen) Appositionen zugerechnet werden (Krifka 1983: $32 \mathrm{f.}$.). Er fast sie als "Adnominal-Nomen-Konstruktion" (ebd. 32), folglich als attributive Konstruktion, auf:

\begin{tabular}{|c|c|c|c|c|c|c|c|}
\hline$(5.41)$ & $\begin{array}{l}\text { three } \\
\text { drei }\end{array}$ & $\begin{array}{l}\text { glasses } \\
\text { Glas/Glasser } \\
\text { ROPF }\end{array}$ & $\begin{array}{l}\text { of beer } \\
\text { Bier } \\
\text { ATTR }\end{array}$ & $(5.42)$ & $\begin{array}{l}\text { san } \\
\text { drei }\end{array}$ & $\begin{array}{c}\text { satsu-no } \\
\text { Flachding-GEN } \\
\text { ATTR }\end{array}$ & $\begin{array}{l}\text { hon (jap.) } \\
\text { Buch } \\
\text { KOPF }\end{array}$ \\
\hline
\end{tabular}

24 Die Rategorienmarkierung bedarf der Erlăuterumg: "nt" = 'titulierter Name'; "np" = 'persönlicher Name'. Dabei wird "np" als Teilmenge der Namen ( $n$ ) angesehen.

25 Dies soll laut Krifka (1983: 113) etwa bedeuten 'persónlicher Name als enthalten in der Klasse der Namen'. Insgesamt ist mir dieses Beispiel nicht ganz verständlich. 
Nach Rrifka spezifizierten im Engl. (und somit auch im Deutschen) die Nomina die Rlassifikatoren, im Japanischen sei es ungekehrt.

Zudem beleuchtet Krifka (1983: 22 f., 39, 71 f.) die sog. "charakterisierende Apposition", gemeint ist damit unser "Prototyp":

(5.43) Emil Schmidt, der Preisträger, (...).

Irreführenderweise fiele auch sie unter das Rriterium der Rategorienkonstanz, denn die Kategorie der Bezugs-NP bleibt nach der Hinzufügung die gleiche. Da jedoch "intuitiv" keine Spezifikation vorliege (Rrifka 1983: 22), müsse diese Ronstruktion anders behandelt werden. Der Weg zu Rrifkas Lösungsvorschlag wird etwas erschwert, weil er unter der Oberschrift "Die diskurspragmatischen Aufgaben der Attribution" der syntaktischen (!) Attribution zwei diskurspragmatische Aufgaben, nämlich "Identifizierung" und "Charakterisierung", zuschreibt, wobei er charakterisierende (lockere) Appositionen und nicht-restriktive Relativsatze auffürt (beider Funktion sei es, "die Zuschreibung von Eigenschaften zu bereits identifizierten Parametern" (ebd. 71) zu leisten); im gleichen Abschnitt schliest er sie jedoch von den Attributionen aus (ebd. 72). Dann wird aber deutlich, daß S.E. Apposition offensichtlich ein Phănomen sui generis ist, jedenfalls nicht unter "Attribution" fallen kann.

Interessant sind in diesem Zusammenhang zwei spãtere Bemerkungen Rrifkas (ebd. 161-163), nämlich daß (a) nicht-restriktive Relativsatze als besondere Art von charakterisierender Apposition verstanden werden konnen 26 und daB (b) auch parenthetische Sătze zu den Appositionen gerechnet werden können. Appositive Relativsătze und charakterisierende Appositionen bezögen sich dabei auf Nomina, Parenthesen auf den Restsatz.

Fazit: In der Rategorialgrammatik fallen unter Spezifikation nur die sog. "engen Appositionen", die "lockeren" (NP-Appositive, nichtrestriktive Relativsătze, Parenthesen) werden nicht als Adnominale (bzw. Attribute) der Bezugs-NP resp. der Bezugsphrase eingestuft.

\subsection{Modellvorstellung: Elenentarsatz, Gastsatz und Bospitanten}

Meine Modellvorstellung geht aus von einer deutlichen syntaktischen und semantischen Unterscheidung zwischen (a) dem sog. Elementarsatz, der das Prădikat, die Satzglieder, deren Gliedteile (Attribute) und ggf. (Grad-, Modal-, Negations-)Partikeln umfaBt und (b) einem um "Găste" erweiterten Satz (Yaximalsatz), der beispielsweise Anreden, Schaltsátze, eingeklammerte zusătze diverser Art (Abkürzungen etc.) oder eben auch "Appositive" enthălt. Die Glieder des Elementarsatzes nenne ich Partizipanten, die Gaste heiBe ich Bospitan-

26 Vgl. auch Krifka (1983: 162): Nicht-restriktive Relativsătze missen in einer Rategorialgrammatik als Nomen, nicht als Adnominale, analysiert werden". 
$\underline{\text { ten }}{ }^{27}$ der Elementarsatz, wenn er Găste enthălt, sei Gastsatz genannt. Man vgl. einen Elementarsatz (5.44) und den entsprechenden Maximalsatz (5.45), der zusătzlich drei Hospitanten (Anrede, Schaltsatz, "Appositiv") enthălt:

(5.44) Heute hat ja sogar Pia dem Kerl heimlich geholfen.

(5.45) Heute, meine Lieben, hat ja sogar Pia - sie ist eben eine gute Seele - dem Kerl, ein dubioses Individuum, geholfen.

Die Hospitanten kann man in EINSCHOBE (meine Lieben bzw. sie ist eben eine gute Seele) und ZUSATZE (ein dubioses Individuum) aufteilen, je nachdem, ob ein Bezugselement unterhalb der Satzebene, also eine Nortgruppe vie NP (dem Kerl), Adjp etc. vorhanden ist, oder ob kein Satzgliedbezug besteht (höchstens ein allgemeiner Bezug auf den gesamten Gastsatz).

Der Elementarsatz besteht aus dem Minimalsatz, der mindestens den primären Valenzträger (das Prădikat) plus dessen notwendig geforderte Ergănzungen aufweist. ${ }^{28}$ Darüber hinaus finden sich z.T. sekundăre Valenztrăger (z.B. Adj, N), die weitere (primăr attributive, allerdings hăufig fakultative) Ergănzungen in den Satz einbringen können. Zudem kőnnen im elementaren Satz freie Angaben zum Satz, zum Prädikat und zu den einzelnen Gliedern (nicht valenznotwendige Attribute) vorkommen. Neben klar satzgliedhaften Angaben wie den Adverbialen werden auch die nichtsatzgliedhaften Partikeln als freie Angaben gevertet. 29

Es erscheint, wie in weiteren Verlauf dieses Kapitels und dieser Arbeit deutlich werden wird, nicht zweckmäbig, auch die Hospitanten zu den freien Angaben $z u$ rechnen und sie dadurch auf eine (funktionale) stufe mit Satzgliedern wie den Adverbialen zu stellen.

Um den Begriff des Elementarsatzes stărker zu konturieren und um dessen allgemeine syntaktische und semantische Charakteristika aufzuzeigen, seien zunăchst einige Aussagen angefūhrt, die sich bestimmend für mein Modell ausgewirkt haben:

"Ein $S$ a $t z$ besteht aus einem Prádikatsausdruck und mindestens einer, höchstens 4 Ergănzungen, ferner (nichtobligatorisch) aus einer oder mehreren Angaben" (v. Polenz 1985: 83).

"Das Hauptverb legt einen wesentlichen Teil der Satzstruktur fest. Es bildet seinerseits den Kern eines engeren Konstrukts, in dem die Satzglie-

27 Die Ronnotationen des Fremdwortes Bospitant 'Gasthörer an einer Bochschule' bzw. 'unabhängiges Gastmitglied einer parlamentarischen Fraktion' sind durchaus beabsichtigt, da sie wesentliche Eigenschaften der Mementarsatzerweiterungen konturieren: die relative Unabhangigkeit und den Status, zwar dazuzugehören, jedoch nicht im eigentlichen Sim (vie z.B. der normale Abgeordmete).

28 Die sog. "Satzbauplăne" beziehen sich auf die Struktur und den Kindestumfang minimaler Sătze. S. 2.B. Engelen (1975; 1986).

29 Bierzu 2.B. Jacabs (1982; z.B. 227) und Jacobs (1983; v.a. 58 f.), der u.a. die Negationspartikel nicht, die Grad- und die Modalpartikeln zu den "Adsententialen" ("satzbezogenen Adverbialen") zăhlt, zu denen er z.B. auch temporale Adverbiale rechnet. Nichtsatzgliedhaft sind Partikeln insofern, als die "klassischen" Satzgliedtests wie Vorfeldfähigkeit, Erfragharkeit, Ersetzbarkeit etc. nicht auf sie anmendbar sind. 
der als seine Satelliten fungieren. (...) Satzglieder werden in Ergănzungen und Angaben unterteilt. (...) Neben den Erganzungen zum Verb (...) gibt es Ergănzungen zu anderen Wortklassen. (...) Auch Angaben gibt es (..) zu anderen Wortklassen, vor allem zu Nomina und Adjektiven (...)" (Engel 1988: 183).

"Der Satz ist die kleinste in sich gegliederte sprachliche Einheit, mit der sich ein außersprachlicher Sachverhalt beschreiben läbt. (...) Sachverhalt = Geschehen/Sein + Rollen" (Schulz/Griesbach 1982: 371).

"Jeder wahrnehmbare Sachverhalt ist von bestimmten Umstanden begleitet, die bei der Beschreibung des Sachverhaltes mit genannt werden können, damit der Hörer z.B. weiB, wo der Sachverhalt zu lokalisieren und wie er zeitlich einzuordnen ist" (Schulz/Griesbach 1982: 432).

Die Angaben zum Verb resp. zum Satz möchte ich (da dies für meine zwecke genügt) nach Engel (1988: 219 ff.) unterteilen in:

- verbbezogene (modifizierende) Angaben (Modaladverbiale)

- satzbezogene (situierende) Angaben (z.B. Temporal-, Kausaladverbiale)

- negative Angaben (v.a. die Negationspartikel nicht) und

- ăußerungsbezogene (existimatorische) Angaben (Satzadverbiale, GP, MP) . Hier ist zu sehen, daß auch Engel (1988) wie Jacobs (1982; 1983) Partikelfunktionen und Adverbial- bzw. Adsententialfunktionen zusammenfast in eine funktionale Großklasse (nämlich die der Angaben).

Aus dem bislang Erwăhnten ergibt sich eine erste, noch recht allgemeine obersicht über den Zusammenhang von Funktion und Satzbedeutung der Partizipanten:

(5.46) Kategorie/Funktion

Satz =
Prădikat/Valenztrăger +
Ergănzungen
$\quad+$ fakultativ:
Modifizierende Angaben
Situierende Angaben
Negative Angaben
Existimatorische Angabe

INHALT/BEDEUTUNG

SACHVERHALT (SV) =

PRADIRATION: HANDLUNG/VORGANG + REFERENZ: INVOLVIERTE ENTITATEN

EIGENSCHAFT EINES VORGANGS etc. LORALISIERUNG IN RAUM/ZEIT etc. WAHRHEITSWERT DES SATZES ANDERN SPRECHERSTELLUNGNAHME ZUM SV.

Die Partizipanten spielen mithin eine Rolle im sog. Sachverhaltsmodell, als das der Satz ofters expliziert wird. ${ }^{30}$ Die Satzglieder bringen z.B. Geschehen, Entităten und Umstănde lokaler, temporaler etc. Natur zum Ausdruck, die Partikeln haben anders geartete Funktionen und gehoren unzweifelhaft zum Sachver-

30 Dies geschieht wohl ausgehend van Vittgenstein, Ludvig. 1980. Tractatus logico-philosophicus. Logisch-philosophische Abhandlung. 15. Aufl. Frankfurt/1., suhrkami S. $20 \mathrm{ff.,} 33 \mathrm{ff}$. 
haltsmodell dazu. ${ }^{31}$ Dies zeigt sich u.a. daran, das sie offenbar nicht eingeschaltet zu erscheinen vermógen:

(5.47) Pia hat nicht den Affen besucht.

*Pia hat - nicht - den Affen besucht.

(5.48) Pia bat sogar den Affen besucht.

* Pia bat - sogar - den Affen besucht.

Wenn man ein (simples) Propositionsmodell ${ }^{32}$ anlegt, wird mit der Fũllung der Partizipanten im einfachen Satz zunăchst durch die Prädikat-Ergănzung(en)Struktur eine Basisproposition aufgebaut. Diese Basisproposition kann einerseits durch kon- bzw. subjunktional angefügte oder unverbunden folgende sätze expandiert werden, andererseits kann ein Ausbau innerhalb des Satzes (Impandierung) erfolgen, z.B. durch Attribution. 33

Es sei hier nur ganz oberflăchlich auf die Gliedteile (Attribute) hingewiesen: Adnominale fungieren als Referentenidentifikatoren oder -charakterisierungen, Ad-Adjektive z.B. als Graduatoren (sehr schnell) usf. Die Behandlung weiterer Gliedteile oder auch der $\mathrm{N}$-Determinantien kann man hier aussparen.

Im Elementarsatz bestehen zwischen dessen Bestandteilen (relativ) starke syntaktische Wechselwirkungen, die kurz mit stichworten wie z.B. Valenz, Rektion, bestimmte Serialisierung der Glieder usw. umrissen sei. Oberdies sind die Partizipanten (überwiegend) gewissen satzsyntaktischen Operationen wie Topikalisierung, Erfragung, Ersetzung durch Pro-Form, Herausstellbarkeit, Negierbarkeit etc. zugănglich. DaB sich diese Eigenschaften nicht gleichmảig bei allen Partizipanten zeigen, erscheint mir dabei nebensáchlich. 34

Gastsatzelemente (Hospitanten) wie Einschübe (Schaltsätze, Interjektionen und Anreden) oder Zusătze ("lockere Appositionen") treten kaum bis gar nicht in Wechselwirkung mit den Elementarsatzeinheiten. Sie werden nie von einem Partizipanten valenzgefordert. Hinsichtlich der Stellungsmöglichkeiten sind

31 Es fuhrte zu weit, wenn ich auf Partikelfunktionen genau einginge; daher der pauschale Verweis auf die Partikelliteratur, z.B. Altmann (1976; 1978), Jacobs (1982; 1983) und Weydt (1977; 1983).

32 Hierzu z.B. Heidolph u.a. (1981: 70 ff.), Moskalskaja (1985: 70 ff., 146 ff.), v. Polenz (1985: $91 \mathrm{ff.}$ ).

33 In Pia kauft ein Baus. Das Baus gehörte einem Filmstar > Pia kauft ein Baus, das einem Filmstar gehörte > Pia kauft das Haus eines Filmstars vird zumächst expandiert, schließlich impandiert (Genitivattribut). Zu "Impandieren" vgl. Moskalskaja (1984: $147 \mathrm{ff.}$ ).

34 So ist z.B. das Prádikat stellungsgebunden, die Satzglieder sind relativ frei stellbar, die Partikeln sind dies ebenfalls, wobei unstellumgen öfters deutliche semantische Veränderumgen hervorrufen, Satzgliedunstellungen jedoch weniger. Vorfeldfăhig sind die Satzglieder, die Partikeln. und das Prädikat (zumindest dessen finiter Teil) jedoch nicht. Hier erimere ich mich an einen Vorschlag von Jacobs (1983: 60), Vorfeld (un) fahigkeit als relativ idiosynkratisches Merkmal kategorial sonst gleicher Ausdrücke zu behandeln. Erfragbar sind die Satzglieder auch nicht alle (nicht einmal alle "klassischen" Adverbiale sind dies, s. 2.B. konsekutive Adverbiale) etc. Man kơnnte hierzu viel bemerken, doch scheint mir die prinzipielle Zusammengehörigkeit all dieser Mlemente evident genug, un die Brörterung hier abzubrechen. 
die Einschübe wesentlich "freier" als die Partizipanten, wenngleich sie nicht völlig beliebig im Satz auftreten kōnnen; das stellungsverhalten der zusătze ist dagegen relativ $z u$ ihrem Bezugselement $z u$ formulieren. An satzsyntaktischen operationen nehmen sie in nur geringem MaBe teil. Topikalisierbar, erfragbar, durch eine Proform ersetzbar und herausstellbar ${ }^{35}$ sind sie nicht.

Besonders interessant und hervorzuheben ist es, daß Hospitanten i.d.R. nicht von Negationen aus dem Elementarsatz heraus erfabbar sind. Sie haben insofern keinen Einfluß auf den Wahrheitswert des Elementarsatzes. Dieses wichtige und recht wenig berücksichtigte Kriterium ist vor allem von Zemb (z.B. 1968: 296; 1972: 79-81) und von Raabe (1979: 248 ff.) zur Analyse von "Appositionen" eingesetzt worden.

Die "Gäste" bleiben sowohl syntaktisch ("Larvierung" durch umhüllende Interpunktion resp. Intonation) als auch semantisch (keine Wahrheitswertbeeinflussung) "im Hintergrund". Sie stellen zusătzliche Rommentare, Bemerkungen, orientierungen etc. oder auch kommunikationsbezogene AuBerungen (z.B. Anrede) dar. In einem Propositionsmodell des Satzes (vgl. weiter oben) bezeichnete man die "Hospitanten" z.T. als in den Gastsatz zusătzlich eingebrachte Sekundărpropositionen. (5.49) veranschaulicht, wie die Proposition eines Folgesatzes (Expandierung) zunachst als EINSCHUB (5.49b) und schlieblich als zUSATZ (5.49c) impandiert wird. (Ob man in jedem HOSPITANZ-Fall dieses Modell anlegen kann, bleibt offen.)

(5.49a) Kuno liebt Asterix (Prop 1 ). Er ist ein Comicfan (Prop 2 ).

(5.49b) Kuno - er ist ein Comicfan (Prop 2 ) - liebt Asterix (Prop $)_{1}$ ).

(5.49c) Kuno, ein Comicfan (Prop 2 ), liebt Asterix (Prop 1 ).

Aus dem oben Gesagten folgere ich, das man eine eigene syntaktische Relation zwischen einem Hospitanten und dem Gastsatz (bzw. einem Element des Gastsatzes) ansetzen sollte, námlich die Adordination $(5.52 ; \mathrm{vgl}$. Kap. 4$) .36$ Sie bezeichnet eine Beziehung zwischen zwei Einheiten, die ungleichstufig sind, ohne daB jedoch eine von der anderen dependent ist. Die Adordinationsbeziehung ist

35 Dieser Punkt bedarf allerdings noch genaverer Untersuchung. Was liegt in den folgenden Beispielen vor (Herausstellung, genauer: freies Thema, oder etwas anderes?):

(F5.10) Pia hat - kaum zu glauben - gestern sogar kumo verführt.

Apropas kaum zu glauben! Pia hat gestern sogar Kumo verführt.

(15.11) Rumo hat - pfui teufel - gestern bei Tisch gerulpst.

Apropos pfui teufel! Kumo hat gestern bei Tisch gerillpst.

Andere Herausstellumgsmöglichkeiten (z.B. LV, RV) sind allerdings nicht gegeben:

(55.12) Kaum zu glauben, *das/*so bat Pia gestern sogar Kumo verführt.

Pia hat (*das/*es) gestern sogar Runo verführt, kaum zu glauben.

(55.13) Pfui Teufel, *das/*so hat kumo gestern bei Tisch gerillpst.

Rumo hat (*das/*so) gestern bei Tisch gerilpst - pfui teufel!

36 zur Beschreibung syntaktischer Relationen ogl. Raabe (1979: 226 ff.), Matthews (1981) und Lehmann (1983). 
aufzuteilen in die Zusatz- und die Einschubrelation. 37 Die adordinative Relation ist zudem von Subordination (5.50) und Koordination (5.51) abzugrenzen. Die Relationen finden sich im folgenden auf NPn (a-Varianten), auf AdjP (bVarianten) sowie auf Sätze (c-Varianten) angewendet. Bei der subordination ist das Regens kursiv/fett, das Dependens kursiv/unterstrichen markiert; bei der Roordination sind die Ronjunkte kursiv/unterstrichen markiert; bei der Adordination ist sowohl die Bezugswortgruppe (kursiv/fett) des Zusatzes (5.52a, b; kursiv/unterstrichen) als auch der Gastsatz (kursiv/fett) des Einschubs (5.52c; kursiv/unterstrichen) markiert:

(5.50a) Die Annabne einer 5. Dimension war kühn.

(5.50b) Der des Mundraubs verdachtige Garfield schmollte.

(5.50c) Pia nahn an, (dab) eine 5. Dimension existiere.

(5.51a) Pia verdrießen Annahmen und Behauptungen.

(5.51b) Der verdächtige, alibilose Angeklagte schwieg.

(5.51c) Pia ging ins Büro und Runo (ging) in die Kneipe.

(5.52a) Diese Annabne, eine gewagte Hypothese, erwies sich als korrekt. Die Vögel, ferner die Fledermäuse, erhoben sich in die Lufte. Die Orthographie (Rechtschreibung) fällt Pia leicht.

(5.52b) Der paranoische (geistesgestörte) Patient taumelte umber. Bunt, vor allem rot und blau, waren die Lichter.

(5.52c) Die Annahne = alle waren davon überrascht - war korrekt. Die Annahne, meine Damen und Herren, erweist sich als falsch. Dann sprang - o schreck! - ein Lowe aus den Busch.

Die sog. "Herausstellungen" sind nicht gar so einfach in das Modell zu integrieren. Bei ihnen handelt es sich vorwiegend um eher kommunikationspragmatische Erscheinungen, da sie z.T. Bezüge zu Satzgliedern aufweisen (LV, RV) und entweder vorweg (LV; 5.53) oder nachtrăglich (RV; 5.54) eine semantisch "leere" Pro-Form inhaltlich spezifizieren:

(5.53) Die Yuppies, die kann er gar nicht leiden.

(5.54) Er kann die gar nicht leiden, die Yuppies.

vielleicht zahlt die Linksversetzung ebenfalls zu den adordinativen Beziehungen. Die mögliche Einordnung muß leider unerörtert bleiben. Zu den Details und den restlichen Herausstellungsoptionen verweise ich auf Rap. 10.

37 Denkbar wäre auch ein anderer Ansatz, der unter Adordination nur die Zusätze faßt und bei den Binschuben keine Beziehung zu dem Satz annimmt, in den die Binschaltung erfolgt. Dann mürde man zwischen Einschub und Gastsatz Relationslosigkeit annehmen. Vgl. Lehmann (1983: 340): "Die Soziation [Roordination in meiner Terminologie, W.S.] grenzt an die Relationslosigkeit, auf Satzebene an die Parataxe, auf Syntagmenebene an die Parenthese". Die für diese Arbeit vorgeschlagene Taxonomie syntaktischer Relationen folgt solchen Ansătzen aus praktischen Grimden nicht, da sie folgende "Winimalpaare" erfassen móchte:

(F5.14a) Pia, seit gestern Institutsleiterin (ZUSATZ), begribte alle Mitarbeiter.

(55.14b) Pia, sie ist seit gestern Institutsleiterin (EnschuB), begriste alle Mitarbeiter.

(55.15a) Barry Birsch - er gehört der FDP an (EnNSCHUB) - füllte das Somerloch.

(F5.15b) Barry Birsch (FDP) (ZUSATZ) fullite das Sammerloch. 


\section{DIE BIGENSCHAFTEN DES APPOSITIONSPROTOTYPEN}

Ebenso vie Raabe (1979: 223) geht diese Arbeit davon aus, daB mindestens eine Spracherscheinung existiert (s. 6.1a-e), die in jeder sprachdeskription als ein Fall von "Apposition" angesehen wird. ${ }^{1}$ M.a.W.: Es gibt eine sprachliche Konstruktion im Deutschen, die nicht mit den bereits vorhandenen grammatischen Termini wie z.B. Attribut, Adverbial, Parenthese etc. erfabt werden kann. Diese Erscheinung nenne ich Prototyp (der Apposition), womit allerdings nicht nahegelegt werden soll, daB dieses Muster "im Zentrum" appositiver Konstruktionen (Zusätze) steht, also den typischsten Vertreter darstellt, und weitere, verwandte Muster "peripher" wăren. Vielmehr befand er sich bislang im Zentrum des Untersuchungsinteresses, wenn es um die Erforschung von "Apposition" ging.

Der Prototyp ist kein ohne weiteres eindeutiges Muster; eigentlich sollte er sich erst nach den Abgrenzungsdiskussionen gegen Ende der Arbeit herauskristallisieren. Wie komme ich auf die sechs Beispiele (6.1a-f)? Warum schiebe ich die Muster unter (6.2) beiseite? Erste Oberlegung: Das "lockere Muster" (mit nominalem Appositiv; z.B. 6.1a) findet sich ausnahmslos in jeder Appositionsdeskription. Zweitens: Die Abgrenzungsproblemfalle wie "Rechtsversetzung" 2 (vgl. 6.2a) und "Nachtrag", die vor allem Altmann (1981) diskutiert hat, werden zunăchst ausgeklammert, was nicht bedeutet, daß ich gelegentlich auf sie Bezug nehme, um offensichtliche Unterschiede zu demonstrieren. Ebenso werden Problemfälle wie z.B. $(6.2 b$, c) vorlăufig ausgespart. Drittens: Es besteht bei 3 den Appositionsbeschreibungen, die mit dem Merkmal der Paraphrase argumentieren, Einigkeit darüber, daB typische Appositionen in systematischen Beziehungen zu Umschreibungen mit der Kopula sein stünden (vgl. Abs. 5 dieses Rapitels). Somit wurden nur Muster mit einbezogen, die solche Umschreibungen zulassen und alle sich anders verhaltenden Typen ausgeschlossen. Viertens: Wie 4 Altmann (1981) und Engel (1986; 1988) nachgewiesen haben, sind Appositive nicht auf die Kategorie NP beschränkt; auch AdjP und PP sind möglich. Daher werden zwei Beispiele mit derartigen Appositionen $(6.1 e$, f) aufgenommen.

Möglicherweise werden jetzt methodische Bedenken erhoben gegen mein Vorgehen, mit diesen vier Oberlegungen den Prototypen vorlăufig zu umgrenzen. Wáre es nicht sinnvoller gewesen, alle verdăchtigen Muster (aus Kap. 2) mittels einer Testbatterie abzuprüfen und dann all jene, deren Merkmale sich gleichen, zusammenzufassen? Vielleicht! Doch schien mir der unreflektierte "Merkmalismus" zu bedenklich, als daB ich ihm die aussagekrăftigsten Ergebnisse zumutete. Wie wichtig die relative Gewichtung der Eigenschaften von Mustern im Bereich der appositionsverdăchtigen Ronstruktionen ist, wird der Leser im Verlauf der Arbeit immer wieder feststellen; zudem hat er bereits wahrnehmen können, wie unterschiedliche Gewichtung $z u$ verschiedenen Appositionsbegriffen

1 Das bedeutet nicht, daß sie in jeder Sprachdeskription gleich erklärt wird. 
(Kap. 2, 3) führen kann. M.E. ist die Ropulaparaphrase ein hoch zu wertendes Merkmal - wesentlich höher z.B. als die Stellungseigenschaften einzelner Submuster (vgl. Abs. 3). Beurteilte man stellungsverhalten und Paraphrasemöglichkeit gleich, so müBten mindestens $(6.1 \mathrm{e}, \mathrm{f})$ einer eigenen Klasse zugeordnet werden, weil sie nicht vom Bezugselement entfernt werden dürfen; ein Appositiv wie (6.1d) ist der Distanzstellung eher zuganglich. Damit ist meine "VorausSetzung" offengelegt. Im übrigen hätte mein Verfahren spătestens bei der Untersuchung der im engeren Sinne appositionsverdächtigen strukturen (Kap. 1215) Schiffbruch erlitten, wenn die vorgeschlagene Voraus-Setzung des Prototypen unzulänglich gewesen wäre. Er stellte sich allerdings als deutlich von den restlichen Mustern abgrenzbarer Typ heraus.

Im folgenden soll eine Obersicht über die wichtigsten Merkmale und Verhaltensweisen des Appositionsprototypen gegeben werden, um eine Kriterienliste zur Abgrenzung von verwandten, ähnlichen oder auch fernerstehenden, bislang jedoch in den "Appositionstopf" geworfenen Konstruktionen zur Hand $z u$ haben. Dabei wird über die in Kap. 3 erörterten Appositionsmerkmale hinausgegangen; die zusătzlichen Kriterien werden in diesem Kapitel erläutert. Die Durchnumerierung der Kriterien entspricht derjenigen in Kap. 3.

(6.1a) Der Tabak, ein typisches GenuBgift, mundete pia nicht.

(6.1b) Diese Frau, Dozentin in Bonn, schwärmt von Tabak.

(6.1c) Der Kuno, früher mein bester Freund, ist jetzt mein Widersacher.

(6.1d) Jener Graf, übrigens vermutlich ein Vampir, betöt alle Frauen.

(6.1e) Runo, spitznasig und dürr, lugte ins Zimmer hinein.

(6.1f) Das Rathaus, am Marktplatz des Orts, wird restauriert.

Nicht zum Prototypen gehören u.a. folgende ăhnlich aussehenden Beispiele:

(6.2a) Sie, (ich meine) deine Oma, kocht gut.

sie kocht gut, (ich meine) deine oma.

* Sie - sie ist deine Oma - kocht gut.

(6.2b) Der Bundeskanzler, Helmut Kohl, hălt eine Rede.

* Der Bundeskanzler halt eine Rede, Helmut Rohl.

? Der Bundeskanzler häl eine Rede, ich meine Helmut Kohl.

(?) Der Bundeskanzler, ich meine Helmut Kobl, halt eine Rede.

Der Bundeskanzler, er heiBt Helmut Kohl, halt eine Rede.

?? Der Bundeskanzler, er ist Helmut Kohl, hält eine Rede.

(6.2c) James Bond (Sean Connery) schlug wieder $z u$.

In (6.2a) liegt eine Rechtsversetzung vor; ${ }^{2}$ man kann dies anhand des zusatzes ich meine (beim Prototypen unmöglich) und der Inakzeptabilităt der Kopulasatzumschreibung deutlich machen. Die Rechtsversetzung wird in Kap. 12 und 15 im Ggs. zum "Prototypen", der dort "prădizierender Zusatz" genannt wird, als "referenzklärender Zusatz" eingestuft. Aus meinen Analysen resultiert am Ende der

2 S. Altmann (1981: $54 \mathrm{ff.}$ ). 
Arbeit, das "Prototyp" und "Rechtsversetzung" zwei von mehreren Subtypen einer allgemeinen funktional-syntaktischen Relation (vgl. Kap. 4 (c), Kap. 5.5) darstellen, die ich ZUSATZ(Relation) nenne. In (6.2b) findet sich eine Konstellation, die eine gewisse Năhe zur Rechtsversetzung hat. Es findet jedoch keine Referenzklărung statt (fast jeder weiB, daß der derzeitige Ranzler Helmut Kohl heiBt), die Funktion ist vielmehr etwas wie NAMENSANGABE (IN-ERINNERUNG-RUFEN DES NAMENS $0 . a \overline{)}$. Dieser Konstruktion begegnet man hăufig in Tageszeitungen: ${ }^{3}$

(6.3) Der Sprecher des sowjetischen AuBenministeriums, Gerassimow, (...). Man sieht, daB die weite Rechtsversetzungsstellung ohne die Rechtsversetzungsfloskel ich meine inakzeptabel ist. Die Variante mit Rechtsversetzungsfloskel ist m.E. auch nicht gerade annehmbar. Die heiBen-Paraphrase hingegen zeigt den Konstruktionssinn am besten, die Kopula-Paraphrase ("Prototyp") ist wahrscheinlich inakzeptabel, zumindest aber sehr unpassend. (6.2c) scheint mir ein ganz ähnlich gelagertes Beispiel zu sein; auch hier ist die Funktion so etwas wie NAMENSANGABE. Diese Fălle gehören in den Bereich der "Rundklammerzusătze" bzw. der "uneingeleiteten Zusätze", der in Kap. 13. untersucht wird.

1) Rategoriale Füllung: Die Basis ist stets eine Voll-NP (N3; auch innerhalb einer PP), und $z$ war entweder ein Syntagma aus "Det $+N "$, ein NP-wertiges Nomen (z.B. EN, SN, Abstraktum) oder ein Pronomen. Attributive Erweiterungen des Kerns der Basis sind möglich. Nach Engel (1986: 199; 1988) sind Personal-, Possessiv-, Demonstrativ- und Indefinitpronomen möglich. Ich betrachte einige solcher Fålle:

(6.4a) Dich, (du bist übrigens) unser bestes stück, schätzen wir. Sie, (sie sind übrigens) Köche aus Passion, servierten Leckeres. Sie, (ich meine) die Köche aus Passion, schätze ich sehr.

(6.4b) Meiner, (meiner ist übrigens) ein 57er Baujahr, făhrt tadellos. Eure, (eure/ sie sind) Beamte wie wir, streiken jetzt auch. Thre, (ich meine) ihre Ringe, sind aus Gold.

(6.4c) Jene, (jene sind übrigens) aus München, brüllten nach Bier. Dies, ubrigens eine Rarităt, kannst du nicht kaufen.

Dies, (ich meine) das Perpetuum mobile, kannst du nicht kaufen.

(6.4d) Einige, (übrigens) humorvoll und gebildet, kannten viele vitze. Einige, (ich meine) die Philosophen und die Psychologen, redeten unverständiches Zeug.

(6.4e) ?Viele, (übrigens) Dozenten aller Fakultaten, streikten auch. ?Alle, aufgekratzt und lärmend, blieben bis zum Morgengrauen.

(6.4f) ^Die Chirurgin, die, Chefarztin der Abteilung, das beschloß, erinnerte sich.

(6.4g) *Wer, Chefäztin der Abteilung, erinnerte sich?

3 Beispiel aus der Suddeutschen Zeitung vam 17.11.1988, 44. Jahrgang, Nr. 265, S. 1. 
Generell lảst sich feststellen, daB "Prototyp"- und Rechtsversetzungslesart je nach Kontext moglich sind. Ist die Pro-Form bereits im Vortext spezifiziert (m.a.W.: eine anaphorische Relation liegt vor), so tritt die erste Lesart ein; ist sie es nicht (keine Anaphorik), tritt die zweite ein.

Interrogativ- (6.4g) und Relativpronomina (6.4f) fallen als Bezugselemente fort. Bei den Indefinitpronomina kommen mir die Bezugsmöglichkeiten teils fraglich $(6.4 \mathrm{e})$, teils akzeptabel vor $(6.4 \mathrm{~d}) .^{4}$ Die restlichen Pro-Elemente können augenscheinlich problemlos als Basen dienen $(6.4 \mathrm{a}-\mathrm{c})$.

Das Appositiv kann von einer NP bzw. einem Nominal einer X-Bar-Stufe unterhalb von N3 (vgl. z.B. 6.1b) repräsentiert sein; ebenfalls möglich sind Adjektivphrasen oder PPn.

Bei nominalen Köpfen ist die Betrachtung der Determinantienverhăltnisse bei Basis und Appositiv von Interesse. Wenn man vor allem die materialreichen Arbeiten (z.B. Rusmin 1960, Molitor 1979, Engel 1986) durchsieht, dann fällt auf, daß nur ganz bestimmte Determinantienverhältnisse anzutreffen sind: Neben Artikellosigkeit, unbestimmtem und bestimmtem Artikel finden sich eigentlich nur noch Kardinal-Quantoren (zwei, drei etc.) und Possessivpronomina; mit der Basis (nicht mit dem Appositiv) kommen manchmal Demonstrativpronomina vor $(6.5 \mathrm{a})$. Auch Indefinitpronomina als Basis-N-Begleiter scheinen tendenziell unproblematisch $z$ u sein $(6.5 b)$ :

(6.5a) Die/ Drei/ Diese/ Jene/ Unsere Musiker, Konnner auf ihren Instrumenten, verzauberten uns.

(6.5b) (?) Alle/ Viele/ Manche Musiker, Könner auf ihren Instrumenten, verzaubern uns.

(6.5c) ?Die Musiker, alle Gitarristen, begeisterten uns.

?Die Musiker, manche Violinisten und Cellisten, begeisterten uns.

(6.5d) Die Musiker, diese Rlangzauberer, verzaubern viele Menschen. Indefinitpronomina beim Appositiv bewirken Interpretationsschwierigkeiten und sind beim "Prototypen" eventuell gar nicht möglich. Die sătze in (6.5c) wären sofort klar, wenn es z.B. hieße allesamt Gitarristen oder manche von ibnen Violinisten und Cellisten oder aber genauer gesagt alle Gitarristen bzw. vor allem manche Violinisten und Cellisten. Im ersten Fall wären prădikative Umschreibungen möglich (sie waren allesamt.../ manche von ihnen waren...), was auf den Prototypen schließen ließe; im zweiten Fall lage ein Nicht-Prototyp vor, nămlich ein spezifizierender Zusatz (ăhnlich dem "Nachtrag" bei Altmann 1981; vgl. Kap. 12, 15). (6.5d) kann nicht durch einen Kopulasatz umschrieben werden (??Die Musiker sind diese Klangzauberer); was hier vorliegt, ist nicht ganz klar - möglicherweise kommt ein Alternativen nennender Zusatz (m.a.W. diese Klangzauberer; vgl. Kap. 12, 15) in Frage.

Wenn das Appositiv einen adjektivischen Kopf aufweist, muB das Adjektiv i.d.R. erweitert sein: entweder durch ein weiteres, konjunktorsyndetisch an-

4 Nähere Untersuchungen sind wünschenswert, müssen jedoch leider unterbleiben. 
gefügtes Adj oder durch eine Anreicherung um Adjektivergănzungen oder -angaben. 5 Das Appositiv kann von Gradpartikeln, Satzadverbien, temporalen Adverbien und den beiden "Floskeln" ("Appositionslexemen") übrigens und bekanntlich begleitet werden. 6

Daß ubrigens hinzufügbar ist, darf nicht als ausschließliches Merkmal unseres Appositionsprototyps gelten:

(6.6a) Viele Haie, (sie sind) übrigens gefürchtete Meeresräuber, werden dort gejagt.

(6.6b) Viele Haie (sie sind übrigens besonders gefräBig) werden dort gejagt.

Obrigens kennzeichnet " (..) eine bestimmte Teilinformation als Abweichung vom geraden Verlauf eines Diskurses, als zum Verstăndnis der umgebenden Information nicht notwendigen Einschub" (Altmann 1981: 97). Diese Markierung kann nicht nur auf die "Appositive", die als reduzierte Ropulasătze expliziert verden können, angewendet werden, sondern auch auf weitere Zusătze zu NPs (und anderen Phrasen):

(6.7) Viele Haie, übrigens vor allem die Blauhaie, werden gefürchtet. *Viele Haie, sie sind vor allem die Blauhaie, werden gefürchtet. Hier liegt nicht das "prototypische" prädizierende Verhăltnis zwischen "Appositiv" und Basis vor. Deshalb sollte übrigens nicht per se als "Appositionsmarker" gewertet werden; weitere Merkmale wăren zu berücksichtigen.

Es ist auch nach der "Satzwertigkeit" der Appositionen zu fragen. Sie ist durch den möglichen zusatz von Satzadverbien, Gradpartikeln und freien Adverbialen schon weitgehendst nahegelegt. 7 Ich prufe nun, ob Appositive von illokutionsmodifizierenden, adsententialen Modalpartikeln begleitet werden können, die gewŏnnlich nur in (konstativen, interrogativen, imperativen etc.) sătzen und nicht in Wortgruppen auftreten: 8

(6.8a) Die Pia, (sie ist) eben doch eine nette Nachbarin, backt schon wieder für uns.

(6.8b) Den Kuno, (er ist) ja bloB ein Papiertiger, nehme ich nicht ernst.

(6.8c) Die Pia, (sie ist) nun mal fleiBig und gründlich, schafft das.

(6.8d) Der Runo, (er ist) wohl noch Student, wobnt zu Hause.

Die Syntagmen erscheinen akzeptabel. Offensichtlich kŏnnen die eigentlich satz- bzw. illokutionsbezogenen Modalpartikeln das Appositiv begleiten - ein

5 Vgl.: Pia, ?lustig/ vom Weine lustig/ lustig wie immer/ Iustig und fidel, tanzte auf dem Tisch.

6 Andere "Appositionslexeme" wie z.B. genauer gesagt, práziser, vielmehr, bzw., d.h. koumen später zur Sprache. Es wird sich erweisen, daß sie nicht zu den Begleitern des Prototypen zăhlen.

7 Vgl. auch Altmann (1981: 97 ff., v.a. 99).

8 Man vgl. folgende Unformungsbeziehung Satz-Hortgruppe:

(F6.1) Cäsar eraberte mal eben schnell Gallien.

(F6.2) *Die mal eben schnelle Eraberung Galliens durch Cásar. 
gewichtiges Indiz für die Satzwertigkeit und den Einschubcharakter d:r Appositive!

Als zusătzlicher Prüfstein für "Satzwertigkeit" kann der mögliche Zusatz von Textkonnektoren, auch Ronjunktionaladverbien geheiBen, ${ }^{9}$ betracht.t werden. Wenn Appositionen "satzartig" wären, dann erwartete man, daß neben feien Adverbialen, Gradpartikeln etc. auch Konjunktionaladverbien im Appositv auftreten können. Mit entsprechenden Kontexten erhält man derartige Beispille:

(6.9a) Das war eine lahme Fete. Pia, wenigstens/ trotzdem/ dennoc/?allerdings kein Rind von Traurigkeit, hat immerhin dreimal getazt.

(6.9b) Gestern war schon ein harter Tag. Pia, ?jedenfalls/ deswegn/ trotzdem/ dennoch /allerdings keineswegs müde und faul, hat heue wieder viel gearbeitet.

(6.9c) Heute war ein harter Tag. Pia, folglich/ dadurch/ deshalb/infolgedessen ziemlich geschafft, gönnte sich einen ruhigen Abend

Trotz gelegentlicher Einschrănkungen dürften diese zusätze möglich sin, ein weiteres Indiz für die Satzwertigkeit von Appositiven. Auch die Anweenheit von Subjunktionen scheint nicht inakzeptabel:

(6.10a) Pia, (?) obwohl (übrigens) schon eine ältere Dame/ müde un' lustlos, ging auf die Mitternachtsfete.

(6.10b) Pia, ?weil (übrigens) schon eine ältere Dame/ müde und lutlos, blieb zu Hause.

(6.10c) Pia kann SpaB vertragen. Pia, (?) insofern ein guter Kumpe, hat folglich viele Freunde.

Handelt es sich hier noch um unseren Prototypen? Liegen hier evtl. eliptische Verbendsătze (reduzierte Schaltsătze) vor? Gehören solche Sătze zum tandarddeutschen? Man sieht, daß hier ein interessanter Aspekt der Appositinsdiskussion vorliegt. Unzweifelhaft nach dem Bisherigen ist allerdings die atzwertigkeit der Appositive, die auch Altmann (1981: 63, 336 f.) konstatirt hat.

Nun noch ein Aspekt, der in der Appositionsdiskussion noch keine eachtung gefunden hat: Können Appositive Parenthesen (insonderheit Schaltsätz) enthalten? Parenthesen sind $j a$ in einen "Trăgersatz" eingebettet und konne somit als Indikatoren für "satzwertige Konstrukte" herangezogen werden. 10

(6.11a) Der Graf, wahrscheinlich - jedenfalls behaupten das viele- ein Vampir, betörte alle Damen.

(6.11b) Der Kuno, seit langem - wie man weiB - ein fürchterlicherSchluckspecht, treibt es gar zu toll.

(6.11c) (?) Pia, auch heute wieder - darauf haben alle gehofft - gistreich und humorvoll, rettete die stimmung.

(6.11a-c) demonstrieren, das dies weitgehendst der Fall ist, sofern ie Apposition von weiteren Einheiten (z.B. Satzadverb, Adverbial) begleitetwird, so

9 zur Definition von Konjunktionaladverb s. Helbig/Buscha (1980: $306 \mathrm{f.}$ ).

10 vgl. z.B. Bassarak (1985). 
daß sich die Schaltsătze in den "Parenthesenischen an den Satzgliedgrenzen" einnisten können. Aber sogar zu unbegleiteten Appositiven vermögen sich Schaltsătze zu gesellen, was wiederum auf "Satzwertigkeit" hinweist:

(6.12a) Der Graf, ein Vampir (jedenfalls behaupten das viele), betorte alle Damen.

(6.12b) Der Kuno, ein fürchterlicher Schluckspecht (wie man weiB), treibt es gar zu toll.

(6.12c) Pia, geistreich und humorvoll (darauf baben alle gehofft), rettete die stimmung.

Bei den Rechtsversetzungen (nach Altmann) sind die gerade diskutierten Zusatzmöglichkeiten eher unmöglich (man versuche, ich meine einzufügen):

(6.13a) ?Eine nette Nachbarin, eben die Pia, backt schon wieder für uns.

(6.13b) *Ein Papiertiger, ja bloB der Kuno, wird nicht ernst genommen.

(6.13c) * Sie, halt doch die Pia, kann jeder leiden.

(6.13d) *Eine Studentin, trotzdem/ allerdings/ dennoch die Pia Müller, gewann den Nettbewerb.

(6.13e) *Ein Lümmel, weil/ obwohl Kuno Knall, hat sie belästigt.

(6.13f) Eine Studentin, Pia Müller (das weiB doch jeder), gewann den Wettbewerb.

(6.13g) Ein Lümmel, Kuno Knall (jedenfalls weist alles auf ihn hin), hat sie belastigt.

Lediglich der Zusatz von Parenthesen $(6.13 \mathrm{f}, \mathrm{g})$ scheint akzeptabel, was als Hinweis auf mögliche Satzwertigkeit der Rechtsversetzung gewertet werden kŏnnte. Deren Satzwertigkeit ist umstritten. Altmann (1981: 337) ăuBert, das manches dagegen spricht: Satzadverbien, Gradpartikeln, Adverbiale nicht hinzufügbar, intonatorische Integration der Rechtsversetzung in den "Bezugssatz". Dennoch sprăche dies "nicht generell gegen Satzwertigkeit" (ebd. 337).

2) Abtrennung: Das Appositiv ist in der Schriftsprache von Rommas, manchmal auch von runden Klammern oder Gedankenstrichen umgeben; in der gesprochenen Sprache wird es von deutlichen Pausen umhullt und evtl. etwas "zuruckgenommen" (d.h. mit geringerem Schalldruck als die Basis) intoniert (Details bei Altmann 1981: 202-204; vgl. zudem Winkler 1969 und DUDEN 1984: 747).

3) Stellung: In der Regel folgt das Appositiv unmittelbar rechts auf die nominale Basis ("unmittelbare Adjazenz"):

(6.14a) Der Tabak (*, ein typisches GenuBgift,) des Ostens (, ein typisches Genußgift, ) mundet.

(6.14b) Der Kuno (*, spitznasig und dürr,) mit dem Knebelbart (, spitznasig und dürr,) tobte herum. 
(6.14c) Die Hoffnung (*, eine trügerische Hoffnung), daB man im Lot to gewinnt, (eine trügerische Hoffnung,) hegen viele.

(6.14d) Der Baum (*, eine alte Eiche,), der vor dem Haus steht, (eine alte Eiche,) soll gefält werden.

Hierbei trennen dem nominalen Kern folgende Attribute (Genitiv-, Präpositionalattribut, Relativsatz, daB/ob/wie-Satz-Attribut etc.) ein Appositiv von der Basis. Arbeiten, die die Wortstellung in Substantivgruppen behandeln, verorten die "lockeren Appositionen" auch am rechten Rand der NP - teils aus morphologischen Erwăgungen (grob zusammenfasbar in: Genitiv-) Prāpositional-) Relativsatzattribut, Apposition), teils aus dependenziellen (Valenzkomplemente vor restriktiven vor appositiven). 11 Auch bzgl. satzformiger Komplemente (die nichtsatzformatigen prinzipiell rechts nachfolgen müssen) werden Appositive rechts von diesen angesiedelt. 12

Interessant und m.W. nicht untersucht ist die Position der Appositive bei PPn mit Zirkum- oder Postpositionen:

(6.15a) Dieser Frau (*, Dozentin in Bonn,) zuliebe (, Dozentin in Bonn,) tut Kuno alles.

(6.15b) Um des Tabaks (?, eines typischen GenuBgifts/*, ein typisches GenuBgift,) willen (, ein typisches GenuBgift/ ?eines typischen Genusgifts,) gehen viele meilenweit.

(6.15c) Um Kunos (*, ein guter Freund/?, eines guten Freundes,/*, ubrigens ein guter Freund,) willen (, ein guter Freund/ ? eines guten Freundes, ) tut pia alles.

(6.15d) Um Kunos (*, früher mein bester Freund,) willen (, früher mein bester Freund,) tut Pia alles.

(6.15e) Kuno (*, spitznasig und dürr,) zuliebe (, spitznasig und dürr,) verzichtete man darauf.

(6.15f) Um des Rathauses (*, am Marktplatz des Orts,) willen (, am Marktplatz des orts,) verzichteten sie auf den Supermarkt.

Wie zu sehen ist, müssen die Appositive - anders als die Attribute! - den Postpositionen resp. den zweiten Teilen der Zirkumpositionen nachfolgen. Nicht ganz eindeutig sind die kongruenten Beispiele $(6.15 \mathrm{~b}, \mathrm{c})$; sie sind vielleicht måig akzeptabel, obwohl die inkongruenten (und evtl. erweiterten) Appositive an dieser stelle eindeutig inakzeptabel sind. Das erklăt sich dadurch, daB die kongruenten Beispiele den Eindruck entstehen lassen, das kongruente Appositiv sei lediglich nur eine "anders formulierte" Basis, und zwar als VERALL-

11 Siehe z.B. Sommerfeldt (1971); Teubert (1979); Rolde (1985; darin auf S. 265 ff. ein knapper Exkurs zur Darstellung der NP-Giedfolge in deutschen Grammatiken); Vater (1986). ")" bedeutet hier 'aufgrund seiner (morphosyntaktischen) Rategorie năher an Rernnomen als'.

12 Stellvertretend Teubert (1979: 209): "Die lose appositionelle Fugung mit substantivischem, adjektivischem und partizipialem Rern tritt meist an den Schlub des Nominalkomplexes, und zwar auch dann, wenn zum Nachfeld eine satzförmige Ergănzumg gehört". 
GEMEINERUNG ('Pia tut um Runos willen, aber generell um jedes guten Freundes willen alles' etc.). Dabei geht das "appositionstypische" prådikative Verhăltnis zwischen B und A verloren (Um des Kunos willen, er ist ein guter Freund, tut Pia alles).

Nun werfe man einen Blick auf die Stellung des "Prototyps" bzgl. Konjunktionaladverbien und postponierten Gradpartikeln (die das Kernnomen des Bezugselements fokussieren):

(6.16a) Diese Frau (jedoch), Dozentin in Bonn, (??jedoch) kannte Kuno nicht.

Diese Fr a u (sogar), Dozentin in Bonn, (*sogar) kam zur Party.

(6.16b) Das Rathaus (jedoch), am Marktplatz des Orts, (??jedoch) wurde be schädigt.

Das $R$ a thaus (nur), am Marktplatz des Orts, (*nur) wurde beschä digt.

(6.16c) Kuno (jedoch), spitznasig und dürr, (??jedoch) lachte nur. $K$ u no (allein), spitznasig und dürr, (*allein) kann dies wissen.

(6.16d) Der Tabak (hingegen), ein typisches Genußgift, (??hingegen) erfreut sich ungebrochener Beliebtheit.

Der $T$ a bak (nur), ein typisches GenuBgift, (*nur) vermochte zu be ruhigen.

Gradpartikeln können lediglich unmittelbar der Basis folgen, nicht aber nach dem Appositiv stehen. Bei den Satzkonnektoren sind die Verhălnisse wahrscheinlich ebenso zu formulieren, wobei mir die Inakzeptabilităt der Nachstellung nicht gar so $\mathrm{kraB}$ erscheint. Im übrigen verăndert auch die Rongruenz die Verhältnisse nicht (die VERALLGEMEINERUNGS-Lesart sei ausgeschlossen!):

(6.17a) Des $T$ a baks (sogar), eines typischen GenuBgifts, (*sogar) enthielt er sich.

Des Tabaks (jedoch), eines typischen GenuBgifts, (*jedoch) enthielt er sich.

(6.17b) Dem $K$ u no (allein), einem begnadeten Erfinder, (*allein) gebührte der Preis.

Nun ist die Möglichkeit der Distanzstellung zu untersuchen. Dabei seien folgende Varianten geprüt:

(1) Basis im Vorfeld, Appositiv nach dem Klammerschluß;

(2) Basis im Mittelfeld vor, Appositiv nach dem KlammerschluB;

(3) Basis + weiteres Satzglied vor, Appositiv nach RlammerschluB.

Hinsichtlich des ersten Tests beurteile ich die Ergebnisse folgenderweise:

(6.18a) ??Das Rathaus haben sie neu angestrichen, am Marktplatz des orts.

$(6.18 \mathrm{~b})$ *Der Kuno ist Beamter geworden, spitznasig und dürr. ${ }^{13}$

13 In diesem Beispiel erscheinen Appositiv und Trăgersatz (bedeutung) nicht sinnvoll zueinander zu passen, weshalb der Satz wohl inhaltlich (pragmatisch) abweichend wirkt. (Diesen Hinweis verdanke ich Marie LeBras.) Demonstrieren soll er lediglich eine syntaktische Inakzeptabilităt. Man vgl. 
(6.18c) *Diese Frau ist zur Party gekommen, Dozentin in Bonn.

(6.18d) (?) Der Graf hat die Damen begeistert, übrigens vermutlich ein Vampir.

Der Graf hat die Damen begeistert, er ist ubrigens vermutlich ein Vampir.

(6.18e) Dem Tabak wird Pia nie entsagen, ?? ein typisches GenuBgift/ ??einem typischen Genußgift.

(6.18f) ??Kuno hat das nicht gewust, früher mein bester Freund.

(6.18a) ist - wenn überhaupt - nur so interpretierbar, daB die PP ein nachgetragenes Lokaladverbial reprãsentiert. Die appositionstypische Prädikationsrelation zwischen der PP und der NP im Vorfeld bleibt nicht erhalten. (6.18b) ist nicht sinnvoll lesbar. Der Versuch, die AdjP als nachgetragenes prädikatives Attribut zu interpretieren ('als Kuno Beamter geworden ist, war er spitznasig und dürr') scheitert. (6.18c) ist deutlich inakzeptabel. (6.18d) scheint einigermaBen annehmbar, doch könnte die Konstruktionen auch so verstanden werden, das eine Reduktion eines asyndetisch angereihten Satzes vorliegt (2. Beispiel unter 6.18d) und nicht ein Appositiv nach dem klammerschließenden Element. Wahrscheinlich mus man auch eine Regularität annehmen, nach der nach rechts verlagerte "Appositive" der Kategorie NP je akzeptabler sind, desto mehr Elemente diese begleiten. (6.18e, f) sind kaum annehmbar.

Die Urteile bzgl. der Stellungstests (2) und (3) sind nicht leicht $z u$ rechtfertigen, da sie idiolektabhăngig sein dürften:

(6.19a) ?? Sie haben das Rathaus angestrichen, am Marktplatz des Orts. ?? Sie haben das Rathaus gestern angestrichen, am Marktplatz des orts.

(6.19b) *Beamter ist der Kuno geworden, spitznasig und dürr.

*Beamter ist der Kuno gestern geworden, spitznasig und dürr.

(6.19c) ??Zur Party ist diese Frau gekommen, Dozentin in Bonn.

*Zur Party ist diese Frau gestern gekommen, Dozentin in Bonn.

(6.19d) Die Damen hat jener Graf begeistert, ubrigens vermutlich ein Vam pir.

Die Damen hat jener Graf gestern begeistert, ubrigens vermutlich ein Vampir.

(6.19e) Pia wird dem Tabak entsagen, ?ein typisches GenuBgift/ ?einem typi schen Genusgift.

Pia wird dem Tabak morgen entsagen, ?? ein typisches GenuBgift/ ?? einem typischen GenuBgift.

(6.19f) ?Das hat Kuno gewuBt, fruber mein bester Freund.

? Das hat Kuno seit langen gewuBt, früher mein bester Freund.

Insgesamt gesehen scheint die stellung hinter dem Klammerschlus umso akzeptabler, je ausführlicher (erweiterter) das Appositiv ist (vgl. mit abnehmender

das pragmatisch plausible Beispiel Der Kuno (, erfolgreich wie immer,) ist Beamter geworden (*, erfolgreich wie imner). 
Akzeptabilitat z.B. 6.19d, e und c). Bei den ubrigen Beispielen liegt Inakzeptabilităt vor oder aber wahrscheinlich Ronstruktionsverănderung (reduzierter zweiter Satz, Nachtrag etc.).

Für kaum üblich halte ich die Möglichkeit, die Basis im Vorfeld und das Appositiv im Mittelfeld zu plazieren; dennoch finden sich solche Sătze. Ob man sie zu den Möglichkeiten des Standarddeutschen rechnen oder als stilistische Besonderheiten (Ausnahmen) ansehen sollte, kann ich hier nicht klären:

(6.20) Er hat, ein weiser Schüler des Ptolemäus und der griechischen Philosophen, die neuen sterne beobachtet. 14

Zudem ist die stellungsvariante zu berücksichtigen, in der das Appositiv allein vorfeldfüllend aufritt (Beispiele aus Molitor 1979: 134):

(6.21a) Schöpfer des Tunnels, war er zu seinem Sklaven geworden.

(6.21b) Ein geiziger Mann, kam Onkel Erich nicht zur Familienfeier (...). Diese Variante ist recht eigenartig. Sie ist m.E. nicht systematisch möglich! ${ }^{15}$ Immer muB eine kausale, konzessive oder komparative Interpretation möglich sein (z.B. 'obwohl er der Schöpfer des Tunnels war' oder 'weil onkel Erich ein geiziger Mann war'). Zudem besetzt das Appositiv als ehemaliger "Gliedteil"(?) eine Satzgliedstelle (das Vorfeld), 16 sogar dann, wenn ein Nominal kleiner als N3 vorliegt. 17 Eine Rlärung dieser Variante kann hier nicht geleistet werden. Eine m.E. plausible Deutung der Varianten als verkürzte adverbiale Nebensătze möge als erste Erklărung dienen. 18

Trotz meines Unbehagens darf die Distanzstellung des Appositivs nicht ohne weiteres verworfen werden, finden sich doch Beispiele wie die folgenden: 19

(6.22a) Jetzt werde ich von Pater Schimmelpreester sprechen, einem nicht alltăglichen Manne.

(6.22b) Aber das Schicksal hatte Allan vor die Front geschickt, einen kalten Fechter, der nicht wich.

(6.22c) Ronsistorialrat Pieper stand, ein langer schwarzer strich, vor seinem Stubl.

(6.22d) Die neuen Mieter kamen herein, große ruhige Menschen mit einem stillen Kind.

14 Beispiel aus Sorinski, Bernhard. 1978. Deutsche Stilistik. Frankfurt/M., Fischer, S. 127.

15

So sind z.B. inakzeptabel (weitere Beispiele sind leicht zu kanstruieren):

(F6.3) *Spitzmasig und dürr, ist Kumo ein guter Pä́dagoge.

(F6.4) ?? Beute eine Medizinstudentin, war Pia friher nur Bausfrau.

16 Vielleicht ist die Apposition ja gar kein Gliedteil, sondern eher ein gliedassoziiertes Mlement; aber auch typische Gliedteile vie ein Präpositionalattribut zeigen dieses Verhalten (Auf bessere Zeiten sollte man die Boffnung nie aufgeben).

17 Normalerweise kann eine ON-Phrase (NO, M, N2) nie determinantienlos im Vorfeld stehen.

18 So z.B. Molitor (1979: $134 \mathrm{ff}$. ).

19 Beispiele aus Rusmin (1960: 72-85), allesamt von jenem aus literarischen Werken zitiert. 
Hier wird das Appositiv durch den KlammerschluB bzw. den KlammerschluB und ein Satzglied von der Basis getrennt (vgl. die Beispiele unter 6.18). Sogar für die Abfolge "Basis im Vorfeld, Appositiv nach KlammerschluB" (vgl. 6.17) 1aBt sich ein Beispiel finden. Oberdies gibt es Beispiele für die Variante "Basis im Vorfeld, Appositiv im Mittelfeld"! In eine Diskussion darüber, inwieweit solche Konstruktionen stilistische Eigenheiten bzw. Freiheiten darstellen, möchte ich mich hier nicht einlassen. Exakte Feststellungen (bzw. empirische Datenerhebungen) über die Möglichkeiten der Distanzstellung von Basis und Appositiv müssen aus Zeitgründen unterbleiben. Daher verbleibt ein nicht ganz zufriedenstellendes Fazit, das vorlāufig genügen muB:

- Die übliche Stellungsbeziehung zwischen Basis und Appositiv ist "Appositiv rechts von Basis", wobei sich zwischen Basis und Appositiv diverse Attributstypen schieben können;

- Prinzipiell unmöglich sind Distanzvarianten nicht. Sie sind jedoch an Sonderbedingungen gebunden, und zwar vornehmlich an die kategoriale Füllung des Appositivs (N3 ist am zugånglichsten, N2/N1/NO sind wenig zugänglich; PP andern meist ihre Interpretation; AdjP werden in Distanzstellung i.d.R. inakzeptabel) und an das Auftreten von erweiternden Elementen wie "Appositionslexeme", Gradpartikeln, (Satz)Adverbien etc. Am ehesten akzeptabel scheinen umfangreiche Appositive nach dem Klammerschluß, sofern die Basis im Mittelfeld (möglichst dem KlammerschluB nahe) steht. Unübliche, evtl. aus Gründen "stilistischer Freiheit" $0 . a ̊$. aber durchaus einmal mögliche Varianten mit Basis im Vorfeld und Appositiv im Mittelfeld oder nach dem Klammerschluß sind - statistisch betrachtet - ziemlich selten.

Altmann (1981: $298 \mathrm{ff.} 297 \mathrm{ff.}$ ) führte bereits Untersuchungen zum "Herausstellungsverhalten" der zur Diskussion stehenden Muster durch. Diesbezügliche Analysen habe ich (in der begutachteten Fassung der Diss.) ebenfalls durchgeführt, doch aufgrund des für diese Veröffentlichung begrenzten Raumes mũssen sie fortgelassen werden. (Eine gesonderte Veröffentlichung wird erwogen.)

Jetzt möchte ich noch einige syntaktische Permutationsoperationen in Betracht ziehen. Wie verhalten sich Basis-Appositiv-Typen bei CLEFT (6.23a) und PSEUDOCLEFT $(6.23 \mathrm{~b})$ bei Linksverschachtelung $(6.23 \mathrm{c})$ und bei Satzverschrănkung $(6.23 d) ?^{20}$

$20 \mathrm{Zu}$ CIRFT/PSEUDOCLWFT Altmann (1981: $176 \mathrm{ff}$. mit Literaturhinweisen), Engel (1988: $297 \mathrm{f}$. ). Bei CIBrT wird aus einem zugrundeliegenden Satz ein Satzglied herausgelost und in einen Kopulasatz der Struktur Es war $X$ eingefügt, wobei der Restsatz relativsatzartig rechts angeschlossen wird. Bei PSEDDOCLRT wird ebenfalls ein Satzglied ausgelöst und in einen Kopulasatz der Struktur ist $X$ eingefugt; der Restsatz steht links von diesem, mobei das ausgelöste Glied durch ein entsprechendes Interrogativ-plement vertreten wird.

Zur SATZVBRSCHRANKUANG s. Andersson/Kvam (1984), Engel (1988: 297 ff.); kurz auch in Eisenberg (1986: 367). Hierbei wird aus einem dab-Satz, der zumeist von einem Verbum dicendi abhăngt, ein Satzglied herausgenormen und ins Vorfeld des Obersatzes gestellt, während der Restsatz abzüglich des entfernten Teils rechts verbleibt. 
(6.23a) Es war Pia, Dozentin in Bonn, die zur Party kam.

Es war jener Graf, übrigens vermutlich ein Vampir, der die Frauen betörte.

Es war Kuno, spitznasig und dürr, der ein guter Pädagoge wurde. Es war das Rathaus, am Marktplatz des Orts, das renoviert wurde.

(6.23b) Ner zur Party kam, war Pia, Dozentin in Bonn.

Wer mich besuchen kommt, ist Kuno, früher mein bester Freund.

Was renoviert wurde, war das Rathaus, am Marktplatz.

(6.23c) Die Pia, Dozentin in Bonn, hoffte Kuno zu treffen.

Jenen Grafen, übrigens vermutlich ein Vampir, glaubte Pia zu kennen.

Den Kuno, spitznasig und dürr, hoffte Pia nie wieder zu sehen.

(6.23d) (?) Die Pia, Dozentin in Bonn, vermute ich, daB man auf der Party treffen wird.

(?) Den Tabak, ein beliebtes GenuBgift, glauben Wissenschaftler, daB man verbieten sollte.

(?) Dem Kuno, spitznasig und dürr, glaube ich, daB man die Exmatrikulation angedroht hat.

Obzwar die Wertung der Beispiele nicht ganz leichtfiel, kann ich die entstehenden Syntagmen überwiegend akzeptieren (mit Einschränkungen bei der Satzverschränkung). Falls man nur die Basis extrahiert und das Appositiv im Restsatz beläBt, muten die entstehenden syntagmen inakzeptabel an:

(6.24a) *Es war jener Graf, der, übrigens vermutlich ein Vampir, die Frauen betörte.

(6.24b) * Wer die Frauen betörte, übrigens vermutlich ein Vampir, war der Graf.

(6.24c) ??Jenen Graf glaubte Pia zu kennen, übrigens vermutlich ein Vampir.

(6.24d) * Jenen Graf vermute ich, das man auf der Party treffen wird, übrigens vermutlich ein Vampir.

4) Funktion des Appositivs: Hinsichtlich der pragmatischen Funktionen móge der pauschale Hinweis genügen, daß das Appositiv der Basis eine zusătzliche, sekundảre Information (eine Charakterisierung) hinzufūgt. ${ }^{21}$ Semantisch betrachtet liegt Eigenschaftszuschreibung (6.25a), Klassenzuordnung resp. -einordnung (6.25b), Gleichsetzung (6.25c) oder "Lokalisierung" (6.25d) vor. Das Verhaltnis zwischen Basis und "Appositiv" kann als prädizierend bezeichnet werden (verdeutlicht durch die Kopula-Paraphrase):

(6.25a) Pia, (sie war) Iustig und fidel, ermunterte alle Anwesenden.

(6.25b) Pia, (Pia ist) eine Dozentin, widerlegte diese These.

Zur LDKSVDRSCHACHIVIUNG Kvam (1979), kurz auch Eisenberg (1986: 366). Sie funktioniert analog zur Satzverschränkung mit dem Unterschied, daß das Satzglied nicht aus einem Nebensatz sondern aus einer abhăngigen Infinitivgruppe herausgelöst wird.

21 Raabe (1979: 237 ff.) gibt einen Unriß der möglichen pragmatischen Punktionen van Appositiven. 
(6.25c) Der Kultusminister von Baden-Nürttemberg, (er ist übrigens zugleich) der Prásident des VFB stuttgart, steht dort.

(6.25d) Pia, (sie war) gerade im Reller, hörte ein seltsamen Gerăusch. Unter dem Gesichtspunkt von "Kompressions- resp. Verdichtungserscheinungen im einfachen Satz" läßt sich sagen, daß die Apposition ermöglicht, der Basisproposition des Gastsatzes (vgl. Rap. 5.5) eine oder mehrere zusatzliche Propositionen (als "sekundare Prädikationen") hinzuzufügen. Beim Prototyp wird die Proposition eines Kopulasatzes in den Gastsatz integriert (vgl. Kuno liebt Asterix + Kuno ist ein Comicfan > Kuno, ein Comicfan, liebt Asterix).22

Etwas andere Funktionen haben folgende "Zusătze", die der Apposition ăhneln und nicht selten mit ihr in eins gesetzt worden sind: Durch eine Rechtsversetzung (6.26a) wird hingegen eine referenzunklare NP verdeutlicht (ausbuchstabiert). Die spăter von mir spezifizierende Zusătze genannten Einheiten (6.26b) nennen präzisierend (hervorhebend etc.) eine Teilmenge der Basis. In (6.26c) nennt der zusatz verallgemeinernd eine obermenge (spăter: nicht-spezifizierende Zusatze; 6.26c); in (6.26d) kann er als Ausdruck aufgefast werden, der alternativ zur Basis gilt (spăter: Alternativen nennende Zusätze):

(6.26a) Sie, die Pia, ermunterte alle Anwesenden.

(6.26b) Die Affen, vor allem die Paviane, belustigten die Rinder.

(6.26c) Die Paviane, allgemeiner alle Affen, belustigten die Kinder.

(6.26d) Die Ledigen, m.a.W. die Unverheirateten, tanzten am hăufigsten.

Zu jenen "Zusatze" in (6.26) existiert keine Kopulasatzumschreibung; sie prădizieren nichts über ihr Bezugselement. Zu ihren Funktionen s. Kap. 12, 15.

5) Paraphrase: In der Appositionsforschung wurde öfters erwăhnt, daß für den Prototyp eine Kopulasatzparaphrase angenommen werden kann. Der Kopulasatz kann als ("parenthetischer") Verbzweitsatz oder als nicht-restriktiver Relativsatz (6.27 a, d) erscheinen:

(6.27a) Der Tabak, der Tabak/ er ist ein typisches GenuBgift, schmeckt ihr. Der Tabak - der ein typisches GenuBgift ist - schmeckt ihr.

(6.27b) Jener Graf, er ist übrigens vermutlich ein Vampir, betorte alle Frauen.

(6.27c) Pia, Pia/ sie ist Dozentin in Bonn, kommt zur Party.

(6.27d) Der Kuno, der Kuno/ er ist spitznasig und dürr, besucht mich. Der Kuno - der übrigens spitznasig und dürr ist - besucht mich.

(6.27e) Das Rathaus, es ist am Marktplatz der stadt, wird renoviert. Daß die systematische Beziehung von Appositiven zu Prădikativen (und z.T. zu Subjekten) in Ropulasătzen zurecht angesetzt wird, verdeutlichen die folgenden. Beispiele:

22 Vgl. Moskalskaja (1984: 146 ff.), Sommerfeldt (1984). "Die Impandierung beruht auf der Informationsverdichtumg, darauf, daß ein oder mehrere prădikative Linien als impandierte Propositionen in den Satz eingehen (...)" (Moskalskaja 1984: 147). 
(6.28) Runo, (er ist) von Sinnen/ des Mahnsinns, tobte durch den Raum.

Kuno, (er ist) mit dem Kerl im Bunde, fuhlte sich stark.

Pia, (sie ist) Kompromissen feind, lebt lieber allein.

Dieses Idiom, (es ist) gang und gäbe, wird vielfach verwendet.

Nicht nur prădikative Genitiv-NPn und PPn, sondern auch reine Ropulapartikeln und nur kopulativ verwendbare Nortgruppen sind als Appositive möglich. ${ }^{23}$ Nicht zu finden sind dagegen Adjektivphrasen, die nur attributiv verwendbar sind. Hier wăren keine prådikativen Kopulasatzparaphrasen bildbar:

(6.29) Die Praxis, hell und *ärztlich/ gerăumig, wurde sofort gekauft. Die Praxis, sie war hell und *ärtlich/ geráumig, wurde sofort gekauft.

Man betrachte in diesem Zusammenhang auch:

(6.30) Pia, (sie ist) trotz lauernder Gefahren (RONZ) oft (FREQ) unvorsichtig, geht immer wieder in den Dschungel.

Pia, (sie ist) mit ihrem Freund (KOMIT) im Reller, suchte nach Weinvorräten.

Bei diesen Beispielen finden sich die Adverbiale aus dem Kopulasatz in den Appositionen wieder! Die Annahme systematischer Beziehungen zu Kopulasătzen erklărt z.B. auch die Tatsache, daB keine modalen, instrumentalen und direktionalen Adverbialbestimmungen in Appositiven erscheinen! Diese sind namlich in Ropulasătzen nicht möglich, weil sie nicht wie z.B. TEMP und KAUS "adsentential", sondern "verb- bzw. prădikatsbezogen" fungieren:

(6.31) *Pia, (sie ist) von München nach Bonn (DIR) mies gelaunt, schlief endlich ein.

*Kuno, (er ist) mittels der Săge (INSTR) arbeitsam, machte eine Pause.

*Pia, (sie ist) sehr leise (MOD) arbeitsam, war kaum zu hóren.

Interessant ist auch der Unterschied in folgenden Beispielen:

(6.32) Pia, (sie ist) wieder in der stadt (PTIV), freute sich sehr. ?Pia, (sie ist) wieder in die stadt (DIR) [gegangen], freute sich sehr.

Nur die erste Version, mit einem Ropulasatz paraphrasierbar, ist voll akzeptabel. Die zweite sieht wie ein Satz mit Ropula aus, was die Ellipse des infiniten Vollverbs nahelegt; eigentlich liegt aber eine Perfektform vor (ist = Tempusverb), von der ein Appositiv nicht ableitbar ist. Nicht gănzlich inakzeptabel ist das Beispiel vegen der möglichen Interpretation als elliptischer Adverbialsatz (als/ nachdem sie wieder in die stadt gegangen war).

Auch deutsche Aquivalente der "continuous form" lassen sich mittels der Ropulasatzannahme deuten:

23 Hierzu Engel (1988: 767 ff.). Ir nennt z.B. abhold, plemplem "Ropulapartikeln". Nicht selten werden solche Wörter als "nur prădikativ mögliche Adjektive" bezeichnet (z.B. DUDXN 1984: 272). 
(6.33) Kuno, (er war) gerade am Festerputzen, wurde ans Telefon gerufen. Pia, (sie war) intensiv am Nachdenken, wurde ans Telefon gerufen. Um die Darstellung zur Beziehung zwischen unserem "Appositionsprototyp" und Kopulasătzen abzuschließen, sei noch darauf hingewiesen, daß sich bei der "Apposition" durchaus Erscheinungen feststellen lassen, die man nicht "eins zu eins" mit den Verhăltnissen in Kopulasätzen setzen kann, z.B.:

(6.34a) Pia, (sie ist) Köchin aus Passion, servierte einen Karpfen.

(6.34b) Louis de Funès, er ist ??genialer Grimassenschneider/ ein genialer Grimassenschneider, hupfte wild herum.

(6.34c) Louis de Funès, (ein) genialer Grimassenschneider, hupfte wild herum.

Es ist möglich, artikellose Nominale (z.B. N2), die in Kopulasätzen vorkommen, nach Tilgung von Subjekt und Kopulaverb in ein Appositiv zu verwandeln (6.34a). Bei dem Beispiel in (6.34b) jedoch muB im Kopulasatz wohl ein Artikel gesetzt werden - als Appositiv kann die entsprechende NP dagegen artikellos verwendet werden. Appositive NPn weisen hinsichtlich der Artikelbegleitung eine gröBere Freiheit auf: Sie können sowohl mit als auch ohne Artikel erscheinen. Hier zeigt sich die bekannte Erscheinung, daß zwei sprachliche Konstruktionen, die ("transformationell") aufeinander bezogen werden könn(t)en, jeweils ihre "Idiosynkrasien" entwickeln.

Eine Parallele kann zwischen der "lockeren" Apposition und den als-Translativphrasen (dazu auch Kap. 7.B) gezogen werden; diese besteht in dem X-BarStatus der möglichen "Appositive". Auch in als-Phrasen können Nominale unterhalb der Stufe N3 (= NP) auftreten (6.35a); allerdings ist ihre Erweiterbarkeit stark eingeschränkt (6.35b). "Appositive" mit adjektivischen oder prăpositionalem Kopf sind als als-Komplemente unmöglich (6.35c):

(6.35a) Der Tabak als ein typisches GenuBgift (N3) schmeckt vielen Leuten. Pia als junge Dozentin (N2) ist sehr beliebt.

Pia als Fachfrau (NO) wurde um Rat gebeten.

(6.35b) * Runo als fruher mein bester Freund besucht mich morgen.

* Jener Graf als übrigens vermutlich ein Vampir betörte alle Frauen.

$(6.35 \mathrm{c})$ * Kuno als spitznasig und dürr ist ein guter Pädagoge.

* Das Rathaus als am Marktplatz des orts wird renoviert.

Im ubrigen kann der Artikel in als-Phrasen ebenfalls eher entbehrt werden als in den entsprechenden Kopulasătzen:

(6.36) Louis de Funes ist ein genialer Grimassenschneider/ ??genialer Grimassenschneider.

Louis de Funds als ein genialer Grimassenschneider/genialer Grimassenschneider brachte uns zum Lachen.

6) Rasuskongruenz: Sie wird m.E. als notwendiges Appositionskriterium überbewertet. Sie ist allerdings unverzichtbar (sofern eine unerweiterte Voll-NP 
vorliegt), wenn mehr als eine mögliche Basis zu einem Appositiv in Frage kommt :

(6.37) Er hat dem Freund des Onkels, ein stämmiger Mann/ einem stämmigen Mann/ eines stämmigen Mannes, geholfen.

Unmarkierte bzw. nominativisch markierte Appositive können bezugsunklar sein! Die Zuordnung erfolgt dann "pragmatisch": Die erste mögliche links gelegene Bezugsphrase wird im Zweifelsfall als Basis angenommen (sofern der Kontext nicht dagegen spricht). Nur die Kasuskongruenz sichert eine eindeutige Zuordnung zu einer Basis.

Die Regularitäten der Kongruenz werden nur kurz behandelt; speziellere Aussagen zu diesem Komplex (nicht nur hinsichtlich des Appositionsprototypen) sind in den zum Phänomenbereich ausgewählten Arbeiten (s. Kap. 2, 3) und in zahlreichen Publikationen nachzulesen. ${ }^{24}$ Bei unserem Prototypen

- tritt in jedem Fall Kasuskongruenz auf, wenn das Appositiv durch eine nicht durch Zusătze, Floskeln etc. erweiterte NP repräsentiert wird und eine eindeutige Zuordnung zur Basis (aus Disambiguierungsgründen) notwendig ist;

- kann Kasuskongruenz immer dann auftreten, wenn das Appositiv eine unerweiterte NP ist. Es kann jedoch auch der Nominativ ("der Nullkasus") gewăhlt werden (Das Leben Kunos, ein Abenteurer/ eines Abenteurers,). Selbst wenn de facto die Kasuskongruenz meist realisiert wird, so liegt doch auBer der Vermeidung von Ambiguität keinerlei grammatische Notwendigkeit dazu vor! ${ }^{25}$ Das Appositiv muß also nicht notwendigerweise mit dem "Bezugselement" im Kasus kongruieren; hier zeigen sich eine gewisse Unabhängigkeit vom Bezugselement sowie der eher parenthetische als attributive Charakter des Appositivs. (Speziellere Abweichungen wie z.B. "Dativapposition" sind unter Sonderbedingungen möglich, die man in der angegebenen spezialliteratur nachlesen kann.)

- kann nie Kasuskongruenz vorkommen, wenn das Appositiv ein Nominal der XBar-Stufen N2, N1 oder NO ist, also determinantienlos vorkommt (vgl.: das Leben Kunos, (kühner) Abenteurer/ *(kühnen) Abenteurers (aus Passion));

- kann nie Kasuskongruenz auftreten, wenn das Appositiv von Gradpartikeln, Satzadverbien, adverbialen Zusätzen oder Floskeln begleitet ist (Das Leben Runos, früher/ ubrigens/ auch/ vermutlich ein Abenteurer/ *eines abenteurers,).

7. Referenzidentität: Auch sie spielt m.E. bei der Appositionsdefinition keine entscheidende Rolle. In Făllen mit "Identitätsprädikation" wie unsere Nachbarin, (übrigens) die Ehefrau des Musikprofessors Brummel, können sich beide NPn

24 Ljungerud (1955; v.a. 106 ff.); Schönmann (1959); Winter (1966); Seibicke (1968, 1969); Sievers (1969a, 1969b); Admoni (1970: 111, $261 \mathrm{ff}$ ); Bănzinger (1970: $94 \mathrm{ff} ., 120 \mathrm{ff.}$ insb. 131 ff. u. $151 \mathrm{ff}$. ); Förster (1972); Waterman (1972); Rieffer/Aron (1974); Leirbukt (1978); Gippert (1981); Ostrowski (1982); Abraham (1983); Starke (1983b); Bergenholtz (1985); Duden (1985: 68 ff.); Bisenberg (1985; v.a. TYp Ein Glas Wein); Heringer (1988: 276 f.).

25 Nach Bergenholtz (1985) findet sich die Rongruenz in 96,48 aller möglichen Fălle. 
auf den gleichen Referenten beziehen. Im Fall von unsere Nachbarin, Ehefrau des Musikprofessors Brummel, referiert das Appositiv nicht (zumindest verhält es sich in den mir bekannten Theorien sprachlicher Referenz so, daB determinantienlose singularische Nominale nicht referieren) und somit kann von Referenzidentität keine Rede sein.

Bei Ausdrücken wie Pia, lustig und fidel, oder Pia, gerade im Keller, ist es m.E. sinnlos, von Referenzidentität zu sprechen, außer man meint einen sehr allgemeinen Bezug zweier sprachlicher Einheiten auf die selbe Entităt in der außersprachlichen Realităt. Inwiefern ein solcher Referenzbegriff sinnvoll und vor allem definitorisch fasbar sein soll, ist mir jedoch unklar.

8. Heglaßbarkeit: Appositive sind syntaktisch betrachtet i.d.R. entbehrlich; ihr Auftreten ist in keinem Fall syntaktisch notwendig (z.B. valenzgefordert). Im Einzelfall kőnnen unter Sonderbedingungen allerdings störungen auftreten:

(6.38) Pia, ein Rechengenie, es wird seine Fähigkeiten heute unter Beweis stellen, verlangt mehr Geld.

*Pia, es wird seine Fähigkeiten heute unter Beweis stellen, verlangt mehr Geld.

9. Appositionslexeme: Als Zusătze zum Prototypen seien zunăchst nur zugelassen: Gradpartikeln, Satzadverbien, freie Angaben (temporale Adverbphrasen usw.) und die Floskeln übrigens und (seltener) bekanntlich. Andere zusätze (wie und zwar/d.h./ präziser usf.) werden in Kap. 12 untersucht, da sie bei der Unterscheidung von "Prototyp" und verwandten Funktionen bedeutsam werden.

10. Appositiv vertritt Basis: Dieses Kriterium ist unbrauchbar, da nur bei gleicher kategorialer Füllung von Basis und Appositiv eine wechselseitige Vertretung überhaupt möglich ist. Dann ist sie natürlich meist auch nur syntaktisch möglich. Bei erweiterten Appositiven treten auch syntaktische störungen auf, da hier keine komplexe Phrasenkategorie (etwa NP) vorliegt, sondern eine Folge mehrerer Phrasen. Auch semantisch-logisch betrachtet ist die Vertretung öters unmőglich:

(6.39) Die Pia, eine kesse Dame,/ Die Pia/ Eine kesse Dame tanzte wieder auf dem Tisch.

Die Pia, sicher eine kesse Dame,/ Die Pia/ *Sicher eine kesse Dame tanzte wieder auf dem Tisch.

Die Pia, begeisterte Köchin,/ Die Pia/ *Begeisterte Köchin ist jetzt Runos Frau.

Die Pia, eine altere Dame,/ Die Pia/ *Eine ältere Dame war dreimal verheiratet.

Im letzten Beispiel ist die Vertretungsvariante semantisch nicht äquivalent mit dem Ausgangssatz. Pia muß nicht notwendigerweise als altere Dame dreimal verheiratet gewesen sein. 
11. Satzgrenzenbestimmtheit: Wegen der notorischen Unbestimmtheit des Satzbegriffs - man kann ihn nur theorieabhăngig und nicht a priori festzulegen - ist dieses Merkmal wenig brauchbar.

12. Attribution: Die hier besprochenen lockeren Appositive konnnen ohne weiteres attributiv erweitert werden:

(6.40a) Frank Zappa, ein innovativer Musiker aus den USA, der abwechslungsreiche Musit produziert, ist Pias Liebling.

(6.40b) Der Kerl, des Mundraubs äuBerst verdąchtig, wurde verhórt.

13. Textnotwendigkeit: trăgt kaum zur Abgrenzung bei (vgl. Kap. 3).

14. Sprecherdivergenz und Sprecherpräsenz: Bei deutlichen Redewiedergabeindikatoren (Anführungszeichen) läßt sich zeigen, daß das Appositiv sowohl als vom aktuellen Sprecher (6.41a) als auch vom referierten sprecher (6.41b) geäuBert aufgefast werden kann:

(6.41a) Pia sagte: "Otto", (übrigens) ein Trinker/ lustig und fidel/ zur Zeit in Bonn, "hat gestern Hugo angerufen".

(6.41b) Pia sagte: "Otto, (übrigens) ein Trinker/ Iustig und fidel/ zur Zeit in Bonn, hat Bugo angerufen".

(6.41c) Pia sagte, Otto, ein Trinker/ lustig und fidel/ zur Zeit in Bonn, hat/ habe Hugo angerufen.

In (6.41c) mit referierter Rede ohne interpunktionelle Viedergabeindikatoren ist es unklar, welchem Sprecher das Appositiv zuzuschreiben sei. Doch auch dieses Rriterium wird im weiteren nicht berücksichtigt. Raabe (1979: 268 ff.) behauptet, daß der Zusatz des Appositionslexems übrigens vereindeutige, daß das Appositiv eine Außerung des aktuellen Sprechers sei. Ich kann es (zugegeben mit etwas Mưhe) auch für zutreffend halten, das es vom referierten sprecher stammt:

(6.42) (?) Der Verdächtige äuBerte wörtlich, das otto, ubrigens ein Trinker, den Kerl angerufen hat/ habe, doch m.E. ist otto gar kein Trinker.

Entscheidender ist jedoch, daß die Trennschärfe dieses Rriteriums innerhalb der appositionsverdächtigen strukturen relativ gering sein dürfte. Man prüfe das selbst nach, hier nur ein Beispiel (Rechtsversetzung); wer äußert der Trinker?

(6.43) Der Verdăchtige sagte, daß er, der Trinker, den Hugo angerufen habe/ hat.

15. Adordinativität: Dieses Merkmal wird vernachlăssigt (vgl. Kap. 3 u. 5).

16. Tragweite von Negation und Frage: Dieses selten in der Appositionsbestimmung behandelte Merkmal ist von großem Interesse. Das Appositiv wird vom Skopus einer Negation oder einer Frage, die den Satz betrifft, nicht affiziert. 
So kann man $z$.B. in den nachstehenden Beispielsătzen bestimmte Folgerungen nicht ziehen (Asterisk!):

(6.44a) Es ist nicht der Fall, daB otto, ein Trinker, Hugo anrief. *Es ist nicht der Fall, daB otto ein Trinker ist.

(6.44b) otto, ein Trinker, steht an der Bar. * Otto ist aber gar kein Trinker.

(6.44c) Ist es der Fall, daB otto, ein Trinker, Hugo anrief?

*Ist es der Fall, daß otto ein Trinker ist?

Es ist weder möglich, das Appositiv aus dem Trăgersatz heraus zu negieren, noch kann man es "in Frage stellen", geschweige denn durch eine Gliedteilfrage erfragen (zur Verdeutlichung und Abgrenzung werden vergleichbare "enge strukturen" angeführt):

(6.45a) *Nicht otto, ein Trinker, torkelte herbei, sondern otto, ein Lachgasschlucker.

Nicht ein Trinker otto torkelte herbei, sondern ein Lachgasschlukser otto.

(6.45b) Torkelte otto, ein Trinker, herbei oder otto, ein Lachgasschlucker? Torkelte ein Trinker ot to herbei oder ein Lachgasschlucker otto?

(6.45c) Welcher/ Was für ein otto torkelte herbei? *otto, ein Trinker/ Ein Trinker otto.

Es ist übrigens gleichgütig, ob man eine kontrastierende Negation (6.46a) oder eine Satznegation $(6.46 \mathrm{~b})$ anwendet; es folgt zwar stets, das der Satz (Sachverhalt) verneint wurde, nie jedoch eine der beiden weiteren möglichen Verneinungen $(6.46 \mathrm{c})$ :

(6.46a) Nicht otto, ein Trinker, torkelte herbei (, sondern Kuno).

(6.46b) otto, ein Trinker, torkelte nicht herbei.

(6.46c) Es ist nicht der Fall, das otto herbeitorkelte.

*Es ist nicht der Fall, daB ein Trinker herbeitorkelte. ${ }^{26}$

* Es ist nicht der Fall, daB otto ein Trinker ist.

Nun komme ich zum Verhalten bei weiteren (im Gastsatz) bereichsbildenden Ausdrücken wie Gradpartikeln, Satzadverbien und "quantifizierenden" Adverbi(al)en. Der Prototyp bleibt (im Ggs. zum "engen Typ") stets unaffiziert:

(6.47a) Sogar otto, ein Trinker, torkelte herbei $\left(^{*}\right.$, nicht nur otto, ein Lachgasschlucker).

Sogar ein Trinker otto torkelte herbei (, nicht nur ein Lachgasschlucker ottol.

(6.47b) otto vermutlich, ein Trinker, wird dort saufen.

*Es ist vermutlich der Fall, daB otto ein Trinker ist

Ein $\operatorname{Tr} i$ nker otto vermutlich wird dort saufen.

Es ist vermutlich der Fall, das otto ein Trinker ist.

26 Man bedenke, daß ein Trinker als Klasseneinordnung (oder Eigenschaftszuschreibung) über Ot to prädiziert ist. Wenn nicht otto herbeitorkelt (sondern Udo), dann besteht logisch dennoch die Möglichkeit, daß es sich um einen Trinker handelt. 
(6.47c) Pia, eine àltere Dame, hat dreimal geheiratet.

*Eine altere Dame hat dreimal geheiratet.

$(6.47 \mathrm{c})$ ist insofern abzulehnen, als Pia bereits als junge Dame ein- oder zweimal geheiratet haben könnte.

Intensionale Adjektive, die die Basis begleiten, können sich auf das Basisnomen beziehen, nicht aber auf eine lockere Apposition. Nur wenn vermeintlich innerhalb des Appositivs vorkommt, "operiert" es auf dem Appositiv-Nomen:

(6.48) Der vermeintliche Mörder, ein brutaler Mensch, beschwerte sich bei der Polizei.

$X$ ist vermeintlich (nicht unbedingt tatsächlich) ein Mórder.

*Der Mörder ist vermeintlich ein brutaler Mensch.

(6.49) Der Mörder, vermeintlich ein brutaler Mensch, (...).

Der Mörder ist vermeintlich ein brutaler Mensch.

17. Wahrheitswertkonstanz: Das Appositiv tangiert den Wahrheitswert der Aussage bzw. des Satzes nicht. Diese gewiß zutreffende Aussage trägt nicht viel zur Appositionsbestimmung und -abgrenzung bei, weil sich die Hospitanten generell den Gastsatz-Wahrheitswert unbeeinfluBt lassen. Zudem reicht hier die Durchführung der Weglaßprobe aus.

Jetzt sollen noch einige weitere Merkmale überprüft werden, die man nicht (explizit) in der Appositionsliteratur findet.

18. Rekursivităt und Koordination: Appositive unseres Prototypen können rekursiv erzeugt werden (sofern nicht semantische Restriktionen eine Rolle spielen) und gemăB den üblichen Koordinationsregeln verbunden erscheinen:

(6.50a) Der Tabak, ein typisches GenuBgift, eine Verlockung der Jugend, aus Amerika, seit langem bekannt, wird seit einiger zeit geächtet.

(6.50b) Jener Graf, wahrscheinlich uralt, aus Transsylvanien, vermutlich ein Vampir, betörte alle Frauen.

19. Pronominalisierung und anaphorische Bezüge: Die Basen können wohl regulär, die Appositive hingegen kaum bis gar nicht durch eine Pro-Form ersetzt werden:

(6.51a) Pia/ Sie, Dozentin in Bonn, kommt zur Party. ??Pia, sie, kommt zur Party.

(6.51b) Kuno/ Er, früher mein bester Freund, besucht mich morgen. ?? Kuno, er, besucht mich morgen.

(6.51c) Der Graf/Er, ubrigens vermutlich ein Vampir, betörte die Damen. ??Der Graf, er, betörte die Damen.

(6.51d) Kuno/ Er, spitznasig und dürr, ist ein guter Padagoge. ??Kuno, er, ist ein guter Pădagoge.

(6.51e) Das Rathaus/ ?Es, am Marktplatz der Stadt, wird renoviert. (?) Das Rathaus, dort, wird renoviert. 
Für erweiterte Ausdrücke wie früher mein bester Freund existieren keine Proformen. PP-Ersetzungen durch lokale Pro-Adverbien wirken relativ akzeptabel. Man kann auch Adverbien als "Appositive" auffassen (vgl.: Das Rathaus, es ist dort,), so daB die Appositiv-Kategorien unter Punkt 1. zu erweitern sind.

Es ist teilweise möglich, sich anaphorisch auf Appositive bzw. auf Teile von Appositiven $z u$ beziehen. 27 Dies kann mittels Pro-Formen geschehen oder mittels Synonymen bzw. Ausdrücken mit ähnlicher Bedeutung (Hyperonyme etc. Vgl. 6.52e):

(6.52a) Diese Frau, ein Mathematikgenie, verblüfft mit ihren/*seinen Fäbigkeiten alle Professoren.

(6.52b) Diese Frau, ein Mathematikgenie, verblüft alle Professoren. Ihre/ (?) Seine Fähigkeiten sind wirklich auBerordentlich.

(6.52c) Diese Frau, ein Mathematikgenie, verblüft alle Professoren. Sie/ (?) Es rechnet schneller als der Computer.

(6.52d) Diese Kerle, ein verschworener Haufen, brachen den Rekord. Sie werden noch weitere Rekorde brechen/ (?) Er wird noch weitere Rekorde brechen.

(6.52e) Pia, Dozentin in Bonn, kommt zur Party. Unsere Dozentin/ Unsere Bonnerin/ Unsere Hochschullehrerin wird Zaziki mitbringen.

(6.52f) Pia, neuerdings Besitzerin eines Lowen/ Krokodils, läBt diesen (jenen)/ dieses (jenes) ihr Haus bewachen.

(6.52g) Pia, neuerdings Besitzerin eines Lówen/ Krokodils, läßt dessen/ ??seine Zähne regelmäBig untersuchen.

(6.52h) Pia, neuerdings Besitzerin eines Löwen/ Krokodils, IảBt (?) ihn/ (?) es/ ihr ungewöhnliches Haustier frei herumlaufen.

(6.52i) Pia, neuerdings Besitzerin eines Löwen/ Krokodils, ist zu beneiden. Er/Es/ Jener/ Jenes bewacht nämlich wirksam ihren Garten.

(6.52j) Pia, neuerdings Besitzerin eines Löwen/ Krokodils, hat es schwer. Sein Appetit ist nämlich ungeheuer groß.

Schwierigkeiten ergeben sich bei Possessivpronomina (6.52a, b, g), die nicht ohne weiteres anwendbar sind, vor allem nicht im gleichen Satz, eher dagegen bei einer Satzfolge. Auch Personalpronomina sind bei Satzfolge eher annehmbar als innerhalb eines satzes $(6.52 \mathrm{c}, \mathrm{d}, \mathrm{h}, \mathrm{i})$. Demonstrative Elemente $(6.52 \mathrm{f}, \mathrm{g}$, i) scheinen am wenigsten restringiert. Prinzipiell jedoch ist das Appositiv hier nicht in gleicher Weise "abgeschirmt" wie z.B. bei Anwendung von NEGATIONS- oder GRADPARTIKELN, Skopus einer Frage etc.

Bei nicht-NP-formatigen Appositiven sei nur kurz auf mögliche Anaphern eingegangen; Pro-Adverbien im Folgesatz scheinen einigermaßen akzeptabel zu sein.

(6.53a) Der Kuno, spitznasig und dürr, ist ein guter Pădagoge. ? So (aussehend) wäre er auch nie Fotomodell geworden.

27 Vgl. Raabe (1979: 250 f., 254 f.). Nach Raabe (ebd. 255) "(...) wird ersichtlich, daß das Appositiv im Kontext von Voraussetzungsbedingumgen für nachfolgende Textteile einen unerläßlichen Bezugspunkt darstellen kann, nicht aber für vorausgehende Teile oder seinen Trägersatz". 
(6.53b) Das Rathaus, am Marktplatz des Orts, wird renoviert. (?) Dort wird auberdem noch ein Bürgerhaus gebaut.

20. Passivierung: Basis und Appositiv durchlaufen i.d.R. gemeinsam die Passivtransformation; es wirkt wenig oder gar nicht akzeptabel, wenn das Appositiv am ursprünglichen ort verbleibt (vgl. Beispiele 6.54). Im Ggs. dazu brauchen (per Anaphorik) NP-bezogene Schaltsătze das Bezugselement nicht zu begleiten $(6.54 \mathrm{~b}, \mathrm{~d})$ :

(6.54a) Pia hat den Kerl, übrigens ein alter Macho, beschimpft. Der Kerl, übrigens ein alter Macho, wurde von Pia beschimpft. ??Der Kerl wurde, übrigens ein alter Macho, von Pia beschimpft. *Der Kerl wurde von Pia, übrigens ein alter Macho, beschimpft.

(6.54b) Pia hat den Kerl, er war übrigens ein alter Macho, beschimpt. Der Kerl, er war übrigens ein alter Macho, wurde von Pia beschimpft. Der Kerl wurde, er war übrigens ein alter Macho, von Pia beschimpt. Der Kerl wurde von Pia, er war übrigens ein alter Macho, beschimpft.

(6.54c) Pia hat dem Kind, ein ungezogener Lümmel, etwas vorgelesen. Das Kind (, ein ungezogener Lümmel,) bekam (??, ein ungezogener Lümmel,) von Pia etwas vorgelesen.

*Das Kind bekam von Pia, ein ungezogener Lümmel, etwas vorgelesen. ${ }^{28}$

(6.54d) Das Kind (, es war ein ungezogener Lümmel,) bekam (, es war ein ungezogener Lümmel,) von Pia etwas vorgelesen.

(?) Das Kind bekam von Pia, es war ein ungezogener Lümmel, etwas vorgelesen.

Die Schaltsătze (mit NP-Proform) müssen nicht obligatorisch an der Passivtransformation teilnehmen, weil sie weder Gliedteile der NP, auf die die Passivtransformation operiert, darstellen noch an diese "assoziiert" sind - wie es für die "Appositive" anzunehmen ist ( $\mathrm{g} g \mathrm{l}$. Punkt 22). Restriktionen der Stellungsfreiheit von NP-bezogenen Schaltsătzen ergeben sich aus den Beschrănkungen, die für Pronominalisierungsbeziehungen gelten. Die Appositive verhalten sich bei der PASSIVIERUNG eher gliedteilartig, zumindest irgendwie "gliedassoziiert".

21. Dependenz: Die Dependenz ist als gerichtetes Miteinandervorkommen (Konkomitanz) zweier syntaktischer Einheiten $X$ und $Y$ zu verstehen, wobei $X$ als $R e-$ gens auch allein in einer gröBeren Konstruktion aufzutreten vermag, $Y$ als Dependens jedoch nur zusammen mit X. M.a.W.: $Y$ ist vorkommensgebunden an $X$. Syntaktisch last sich Dependenz durch eine Weglabprobe zeigen: $Y$ ist allein weglaßbar, X nicht. Zudem kann Dependenz anhand der Gerichtetheit von Kongruenz-, Rektions- und Valenzbeziehungen expliziert werden: Das Dependens ubernimmt

28 Der Asterisk markiert das Beispiel als inakzeptables Produkt, weil der Konstruktionssinn verăndert murde. 
evtl. Kongruenzmerkmale, ${ }^{29}$ es ist evtl. (im Kasus) regiert und evtl. valenzbedingt.

Eine bemerkenswerte Explikation im kategorialgrammatischen Paradigma hat Vennemann (1977) unternommen: ${ }^{30}$ Der Kopf einer Konstruktion ist das Regens, das Dependens ist der Spezifikator (als stellenreduzierendes Komplement oder Attribut):

(6.55) $\left((\text { das })_{\mathrm{n} / \mathrm{c}}\left(\left(\text { Buch }_{\mathrm{c}}\left((\text { von })_{\mathrm{c} / \mathrm{c} / / \mathrm{n}}(\text { Alfred })_{\mathrm{n}}\right)_{\mathrm{c} / \mathrm{c}}\right)_{\mathrm{c}}\right)_{\mathrm{n}} \cdot\right.$ So wäre in (6.55) z.B. Alfred Komplement (Dependens) des Kopfes von (Regens) und von Alfred Attribut (Dependens) des Kopfes Buch (Regens).

Wie verhălt es sich nun bei "Basis + Appositiv"? Zunăchst sprechen die Weglabbarkeit des Appositivs (dessen Auftretensgebundenheit an die Basis) sowie die Obernahme von Kongruenzmerkmalen augenscheinlich für Dependenz. Beide Merkmale sollten sehr kritisch betrachtet werden. Teils sind sowohl Basis wie auch Appositiv getrennt weglaßbar (ein schwaches Argument, da auch hier ganz bestimmte Konstellationen vorzuliegen haben), teils ist Kongruenz nur bei bestimmter kategorialer Füllung des Appositivs möglich und selbst dann ist sie meist optional:

(6.56) Pia Müller, die Frau von Kuno,/ Pia Müller/ Die Frau von Kuno liebt Graf Drakula.

Das Leben Kunos, Abenteurer(*s) von Beruf, verlief bewegt.

"Enge Appositive" werden von Krifka (1983: 28 f.) - er arbeitet im kategorialgrammatischen Paradigma - als Spezifikatoren angesehen. Die "charakterisierende Apposition" (unser lockerer Prototyp) ist nach inm weder spezifizierend noch koordinativ; dennoch unterscheidet Krifka in Bezugskonstituente und appositive Konstituente (ebd. 39), wobei mir nicht klar ist, ob nun eine Dependenzrelation anzunehmen ist oder nicht. Festzuhalten bleibt, daß eine spezifikationsrelation verneint wird: Das Appositiv spezifiziert sein Bezugselement nicht! Inwiefern liegt dann überhaupt noch eine operator-Operand-Beziehung vor? ${ }^{31}$ Sollte hier nicht strikt getrennt werden zwischen dem Trăgersatz (sowie den Spezifikationsrelationen innerhalb desselben) und den Einschüben/ Zusätzen/ HOSPITANTEN?

Wahrscheinlich steht und fält die Ansetzung einer Dependenzrelation zwischen Basis und Appositiv mit der Explikation von Dependenz. ${ }^{32}$ Genulgt (teil-

29 Man nehme etwa die Kongruenz des Adjektivattributs mit dem Kernnomen hinsichtlich Kasus, Mumerus und Genus (Bisenberg (1986: 226) nimmt Genusrektion an). Bei Ostrouski (1982) ist nachzulesen, daß Kongruenz nicht nur in Determinationssyntagmen (z.B. in NPs) vorkormt, sondern auch zwischen Phrasen, die nicht einer Ronstruktion angehören, wobei sie dann "den gleichen außersprachlichen Bezug von verschiedenen flektierten nominalen Fugungen deutlich" (Ostrowski 1982: 254) macht.

30 S. auch Vennemann/Jacobs (1982: $98 \mathrm{ff}$.$) .$

31 In Spezifikationsrelationen heißt der dependente Ausdruck Operator, das Regens Operand.

32 Kritisch hierzu äußert sich z.B. Engel (1982: 32): 
weise) Weglaßbarkeit und (teilweise) Obernahme von Kongruenzmerkmalen, dann ist beim lockeren Prototyp das Appositiv dependent. Rektions- bzw. Valenzforderungen der Basis ans Appositiv finden sich allerdings nicht.

Keine Dependenz liegt vor bei Anwendung der Merkmale von Vennemann (1977), weil es sich um keine spezifikationsrelation handelt, die in Kopf und Spezifikator aufzugliedern wăre. Semantisch betrachtet ist es wohl nicht sinnvoll, eine Operator(Appositiv)-Operand(Basis)-Relation anzusetzen.

Wenn Dependenz wie bei Tesniere (1980: $140 \mathrm{f.}$ ) sehr weit gefast wird, dann ist das Appositiv selbstverständlich dependent. Dann allerdings erstreckt sich diese Relation auf sehr viele Ronstruktionen - nach Tesnière (1980: 141) z.B. auch auf Anreden: In lat. Amo te, Domine wăre vom Verb te, von te wăre Domine dependent -, wobei dann fraglich wird, ob dies noch erwünscht (analytisch nüzlich) sei. Insgesamt ist m.E. eine Dependenzrelation zwischen Basis und Appositiv tendenziell auszuschließen, auch wenn das sehr allgemeine Merkmal der Vorkommensgebundenheit des Appositivs besteht. Zumindest ist Dependenz hier nicht im gleichen Sinne $z u$ verstehen wie $z$.B. die Relation zwischen Attribut und Bezugselement. Es läBt sich allerdings ein noch gröBerer "Independenzgrad" feststellen als bei unserem Prototypen, nămlich z.B. bei Schaltsătzen und Interjektionen; sie sind nicht mehr vorkommensgebunden, weil sie im Rahmen einer Satz- bzw. Außerungsfolge dem Gastsatz nachfolgen (und z.T. voraufgehen) könnten.

22. Konstituenz: Die syntaktische Evidenz dafür, daß Basis und Appositiv Konstituenten einer übergeordneten Konstruktion darstellen, ist angesichts des Verhaltens hinsichtlich der "Konstituententests" groß (vgl. Kap. 5). Basis und "Appositiv" sind gemeinsam vorfeldfähig (6.57a), gesamt erfragbar (6.57b), gesamt pronominalisierbar (substituierbar; $6.57 \mathrm{c}$ ) und eher nicht getrennt verschiebbar (6.57d):

(6.57a) Pia, eine Jurastudentin, trat ein.

(6.57b) Wer trat ein? Pia, eine Jurastudentin.

(6.57c) Pia, eine Jurastudentin,/ Sie trat ein. (Sie 1achelte.)

(6.57d) Pia trat ( $*$, eine Jurastudentin,) ein, (??eine Jurastudentin). Bei der Erfragung trifft man allerdings auf Beispiele, deren Akzeptabilitat fragwürdig ist; sie betreffen vornehmlich "Appositive", die nicht als Voll-NPs (N3) realisiert werden:

(6.58a) Pia, (eine) Facharztin, ist eine Bekannte von Kuno. Wer ist eine Bekannte von Kuno? Pia, eine Fachärztin/ ?Fachärztin.

(6.58b) Pia, lustig und fidel, hielt eine Rede.

Wer hielt eine Rede? ? Pia, lustig und fidel.

"Dependenz liegt vor, wenn Ronkomitanz in eine bestimnte (...) R i c h t u n g gebracht worden ist. Aber: Diese Ausrichtung ist keineswegs naturgewachsen oder irgendwie durch "die Sprache" vorgegeben, sie ist ein Artefakt, hervorgegangen aus einer w i $11 \mathrm{k}$ u $\mathrm{r}$ i c h e n Entscheidung des Grammatikers". 
(6.58c) Das Rathaus, am Marktplatz, wird restauriert.

Was wird restauriert? ? Das Rathaus, am Marktplatz.

DaB Basis und Appositiv an syntaktischen Operationen wie PASSIV, CLEFTING etc. gemeinsam teilnehmen, deutet auf Konstituenz hin.

Dennoch erheben sich beim Verf. Bedenken gegen eine derartige Beschreibung, da s.E. Appositive als zusătze anzusehen wären, die zwar relativ strikt bezugselementbezogen sind, jedoch als HOSPITANTEN nicht eigentlich dem Trägersatz angehören und somit auch keine Konstituentenbildung mit ihren Bezugseinheiten eingehen. Dies ist z.B. bei Schaltsätzen, die auf ein Satzglied bezogen sind, offensichtlicher: Sie müssen nicht strikt teilnehmen an operationen, die das "Bezugselement" betreffen und können vom Bezugselement getrennt werden; dabei durchwandern sie den Satz "von vorne nach hinten", eine Akzeptabilitätsminderung tritt nur infolge der Pronominalisierungsrelation auf. 33

Natürlich bedarf es nun einiger Belege gegen die Konstituenzwertung, die allerdings angesichts der starken positiven Evidenz möglicherweise nicht stichhaltig genug erscheinen werden. Zunächst ist es auffällig, daß NEGATION und GRADPARTIKELOPERATIONEN nur das Bezugselement affizieren können - nicht jedoch das Appositiv! Nach Jacobs (1982: 279 ff.) kann die Negationspartikel nicht (bei einteiliger Fokussierung) Konstituenten jeder beliebigen Kategorie unterhalb von $S$ fokussieren (und somit betreffen); Gradpartikeln vermögen dies ebenfalls. ${ }^{34}$ Bei einer komplexen NP ist jede Teilkonstituente betreffbar:

(6.59) Nicht ihre alte Tante in London spinnt, sondern sein junger Neffe aus Bonn.

Zusătze wie z.B. lockere Appositive (nicht-restriktive Relativsătze, Schaltsătze etc.), die an die NP treten, können hingegen weder durch Negationsträger noch durch Gradpartikeln affiziert werden:

(6.60) *Nicht ihre Tante, eine verrückte Mystikerin, sondern ihre Tante, eine verrückte Philosophin, spinnt.

Hierzu passen Beobachtungen, die Bartsch (1978) bei der Untersuchung alternativer Adverbialkonstruktionen gemacht hat. So sind z.B. die Antworten

(6.61) *Er ging, wegen seiner Bauchschmerzen/ weil er Bauchschmerzen hat te, zum Arzt.

auf die Frage Warum ist Hans zum Arzt gegangen? nicht korrekt, weil der Einschub eher als präsupponierte (und nicht als assertierte) Information, die nicht Gegenstand der weiteren Diskussion/Erörterung sein soll, anzusehen ist

33 Vgl.: (F6.5) Pia (- sie kann wirklich alles -) hat (- sie kann wirklich alles -) den Todessalto ((?)- sie kann wirklich alles -) vargeführt (- sie kann wirklich alles).

(F6.6) Kuno (* - sie kann wirklich alles -) hat (?? - sie kann wirklich alles -) Pia (- sie kann wirklich alles - ) verehrt (- sie kann wirklich alles).

34 s. Jacobs (1983: 38 ff.). 
(ebd. 8). 35 Als Merkmal wird angegeben, daß der Einschub nicht durch die Erwiderung Nein, das stimmt nicht oder die Frage Wirklich? betroffen werden kann. Jedoch sollte aufgrund der Fragestellung gerade das Kausaladverbial als Satzglied/PARTIZIPANT erscheinen (als assertierte Information) und nicht als Einschub bzw. HOSPITANT.

Dies läBt sich wahrscheinlich auf lockere Appositionen übertragen, die somit ebenfalls als "Information nebenbei" gelten dürfen und nicht von NEGATION, GRADPARTIKELN, FRAGEN und QUANTIFIKATIONEN (s. auch Punkt 16. dieses Kapitels) aus dem Trägersatz heraus betroffen sind. Die "Resistenz" gegen diese Operationen aus dem Trägersatz heraus legt nahe, das Appositive zumindest nicht den üblichen Konstituentenstatus innehaben.

Ein weiterer Punkt, der gegen Konstituenz spricht, wird in Motsch (1965: 93) angeschnitten, nämlich das Auftreten von Adverbien im Appositiv, die nicht als NP-Konstituenten interpretierbar sind (Peter, früher mein bester Freund,). Auch bei Diese fünf Musiker, ein jeder ein Meister seines Instrumentes, sei die unterstrichene Einheit nicht als Konstituente der nachfolgenden NP aufzufassen, 36 vielmehr müsse man von einem zugrunde liegenden, reduzierten Kopulasatz ausgehen. Wenn nun das Appositiv selbst nicht unbedingt eine Ronstituente - ja nicht einmal eine Phrase, denn ein jeder ein Meister seines Faches kann nach den mir bekannten Phrasenprinzipien keine Phrase sein - darstellt, wie soll man es mitsamt dem Bezugselement zu einer komplexen Konstituente bzw. Phrase zusammenfassen? Wird hier nicht auch dem $\mathrm{X}$-Bar-Phrasenprinzip widersprochen, daß jeder Nicht-Kopf (alles, was nicht No ist) als Phrase zu gelten hat? 37

Folgende Stellungsmerkmale sprechen evtl. auch gegen eine Konstituente "Basis + Appositiv", wobei man einrăumen muß, daß die Regel möglicherweise lautet: Stelle satzformatige resp. -wertige Konstrukte (wie z.B. auch restriktive Relativsatzattribute) hinter die Postposition, den nachgestellten Satzkonnektor, die nachgestellte Gradpartikel. Somit wăre das Argument nicht zwingend.

(6.62) Peter (*, früher mein bester Freund,) zuliebe/ jedoch/ sogar, früher mein bester Freund,

(6.63) Jeder Frau (??, die ihn ansah,) zuliebe/ jedoch/ sogar, die ihn ansah, blieb er treu.

Den Vorfeld-Stellungstest entwertet u.U. auch die Möglichkeit, daß die intonatorisch bzw. graphematisch markierte Apposition eine regulare Parenthesenische zwischen Vorfeldelement und Finitum besetzt:

35 Auf die Präsuppositionsproblematik kann hier nicht eingegangen werden. Ich vill nicht behaupten, daß "Apposition" (Parenthese etc.) gleichzusetzen ist mit "Prăsupposition".

36 Beispiele von Motsch (1965: 93).

37 Hierzu Gremendorf u.a. (1987: $198 \mathrm{ff.,} \mathrm{insb.} \mathrm{202).}$ 
- hol' ihn der Teufel -

$\begin{array}{llll}\text { (6.64) Unser Nachbar, } & \text { ein Philosoph, } & \text { schnarcht } \\ & \text {, er ist ein Philosoph, } & \\ \text { VORFELD } & \text { P A R E N T HE S E N I S C HE } & \text { FINITUM }\end{array}$

Wenn man eine VP-Pro-Formisierung (hier: Verb + Akkusativobjekt) vornimmt, dann scheint das "Appositiv" gar nicht davon betroffen zu sein; 38 zumindest wirkt die Variante mit aufgenommenem Appositiv fragwürdig. Wenn man dagegen die NP mit einem restriktiven Relativsatzattribut versieht, dann liegen die Verhältnisse so, daß das Attribut mit zu dem Sprachmaterial zu zählen ist, das von der VP-Pro-Form aufgenommen wird:

(6.65a) Pia hat den einzigen Gast, ein obskures Individuum, beobachtet, und Kuno tat das auch (, ich meine den einzigen Gast beobachten/ ??ich meine den einzigen Gast, ein obskures Individuum, beobachten).

(6.65b) Pia hat den einzigen Gast, der keinen Hut trug, beobachtet, und Kuno tat das auch (, ich meine den einzigen Gast beobachten, der keinen but trug).

Bei dem "Pro-VP-Test" verhält sich unser Prototyp wie eine Parenthese (die ebenfalls nicht vom Pro-Element erfaBt wird), er verhălt sich hierbei nicht wie eine Konstituente der VP bzw. des Bezugselements (Akkusativobjekt).

Fazit: Die "klassischen" Konstituententests (Vorfeldfähigkeit, Substitution, Erfragbarkeit) und die gemeinsame Teilnahme an syntaktischen operationen diverser Art (PASSIV, CLEFTING etc.) legen in der Tendenz nahe, daB "Basis + Appositiv" eine Konstituente bilden. ${ }^{39}$ Vor allem die "Adjazenzbedingung" (NP-bezogene Appositive dürfen sich kaum vom Bezugselement entfernen, auch nicht beim Durchlaufen syntaktischer Operationen; zu Distanzstellungsoptionen s. Punkt 3.) legt Konstituenz nahe.

Die Anwendung von Negation, Gradpartikeln, Fragen und Quantifizierungen sowie Konstituenten- bzw. Phrasenstrukturprinzipien, die Satzhaftigkeit des Appositivs (Motsch!), 40 die Nicht-Pro-Formisierbarkeit bei "Pro-vp" und viel-

38 Kriterium inspiriert durch McCawley (1982: 96). Has durch die Pro-Form aufgenommen wird, läßt sich durch einen referenzklärenden Zusatz (ich meine...) beschreiben. Der gekünstelt wirkende Ausdruck "Pro-Formisierung" ist notwendig, da Pronominalisierung bedeutet: 'Wiederaufnahme einer nominalen Form'.

39 Dennoch muß dies nicht bedeuten, daß die Konstituenz damit schon bewiesen ist. Man vgl. die kritische Außerumg von McCawley (1982: 97):

"Other tests for constituency of which I am aware - for example, the possibility of topicalizing an expression - provide less information about the constituent structure of sentences containing parentheticals, though the results are consistent with the position that placement of parentheticals changes only order but not constituent structure (...)".

40 Auch die Tatsache, daß Appositive Parenthesen (insonderheit Schaltsätze) enthalten können, demonstriert die Satzhaftigkeit und erschwert zusătzlich die Bildung einer gemeinsamen Konstituente oder Phrase. 
leicht manche stellungseigenschaften sprechen eher gegen Konstituenz; die Eigenschaft, HOSPITANT zu sein, ebenfalls. Aber wie bei der Dependenz gilt, daB es von der Explikation von Konstituenz abhängt, was unter ihren Begriff fällt.

Obwohl es natürlich einfacher wăre, die Prototypen ("lockeren Appositionen") entweder der attributiven Funktion oder der Funktion Parenthese (Einschub, Zusatz) zuzuordnen, erfordert die Lösung unseres Dilemmas möglicherweise neue Begriffe, die den zwitterhaften Status unserer prototypischen Appositive beschreiben, weil sie sich einerseits attributartig, andererseits wie HOSPITANTEN verhalten. Denn so anzweifelbar es ist, das Appositiv als Ronstituente der Bezugs-NP anzusehen, 41 so unbefriedigend scheint mir eine völlige Loslösung des Prototyps von dem "Bezugselement" und dessen Ansiedlung oberhalb des Gastsatzes analog zur Parenthese, die als oberste Ronstituente eines Satzes expliziert wird; ${ }^{42}$ dem obersten Satzsymbol ordnet man dabei als unmittelbare Konstituenten die Parenthese/Apposition und den (Rest)Satz zu.

Als ich uber das Einordnungsdilemma nachdachte, kam mir der folgende Vergleich in den Sinn: Das Appositiv ähnelt einem Kometen, dessen Eigenschaften (z.B. Umlaufbahn) zwar mit Bezug auf das Sonnensystem (= Satz) beschrieben werden müssen, der aber dennoch nicht in der gleichen Veise wie die Planeten (= Satzglieder) zum Sonnensystem gehört, sondern sich nur als zeitweiser Gast in ihm aufhäl. Mir gefiel das Rometen-Bild, so daB ich den Begriff Ronstituentoid (da lacht das Terminologenherz) einführen möchte, definiert als hospitant mit Bezugselement. Der relativ strikten "Assoziiertheit" mit der Bezugs-NP (ủberhaupt einem Bezugselement) wăre damit Rechnung getragen, ohne daB gleichzeitig ein striktes Ronstituenzverhăltnis angenommen wăre. Der Begriff Konstituentoid umfast unseren Prototypen, aber wahrscheinlich auch weitere $\mathrm{Zu}-$ sätze zu Satzteilen: evtl. appositive Relativsatze, Zusătze in Rundklammern (Willy Brandt (SPD)), prăzisierende Zusatze (Die Rrokodile, genauer Alligatoren und Kaimane,) U.a.m. Hierzu vor allem die Kapitel 7.A, 12, 13 und 15. Eine annăherungsweise Beschreibung des Verhăltnisses könnte ich mir folgendermaßen vorstellen; die strichelung reprăsentiert das Konstituentoid-Verhăltnis:

$(6.66)$

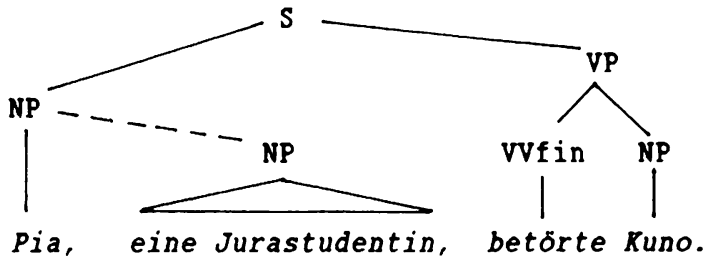

Ein Hospitant ohne Bezugselement (unterhalb der Satzebene) wăre dann eine $\mathrm{Pa}-$ renthese bzw. ein Einschub. Ein Partizipant mit Bezugselement auf syntaktischer Ebene ist ein Attribut (und Konstituente seiner Bezugsphrase), auf mor-

41 Vgl. auch Rap. 5. die Vorschlăge zur Beschreibung der Apposition im Rahmen der Ronstituentenstruktursyntax.

42 Man vgl. die Vorschlăge McCawleys (1982; 2.B. 99). 
phologischer Ebene ist er Determinans eines Kompositums. Die möglichen Bezüge zweier Sătze einer Satzfolge aufeinander sind vereinfachend unter dem Terminus Phorik zusammengefast. Die nachstehende, grobe Abstufung zeigt die grammatischen Bezugsmöglichkeiten zweier Einheiten aufeinander:

\section{PARTIZIPAN TEN}

$\begin{array}{ll}\text { Deteninans - } & \text { Attribur } \\ \text { (Ro-position) } & \text { Ronstituente } \\ 2 \text { Teile einer } & 2 \text { Teile einer } \\ \text { morpholog. } & \text { syntaktischen } \\ \text { Konstruktion } & \text { Ronstruktion }\end{array}$

$$
\begin{array}{lllllllllll}
H & O & S & P & I & T & A & N & T & E & N
\end{array}
$$

Zusatz
Ronstiturentoid
Auf Trägersatz-
einheit bezogener
"Satzrest"(?)

(unselbständiger Textteil?) 45 ständiger Textteil)

\section{(SATZPOLOE)}

Pharik.

Selbstăndiger Textteil (Satz)

mit phorischem Bezug

Folgende Sprachbeispiele mögen diese grobe obersicht verdeutlichen:

(6.67a) Flüssiggas wurde dort gelagert. (Determinans)

(6.67b) Flüssiges Gas wurde dort gelagert. (Attribut)

(6.67c) Gas, flussig und brennbar, wurde dort gelagert. (Zusatz)

(6.67d) Gas, es war flüssig und brennbar, wurde dort gelagert. (Einschub) Gas wurde, es war flüssig und brennbar, dort gelagert.

(6.67e) Dort wurde Gas gelagert. Es war flüssig und brennbar. (Phorik)

43 Wenn man annimnt, daß das Prototyp-Appositiv von einem zugrumdeliegenden Kopulasatz abzuleiten ist. Evtl. fallen unter Zusatz auch die nicht-restriktiven (appositiven) Relativsätze; vgl. dazu' Kap. 7.A.

44 Pro-Porm-Beziehungen auf Gastsatzelemente dürfen vorkammen, dennoch ist selbst dann der Schaltsatz wohl nur dem Cesamtsatz, nicht aber z.B. der "Bezugs-NP" zuzuordnen.

45 Nicht unproblematisch. Gemeint ist, daß Zusătze selbstăndigen Textteilen bzw. - schritten ăhneln (evtl. sind es ja Reduktionsformen von imnen, die in einen anderen Satz eingeschoben werden). 
Dieser Konstruktionstyp ist bislang nicht sehr beachtet worden; speziellere Untersuchungen $z u$ ihm sind rar. ${ }^{46}$ Die nachfolgenden Beispiele (6.68 aus Molitor 1979: 138; 6.69 aus Eichbaum 1978: 29)

(6.68) Die Jugend wendet sich mehr und mehr rechten Gruppen zu, ein Vorgang, der auf Ober- und Hochschulen zu beobachten ist.

(6.69) Tatsächlich wimmelte es am Strande von patriotischen Kindern - eine unnatüliche und niederschlagende Erscheinung.

demonstrieren die Konstruktion. Eine auf einen Deklarativsatz bezogene NP, 47 oft um (emotiv markierte) Attribute erweitert, 48 folgt zumeist rechts des Satzes, abgetrennt durch ein Komma, einen Gedankenstrich, durch strichpunkt, Doppelpunkt und manchmal auch einfachen Punkt. 49 Mündlich ist die NP durch eine deutliche (Satz)Pause vom "Bezugssatz" getrennt, der "Bezugssatz" schliebt mit fallendem Tonmuster ab. Die NP erhalt einen eigenen, deutlichen Akzent und weist ebenfalls fallende Intonation auf: 50

(6.70) /peter will sich $b$ e ssern $\mathrm{ft+}$ ein 1 o benswerter vorsatz $\mathrm{ft+}$.

46 Die ausführlichste ist Fichbaum (1978); s. auch Molitor (1979: $138 \mathrm{ff}$.). Sporadische Brwähnung bei Raabe (1979: 257, 262, 273, 275 f., 292 ff.). S. auch Blumel (1910: 8 f.; 1911: 41 ff.).

47 Bei Interrogativ- und Imperativsätzen z.B. ist diese Konstruktion wohl nicht möglich:

(F6.6) *Die Jugend mende sich mehr und mehr rechten Gruppen zu, ein Vargang, der auf Oberund Hochschulen zu beobachten ist!

(F6.7) * Wimmelte es tatsächlich am Strand von patriotischen Kindern - eine unnatürliche und niederschlagende Erscheinung?

Es handelt sich fast ausschließlich um $\mathrm{N} 3$ ! Eine $\mathrm{N}-$ Phrase unterhalb dieses Levels erscheint sehr selten und klingt markiert (1. Beispiel aus Molitor 1979: 147, das zweite ist kanstruiert):

(F6.8) Seit nevestem langweile ich mich in der Schule, nie dagewesenes, beunruhigendes Symptom.

(F6.9) Die ganze Gruppe warf sich auf den Boden und haderte mit dem Schicksal, ein Bild des Jammers/ (?) Bild des Jammers, das niemand so schnell vergessen wird.

48 Es genügt aber auch, wenn das Kernnomen wertend, emotiv, judikativ o.å. "aufgeladen" ist (das

2. Beispiel von Blitmel 1910: 9):

(F6.10) Am Strand wimmelte es van patriotischen Kindern - ein Ekelbild.

(F6.11) Die Kirche ist von Zypressen umgeben, ein munderbarer Anblick/ ein Traumanblick/ ein Hunschpanorama.

(F6.12) Die Gewässer müssen wieder sauber werden - ein ?? Vorhaben/ Jahrhundertvorhaben.

49 Nach Eichbaum (1978: 33) sind zudem Satzzeichenkombinationen und "Gedankenpunkte" möglich:

(56.13) Pia hat Kunibert verlassen, - ein trauriger Vorfall.

Pia hat Kunibert verlassen. - Ein trauriger Vorfall.

Pia hat Kunibert verlassen...ein trauriger Vorfall.

50 Beispiel aus Molitor (1979: 143); vom Verf. transkribiert. 
Zusätze zur NP sind möglich, jedoch sind Einschränkungen feststellbar, die einer gesonderten Untersuchung bedürften. Jedenfalls ist die Hinzufügbarkeit nicht so frei wie beim Appositionsprototypen:

(6.71a) Peter will sich bessern - übrigens/ ?? bekanntlich ein lobenswerter Vorsatz.

Peter will sich bessern - sicher/ (?) vermutlich/ (?) wahrscheinlich ein lobenswerter Vorsatz.

Peter will sich bessern - wegen seiner Folgen/ ?? seit gestern ein guter Vorsatz.

Peter will sich bessern - *sogar/ ? auch / *halt doch ein lobenswerter Vorsatz.

(6.71b) Hans hat das Fenster eingeschlagen - übrigens/ bekanntlich eine durch nichts bewiesene Behauptung.

Hans hat das Fenster eingeschlagen - sicher/ vermutlich/ wahrscheinlich eine durch nichts bewiesene Behauptung.

Hans hat das Fenster eingeschlagen - wegen fehlender Indizien/ noch immer/ trotz einiger Verdachtsmomente eine durch nichts bewiesene Behauptung.

Hans hat das Fenster eingeschlagen - (?) auch/ ?? nur / *sogar/ ?doch wohl eine durch nichts bewiesene Behauptung.

Die Floskel übrigens kann augenscheinlich einschränkungslos hinzugefügt werden, ebenso Satzadverbien. Auch freie Angaben (z.B. Temporal-, Kausaladverbiale etc.) sind insoweit möglich, als sie semantisch mit der "Satzapposition" verträglich sind. Gradpartikeln sind wohl nicht akzeptabel, bei auch bin ich allerdings nicht sicher. Modalpartikeln sind ebenfalls wenig annehmbar. Statistisch betrachtet treten die Satzappositionen eher selten mit den soeben besprochenen Erweiterungen auf. Die häufigsten Erscheinungsform der "Satzapposition" ist: "unbest. Art + $(\operatorname{Adj})+N+($ attributiver Relativsatz)"; die Rundklammern stehen für die Alternativität prä-bzw. postnominaler Spezifikatoren. Die Attributionsmöglichkeiten dürften keinen Einschränkungen unterliegen.

Die stellungseigenschaften der NP bzgl. des Satzes lassen sich so formulieren: Sie wird am häufigsten dem Bezugssatz nachgestellt, kann aber auch in diesen an den bekannten Parenthesenischen eingefügt sein.

(6.72a) Die Jugend wendet sich mehr und mehr rechten Gruppen $z u$, ein Vorgang, der auf Ober- und Hochschulen $z u$ beobachten ist.

Die Jugend, ein Vorgang, der auf Ober- und Hochschulen zu beobachten ist, wendet sich mehr und mehr rechten Gruppen $z u$. Die Jugend wendet sich, ein Vorgang, der auf Ober- und Hochschulen $z u$ beobachten ist, mehr und mehr rechten Gruppen $z u$. Die Jugend wendet sich mehr und mehr, ein Vorgang, der auf oberund Hochschulen $z u$ beobachten ist, rechten Gruppen $z u$. 
(6.72b) Tatsächlich wimmelte es am Strande von patriotischen Kindern - eine unnatürliche und niederschlagende Erscheinung.

Tatsächlich - eine unnatürliche und niederschlagende Erscheinung wimmelte es am Strand von patriotischen Kindern.

Tatsächlich wimmelte es (- eine unnatürliche und niederschlagende Erscheinung -) am Strand (- eine unnatürliche und niederschlagende Erscheinung -) von patriotischen Kindern.

Die Positionen links vom Satz $(6.73 a, 6.74 a)$ ist inakzeptabel. Eher annehmbar wirkt dagegen die Variante, in der nach dem "Appositiv" links vom Satz ein Doppelpunkt steht (größere Integration; 6.73b, 6.74b). Die Stellung des Appositivs rechts vom Satz mit Punktabtrennung (bzw. fallender Intonation und Satzpause) ist nicht völlig akzeptabel $(6.73 \mathrm{c}, 6.74 \mathrm{c})$; die Satzapposition verhält sich somit nicht wie ein selbständiger Ausdruck (Einschub), sondern eher wie ein Zusatz:

(6.73a) *Ein Vorgang, der auf Ober- und Hochschulen zu beobachten ist. Die Jugend wendet sich mehr und mehr rechten Gruppen $z u$.

(6.73b) ?Ein Vorgang, der auf Ober- und Hochschulen zu beobachten ist: Die Jugend wendet sich mehr und mehr rechten Gruppen $z$.

(6.73c) (?) Die Jugend wendet sich mehr und mehr rechten Gruppen zu. Ein Vorgang, der auf Ober- und Hochschulen $z u$ beobachten ist.

(6.74a) *Eine unnatürliche und niederschlagende Erscheinung. Tatsáchlich wimmelte es am Strande von patriotischen Kindern.

(6.74b) ?Eine unnatürliche und niederschlagende Erscheinung: Tatsáchlich wimmelte es am Strande von patriotischen Kindern.

(6.74c) (?) Tatsachlich wimmelte es am Strande von patriotischen Kindern. Eine unnatürliche und niederschlagende Erscheinung.

Die nachstehenden Satzfolgen sind dagegen ohne weiteres möglich; als mögliche Ableitungsgrundlage wurden die "Appositive" durch Kopulasătze paraphrasiert. Verschöbe man die Kopulasătze in die anderen Sătze, dann hătte man es mit Schaltsătzen (EINSCHOBEN) zu tun.

(6.75a) Es ist ein Vorgang, der auf Ober- und Hochschulen $z u$ beobachten ist. Die Jugend wendet sich mehr und mehr rechten Gruppen $z u$.

(6.75b) Die Jugend wendet sich mehr und mehr rechten Gruppen zu. Das ist ein Vorgang, der auf Ober-und Hochschulen zu beobachten ist.

(6.76a) Es ist eine unnatürliche und niederschlagende Erscheinung. Tatsachlich wimmelte es am Strande von patriotischen Kindern.

(6.76b) Tatsächlich wimmelte es am Strande von patriotischen Kindern. Das ist eine unnatürliche und niederschlagende Erscheinung.

Die Funktion der NP bzgl. des Satzes wird angegeben mit "Einschatzung des Inhalts" (Eichbaum 1978: 29), "Interpretation des vorhergehenden Aussageabschnitts", "Kommentar", "logische Schlußfolgerung" (Eichbaum 1978: 36), "Anzeige, wie der Sprecher die gegebene Proposition verstanden haben will" (Molitor 1979: 140), "Angabe der illokutionăren Rolle der geăuberten Proposition" 
(Molitor 1979: 142) und "Anzeige, wie die Proposition auf den Sprecher wirkt" (Molitor 1979: 144).51 Im wesentlichen handelt es sich um sprechereinstellungen (zur Proposition), die den Geltungsgrad - hierin Satzadverbialen wie vermutlich ăhnlich - resp. die (emotionale) Bewertung (vgl. z.B. das Satzadverb schlimmerweise) zum Ausdruck bringen, oder um Anweisungen an den Hörer, wie die Proposition illokutionăr aufzufassen sei. Grob kann die Funktion auch folgenderweise dargestellt werden:

(6.77) [eine schlimme sache (SCHWANGER (pia))]. 52

DaB die Funktionen z.T. denjenigen der SATZADVERBIALE stark ähneln, veranschaulichen folgende Beispiele:

(6.78) Eine bedauerliche Tatsache: Pia ist krank.

Bedauerlicherweise ist Pia krank.

Es ist bedauerlich/ eine bedauerliche Tatsache, daB Pia krank ist.

(6.79) Eine schlimme Nachricht: Pia ist schwanger.

(?) Schlimmerweise ist Pia schwanger.

Es ist schlimm/ eine schlimme Nachricht, daB Pia schwanger ist.

Die "Satzapposition" ist gleichwohl nicht als Adverbial zu werten, obschon ihre Năhe zu den Satzadverbialen (z.B. als Sprechereinschätzung des Geltungsgrades und der Bewertung eines Sachverhaltes) offenliegt; man vgl. Pia kommt überraschenderweise tatsächlich pünktlich und Pia kommt pünktlich - eine Tatsache, die überraschend ist. Natürlich besteht nur in einem Teil der Fălle eine solche Beziehung; aufgrund ihres differenten grammatischen Verhaltens (Topikalisierung, Erfragung, Substitution, intonatorische Markierung usf.) rechtfertigen aber nicht einmal diese eine Gleichsetzung beider Funktionen. ${ }^{53}$ Man vgl. auch Eichbaum (1978: 36):

"Wie es scheint, lassen sich solche Komponenten mit dem Substantiv im Nominativ als Kernwort in die traditionell aufgestellten Klassen der Satzglieder nicht einreihen (...). Es ist mehr begründet, diese Komponente als ein syntaktisches segment in einem semantisch-strukturellen Gebilde höherer Ordnung zu behandeln (...)".

Umformungsbeziehungen sind vorhanden, und zwar können sowohl selbstăndige Satze - als Folgesatz (6.80a) oder als parenthetisch in den Gastsatz eingeschobener Satz (6.80b) - als auch sog. "weiterführende Nebensätze" (Satzre-

51 Nebenbei: Bei diesem Typ wăre ein Ansetzen von "Referenzidentităt" höchst problematisch. Ein so weites Verstăndnis davon, z.B. daß sich der Bezugssatz auf einen Sachverhalt als dessen "Abbildung" bezoge und das "Satzappositiv" ebenfalls auf den Sachverhalt als Kommentar etc. zu demselben, scheint mir unzweckmäßig.

52 Die "pseudologische" Unschrift soll lediglich die Bezugsverhăltnisse (Skopi) verdeutlichen; nur der Funktor der rumdgeklammerten Satzproposition wird groß geschrieben.

53 In Ggs. zu Satzadverbi (al)en sind "Satzappositionen" z.B. nicht vorfeldfähig und antworten auf keine Entscheidungsfrage. 
lativsătze; $6.80 \mathrm{c}$ ) in Betracht gezogen werden. 54 Es handelt sich wie beim "Prototypen" (Kap. 6) um Umschreibungen mit Kopulaverben, wobei das "Satzappositiv" zum Prădikativ wird.

Zudem sieht man, daB der "Bezugssatz" - entweder zu einem daB-Gliedsatz (6.80d) oder zu einer NP (Nominalisierung; 6.80e) umgeformt - als subjekt in einem Kopulasatz auftreten kann, dessen Prădikativ die "Satzapposition" darstellt:

(6.80a) Pia ist schwanger. Das ist eine schlimme Nachricht.

Pia ist schwanger; (und) das ist eine schlimme Nachricht.

Pia ist schwanger - das ist eine schlimme Nachricht. 55

(6.80b) Pia ist - (und) das ist eine schlimme Nachricht - schwanger.

(6.80c) Pia ist schwanger, was eine schlimme Nachricht ist.

Pia ist - was eine schlimme Nachricht ist - schwanger.

(6.80d) DaB Pia schwanger ist, ist eine schlimme Nachricht.

(6.80e) Pias Schwangerschaft ist eine schlimme Nachricht.

Man könnte die "satzappositive" NP als Tilgungsprodukt analog zum "Appositionsprototypen" - entstanden aus einem Ropulasatz - erklären. Diese Analogie ist ein Hinweis darauf, das "Prototyp" und "Satzapposition" in eine Grosklasse einzuordnen sind. 56 zudem zeigt (6.81) die Möglichkeit, den Satz als Gliedsatz oder Nominalisierung und die Satzappositions-NP als "lockeres Appositiv" in das Vorfeld eines Satzes zu stellen. Mit (6.81b) erhält man sogar eine "prototypische Appositionskonstruktion"!

(6.81a) DaB Pia schwanger ist, eine schlimme Nachricht, beunruhigte alle.

(6.81b) Pias Schwangerschaft, eine schlimme Nachricht, beunruhigte alle. Kasuskongruenz spielt bei dieser Konstruktion keine Rolle. Die isolierte NP steht im Nominativ (Neutralkasus). NeglaBbar sind die "Satzappositionen" in jedem Fall.

Ob hier Satzgrenzenbestimmtheit eine Rolle spielt, ist mir nicht klar. Die deutliche intonatorische Abtrennung der NP (graphematisch durch den Punkt), besonders die fallende Intonation im Bezugssatz vor ihr, spricht für einen elliptischen, eigenståndigen satzwertigen Ausdruck, was durch die Umformungen

$54 \mathrm{Zu}$ den weiterführenden Nebensătzen Scomerfeldt (1983); Bergmann (1985: 61 ff.); die Ausführungen in den Grammatiken des Deutschen sind meist knapp.

55 Schwyzer (1947: 10) bemerkt folgendes: "Besonders einleuchtend ist die Entstehung der vorwiegend literarischen sog. Satzapposition aus alter Endparenthese". Dies wird durch unseren Beispielsatz veranschaulicht. In Schwyzer (1939: 42) wird die Herleitung aus einem opisthothetischen Satz so veranschaulicht (tbersetzung aus dem Griech. von Schwyzer): er erhielt eine unbeherrschte Zumge, das schimpflichste Gebrechen aus das ist das schimpflichste Gebrechen/ was das schimpflichste Gebrechen ist.

56 Die "prădizierenden Zusătze" (s. Kap. 12, 15) haben dabei lediglich unterschiedliche "Bezugselemente" (NP, S). 
z.T. bestätigt wird. 57 Wie aber bereits festgestellt wurde, geht die Selbständigkeit - wie beim Prototypen - nicht so weit, daB man bei Einschaltung in den Bezugssatz von einem Einschub sprechen würde.

Von Negation (6.82a), Gradpartikelanwendung (6.82c) und illokutiven Operationen wie FRAGE (6.82d) ist die "Satzapposition" vom Bezugssatz aus nicht affizierbar (mit "*" markierte Beispiele stellen unzutreffende Schlußfolgerungen aus den Vorgăngersätzen dar; $6.82 \mathrm{~b}$ reprăsentiert informal die Skopusverhältnisse - die "Satzapposition" hat die Satznegation in ihrem skopus):

(6.82a) Pia ist nicht schwanger, eine schlimme Nachricht. Es ist nicht der Fall, daB Pia schwanger ist, eine schlimme Nachricht.

*Es ist nicht der Fall, daß es ein schlimme Nachricht ist, daB Pia schwanger ist.

(6.82b) Pia ist nicht schwanger, eine schlimme Nachricht.

[eine schlimme nachricht (NEG (SCHWANGER (pia))]

(6.82c) Die Kirche ist auch von Zypressen umgeben, ein wunderbarer Anblick. Es ist auch der Fall, daB die Kirche von Zypressen umgeben ist, ein wunderbarer Anblick.

*Es ist auch der Fa11, daB es ein wunderbarer Anblick ist, daB die Kirche von Zypressen umgeben ist.

(6.82d) ^Ist Pia schwanger, eine schlimme Nachricht?

Ist Pias Schwangerschaft, eine schlimme Nachricht, schon bekannt? Die Satznegation nicht bewirkt in $(6.82 \mathrm{a}, \mathrm{b})$, daB der Skopus von eine schlimme Nachricht die negierte Proposition ist. Die Anwendung kontrastierender Negationen ist unzulässig:

(6.83a) *Es ist nicht der Fall, daB Pia schwanger ist, eine schlimme Nachricht, sondern daB Pia schwanger ist, eine gute Nachricht.

(6.83b) *Pia ist nicht schwanger, eine schlimme Nachricht, sondern Pia ist nicht schwanger, eine gute Nachricht.

Die pure Rekursion (Asyndese) wirkt stilistisch etwas unschön, ist jedoch akzeptabel (sofern nicht mehr als zwei Konjunkte erscheinen; vgl. aber Prototyp in (6.50)); die Konjunktorsyndese wirkt unmarkiert:

(6.84a) (?) Pia ist schwanger, eine gute Nachricht, ein langersehntes Ereignis.

(?) Die Kirche ist von Zypressen umgeben, ein schöner Anblick, ein malerisches Bild.

(6.84b) Pia ist schwanger, ein langersehntes Ereignis und eine gute Nachricht.

Die Kirche ist von Zypressen umgeben, ein schöner Anblick und ein malerisches Bild.

57 Fichbaum spricht von einem "eingliedrigen substantivischen Satz" (1978: 32). Zu möglichen Gegenargumenten s. Raabe (1979: $274 \mathrm{ff}$.$) .$ 
Im Folgetext ist es durchaus möglich, sich mit einem Pro-Element auf eine "Satzapposition" zu beziehen. Somit unterliegen Satzappositionen den allgemeinen Pronominalisierungsregeln, ebenso wie der Prototyp:

(6.85) Pia hat bei Tisch gerülpst - ein unerhörter Vorfall. Er/ Dieser Vorfall/ Diese Unerhörtheit beschäftigte die Gäste noch lange.

Sind derartige Appositionen dependent? Und wovon eigentlich? Natürlich sind sie in sehr allgemeiner Weise vom Bezugssatz ("Gastsatz") dependent, insofern als sie an diesen vorkommensgebunden sind. Sonst liegt jedoch keinerlei Hinweis auf Dependenz (etwa Rektion, Valenz, Kongruenz) vor.

Bilden Satzappositionen mit dem "Bezugssatz" eine Konstituente? Wenn ja, dann könnten derartige NPn nur Kokonstituenten des Satzes unter einem gemeinsamen obersten Satzsymbol sein. In einer Satz-Pro-Formisierung mit dem ProSentential das wird das Appositiv allerdings of fenbar nicht mit aufgenommen:

(6.86a) Pia ist schwanger - eine gute Nachricht.

Das freute alle (ich meine, daB Pia schwanger ist/ *daB Pia schwanger ist - eine gute Nachricht).

(6.86b) Pia glaubt, daB es gar keine Appositionen gibt - eine gewagte Hypothese; wir hingegen tun das nicht (glauben, daB es gar keine Appositionen gibt/*glauben, daB es gar keine Appositionen gibt - eine gewagte Hypothese).

Die Satzapposition verhält sich mithin eher wie ein selbständiger Textschritt, auch wenn die syntaktische selbständigkeit nicht gegeben ist (wie z.B. bei der Umformung des Satzappositivs in einen Kopulasatz). Sie ist jedenfalls deutlich selbständiger als der auf ein Satzglied bezogene Prototyp. Eine Konstituentenbildung wird daher (vorläufig) verworfen.

Soll man nun Konstruktionen dieses Typs unter die "Appositionen" bzw. die zUSATZE (und nicht die EINSCHUBE) einreihen? Zunächst ist $z$ u bemerken, daB die Umformungsprodukte (in $6.87 \mathrm{mit}$ zunehmender syntaktischer Fügungsenge)

(6.87a) Pia ist schwanger. [Das ist] Eine gute Nachricht.

(6.87b) Pia ist schwanger, das ist eine gute Nachricht.

(6.87c) Pia ist schwanger und das ist eine gute Nachricht.

(6.87d) Pia ist schwanger, was eine gute Nachricht ist.

(6.87e) Pia ist schwanger, eine gute Nachricht.

(6.87f) Pia, eine gute Nachricht, ist schwanger.

formal nicht sinnvoll unter Apposition einstufbar sind, auch wenn sie funktional nicht unähnlich sind. Syntaktisch betrachtet handelt es sich oben um eine Satzfolge (6.87a), eine asyndetische satzreihung bzw. -verbindung (6.87b), eine konjunktorsyndetische Satzreihung (6.87c) und eine Konstrukt "Satz plus weiterführender Nebensatz", letzterer zwar formal subordiniert, inhaltlich jedoch selbständig. ${ }^{58}$ Erst für $(6.87 e)$ steht kein syntaktischer Begriff zur Ver-

58 Man vergleiche beispielsweise v. Polenz (1985: 264): 
fügung - außer "Satzapposition". (6.87f) zeigt eine Variante von (6.87e) mit Einschaltung.

Satzfolgen und -reihungen möchte ich von vornherein aus dem Appositionsbereich ausschlieben, weil einerseits der Appositionsbegriff nicht auf völlig heterogene Erscheinungen wie Satzfolgen und -reihungen ausgeweitet werden soll und andererseits Apposition nicht rein pragmatisch verstanden werden soll als $x$-beliebige, nichtnotwendige Hinzufügung einer spracheinheit $Y z u$ einer Spracheinheit $X$, die den Wahrheitswert einer Basiseinheit nicht verăndert etc.

Zum weiterführenden Nebensatz bliebe noch einiges zu sagen (immerhin versteht ihn Bergmann (1985: 62) als "im weitesten Sinne appositiv"), eine ausführlichere Behandlung muß hier leider unterbleiben. Ob er eher den ZUSATZEN (zum Satz) oder den EINSCHOBEN zuzurechnen sei, muß of fenbleiben.

Nun noch ein Ausblick darauf, was sich ergibt, wenn man "lockere Appositive" und "Satzappositionen" in folgender Weise kombiniert:

(6.88a) Deine Kleidung, bereits eine arge Zumutung, starrt ja vor Dreck eine Zumutung, die kaum zu ertragen ist.

(6.88b) Der Kuno, ein ulkiger Anblick/ ulkig anzusehen, machte unfreiwillig einen Salto - ein noch ulkigerer Anblick, der uns köstlichst amüsierte.

(6.88c) Seines Dackels Dahinscheiden, ein tragisches Ereignis, wurde gefolgt von seines Hamsters Ableben, ein noch tragischeres Ereignis eine tragische Ereigniskette, wie sie kaum schlimmer hătte kommen können.

Hier erscheint die Ahnlichkeit beider Zusătze sehr groß; man beachte vor allem die Akzeptabilităt von $(6.88 \mathrm{c})$, vorausgesetzt, die Pause zwischen dem zweiten Appositiv und der "Satzapposition" wird deutlich genug artikuliert.

Die "Satzapposition" kann auch jetzt noch nicht als grammatisch befriedigend erforscht gelten. Da in meiner Arbeit die NP-bezogenen ZUSATZE im Vordergrund stehen, muß eine vorlăufige Einordnung genügen. Im Unterschied zu den Satzfolgen kopulativer sătze $\left(6.89 a_{i}\right)$, wo keine grammatischen Integrationserscheinungen wahrzunehmen sind und wo man sieht, daß die Verben ausgetauscht werden könnten, ist die "Satzapposition" $(6.89 \mathrm{~b})$ in den "Bezugssatz" grammatisch teilweise integriert:

"Da ihr [die weiterführ. Nebensätze; W.S.] Beitrag zur Satzbedeutung die Weiterführung des Textverlaufs zu einer neuen Aussage bzw. zu einem neuen Satzinhalt ist, sind sie keine echten Zusătze zu Aussagen, sondern hypotaktisch angeschlossene weitere Textschritte".

In Heidolph u.a. (1981: 787) werden derartige Sătze als "Abwandlungen quasi-koordinativer Verknüpfungen" angesehen. Etwas anders Heringer (1988: 276), der von "nachgestellten Zusătzen, die den Gedankengang weiterführen" spricht. 
(6.89a) Kuno rülpst bei Tisch. Das ist ein Benehmen, das getadelt werden muB.

Kuno rülpst bei Tisch. Das stellt ein Benehmen dar, das getadelt werden mus.

Kuno rulpst bei Tisch. Das werte ich als ein Benehmen, das getadelt werden mus.

(6.89b) Kuno rülpst bei Tisch - ein Benehmen, das getadelt werden muB. Satzfolgen können (mit evtl. variierten Pro-Formen) stellungsmăBig vertauscht werden und sich durchwandern; der "Satzapposition" stehen nicht alle diese Optionen offen. Sie wird daher als hOSPITANT, genauer als zUSATZ zu einem Satz, gewertet (der "Appositionsprototyp" ist ein prădizierender NP-Zusatz). 
Die Abgrenzung zwischen Apposition und Attribut(ion) ist ein kompliziertes Problemfeld, weil die Termini "Attribut" und "Apposition" noch nicht befriedigend expliziert sind. ${ }^{1}$ Traditionell werden die "Appositionen" oft als attributive sub- bzw. Sonderfunktion gewertet und somit in eine Reihe gestellt mit z.B. Genitiv- oder Präpositionalattributen (Unterstreichungen von W.S.):

"Die Apposition ist ein Attribut in substantivischer Form, (...)" (Jung 1982: 110).

"Die Apposition verstehen wir als eine Subkategorie der Rategorie Attribut. Unter Attributen sind Elemente (...) zu verstehen, die nichtverbalen Elementen als nahere Bestimmung zugeordnet werden (năhere Bestimmungen zu Verben oder Sătzen werden anders benannt). Damit ist die Attribution immer ein phraseninterner (intraphrastischer) ProzeB, der unterhalb der Satzebene liegt (...)" (Engel 1986: 189).

" $\mathrm{Zu}$ den Attributen rechnen wir nur die Erweiterungen einer SbG [Substantivgruppe; W.S.], die entweder unmittelbare Konstituenten von SbG (...) oder von $\mathrm{Sb}$ selbst sind $(\ldots)$. (...) Appositionen heiBen traditionell solche Attribute, die entweder mit dem Kern der SbG im Kasus übereinstimmen oder - unabhängig von dessen Kasus - im Nominativ stehen" (Heidolph u.a. 1981: 288, 290).

Aus den Zitaten ist zu erkennen, (a) daB "Apposition" offensichtlich als eine von mehreren möglichen attributiven Subfunktionen (neben GA, AdjA, PA etc.) angesehen wird und (b) daB der Begriff Attribut unterschiedlich weit gefaBt wird. Zum einen werden nur substantivattribute (zitat 3 ), zum anderen Beifugungen zu allen nichtverbalen Elementen (Zitat 2) angenommen. Deswegen ist zunăchst eine terminologische Erörterung notwendig, die für die vorliegende Arbeit klărt, was unter Attribut(ion) zu verstehen sei.

Attribute sind dependenzgrammatisch betrachtet Erganzungen und Angaben $z u$ nichtverbalen Einheiten, und tatsăchlich hăngen nicht nur vom Nomen derartige Satelliten ab ("B" = 'Basis'; "A" = 'Attribut')):
(7.1a) Empfänglichkeit (B) für schóne Dinge (A). empfänglich (B) für schöne Dinge (Prăpositivergănzung). ${ }^{2}$
(7.1b) wahrscheinliche (A) Empfänglichkeit (B). wahrscheinlich (A) empfänglich (B).
(7.1C) wahrscheinlich (A) oft/ heute/ sehr (B). Adjektive nehmen ebenfalls Ergănzungen und Angaben wie Nomina, Adverbien $z . T$. die gleichen Modifikatoren wie Adjektive. Mindestens den Wortarten $N$, Adj und

\footnotetext{
1 Altmann (1981: 278, 289) weist beispielsweise kritisch auf diesen Unstand hin.

2 Terminologie der Adjektivsatelliten nach Engel (1988: 593).
} 
Adv muB - dependenzgrammatisch - zugesprochen werden, daß sie (von sich dependente) Attribute zu sich nehmen können.

Die $X$-Bar-Theorie betont den weitgehend parallelen internen Phrasenaufbau von NP, AdjP, AdvP und PP (!), 3 wobei sie den Terminus Attribut durch Romplement (einer bestimmten $X$-Bar-Stufe) ersetzt hat. Somit werden die Komplemente zu den verschiedenen Wortarten gleichwertig behandelt.

Konstituentenstruktursyntaktisch können alle vom obersten Phrasenknoten (z.B. NP, AdjP, AdvP; bei VP würde man anders werten) dominierten Schwesterbzw. Ko-Konstituenten des Phrasenkerns als Attribute angesehen werden (Determinantien $u . a ̆$. könnte man durch Zusatzbestimmungen ausklammern):

(7.2)

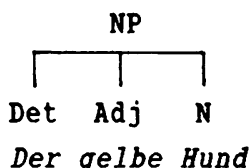

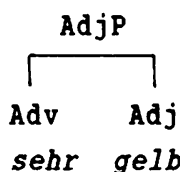

sehr gelb
AdvP

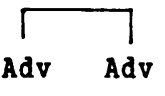

sehr oft

In einer Kategorialgrammatik fallen diese Konstruktionstypen unter die endozentrischen Operator-Operand-Beziehungen, wobei die Attribute (Operatoren), angewendet auf den Basis (Operand), einen komplexen Ausdruck von der Basiskategorie (Operandenkategorie) erzeugen. Zugleich wird "semantisch" die DenotatMenge eingeschränkt, der Denotatstyp (Gegenstand, Eigenschaft etc.) bleibt konstant.

Durch die angeführten Gesichtspunkte wird die Annahme nahegelegt, das die Attribution nicht nur eine adnominale Funktion ist, sondern (mindestens) auch Adjektive und Adverbien, evtl. sogar Präpositionen betrifft. Damit der Leser die folgenden Ausführungen kritisch verfolgen kann, sei sogleich das im folgenden zugrundegelegte Attributverständnis offengelegt (einige Merkmale werden im Folgetext noch vorgefuhrt):

(7.3) ATTRIBUT-Konstruktionen sind in eine Basis einer der Kategorien $\mathrm{N}$, Adj, Adv (und evtl. Prăp) und mindestens einen davon als Ergănzung oder Angabe abhängigen Satelliten strukturierbar. Basis und Attribut sind gesamt vorfeldfăhig, gesamt erfragbar und ersetzbar. Beide sind gesamt permutierbar - bei speziellen Attributskonstruktionen und unter speziellen Bedingungen ist jedoch auch Distanzstellung möglich. ${ }^{4}$ Beide Konstruktionsteile können kontrastierend negiert werden. Das Attribut kann mittels einer Gliedteilfrage (z.B. welch-/ was für ein- + Basis) erfragt werden und es kann den Fokus einer Gradpartikel erhalten. Das Attribut kann explizit als dependent (subordiniert) markiert sein (a) durch morphosyntaktische Mittel, z.B. die Kongruenz des Adjektivattributs mit dem Kernnomen oder die Rektion

3 Hierzu die grundlegende Arbeit von Jackendoff (1977). Zu den PPn auch Munderlich (1984).

4 Vg1.: (F7.1) Auf bessere Zeiten (A) wird sie die Boffnung (B) nie aufgeben. (F7.2) Neikbier (B) hatte er nur altes (A) in Kuhlschrank. 
bei Genitivattributen ${ }^{5}$ und den Adjektivergănzungen, oder (b) durch lexikalische Mittel (Prăposition beim PA und bei gewissen Adjektivergänzungen, Relativpronomen beim attributiven Relativsatz). Eine explizite Kennzeichnung kann aber auch fehlen, so daß Juxtaposition vorliegt, wobei dann feste Folgeregeln $z u$ beachten sind. ${ }^{6}$ Nicht als Attributmarkierung zählt m.E. das "Einschaltungsmuster", das eine Markierung von "Einschüben" oder "Zusătzen" darstellt. 7

Daß die Apposition, und zwar sowohl das "lockere" als auch das "enge" Muster, zur Attribution (vor allem des Nomens) gerechnet wird, erscheint zunachst einleuchtend. Appositionen verhalten sich augenscheinlich nicht wesentlich anders als z.B. Genitiv- oder Prăpositionalattribute: Sie sind Satelliten der Nuklei N (Adj, Adv), gehören gemäB den X-Bar-Ronventionen zur X3-Ebene der Phrasen. ${ }^{8}$ Auch die Konstituentenstruktur (als Teil-Ganzes-Relation) dürfte so aussehen, das die Appositive unterhalb des obersten Phrasenknotens (NP, AdjP, AdvP; evtl. PP) "angehängt" werden. 9 Für diese Explikation sprechen vor allem die bekannten Konstituententests (vgl. Kap. 5).

Hăufig auf Attributkonstruktionen angewendete Konstituententests betreffen folgende Eigenschaften von Basis und Attribut; sie sind

5 Die Frage, ob der Cenitiv vam Nomen regiert wird, lasse ich offen; Eisenberg (1986: 234) nimmt jedenfalls ein Rektionsverhältnis an. Der Dativ erscheint z.B. in Nonstandardfällen (regional?) wie dem Vater sein Haus.

6 Das Adverbattribut (z.B. der Kerl dort/ dort der Rerl (Nonstandard)) steht entweder vor der (determinierten) NP oder unmittelbar nach ihr; evtl. können sich GA und PA dazwischenschieben (die Frau mit hut dort; Der Freund Peters gestern). Die "enge Apposition", in dieser Arbeit den Attributen zugerechnet, muß ummittelbar links oder rechts des Kernnomens stehen (obligatorische umnittelbare Adjazenz; hierzu Löbel 1988a). Genaueres zu den Folgeregeln in NPS S. Sommerfeldt 1971; Teubert (1979: $202 \mathrm{ff}$.$) ; Engel (1988: 606 \mathrm{ff}$.$) .$

7 Man könnte sonst mit Recht - wie 2.B. Jung (1982: 109) oder Vater (1986b: 129-132) - neben den "lockeren Appositionen" auch Parenthesen zu den Adnominalen rechnen und - noch verwirrender - alle mit diesem Muster versehenen Binheiten in folgender Stellung:

(F7.3) Die Frau, eine Studentin,/, sie ist eine Studentin,/, meine Damen und Berren,/, wie schion, /, vermutlich, wird das Buch verstehen.

Es sei noch darauf hingewiesen, daß bei manchen attributiven Mustern das Attribut "abgetrennt" werden kann - z.B. in Tüten (mit Schnipseln) lagen dort herum - wobei dann m.E. keine Attribution mehr vorliegt (keine Gliedteilfrage oder Anwendung von NEG/GP möglich), sondern ein ZUSATZ. Hierzu Năheres in Kap. 11.

8 X3, nach Jackendoff (1977), entspricht der obersten Phrasenebene, also NP, AdjP, AdvP.

9 An dieser Stelle der Binweis auf eine Bemerkung von Engel (1982: 157), der der Ansicht ist, daß "(...) Appositionen nicht Teile der Phrasen sind, denen sie zugeordnet werden". Diese Bemerkung ist von größter Wichtigkeit insofern, als die Selbstverstandlichkeit, mit der die Appositive als Phrasenteile angesehen werden, dadurch eine Erschüttenung erfährt. Es ist unbedingt zu prüfen, inwiefern Appositive tatsăchlich mit ihren "Bezugselementen" eine Phrase bilden (mit ihnen gemeinsam an syntaktischen Prozessen teilnehmen etc.) und nicht etwa - gleich diversen anderen Einschaltungen - dem Satz eigentlich nur sekundăr angehören. 
- gemeinsam vorfeldfähig (zusammen permutierbar; $7.4 a$ ), 10

- gesamt erfragbar (7.4b),

- gesamt ersetzbar (durch ein Pro-Element; 7.4c).

Wenn man nun nachstehend typische Attribute und appositionelle Konstruktionen vergleicht, dann zeigt sich folgendes Verhalten:

Kunos (GA)

an der Tafel (PA)

Kuno (enge APP)

Der Lehrer , Brillenträger, (lockere APP) dozierte monoton.

, ziemlich träge, (lock. APP)

, am Pult, (lock. APP)

(7.4b) Wer dozierte monoton?

Der Lehrer Kunos/ an der Tafel/Kuno.

?Der Lehrer, Brillenträger.

? Der Lehrer, ziemlich träge.

? Der Lehrer, am Pult.

(7.4c) Er dozierte monoton.

Die Vorfeldprobe erbringt zumeist akzeptable Resultate, ganz gleich, ob Attribution oder Apposition vorliegt. Wenn man allerdings genauer hinsieht, dann besagt dies angesichts der nachstehenden Beispiele evtl. nicht sehr viel:

(7.5) liebe Pia (ANREDE),

Der Lehrer, so ist das immer (SCHALTSATZ), dozierte monoton. o mei (INTERJERTION)

SATZGLIED "P A R E N T H E S E" FINITUM

10 Das Kriterium "Rern und Attribut sind nur gemeinsam verschiebbar" ist bekanntlich nicht ausreichend prăzise. So können sich aus NPs z.B. Deteminantien, Adjektiv- und Präpositionalattribute herauslösen, mobei die Trennung des Attributs van Kern keinesfalls nur "in seltenen Fällen"

(Helbig/Buscha 1980: 518) geschicht:

(17.4) Mur ihre Blumen haben heute geblüht. Blumen haben pur ihre beute geblüht.

Schöne Hemden hat es dort gegeben. Bemden bat es dort schóne gegeben.

Die Bloffnung auf bessere Zeiten soll man nie aufgeben.

Auf bessere Zeiten soll man die Boffnumg nie aufgeben.

Zudem sind auch manche Adjektiv- und Adverbsatelliten distanzstellbar:

(F.5) Thres Sieges (Gen.erg. z. Adj) war sich Pia nicht sicher.

(F7.6) Dort [unten] babe ich unten eine Maus geseben.

(F7.7) Hinten [im Regal] habe ich im Regal eine Maus gesehen.

Bei den Adadverbien ist jedoch Vorsicht geboten, weil die Bedeutung der Distanzvarianten sich von derjenigen der Kontaktvarianten unterscheidet. So kann hinten in Regal angegeben werden mit 'in hinteren Teil des Bezugsraumes in Regal' oder mit 'im hinteren Teil des Regals', hinten (...) im Regal dagegen nur mit der ersten Bedeutungsangabe!

Insgesamt gesehen ist also das Permutationskriterium nicht zuverlăssig genug, un als Bxplikans für Attribution zu dienen; es reicht allerdings für diverse andere Abgrenzungen aus, z.B. Attribut versus Satzglied (i.d.R. kann nur ein Satzglied ins VF). Ober "Satelliten mit variabler Positicn" finden sich in Engels Grammatik (1988: $625 \mathrm{ff}$.) weitere Ausführumgen. 
Die Erfragung zeigt, daB Basis und Attribut bzw. enge Apposition (!) als natürliche Antwort aufzutreten vermögen, Nomen und lockeres Appositiv hingegen muten als Antwort fragwürdig (wenngleich nicht unbedingt inakzeptabel) an.

Die gemeinsame substitution ist bei allen Beispielen unproblematisch. Wenn man allerdings nur den Kern (genauer: Det $+N$ ) durch eine Pro-Form ersetzt und betrachtet, was dann noch anfügbar ist, dann bleiben Appositive ohne weiteres hinzufügbar, ${ }^{11}$ das $\mathrm{PA}$ ist weitgehend akzeptabel; GA sowie enge Apposition (!) sind inakzeptabel:

(7.6) Er *Kunos/ (?) an der Tafell *Kuno dozierte monoton.

Er, Brillenträger/ ziemlich träge/ am Pult, dozierte monoton.

Allerdings kann die Basissubstitution nicht als generelles Merkmal attributiver Konstruktionen herangezogen werden. Z.B. ist dies bei Adjektivattributen (der gelbe Hund/ *der gelbe er) nicht möglich.

Erfragung und Substitution lassen erkennen, daB die "Verwandtschaft" von Attribut und enger Apposition größer ist als zwischen Attribut und lockerer Apposition.

Nun möchte ich einige weitere Merkmale von Attributen bzw. attributiven Konstruktionen vorstellen:

- Es ist eine Frage (ohne das vermeintliche Attribut) möglich, auf die mit der Gruppe "Basis und Attribut" geantwortet werden kann, wobei auf dem Attribut dann ein deutlicher Akzent liegt; sie heiBt im folgenden "Gliedteilfrage". Typische Gliedteilfragen sind welch-/ was für ein-/ wie + Basis? Dazu unten die b-Beispiele.

- Es ist möglich, sowohl das Basiselement als auch das Attribut kontrastierend zu negieren. ${ }^{12}$ Das kontrastierte Element erhät in der angefügten sondern-Phrase einen deutlichen Akzent. Dazu unten die c-Beispiele.

- Beide Bestandteile einer attributiven Konstruktion können von einer Gradpartikel fokussiert werden. Die Möglichkeit der Affizierung beider Teile kann durch entsprechende Weiterführungen (unten nicht immer angegeben) verdeutlicht werden. Siehe die d-Beispiele.

(7.7a) Das rote Auto (AdjA) knattert.

(7.7b) Welches Auto knattert? Das rote Auto.

(7.7c) Nicht das rote Auto knattert, sondern das rote Mofa. Nicht das rote Auto knattert, sondern das blaue Auto.

(7.7d) Sogar das rote Auto knattert (, nicht nur das rote Mofa). Sogar das rote Auto knattert (, nicht nur das grüne Auto).

11 Nach den (X-Bar-theoretischen) Explikationen von Vater (1986b) ist dies kein großes Problem; Pro-Fonmen (hier: Pro-IPs) können für unterschiedliche Bar-Ebenen stehen (ebd. 133 f.) und Personalpronomina stehen für N2 (ebd. 134), so daß sie mit appositiven Komplementen verbindbar sind.

12 Zur kontrastierenden Negation ausführlich Jacobs (1982: $269 \mathrm{ff}$. ). 
(7.8a) Das Auto der Oma (GA) knattert.

(7.8b) Welches Auto knattert? Das Auto der Oma.

(7.8c) Nicht das Auto der Oma knattert, sondern das Moped der Oma. Nicht das Auto der Oma knattert, sondern das Auto des opas.

(7.8d) /auch das a uto der oma knattert $\mathrm{ft+}$ /. /auch das auto der o ma knattert $\mathrm{f}++$ /.

(7.9a) Das Auto im Garten (PA) knattert.

(7.9b) Welches Auto knattert? Das Auto in Garten.

(7.9c) Nicht das Auto im Garten knattert, sondern das Mofa im Garten. Nicht das Auto im Garten knattert, sondern das Auto im Bof.

(7.9d) Nur das Auto im Garten knattert (, nicht aber das Mofa im Garten). Nur das Auto im Garten knattert (, nicht aber das Auto im Bof).

(7.10a) Pia ist des Lobes würdig (Genitivergänzung zum Adj). 13

(7.10b) Wessen ist Pia würdig? des Lobes würdig.

(7.10c) Nicht des Lobes würdig ist Pia, sondern des Lobes bedürftig. Nicht des Lobes würdig ist Pia, sondern des Ruhmes würdig.

(7.10d) / sogar des lobes $w$ ü rdig ist pia $\mathrm{ft+}$ /. /sogar des 1 o bes würdig ist pia $\mathrm{ft+}$ /.

(7.11a) Pia wird sehr sicher (graduierende Angabe zum Adj).

(7.11b) Wie sicher wird Pia? Sehr sicher.

(7.11c) Pia wird nicht sehr sicher, sondern sehr frech. Pia wird nicht sehr sicher, sondern ziemlich sicher.

(7.11d) /pia wird sogar sehr $s i$ cher $\mathrm{ft+} /$. /pia wird sogar $s$ e hr sicher $\mathrm{ft+}$ /.

(7.12a) Pia grinst ziemlich oft (Adverbattribut).

(7.12b) Wie oft grinst Pia? Ziemlich oft.

(7.12c) Pia grinst nicht ziemlich oft, sondern ziemlich frech. Pia grinst nicht ziemlich oft, sondern sehr oft.

(7.12d) /pia grinst sogar ziemlich oft $\mathrm{ft+}$ /. /pia grinst sogar $z i$ emlich oft $\mathrm{f}++$ /.

Beim Appositionsprototypen (s. Kap. 6.) ist weder eine Gliedteilfrage noch eine Kontrastnegation der "Beifügung" möglich; auch kann sich der Gradpartikelfokus nicht auf das lockere Appositiv erstrecken:

(7.13a) Das Auto, ein 50er-Jahre-Mode11, knattert.

(7.13b) Welches Auto knattert? *Das Auto, ein 50er-Jahre-Modell.

(7.13c) *Nicht das Auto, ein 50er-Jahre-Modell, knattert, sondern das Auto, ein 60er-Jahre-Mode11.

(7.13d) */sogar das auto $\mathrm{p}+$ ein $f$ ü nfziger-jahre-modell $\mathrm{p}+$ knattert $\mathrm{f}++/$. Eine Zuweisung derartiger Konstruktionen zu der attributiven Relation bedeutete, über wesentliche grammatische Unterschiede hinwegzusehen.

13 Zur Terminologie siehe vieder Engel (1988: $590 \mathrm{ff}$. ). 
Mithilfe weniger Merkmale, die an "typischen" Attributkonstruktionen geprüt wurden, kann man aus unserem Phänomenbereich folgende Muster den Attributskonstruktionen zuweisen:

(a) 1.2.4. den restriktiven Relativsatz (a-Varianten), der sich vor allem in der gesprochenen Sprache deutlich vom nicht-restriktiven Relativsatz (b-Varianten) unterscheidet. Obwohl der restriktive Relativsatz nie den Appositionen zugerechnet worden ist, möchte ich dessen eindeutig attributive Charakteristika kurz demonstrieren, um die Diskussion des nicht-restriktiven Relativsatzes vorzubereiten, der sich deutlich anders verhält. Weil die Erörterung dieses Musters für die Appositionsdiskussion von Bedeutung ist und relativ breiten Raum beansprucht, wird sie in einen Exkurs am Kapitelende (7.A) verlegt.

(7.14a) /pia fand das haus $(\mathrm{p}+)$ das $k$ u no gehörte $\mathrm{ft+}$.

/w e lches haus fand pia st+ das haus $(\mathrm{p}+$ ) das $k$ u no gehörte $\mathrm{ft+}$.

(7.14b) /pia fand das $h$ a us $\mathrm{pt}$ das übrigens $k$ u no gehörte $\mathrm{f}++/$.

* $w$ e lches haus fand pia st+ das haus $\mathrm{p}+$ das übrigens $k$ u no gehörte $\mathrm{f}++/$.

(7.15a) Pia fand nicht das Haus, das Kuno gehörte, sondern das Haus, das Hugo gehörte.

(7.15b) *Pia fand nicht das Haus, das übrigens Kuno gehörte, sondern das Haus, das übrigens Hugo gehörte.

(7.16a) Pia fand sogar das Haus, das $K$ u no gehörte (, nicht nur das Haus, das Hugo gehörte).

(7.16b) *Pia fand sogar das Haus, das übrigens $K$ u no gehörte $($, nicht nur das Haus, das übrigens Hugo gehörte).

(b) 2.1. mit 2.7. die "engen Appositionen". Gerade weil diese Muster traditionell den "Appositionen" zugerechnet werden, bedarf es einer ausführlicheren Besprechung ihrer Eigenschaften (s. Abschnitt 7.1.).

(c) 4.1.1. das nicht-restriktive Adjektivattribut. Neiteres s. Punkt 7.2.

(d) 4.1.3. die aus einer NP "gefloateten" Elemente. Weiteres s. Punkt 7.3.

(e) 4.2.1. die Typen hier am Knie (Adv + PP) bzw. im Stadion auf der Tribüne $(P P+P P)$. Weiteres s. Punkt 7.4 (und Kap. 9).

(f) 5.2. das Muster eine Chefin wie Pia. Weiteres s. Punkt 7.5.

Wăhrend nachstehend sich typische Attributmerkmale der wie-Translativphrase zeigen (a-Varianten), bleibt dagegen das Muster 5.1. Pia als Arztin zunăchst Appositionskandidat, denn die als-Translativphrase zeigt in einigen Punkten 
nicht-attributtypisches Verhalten: ${ }^{14}$ keine Gliedteilfrage möglich (7.17b), nicht kontrastierend negierbar (7.18b), nicht von GP fokussierbar (7.19b):

(7.17a) Eine Arztin wie Pia arbeitet gern.

Was für eine Arztin arbeitet gern? Eine Arztin wie Pia.

(7.17b) Pia als Arztin arbeitet gern.

Was für eine/ Welche Pia arbeitet gern? *Pia als Arztin.

(7.18a) (?) Nicht eine Arztin wie Pia arbeitet gern, sondern eine Frau wie Pia.

Nicht eine Arztin wie Pia arbeitet gern, sondern eine Arztin wie Eulalia.

(7.18b) ?? Nicht Pia als Arztin arbeitet gern, sondern Pia als Frau. * Nicht Pia als Arztin arbeitet gern, sondern Eulalia als Arztin

(7.19a) Sogar eine Arztin wie $P$ i a arbeitet gern.

(7.19b) *Sogar Pia als A rztin arbeitet gern (, nicht nur Pia als Frau).

(7.19c) Pia arbeitet sogar als $A$ rztin gern (, nicht nur als Krankengymastin).

Beispiel (7.19c) zeigt, daB ein GP-Fokus durchaus auf einer Translativphrase lagern kann; allerdings fungiert hier die Translativphrase m.E. als ADVERBIAL (als Satzglied; näheres Kap. 7.B). Aufgrund der wenig akzeptablen als-Beispiele wird der Typ 5.1. hier nicht als attributive Konstruktion ausgeschieden. Die Besonderheiten der als-Translativphrasen erfordern einen Exkurs, der diesem Kapitel angeschlossen wird (7.B).

\subsection{Die "engen Appositionen"}

Die Frage, ob Syntagmen wie Onkel Paul/ Unser Onkel Paul/ Das Laster Trunksucht/ Ein Liter (kühler) Wein zu den appositiven Konstruktionen zăhlen, oder ob sie nicht syntaktisch anders - z.B. als Attributkonstruktionen - zu behandeln seien, scheint derzeit noch nicht klar entschieden. Moglicherweise kann man insofern von einer Tendenz "contra Apposition" sprechen, als in einigen neueren Arbeiten, die sich eingehender mit der Apposition bescháftigen (Z.B. Raabe 1975, 1979; Altmann 1981; Engel 1986, 1988), die "engen Appositionen" anders analysiert werden, und zwar als "determinative Syntagmen" (Raabe 1975: 315 f., Altmann 1981: 57) bzw. als Attributkonstruktionen (z.B. Engel 1988: $606 \mathrm{ff.}$.). Dennoch findet sich in anderen neveren Arbeiten (z.B. Hackel 1978; Molitor 1979; Lobel 1986a, 1986b, 1988b) die "enge Apposition" als syntaktische Funktion unverăndert. Nachstehend gebe ich einige Begründungen dafür an, die zur Diskussion stehenden Muster als "Appositionen" einzustufen:

14 Aufgrund des unklaren Wortartenstatus von als und wie wurde der Terminus "Translativ" für diese Ausdrücke vorgeschlagen, so z.B. in Heringer (1973). Das Konstrukt aus als/wie + Nomen bzw. NP sei daher Translativphrase genannt. S. dazu auch Rap. 7.B. 
(a) Muster wie der König Herodes lassen sich - z.B. nach Wunderlich/Reis (1925: 178) - diachron zurückführen auf "Nachtragskonstruktionen" wie Herodes(,) der König(,). Die nachgestellte, "hinzugefügte" Einheit rückt vor die Bezugs-NP (bzw. das Bezugsnomen); evtl. vorhandene Abtrennungsmarkierungen fallen weg, der Komplex wird nunmehr als eng zusammengehörig empfunden (Beispiele Wunderlich/Reis 1925: 180):

(7.20) in den Tagen Hêrôdis des kuniges.

(7.21) Dô abir der kunig Hêrôdes diz hôrte.

Wunderlich/Reis (1925: 181) kommentieren dies wie folgt:

"Diese Verbindungen mit vorangestellter Titelbezeichnung zeigen, wie durch eine solche Voranstellung eine ursprüngliche Apposition mit dem Bezugswort zu einer Einheit verschmilzt und so schon zum Attribut überleitet".

(b) Enge Appositionen ähneln "lockeren Appositionen"; so erscheint z.B. das gleiche Wortmaterial, z.T. die gleiche Wortfolge:

(7.22) Mein Bruder Kuno/ Mein Bruder, Kuno,/ Kuno, mein Bruder. Dabei wird jedoch der durch das "Einschaltungsmuster" markierte Unterschied verkannt: Gliedteil (enges Muster) versus "Zusatz" (lockeres Muster)!

(c) Enge und lockere Appositionen werden in der generativen Grammatik (s. z.B. Chomsky 1957; 1965) aus gleichen Tiefenstrukturen (7.24) abgeleitet:

(7.23) Der Physiker Max Plank/ Der Physiker, Max Plank, löste das Problem.

(7.24) Max Plank - Max Plank ist ein Physiker - löste das Problem.

Meist werden als gemeinsame Ableitungsbasis für lockere und enge Appositionen parenthetische Kopulasätze oder nicht-restriktive Relativsätze angesetzt (einen Oberblick über gTG-Versuche zur Apposition bietet Raabe 1979: $154 \mathrm{ff}$; zum Deutschen s. Motsch 1965).

(d) Die appositionstypische "Referenzidentität" beider Konstruktionsteile wird z.T. auch beim "engen" Typ angenommen. So könnten sich etwa beide Teile der folgenden Konstruktionen auf den gleichen Referenten beziehen:

(7.25a) (Der Philosoph) (, Platon,).

(7.25b) (Der Philosoph) (Platon).

Raabe (1979: 111 ff.) demonstriert, daB der "enge" Typ insgesamt auf einen Referenten bezogen ist; Muster wie die Universitat Erfurt/ das Laster Trunksucht/ zwei Liter Bier verdeutlichen dies. Dagegen darf man beim "lockeren" Typ manchmal von Referenzidentitat sprechen. ${ }^{15}$

Der Untersuchung sog. "enger Appositionen" widmete sich vor allem Hackel, und zwar in seiner Dissertation (Hackel 1969) sowie einer Reihe anschliebender Aufsătze (Hackel 1972, 1973, 1975, 1978 und 1986). Aus diesen Arbeiten geht eine differenzierte Subklassifizierung dieser Fügungen hervor.

15 Vgl. Raabe (1979: 115):

"Tassen wir zusammen, so läßt sich feststellen, daß das Identitătsdefiniens in einer Definition der engen "Apposition" eine Absurdität ist, daß es aber (...) für die lockere Apposition gilt, wenn auch in zu differenzierender Sichtweise (...)". 
Von den neueren Arbeiten sei auf Kusmin (1960), Molitor (1979) und Weinrich (1982: $383 \mathrm{ff.}$ ) und die Darstellung in der Grammatik von Eisenberg (1986: 242 ff.) verwiesen. In der jüngsten zeit sind vor allem syntagmen des "quantitativen Typs" (z.B. zwei Liter Bier/ ein Kilo frische Butter/ eine Herde Kühe) in den Vordergrund gerückt: Katz (1982), Flückiger-studer (1983: 124 ff.), Eisenberg (1985, 1986: $245 \mathrm{ff.})$, Krifka (1986) und v.a. Löbel (1986a, 1986b). An älteren Untersuchungen wăren Blümel (1910, 1911) und Fey (1912) zu nennen.

Während Attributkonstruktionen eine eindeutige head-modifier-Aufteilung zeigen, wurde dies bei den "engen Appositionen" bezweifelt, so daß diese nicht der naheliegenden attributiven Relation zugeordnet wurden:16

(7.26a) Der Nachbar (head) des Vitzliputzli (modifier; GA).

(7.26b) Der Nachbar (head? enge APP?) Vitzliputzli (head? enge APP?). Gefragt wurde nach (morpho)syntaktischen und semantischen Indizien für eine struktur- und Funktionszuweisung. Vor allem die Kasuskongruenz sowie die Veglabbarkeit beider Konstituenten bei einigen engen strukturen legt eine "double-head"-Interpretation nahe; ${ }^{17}$ zudem ist nicht so einfach wie bei attributiven Konstruktionen festzustellen, welche Konstituente velche modifiziert (oder ob überhaupt Modifikation vorliegt?). Wie sich allerdings in den nachfolgenden Ausführungen herausstellen wird (Testergebnisse!), zeigen "enge APP" Attributverhalten und sind in head und modifier strukturierbar.

Wie Konstruktionen mit Genitiv- oder Präpositionalattribut sind "enge Appositionen" gesamt vorfeldfăhig, erfragbar (7.27a) und ersetzbar (7.27b). AuBerdem sind Gliedteilfragen anwendbar; dabei zeigt sich, daB ein Grosteil der Konstruktionen, zu denen eine sein/heiBen-Prädikation gebildet werden kann, Gliedteilfragen bzgl. beider Konstruktionsteile zuläBt - eine Besonderheit, die attributive Konstruktionen sonst nicht aufweisen (7.27c)!

Beide Konstruktionsteile sind kontrastierend negierbar (7.27d) und kónnen einen Gradpartikelfokus auf sich ziehen (7.27e):

(7.27a) Sie sah die Universität Erfurt/ den Tenor Krause.

Was sah sie? Die Universität Erfurt.

Wen sah sie? Den Tenor Krause.

(7.27b) Sie sah die Universität Erfurt/ sie.

Sie sah den Tenor Krause/ ihn.

16 Die modifier-head-Debatte findet sich bei Raabe (1979: $117 \mathrm{ff}$.) zusammengefaßt.

17 Das Prinzip lautet: "Die Flexionsmerkmale eines Syntagmas werden (mindestens) am Kopf ausgedrückt"; wo also ist der Kopf: statt Peters (head?) des Groben (head?). Oder aber: "Der Kopf eines Syntagmas kann anstatt der ganzen Gruppe stehen"; wo ist der Kopf: Weine Tante Bea/ Weine Tante/ Bea kamat zu Besuch. Siehe z.B. Bisenberg (1986: 244-248). Das semantische Prinzip lautet etwa: "Der Satellit modifiziert den Kopf". 
(7.27c) Welche Universität sab sie? Die Universität Erfurt.

Welchen Tenor sab sie? Den Tenor $\mathrm{Kr}$ a use.

Velchen Krause sab sie? Den Ten or Krause.

(7.27d) Sie sah nicht die Universität $E$ rfurt, sondern die Universität L e ipzig.

Sie sah nicht die Universit ä $t$ Erfurt, sondern das $F$ o rschungsinstitut Erfurt.

Sie sah nicht den Tenor Rr a use, sondern den Tenor $M$ ü ller.

Sie sah nicht den Ten or Krause, sondern den B a riton Krause.

(7.27e) Sie sah auch die Universität $E$ rfurt.

Sie sab auch die Universit ä $t$ Erfurt.

Sie sah sogar den Tenor Kr a use.

Sie sah sogar den Ten or Krause.

Die Normalbetonung, die unmarkierte Betonung in "Normalkontexten" (ohne Emphase, Kontrast etc.), liegt auf dem zweiten Nomen. Dies jedoch nicht etwa deshalb, weil es der Satellit ist, sondern weil hier eine "normale" Nominalgruppenbetonungsregel wirkt (Lötscher 1983: 193; Beispiele 7.28 ebd. 198):

"Generell wird (...) festgestellt, das bei neutraler Akzentuierung Endakzentuierung gilt, das also das letzte Wort am stärksten akzentuiert ist (...). Dabei kommt es (...) of fenkundig nicht auf die innere struktur der Nominalgruppe oder auf die syntaktische Funktion des einzelnen Wortes an".

(7.28) Karl der Gr o Be. Der große $R$ a rl. Mein Schwager Fr $i t z$.

Das Substantiv "W e gwerfpackung". Frau Doktor $M$ a ier.

Das bedeutet, daß die standardintonation einer nichtfunktionellen Regel folgt und somit nicht als Kriterium beispielsweise zur Nukleus-Satellit-Differenzierung (Kern-Attribut-Bestimmung) herangezogen werden kann.

Nun soll eine Subklassifizierung der Syntagmen vorgenommen werden. Die Klassifikationskriterien sind (a) die vorkommenden unterschiedlichen Kategorien (hier soll noch keine interne Klammerung der Kategorien zu möglichen Konstituenten vorgenommen werden), (b) die zunehmende Romplexität und (c) systematische Beziehungen zu äquivalenten Syntagmen (i.d.R. Reduktionsbeziehung).

zu den Tests im einzelnen: In den ersten Spalten wird geprüft, ob sich das jeweilige Syntagma in ein Determinativkompositum (KP) bzw. in eine attributive Konstruktion (Spalten 2-4) umformen läBt. Dann wird versucht, verbale Paraphrasen zu bilden (Spalten 5,6), die die semantische Relation zwischen den Nomina prădikativ zum Ausdruck bringen sollen; hierbei wird auf Detailprobleme (z.B. Wechsel des Artikels von bestimmt zu unbestimmt etc.) keine Rücksicht genommen.

Es wird auch überprüft, ob die "engen Syntagmen" in lockere umgeformt werden könnten und ob dabei die Reihenfolge der Nomina umgekehrt werden kann. Dann wird versucht, das enge syntagma in eine Konstruktion mit als (vgl. der Fakir Krause, Krause als Fakir) oder wie (ein Fakir wie Krause) umzuformen, 
wobei Detailprobleme wie Artikelwechsel oder -wegfall wiederum unbeachtet bleiben. Zudem wird angegeben, welche Nomina erfragt werden können, 18 ob auf beide Konstruktionsbestandteile Negationspartikeln bzw. GP angewendet werden können (Neg/GP) und ob Kasuskongruenz zwischen N1 und N2 (i.d.R.) vorliegt. Die zu prüfenden Konstruktionen sind:
(a1) Pia Huber
(EN EN)
(a2) Tante Pia. Doktor Huber
(CN EN)
(a3) Hamburg Hauptbahnhof
(EN CN)
(a4) Der Fakir Krause
(Det CN EN)
(a5) Die Stadt Erfurt
(Det CN EN)
(a6) Das Drama "Faust"
(Det CN "EN")
(a7) Die Arbeitsgruppe Sport
(Det CN EN)
Das Fach Latein
(a8) Die Brigade Krause
(Det CN EN)
Die Regierung Kohl
(a9) Das Laster Trunksucht
(Det CN EN)
(a10) Die Universität Erfurt
(Det CN EN)
(a11) Der Vogel StrauB
(Det CN CN)
(a12) Der Kampf Clay-Frazier
(Det CN EN-EN)
(a13) Zwei Liter (kühles) Bier
(Num QN N)
Zwei Klumpen (edles) Gold
(a14) Wir (armen) Lehrer
(Pron N)

KP Adj GA PräpAttr N1 VB N2 N2 Vb M APPlocker

\begin{tabular}{|c|c|c|c|c|c|c|c|}
\hline Pia Huber & - & - & - & ??namens & ?heißt & - & ? \\
\hline Tante Pia & - & - & - & - & - & ist & ?Pia, Tante, \\
\hline Die Tante Pia & - & - & - & namens & heist & ist & $\mathrm{N} 1, \mathrm{~N} 2 / \mathrm{N} 2, \mathrm{~N} 1$ \\
\hline Der Fakir Krause & - & - & - & namens & heist & ist & $\mathrm{M} 1, \mathrm{~N} 2 / \mathrm{N} 2, \mathrm{M} 1$ \\
\hline Die Stadt Erfurt & - & - & - & namens & heist & ist & $\mathrm{N}, \mathrm{N} 2 / \mathrm{N} 2, \mathrm{~N}$ \\
\hline Das Drama "Taust" & $(-)$ & - & - & namens & heist & ist & $\mathrm{N} 1, \mathrm{~N} 2 / \mathrm{N} 2, \mathrm{~N} 1$ \\
\hline Der Vogel Strauß & $(-)$ & - & - & namens & heist & ist & ? \\
\hline Das Fach Latein & - & - & - & namens & heiBt & ist & ? \\
\hline Das Laster Trunksucht & $(-)$ & - & + & ?mamens & ?heißt & ist & $(-)$ \\
\hline $\begin{array}{l}\text { Die Arbeitsgruppe } \\
\text { Sport }\end{array}$ & N21 & - & - & - & $\begin{array}{l}\text { befaßt } \\
\text { sich mit }\end{array}$ & - & - \\
\hline Die Brigade Krause & N21 & $(+)$ & + & $\begin{array}{l}\text { von } \\
\text { (unter) }\end{array}$ & - & leitet & - \\
\hline Die Regierung Kohl & $\mathrm{N} 21$ & $(+)$ & + & $\begin{array}{l}\text { von } \\
\text { (unter) }\end{array}$ & - & leitet & - \\
\hline $\begin{array}{l}\text { Die Universităt } \\
\text { Erfurt }\end{array}$ & - & + & + & von/in & $\begin{array}{l}\text { befindet } \\
\text { sich in }\end{array}$ & - & - \\
\hline
\end{tabular}

18 2.B. Welcher Fakir? Der Fakir Kr a use. Welcher Krause? Der $F$ a kir Krause. Anders allerdings: Welche Brigade? Die Brigade Kr a use. *Nelche(r) Krause? *Die Brig a de Krause. Hier ist nur das N2 erfragbar. 


\begin{tabular}{|c|c|c|c|c|c|c|}
\hline $\begin{array}{l}\text { Der Kampf } \\
\text { Clay-Frazier }\end{array}$ & + & $(-)$ & $(-)$ & $\begin{array}{l}\text { zwischen } \\
\text { von }\end{array}$ & $\begin{array}{l}\text { findet } \\
\text { statt zwischer }\end{array}$ & (tragen aus) \\
\hline $\begin{array}{l}\text { Der Raum } \\
\text { Halle-Leipzig }\end{array}$ & $(+)$ & $?$ & $(-)$ & $\begin{array}{l}\text { zwischen } \\
\text { um. .herum }\end{array}$ & $\begin{array}{l}\text { liegt } \\
\text { zwischen }\end{array}$ & $\begin{array}{l}\text { ?bilden } \\
\text { ?begrenzen }\end{array}$ \\
\hline $\begin{array}{l}\text { Zwei Liter (kihles) } \\
\text { Bier }\end{array}$ & $(-)$ & - & $(+)$ & (von) & $?$ & - \\
\hline Zwei Klumpen Gold & + & - & $(+)$ & aus & $\begin{array}{l}\text { bestehen } \\
\text { aus }\end{array}$ & bildet \\
\hline Wir (armen) Lehrer & - & - & - & - & sind & sind \\
\hline Ludwig das Kind & - & - & - & - & - & - \\
\hline Hamburg Hauptbahnhof & - & - & - & - & - & - \\
\hline
\end{tabular}

N2alsN1 MwieN2 Frage Neg/GP Kongr

\begin{tabular}{|c|c|c|c|c|c|}
\hline Pia Huber & - & - & $\mathrm{M} 2$ & + & - \\
\hline Tante Pia & + & - & $\mathrm{N} 12$ & + & - \\
\hline Die Tante Pia & + & + & $\mathrm{N} 12$ & + & - \\
\hline Der Fakir Krause & + & + & $\mathrm{M} 2$ & + & - \\
\hline Die Stadt Erfurt & + & + & $\mathrm{N} 2$ & + & - \\
\hline Das Drama "Taust" & + & + & N?12 & + & - \\
\hline Der Vogel Strauß & + & + & $\mathrm{N} 12$ & $(+)$ & - \\
\hline Das Fach Latein & + & + & $\mathrm{N} 2$ & + & - \\
\hline Das Laster Trunksucht & $(+)$ & $(+)$ & N2 & + & - \\
\hline Die AG Sport & - & - & $\mathrm{N} 2$ & + & - \\
\hline Die Brigade Krause & - & - & $\mathrm{N} 2$ & + & - \\
\hline Die Regierung Kohl & - & - & $\mathrm{N} 2$ & + & - \\
\hline Die Universität Erfurt & - & - & N2 & + & - \\
\hline Der Kampf Clay-Frazier & $?$ & $?$ & $\mathrm{~N} 2$ & + & - \\
\hline Der Raum Halle-Leipzig & $?$ & ? & $\mathrm{N} 2$ & + & - \\
\hline Zwei Liter (kuhles) Bier & - & - & $\mathrm{M} 1 ? 2$ & + & + \\
\hline Zwei Klumpen Gold & - & - & $\mathrm{M} 12$ & + & + \\
\hline Wir (armen) Lehrer & Malsk2 & N2wien & $\mathrm{M} 1$ & + & + \\
\hline Ludwig das Kind & - & - & N2 & + & + \\
\hline Hamburg Hauptbahnhof & - & - & $\mathrm{M} 1$ & + & - \\
\hline
\end{tabular}

Einige weitere Tests sollen zusätzlich das Vorliegen einer head-modifierstruktur erhärten; dabei soll gezeigt werden, daß vornehmlich oder ausschließlich ein Konstruktionsteil, eben der Kopf, (a) die Pro-Form-Beziehungen steuert, und zwar (a1) beim Possessivpronomen (7.29a), (a2) beim Relativpronomen (7.29b), (a3) beim Personalpronomen (7.29c) und (a4) beim Personalpronomen in einer Parenthese (7.29d):

(7.29a) Das Schachgenie Anna hat seinen/ ihren Pelz verkauft.

(7.29b) Das Schachgenie Anna, das/??die einen Pelz verkauft.

(7.29c) Das Schachgenie Anna kam nicht. Es/(?) Sie war krank.

(7.29d) Das Schachgenie Anna kam - es/ ??sie war sehr krank - gestern nicht. 
(b) die Verbkongruenz steuert:

(7.30a) Drei Krüge dunkles Bier erfrischten/*erfrischte Kunibert.

(7.30b) Drei Ellen Stoff lagen/ ? lag im Schrank. ${ }^{19}$

(c) einen regierten Kasus flexivisch realisiert:

(7.31) statt des Fakirs Krausel *des Fakir Krauses/ *des Fakir Krause. 20

(d) bei Umformung in eine (åquivalente) Attributskonstruktion Nukleus ist bzw.

bei Umformung in ein Determinativkompositum das Grundwort (7.32b):

(7.32a) Der Fakir (namens) Krause. Die Universitat (in) Erfurt.

Ein Gramm reines Gold/ reinen Goldes.

(7.32b) Die Brigade Krause. Die Krausebrigade.

(e) das Gesamtsyntagma ohne Bedeutungsveränderung vertreten kann:

(7.33) Die Brigade Krause/ Die Brigade/ *Krause tritt an.

(f) von Adjektivattributen modifiziert werden kann; für diesen Test eignen sich insbesondere intensionale Adjektive:

(7.34) Der vermeintliche Fakir (Krause). Die ehemalige Brigade (Müller). In (7.34) ist es vermeintlich der Fall, daß der Referent ein Fakir ist, nicht jedoch, daB er Krause ist (heiBt). Ehemals bestand die Brigade, nicht etwa Müller.

Zeichenerklärung: "0" = nicht spezifiziert bzw. spezifizierbar. "?" = evtl. möglich, jedoch nicht ganz klar.
(a1)
(a2)
(a3) (a4)
(b) (c)
(d) (e)

Pia Huber

Tante Pia

Die Tante Pia

0

0

\section{0}

0
$\mathrm{~N} 12$

N12 0

Der Fakir Krause

N12

N12

N12

0

Die Stadt Erfurt

N12

N1

N12

N1

N1 $21 \quad N 1^{22} N 1$

N2 0

Das Drama "Faust"

N1 N1

N12

N1

N1 N1 N1

N12 N1

N1

N1 N1

N1 N1 N1

N12 N1

N1 N1 N1 N1

$\mathrm{N}^{23} \mathrm{~N} 1 \quad \mathrm{~N} 1$

N12 N1

N12 N1

19 Beispiel von Krifka (1986: 9). Für inn sind beide Kongruenzoptionen gleich akzeptabel (ergo: double-headed?); in meinem Idiolekt ist die Rongruenz mit dem $\mathrm{N} 2$ fragwildig.

20 Und so fort. Besonderheiten der NP-Flexion des Deutschen wie statt meines Onkel(s) Kuno oder "săchsische" Varianten wie des ankel Kunos Heldentaten anstelle von "analog" (?) des Onkels Kuno Heldentaten (des onkels Kuno) sind zwar interessant, werden aber hier vernachlässigt.

21 Z.B.: Die Fakire (pl.) Krause (sg. Der Pl. wäre Krauses) > treten (pl.) auf.

22 Daß der Deterninator ebenfalls die Flexionsmerkmale realisiert und $2 \mathrm{~mm} \mathrm{M}$ gehört, sei hier wie in folgenden selbstverständlich.

23 S.: Der Thriller "Die Vögel" war/ twaren sehr spannend. 


\begin{tabular}{|c|c|c|c|c|c|c|c|c|c|}
\hline & (a1) & (a2) & (a3) & $(24)$ & (b) & (c) & (d) & (e) & (f) \\
\hline Der Vogel straus & N1 & N1 & N1 & N1 & N1 & N1 & N1 & $0^{24}$ & N1 \\
\hline Das Fach Latein & N1 & N1 & N1 & N1 & 0 & N1 & N1 & 0 & N1 \\
\hline $\begin{array}{l}\text { Das Laster } \\
\text { Trunksucht }\end{array}$ & N1 & N1 & $\mathrm{N1}$ & $\mathrm{N} 1$ & 0 & $\mathrm{~N} 1$ & N1 & 0 & N1 \\
\hline $\begin{array}{l}\text { Die Arbeitsgruppe } \\
\text { Sport }\end{array}$ & N1 & N1 & N1 & N1 & $\mathbf{N} 1$ & N1 & N1 & N1 & N1 \\
\hline Die Brigade Krause & N1 & N1 & N1 & $\mathbf{N} 1$ & $\mathrm{~N} 1$ & $\mathrm{~N} 1$ & $\mathrm{~N} 1$ & $\mathrm{~N} 1$ & N1 \\
\hline Die Regierung Kohl & $\mathrm{N} 1$ & N1 & $\mathrm{N} 1$ & $N 1$ & N1 & $\mathrm{N} 1$ & N1 & ?N1 & N1 \\
\hline $\begin{array}{l}\text { Die Universităt } \\
\text { Erfurt }\end{array}$ & N1 & N1 & N1 & $N 1$ & $\mathrm{~N} 1$ & N1 & N1 & N1 & N1 \\
\hline $\begin{array}{l}\text { Der Kampf } \\
\text { Clay-Frazier }\end{array}$ & N1 & $\mathbf{N 1}$ & N1 & $N 1^{25}$ & N1 & N1 & N1 & $\mathrm{N} 1$ & N1 \\
\hline $\begin{array}{l}\text { Der Raum } \\
\text { Halle-Leipzig }\end{array}$ & N1 & N1 & N1 & $\mathrm{N} 1$ & N1 & N1 & N1 & N1 & N1 \\
\hline $\begin{array}{l}\text { Zwei Liter (kühles) } \\
\text { Bier }\end{array}$ & N12 & N12 & $\mathrm{N} 12$ & N12 & N1 & $\mathrm{N} 12^{26}$ & N1 & $\mathrm{N} 12^{27}$ & N1 \\
\hline $\begin{array}{l}\text { Zwei Klumpen } \\
\text { (glănzendes) Gold }\end{array}$ & N1 & N1? 2 & N12 & $\mathrm{N} 12$ & N1 & $\mathrm{N} 12$ & N1 & N12 & \\
\hline Wir (armen) Lehrer & N1 & 0 & $\mathrm{~N} 1^{28}$ & N1 & N1 & N12 & 0 & N1 & $\mathrm{N} 2$ \\
\hline Ludwig das Kind & N1 & N1 & N1 & N1 & 0 & N12 & 0 & 0 & 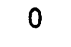 \\
\hline Hamburg Hauptbhf. & 0 & 0 & 0 & 0 & 0 & 0 & 0 & 0 & \\
\hline
\end{tabular}

Nach den Testergebnissen kristallisieren sich folgende Gruppen heraus:

(A) eine Grosgruppe, in der verbale Paraphrasen mit sein/heiBen, die Präp namens und zumeist die Umformungen mit als/wie möglich sind. Die Syntagmen sind nicht via Reduktion aus Attributkonstruktionen entstanden. Ein Grobteil dieser Syntagmen läßt Gliedteilfragen nach beiden Nomina $z u$ - eine Besonderheit bei attributiven Gruppen (i.d.R. nur Satellit erfragbar)! 29

24 Beim Subtyp ( (D CN) (CN)) ist das N2 als CN nicht in der Lage, die Gruppe zu vertreten; die Vertretung durch D N führt zu primär generisch interpretierbaren Aussagen, wodurch die semantische Aquivalenz gefăhrdet wird: Der Vogel Strauß/ ??Der Vogel steckt seinen Kopf in den Sand.

25 Möglicherweise auch N2: Der Kampf Clay-Frazier zog - (?) sie waren beide Klasseboxer - viele Zuschaver an.

26 Wenn N2 unbegleitet ist, dann flektiert nur (Det $\mathrm{N} 1$ ); sobald es von AdjA begleitet wird, kann N2 Flexive annehmen.

27 Wenn man Beispiele wie Kuno trank zwei Liter/kühles Bier heranzieht; in bestimnten Verwendungen ist (ohne entsprechende Anpassungen) jedoch nur N2 weglaßbar: Zwei Liter/ *Kulhles Bier erfrischten Kuno.

28 S.: Du Idiot machst alles kaputt. Hast Du/ *Hat er den Verstand verloren?

29 Viewohl keine "Ausnahme". Man vgl. auch den Typ oben im Schrank/ im Stadion auf der Tribüne. 
(B) eine Großgruppe, die reduktiv aus Genitiv- bzw. Prăpositionalattributen entstanden ist; ${ }^{30} \mathrm{z} . \mathrm{T}$. ist Konstruktionsvariation heute noch möglich (Drei Humpen edlen Weines/ edler Wein; das Laster (der) Trunksucht). Wie bei den Attributkonstruktionen sind bei vielen dieser Muster Gliedteilfragen nur nach dem Satelliten (N2) möglich; der Typ Ein Liter Bier tanzt hier aus der Reihe.

(C) Den Rest bildet eine heterogene Gruppe von Einheiten, bei denen Kasuskongruenz möglich oder gar die Regel ist (dich Idioten; Ludwigs des Kindes) sowie die Typen die Arbeitsgruppe sport und der periphere, wohl veraltende Typ Hamburg Hauptbahnhof.

Nun sollen die Subtypen etwas năher betrachtet werden:

(Aa) Die (EN EN)-Gruppe, also komplexe Namen, i.d.R. Personennamen. 31 Verbindungen aus Vor- bzw. Rufnamen und Nachnamen entstanden historisch aus diversen Konstruktionen, in denen der Nachname den Rufnamen năher bestimmte, weil der Rufnamenschatz etwa seit dem 10 . Jahrhundert nicht mehr ausreichte, um Personen zu identifizieren: 32

(7.35a) Hans, der Schmi (e)d, (lockere APP) > Hans Schmi(e)d.

(7.35b) Heinrich mit dem Zahn (ATTR) > Heinrich Zahn.

Diachron ist also der Vorname primăr. Synchron verhălt sich die Sachlage syntaktisch anders. Vor- und Nachname sind durch eine Gliedteilfrage erfragbar, negierbar, durch Gradpartikeln affizierbar; sie verhalten sich folglich attributartig.

Nur der (letzte) Nachname nimmt ein Kasusflexiv an, so daB man ihn als Nukleus ansehen könnte. Man kann auch die Position vertreten, daB sich Namensyntagmen wie Nominalkomposita (mit Endflexion) verhalten, so daB automatisch das letzte Element das Flexiv annimmt. ${ }^{33}$ Interessant ist auch die sicht von Eisenberg (1986: $243 \mathrm{f}$. ), der die These vertritt, das der Nachname den Vornamen hinsichtlich der Merkmale 'Nominativ' und 'Singular' regiere; in (7.36) regierte nach Eisenberg Opa den Genitiv bei Bierbichlers (das Rernnomen regiert den Genitiv seines GA) und der Nachname Bierbichler(s) regierte bei Pia Eulalia Nom. und $\mathrm{Sg}$. :

(7.36) (( ((Pia) VN $\left.\left.(\text { Eulalia) VN })_{\text {VNnom }}(\text { Bierbichlers })_{\text {NN }}\right)_{\text {NPgen }}(O p a)_{N}\right)_{\text {NPnom }}$.

30 Nach Hackel (1972; 1973; 1975; 1986: 9 f.). In Starke (1983b) werden Konstruktionen wie auf Basis Formaldehyd, die "weder normgerecht noch akzeptabel" seien (ebd. 174), abgelehnt. Hier ufert der "Abbau attributiver Markierungen" zugunsten purer Juxtaposition z.T. aus.

31 Speziellere Betrachtumgen zu diesem Typ s. Blümel (1910, 1911); Vimmer (1973: 63 ff.);

Ranseyer (1976); Kalverkämper (1978); Kuhn/Serzisko (1982); Weinrich (1982: 383 f.); Hackel (1978, 1986). S. auch die entsprechenden Passagen in den ausgewählten Grammatiken und Monographien.

32 Dazu Blưmel (1910, 1911), Ramseyer (1976), Kalverkämper (1978: 152, 204) und Weinrich (1982: $387 \mathrm{f}$. ..

33 So argumentieren z.B. Kalverkămper (1978: $198 \mathrm{ff.}$ ) und Kolitor (1979: $93 \mathrm{ff.}$. . 
Distributionell können sowohl Vor- als auch Nachname die Namengruppe vertreten; dies ist ein primär syntaktisches Argument, da pragmatisch keinesfalls Vor- und Nachnamen frei wăhlbar sind. Ein eher pragmatisches (bzw. textlinguistisches) Argument von Kalverkämper (1978: 147) besagt, Vornamen signalisierten, daB in ihrem Nachbereich ein als Nachnamen aufzufassendes Wort folgte, was man sich anhand (7.37) vor Augen führen kann:

(7.37) Ich gehe heute zum (Robert-)Koch-Institut. /pia suchte die (alfred) lange straße $\mathrm{f}+\mathrm{t} /$.

Nach meinen Explikationen liegen Attributkonstruktionen vor, wobei der Nachname (vgl. Eisenberg) als Basis und der Vorname als Attribut zu werten ist. In diesem Sinne löst auch die Grammatik von Engel (1988: 610) die Namensequenz auf und fast den Vornamen als vorangestellten Eigennamen-Satellit (Fachterminus: "Nomen invarians") auf.

(Ab) Die (CN EN) -Gruppe, also "Titel"/"Beruf"/"Verwandtschaftsbezeichnung" + Eigenname. ${ }^{34}$ Die Syntagmen dieses Typs verhalten sich ähnlich wie die Namensequenzen in ( $A a)$, jedoch ist das $C N$ eindeutig als Attribut auszumachen; nur der EN kann das Syntagma vertreten, nur er flektiert. Von gewissem Interesse ist die Möglichkeit von Häufungen: 35

(7.38) [(Herrn Genossen) Dekan(*s) Professor(*s) Kuno(*s)] Knalls Vorträge ergötzten das Auditorium.

Dabei erweist sich wiederum der Nachname als head; alle übrigen Elemente (Anredenomina, Funktionstitel, akad. Titel, Vorname) sind Satelliten. Die Anredenomina (Herr, Genosse) kongruieren mit dem Kopf; nach Engel (1988: 610) nenne ich ein solches N1 "Nomen varians" (vorangestellt). Ein unflektiertes N1 (Professor, Dekan) wird gleich den Vornamen "Nomen invarians" genannt.

(AC) Eine große Gruppe (Det CN EN) mit diversen Kombinationen von Determinantien plus Berufe/ Verwandtschaftsbezeichnungen/ Objekte/ Klassennamen (N1) etc. und Eigennamen (N2): die Tante Pial der Fakir Krause/ die Stadt Erfurt/ das Drama "Faust". Die Muster dieser Gruppe, zu der auch ein Teil der ((Det CN) (CN))-strukturen gehört, nämlich z.B. das Fach Latein, der Vogel StrauB, 36 lassen verbale Umformungen mit sein/heiBen, 37 Umformungen in Translativphrasen oder Syntagmen mit namens zu. Auch sie dürfen als Attributkonstruktionen mit N1 als Kopf aufgefaßt werden. Das N2 sei mit Engel (1988: 616 f.) als nachgestelltes "Nomen invarians" bezeichnet. Ein interessanter Aufsatz zu derartigen

34 Spezieller hierzu: Die ausgewählten Grammatiken und Monographien sowie Blümel (1910, 1911); Thnderlich/Reis (1925: 187 ff.); Hackel (1969, 1975, 1978, 1986); Doerfer (1973: 199 ff.); Kalverkämper (1978: $239 \mathrm{ff}$. et pass.); Weinrich (1982: 390-392).

35 Zur Abfolge Sommerfe].dt (1971); Kalverkămper (1978: $251 \mathrm{ff.}$ ).

36 Dazu Blümel (1910, 1911); Schwyzer (1947); Hackel (1969, 1972, 1975, 1986); Weinrich (1982: $390 \mathrm{ff.}$ ) und die ausgewählten Gramatiken/Konographien. Marginal Zoeppritz (1984).

37 Zur "Nominalrelation" s. auch Rahmstorf (1983: 317 f.). 
Mustern liegt mit Jackendoff (1984) vor (Jackendoffs Beispiele von W.S. ins Deutsche übertragen):

(7.39a) Die Phrase die Phrase (objektsprachliches Beispiel).

(7.39b) Die Farbe Rot (Rot ist eine Farbe).

(7.39c) Der Buchstabe A. Das Symbol $S$. (A ist ein Buchstabe etc.).

(7.39d) Das Geräusch Pfft (Beispiel für ein Geräusch).

Nach Jackendoff (ebd. 30) hat das $N 2$ "(...) the function of uniquely specifying some individual of the category named by (...)" N1. Jackendoff sieht das N2 bei "enger" Anfügung nicht als appositives Komplement an; bei "lockerer" Anfügung läge allerdings eine Apposition vor:

(7.40) Das Geräusch des Reifens, Pfft, beunruhigte Pia. Jackendoffs Argumente für die Zuordnung von (7.39) entweder zur X-Bar-Ebene N1 (Valenzkomplemente) oder zur Ebene $N 2$ (restrictive modifiers) widersprechen sich z.T. und führen zu keiner eindeutigen Evidenz:

Reihenfolgebeziehungen sprechen entweder für X-Bar-Ebene N1 (unmittelbare Adjazenz ist notwendig), oder dafür, Elemente wie die Phrase als topologisch erstes Element der Ebene $\mathrm{N} 2$ einzuführen (ebd. $30 \mathrm{f.}$ ), denn es kann nur vor weiteren restrictive modifiers stehen. Falls die betreffenden Einheiten hinter ein weiteres N2-Komplement treten, müssen sie abgetrennt werden und wechseln somit in die Funktion "lockeres Appositiv" über:

(7.41a) Die Phrase die Phrase im Deutschen.

Die Phrase im Deutschen (*die Phrase/ ?, die Phrase,).

(7.41b) Das Geräusch (*Pfft) des Reifens (, Pfft,) beunruhigte Pia.

Teilweise mögliche Umformungen in typische restrictive modifiers sprechen für N2 (ebd. 31; z.B. der Schauspieler (namens) John Gielgud). Eher für N1 spricht die Reihenfolgebeziehung (ebd. 32):

(7.42) Der Romponist berühmter Lieder (N1) *Franz Schubert/, Franz Schubert.

Schließlich nimmt Jackendoff (1984: $35 \mathrm{f.}$ ) drei mögliche Strukturen an:

(7.43)

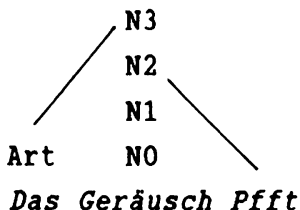

HEAD

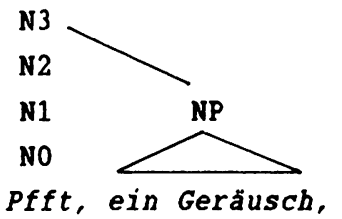

HEAD APPOSITIV

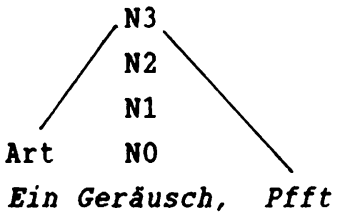

HEAD APPOSITIV

Die Entscheidung fallt in der Tendenz zugunsten von $N 2$ als restriktivem Attribut aus; die lockeren Strukturen stellen dagegen tatsăchlich "Appositionen" (N3-Ebene) dar.

Vater (1986b: 131, 142) dagegen siedelt im X-Bar-Rahmen "enge Appositive" zunăchst auf der $\mathrm{N} 1$-Stufe an, 38 weil sie unerweiterbar sind und keine Determi-

38 Vermutlich sieht Elisabeth Löbel (1988a: 5) die Struktur ebenso. 
nantien zu sich nehmen. Vor dem engen "Appositiv" darf kein restriktives Komplement (N2) stehen:

(7.44) die Königin (*von Schweden) Christine (von Schweden). Vater rảumt jedoch ein (ebd. 132), daB "(...) zur struktur appositiver syntagmen (...) noch nicht das letzte Wort gesprochen (...)" sei. Er schließt seinen Aufsatz ab mit der Bemerkung (ebd. $143 \mathrm{f}$. ), das

"(...) enge Appositionen den gleichen Status haben wie das Kern-N, nämlich

$N(\ldots)$; sie unterscheiden sich vom Kern-N lediglich dadurch, daB sie fa-

kultativ sind".

Mit (AC) verwandt (heiBen/sein; namens; als/wie) ist der Typ das Laster Trunksucht, der zum "Genitivus definitivus/explicativus" in Beziehung steht. Dieses Muster taucht in der Literatur zur "engen Apposition" gar nicht auf! Es steht zwischen den GroBgruppen (A) und (B). Die Umformungsbeziehung besteht nicht systematisch:

(7.45a) Der Grundsatz (der) Partnerschaft. Die Todsünde (der) Hoffahrt. Die Bewegung (des) Pietismus. Das Problem (der) Bildung.

(7.45b) Der Unfug des Sterbens. ??Der Unfug Sterben.

Das Glück eines Lottogewinns. *Das Glück Lottogewinn.

Als head kommt nur (D)N1 in Frage. Gerade aus der möglichen Beziehung auf Attributstrukturen läBt sich ein weiteres, gewichtiges Argument dafür ableiten, daß die N2 aller "engen Appositionen", die von einem Determinans eingeleitet werden, attributive satelliten sind!

(Ba) die ((D CN) (EN))-Typen, die zu Genitiv- und/oder Präpositionalattributen in Beziehung stehen: ${ }^{39}$ die Brigade Krause(s), die Regierung (unter) Koh1, die Universität (in) Erfurt. Sie können ofters auch in Konstrukte mit N2 als Adjektivattribut zu N1 (die Erfurter Universitát; die Kohlsche Regierung) transformiert werden. Wieder ist (D) N1 Nukleus, N2 ist wie im entsprechenden attributiven Syntagma Satellit ("Nomen invarians").

(Bb) der Typ ((D CN) (N-N)), der bezogen werden kann auf attributive strukturen mit Práposition nach $N 1$ und zwei mittels und koordinierten Nomina: 40

(7.46) Der Kampf (zwischen) Clay (und) Frazier. Die Krieg (zwischen) Iran (und) Irak. Die Folge (von) Dativ (und) Akkusativ.

Die bindestrichgefügten Nomina sind als Satelliten (Attribute) zu werten.

(BC) Der Typ ((Num $Q N$ ) (N)), dessen subtypen ebenfalls zumeist zu Genitivbzw. Präpositionalattributen in Beziehung stehen. 41 wăhrend die erste Konstituente eine Voll-NP ist, differiert der status der zweiten:

39 s. Hackel (1969; 1972: 341 f. u. 346 f.; 1973: 21; 1975: 44; 1986: 9f.). 2u den Unformungsbeziehungen auch sugarewa (1974).

40 Hierzu Hackel (1969, 1972); insb. Hackel (1973).

41 Hierzu natürlich die ausführliche Arbeit van Löbel (1986a); kurz zusammengefaßt in Löbel (1986b). Empfohlen auch Katz (1982), Fückiger-Studer (1983) und Eisenberg (1985; 1986: 242 ff.). Speziell zu diesen Konstruktionen auch Krifka (1986). Interessant auch die X-Bar-theoretischen 
(7.47a) ((Ein Glas) $)_{\mathrm{N} 3}\left(\right.$ Wein) $\left.\left.\mathrm{N}_{3}\right)\right)_{\mathrm{N} 3}$.

(7.47b) ((Ein Stück) $\mathrm{N} 3$ (Knochen) $\left._{\text {NO }}\right)_{\mathrm{N} 3}$ lag da herum.

$\left((\text { Ein Stück) } \mathrm{N} 3 \text { (morscher Knochen) } \mathrm{N} 2)_{\mathrm{N} 3}\right.$ lag da herum.

Die interne Vielfalt dieses Typs ist beachtlich. Die folgende Auflistung deutet sie nur an und ist keinesfalls vollständig:

- "Num + Numeralklassifikator $42+N ":$ Zwei stück süBe/ *süBer Torte.

- "Num + Numerativ + N": Zwei Scheiben altes Brot/ (?)alten Brotes.

- "Num + Mensurativnomen + N": Zwei Kilo frische/ (?) frischer Himbeeren.

- "Num + Behälternomen + N": Drei Kisten altel (?) alter Apfel/ mit alten Apfeln.

- "Num + Kollektivnomen + N": Vier Berden wilde/ wilder stiere.

Die Kern-Satellit-Verteilung ist aufgrund der möglichen Kongruenz beider Nomina, der (öfters) möglichen Pro-Form-Beziehung auf beide Nomina, der Modifizierbarkeit beider Nomina etc. nicht ganz einfach. (Adj)N2 ist kaum erfragbar, so daß eher der erfragbare Teil "Numerale +N1" als Gliedteil zu gelten hătte:

(7.48a) Pia trank zwei Liter Bier.

Wieviel Bier trank Pia? Zwei $L$ i ter (Bier).

Wovon trank Pia zwei Liter? ?Bier/ ?? Zwei Liter B $i$ er.

? Was für zwei Liter trank Pia? *Bierl ??Zwei Liter B $i$ er.

(7.48b) Pia fand zwei Klumpen Gold.

Wieviel Gold fand Pia? Zwei Klumpen (Gold).

Wovon fand Pia zwei Klumpen? ? Gold/ *Zwei Klumpen $G$ o ld.

Was für $z$ wei Klumpen fand Pia? ??Gold/ ?Zwei Klumpen G o ld.

In attributiven Varianten ist (Adj)N2 hingegen klar Satellit (Genitiv- oder Präpositionalattribut), bei einigen Typen Bestimmungswort eines Determinativkompositums (N1 ist das Grundwort):

(7.49) Zwei Stapel (mit) Holz, Zwei Holzstapel.

Zwei Sträuße Rosen > Zwei Rosenstrăuße.

Numerale + N1 steuert (bei den meisten Subtypen) die Kongruenz mit dem Prădikat. Bei Herausstellungen (7.50a zeigt Links-, 7.50b Rechtsversetzung, 7.50 c ein freies Thema) steuert das $\mathrm{N} 1$ die anaphorische Beziehung:

(7.50a) Ein Löffel Milch, der/*die hat noch keinem geschadet.

Zwei Stapel altes Holz, die hatte/ ??das hat der Forster geschlagen.

(7.50b) Ich habe ihn/??sie erhalten, einen Beutel frische Früchte. Ich habe (?) es/ *sie gegessen, ein Stück Torte.

(7.50c) Apropos ein Schwarm Bienen! Hast du ihn/ ?sie dort gesehen? Zwei Liter Bier? Gestern habe ich die/ ?das nie getrunken.

Ein weiteres Argument ist das Verhalten bei der Weglaßprobe. Hier erweist sich das $\mathrm{N} 1$ als die unverzichtbare Komponente der Gruppe:

Ausführungen in Jackendoff (1977), Selkirk (1977) u. Qehrle (1977). Zudem Blümel (1910, 1911); Hackel (v.a. 1969; 1975), Birkenmaier (1978) und Starke (1985).

42 zur Begrifflichkeit s. Löbel (1986a, 1986b). 
(7.51) Ein Kubikzentimeter verdünntes Gas/ *Verdünntes Gas/ Ein Kubikzentimeter enthäl bei Zimmertemperatur und 1/30 Atmosphäe Druck etwa 1018 Moleküle.

Insgesamt ist die von Löbel (1986a: $94 \mathrm{f}$.$) vorgeschlagene Trennung von syntak-$ tischem (Num + N1) und semantischem (N2) Nukleus wohl die plausibelste Lösung für die strukturierung derartiger Konstruktionen. Somit fungiert N2 syntaktisch als nachgestelltes Attribut ("Nomen varians"; Engel 1988: 618).

Der Typ die Arbeitsgruppe Sport steht in systematischen Beziehungen zu Determinativkomposita (die Sportarbeitsgruppe), nicht jedoch zu Genitiv- bzw. Präpositionalattributen. ${ }^{43}$ Zwischen den Komponenten liegt keine sein/heiBenRelation vor. Sie stellt somit einen Typ dar, der nur peripher der Grobgruppe (B) angehört. Das $\mathrm{N} 1$ ist der Nukleus der Gruppe, das N2 "Nomen invarians".

Der Typ Ich (armer) Idiot/ Uns (treuen) Beamten besteht aus einem Personalpronomen der 1./2. Person (Sg./P1.) und einem Nominal beliebiger X-Bar-Stufe. 44 Nach Engel (1988: 650) bildet man diese Pronominalphrasen, "(..) indem einem Pronomen eine mehr oder weniger vollständige Nominalphrase als Attribut zugeordnet wird". Wenn an die zweite Komponente ein Determinans tritt, muß eine "lockere Apposition" gewăhlt werden:

(7.52) Uns armen Irren/ *Uns den armen Irren/ Uns, den armen Irren, hat man etwas vorgegaukelt.

Zu korrigieren ist das Urteil, daB ausschlieblich emotiv markierte Nomina zu den Pronomina hinzuträten ${ }^{45}$ zutreffend ist vielmehr, daß dies zwar häufig der Fall ist (7.53a), aber keine Notwendigkeit darstellt (7.53b):

(7.53a) Du Esel. Ihr ewigen Studenten. Euch Idioten. Du alter Nörgler.

(7.53b) Nir Schuster/ Oberinspektoren/ Bäcker haben es nicht leicht.

Die struktur dieser Syntagmen ist umstritten. Eine Argumentationsrichtung verwendet die Austauschbarkeit der Pronomina mit Determinantien als Indiz dafür, daß eigentlich eine völlig "normale" NP vorliegt. 46 Diese Analyse kann man augenscheinlich durch das Verhalten in PPs stützen:

(7.54) Mit uns/ den armen Irren kann man das ja machen.

Wegen euch/ der Linguisten muß man obskure Dissertationen lesen.

Möglicherweise ist dieser Befund auch anders erklärbar, nämlich indem man annimmt, daB das Pronomen der syntaktische Nukleus ist und die Nominale Attribute darstellen. Schlieblich können die Pronomina die Gruppe allein vertreten, sie steuern die Prädikatskongruenz hinsichtlich der Kategorie 'Person' und sie steuern anaphorische Bezüge. Nicht für die Ansicht spricht evtl. die Kasuskon-

43 Weitere Literatur: Hackel (1969, 1972, 1975); auch Blưmel (1910, 1911).

44 Hierzu noch: Delorme/Dougherty (1972); Lange (1974); Schapiro (1977); Engel (1988: 649 ff.).

45 S. Lange (1974: 124).

46 So Kusmin (1960: 120). Schapiro (1977: $77 \mathrm{ff}$.) analysiert z.B. so: ((me) Det (men) N) NP. Ahnlich auch Molitor (1979: 127). 
gruenz (double-head?) und die Tatsache, daB Gliedteilfragen nur auf das Pronomen anwendbar sind:

(7.55) Welcher Idiot tut sowas? I ch Idiot.

Welchen Beamten soll man mehr zahlen. U ns Beamten.

Dennoch erscheint es mir sinnvoll, die vermeintliche Apposition als Attribut anzusehen; man vgl. auch:

(7.56) Wir armen Irren/ in der stadt (PA) / mit den dicken Autos (PA)/, die wir in der stadt leben, (attr. Rels) etc.

Hingewiesen sei noch auf eine (Schnaps?) Idee: Könnte man nicht infolge der Verwandtschaft derartiger Einheiten mit Vokativischen NPn annehmen, daB hier "in den Satz integrierte Vokativische NPn" vorliegen? 47 Sogar als Komplemente einer Präposition können diese strukturen vorkommen (7.57b):

(7.57a) Du Idiot (du)! Hast du (Idiot (du)) denn das wieder vergessen? Ich Trottel (ich)! Wieder einmal habe ich (Trottel (ich)) nichts gemerkt.

(7.57b) Du Idiot Du! Man kann mit dir (Idiotem (dir)) nichts anfangen. Ich Trottel (ich)! Durch mich (Trottel (mich)) wurde alles verpatzt.

Obrig bleiben noch die heute wohl nicht mehr produktiven Namenfügungen des Typs Karl der Kahle etc. ${ }^{48}$ Diese Fügungen stehen funktionell den Eigennamenfügungen (vgl. oben (Aa)) nahe. Zudem verbleibt der ebenfalls periphere, zweigliedrige Typ mit vorangestelltem EN: Hamburg Bauptbahnhof/München Süd/ Dettelbach stadt. ${ }^{49}$ Hier könnte man ebenfalls Reduktionen annehmen, vielleicht folgender Art:

(7.58) Der Zug hält (in) Hamburg (am) Hauptbahnhof und nicht (in) Dettelbach (in der) stadt.

Oder man faßt die zweigliedrigen Muster als eine Art Eigenname auf, ohne daB Tilgungen angenommen würden. Dann bestünde eine Nähe zur Gruppe (Aa). Wegen ihrer marginalen Rolle werden beide Konstruktionstypen nicht năher behandelt.

\subsection{Das nicht-restriktive Adjektivattribut}

Seiler (1960: $10 \mathrm{f}$.$) erweist das Konstrukt "kongruierendes Adjektiv + Nomen$ innerhalb einer NP" als Nukleus-Satellit-Syntagma; das Nomen kann anstelle der ganzen Gruppe stehen (7.59), so daß man eine endozentrische Ronstruktion ansetzen darf:

47 Zur Vokativischen NP s. Altmann (1981: 51 f., 56 f.).

48 Hierzu s. die in Rap. 2 ausgewählten Arbeiten; s. auch Blümel (1911: 4 f.); Schwyzer (1947); Kusmin (1960: $50 \mathrm{ff}$ ); Kalverkämper (1978: 265 ff.); Eisenberg (1986: $242 \mathrm{ff.}$ ).

49 Hierzu neben der in Rap. 2 angegebenen literatur auch: Blümel (1910: 5, 28 f.; 1911: 10); Kalverkämper (1978: 265 ff.). 
(7.59) Dort blühen ((rote/ gelbe/ langstielige) Rosen).

Aufgrund der variierenden Semantik bei wechselnden Akzentmustern, die eine Nominalgruppe suprasegmental überlagern können, nimmt Seiler (1960: 19 ff.) folgende Relationen zwischen "Klasse" (Grund- bzw. Basismenge) und "Selektor" (Schnitt- resp. Submengenbilder) an: 50

(7.60) $1 \quad 2 \quad 2 \quad 1$
(a) böse Hunde
(b) böse Hunde
(c) böse Hunde
(d) böse Hunde

In (a) fungiert der Nukleus Hunde als Klasse, das Adjektiv als Selektor; diese Beziehung nennt Seiler "Spezifikation". In (b) ist das Adj Klasse und der Nukleus Selektor: Aus der Menge alles Bösen werden die bösen Hunde herausgeschnitten; dieses Verhăltnis nennt Seiler Charakterisierung. Die Fälle (c, d) stellen nach Seiler gar keine Klasse-Selektor-Relation dar und bleiben bei ihm ununtersucht (und hier unberücksichtigt). 51

Der (a)-Fall ist nach Seiler (1960: $34 \mathrm{ff.}$ ) ein Beispiel für Attribution, der (b)-Fall eines für Apposition. Attribut wie Apposition sind zwar Satelliten des Kernnomens, unterscheiden sich jedoch in der spezifizierenden bzw. charakterisierenden Relationsbedeutung, die mit entsprechenden Intonationsunterschieden formal korreliert. Somit erweist sich Seilers Appositionsbegriff als ein semantischer: zentral ist das Merkmal [ $+/-$ charakterisierend], angewendet auf Nukleus-Satellit-strukturen. 52

Auch Lehmann (1984: 275-277) versteht charakterisierende adnominale Adjektive als (enge) Apposition, 53 wobei gilt: Das Appositum ist Satellit eines Nomen und strukturell entbehrlich; es hat nicht den Hauptakzent der Gruppe; das gesamte Syntagma hat dieselbe Referenz wie der Nukleus ohne Satellit. Er weist (ebd. 275) allerdings auf einen möglichen Unterschied hin: Wăhrend die "enge Apposition" vom Nukleus dependent sein dürfte, liegt bei der lockeren lediglich Juxtaposition ohne Dependenz vor.

In den Grammatiken des Deutschen wird dieser Typ von adnominalem Adjektiv als Attribut gefüht, wobei sich immer wieder Bemerkungen zum Unterschied von den restriktiven Typen finden. ${ }^{54}$ In der X-Bar-Syntax werden die Adjektivphrasen trotz semantischer Nicht-Restriktivität nicht auf der N3-Stufe (appositive

50 "1" bezeichnet einen Hauptakzent, "2" einen Neben- bzw. Schwachakzent.

51 Vgl. Seiler (1960: 22): "Bei der Nicht-Abstufung des Akzents wird also uber die Relation Klasse-selektor nichts ausgesagt".

52 Vgl. auch Raabe (1979: 126-129).

53 Man s. auch Lehmann (1984: 276): "Das appositive Adjektiv fungiert nomalerweise als informationsloses Epitheton (...)".

54 Heidolph u.a. (1981: 826 ff., 835 ff.) unterscheiden zwischen restriktiver und appositiver Attribution; Schulz/Griesbach (1982: 146) differenzieren zwischen "erklärendem" und "unterscheidendem" Adjektivattribut. In Engel (1988: 608) wird auf die restriktive bzw. nicht-restriktive Verwendbarkeit von Adjektiven bei der Attribution hingewiesen. 
Komplemente) angesiedelt, sondern aus syntaktischen (!) Gründen unterhalb derselben; Jackendoff (1977: $72 \mathrm{f.}$ ) setzt N2 an, Vater (1986b: $140 \mathrm{f.}$ ) N1.

DaB semantisch nicht-restriktive Adjektive dennoch syntaktisch betrachtet typische Attribute darstellen, sei nachstehend an Beispielen angedeutet und in einer Merkmalsmatrix zusammengefaßt. ${ }^{55}$ Gezeigt werden die klaren Unterschiede zwischen nicht-restriktiven Adjektivattributen und einer nachgestellten Variante (7.61b), die als "Appositionsprototyp" aufgefaBt wird (Kap. 6, 15):56

(7.61a) Die fleiBigen und unergründlichen Chin e sen sind ein Phänomen. Die sparsamen und tüchtigen Schw a ben sind ein Phänomen.

(7.61b) Die Chin e sen, fleiBig und unergründlich, sind ein Phänomen. Die Schw a ben, sparsam und tüchtig, sind ein Phänomen.

(a)

Kongruenz
Position
Erfragung
Negation
Gradpartikeln
vor der NP

Intonation

Satzzeichen

Paraphrase

Semantik

Sprecherdivergenz
Kopulasatz

[+/- restriktiv]

nie möglich (b)

\author{
nach Nukleus

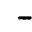 \\ $-$ \\ affizieren Adj nicht
}

evtl. Nebenakzent, von Pausen umhüllt

von Kommata (evtl. Rundklammern, Gedankenstrichen) umgeben Kopulasatz 57

nur [- restriktiv]

möglich. 58

55 Unberücksichtigt bleibt der archaisierende, volkstümliche Typ Ein garstig(es) Lied, lieb(es) miltterlein etc. (z.B. Duden 1984: 267), der aufgrund seiner Nicht-Flektiertheit dem postponierten appositionsverdăchtigen Typ ähnelt, ebenso der TỴ Röslein rot, o Täler weit! (Duden 1984: 267). In Ausbreitung begriffen scheint der zunăchst fach-, werbe- und kulinarsprachliche TYp Eiche mittelbraun, Genitiv maskulin, Henkell trocken, Schauma mild, Forelle blau, Beefsteak rosa. Dieser restriktive TYp, äquivalent zum präponierten (mittelbraune Eiche, trockener Henkell, rosa Beefsteak), findet über diese spezielleren Verwendungen hinaus immer mehr Anwendung (z.B. Pressesprache): Leben pur, Fubball brutal.

56 Die Sperrung unterstreicht die von Seiler herausgehobene Akzentuierung auf dem Mukleus.

$57 \mathrm{Vgl}$.: Die Chinesen sind ein Phänomen. Die Chinesen sind fleibig und unergrindlich) Die (fieiBigen und unergründlichen) Chinesen (, fleiBig und unergründlich,) sind ein Phánomen.

58 Vgl.: *Pia sagte: "Die" fleiBigen und unergründlichen "Chinesen sind ein Phänomen". Pia sagte: "Die Chinesen", fleißig und unergründlich, "sind ein Phänomen". 
Wăhrend sich bei (a) typische Attributcharakteristika finden (Kongruenz, Gliedteilfrage, von NEGATION oder Gradpartikeln erfaßbar, kein Einschaltungsmuster, potentiell auch restriktive Lesart, keine Sprecherdivergenz möglich), zeigt (b) ein davon klar abweichendes Verhalten (weiteres Kap. 6, 12, 15).

\subsection{Gefloatete NP-Elemente}

Hier genügt eine sehr knappe Behandlung. Unter "gefloateten" NP-Elementen kann man folgende Syntagmen verstehen, die offensichtlich erfragbar und kontrastiv negierbar sind und sich somit wie Attribute verhalten:

(7.62a) Die Blumen haben alle geblüht.

Welche/ Wie viele Blumen? Alle.

Die Blumen haben nicht a lle geblüht, sondern nur m a nche.

Die Blumen haben sogar a lle geblüht.

(7.62b) Obst kaufte sie nur fr $i$ sches.

Obst kaufte sie nicht fr $i$ sches, sondern verg a mmeltes.

Was für Obst kaufte sie? Fr i sches (Obst).

Demnach wären "gefloatete" Adjektive (ebenso wie bei Kontaktstellung vor dem Bezugsnomen) und möglicherweise auch einige der gefloateten Quantoren Adnominale. 59 Die gefloateten Quantoren zählt man aus vielerlei Gründen auch zu den Determinantien bzw. Artikelwörtern, 60 doch diese Diskussion muß hier nicht geführt werden, da mir der Nachweis genügt, daß derartige Strukturen keine Kandidaten für einen sinnvollen Appositionsbegriff darstellen. Die Verhältnisse bei vom Nukleus entfernten Satelliten sind befriedigend mit dem Begriff "(Attribut-/Determinatien-)Floating" erfaBt. Eine Notwendigkeit, adjazente Quantoren, Adjektive etc. wie Schulz/Griesbach (1982: 191, 196, 363) als Attribute und entsprechende Distanzvarianten als Appositionen anzusehen, erscheint aufgrund des (weitgehend) gleichen syntaktischen Verhaltens (zu möglichen Bedeutungsveränderungen $\mathbf{s}$. die spezialliteratur) entbehrlich.

\subsection{Adverbial-Komplexe in Vorfeld}

Die syntaktische Wertung von vorfeldbesetzenden Mustern (a-Varianten), die nicht selten in Beziehung $z u$ getrennt permutierbaren Adverbialen gleicher Subklasse (in den Beispielen handelt es sich jeweils um zwei Lokaladverbiale) gesetzt werden (b-Varianten), ist kontrovers.

59 Zum Quantorenfloating s. Link (1974), Vater (1981) und Kniffka (1986).

60 Hierzu die entsprechenden Aufsătze in Vater (1986a). 
(7.63a) Oben im Schrank stank es.

(7.63b) Es stank oben furchtbar im Schrank.

Oben stank es im Schrank.

(7.64a) Im Stadion auf der Tribüne stank es.

(7.64b) Es stank im Stadion furchtbar auf der Tribüne.

Im Stadion stank es auf der Tribüne.

Als Appositionskonstruktionen werden sie von Steinitz (1969: $47 \mathrm{ff}$. ) und Droop (1977: $40 \mathrm{ff.}$ ) behandelt; Heidolph u.a. (1981: 451) und Eisenberg (1986: 196) räumen diese Möglichkeit neben der auch in Frage kommenden Einstufung als Attribut ein.

Als Attributkonstruktion werden derartige Muster von Helbig/Buscha (1980: 537), Jung/Starke (1982: 108) und Schulz/Griesbach (1982: 361) eingestuft. In diesem Sinn darf man wohl auch Wunderlich (1984) verstehen, der beide (!) oben erwähnten Typen - er versteht Adverbien als "intransitive" Prápositionen (vgl. ebd. $82 \mathrm{ff.}$ - - innerhalb einer komplexen Präpositionalphrase behandelt; somit bilden für ihn beide Konstruktionsteile (zumindest bei gemeinsamer Vorfeldstellung) eine Konstituente!

Von "zusammengerückten Satzgliedern" (mit u.U. daraus resultierenden Eigenheiten der Konstruktion) sprechen Brinker (1972: v.a. 130 ff.), van de Velde (1978: $134 \mathrm{f.}$ ) und Lehmus (1983: $147 \mathrm{ff.}$ ). Clément/Thümmel (1975: $173 \mathrm{ff.;}$ Zitat S. 182) sprechen von "Lokalisationskomplexen", in denen "(...) mehrere lokalisationspartikeln und lokalisationsnominale beliebig miteinander wechselnd aufeinander folgen können".

Altmann (1981: 285 ff. Zitat 285 f., Unterstreichung von w.S.) entwickelt die Problematik folgenderweise:

"Wie eben erwähnt, sind die Beziehungen adverbialer PPn untereinander denkbar vielschichtig und variabel. Das Hauptproblem der Untersuchung ist, daB zwischen attributiver Beziehung und bloser Nebenordnung i.d.R. nur ein geringfügiger Bedeutungsunterschied festzustellen ist $(\ldots)$. Bei gemeinsamer Vorfeldposition von zwei adverbialen PPn kann man gewöhnlich annehmen, daB die zweite PP Attribut zur ersten ist".

Hier liegt ein interessantes, nuancenreiches Muster vor und ich mus bedauern, dieses Muster nicht ausführlicher erörtern zu können. Für meine zwecke genügt die Darlegung, daß diese Muster keine sinnvollen Kandidaten für die Annahme einer "Appositionskonstruktion" sind und am ehesten den attributiven Konstruktionen zugeordnet werden können (ohne daß dies für alle varianten damit schon "bewiesen" ist). Doppelte Vorfeldbesetzung liegt bei den Mustern (7.63/7.64) nicht vor, man vgl. dazu Kap. 9.

Ich möchte vier Muster auswählen, deren semantische Interpretation(en) die über das Konzept von räumlichen (zeitlichen) Bezirken und deren Inklusions-bzw. Spezifikationsbeziehungen erfaBt werden - und deren syntaktische Charakteristika, vor allem hinsichtlich der "Attributtests", im folgenden vor- 
geführt werden. 61 Ich beschränke mich auf "lokale" Beispiele, "temporale" (PKT, DUR, FREQ) wären bei diesem Muster z.T. auch möglich.

Unter den a-Beispielen wird neben dem jeweiligen lokalen Muster (Sperrung zeigt den Hauptakzent), der Anwendung einer Frage nach der ganzen Gruppe 62 und der semantischen Erläuterung (wenn möglich) ein ähnlich gelagerter temporaler Fall angegeben. In den b-Beispielen wird geprüt, ob in attributtypischer Weise Gliedteilfragen, Negationen und Gradpartikeln anwendbar sind. Unter den cBeispielen wird geprūft, inwiefern Herausstellungen (Links-, Rechtsversetzung: Freies Thema; Nachtrag) den ganzen Komplex bzw. die Konstruktionsteile (bei Rechtsversetzung, Nachtrag) betreffen. Schlieblich wird noch geprüf, ob gewisse Rechtsversetzungs- bzw. Nachtragsvarianten stellvertretend möglich wären und ggf. ob Distanzstellung die Konstruktionssemantik veränderte. Zuerst werden Muster mit Akzentuierung des ersten Bestandteils $(7.65,7.66)$, dann jene mit Akzent auf dem zweiten $(7.67,7.68)$ getestet:

(7.65a) O ben im Schrank/ Dort/ Da stinkt es.

Wo stinkt es? 0 ben im Schrank.

'im oberen Teil des Schranks'. 63

'im Raumbezirk "Schrank" im Unterbezirk "oben"'.

$D$ a mals im Sommer stank es. 64

'im Zeitbezirk "Sommer" im Unterbezirk "damals"'.

(7.65b) Wo im Schrank stinkt es? O ben (im Schrank).

Nicht o ben im Schrank stinkt es, sondern u nten im Schrank.

/sogar o ben im schrank stinkt es (, nicht nur u nten)/.

(7.65c) 0 ben im Schrank, da stinkt es.

Apropos o ben im Schrank! Es stinkt da fürchterlich.

Es stinkt dort, ich meine o ben im Schrank.

*Es stinkt o ben, ich meine im Schrank.

Es stinkt, und zwar o ben im Schrank.

* Es stinkt o ben, und zwar im Schrank.

(7.65d) *O ben, (ich meine) im Schrank, stinkt es.

$\star 0$ ben, (und zwar genauer) im Schrank, stinkt es.

61 so kann z.B. innerhalb eines (größeren, vageren) Raum- oder Zeitbezirks ein (kleinerer, präziserer) "Unterbezirk" (Raum- bzw. Zeitpunkt o.ă.) angegeben werden, teilweise mit allmählicher Annäherung:

(F7.8) Bei uns im Keller hinten unter dem Hängeregal am Boden ist ein Weinfleck.

(m.9) Drei Jahre später am 1. Juli am Morgen kurz vor Anbruch des Tages merkten sie das.

62 Was sich zusammen erfragen läßt, ist (wahrscheinlich) eine Konstituente.

63 Auch die Phrase im Schrank o ben kann so interpretiert werden! Sie ist ambig, die andere, typisch attributive Lesart ist 'im Schrank, der sich oben befindet'.

64 Ein ambiges Beispiel: Es könnte auch als 'im Zeitbezirk "damals" im Unterbezirk "Sammer"' gelesen werden. 
(7.65e) ?O ben stinkt es im Schrank.

Im Schrank stinkt es o ben.

In den mit "*" markierten Sätzen kommt eine andere semantische Interpretation zum Ausdruck, nämlich die von (7.67a).

(7.66a) Im St a dion auf der Tribüne/ Dort/ Da stinkt es.

Wo stinkt es? Im St a dion auf der Tribüne.

'Im Raumbezirk "Stadion" im Unterbezirk "Tribüne"' .

Am 1. J u li im Sommer stank es.

'im Zeitbezirk "Sommer" im Unterbezirk "1. Juli"'.

(7.66b) Wo auf der Tribüne stinkt es?

?? Im St a dion (auf der Tribüne).65

(?) Nicht im st a dion auf der Tribüne stinkt es, sondern in der H a lle auf der Tribüne.

(?)/sogar im st a dion auf der tribüne stinkt es ( $\mathrm{f}++$ nicht nur in der $h$ a lle auf der tribüne $\mathrm{f}++/$.

(7.66c) (?) Im St a dion auf der Tribüne, da stinkt es.

(?) Apropos im St a dion auf der Tribüne! Es stinkt da entsetzlich.

(?) Es stinkt dort, ich meine im st a dion auf der Tribüne.

*Es stinkt im st a dion, ich meine auf der Tribüne.

(?) Es stinkt, und zwar im st a dion auf der Tribüne.

Es stinkt im St a dion, und zwar auf der Tribüne.

(7.66d) *Im St a dion, (ich meine) auf der Tribüne, stinkt es.

(?) Im St a dion, (und zwar genauer) auf der Tribüne, stinkt es.

Dieses Muster mutet bei mehreren seiner Variationen nicht voll akzeptabel an, z.T. führen die Abwandlungen zur Inakzeptabilität. Dies liegt m.E. an der Konstruktionssemantik: Wenn man in der Abfolge 'gröBerer Raumbezirk - kleinerer Unterbezirk' den gröBeren Bezirk intonatorisch hervorhebt, dann nur deshalb, weil man einen Kontrast $z$ u einem anderen Grobbezirk (z.B. in der $H$ a lle auf der Tribüne) erzielen will. Eine Spezifikationslesart wie z.B. in im stadion auf der Trib ü ne (mit Hervorhebung des 'Unterbezirks') liest sich leichter. Die Rechtsversetzungslesarten geben nicht die semantik der Fügung wieder.

(7.67a) Oben im Schr a nk/ Dort/ Da stinkt es.

Wo stinkt es? Oben im Schr a nk.

'im Raumbezirk "oben" ("über uns") im Unterbezirk "Schrank"' Damals im $S$ o mmer stank es.

'im Zeitbezirk "hinter uns/ Vergangenheit" im Unterbezirk "Sommer"' .

(7.67b) Wo ((?)oben) stinkt es (oben)? (Oben) Im Schr a nk.

Nicht oben im Schr a nk stinkt es, sondern oben in der $\operatorname{Tr} u$ he. /sogar oben im schr a nk stinkt es (f++ nicht nur oben im $z$ i mmer $\mathrm{f}++/$.

65 Man erwartet hier die Angabe eines Unterbezirks, z.B. Bei den St $u$ fen/ Hinten (auf der Tribüne). 
(7.67c) Oben im Schr a nk, da stinkt es.

Apropos oben im Schr a nk! Stinkt es da etwa?

Es stinkt dort, ich meine oben im Schr a nk.

*Es stinkt im Schr a nk, ich meine oben.

Es stinkt, und zwar oben im Schr a nk.

*Es stinkt im Schr a nk, und zwar oben. 66

(7.67d) Oben, (ich meine) im Schr a nk, stinkt es.

oben, (und zwar genauer) im Schr a nk, stinkt es.

Die Rechtsversetzung sowie der Nachtrag von oben geben nicht die Konstruktionssemantik wieder.

(7.68a) Im Stadion auf der Trib ü ne/ Da/ Dort stinkt es.

Wo stinkt es? (Im Stadion) auf der Trib ü ne.

'Im Raumbezirk "Stadion" im Unterbezirk "Tribüne"'

Im Sommer am 1. $J$ u $1 i$ stank es.

'im Zeitbezirk "Sommer" im Unterbezirk "1. Juli"'.

(7.68b) Wo im stadion stinkt es? (Im stadion) auf der Trib ü ne.

Nicht im Stadion auf der Trib ü ne stinkt es, sondern im stadion auf dem $R$ a sen.

lauch im stadion auf der trib ü ne stinkt es (f++) nicht nur im stadion auf dem $r$ a sen $\mathrm{ft+l}$.

(7.68c) Im Stadion auf der Trib ü ne, da stinkt es.

Apropos im Stadion auf der Trib ü ne! Stinkt es da etwa?

Es stinkt dort, ich meine im Stadion auf der Trib ü ne.

*Es stinkt auf der Trib ü ne, ich meine im stadion.

Es stinkt, und zwar im stadion auf der Trib ü ne.

*Es stinkt auf der Trib ü ne, und zwar im Stadion.

(7.68d) *Im Stadion, (ich meine) auf der Trib ü ne, stinkt es.

Im Stadion, (und zwar genauer) auf der Tribüne, stinkt es.

Rechtsversetzung und Nachtrag von im Stadion geben nicht die

Konstruktionsbedeutung wieder, auch nicht die Rechtsversetzung von auf der Trib ü ne.

Abschließend verdeutlicht eine Obersicht, daß sich die erörterten Konstellationen in der Tendenz attributartig verhalten ("lock" = "locker"):

GT-Prage KNDG GPn LV FT RVg RVt NTg NTt RVlock NTlock

0 ben im $S$.

O. im Schr a nk

$(+)$

Im St a dion a. d. T.

Im S. a. d. Trib ü ne

?

$+$

$+\quad++$

$+\quad+$

$+++-+-t+$

$+\quad+++t-t ?$ ?

66 Hier Lesartveränderung (= 'im oberen Teil des Schrankes'). 
Neben der gesamten Erfragbarkeit und Ersetzbarkeit bestehen alle Konstruktionen (im großen und ganzen) die drei "Attributtests" Gliedteilfrage, Kontrastnegation und Gradpartikelanwendung. 67 Auch die Konstruktionssemantik widerspricht nicht einer solchen Einstufung: Bei "typischer Dingattribution" (z.B. im Stadion auf dem Berg) wird aus einer Entitätenklasse durch Hinzufügung restringierender Eigenschaften etc. eine Subklasse oder eine Einzelentität identifiziert; bei dem vorliegenden Typen wird ein Ort (ein Zeitpunkt) schrittweise identifiziert, indem zuerst ein "Grobraum", dann ein präziserer "Unterraum" angegeben wird. Die Funktion "Identifikation durch Einschränkung (bzw. Approximation)" gilt mithin in beiden Fällen.

Die "eigenartigste" Konstruktion ist dabei im st a dion auf der Tribüne. Die akzeptablen Herausstellungen der Gesamtkomplexe (und natürlich die gemeinsame Vorfeldbesetzung) unterstreichen den "konstituentenhaften" Status der Konstellationen und somit deren Attributhaftigkeit. Aus den referierten grammatischen Eigenschaften wird auch verständlich, daß Wunderlich (1984) derartige Muster als komplexe PPn behandelte. Wenn man davon ausgeht, daB ein Verb wie z.B. Wohnen eine thematische Rolle (LOKATIV) nur einmal an seine Aktanten vergibt, dann sollte man eher von "Mehrfachbesetzung" einer syntaktischen Funktion (mit interner strukturierung) sprechen als von zwei satzgliedern resp. "doppelter Vorfeldbesetzung":

\section{(7.69) Oben auf dem Berg (in Audorf) wohnt Pia.}

Die Einstufung als attributive Konstruktionen von oben im Schrank/ im Stadion auf der Tribüne etc. ist augenscheinlich auch durch folgende Beispiele gerechtfertigt, bei denen der "attributverdächtige" Komplex innerhalb einer komplexen adnominalen Attribution auftritt: 68

(7.70a) Der Fleck oben im Schrank von vorgestern muB weg.

(7.70b) Der Brand im Stadion auf der Tribüne von vorgestern war harmlos. Man vergleiche hierzu die "lockeren" Entsprechungen:

(7.71a) *Der Fleck oben, im Schrank, von vorgestern muB weg.

(?) Der Fleck oben von vorgestern, im Schrank, muß weg.

(7.71b) *Der Brand im Stadion, auf der Tribüne, von vorgestern war harmlos. (?) Der Brand im Stadion von vorgestern, auf der Tribüne, war harmlos.

Völlig akzeptabel sind nur die "engen" Varianten des komplexen lokalen Präpositionalattributs. Die "lockeren" Strukturen sind nicht in die NP einfügbar; als einschaltungsmarkierte Zusätze folgen sie einer NP stets nach.

67 Eine Eigenschaft haben sie mit attributiven Fügungen nicht gemein (s. Droop 1977: 59 f.): die Relativsatzparaphrase. Vgl. auch Heidolph u.a. (1981: 287 ff., 296 ff.; insb. 835-838), die diverse Attributtypen aus zugrundeliegenden Relativsätzen ableiten:

(F7.10) Im Stadion auf dem Berg (das sich auf dem Berg befindet) stank es.

(F7.11) Im Stadion auf der Tribüne (*das sich auf der Tribüne befindet) stank es. 
Trotz relativ deutlicher Evidenz dafür, daß die eben behandelten Konstrukte zu den attributiven strukturen gezăhlt werden sollten, sei darauf hingewiesen, das das Muster "juxtaponierte Adverbiale gleicher Subklasse" noch nicht als ausreichend erforscht gelten kann; hier wäre noch einiges an Arbeit zu tun.

\subsection{Translativphrasen nit wie}

"Appositionsverdăchtige" Konstruktionen (7.72) bestehen aus einer Voll-NP (Basis) und einer mit wie angefügten Voll-NP. ${ }^{69}$ Anders verhält es sich beim Translativ als, das auch N2- bzw. NO-Komplemente zuläBt (näheres in 7.B.).

(7.72a) Ein Auto wie der VW Käfer verkauft sich immer.

(7.72b) Ein Arzt wie Kuno hätte das wissen müssen.

(7.72c) Ein helles Lachen wie der Schrei einer Möwe erklang.

(7.72d) Kategorien wie Laster und Dekadenz sind Ansichtssache.

MaBgeblich für die Einstufung als Apposition ist insbesondere die Kasuskongruenz der wie-Komplement-NP mit der Bezugs-NP. 70 Dabei wird in Heidolph u.a.

(1981: 294) darauf hingewiesen, daB appositionstypische Grundstrukturen - parenthetische Kopulasătze und nicht-restriktive Relativsätze - nicht plausibel seien, sondern nur restriktive Relativsätze:

(7.73) Für diese Aufgabe brauchen wir einen Mitarbeiter wie dich/, der so ist, wie du bist/ *er ist (*übrigens) so wie du.

Dieses Faktum spricht eher gegen die Wertung der wie-Phrase als Apposition und für eine Einordnung als attributives Gliedteil. Auch weitere Merkmale erweisen die fraglichen Phrasen als Attribute: (a) Gliedteilfragen sind möglich (z.B. Was für ein Auto verkauft sich immer? Ein Auto wie der VW Käfer.); (b) Negations- und Gradpartikeln operieren auch über die wie-Phrase (z.B. Sogar Kategorien wie Laster und Dekad e nz sind Ansichtssache, nicht nur Kategorien wie Schuld und $S$ ü hnel; (c) semantisch fungieren wie-Phrasen als restriktive Modifikatoren ihrer nominalen Basen (daher auch die Möglichkeit der Gliedteilfrage; "Appositive" sind jedoch nie restriktiv).

Wie-Phrasen können nicht um appositionstypische zusätze erweitert werden (7.74); zudem ist sprecherdivergenz ausgeschlossen (7.75):

(7.74a) Ein Arzt wie *übrigens/*vermutlich/*sogar Kuno hätte das wissen müssen.

(7.74b) Ein helles Lachen wie *übrigens/ *wegen des Klangs/ *sogar der Schrei einer Möwe erklang.

(7.75) ^Pia sagte: "Ein Arzt" wie Kuno "hätte das wissen müssen".

69 Mehr zu den Funktionen von wie-Phrasen: Engel (1982: 185 f.); Schulz/Griesbach (1982: 391 f.); vor allem Helbig (1984a); Fisenberg (1986: 313, 323 ff.).

70 s. z.B. Heidolph u.a. (1981: 294), Schulz/Griesbach (1982: 362 f.) und Duden (1984: 593). 
Es gibt folglich keine zwingenden Gründe, Translativphrasen dieser Art den appositiven strukturen - und nicht den attributiven, wo sie offensichtlich hingehören - zuzuordnen. Das Kriterium der Kasuskongruenz allein, auch in Verbindung mit (wohl eher zweifelhaften) Annahmen über Referenzidentität (Kuno und ein Arzt bezögen sich auf den gleichen Referenten), reicht als Appositionskriterium nicht aus.

\section{A. DER NICHT-RESTRIRTIVE RELATIVSATZ}

Relativsätze (darunter verstehe ich durch Relativpronomen bzw. -adverbien eingeleitete Verbendsătze) lassen sich in Anlehnung an Bergmann (1985: 64) wie folgt einteilen (zur Relativsatzklassifikation auch Lehmann (1984)):

\section{determinativ resp. restriktiv}

A. ohne Bezugsglied (GLIEDSATZ)
B. mit Bezugs- glied (GLIEDTEIL)

\section{nicht-restriktiv/explikativ/appositiv}

D. auf einen Satz bezogen. (GLIEDSATZ?).

- A: Wer wagt, gewinnt.

- B: Diejenigen Bäume, die krank sind, werden gefällt.

- C: Die Bäume, die heute bekanntlich von Kablschlag und saurem Regen bedroht sind, hätten es ohne den Menschen besser.

- D: Viele Bäume sind bereits erkrankt, was niemanden mehr wundern sollte.

Für unsere Analysen sind Typ B und C (mit nominalem Bezugsausdruck) von Belang. Der Typ A zeigt Satzgliedverhalten (fungiert als subjekt) und wird bisweilen auch "freier Relativsatz" genannt. 71 Den Typ D, Z.T. als "weiterführender Nebensatz"72 oder "weiterführender Relativsatz"73 bezeichnet, schlug man m.W. bislang nicht den Appositionen zu. ${ }^{74}$ Man könnte ihn wegen seines Bezugs auf einen Verbzweit-Satz auch "Satzrelativsatz" nennen (vgl. Kap. 6.A.).

71 so z.B. bei Eisenberg (1986: 220).

72 Z.B. Helbig/Buscha (1980: 574, 596) und Heidolph u.a. (1981: 827).

73 S. DUDEN (1984: 672, 676).

74 Jedenfalls nicht in Arbeiten zum Deutschen. In der englischen Grammatik von Quirk u.a. (1976: 648) findet sich allerdings zu folgendem Beispiel ( 7.12 ; 7.13 meine tbersetzung)

(F7.12) He sold his shop, which surprised me.

(17.13) Er verkaufte seinen Laden, was mich überraschte. die Erlauterung: "(...) the non-restrictive clause with the previous clause as its antecedent is then said to be appositive to that previous clause". 
Die Relativsätze des Typs $C$ und $D$ werden traditionell als adnominale Attribute klassifiziert, wobei nicht auf syntaktischer, sondern auf semantischer Ebene zwischen restriktiven (7.76a) und nicht-restriktiven bzw. appositiven (7.76b) Relativsatzen unterschieden wird. Es ist allerdings auf (mindestens) einen "Zwischentyp" aufmerksam zu machen, den Ebert (1973: 2 ff.) als "reidentifying" bezeichnet ( $7.76 \mathrm{c})$. Dieser ebenfalls nicht-restriktive Typ wird im folgenden nicht weiter diskutiert. Er läßt sich meiner Meinung nach u.a. durch den einleitenden Zusatz du weiBt schon vom "appositiven" Rels unterscheiden, in dem fakultativ die "Abtöner" übrigens, bekanntlich, ja erscheinen. 75 Daß beide "Zusätze" miteinander unverträglich sind (was auf die Inkompatibilität der pragmatischen Funktionen hindeutet), zeigt (7.76d): 76

(7.76a) Manche Hunde, die (*übrigens) bellen, beiBen nicht.

(7.76b) Diese Hunde, die übrigens laut bellen, beiBen nicht.

(7.76c) A: Hast du von dem schlimmen Unfall, (du weiBt schon,) der sich gegen Mittag ereignet hat, gehört?

B: Ja.

(7.76d) A: Hast du von dem schlimmen Unfall, (*du weiBt schon,) der sich übrigens gegen Mittag ereignet hat, gehört?

Der nicht-restriktive Relativsatz wird in der einschlägigen Literatur nur selten direkt der syntaktischen Funktion APPOSITION zugerechnet. In den von mir in Kap. 2 ausgewerteten Arbeiten nehmen eine solche Einordnung nur Raabe (1979: $290 \mathrm{f.}$ ) und Seiler (1960: $35 \mathrm{f.}$ ) vor. 77 Doch weist z.B. auch Engel (1988: 293) auf eine "Nähe" von nicht-restriktiven Relativsätzen, die er sie als ATTRIBUTE einstuft, und (lockeren) Appositionen hin (Beispiele ebd. 293): "Sie [die nicht-restriktiven Rels; W.S.] spielen eine ähnliche Rolle wie die Appositionen (...), mit denen sie auch kommutieren (...)".

(7.77) Mein Urgrobvater, der Chemiker war, lebte (...) in Geldsorgen.

(7.78) Mein Urgrobvater, von Beruf Chemiker, (...).

Restriktive Relativsätze schlieBen sich an NPn - genauer: an Nomina - an, die allein noch keine Referenten identifizieren können; typischerweise sind dies Gattungsnamen (CN) mit indefiniten oder quantifizierenden Determinantien (ein, jeder, alle, einige, kein usf.) oder indefinite bzw. quantifizierende Pronomina. Appositive Rels treten i.d.R. an (re-)identifizierende NPn (also nicht an Nomina; vgl. Ausführungen weiter unten) wie beispielsweise Eigennamen, Personal- oder Demonstrativpronomina bzw. CN mit demonstrativen Determinantien usf.

75 Zu weiteren Unterscheidungskriterien s. Ebert (1973).

76 Für den Hinweis auf den "re-identifying-Typ" danke ich Berrn Shin Tanaka. Auch Hans Altmann weist im Korreferat zu meiner Dissertation auf einen TYp zwischen restriktivem und appositivem Relativsatz hin: den charakterisierenden Relativsatz, der semantisch nicht-restriktiv ist und syntaktisch als Attribut fungiert.

77 Für das Englische rechnet z.B. Schapiro (1977: 4) die nicht-restriktiven Relativsätze zu den Appositionen; für das Französische z.B. Dupont (1985: 332). 
Wăhrend die restriktiven Rels sich auf $\mathrm{CN}$-wertige Phrasen (X-Bar-theoretisch gesprochen: auf die $\mathrm{N}^{2}$-Ebene) beziehen, knüpfen appositive nur an NP-wertige Phrasen (N3-Ebene) an. Man vgl. hierzu Lehmann (1984: 270):

"Der appositive RS bezieht sich auf ein vollständig determiniertes NS [Nominalsyntagma; entspricht NP; W.S.], das er nicht mehr restringieren kann. Er fungiert daher wie eine Parenthese, in der etwas zusätzliches über das Bezugs-NS gesagt wird."

Daher befinde sich der appositive Rels - er ist Ko-Konstituente der determinierten NP - auch nicht im Skopus eines Determinators (ebd. $261 \mathrm{ff.}$ ), der restriktive hingegen schon; demnach ist er Ko-Konstituente des Basis-Nomens.

Die Unterscheidung restriktiver und appositiver Relativsätze nimmt also darauf Bezug, daß im ersten Fall "die NGr [Nominalgruppe; W.S.] mit Relativsatz extensional eingeschränkt ist gegenüber der ohne Relativsatz", wogegen im zweiten Fall "der Relativsatz nichts an der Extension der NGr, in der er enthalten ist, ändert" (Zitate Eisenberg 1986: 217). Man bemerkt eine submengenbildung in (7.79); der attributiv fungierende Relativsatz kann mittels Gliedteilfrage erfragt (d-Fälle), kontrastiv negiert (b-Fälle) und von Gradpartikeln affiziert werden. Im Falle der "appositiven" Beispiele in (7.80) besteht keine dieser drei Möglichkeiten, weil der Rels nicht submengenbildend wirkt. Kategorialgrammatisch wären restriktive Relativsätze somit als CN/CN (bilden komplexe, extensional eingeschränktere Gattungsnamen) zu beschreiben, "appositive" hingegen als NP/NP (operieren ohne extensional einzuschränken auf NPn).

Auf der pragmatischen Beschreibungsebene ist festzustellen, daß "appositive" Rels im Gegensatz zu restriktiven RelS Ausdrücke enthalten können, die Sprechakte indizieren (e-Beispiele).

(7.79a) Die Trinker, die dem Spiritusgenuß frönen, leben kürzer als die Trinker, die dem MalzbiergenuB frönen.

(7.79b) Pia verachtet nicht die Trinker, die dem M a lzbiergenuß frónen, sondern die Trinker, die dem $S p$ i ritusgenus frönen.

(7.79c) Pia verachtet sogar die Trinker, die dem M a lzbiergenuß frönen, nicht nur die Trinker, die dem $S p$ i ritusgenub frönen.

(7.79d) Was fü/ Welche Trinker leben kürzer als die Trinker, die dem Malzbiergenus frönen? Die Trinker, die dem Spiritusgenuß frönen.

(7.79e) *Dasjenige/ *Jedes Hemd, das ich hiermit $z u$ waschen verspreche, starrt vor Dreck.

(7.80a) Die Kobras, die bekanntlich sehr $g$ i ftig sind, werden von den Einheimischen gefürchtet.

(7.80b) *Nicht die Kobras, die bekanntlich sehr $g$ i ftig sind, werden von den Einheimischen gefürchtet, sondern die Kobras, die (bekanntlich) sehr aggress $i v$ sind.

(7.80c) *Auch die Kobras, die bekanntlich sehr $g$ i ftig sind, werden von den Einheimischen gefürchtet, nicht nur die Kobras, die (bekanntlich) sehr aggress $i v$ sind. 
(7.80d) Was für/ Welche Kobras werden von den Einheimischen gefürchtet? *Die Kobras, die bekanntlich sehr $g$ i ftig sind.

(7.80e) Mein Hemd, das ich hiermit $z u$ waschen verspreche, start vor Dreck. Im Deutschen werden diese beiden Relativsatztypen nicht graphematisch auseinandergehalten; im Englischen und im Französischen wird der nicht-restriktive Typ durch Kommata abgetrennt: 78

(7.81a) The lady who usually types my letters has fallen $i 11$. Miss Evans, who usually types my letters, has fallen ill.

(7.81b) Les enfants qui avaient fini de déjeuner quittèrent la salle. Les enfants, qui avaient fini de déjeuner, quittèrent la salle. Der appositive Relativsatz, in dem sich manchmal Modalpartikeln und/oder Satzadverbien befinden, die ihn als zusätzliche (beiläufige), nicht-identifizierende Hinzufügung kennzeichnen, kann im Gegensatz zum restriktiven auch in Rundklammern oder Gedankenstriche gesetzt werden:

(7.82a) Pia hat jenen Kerl (der ihr übrigens immer nachstellt) angezeigt. Pia hat jenen Kerl - der ihr übrigens immer nachstellt - angezeigt.

(7.82b) Pia hat jeden Kerl, der ihr nachstellt, angezeigt.

??Pia hat jeden Kerl (der ihr nachstellte) angezeigt.

* Pia hat jeden Kerl - der ibr nachstellte - angezeigt.

In der gesprochenen Sprache entspricht dieser Markierung ein Intonationsunterschied: Der nicht-restriktive Typ wird von deutlichen Pausen umrahmt und etwas "zurückgenommen" artikuliert. Diese parenthetische Intonation gibt es auch im Deutschen. 79 Der nicht-restriktive Relativsatz hat einen vom Bezugssatz unabhăngigen eigenen Satzakzent (Rhemaakzent). Sowohl die "Bezugs-NP" als auch der Satz selbst könnten einen Rhemaakzent (Starkakzent) tragen. Der nicht-restriktive Relativsatz besitzt eine eigene Thema-Rhema-Gliederung. 80

Der restriktive Relativsatz hingegen weist keine deutlichen Pausen um sich herum auf, er bildet keine eigenständige Tongruppe und ein auf dem Satz lagernder Akzent ist insofern vom Bezugselement abhängig, als er das Glied des Relativsatzes hervorhebt, welches das entscheidende spezifizierende Merkmal

78 Englische Beispiele aus: Sutton/Beilhardt (1977: 88), französische aus: Klein/Strohmeyer (1980: 189).

79 Z.B. Helbig/Buscha (1980: 597): "Der nicht-restriktive Attributsatz hat Parenthese-Intonation (...)". Zur Intonation vgl. auch Lötscher (1972: 53 ff.), Pheby (1975: 110 f.), Jackendoff (1977: 172), Heidolph u.a. (1981: 860 f.) und Lehmann (1984: 263).

80 s. Lötscher (1972: 55):

"Restrictive clauses behave in stress and some word order rules and above all semantically as integral parts of the overall thema-rhema structure of a sentence. Nonrestrictive relative clauses on the other hand seem to be totally seperated from such a structure and rather to take their own thema-rhema within their own sentence boundaries. This indicates that whereas restrictive relative clauses seem to have the status of embedded sentences (...), nonrestrictive relative clauses arise through some kind of sentence conjunction". 
zur Identifikation des NP-Referenten nennt (vgl. 7.83a, b).81 (Die skizzierten Merkmale treten in der gesprochenen sprache allerdings nicht immer mit voller Deutlichkeit auf.)

(7.83a) Kuno liebt die Frau, die $n$ e ben der Säule steht (, und nicht etwa die, die $v$ or der Säule steht).

(7.83b) Pia liebt die Frau, die neben der $S$ ä ule steht (, und nicht etwa die, die neben der $T$ ü $r$ steht).

Zur Relativsatztopologie bemerkt Lötscher (1972: 54):

"On the whole, it is only possible to extrapose a nonrestrictive relative clause across a final verbal part. (...) But any movement across longer constituents is impossible".

Für das Deutsche ausbuchstabiert heiBt dies: Wăhrend der restriktive Rels sowohl adjazent zur Basis stehen als auch (sogar über mehrere satzglieder hinweg) extraponiert werden kann, ist der "appositive" auf die Adjazenz beschränkt; nur wenn sich die Basis direkt vor dem KlammerschluB befindet, darf extraponiert werden. Die eingeschränkteren Stellungsoptionen des appositiven Rels lieben sich vergleichen mit den zumeist stark eingeschränkten Möglichkeiten bei Appositionen bzw. Zusätzen (vgl. Kap. 6; Kap. 12, 15). Eine genauere Analyse zur Rels-Topologie muB leider unterbleiben. Abschließend noch ein markantes Beispiel für einen appositiven Rels (Basis-NP: unserer...Intelligenz):

(7.84) Es bedurfte drastischerer Signale, um uns aus dem (...) Traum von der (...) überlegenen Kraft unserer wissenschaftlich-technischen Intelligenz aufschrecken zu lassen. (Der wir andererseits nun aber nicht (...) gleich wieder abschwören dürfen $(\ldots).)^{82}$

Hinsichtlich der Frage, ob restriktive und appositive Relativsätze in zwei syntaktisch differenzierbare Funktionsklassen fallen, gehen die Meinungen auseinander. In v. Gestel (1986: 109) wird ein Unterschied - im Rahmen einer XBar-Syntax - verneint. In Lötscher (1972: 50) wird erwăhnt, daß " (...) the common opinion is that these two types of relative clauses cannot be distinguished syntactically". Nach Lötscher rechtfertigen jedoch mindestens die intonatorische Verschiedenheit und die unterschiedliche Extraponierbarkeit einen syntaktischen Unterschied (ebd. $53 \mathrm{ff.,} v \mathrm{gl}$. auch Lótschers Fazit $55 \mathrm{ff.}$ ). Auch Lehmann (1984: $261 \mathrm{ff.}$ ) arbeitet syntaktische, semantische und pragmatische Unterschiede $z$ wischen beiden Typen heraus. Die Grammatiken verhalten sich nicht einheitlich. Teils weisen sie auf Unterschiede hin, teils bleiben sie unerwähnt. 83

Im Verlauf der bisherigen Erorterungen ist wohl deutlich geworden, das der restriktive Relativsatz das Verhalten eines Attributs an den Tag legt und da-

81 Vgl. Heidolph u.a. (1981: 860).

82 Aus: v. Ditfurth (1988), s. 366. Meine Unterstreichung.

83 Unterschiede z.B. in Helbig/Buscha (1980: 596 f.); keine Angaben z.B. in Jung/Starke (1982: 35 f.; 1099). 
her mit Erscheinungen wie Genitiv- und Prăpositionalattribut zusammengefaßt werden kann. Man vgl. nochmals die markantesten Unterschiede (und Kap. 7):

restriktiver Rels

Bezug:

Kategorialgramm. :

Schreibzeichen:

Pausen vor/hinter:

Akzente:

Gliedteilfrage:

Kontrastnegation:

GP-Affizierung:

Topologie:

Illokution:

Funktion:

\author{
$\mathrm{CN}$-wertige Phrasen $\left(\mathrm{N}^{2}\right)$ \\ $\mathrm{CN} / \mathrm{CN}$ \\ nur Kommata \\ keine (deutlichen) \\ nur Rels, nicht Basis \\ welch-/ was für ein-? \\ möglich \\ möglich \\ adjazent, Extraposition \\ über satzglieder hinweg \\ nein (keine Indikatoren) \\ Identifikation, Mengen- \\ subsektion
}

\section{appositiver Rels}

NP-wertige Phrasen $\left(N^{3}\right)$

NP/NP

auch Klammern, Gedankenstriche deutliche Pausen 84

Basis und Rels

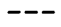

adjazent, über Klammerschluß extraponierbar

ja (Indikatoren möglich)

Ins-Gedächtnis-Rufen v. Hintergrundwissen, Charakterisierung

Weil sich der appositive Relativsatz in seinem Verhalten mit den HOSPITANTEN berührt, mit den "Appositionen", evtl. sogar mit den "Parenthesen", erscheint mir dessen Zuordnung zur attributiven Funktion sehr fraglich. ${ }^{85}$ Die Attributwertung erschüttert auch ein Test von McCawley (1982: 96), der zeigt, daB sich appositive Relativsätze (gleich lockeren Appositionen und auch Schaltsätzen) so verhalten, als ob sie kein VP-Bestandteil wären. Wenn man die VP (mittels des Pro-VP-Elements das) anaphorisch aufnimmt, werden augenscheinlich nur Verb und Akkusativobjekt (evtl. mit restriktivem Relativsatz; $7.85 \mathrm{c}$ ) wieder aufgenommen, aber keine Parenthesen $(7.85 \mathrm{~b})$ bzw. appositive Relativsătze (m.a.W.: keine Hospitanten):

(7.85a) Pia hat die Gäste, die sich übrigens langweilten, beobachtet, und Kuno tat das auch (, ich meine die Gäste beobachten/ ??die Gäste, die sich übrigens langweilten, beobachten).

(7.85b) Pia hat die Gäste - sie langweilten sich übrigens - beobachtet, und Kuno tat das auch (, ich meine die Gaste beobachten/*ich meine die Gäste - sie langweilten sich übrigens - beobachten).

84 Hier werden nur die intonatorischen Verhältnisse in Satz besprochen, die bei Extraposition sind u.U. komplizierter bzw. anders zu formulieren.

85 Auf die Ahnlichkeit mit Parenthesen wird in Lehmann (1984: 263, 270 ff.) hingewiesen. Für das molische wird die Verwandtschaft appositiver RelS mit Schaltsătzen z.B. von Enonds (1979), McCawley (1982) und - mit kritischen Anmerkungen zu McCawley - Safir (1986) herausgearbeitet. 
(7.85c) Pia hat alle Gäste, die sich langweilten, beobachtet, und Kuno tat das auch (ich meine alle Gäste beobachten, die sich langweilten/ ?? alle Gäste beobachten).

Daher sei nun ein Vergleich von Relativsatz, Apposition und Parenthese angestellt, um zu erfahren, inwiefern sich diese Konstruktionen ähneln bzw. unterscheiden. ${ }^{86}$ Gemeinsam ist allen drei Konstruktionen: (a) graphematische bzw. intonatorische Abtrennung, (b) nicht per Gliedteilfrage erfragbar, (c) weder von Kontrastnegation noch von Gradpartikeln affizierbar und (d) stellung bei Adjazenz am Ende der Bezugs-NP nach Genitiv-, Präpositionalattributen sowie nachgestellten Adpositionen, postponierten GPn oder Konjunktionaladverbien (vgl. Kap. 6, Punkt 3).

\section{Relativsatz, appositiv lockere Apposition Parenthese/Schaltsatz}

Stellung zur Basis: stark adjazent relativ zueinander: RelS/APP; RelS/SCHALI 87 Distanzstellung über Klammerschluß: möglich mehrere Satzglieder: nicht möglich Unformungsbeziehung: evtl. Schaltsatz Sprecherdivergenz: fragwürdig ${ }^{89}$ Rekursivität und Koordinierbarkeit: nein Illokution: illokutionäre Selbstän- keine Illokution digkeit (in Grenzen?) stark adjazent

RelS/APP; APP/SCHALT

beschränkt möglich 88

kaum möglich

Satz mit Kopula sein

möglich

ja sehr variabel

RelS, APP/SCHALT

möglich

unproblematisch

???

möglich

fragwïrdig 90

illokutionsvariabel

86 Ich ziehe hier besonders "elementbezogene" Schaltsätze in Betracht. Z.B.: Pia, sie besitzt einen Kakadu, liebt Tiere. Vgl. dazu den Relativsatz Pia, die einen Kakadu besitzt, liebt Tiere und die Apposition Pia, Besitzerin eines Kakadus, liebt Tiere.

Zu den geprüften Merkmalen vgl. Kap. 6 sorie Kap. 3 und 15.

87 Vgl. auch Kap. 15. Punkt 3. Sonderbedingungen - vgl. auch Teubert (1979: 210) - seien hier ausgeblendet. Bezieht sich beispielsweise das Appositiv auf Bezugsnominal + Relativsatzattribut, dann steht es notwendigerweise am Ende der NP (Der Mohn, der in südlichen Ländern gedeiht, übrigens ein Narkotikum, (...)) etc.

88 Hierzu auch Kap. 6. Punkt 3. Die Beschränkungen resultieren vor allen daraus, daß etliche "Appositive" (besonders in Form von PP, AdjP) bei Distanzposition nach dem Klammerschlub eher als Nachträge, zumindest aber kaum mehr als "Appositive" interpretiert werden.

$89 \mathrm{Vgl.:}$ (F.14) Runo sagte: "Otto", übrigens ein Trinker, "ist gerade auf Entzug".

(П7.15) Kuno sagte: "Otto", er ist übrigens ein Trinker, "ist gerade auf Entzug".

(7.16) ?Kuno sagte: "Otto", der übrigens ein Trinker ist, "ist gerade auf Entzug".

90 Man vergleiche die nachstehenden Beispiele:

(F7.17) Jener Graf, vermutlich uralt, aus Transsylvanien, ein Centlemen, betörte alle Damen.

(F.18) * Jener Graf, der vermutlich uralt war, der aus Transsylvanien stamte, der sich als ein Gentlemen ervies, betörte alle Damen.

(F.19) ? Jener Graf, er war vermutlich uralt, er stammte aus Transsylvanien, er erwies sich als ein Gentlemen, betörte alle Damen. 
Die bisherigen Analysen und die obersichten oben lassen erkennen, daB sich der appositive Relativsatz nicht wie ein PARTIZIPANT, sondern wie ein HOSPITANT verhält. Die "lockeren Appositive" und die nicht-restriktiven Relativsätze differieren in einem Punkt entscheidend:

(7.86a) Pia, die übrigens eine gute Köchin ist, besucht mich morgen. Pia, übrigens eine gute Köchin, besucht mich morgen.

(7.86b) Pia, die übrigens einen Kakadu besitzt, besucht mich morgen. *Pia, übrigens einen Kakadu, besucht mich morgen. 91

Während Relativsätze mit Kopulaverb und lockere Appositive funktional austauschbar sind, ist ein funktionaler Wechsel zwischen Relativsatzen mit Vollverben und lockeren Appositiven unmöglich. Im Gegensatz zum "Appositiv" ist der Relativsatz in seinen Ausdrucksmöglichkeiten vielfältiger (nicht nur Kopulaverben möglich, z.T. eigene Illokution etc.); er ist nicht wie die "Apposition" rekursiv anfügbar und eine sprecherdivergenz kann wahrscheinlich nicht vorliegen.

Vielleicht ist der appositive Relativsatz eher eine besondere Parenthesebzw. Schaltsatzform. Immerhin trägt er im Ggs. zur Apposition u. U. eine eigene Illokution, ein zentrales Definiens für Parenthesen bzw. Einschübe in Kap. 11 und 16. Der appositive Relativsatz unterscheidet sich allerdings durch seine strikte Adjazenz zum Bezugselement deutlich von den Schaltsätzen, die einen Satz relativ frei durchwandern können. Hinsichtlich der Möglichkeit der Extraposition ist der appositive Relativsatz sogar enger als der restriktive an die Bezugs-NP gebunden. Im Vergleich mit einer Parenthese ist ein appositiver Rels nicht nur stellungsmäBig deutlich stärker nukleusgebunden. Auch z.B. hinsichtlich der Pronominalisierungsrelation manifestiert sich ein Unterschied, insofern als Relativpronomina strikt "Genuskongruenz" aufweisen müssen (Kontext: Fasching, Maskenbal1):

(7.87a) Unsere Diva - sie/ "er"/ die/ (?) "der" hat sich wirklich perfekt zurechtgemacht - hatte einen tollen Auftritt.

(7.87b) Unsere Diva - die/ *"der" sich wirklich perfekt zurechtgemacht hat - hatte einen tollen Auftritt.

Eine voll befriedigende grammatische Einordnung des appositiven Relativsatzes (sowie noch möglicher weiterer Muster wie der "re-identifying-Typ") bleibt eine Forschungsaufgabe. Er zahlt m.E. wie der Appositionsprototyp zu den HOSPITANTEN, genauer: zu den NP-ZUSATZEN (Konstituentoiden), wobei die Assoziation an sein Bezugselement noch etwas stărker ausgeprägt ist als beim Appositiv.

Der Schaltsatz mit pronominalem Bezug auf eine NP stellt m.E. einen EINSCHUB dar (vgl. Rap. 11). Er ist in wesentlich geringerem MaBe an die Bezugsphrase "assoziiert" (eigentlich nur durch die "phorische Relation") und in nur geringem Maße in den Trăgersatz integriert, weshalb hier keine syntaktische Relation zwischen beiden Elementen angesetzt wird.

91 Die Kasuskongruenz in Pia, übrigens ein Kakadu, (...) veränderte den Sinn fundamental. 
Zusatz während der uberarbeitung: In dem mir soeben bekannt gewordenen Aufsatz von Thorne (1988; Zitat S. 424) wird für das Englische die Ansicht vertreten, daB "(...) non-restrictive relative clauses are $S$ (in Jackendoff's theory $v^{3}$ ) complements". Nach Jackendoff (1977) sind u.a. auch "parentheticals", also Parenthesen, der V3-Ebene zuzuordnen. Parenthesen sind, wie festgestellt, äuBerst positionsvariabel; appositive Relativsätze (die in meiner Grammatikvorstellung unter die Funktion zUSATZ fallen) assoziieren sich im Deutschen dagegen eng an ihre Basis. Warum wohl? Ich will nicht ausschließen, daB für das Englische eine Hypothese Erfolg verspricht, die einen appositiven Relativsatz direkt dem Satz und nicht der NP (konstitutionell) zuordnet. Für das Deutsche allerdings nehme ich vorläufig diese Möglichkeit nicht an, sondern verweise auf mein Konstituentoid-Verhältnis; zukünftige Analysen sollten diese Fragen zu klären versuchen.

\section{B . TRANSLATIVPHRASEN}

Aufgrund des unklaren Wortartstatus von als und wie erschien es notwendig, neue Termini für diese Ausdrücke zu prägen. "Identifikationstranslativ" ist ein Vorschlag von Heringer (1973: 205), der die gleichsetzende Funktion dieser Partikeln hervorhebt; ein anderer Vorschlag stammt von Kolde (1971: 200): "Subsumtionstranslativ".92 Abraham (1983: 76) bemerkt, nachdem er die Einstufung als Präposition verworfen hat, daß "als (...) sich demnach als lexikalischer Andeuter für ein nachgestelltes, erweiterndes oder einschränkendes Spezifikanz [sic!]" erweise.

Eine ausführliche Diskussion über den Wortartenstatus kann hier nicht geführt werden; sie ist auch nicht notwendig. Einige Argumente zur möglichen Einordnung müssen ausreichen. Für Präposition spricht, daß als von bestimmten Verben wie eine Präposition bei Präpositionalobjekten regiert wird (vgl.: denken an, gelten als) und N/NP-Komplemente ${ }^{93} \mathrm{zu}$ sich nimmt. 94 Dagegen spricht die fehlende feste Kasusrektion, die wiederum eher auf die Wortart Konjunktor hindeutet (Konjunkte nehmen den gleichen Kasus an): Für Konjunktor spricht die Kasusidentität zwischen dem als-NP-Komplement und einer "Bezugs-NP". 95 Aber

92 Identität liegt z.B. vor bei Willy Brandt als der vierte Bundeskanzler, subsumtion bei Willy Brandt als erfahrener Politiker (Binordnung in die Menge der erfahrenen Politiker).

93 Manchmal auch AdjP-Kamplemente wie in Diese Vase gilt als sehr wertvoll; dies ist bei NP-adjazenten Phrasen jedoch unmöglich: *Diese Vase als sehr wertvall etc.

94 Zur Einordnung als Präposition z.B. Kolde (1971: 186) mit folgendem Beispiel (F7.28); man vgl. zudem Helbig/Buscha (1980: $363 \mathrm{ff.}$ ), Wunderlich (1984: 73, $75 \mathrm{f.,} 83 \mathrm{f.}$ ) und Fries (1988: $80 \mathrm{f.}$ ). (F7.20) Es sah das für seine Pflicht/ als seine Pflicht an.

95 Hierzu Eisenberg (1986: 313, 323 ff.). Auch Bergenholtz/Schaeder (1977: 129 ff.). 
auch Konjunktor befriedigt als Einordnung nicht, so daB einige Linguisten diese Partikel als eigene Klasse/Sonderklasse führen. 96 Diesem Usus - ich übernehme den Vorschlag "Translativ" - will ich mich anschließen.

Translativphrasen sollen diejenigen Konstrukte heißen, die aus als und einer zugeordneten $\mathrm{N}$-Phrase einer beliebigen X-Bar-Stufe bestehen. Als Komplemente von als erscheinen die gleichen $\mathrm{N}$-Kategorien wie beim prototypischen $\mathrm{Ap}-$ positiv (nicht nur Voll-NPs, sondern auch Nominale niedrigerer X-Bar-Stufe):

(7.87) Pia als die behandelnde Arztin/, die behandelnde Arztin, (N3).

Pia als behandelnde Arztin/, behandelnde Arztin, (N2).

Pia als Arztin/, Arztin, (NO).

Das Translativ wie kann hingegen als Komplement nur N3 (NP) zu sich nehmen (Ein Kerl wie dieser alte Trottel/ *alter Trottel/ *Trottel).

Die wie-Phrasen scheiden aus dem appositionsverdächtigen Bereich aus, da sie eindeutig attributiv fungieren (vgl. 7.88a; dazu auch Kap. 7.5.). Unterschiede $z$ wischen den als- und wie-Phrasen lassen sich deutlich erkennen:

(7.88a) Was für eine Dozentin? Eine Dozentin wie $P$ i a.

Was für eine/ Welche Pia? *Pia als Doz e ntin.

(7.88b) Nicht eine Dozentin wie $P$ i a, sondern eine Dozentin wie $H$ e lga suchen wir.

* Nicht Pia als Doz e ntin arbeitet hier als Aushilfe, sondern Pia als stud e ntin.

(7.88c) Wer hät te das wissen müssen? Eine Dozentin wie Pia.

Wer hätte das wissen müssen? ?Pia als Dozentin.

Im Ggs. zu den attributiven wie-Phrasen sind die als-Phrasen weder mit einer Gliedteilfrage erfragbar (7.88a) noch kontrastierend negierbar $(7.88 \mathrm{~b})$; sie sind auch nicht von GPn erfaBbar. Die Konstruktion Basis plus als-Phrase antwortet auf eine Satzgliedfrage mit eher geringer Akzeptabilität (7.88 $)$, das analoge Muster mit der wie-Phrase ist diesbezüglich problemlos. uberdies kann die als-Phrase nie als restriktiver Modifikator der Bezugs-NP fungieren! 97

Welche Merkmale legten es nahe, als-Phrasen evtl. den Appositionen zuzurechnen? Vor allem die Möglichkeit der Kasuskongruenz zwischen Translativphrase und Bezugs-NP98 sowie die Möglichkeit einer Kopulasatzparaphrase: ${ }^{99}$

(7.89a) Dir als meinem Freund verrate ich das Geheimnis.

(7.89b) Dir - du bist mein Freund - verrate ich das Geheimnis.

Hier ist $z$ u beobachten, wie ein Appositionsbereich dadurch konstituiert wird, daB man ein oder zwei Kriterien ansetzt und sämtliche weiteren Verhaltensei-

96 2.B.: Engel (1988: 775).

97 Jeder Attributstyp kann restriktiv verwendet werden, die "appositive" als-Phrase nicht. Vgl. auch Pütz (1988: $198 \mathrm{f.}$ ).

98 Z.B. DUDWN (1984: 593), Schulz/Griesbach (1982: 362 f.).

99 Z.B. Heidolph u.a. (1981: 292 f.), Motsch (1965: 117 ff.). 
genschaften beiseite läßt. In Darstellungen, die die orthographische bzw. intonatorische Abtrennung hervorheben, zählt die Translativphrase natürlich nicht zu den Appositionen. 100

Bei Motsch (1965: $117 \mathrm{ff.}$ ) ist für die Einordnung als Apposition entscheidend, daß Phrasen mit als (und z.T. auch wie) in einer Umformungsbeziehung zu Kopulasätzen stehen: 101

\section{(7.90a) Peter wurde als völlig geheilter Mensch aus dem Krankenhaus entlas- sen. \\ (7.90b) Peter wurde, er war ein völlig geheilter Mensch, aus dem Kranken- haus entlassen. \\ (7.90c) Peter, ein völlig geheilter Mensch, wurde aus dem Krankenhaus ent- lassen.}

Das letzte Beispiel - eine prototypische Apposition - ist im Ggs. zum ersten "mit stilistischer Konnotation" (Motsch (1965: 118)) behaftet. Das heiBt vermutlich, daß ein gewisser Bedeutungsunterschied vorliegt. Vor allem aber liegt ein evidenter syntaktischer Unterschied vor: Während im ersten Beispiel ein permutables, vorfeldfähiges und erfragbares satzglied vorliegt, enthält das letzte Beispiel ein unselbständiges, nicht allein vorfeldfähiges Gliedteil. 102 Motsch (1965: 122) zählt sogar obligatorische verbergänzungen wie in (7.91) $\mathrm{zu}$ den Appositionen, nur daB sie strukturell enger als andere zum Verb gehörten:

(7.91) Kant gilt als bedeutender Philosoph. Die Kiste diente als Stuhl. Pias Urgrobvater gab sich als Nachfahre des Marquis de Sade aus.

Heidolph u.a. (1981: 292) setzen als "Grundstrukturquellen" für derartige Ronstruktionen wie Motsch (1965) Kopulasätze an:

(7.92) Du als (der) Brigadier darfst das nicht dulden. Du - du bist doch der Brigadier/ da du doch der Brigadier bist darfst das nicht dulden.

100 Z.B. Ingel (1986). Zum Einordnungsproblem vgl. auch Helbig (1984a: 79):

"In der Grundstruktur kann ein Kopulasatz angenormen werden (...). Deshalb kann man die alsGruppen (...) als Apposition einstufen (Referenzidentităt ist bei der Apposition in Unterschied zum Attribut vorausgesetzt) - falls die als-Gruppen überhaupt in die Appositionen eingeschlossen werden. Wenn das nicht geschieht, handelt es sich um Attribute (...)".

101 s. Motsch (1965: 117 ff.). Pütz (1988: 199) paraphrasiert diesen TYp mit einem nicht-restriktiven Relativsatz:

(F7.21) Sie mïssen mich als ihren Verteidiger genavestens mit dem Hergang des Unfalls vertraut machen.

(F7.22) Sie müssen mich, der ich ja ihr Verteidiger bin, genavestens mit dem Hergang des unfalls vertraut machen.

102 Es sei denn, man erkennte Bin völlig geheilter Mensch, wurde Peter aus dem Krankenhaus entlassen als voll akzeptabel und vor allem als voll äquivalent an; dann jedoch mư man zeigen können, daß diese Permutation ohne Bedeutungs- und Funktionsveränderung vonstatten geht und dies ist m.E. nicht der Fall. 
Schulz/Griesbach (1982: 363) behandeln derartige Konstruktionen als "abrückbare Appositionen" und nehmen hierzu noch abrückbare pronomina und gefloatete Quantoren:

(7.93) Dr. Müller (als Chefarzt) trägt (als Chefarzt) viel Verantwortung. Wir (alle) sind (alle) zu Hause.

Jung/Starke (1982: 111) sprechen davon, daß die hier angesprochenen Konstruktionen "der Apposition vergleichbar" seien. Wegen des Kongruenzverhaltens rechnet die DUDEN-Grammatik (1984: 593) die als- und wie-Gruppen den Appositionen $z u$.

Eisenberg (1986: $323 \mathrm{ff.}$ ) faBt als und wie als koordinierende Konjunktionen auf und hält die Wertung als Attribut oder Apposition in Beispielen wie (7.94) offen ("eine terminologische Frage"; ebd. 324).

(7.94) Herr Schulze (als erfahrener Lehrer) weiB (als erfahrener Lehrer) sowas.

Speziellere Untersuchungen zu Translativphrasen liegen mit Pütz (1982; 1988) und Helbig (1984a) vor. Erwähnt seien auch: Kolde (1971), Engelen (1975: 148 ff.), Smith (1977) und Helbig (1984c). Pütz und Helbig gelangen zu einer feinen (funktionalen) Ausdifferenzierung der als-Translativphrasen: 103

(7.95a) Er arbeitet (nicht) als Schlosser in diesem Betrieb.

(7.95b) Er als Schlosser arbeitet (nicht) in diesem Betrieb.

(7.95c) Der Betrieb stellte ihn als Schlosser ein.

(7.95d) Der Professor gilt als Experte auf diesem Gebiet.

(7.95e) Wir betrachten den Professor als Experten.

(7.95f) Er starb als junger Mann/ jung.

$(7.95 \mathrm{~g})$ Wir trafen ihn als verzweifelten Mann/verzweifelt an.

(7.95h) Die Verwendung dieses Zitats als (ein) Beleg.

(7.95i) Die Verwendung dieses Zitats als eines Belegs.

Ich teste diese Sätze zusätzlich zu Helbigs Kriterien - Bezug, Valenz, Eliminierbarkeit und Grundstrukturen (einige weitere werden nicht herangezogen) hinsichtlich: Erfragung, Phrasenersetzung, Topikalisierung, Anwendung von Negationsträgern (nicht) und Gradpartikeln auf die Translativphrasen und Satzgliedhaftigkeit (die sich aus den Testergebnissen ableiten läßt). Die Vorschläge in der spalte "Funktion" scheiden entweder die appositionsunverdächtigen Satzglied-bzw. Gliedteilfunktionen aus oder lassen die Einordnung noch offen ('PTIV SBJ/OBJ' = 'Prädikativ zum subjekt/Objekt'; 'PATTR SBJ/OBJ' = 'prådikatives Attribut mit subjekts-/Objektsbezug'):

103 Die nachstehenden beiden Ubersichten über die Funktionen von als-Phrasen sind Zusammenfassungen von mir. Die Beispiele stammen überwiegend aus helbig (1984a) und Pütz (1988). 


\begin{tabular}{|c|c|c|c|c|c|c|c|c|c|}
\hline & a & b & c & $d$ & e & f & g & $\mathrm{h}$ & i \\
\hline Bezug & $\mathrm{V} / \mathrm{N}$ & N & $\mathrm{V} / \mathrm{N}$ & $\mathrm{v}$ & $\mathrm{v}$ & $N /(V)$ & $N /(V)$ & $\mathrm{N}$ & N \\
\hline $\begin{array}{l}\text { Bezug + Tr. } \\
\text { phrase im VF? }\end{array}$ & 104 & + & $(+)$ & - & - & - & - & + & + \\
\hline Frage & $\begin{array}{l}\text { als } \\
\text { was? }\end{array}$ & $?$ & $\begin{array}{l}\text { als } \\
\text { was? }\end{array}$ & $\begin{array}{l}\text { als } \\
\text { was? }\end{array}$ & $\begin{array}{l}\text { als } \\
\text { was? }\end{array}$ & wie? & wie? & $\begin{array}{l}\text { was } \\
\text { für ein- }\end{array}$ & ? \\
\hline substitut & $?$ & $?$ & $?$ & $?$ & $?$ & so & so & $?$ & $?$ \\
\hline Kopulasatz & - & + & - & - & - & $(t)$ & $(+)$ & - & + \\
\hline Negation & + & - & + & + & + & + & + & + & - \\
\hline Gradpartikel & + & - & + & + & + & + & + & + & - \\
\hline Valenz & frei & frei & frei & obl & obl & frei & frei & frei & frei \\
\hline Funktion & ADVB & ATTR & $A D V B$ & PTIV & PTIV & PATTR & PATTR & ATTR & ATTR \\
\hline & & APPOS & & SBJ & $\mathrm{OBJ}$ & SBJ & OBJ & & APPOS \\
\hline Satzglied & $?$ & $?$ & + & + & + & + & + & - & $?$ \\
\hline
\end{tabular}

Wenn man das Topikalisierungskriterium betrachtet, dann zeigt sich, daB es mindestens in (7.95d, e, f, g) nicht erfüllt wird. In (7.95f, g) liegen Fälle von prädikativen Attributen vor, die als Satzglieder (erfragbar, relativ frei permutierbar, allein vorfeldfähig, ersetzbar durch das Modaladverb so etc.) mit "semantischen subjekt- bzw. Objektbezug" zu werten sind; man beachte die Kommutationsmöglichkeit mit den Adjektivphrasen, die typischerweise als prädikative Attribute fungieren.

In $(7.95 \mathrm{~d}$, e) hat man es mit verbdependenten obligatorischen Ergänzungen $z u$ tun. Als wird gefordert wie eine Präposition bei einem Präpositionalobjekt (was übrigens bei wie nie der Fall ist). Die Ergänzungen in (7.95d, e) nenne ich mit Helbig (1984a: $76 \mathrm{f}$. ) Prädikative zum Subjekt bzw. Objekt.

(7.95c) erlaubt zwar eine Kontaktstellung im Vorfeld (Ihn als Schlosser stellte der Betrieb ein), doch es handelt sich um eine Konstruktionsveränderung. Während (7.95c) aussagt, daB jemand 'in der Funktion eines Schlossers' eingestellt wurde (der eigentlich gar kein Schlosser sein muB), muB die Vorfeld-Kontaktvariante anders interpretiert werden, nämlich daß jemand eingestellt wurde, da er Schlosser ist (und nicht etwa Dreher). Daher wird (7.95d) als Adverbiale aus dem Appositionsbereich ausgeschlossen. Nur Kontaktvarianten wie (7.95b) gehören u.E. zu den "appositionsverdächtigen" Mustern. (7.95a) sieht wie eine Permutationsvariante von (7.95b) aus, doch liegt auch hier eine adverbiale als-Phrase vor (weitere Argumente folgen noch). Die Testmatrix zeigt bereits auffällige Unterschiede (Frage, Kopulasatzparaphrase, Negation)!

$(7.95 \mathrm{~h})$ steht als Nominalisierung in Beziehung zu verbalen Syntagmen wie Dieses Zitat wird als Beleg verwendet. Die als-Phrase verhält sich wie ein Attribut (Gliedteilfrage, Negation möglich). (7.95i) läBt sich dagegen nicht

104 Die Unstellung der Translativphrase ins Vorfeld zu Er bewirkt eine Konstruktionsveränderung. 
zu einem verbalen Syntagma in Beziehung setzen; eine Umformung würde eher einen (auf "Appositionsverdacht" hinweisenden) Kopulasatz beinhalten: Dieses $z i-$ tat - es ist ein Beleg - wird verwendet $(z u X)$. Zudem ist keine Gliedteilfrage (nach der als-Phrase) möglich, von einer Negation bleibt die Translativphrase unberührt. Es liegt somit ein Muster vor, das (7.95b) vergleichbar ist und als "appositionsverdächtig" gelten kann.

Nun nehme ich noch einige weitere Typen in Augenschein, die Pütz (1988: 195 f.) anführt. Es zeigt sich, daß die Translativphrase vielfältige Funktionen auszuüben vermag, deren (adverbialer) status noch genauer zu erforschen wäre. Hier müssen pauschale Einstufungen ausreichen - wichtig ist allein die Ausscheidung aus den "appositionsverdächtigen" Mustern:

(7.95j) Viele hatten Willy als Minister/ damals ('als er Minister war') mehr geschätzt (als heute, wo er Kanzler ist).

(7.95k) Als Kollegen schätze ich Peter (, aber als Freund nicht). Inwiefern schätze ich Peter? Als Kollegen.

(7.951) Peter hat den VW als Cabrio gekauft (und nicht als Roadster). Peter hat den VW in der Cabrioausführung gekauft.

(7.95m) Als Ingenieur kann ich sie einstellen, sonst gar nicht. Falls sie Ingenieur sind, kann ich sie einstellen.

(7.95n) Gründgens hat den Mephisto als derben Spaßmacher/ so gespielt.

(7.950) Viele Touristen scheinen ihre Personenwagen als Lastwagen/ so $z u$ miBbrauchen.

$\begin{array}{lcccccc} & j & \mathrm{k} & \mathrm{l} & \mathrm{m} & \mathrm{n} & \text { 0 } \\ \text { Bezug }+\mathrm{Tr} . & ? & ? & ? & ? & ? & ? \\ \text { phrase im VF? } & & & & & & \\ \text { Frage } & \text { wann? } & \text { inwie- } & \text { als } & \text { als } & \text { wie? } & \text { als was? } \\ & & \text { fern? } & \text { was? } & \text { was? } & & \text { (wie?) } \\ & & & \text { (wie?) } & & & \\ \text { Substitut } & \text { damals } & \text { insofern } & \text { (so) } & \text { (dann) } & \text { so } & \text { (so) } \\ \text { Kopulasatz } & ? & ? & ? & + & - & - \\ \text { Negation } & + & + & + & - & + & + \\ \text { Gradpartikel } & + & + & + & ? & + & + \\ \text { Valenz } & \text { frei } & \text { frei } & \text { frei } & \text { frei } & \text { frei } & \text { frei } \\ \text { Funktion } & \text { TEMP } & \text { RESTR } & \text { ADVB } & \text { ADVB } & \text { MOD } & \text { (MOD) } \\ \text { Satzglied } & + & + & + & + & + & +\end{array}$

Infolge relativ klarer Ergebnisse bei Satzgliedfrage und Substitution lassen sich $(7.95 \mathrm{j})$ als temporales, $(7.95 \mathrm{k})$ als restriktives ${ }^{105}$ und $(7.95 \mathrm{n}, 0)$ als

105 S. hierzu auch Engel (1988: 225). 
jeweils modales Adverbial einstufen. $(7.95 \mathrm{~m})$ darf wohl als konditionales Adverbial gelten, die Topikalisierung Sie als Ingenieur kann ich einstellen verändert wieder einmal den Konstruktionssinn ('da sie Ingenieur sind'). (7.951) ist m.E. im herkömmlichen Adverbialsystem nicht zu verorten; ${ }^{106}$ den VW als Cabrio habe ich gekauft kann man als bedeutungsäquivalente stellungsvariante nicht akzeptieren ('da er ein Cabrio ist'), vielleicht ist die Permutation ohnedies unannehmbar.

Nachfolgend untersuche ich als "appositionsverdächtige" als-Phrase den Typ (7.95b): (7.95i) wäre ein weiteres Beispiel dafür. Zur kategorialen Füllung wurde bereits erwähnt, daß als Komplemente unterschiedlicher N-Bar-stufen zu sich nimmt; diese können jedoch nicht - wie z.B. der "Prototyp" - um Gradpartikeln, Sadv, freie ADVB, Appositionsfloskeln oder Modalpartikeln erweitert werden. (7.97) ist kein Gegenbeispiel, da sich die Zusatzlexeme auf das Adjektiv beziehen; als Adjektivangaben sind sie Konstituenten der Adjektivphrase:

(7.96a) Pia als *sogar/*nur/*auch/*glücklicherweise/ *vermutlich/ *bekanntlich Arztin hat die Verantwortung übernommen.

Pia, sogar/ nur/ auch/glücklicherweise/ vermutlich/bekanntlich Arztin, hat die Verantwortung übernommen.

(7.96b) Den Kuno als *übrigens/ * früher/ *ehemals einen bekannten Politiker kennt heute kein Schwein mehr.

Den Kuno, übrigens/ früher/ ehemals ein bekannter Politiker, kennt heute kein Schwein mehr.

(7.96c) Die Pia als *eben doch/*ja wirklich/ *nun mal eine nette Nachbarin hat wieder einen Kuchen gebacken.

Die Pia, eben doch/ (?) ja wirklich/ nun mal eine nette Nachbarin, hat wieder Kuchen gebacken.

(7.97) Pia als übrigens/ sogar/ vermutlich/ seit langem erfahrene Arztin hat die Verantwortung übernommen.

Eine intonatorische/graphematische Kennzeichnung liegt nicht vor. Dennoch können als-Phrasen ohne weiteres als Einschaltungen markiert werden ( 7.98 a) aus: Abendzeitung Nr. 267/46, Fr. 18.11.1988, S. 3):

(7.98a) Es ist sicher $z u$ früh, um vorauszusagen, daß Benazir Bhutto - als selbständige Frau und als Erbin und Vollstreckerin der politischen Absichten ihres Vaters - die Zukunft ihres Landes maBgeblich bestimmen wird.

(7.98b) Pia, als Arztin, übernahm die Verantwortung.

Wenn man davon ausgeht, daB ein ZUSATZ wie die "prototypische Apposition" gerade durch das Einschaltungsmuster als HOSPITANT gekennzeichnet wird und die unmarkierten Satzglieder sowie Gliedteile durch eine Einschaltungsmarkierung aus dem Trägersatz "ausgegliedert" werden könnten (vgl. Kap. 11), dann scheint

106 Damit meine ich z.B. die Vorschläge von Grammatiken wie z.B. Heidolph u.a. (1981) oder DUDEN (1984). 
es sich bei dem diskutierten Muster nicht um etwas Appositionsartiges zu handeln. Es liegt wohl eher ein Satzgliedteil vor, denn ein solches kann unter gewissen Voraussetzungen (Kap. 11) als Einschaltung markiert werden. 107

Die appositionsverdăchtigen Translativphrasen stehen unmittelbar rechtsadjazent zur Bezugs-NP; evtl. schieben sich restriktive Attribute dazwischen (Der Onkel deiner Schwester als Jurist hat mir sehr geholfen). Interessant ist, daB sich die als-Phrase offensichtlich nur auf den Nukleus einer komplexen Nominalgruppe zu beziehen vermag, sofern keine Nominalisierungen vorliegen (vgl. 7.95i; ein lockeres Appositiv hat zwei Bezugsoptionen):

(7.99a) Den Bruder deines onkels als den zuständigen Juristen/, den zuständigen Juristen, hätte man informieren müssen.

Den Bruder deines Onkels als *des zuständigen Juristen/, des zuständigen Juristen, hätte man informieren müssen.

(7.99b) Dem Freund deines Vaters als einem erfahrenen Mann/, einem erfahrenen Mann, sollte man besser zuhören.

Dem Freund deines Vaters *als eines erfahrenen Mannes/, eines erfahrenen Mannes, sollte man besser zuhören.

"Lockere Appositive" (auch appositive Relativsätze) folgen der als-Phrase stets nach - wiederum ein Hinweis auf deren stärkere Anlehnung an die Basis:

(7.100) Pia (*, eine kluge Frau,) als Arztin (, eine kluge Frau,) weiB das sicher.

(7.101) Pia (*, die lange studiert hat,) als Arztin (, die lange studiert hat,) weiB das sicher.

Die Distanzstellung der adjazenten Translativphrase führt zu einer anderen Lesart: Es liegt dann eine als-Adverbiale vor, die eine temporäre Funktion angibt und z.T. eine "kausale Nuance" annimmt (7.102; vgl. Kap. 8.1.2). Bei gleichzeitiger Anwesenheit einer als-Adverbiale führt die Dislokation der adjazenten Translativphrase zu inakzeptablen Resultaten (7.103):

(7.102) Pia als Arztin (sie ist ja Arztin/ *in der Funstion einer Arztin) arbeitet jetzt bei Dr. No.

Pia arbeitet jetzt als Arztin (in der Funktion einer Arztin/ *sie ist ja Arztin) bei Dr. No.

(7.103) Pia als Arztin arbeitet jetzt als Graphikerin in einem Büro. * Pia arbeitet als Arztin jetzt als Graphikerin in einem Büro. *Pia arbeitet jetzt als Arztin als Graphikerin in einem Büro.

(7.104) Als Arztin (da sie ja Arztin ist/ in der Funktion einer Arztin) arbeitet Pia jetzt bei Dr. No.

(7.105) *Als Arztin (*da sie ja Arztin ist/*in der Funktion einer Arztin) arbeitet Pia jetzt als Graphikerin (in der Funktion einer Graphikerin) in einem Büro.

107 Vgl.: Pia (aus Mexiko/ mit ihrer Prozeßerfahrung) kannte alle Tricks. Pia (als deine Anwalltin) kannte alle Tricks. 
Interessant ist, daß bei Spitzenstellung von Translativphrase und Bezugselement offensichtlich die Semantik der Kontaktvariante (prädikative Lesart) bewahrt werden kann (wobei auch die adverbiale Lesart möglich ist; 7.104).108 Man beachte, daB hier deutlich eine kausale Nuance mitschwingt. (7.105) demonstriert, daß die Hinzufügung einer als-Adverbiale zu (7.104) einen inakzeptablen Satz zur Folge hat; dieses eigenartige Phänomen ist mir unerklärlich, denn es wäre ja denkbar, daß zwar die "Funktionslesart" wegfiele, die "Kopulalesart" jedoch bestehen bliebe. 109

Somit ist unser appositionsverdächtiger Typ wahrscheinlich auf die unmittelbare Kontaktstellung beschränkt. Inwiefern die Möglichkeit der Topikalisierung der Phrase (7.104) eine gleichwertige, voll akzeptable Alternative darstellt, ob also die prädikative Lesart erhalten bleibt oder ob ein Adverbial (evtl. mit kausaler Nuance) vorliegt, bleibt offen.

Das Stellungsverhalten hinsichtlich von Adpositionen, Gradpartikeln oder Konjunktionaladverbien sieht folgenderweise aus: Die Beispiele mit Nachstellung der Translativphrase sind nicht immer völlig inakzeptabel (um...willen, Konjadv), jedoch ist deren Voranstellung eindeutig die vorzuziehende Variante, in einigen Fällen sicher auch die einzig akzeptable (zuliebe, GP).

(7.106) Pia (als Arztin) zuliebe (*als Arztin) verzichtet Kuno auf das Rauchen.

Um ihrer (als Arztin) willen (?als Arztin) verzichtet Kuno auf das Schmausen.

(7.107) Pia (als Arztin) sogar (??als Arztin) kam zur Patientenversammlung.

(7.108) Pia (als Arztin) jedoch ((?)als Arztin) wuBte es besser.

Bei CLEFT/PSEUDOCLEFT (7.110/7.111), SATZVERSCHRANKUNG (7.113) und LINKSVERSCHACHTELUNG (7.115) legt die Konstruktion folgendes Verhalten an den Tag:

(7.109) Pia als Arztin arbeitete bei Dr. No.

(7.110) Es war Pia (*als Arztin/ aus U1m), die bei Dr. No arbeitete.

(7.111) Wer bei Dr. No arbeitete, war Pia (*als Arztin/ aus UIm).

(7.112) Ich vermute, daB jene Pia als Arztin bei Dr. No arbeitet.

(7.113) Jene Pia (*als Arztin/ aus Ulm) vermute ich, daB bei Dr. No arbeitet.

(7.114) Kuno hoffte, jene Pia als Arztin zur Assistentin ernennen zu können.

(7.115) Jene Pia ?als Arztin/ aus U1m hoffte Kuno zur Assistentin ernennen zu können.

108 So auch Pütz (1988: 199).

109 Bin Beispiel wie Als Arztin (da sie ja Arztin ist/ * in der Funktion einer Arztin) arbeitet Pia jetzt als Leiterin (*da sie ja Leiterin ist/ in der Funktion einer Leiterin) in einem Krebsforschungsinstitut bringt meine Argumente jedoch ins Wanken (Hinweis Marie LeBras); dazu mein $\mathrm{Zu}$ satz am Ende dieses Kapitels. 
Auffallend ist, daB Attribute (aus Ulm) und der "Appositionsprototyp" (vgl. Kap. 6.) das Bezugselement begleiten können, als-Phrasen jedoch nicht. Nach meinem Sprachgefühl sind die Resultate - nicht ganz sicher bin ich mir bei der Bewertung der Linksverschachtelungsvariante - völlig inakzeptabel, ohne daB ich einen Grund dafür wüßte. 110 Dabei ist der Grad der "Fügungsenge" (der Grad der Assoziiertheit von Basis und "Appositiv") beim "Prototypen" geringer als bei als-Phrasen, so daß zu erwarten wäre, daß diese ihr Bezugselement eher begleiten (müssen) als jener. Jedenfalls scheint das Muster "NP + adjazente Translativphrase" außerst stellungsrestringiert und besonderen stellungskonstruktionen kaum zugänglich zu sein!

Die semantische Funktion adjazenter Translatiophrasen ist die zusätzliche (nichtrestriktive) Angabe einer dauerhaften, "wesentlichen" Eigenschaft des Referenten der Bezugs-NP resp. die Bezeichnung einer Klasse von Entitäten, in die der Referent der Kern-NP eingeordnet wird;111 s. (7.117). Manchmal liegt auch Referenzidentität von Bezugs-NP und als-NP vor (dagegen referiert in 7.117 die als-NP nicht!); semantisch handelt es sich dann um eine Gleichsetzung (7.116):

(7.116) Willy Brandt als der vierte deutsche Bundeskanzler gefiel Pia.

(7.117) Willy Brandt als mutiger Politiker gefiel Pia.

Als Paraphrase kann ein nichtrestriktiver Relativsatz (besonders plausibel mit Modalpartikeln, welche die Beiläufigkeit der Information anzeigen) dienen: 112

(7.118) Kuno als Schlosser/, der ja (eigentlich) Schlosser ist, arbeitet jetzt als Maler in diesem Betrieb.

110 Daß sich als-Phrasen bzgl. der eben behandelten Stellungseigenschaften nicht wie Attribute verhalten, sei hier kritisch vermerkt, denn ich werte Translativphrasen später als (wenn auch untypische) Attribute.

111 Pia als Lehrerin kann expliziert werden durch 'Lehrerin zu sein, ist eine Eigenschaft Pias' oder 'Das Individuum Pia ist ein Blement der Menge der Lehrerinnen'.

112 So z.B. Pütz (1988: 199); nach Pütz dienen sowohl diese Paraphrase (F7.23) als auch die Nichtdislozierbarkeit (F7.24) und die gemeinsame Vorfeldfähigkeit (F.25) zur Abgrenzung dieses Typs von den OBJEXTSPRADIKATEN (OPRAD); man betrachte (angelehnt an Pütz' Beispiele ebd. 198 f.):

(F7.23) Sie müssen mich als Thren Verteidiger (, der ich ja Thr Verteidiger bin), genauestens mit dem Hergang des Unfalls vertraut machen.

Ich habe meine Höhensonne als Heizung ( $*$, die ja eine Beizung ist,) verwendet (OPRAD) .

(F7.24) * Sie müssen mich genauestens als Ihren Verteidiger mit dem Hergang des Unfalls vertraut machen.

Ich habe meine Höhensonne gestern als Beizung venwendet.

(F.25) Mich als Inren Verteidiger müssen Sie genavestens mit dem Hergang des Unfalls vertraut machen.

* Meine Höhensanne als Heizung habe ich gestern verwendet. 
Nach Pütz (1982: $357 \mathrm{f.}$ ) können derartige als-Konstruktionen aus zugrundeliegenden Kopulasätzen via GLEICHE-NP-TILGUNG abgeleitet werden; dieser Ableitungsweg bestünde auch bei "lockeren Appositiven": 113

(7.119a) Ich habe mir einen VW (der VW ist ein Cabriolet) gekaut.

(7.119b) Ich habe mir den VW als Cabriolet gekauft.

(7.119c) Ich habe mir den VW, ein Cabriolet, gekauft.

Hier erhebt sich auch die Frage, inwieweit Translativphrasen zu "lockeren Appositionen" (der Kategorie Nominal; nicht möglich sind adjektivische oder präpositionale Einheiten) äquivalent sind:

(7.120a) Kuno als Schlosser/, ein Schlosser, arbeitet jetzt als Koch in diesem Betrieb.

Den VW als Cabriolet/, ein Cabriolet, habe ich mir gekauft.

(7.120b) Pia als frischgebackene Jurastudentin/, frischgebackene Jurastudentin, ('da sie ja frischgebackene Jurastudentin ist') betrat den Hörsaal.

(7.120c) Pia, seit gestern Jurastudentin, / *als seit gestern Jurastudentin betrat den Hörsaal.

Eulalia, übrigens keine Jurastudentin, / *als übrigens keine Jurastudentin betrat den Hörsaal.

Kuno, nicht gerade eine Schönheit,/ *als nicht gerade eine Schönheit zieht die Frauen dennoch an.

(7.120d) Den Bruder deines Onkels als den zuständigen Juristen (, den zuständigen Juristen, / *als des zuständigen Juristen (, des zuständigen Juristen,) hätte man unbedingt informieren müssen.

Dem Freund deines Vaters als einem erfahrenen Mann 1, einem erfahrenen Mann, / *als eines erfahrenen Mannes (, eines erfahrenen Mannes,) sollte man besser zuhören.

Sicher nicht gleichwertig sind die Umformungsbeziehungen dann, wenn die lockeren Appositive um Floskeln, Adverbiale usf. erweitert sind. Ansonsten scheint weitgehend Aquivalenz zu bestehen; sogar die kausale Nuance in (7.120b) scheint in beide Konstruktionsmuster hineinlesbar. Ein wichtiger Unterschied manifestiert sich in (7.120d): Hier zeigt sich, das "lockere Appositive" sich sowohl dem Nukleus der Attributskonstruktion als auch dem Satellitennomen (Nukleus des Genitivattributs) zuordnen lassen. Translativphrasen jedoch lediglich dem dependenziell obersten Nomen! ${ }^{114}$ Dies 1 äBt eine eine sehr enge Bindung der Translativphrase ans Bezugsnomen erkennen, wăhrend die Bindung eines "Appositiv" sich deutlich lockerer ausnimmt.

113 Sicher nicht bei allen Typen; hierzu insb. Raabe (1975). Das Problem differierender Determinantien (7.119a) bleibt außer Betracht, ist aber evtl. nicht ganz umproblematisch.

114 Diese Regel gilt allerdings nur für nicht-deverbative Kernnomina, m.a.W. für NPn, die sich nicht als Nominalisienungen verbaler Syntagmen erklären lassen. 
Die Regeln der Kasuskongruenz zwischen Bezugs-NP und als-Komplement lassen sich (grob) auf folgenden Nenner bringen: ${ }^{115}$ Den Regelfall stellt wohl die Kasuskongruenz dar, vor allem wenn das Komplement von als eine Voll-NP ist. Wenn die Basis genitivisch markiert ist und das als-Nominal keinen Determinator bei sich hat, dann wird der Nominativ gewählt, da der Kongruenzgenitiv bzgl. der Akzeptabilităt eingeschränkt wirkt (7.121b). Ist kein Determinator, aber ein Adjektivattribut vorhanden, dann erscheinen mir Genitiv- und Nominativmarkierung etwa gleichberechtigt, wobei beide "unelegant" klingen und man eher zur Vermeidung solcher Konstellationen tendierte (7.121b). Abweichungen in den Dativ (Stichwort "Dativapposition") sind bei nominativisch und akkusativisch markierten Basen ungrammatisch (7.121a, d) und bei Basen mit Genitivflexiv möglich (7.121b). Abweichungen in den Nominativ sind nicht leicht zu beurteilen: Sie erscheinen nicht völlig akzeptabel, aber dennoch mutet der Grad der Abweichung eher gering an $(7.121 b, c, d)$. Eine Absicherung dieser Urteile müBte allerdings wesentlich mehr an Material berücksichtigen.

(7.121a) Das Kind als das jüngste Familienmitglied wurde verwöhnt. * Das Kind als dem jüngsten Familienmitglied wurde verwöhnt. Das Kind als jüngstes Mitglied wurde verwöhnt.

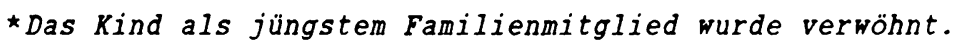

(7.121b) Sie bemächtigten sich des Kindes als des jüngsten Mitglieds. Sie bemächtigten sich des Kindes als dem jüngsten Mitglied.

(?) Sie bemächtigten sich des Kindes als das jüngste Mitglied.

(?) Sie bemächtigten sich des Kindes als jüngsten Mitglieds. Sie bemächtigten sich des Kindes als jüngstem Mitglied.

(?) Sie bemächtigten sich des Kindes als jüngstes Mitglied. Sie bemächtigten sich des Kindes als Mitglied/ ?? Mitglieds.

(7.121c) Sie halfen dem Kind als dem jüngsten Mitglied.

(?) Sie halfen dem Kind als das jüngste Mitglied.

Sie halfen dem Kind als jüngstem/ (?) jüngstes Mitglied.

(7.121d) Sie tranken den Tee als den besten Aufwärmer.

* Sie tranken den Tee als dem besten Aufwärmer.

(?) Sie tranken den Tee als der beste Aufwärmer.

Sie tranken den Tee als besten/*bestem/ (?) bester Aufwärmer.

Aus (7.121) folgt einerseits, daB das Urteil des "Zweifelsfälle-DUDENS" (1985), das Ausweichen auf den Nominativ in Fällen wie

(7.122) mit Französisch als zweiter (Dat.)/ zweite (Nom.) Fremdsprache sei "nicht korrekt" (ebd. 71), zu kraB formuliert sein dürfte. Andererseits ist die Abweichung in den Nominativ bei der als-NP keineswegs so akzeptabel wie die beim "Appositionsprototypen" (vgl. Kap. 6 Punkt 6). Das kann als In-

115 Hierzu z.B. Schönmann (1959); Winter (1966: 25 ff.); Seibicke (1968); Sievers (1969); Bänzinger (1970: 104 ff.); Förster (1972); Leirbulkt (1978); Heidolph (1979); Teubert (1979: 143 f.); Gippert (1981); Jumg (1982: $111 \mathrm{ff.,} \mathrm{266);} \mathrm{Abraham} \mathrm{(1983:} 71 \mathrm{ff}$.$) ; Bergenholtz (1985); Duden$ (1985: 71 ff.). 
dikator für eine stărkere Anbindung der Translativphrase an die Bezugs-NP gewertet werden (und als Indiz für gröBere syntaktische Integration in den Trägersatz bzw. die Trägerphrase).

Weglabbar sind die Translativphrasen ohne weiteres, ihre Basis können sie syntaktisch nie vertreten. Dies unterscheidet sie von "prototypischen Appositiven", die wenigstens manchmal ihre Basis syntaktisch vertreten könnten:

(7.123) Pia als die Teamchefin/ Pia/ $\star A l s$ die Teamchefin gab die Anweisungen.

(7.124) Pia, die Teamchefin,/ Pia/ Die Teamchefin gab die Anweisungen. Wenn man die "Sprecherpräsenz und -divergenz" mit dem Prototyp vergleicht, dann stellen sich folgende Unterschiede heraus:

(7.125a) *Pia sagte: "Otto" als Trinker "hat wieder weiBe Măuse gesehen".

(7.125b) Pia sagte: "Otto als Trinker hat wieder weiBe Mäuse gesehen".

(7.125c) (?) Pia sagte: "Otto", als Trinker, "hat wieder weiBe Mause gesehen".

(7.125d) Pia sagte: "Otto", ein Trinker, "hat wieder weiBe Măuse gesehen". Während man das lockere Appositiv (7.125d; eine als Einschaltung markierte Translativphrase erscheint ebenfalls nicht inakzeptabel, wenngleich etwas markiert; $7.125 \mathrm{c}$ ) als vom aktuellen sprecher geăuBert auffast, ist die einzig mögliche Interpretation der unmittelbar angeschlossenen Translativphrase: AuBerung des referierten Sprechers (Pia; 7.125b); die gegenteilige Interpretation ist inakzeptabel (7.125a).

Die Anwendung von Negation und von Gradpartikeln auf die adjazente Translativphrase ist nicht möglich! Die inakzeptablen Resultate und Folgerungen veranschaulichen (7.126-7.129); (7.127) zeigt überdies ein Beispiel dafūr, daß die als-Adverbiale dagegen wie jedes Satzglied der Negation zugänglich ist:

(7.126) Es ist nicht der Fall, daB Pia als Arztin in einer Backerei arbeitet.

[Daraus folgt:] Es ist nicht der Fall, daB Pia in der Funktion einer Arztin dort arbeitet/ $\star d a B$ Pia Xrztin ist.

(7.127) *Nicht Pia als A rztin, sondern Pia als $K r$ a nkenschwester arbeitet in dieser Klinik.

Pia arbeitet nicht als Arztin, sondern als Rrankenschwester (FUNRT) in dieser Klinik.

(7.128) *Nicht $P i$ a als Arztin, sondern $B$ e lga als Arztin arbeitet in dieser Rlinik.

(7.129) *Sogar Pia als A rztin arbeitet dort, nicht nur Pia als Kr a nkenschwester.

* Sogar $P$ i a als Arztin arbeitet dort, nicht nur $B$ e lga als Arztin.

Auch Fragen beeinflussen die identifizierende (subsumierende) Beziehung zwischen Bezugs-NP und Translativphrase nicht (7.130). Gliedteilfragen sind nicht anwendbar (7.131). Die Antwort der gesamten Konstruktion auf eine Satzglied- 
frage ist inakzeptabel, was besonders erstaunt, wenn man (akzeptable) appositive Relativsätze oder lockere Appositionen vergleicht (7.132):

(7.130) Arbeitet Pia als Arztin bei Dr. No? (Nein - als Krankenschwester.) *Ist Pia Arztin?

(7.131) Welche/ Was für eine Pia arbeitet jetzt (als Graphikerin) in diesem Büro? *Pia als A rztin.

(7.132) Wer arbeitet jetzt (als Graphikerin) in diesem Büro? Pia /, die ja eigentlich Arztin ist,/, eigentlich eine Arztin,/ *als Arztin.

Angesichts dieser Verhaltensweisen erscheint die Einstufung als Attribut nicht naheliegend! Doch verhält sich die Translativphrase ebenfalls anders als der Appositionsprototyp! Möglicherweise handelt es sich um eine Konstruktion eigener Art.

Nun möchte ich noch die Anwendung von Satzadverbien und quantifizierenden Ausdrücken streifen:

(7.133) ((?) Vermutlich) Pia als Arztin übernimmt (vermutlich) die Verantwortung.

^Es ist vermutlich der Fall, daB Pia eine Arztin ist.

Es ist vermutlich der Fall, daB Pia die Verantwortung übernimmt, denn sie ist ja Arztin.

(7.134) Pia als eine altere Dame (/, eine altere Dame,) hat dreimal geheiratet.

Pia, die ja eine allere Dame ist, hat dreimal geheiratet.

* Eine altere Dame hat dreimal geheiratet.

In diesen Fällen verhält sich die Konstruktion wie jene mit "lockerem Appositiv"; sie ist aus dem Trägersatz heraus nicht affizierbar und zeigt den Charakter einer sekundären zusatzprådikation. Der Wahrheitswert des satzes wird (wie bei lockeren Appositiven) durch die Translativphrase nicht affiziert.

Translativphrasen lassen sich nicht wie z.B. der "Prototyp" rekursiv erzeugen bzw. koordinieren, sofern als wiederholt wird (7.135). Das N-Komplement des Translativs kann koordinativ erweitert werden (7.136):

(7.135) *Pia als Chirurgin als Oberärztin hätte dies wissen müssen. Pia, Chirurgin, Oberärztin, hätte dies wissen müssen.

(7.136) Pia als Chirurgin, Oberärztin und Freundin des Klinikchefs bätte dies wissen müssen.

Nun zum Verhalten bei pronominalen/anaphorischen Bezügen. Offensichtlich kann man nicht wie beim Prototyp derartige Bezüge auf die als-Phrase vornehmen:

(7.137) Diese Frau als Mathematikgenie verblüfft mit ihren/ *seinen Fähigkeiten alle Professoren.

Diese Frau als Mathematikgenie verblüft alle Professoren. Ihre/ * Seine Fähigkeiten sind wirklich auBerordentlich.

Diese Frau als Mathematikgenie verblüft alle Professoren. Sie/ ${ }^{\star E S}$ rechnet schneller als der Computer. 
In dieser Hinsicht erscheint die als-NP stärker "absorbiert" als eine lockere nominale Apposition.

Als-Phrasen durchlaufen mitsamt ihrer Bezugs-NP die Passivtransformation. Die Distanzstellung in (7.138) wirkt syntaktisch abweichend:

(7.138) Pia als deine Anwältin sollte den Prozeb vertagen.

Von Pia als deiner Anwaltin sollte der ProzeB vertagt werden.

*Von Pia sollte als deiner Anwälin der Prozeß vertagt werden.

Ist die Translativphrase als Dependens zu betrachten? Dafür spricht, daß sie ohne Bezugs-NP nicht auftritt und nicht selten Kongruenzerscheinungen auftreten. Dagegen sprechen das Fehlen von Rektions- und Valenzforderungen. 116 Auch die von Vennemann (1977) als Spezifikationsrelation explizierte Dependenzrelation liegt nicht vor; die Translativphrase ist wohl schwerlich als operator aufzufassen.

Die Evidenz für das Vorliegen von Konstituenz - Bezugs-NP und Translativphrase als eine komplexe Konstituente - ist kontrovers: Die Unmöglichkeit einer Auseinanderstellung der Gruppe (sonst Funktionswechsel!), die Bevorzugung des unmittelbaren Kontakts auch bei Postpositionen, nachgestellten GP etc., die gesamthafte Ersetzbarkeit (Pia als Arztin/ Sie weiB das) sprechen eher dafür, dagegen spricht z.B. die Erfragung:

(7.139) Wer arbeitet in diesem Betrieb? *Kuno als Schlosser. Wer sollte den Prozeß vertagen? ? Pia als deine Anwältin.

Sie scheint nicht resp. kaum möglich zu sein. Zudem legen die Widerspenstigkeit gegen einige syntaktische Operationen (Clefting, Linksverschachtelung) und die Nichtanwendbarkeit von Negationsträgern und Gradpartikeln Nicht-Konstituenz nahe.

Nun erhebt sich die schwierige Frage, wie die "appositionsverdachtigen" adnominalen als-Phrasen einzustufen sind. Die Resultate stellen sich im Oberblick folgenderweise dar:

\section{"als"-Phrasen Attribute Prototyp}

Basis $+X$ im Vorfeld Distanzstellung

Stellung zur Basis stellung relativ

Stellung bzgl. nachgestellter Präp, Konjadv Verhalten bei CLEFT PSEUDOCLEFT ja

Lesartveränderung , Funktionswechsel rechtsadjazent nach ATTR, vor Prototyp davor inakzeptabel inakzeptabel ja manchmal bei kaum AdjA, PA rechtsadjazent rechtsadjaz. vor als-Phrase nach Attr und und Prototyp als-Phrase davor danach

akzeptabel akzeptabel akzeptabel

akzeptabel

116 Es ist fraglich, ob eine als-Phrase jemals eine naminale Valenzstelle abzubinden vermag. 


\begin{tabular}{|c|c|c|c|}
\hline & "als"-Phrasen & Attribute & Prototyp \\
\hline SATZVERSCHRANKUNG & inakzeptabel & akzeptabel & akzeptabel \\
\hline LINRSVERSCHACHTELUNG & inakzeptabel & akzeptabel & akzeptabel \\
\hline Substitution Basis $+x$ & ja & ja & ja \\
\hline Erfragung Basis $+X$ & kaum & ja & $\mathrm{ja} / \mathrm{nein} 117$ \\
\hline Gliedteilfrage nach $X$ & nein & ja & nein \\
\hline Kasuskongruenz & möglich/notwendig & kaum 118 & möglich 119 \\
\hline Anwendung NEG/GP & nein & ja & nein \\
\hline Abtrennung & möglich & z.T. möglich ${ }^{120}$ & notwendig \\
\hline $\begin{array}{l}\text { Umformungsbeziehung } \\
\text { restriktiv möglich }\end{array}$ & $\begin{array}{l}\text { Kopulasatz } \\
\text { nein }\end{array}$ & $\begin{array}{l}\text { diverse sätze } \\
\text { ja }\end{array}$ & $\begin{array}{l}\text { Kopulasatz } \\
\text { nein }\end{array}$ \\
\hline Kategorie $\mathrm{X}$ & NO-N3 & $\begin{array}{l}\text { NP, Adj, PP, } \\
\text { V-E-Satz usw. }\end{array}$ & $\begin{array}{l}\text { NO-N3, Adj, } \\
\text { PP }\end{array}$ \\
\hline Floskeln & keine & keine & übrigens \\
\hline GPn, Sadv, freie ADVB & keine & keine & ja \\
\hline Funktion & $\begin{array}{l}\text { Eigenschaft/ } \\
\text { Klasse/Identifikat }\end{array}$ & diverse & $\begin{array}{l}\text { Klasse/Eigen- } \\
\text { schaft/Ident. }\end{array}$ \\
\hline Spezifikation 121 & nein & ja & nein \\
\hline X weglaßbar & ja & z.T. & ja \\
\hline$X$ vertritt Basis & nie & selten $^{122}$ & selten \\
\hline Sprecherdivergenz & unmöglich & unmöglich & möglich \\
\hline mehrfache Anwendung 123 & nein & ja & ja \\
\hline $\mathrm{X}$ dependent & ?? & ja & ?? \\
\hline Konstituente der Basis & ? & ja & ?? \\
\hline
\end{tabular}

Wie man sieht, weisen die "Translativbeifügungen" etwas mehr Gemeinsamkeiten mit den "lockeren Appositionen" (Funktion, nicht-restriktiv, fragliche Dependenz u. Kongruenz, Kopulasatzparaphrase, NO-N3 möglich, keine NEG/GP-Anwendung, keine Gliedteilfrage) auf als mit attributiven Beifügungen (keine Flos-

117 Von der Kategorie des Appositivs abhăngig.

118 In Frage komen nur die wenigen Typen aus dem Bereich der von mir als Attribut eingestuften "engen Apposition" (bei einem Glas gutem Wein, Karls des Kahlen etc.).

119 Absolut notwendig ist die Kongruenz nur dann, wenn mehr als eine NP als mögliche Basis vorhanden ist (Disambiguienung).

$120 \mathrm{Vgl}$. z.B. Bine Riste (mit Pulvertüten) stand auf dem Dachboden herum. Vgl. Winkler (1969) und Kap. 11.

121 cemeint ist eine operator-operand-Beziehung in Rahmen einer Kategorialgrammatik.

122 Auch hier kommen lediglich wenige "enge Appositionen" in Frage.

123 Dies betrifft den Punkt "Rekursive Brzeugung". 
keln/ GPn/ Sadv/ freie ADVB, Abtrennung nicht notwendig, stellungsverhalten, keine Sprecherdivergenz). Daraus allerdings bereits eine Einstufung als Apposition abzuleiten, erscheint mir vorschnell.

Trotz ähnlicher Semantik/Pragmatik von als-Phrase und "Appositiv" und trotz der attributsuntypischen Nichtanwendbarkeit von Gliedteilfrage, NEG und GP steht m.E. die Translativphrase der Funktion Attribution näher als der Funktion Apposition bzw. Zusatz. Maßgebend für die Einstufung als Attribut ist bei diesem Muster, daß keine Abtrennung vorliegt und daB diese nachträglich vorgenommen werden kann. Die Einschaltungsmarkierung wird in dieser Arbeit als wesentlichster Indikator für nicht-attributive, lediglich "gliedassoziierte" Elemente (Zusätze) und Einschübe angesehen.

Ebenfalls eher für Attribut spricht das Stellungsverhalten (Adjazenz!). Die Translativbeifügungen sind untypische Attribute und daher "am Rande der attributiven Funktion" anzusiedeln. Ich stelle mir das in einer skala der möglichen Bezüge zwischen zwei Spracheinheiten so vor (die syntaktische Fügungsenge nimmt von oben nach unten $a b): 124$

\section{Bezugselement \\ Grundwort \\ Wortgruppenkern}

Satzglied

\section{"bezogenes" Element \\ Bestimmungswort}

Attribute (AdjA, GA, PA etc.)

Translativbeifügung 125

appositiver Relativsatz

Zusătze aller Art (s. Kap. 15)

Schaltsatz mit phorischem Gliedbezug

Folgesatz mit phorischem Gliedbezug
BEZIEHUNG

KOMPOSITION

ATTRIBUTION

ZUSATZ

EINSCHUB

SATZFOLGE

Zusatz während der Oberarbeitung: Man kann daran zweifeln, daB die "appositionsverdächtigen" als-Phrasen als (wenn auch untypische) Attribute fungierten. Zwei Alternativlösungen, die von zukünftigen Untersuchungen in Betracht gezogen werden kōnnten, stelle ich mir so vor: (A) die "attributive" als-Phrase labt sich von der als-Adverbiale ableiten und besitzt eine für adverbiale Glieder ungewöhnliche stellungsoption (mit einer NP im Vorfeld), die zu einer leichten Veränderung ihrer Bedeutung führt. (B) Nach Engel (1988: 628 ff.) sind unsere distanten als-Phrasen dislozierbare Satelliten des Nomens bzw. Adjunkte (er rechnet übrigens auch die prädikativen Attribute hierzu), die "ein Merkmal neu einführen", wogegen Attribute "dieses Merkmal als bekannt, als voraussetzend ausweisen".

$124 \mathrm{Zu}$ "Tügungsenge" Lehmann (1984: $207 \mathrm{ff}$.). Die Fügungsenge läßt sich mithilfe von Ausdruckskorrelaten wie Stellung (adjazent - distant), Fügungsmittel (Kasus - Lexeme) etc. explizieren.

125 vielleicht ist als Bezugselement auch "Satzglied" anzunehmen; da die "typischen" Translativphrasenbeispiele nicht bei av(-Phrasen) auftreten, sondern bei Eigennamen, Pronamina, Stoffnamen etc., erscheint es mir unsicher, ob sie sich - wie Attribute - auf den Wortgruppenkern beziehen. 


\section{SATZGLIBDER UND PARTIRELFUNRTIONBN}

Satzglieder und Partikeln werden zusammen abgehandelt, obwohl sie keinesfalls in eins gesetzt werden sollten, da Partikeln sich überwiegend nichtsatzgliedhaft verhalten; dennoch zăhlen sie zum "Grundbestand" des Satzes, auch wenn ihr Auftreten nie durch Valenz, Rektion oder andere Abhängigkeitsverhältnisse notwendig wird, sondern durch semantisch-pragmatische Faktoren.

\subsection{Satzglieder}

Satzglieder sind Ergănzungen und Angaben zum Verb bzw. Angaben zum Satz, die (a) vorfeldfähig, (b) gesamt ersetzbar und (c) mittels Satzgliedfragen (wer? wo? wie? usw.) erfragt werden können. ${ }^{1}$ sie stellen semantisch Entităten, die in den vom Satz zum Ausdruck gebrachten Sachverhalt involviert sind, oder situierungen des Sachverhaltes (zeitlich, lokal, kausal etc.) dar. Satzglieder können als Teile des Sachverhaltes kontrastierend negiert werden, wobei sprachlich in einer sondern-Phrase spezifiziert werden kann, was anstelle des Verneinten anzusetzen ist, damit einem Satz der Wahrheitswert "wahr" zukommt:

(8.1) Nicht den Hund liebt er, sondern den Affen (AKKO).

Nicht wegen des Regens kam sie, sondern trotz des Regens (KAUS). Satzglieder können von einer Gradpartikel betroffen werden und den Gradpartikelfokus auf sich ziehen:

(8.2) Sogar dem 0 pa gefiel die Show (, nicht nur der Oma; DATO).

Nur am $S$ o nntag war es schön (, nicht unter der Woche; TEMP). Verglichen mit den durch das Einschaltungsmuster "abgetrennten" Hospitanten sind Satzglieder weder intonatorisch noch graphematisch besonders markiert. Gerade das Einschaltungsmuster bewirkt ja eine "Herausnahme" bzw. "Einschaltung" eines Elements in einen Satz. ${ }^{2}$ (8.3a) zeigt die Unmöglichkeit kontrastierender Negation bei einer "lokalen Angabe mit Einschaltungsmarkierung", (8.3b) demonstriert, das zwar Angaben, nicht jedoch valenznotwendige Ergänzungen (Teile des Minimalsatzes; vgl. Kap. 5.5) "abgetrennt" werden dürfen:

(8.3a) Pia hat nicht in der Wanne gesungen, sondern in der Küche. * Pia hat nicht, in der Wanne, gesungen, sondern in der Küche.

1 Satzadverbiale wie wahrscheinlich, glücklicherweise usw. bilden eine gewisse Ausnahme; sie können durch Entscheidungsfragen erfragt werden. Die Erfragung mit einer Satzgliedfrage kann in einigen wenigen Fälen entweder sehr umständlich oder gar ummöglich sein:

(F8.1) Kumo öffnete die Band, so daß die Kirschen zu Boden fielen (mit welcher Folge? KONSEX).

(F8.2) Pia ging trotz des Sturmes (Prage?? Karz) in den Carten.

(F8.3) Chne Reve (Frade?? RarITMTTV?) verschlang Pia das Grras.

2 Folglich können z.B. obligatorische Funktionen wie Subjekt, Objekt, obligatorische Adverbiale etc. keine Einschaltungsmarkierung amehmen, weil der Satz sanst defizient wird. 
(8.3b) Pia hat wieder, mit Rechenschiebern (INSTR), gerechnet.

*Pia hat wieder, mit dem Allerschlimmsten (PO), gerechnet.

Satzglieder können einen rhematischen Akzent (Satzakzent) auf sich ziehen, wenn sie als Information hervorgehoben werden sollen (bei Satzadverbialen allerdings kaum möglich) $i^{3}$ solcher Akzentuierung sind "Einschaltungen" prinzipiell nicht zugänglich - es sei denn, sie sind selbst satzformatig und weisen eine eigenständige Thema-Rhema-Gliederung auf.4

Nach diesen Explikationen von "Satzglied" kann man aus dem Bereich appositionsverdăchtiger Konstruktionen folgende Muster ausgliedern:

- 3.6.1.2. nicht als Einschaltung markierter Adverbialsatz;

- 5.1. als-Adverbialphrasen mit "Konnexion zu einem Bezugselement (SBJ, OBJ)", sofern sie nicht mit dem Bezugselement zusammen im Vorfeld stehen. ${ }^{5}$

- 6. alle prädikativen Attribute.

\subsubsection{Die adverbialen Gliedsātze}

Adverbiale Gliedsätze können m.E. in bestimmten Positionen, vor allem im Satzmittelfeld, als Satzglied oder aber als Einschaltung interpretiert werden (s. auch Kap. 11). In der Schriftsprache kann man nur dann genau $z$ wischen einem "eingeschalteten Adverbialsatz" (er kann u.a. nicht erfragt werden und ist vom "Gastsatz" aus weder negierbar noch von einer Gradpartikel in einem Matrixsatz affizierbar; er ist somit nicht satzgliedhaft) und einem Gliedsatz, der als Adverbial fungiert, unterscheiden, wenn typische einschaltungsmarkierende Satzzeichen - Gedankenstriche oder Rundklammern - dies anzeigen (8.4a); Kommata lassen beide Interpretationsmöglichkeiten offen (8.4b):

(8.4a) Sie ging nicht - als es klingelte - zur Tür (*, sondern als es klopfte; PAR).

sie ging nicht (als es klingelte) zur Tür (*, sondern als es klopfte; PAR).

(8.4b) Sie ging nicht, als es klingelte, zur Tür (, sondern als es klopfte) (ADVB).

3 Zur Zugänglichkeit der Satzglieder für Akzente s. Heidolph u.a. (1981: 741 ff., 866 ff.).

4 Der Unzulänglichkeit meiner Aussagen bin ich mir bernußt, daher sind sie höchstens als "Taustregeln" zu nehmen. Eine genaue Explikation von "Satzakzent", "rhematischer Akzent", "Thema-RhemaGliederung" sorie der Satzintonationsverhältnisse muß aus Platzgründen unterbleiben. Weiterführende Literatur: Bierwisch (1966), Heidolph u.a. (1981: 866 ff.) und Höhle (1982).

5 Vgl. Kap. 7.B. Als Appositionskandidaten interessieren nur diese, und zwar sowohl die Kontaktals auch die Distanzvarianten. Einen tberblick über die syntaktischen Möglichkeiten von als-Phrasen bieten Helbig (1984a), Pütz (1988: 195 ff.). Zu weiteren, z.T. "appositionsverdăchtigen" alsPhrasen s. Kap. 7, insb. 7.B. 
Sie ging nicht, als es klingelte, zur Tür (*, sondern als es klopf-

te) (PAR).

In gesprochener Sprache besteht ein merklicher Unterschied, je nachdem, ob ein adverbiales Satzglied ( $8.5 \mathrm{a})$ mit enklitischer Intonation oder ein Einschub mit Parentheseintonation $(8.5 \mathrm{~b}$; er bleibt "appositionsverdächtig", weiteres Kap. 11.) vorliegt: 6

(8.5a) /sie ging nicht als es klingelte zur tür p+ sondern als es klopfte $\mathrm{f}+\mathrm{t}$.

(8.5b) */sie ging nicht $\mathrm{p}+$ als es klingelte $\mathrm{p}+$ zur tür $\mathrm{p}+$ sondern als es slopfte $\mathrm{ft+l}$.

Als Satzglied kann der Adverbialsatz im Satzvorfeld, extraponiert und im Satznittelfeld vorkomen, wobei nur die Mittelfeldvariante und die Extraposition (Satzglied? Nachtrag?) mehrdeutig sein kann. ${ }^{7}$ Im Vorfeld ist ein adverbialer Gliedsatz stets Satzglied, nie Einschaltung! Einschaltungen können Felder eines Satzes nicht allein belegen:

(8.6a) Als es klingelte, ging sie zur Tür.

* - Als es klingelte - ging sie zur Tür.

(8.6b) Pia, das wubte jeder/ glücklicherweise, meisterte das.

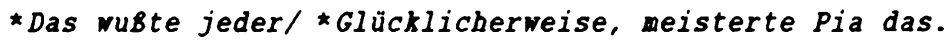

Es ist fraglich, ob Einschaltungen überhaupt sinnvoll einem Satzfeld zugerechnet verden können oder ob sie nicht besser als auBerhalb der Satzfelder stehend anzusehen sind.

\subsubsection{Adverbiale als-Phrasen}

Sie sind am ehesten als Adverbiale mit besonderen Bezugsverhăltnissen zu erklăren; man beachte die Unterschiede zu den Beispielen mit "attributartigen" als-Phrasen, die zusammen mit ihrem Bezugselement im Satzvorfeld stehen, und bei Dislozierung entweder eine andere Bedeutung annehnen oder aber nicht mehr in den Satz passen $(8.7 \mathrm{~b}):^{8}$

6 Hier vie im witeren Verlauf dieses Rapitels bedeutet "adverbial" nicht 'auf das Verb bezogen', sandern 'die syntaktische Funtion Adverbial ausibend'.

7 An dieser Stelle ist darauf hinzumeisen, daß man alle Gliedsătze in Kittelfeld als Parenthesen werten könnte. Vgl. 2.B. Altmann (1981: 168):

"Doch ist vermutlich die Mittelfeldposition aller Gliedsătze im Deutschen inakzeptabel. Soweit Gliedsătze in Yittelfeld auftreten, kam es sich mur um nicht-obligatorische Funktionen bandeln; das spricht dafür, daß diese Verb-Letzt-Sätze parenthetisch eingefügt sind".

$8 \mathrm{zu}$ den hier interessierenden als-Phrasen vor allem Belbig (1984a) und Pütz (1988); allgemein zu den als-Phrasen in diversen syntaktischen Funktionen Rolde (1971), Smith (1977), Heidolph (1979), Pütz (1982) und Belbig (1984c). 
(8.7a) Kuno arbeitet als Schlosser ('in der Funktion eines Schlossers'/ * 'der ja Schlosser ist') in diesem Betrieb.

(8.7b) Kuno als Elektriker ( 'in der Funktion eines Elektrikers'\% 'der ja Elektriker ist') arbeitet als Schlosser in diesem Betrieb.

* Kuno arbeitet als Elektriker seit heute als Schlosser in dem Betrieb.

(8.7c) (Nicht) Kuno als Schlosser arbeitet (nicht) in diesem Betrieb. * Nicht Kuno als Schlosser arbeitet hier, sondern Kuno als Koch.

(8.7d) Runo arbeitet nicht als Schlosser in diesem Betrieb, sondern als Koch.

Kuno arbeitet nur als Schlosser hier, nicht auch als Koch.

(8.7e) Kuno arbeitet als Schlosser nicht in diesem Betrieb.

(8.7f) Sogar Kuno als Schlosser arbeitet in diesem Betrieb (*, nicht nur Kuno als Installateur).

Sogar als Schlosser arbeitet Kuno in diesem Betrieb (, nicht nur als Installateur).

Die Kontaktvariante $(8.7 \mathrm{~b}, 8.7 \mathrm{c}, 8.7 \mathrm{f})$ mit gemeinsamer Vorfeldposition spezifiziert eine dauernde, wesentliche Eigenschaft des subjektreferenten, was durch eine Kopulasatz-Paraphrase verdeutlicht werden kann; dabei ist die alsPhrase weder einer Negation noch einer Gradpartikelanwendung zugänglich.

Die Distanzvarianten ( $8.7 a, d, e, f)$ bringen eine temporäre, akzidentelle Eigenschaft bzw. Funktion des subjektsreferenten zum Ausdruck und sind sowohl der Negation als auch der Gradpartikelanwendung zugănglich; sie sind eher umständlich erfragbar und ersetzbar (Fragen: in welcher Funktion? als was? ProForm: ?als solcher). Zudem weisen die adverbialen Translativphrasen ein satzgliedtypisches stellungsverhalten auf.

Die Rontakt- resp. Exklusionsprobe in (8.7b) erweist, das Kontakt- und Distanzvariante gemeinsam in einem Satz aufzutreten vermögen (schon dies spricht eher für zwei unterschiedliche Funktionen), wobei die Dislozierung der adjazenten als-Phrase - so daB sie ebenfalls als eine Art Satzglied und nicht mehr "attributiv" fungierte - zu inakzeptablen Resultaten füht; sie ist im Ggs. zur adverbialen Phrase auf die Kontaktstellung beschrankt.

Die adverbiale als-Phrase ist als freie Angabe anzusehen, da sie augenscheinlich nicht vom Satzverb valenzgefordert ist. In die traditionell etablierten Adverbialklassen (kausal, lokal, modal, temporal) ist sie jedoch schwer einzupassen. Naheliegend scheint eine Einstufung als Modaladverbial, auch wenn das entsprechende Erfragungs- und Ersetzungsverhalten untypisch ist (Wie arbeitet Kuno in diesem Betrieb? FleiBig/ ?? Als Schlosser. Kuno arbeitet als Schlosser/??so in diesem Betrieb).

Auch hinsichtlich der Bedeutung unterscheidet sich die als-Phrase von Modaladverbialen, die eine Eigenschaft einer Handlung/ eines Vorgangs etc. angeben. Andererseits labt sich eine für Modaladverbiale typische Erscheinung beobachten: Es gibt Vollverben, welche die Bedeutung des Komplexes "Modaladver- 
bial + Vollverb" zusammenfassen (vgl. 8.8a; es ist aber fraglich, ob wirklich adáquate Paraphrasen vorliegen):

(8.8a) Pia lief schnell/ rannte nach Hause.

Pia rief laut/ schrie nach ihrem Hund.

(8.8b) Pia arbeitet als Schneiderin/ ?schneidert in diesem Betrieb. Kuno arbeitete als Moderator in dieser Sendung/ ?moderierte diese Sendung.

\subsubsection{Die prădikativen Attribute}

Sie verhalten sich bei einer Satzgliedfrage oder bei der Ersetzung durch eine Pro-Form ähnlich wie Modaladverbiale: ${ }^{9}$

(8.9a) Pia lief schnel1 (MOD)/ wütend (PATTR) nach Hause.

(8.9b) Wie lief Pia nach Hause? Schnell/Wütend.

Pia lief wütend/ schnell/ so nach Hause.

Wie die verbbezogenen Modaladverbiale (8.10a) - und anders als satzbezogene Angaben wie z.B. Temporal- und Kausaladverbiale (8.10b) - sind prädikative Attribute bei "Normalbetonung" kaum als erstes Satzglied eines Verbend-Satzes möglich (8.10c):

(8.10a) Pia sah, daB (*schnell) Kuno (schnell) den $R$ o twein entkorkte. Pia weiB, daB (??als Schlosser) Kuno (als Schlosser) in $d$ i esem Betrieb arbeitet.

(8.10b) Pia sah, daB um elf Uhr ein Kerl ins $Z$ i mmer spahte. Kuno abnte, daß wegen des Regens der Platzwart das E ndspiel verlegte.

(8.10c) Kuno sah, daB (*wütend) Pia (wütend) nach H a use lief. Die prädikativen Attribute können sowohl von einer kontrastierenden Negation als auch von Gradpartikeln betroffen werden.

Im Ggs. zu den Modaladverbialen im engeren Sinn (wie laut (rufen), schnell (fahren); der Terminus Modaladverbial ist keineswegs völlig klar, nicht selten werden auch instrumentale, restriktive und komitative Adverbiale hinzugerechnet), die das Prädikat modifizieren, indem sie dessen Intension anreichern und dessen Extension einschränken ('Eigenschaft einer Handlung/eines Vorgangs etc.'), drücken prädikative Attribute temporäre Eigenschaften der subjektbzw. Objektreferenten aus, die diesen zukommen, solange die Prädikation des Satzes gilt.

9 Auch z.B. Eisenberg (1986: $208 \mathrm{ff}$.) behandelt die prädikativen Attribute unter "Adjektive als Adverbiale zum Verb" zusammen mit den typischen Modaladverbialen. 


\subsection{Partikelfunktionen}

Partikeln verhalten sich in vielerlei Hinsicht (z.B. Erfragung, Substitution, Vorfeldfähigkeit, Negierbarkeit) nicht wie Satzglieder. Ihre Stellungsmöglichkeiten sind - verglichen mit typischen Satzgliedern - eingeschrănkt. Modalpartikeln stehen i.d.R. im Mittelfeld nach thematischen und vor rhematischen Satzgliedern, jedoch weder im Vor- noch im Nachfeld. Wenn die Negationspartikel nicht oder eine Gradpartikel ein Satzglied affiziert (fokussiert), dann muß sie - sehr grob gesprochen - in der Nähe ihres Bezugselements verbleiben. "Herausstellungen" von Partikeln (Satzglieder sind i.d.R. "herausstellbar") sind nicht möglich. 10

Partikeln können nie eingeschaltet erscheinen, ebensowenig wie obligatorische syntaktische Funktionen (SBJ, OBJ etc.), da sie am Sachverhaltsmodell, das der Satz zum Ausdruck bringt, und am Wahrheitswert des Satzes z.T. direkt beteiligt sind (die Negation und z.T. Gradpartikeln; die Modalpartikeln weniger). Durch die Einschaltungsmarkierung wird die "Einwirkung" der Partikeln auf den Bezugssatz resp. ihr Bezugssatzglied verhindert (ihr skopus wird gewissermaßen absorbiert). Sie gehören mithin zwar nicht zum (vornehmlich valenzkonstituierten) Minimalsatz, doch bestimmt zum Elementarsatz.

Partikeln fungieren als (a) Wahrheitswertveränderer (Negationspartikel nicht), als (b) Quantifizierer (Gradpartikeln, z.B. nur, auch) oder Skalenbilder (z.B. die Gradpartikel sogar) in bezug auf die betroffenen Phrasen(teile) und (c) als "Abtöner" der Satzillokution (Modalpartikeln). 11

Obwohl die Partikeln keine Satzglieder darstellen, müssen sie dennoch als elementare Glieder des Satzes - aber eben nicht als Satzglieder - angesehen werden und nicht als Einschaltungen, Einstreusel oder "Würzwörter". DaB Gradpartikeln und Negationselemente in bestimmten Verwendungsweisen keine Attribute zu einer Phrase bzw. einem Wort darstellen, hat Jacobs (1982; 1983) nachgewiesen:

(8.11a) Nicht/ Sogar Pia ärgerte Udo.

(8.11b) Udo wurde (nicht/ sogar) von (*nicht/*sogar) Pia geärgert. Falls Negations- oder Gradpartikeln als Attribute der Vorfeld-NP fungierten, müBten sie dies auch in Umgebungen tun, in denen sie unzweifelhaft der NP attributiv zugehören: nämlich als Komplemente einer Präposition in einer PP. Diese Beispiele sind jedoch ungrammatisch (8.11b).

Die Partikeln scheinen mithin weder Gliedteile noch Satzglieder darzustellen; sie stellen folglich eine dritte elementare Funktionsgrobklasse im fol-

$10 \mathrm{Vgl}$. Altmann (1981: 340; Obersicht).

11 Genaueres zur Partikelsyntax und -semantik in: Altmann (1976; 1978), Jacobs (1982; 1983), Weydt (1977), 1983) und Hentschel (1986). 
genden Rahmen dar: Satzgliedfunktionen ${ }^{12}$ - Partikelfunktionen ${ }^{13}$ - Gliedteilfunktionen. Somit entfällt die Notwendigkeit der Annahme, daß Partikeln (speziell Gradpartikeln) zu den von ihnen fokussierten Konstituenten in einer appositiven Beziehung stünden. Erben (1972: 175) spricht bei einer Konstruktion wie

(8.12) Die Wissenschaft allein (nur/ bloB/ gerade) kann (...) Antwort geben.

von einer "appositiven Nachstellung" der Beiwörter [der Gradpartikeln; W.S.] Abgezielt ist of fensichtlich hierbei auf das Verhälnis "Juxtaposition nach dem Bezugselement" und die gemeinsame Vorfeldposition. Zudem spielt es vermutlich eine Rolle, daB zwischen Partikel und Bezugselement weder eine subordinative noch eine koordinative Beziehung auszumachen ist, so das "appositiv" als Terminus gewählt wird, um die besondere Beziehung zwischen beiden Einheiten zu benennen (vgl. Kap. 4). Es genũgt m.E. allerdings, wenn man mit den von Jacobs (1982, 1983) verwendeten Relationen - syntaktischer Bereich, semantischer Bereich (Skopus) und Fokussierung - die Verhăltnisse beschreibt. Man spricht dann z.B. davon, das der syntaktische Bereich einer Partikel eine verbalphrase ist, daB ihr Skopus den Gesamtsatz umfaBt oder daB sie ein Adjektiv (Modaladverbial) fokussiert usf.

12 Nebenbei bemerkt: Wenn die (Grad)Partikeln nicht zu den Satzgliedern zăhlen, dann darf man bei der Konstellation "Partikel + (Bezugs) Satzglied im Vorfeld" die (problematische) Annahme fallenlassen, es handle sich um zwei Satzglieder im Vorfeld (Stichwort: "doppelte Vorfeldbesetzung") und Verbdrittstellung. Partikeln sind seltenst vorfeldfähig (Beispiele wie Udo hatte die beiden Haupteinwande gut bedacht; nicht hat er jedoch den Einwand des opas bedacht, sie kommen ohnehin kaum vor, scheinen mir keine systematische standardsprachliche option darzustellen). Im Deutschen existiert m.E. genau ein Fall doppelter Vorfeldbesetzung (Aus meiter Ferne her mit müden Schwingen kam Musik geflogen), alle weiteren verdächtigen uster kömen nit Begriffen wie Parenthese, Her- . ausstellung u.a.m. erklärt werden (dazu ausführlich Kap. 9).

13 Is sei kurz darauf hingevriesen, daß die Kategorie/Funktion NJGATIor hier recht eng angesetzt wird, also eigentlich nur die Partikel nicht betrifft. Andere "Negationswörter" haben andere Funktionen; so ist keineswegs ein Satzadverbial (Antwort auf Entscheidungsfrage!), niemand eine NP resp. ein Indefinitpronomen (als SBJ, OBJ) usw. Genaueres hierzu Jacobs (1982; Obersicht 226 f.). Zu den möglichen Funktionen van Gradpartikeln s. Jacobs (1983: 122 f.). 


\section{MEHRFACHE VORFELDBESETZUNG}

Die doppelte bzw. mehrfache Vorfeldbesetzung stellt trotz etlicher Veröffentlichungen zum Thema eine noch nicht hinreichend beschriebene syntaktische Erscheinung dar. ${ }^{1}$ In (9.1) wird das (vermeintlich) vorfeldbesetzende Material durch Unterstreichung hervorgehoben (Funktionsangaben in folgenden von W.S.): ${ }^{2}$

(9.1a) Unten (LOK) in der Stadt (LOK) ['im Tal in der Stadt'] stant es.

In der Stadt (LOK) unten (LOK) ['in der Stadt in ihrem unteren Teil'] stank es.

(9.1b) Sogar bei uns hinten in Keller sind Mäuse.

(9.1c) In Zentrum (LOK) auf der Kreuzumg (LOK) ereignete sich der unfall.

(9.1d) Aus unbestimmter Ferne her (DIR) mit miden Schwingen (10D) kam Musik geflogen.

(9.1e) Oben (LOK), im Schrank (LOK), ist es feucht.

Im Zentrum (LOK), auf der Kreuzung (LOK), stank es.

(9.1f) Von dort aus (LOK), von jener pinute an (TMP), liefen wir curch unbelebte Straben.

(9.1g) Mein Talent (SBJ), und darin bin ich genial (SATZ), ist die Bosbeit.

(9.1h) Nach einer Viertelstumde (TDYP) - Winter schluchzte noch immer (SATZ) - war er wieder auf der Bricke.

Zwei Positionen markieren die Pole, zwischen denen sich Analysevorschlăge bewegen: Einerseits das topologische "Axion", das vor einem Finitun in zweitstellung nur ein Satzglied stehen kann; ${ }^{3}$ andererseits die Meinung, das diese einfache Regel, deren heuristischer Hert bei der Satzgliedanalyse dadurch keinesfalls geschmălert wird, mindestens zu modifizieren, wenn nicht gar umuformulieren ist. "Beim Phănomen "mehrfache Vorfeldbesetzung" dreht es sich u.a. um folgende Fragen: (a) Können im Vorfeld eines Verb-zweit-Satzes mehrere Satzglieder stehen? Ist die Regel "ein Satzglied pro Vorfeld" korrekturbedürftig? (b) Ist der Vorfeldbegriff zu modifizieren (Unterfelder etc.)? (c) Kann ein Finitum auch in Dritt- oder Viertstellung vorkommen? (d) Kenn die Regel "ein Satzglied pro Vorfeld" stimmt, wie ist dann die Beziehung zwischen nehreren Vorfeldelementen aufzufassen: attributiv, appositiv oder parenthetischer Einschub eines Gliedes?

Gerade Frage (d) berührt die Appositionsproblematik, denn es erscheint zunăchst naheliegend, die Vorfeldregel (und die Verbzweitstellung) durch die Annahme besonderer Beziehungen zwischen den Vorfeldelementen zu "verteidigen".

1 Literatur (Auswahl): Steinitz (1969); Brinker (1972); Droop (1977); van de Velde (1978); Lee (1979); Lehmus (1983); Mmderlich (1984) und Lulhr (1985).

Anmerkung: Kap. 9 murde während der Oberarbeitung stark gekürzt. Moglicherweise wird eine ausfuhrliche Fassung irgendwann als Aufsatz exscheinen.

2 (9.1a) angelehnt an Droop (1977: $46 \mathrm{ff.}$ ), (9.1b) an Thmderlich (1984: $79 \mathrm{f.}$ ), (9.1c) an Droop (1977: 40), (9.1d) an van de Velde (1978: 136), (9.1e) an thunderlich (1984: 83) und Droop (1977: 40), (9.1f) an van de Velde (1978: 137), (9.1g, h) an ebd. 133.

3 Z.B. Schulz/Griesbach (1982: 393); Engel (1988: 310).

4 S. z.B. Lee (1979); Luthr (1985). 
Von derartigen Strukturen wurde in der Forschung bislang vor allem die Häufung mehrerer Adverbiale im Vorfeld als mögliche appositive Konstruktion untersucht $(9.1 \mathrm{a}-\mathrm{f})$. Satzförmige Einschübe ohne Bezug zum vorigen Element sind dagegen wohl generell als Parenthesen anzusehen (9.1g, h; s. Kap. 11).

Die Vorschläge von seiten der Spezialliteratur (s. Fußnote 1) lassen sich folgendermaßen zusammenfassen: Steinitz (1969: $47 \mathrm{ff.}$ ) wertet Muster wie in BerIin am Alexanderplatz und am Bahnhof, im Kosmetiksalon, als appositive Adverbiale, wobei (a) zwei Adverbiale gleicher subklasse vorliegen müssen, (b) die zweite PP der ersten subordiniert sei, 5 (c) eine (transformationelle) Herleitung weder aus Relativsătzen noch aus parenthetischen Kopulasătzen möglich sei, (d) jedes Adverbial für sich die gemeinsame Satzgliedstelle auch allein ausfüllen könnte und (e) beide auf denselben Sachverhalt referierten - innerhalb desselben möglicherweise auf verschiedene "Gegenstănde", sofern zwischen diesen eine "Enthaltensein-Relation" bestünde.

Nach Brinker (1972: $130 \mathrm{ff.}$ ) bestehen drei Möglichkeiten des Verhăltnisses zwischen zwei Adverbialen, wobei (9.2c) den Fall repräsentiert, in dem beide Adverbiale nicht gemeinsam ins Vorfeld rücken kőnnen (s. dazu 9.11a, b!):

(9.2a) In der Toilettenanlage, (in) einem fensterlosen, ungelüfteten Raum, rasiere ich mich.

(9.2b) In der Toilettenanlage im Südbahnhof rasierte er sich. Das Kind lehnte hinter der Frau am stuhl.

(9.2c) Sie saßen am Vormittag im Garten. *Am Vormittag im Garten saBen sie.

Apposition (9.2a) Zusamenrichng (9.2b) Satzglied (9.2c)

Substituierbarkeit: nur geschlossen Permutierbarkeit: geschl. + getrennt Satzglied: Semantik:

Abtrennung: ein Satzglied Referenzidentität

durch besondere Zeichen geschlossen + getrennt geschl. + getrennt zwei Satzglieder Enthaltensein; Teil-von; Relation der Nähe. keine nur getrennt geschl. + getrennt zwei Satzglieder unterschiedliche semantische Rlassen keine

Droop (1977: $40 \mathrm{ff}$. ) bezeichnet Konstruktionen wie

(9.3a) Im Zentrum auf der Kreuzung ereignete sich der Unfall.

(9.3b) Unten in der Stadt/ In der Stadt unten stank es.

als "appositive adverbiale Romplexe". Beide Komplexteile können (allein) die gleiche syntaktische Position einnehmen und müssen der gleichen Adverbialklas-

5 Die "Subordination" - typisch für attributive Muster - widerspricht eigentlich dem "appositiven" Verhältnis in seiner traditionellen Fassung als gleichberechtigte Nebenordnung (oder Juxtaposition) zweier Spracheinheiten. 
se angehören. Eine Relativsatzparaphrase wie bei der attributiven Relation (z.B. Der Zug aus Köln/, der aus Köln kommt, - vgl. Steinitz 1969!) ist nicht möglich. Die semantischen Relationen zwischen appositionellen Konstruktionsteilen sind nach Droop (1977: 60 et pass.) zum einen Charakterisierung (Identităt (9.4a) und Inklusion (9.4b)), zum anderen Fixierung einer kleineren Einheit innerhalb einer größeren (9.4c):

(9.4a) Nach Paris, (in) die schöne stadt an der Seine, zog er.

(9.4b) In GroBbritannien, in England, Schottland, Nales und Nordirland, wurde der EG-Beitritt begrüBt.

(9.4c) In der Küche auf dem Tisch liegt noch Brot.

Bei van de Velde (1978) seien folgende Fălle "mehrfacher Vorfeldbesetzung" herausgegriffen: 6 (a) "Zwischen das eigentliche Vorfeldelement und das Finitum wird ein Element eingeschoben. Es ist (...) nach der Form der Parenthese zu unterscheiden" (ebd. 133; Beispiele $133 \mathrm{f.}$ ):

(9.5a) Und manchmal (FREQ), plötzlich (PKT), zieht ein jäher Sog von Luft durch die zimmer (...).

(9.5b) Nach einer Viertelstunde (PKT) - Winter schluchzte noch immer (SCHALTSATZ) - war er wieder auf der Brücke.

(9.5c) Mein Talent (SBJ), und darin bin ich genial (SCHALTSATZ), ist die Bosheit.

(9.5d) Und dieser (SBJ), nachdem er meine Arbeiten studiert hatte (ADVBSATZ als SCHALTSATZ?), sicherte mir zu, daB (...).

In (9.5a) nimmt er eine nichtverbale Parenthese an, in $(9.5 b, c)$ Vollsătze, wobei in (9.5c) vermutlich ein nebengeordneter (Folge)Satz in sein "Antezedens" hineingerückt wird. (9.5d), ein eingeschobener Nebensatz, kann mit Relativsatzkonstruktionen verglichen werden. Diesen Beispielen ist gemeinsam, daB die Funktion der vorfeldbesetzenden Glieder nicht gleich, sondern nur ähnlich oder gar sehr verschieden ist; zudem sind sie graphematisch (Kommata) voneinander abgesetzt. Die Einordnung als Parenthese ist ein neuer Aspekt der Erklärung (s. auch Kap. 11).

(b) Als "Kombination von Elementen" bezeichnet van de Velde (ebd. 134) folgende Beispiele:

(9.6a) Am Sonnabendmorgen (TEMP) im frühesten Zug (LOK? TEMP?) war er unausgeschlafen $(\ldots)$.

(9.6b) Am Nachmittag (PKT) in einem Gang zwischen Ladenhäusern (PKT? LOK?) lieb B. sich das Flugticket geben (...).

Er wertet diese Erscheinung jedoch nicht als attributive bzw. appositive Konstruktion, sondern als zwei Satzglieder im Vorfeld (ebd. 135); die gemeinsame Vorfeldbesetzung bewirke zwar eine engere Beziehung der Glieder aufeinander

6 Bei van de Velde erörterte Fälle mit Konjunktionaladverbien (Jedenfalls: Berr X war zufrieden/ Herr $X$, jedenfalls, war zufrieden) bleiben hier ausgeklammert. Zur Syntax und Semantik derartiger "Satzkonnektoren" (Konjunktionaladverbien) s. Thim-Mabrey (1985). Thenfalls ausgespart bleiben die Fälle mit LnNSvirssizung (Aber Celestina, die gibt nichts mehr her, ebd. $132 \mathrm{f}$.). 
als gemeinsame Mittelfeldposition, im Sinne einerseits eines Satzbezugs beider Glieder und andererseits einer besonderen Beziehung des zweiten VF-Elements zum ersten - jedoch läßt van de Velde die Frage offen, ob beide Einheiten im Vorfeld eine Konstituente bildeten.

(c) Nicht von Rombination, sondern von "Reihung" von Elementen im Vorfeld spricht van de Velde (ebd. 136) bei (9.7). Zwischen den "gereihten" Elementen besteht nicht der inhaltliche Zusammenhang, den die "kombinierten" zeigen: Kombination liegt vor bei gleichen (ăhnlichen) Adverbialfunktionen, Reihung bei ungleichen. Im Ggs. zu den oben erwăhnten Parenthesen handelt es sich hierbei um zwei gleichwertige Vorfeldelemente:

(9.7a) Aus unbestimmter Ferne her (DIR) mit müden Schwingen (MOD) kam Musik geflogen.

(9.7b) Von dort aus (DIR), von jener Minute an (PRT? DUR?), liefen wir durch unbelebte straBen.

Die Arbeit von Lee (1979) argumentiert mit dem Permutationskriterium: Sofern ein Komplex mehrerer Einheiten im Vorfeld sich getrennt ins Mittelfeld permutieren läst, liegen sowohl mehrere satzglieder als auch besondere Verbstellungstypen vor, und $z$ war Verb-Dritt $(9.8 \mathrm{a})$, Verb-Viert $(9.8 \mathrm{~b})$ und Verb-Fünt (9.8c; Beispiele Lee 1979: 124, 129, 135, 147):

(9.8a) Und plötzlich (TEMP), dicht ror ihn (LOK), entstand ein stolpern, Rutschen und stūrzen.

Reinhard (SBJ), nachdem er alles erfahren hatte (TEMP), stand auf (...).

(9.8b) Das Bild (SBJ), in der Tat (SADV), je langer sie es ansah (DUR), hatte eine auffallende Ahnlichkeit mit ihm.

(9.8c) Und damit (MOD), ehe ich noch recht begriffen, was sie sagt (TEMP), auf dem Platz (LOR), vor Erstaunen sprachlos (PATTR), labt sie mich stehen.

Lee wertet "mehrfache Vorfeldbesetzung" als eigenes topologisches Muster und er nimmt Verbstellungstypen an, die über Erst-, Zweit- und Endstellung hinausgehen. Somit ist bei ihm veder eine Vorfeldregel im Sinne von "enthălt genau ein Satzglied" vonnoten, noch mus er diskutieren, ob mehrere Glieder im Vorfeld in besonderer (z.B. appositiver) Beziehung zueinander stünden.

Ursula Lehmus (1983; vgl. $147 \mathrm{ff.}$ ) wertet Adverbialbestimmungen gleicher Subklasse im Vorfeld als "zusammenrückbare Adverbiale", sofern sie untereinander permutierbar (9.9a, b), getrennt im Satz verschiebbar (9.9c) und getrennt weglasbar (9.9d) sind:

(9.9a) Unter der Stiege (, und zwar) zwischen den Krampen und Schaufeln (,). hatte die Katze gekauert.

(9.9b) Zwischen den Krampen und Schaufeln unter der stiege hatte (...).

(9.9c) Unter der Stiege hatte die Katze zwischen den Krampen und Schaufeln gekauert. 
(9.9d) Unter der Stiege hatte die Katze gekauert.

Zwischen den Krampen und Schaufeln hatte die Katze gekauert. Semantisch liegt entweder eine "Teil-von-Relation" (9.9b) oder eine "spezifizierende Funktion" vor (9.9a; und $z$ war einfügbar). Es liegen zwei Satzglieder im Vorfeld vor (ebd. 153); Apposition wird wegen der fehlenden Referenzidentităt der beiden Adverbiale ausgeschlossen (ebd. 151).

Wunderlich (1984: $79 \mathrm{ff.}$ ) behandelt Prăpositionalphrasen, deren beide PPn einer thematischen Rolle zuzuordnen sind. Eine PPn fungiert bzgl. der anderen als (restriktiver) Modifikator, wobei jede PPn für sich topikalisierbar (und mithin permutierbar) ist (vgl. 9.1b, 9.10). Nunderlich bietet (nicht für alle denkbaren Variationen) zwei mögliche strukturierungen an: 7

(9.10)
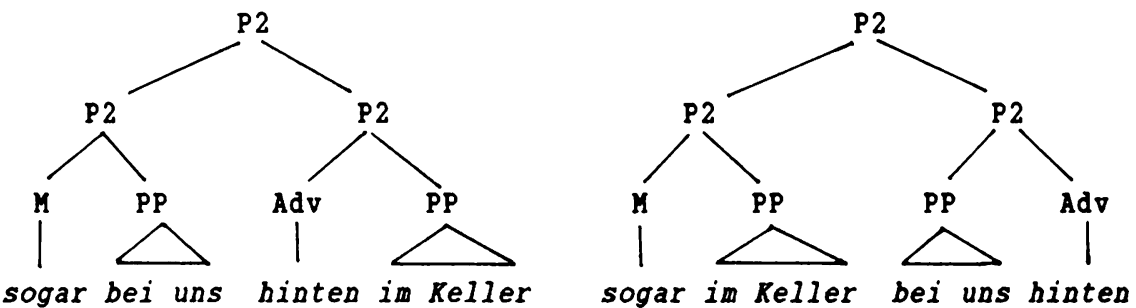

Wunderlich behandelt viele der traditionellen Adverbien als "intransitive Prăpositionen" (ebd. 82). Das Beispiel Oben im Schrank liegen die Hemden (...) (ebd. 83) sei zweifach lesbar: 'im oberen Teil des Schranks' und 'über uns im Schrank'.

Da nach Wunderlich nur eine thematische Rolle vorliegt, beide PPn (bzW. Adv und PPn) zusammen eine Konstruktion bilden $(9.10)$, eine Modifikationsbeziehung vorzufinden und gemeinsame Topikalisierung möglich ist, schliebe ich auf das Vorhandensein einer attributiven Relation zwischen beiden Konstituenten.

Rosemarie Lühr (1985) mist der Vorfeldbesetzung durch zwei (oder mehr) Satzglieder 2.T. "stilistische Funktion" zu (ebd. 10, 11, 14). Besondere Bezuge zwischen den Vorfeldelementen werden nicht angenommen. Vielmehr folgert Lühr (ebd. 19), daB "(...) das spitzenstellungskriterium zur Satzgliedermittlung nur eingeschrănkt brauchbar ist (...)" und das die deutsche verbstellung mit den Ausdrücken "Achsenstellung" bzw. "Mittelstellung" wohl besser zu beschreiben sei.

Wie sind die Fălle (9.1.a-h) zu werten? Die "engen" PP-PP- bzW. Adv-PP(-Adv)Strukturen (9.1a-c) fasse ich als attributive Relationen auf. Die Ronstruktionsteile können gesamt erfragt, ersetzt und topikalisiert verden (vgl. hierzu Kap. 7.4.); die Anwendbarkeit von Kontrastnegation und Gradpartikeln auf beide Teile sowie die gemeinsame thematische Rolle deuten darauf hin, das hier

$7 \mathrm{Ganz}$ ähnliche Strukturierumgsvorschlăge unterbreitet die interessante Arbeit van Fries (1988: 43), die ich leider zu spăt in die Hănde bekam, um sie hier noch ganz einarbeiten zu boumen: (( (hinten) p0)p1 (auf dem Tisch)p1)p1. In derartigen Phrasen kann "(...) jede p1-Phrase restriktiver Modifikator [im Original kursiv. M.S.] der jeweils anderen p1-Phrase sein (...)". 
eine komplexe Konstituente vorliegt (Wunderlich 1984!) und nicht die Füllung eines Vorfelds mit mehreren Satzgliedern. Man mag einwenden, daß die getrennte Permutierbarkeit (z.B. in Im Zentrum hat es auf der Kreuzung gestunken oder in Im Schrank findest du die Karte oben) möglicherweise doch auf das Vorliegen zweier Satzglieder (mit besonderen Eigenschaften) hinweist. Diesem Einwand ist entgegenzuhalten, daß typische Attributkonstruktionen ein vergleichbares Verhalten an den Tag legen (z.B. Auf bessere Zeiten sollte man die Hoffnung nie aufgeben), wobei allerdings nur die Topikalisierung des Satelliten völlig akzeptabel ist (vgl.: ?? Die Hoffnung sollte man auf bessere Zeiten nie aufgeben).

Ein weiteres "enges." Muster (vgl. 9.1d) wird gebildet von zwei Adverbialen verschiedener Subklasse (9.11a: DIR - MOD; 9.11b: FREQ - DIR):

(9.11a) Aus unbestimmter Ferne her mit müden Schwingen kam Musik geflogen.

(9.11b) Manchmal tief aus dem Kehlkopf holte der Greis seine stimme. Schon aufgrund der Unmöglichkeit gesamter Erfragung und Ersetzung (9.12a; getrennt kann man fragen) ist es wahrscheinlich, das dieses Muster nicht attributiv ist und beide Angaben keine Konstruktion bilden - trotz gemeinsamer Vorfeldposition. In ( $9.12 \mathrm{~b})$ ist die erste Frage sogar ungrammatisch, weil die obligatorische Adverbiale fehlt. Auch die Herausstellungen LINKS- und RECHTSVERSETZUNG sind im Ggs. zu den attributiven strukturen $(9.1 \mathrm{a}-\mathrm{c})$ bei beiden Adverbialen unmöglich (vgl. 9.12c):

(9.12a) Woher kam Musik geflogen? Aus unbestimmter Ferne her (*mit müden Schwingen).

Wie kam Musik geflogen? (?? Aus unbestimmter Ferne her) mit müden Schwingen.

Aus unbestimmter Ferne her mit müden Schwingen, *dorther/ ??so/ ?da sam Musik geflogen.

(9.12b) * Wie of holte der Greis seine Stimme?

Woher holte der Greis seine Stimme? *Manchmal tief aus der Kehle. Manchmal tief aus der Kehle, *dorther/ *so oft/??da holte der Greis seine stimme.

(9.12c) Wo stank es? Im Stadion auf der Tribüne.

Im Stadion auf der Tribüne, da wohnte ein specht.

Auch die abwesende Spezifikationsbeziehung $z$ wischen beiden Elementen spricht dafür, daB zwei Satzglieder (Adverbiale) gemeinsam das Vorfeld besetzen:

(9.13) * Manchmal, und zwar (genauer) tief aus der Kehle, holte der Greis seine stimme.

*Aus unbestimmter Ferne her, und zwar (genauer) mit müden Schwingen,. kam Musik geflogen.

Zudem sind weder kontrastive Negation noch Gradpartikeloperationen uber beide Einheiten gleichzeitig möglich: 
(9.14) Nicht manchmal tief aus der Kehle holte der Greis seine stimme, sondern ??häufig tief aus der Kehle/ ??manchmal tief aus dem Rachen. Nicht aus unbestimmter Ferne her mit müden Schwingen kam Musik geflogen, sondern *aus der Năhe mit müden Schwingen/ *aus unbestimmter Ferne her mit lockeren Schwingen.

Ich schliebe eine attributive Konstruktion aus, andererseits kann jedoch auch keine Struktur angesetzt werden, die in "Bezugselement plus ZUSATZ" zu gliedern wäre, da mindestens die zusatztypischen Abtrennungsmarkierungen fehlen. (ubrigens liegen auch nicht die zusatztypischen semantischen verhältnisse vor; vgl. Kap. 12, 15.) Die völlig unproblematische Distanzstellung und alleinige Vorfeldbesetzung eines der beiden Elemente weist überdies darauf hin, daß es sich bei zwei juxtaponierten PPn (bzw. PP plus Adverb) unterschiedlicher semantischer Klasse - also dem Fall (9.1d) - tatsăchlich um DOPPELTE bzw. MEHRFACHE VORFELDBESETZUNG handelt! Dabei scheint es mir unnötig, zwischen den Vorfeldelementen ein "appositives" oder sonstwie geartetes Sonderverhältnis anzusetzen.

Wie sind nun Konstruktionen zu beurteilen, die den obigen kategorial gleichen (9.1e, f), jedoch mit dem "Einschaltungsmuster" versehen sind? In (9.1f) zăhle ich das einschaltungsmarkierte "Adverbial" zu den ZUSATZEN zum Satz (traditionell würde wohl Parenthese angenommen); sie werden in Kap. 11 (und 16) kurz besprochen. Der Typ (9.1e) stellt einen ZUSATZ zu einem Satzglied dar. Im Ggs. $z u$ den engen Mustern sind sie kontrastierender Negation und der Anwendung von Gradpartikeln aus dem Gastsatz heraus nicht zuganglich (9.15), zudem ist eine Erfragung kaum möglich (9.16):

(9.15) *Nicht oben, im Schrank, ist es feucht, sondern oben, im Regal. * Nur im Schrank, oben, ist es feucht, nicht im Regal, oben.

* Sogar auf der Kreuzung, im Zentrum, stank es, nicht nur am Straßenrand im Zentrum.

* Nicht im Zentrum, auf der Kreuzung, stank es, sondern im Zentrum, auf dem Wochenmarkt.

(9.16) Wo oben ist es feucht? Im Schrank oben. ? Im Schrank, oben.

Wo im Schrank ist es feucht? Oben im Schrank. *Oben, im Schrank.

Wo im Zentrum stank es? *Im Zentrum, auf der Kreuzung, stank es.

Diese Eigenschaften genügen m.E. bereits, um ATTRIBUTION völlig auszuschlieBen. Es handelt sich um die in Kap. 12, 15 ausführlich dargestellten ZUSATZE, und zwar Um SPEZIFIZIERENDE ZUSATZE (z.B. 9.17a) SOWIe Um ALTERNATIVEN NENNENDE ZUSATZE (m.a.W.): 8

(9.17a) Oben, (und zwar genauer/ vor allem) im Schrank, ist es feucht. Im Schrank, (und zwar genauer/ besonders) oben, ist es feucht.

8 Nach Altmann (1981) könnte hier das Herausstellungsmuster NACHTRAG (und zwar!) angencumen werden. Vgl. auch Kap. 14. 
(9.17b) Im Zentrum, (und zwar vor allem) auf der Kreuzung, stank es. Auf der Kreuzung, (m.a.k.) im Zentrum, stank es.

Nun bleiben noch die eher appositionsunverdăchtigen strukturen $(9.1 \mathrm{~g}, \mathrm{~h}$ ) ubrig, die keinesfalls als MEHRFACHE VORFELDBESETZUNG zu erklăren sind, da die Einschaltungsmarkierung die "ausgeklammerten" Einheiten als HOSPITANTEN ausweist. Sie sind von keiner der üblichen Satzglied-bzw. Gliedteiloperationen affizierbar. Es liegen allerdings Indizien vor, die Muster auch nicht als $2 U-$ SATZE einzustufen: In $(9.1 \mathrm{~g}, \mathrm{~h})$ vermag die betreffende Einheit den Satz vollig frei zu durchwandern (vgl. 9.18a, b) und inm gar frei voraufzugehen oder nachzufolgen (womit Satzfolge vorliegt) - Restriktionen entstehen allein aufgrund von anaphorischen Beziehungen oder sie entstünden dann, wenn man zwischen das Prădikat und syntaktisch sehr eng zum Prädikat gehörige Elemente (z.B. obligatorisches Direktionaladverbial etc.) einzufügen versuchte: ${ }^{9}$

(9.18a) ((?) Und darin bin ich genial:) Mein Talent (, und darin bin ich genial,) ist (, und darin bin ich genial,) die Bosheit. (Und darin bin ich genial.)

(9.18b) (Winter schluchzte noch immer.) Nach einer Viertelstunde (- Winter schluchzte noch immer -) war (- Winter schluchzte noch immer -) er (- Winter schluchzte noch immer -) wieder (- Winter schluchzte noch immer -) auf der Brücke. (Winter schluchzte noch immer.)

An diesem Verhalten erkennt man typische EINSCHOBE (hier: Schaltsătze), die traditionell als PARENTHESEN bezeichnet werden. Damit scheiden solche Muster eindeutig aus dem appositionsverdăchtigen Bereich und dem Bereich möglicher mehrfacher Vorfeldbesetzung aus (ausfühlicher dazu Kap. 11).

9 Man betrachte beispielsweise folgende Fălle:

(F9.1) Pia (, sie trinkt gerne,) hat (, sie trinkt gerne,) den Sekt (, sie trinkt gerne,) in den Kuhlschrank (?, sie trinkt gerne,) gelegt.

(F9.2) Pia (, sie haßt Insekten,) hat (, sie hast Insekten,) dem Moskito (, sie haßt Insekten,) den Garaus (??, sie hast Insekten,) gemacht. 
Um Herausstellungskonstruktionen von "appositionsverdăchtigen" Mustern abzutrennen, wird weitgehend auf die in Altmann (1981) vorgestellten Kriterien Bezug genommen. Die Abgrenzungsdiskussion kann bis auf den Nachtrag knapp gehalten werden, da sich bei Altmann die Details für den interessierten Leser dargestellt finden. Als Herausstellungen werden von Altmann (1981: 47) angesehen: (a) Freies Thema (FT), (b) Vokativische NP (VokNP), (c) Linksversetzung (LV), (d) Rechtsversetzung (RV) und (e) Nachtrag (NT).

Mit "Herausstellung" verwechselbar sind nach Altmann (f) die Ausklammerung (AUS; auch: Nachfeldbesetzung) und (g) die Extraposition (EXT; Glied-bzw. Gliedteilsätze werden hinter den rechten Rlammerteil geschoben); bei diesen Einheiten handelt es sich um Permutationen "normaler" Satzglieder oder Satzgliedteile; die syntaktische Integration der ausgeklammerten und extraponierten Glieder in den Matrixsatz ist maximal. 1

Ebenfalls den "Herausstellungen" ähnlich sind (h) Apposition (APP) und (i) Parenthese (PAR); bei diesen Mustern kann von syntaktischer Integration in den Gastsatz weniger (APP) bzw. kaum (PAR) die Rede sein. ${ }^{2}$ Die nachstehenden Beispiele, deren Rleinbuchstaben der obigen Einteilung entsprechen, zeigen die betreffenden Ausdrücke:

(10.1a) Apropos Pia! Der Kuno kennt auch eine Frau mit diesem Namen.

(10.1b) Du Rindvieh! So kannst du doch keine Dissertation schreiben.

(10.1c) Die Pia, die kennt der Kuno auch.

(10.1d) Die kennt der Kuno auch, die Pia.

(10.1e) Der Kuno hat eine Frau kennengelernt, (und zwar) eine nette.

(10.1f) Der Kuno hat eine Frau kennengelernt gestern.

(10.1g) Er hat den Affen gesehen, der mit Bananen schmeiBt.

(10.1h) Die Pia, eine nette Frau, studiert Jura.

(10.1i) Die Pia - sie ist eine nette Frau - studiert Jura.

"Herausstellungen" besitzen nach Altmann (1981: $46 \mathrm{f.}$ ) folgende Merkmale: (a) sie sind keine obligatorischen Satzbestandteile (stets entbehrlich). (b) Entweder erfüllen sie im GASTSATZ keine syntaktische Funktion (FT, VokNP, NT)

1 Kerkmale, die syntaktische Integration anzeigen, sind vor allem (a) morphologische Markienmgen, (b) Reihenfolgebeziehungen und (c) intonatorische Karkienungen. Ausgeklamaerte und extraponierte Elemente haben hinsichtlich des Bezugssatzes stets eine syntaktische Punktion, ein freies Thema oder ein Nachtrag hingegen nicht.

2 Während Appositionen auf Elemente des Gastsatzes bezogen sind und diesen i.d.R. ummittelbar nachfolgen (die strikte Nachstellung ist als syntaktische Markierung anzusehen) und mit einer nominalen Basis z.T. kongruieren, können Parenthesen i.d.R. den Gastsatz frei durchwandern, da sie kein direktes Bezugselement haben, selbst wenn sie phorisch auf ein Gastsatzelement verweisen; entstehende Stellungseinschránkungen ergeben sich dann aufgrumd der Abfolgerestriktionen zwischen Pro-Formen und ihren Bezugseinheiten. Rangruenz spielt bei Parenthesen keine Rolle. 
oder es liegt Doppelbesetzung einer syntaktischen Funktion vor (LV, RV), wobei die HERAUSSTELLUNG dann bezogen ist auf das eigentliche satzglied (i.d.R. eine Pro-Form). (c) Die syntaktischen Verknüpfungen der "herausstellungen" mit dem Restsatz sind unterschiedlich stark; nie jedoch sind sie völlig in diesen integriert. (d) Sie füllen nicht allein ein Stellungsfeld des Satzes aus. (e) Außer bei einigen Ausprăgungen des FT sind die Herausstellungsausdrücke nicht satzformatig; sie können aber durchaus satzwertig sein. ${ }^{3}$ (f) Die semanto-pragmatische Funktion wird den Herausstellungen durch den GASTSATZ zugewiesen. (g) Sie stehen links oder rechts vom Bezugssatz, z.T. aber auch parenthetisch (als Einschaltung) in diesen eingefügt.

HERAUSSTELLUNGEN, PARENTHESEN und APPOSITIONEN (dazu Kap. 11, 12, 15) sind nicht leicht voneinander zu trennen. Die PARENTHESE kann man als sprachliche Nebenhandlung (in den "Hintergrund" gesetzte Erläuterung) bezeichnen. 4 Der Sprecher kommentiert mitunter damit den dargestellten Sachverhalt oder er ruft Hintergrundwissen, Weltwissen usf. in Erinnerung. "Appositionen" im bisherigen Verständnis stellen zusätze zu Satzgliedern dar, die ebenfalls kommentierend, erinnernd o.ä. sein können und etwas zu dem Bezugsausdruck "prädizieren".

Herausstellungen hingegen haben nichts von Kommentar, distanziertem Zusatz o.a. an sich. Sie übernehmen primăr Aufgaben im Bereich der Thema-Rhema-Gliederung des Satzes. ${ }^{5}$ Ihre syntaktische Integration in den zugeordneten Satz ist etwas (VokNP, FT), i.d.R. aber erheblich gröBer (LV, RV, NT) als bei den Parenthesen. Auf keinen Fall können sie den Gastsatz frei durchwandern, d.h. jede "Parenthesenische" besetzen. Herausstellungen sind aber nur selten stărker integriert als der Appositionsprototyp (betrifft wohl nur die LV). 6
(10.2)
(1) Di
(2) hat (3)
(3) gestern (4)
(4) den Kuno
(5) getroffen (6).
FT (Apropos Pia): (1).
VokNP (Du Unwissender): (1), ?(2), ?(3), ?(4), ?(5), (6).
LV (Die Pia): (1).
RV (ich meine die Pia): (2), (6).

3 Sie käunen möglicherweise als Reduktionsprodukte von Sätzen angesehen werden, was durch den möglichen Zusatz z.B. von satzbezogenen Adverbialen etc. unterstrichen werden kann.

4 Vgl. Altmann (1981: 63-65), Bassarak (1985: 373 ff.) und Heringer (1988: 276 f.). "Hintergrumd" ist hier anders gemeint als im Begriffspaar "Tokus - Hintergrund" (vgl. Jacobs 1984).

5 Vgl. auch v. Polenz (1985: 297). FT und LV dienen der (emphatischen) Thematisierung. Die RV spezifiziert ein als unklar empfundenes Thema (eine Pro-Form, die zu den thematischen Blementen des Satzes gerechnet werden kann; vgl. dazu Heidolph u.a. 1981: 731 ff.)). Der MT ermöglicht ein mehrteiliges Rhema.

6 Das FT wird van Altmann (1981: $49 \mathrm{f}$.) als relativ selbständiger satzwertiger Ausdruck behandelt. Die VokNP ist "syntaktisch noch weniger als ein freies thema in den folgenden Satz integriert" (Altmann (1981: 52)). Es ist zu uberlegen, ob die VokdP überhaupt in den Bereich "Herausstellung" gehört oder eher zu den kammikationsorientierten Funktionen wie ANRBDE usf. 
APP (Dozentin in Bonn): (2).

PAR (Das weiB doch jeder): (1), (2), (3), (4), (?) (5), (6).

FLUCH (Kruzifix): (1), (2), (3), (4), (?) (5), (6).

Man sieht, daß die HERAUSSTELLUNGEN (wie die APPOSITIONEN) i.d.R. über nur eine Strukturposition verfügen. Die PARENTHESE (vor allem als Schaltsatz) kann nahezu ungehindert vor bzw. hinter dem Satz sowie zwischen den Satzgliedern auftreten; lediglich unmittelbar vor einem verbalen KlammerschluB (und nach einem eng zum Prädikat gehörigen Satzglied) treten möglicherweise Akzeptabilitätsminderungen auf. Auch Flüche oder Interjektionen bewegen sich frei im Satz, denn ihr Auftreten ist nicht syntaktisch, sondern "kommunikativ-pragmatisch" bedingt. Interjektionen und schaltsätze finden sich manchmal sogar in komplexen Nominalphrasen:

(10.3a) Die - ich will mich vorsichtig ausdrücken - etwas unterentwickelte Höflichkeit der Deutschen wird im Ausland bemängelt.

(10.3b) Die - na ja - etwas unterentwickelte Höflichkeit der Deutschen wird im Ausland bemängelt.

Auf die HERAUSSTELLUNGEN treffen weder die Satzglied- noch die Gliedteilkriterien (Erfragung, Anwendung von Negationen/Gradpartikeln aus dem Bezugssatz heraus etc.) $z u$. Ich werfe nun einen Blick darauf, weshalb "herausgestellte Elemente" (und deren "Bezugselemente" im Satz) als mögliche Beispiele von "Apposition" in Frage kommen sollen.

\subsection{Linksversetzung}

Die Linksversetzung trifft man im (französischen) Appositionsbereich von Raabe (1979; "Das Appositiv als Nebensatz", $290 \mathrm{ff.;}$ Beispiel S. 291) an. Das frz. Muster dürfte in (10.4) mittels dieser Konstruktion adäquat wiedergegeben sein, da eine Interpretation als freies Thema aufgrund der Nichtanwendbarkeit von FT-Floskeln (wie d'ailleurs, a propos) wohl ausscheidet:

(10.4) Qu'il s'est trompé, je le crois. Daß er sich getäuscht hat, das glaube ich.

Raabes neun Appositionskriterien scheinen auf die Linksversetzung zuzutreffen. 7 Es handelt sich um zwei Einheiten (Basis: das; "Appositiv": daB-Satz), wobei das "Appositiv" graphematisch abgetrennt ist, die Satzgrenze nicht überschreitet, eine gewisse Referenzidentität zwischen dem dab-Satz und der ProForm das vorliegt und die Eliminierung des "Appositivs" augenscheinlich den Wahrheitswert des Satzes unangetastet läBt. Was aber geschieht, wenn man zu negieren versucht?

7 Raabe (1979: 248 ff.): Wahrheitswertkonstanz, Textnotwendigkeit, Referenzidentităt, Tragmeite von Negation/Affirmation, Sprecherdivergenz, Sprecherpräsenz und Sprecherlexeme, Satztypdivergenz, Satzgrenze, prosodische bzw. graphematische Markierung. S. auch Kap. 3 dieser Arbeit. 
(10.5) Daß er sich getáuscht hat, das glaube ich nicht, sondern daß er getăuscht wurde, (das glaube $i c h$ ).

Kontrastierende Negation ist anwendbar. Die Merkmale Sprecherdivergenz (10.6) und Hinzufügbarkeit von "Appositionslexemen" (10.7) treffen nicht zu:

(10.6) *Paul sagte: Daß er sich getäuscht hat, "das glaube ich". DaB er sich getáuscht hat, glaubst du das?

(10.7) *Obrigens daB er sich getáuscht hat, das glaube ich.

Henn man akzeptiert, daB Raabes Beispiel im Deutschen adäquat als Linksversetzung wiederzugeben ist, dann stellt sich heraus, daB nicht einmal alle von Raabe selbst aufgestellten Kriterien auf sie zutreffen.

Es gibt darüber hinaus weitere Gründe, die einer Einstufung der Linksversetzungen als "Appositive" entgegenstehen: (a) die stellung: Prototypische Appositive und zusătze, die appositionsähnlich sind, stehen i.d.R. rechts vom Bezugsausdruck; (b) die Permutierbarkeit: Lockere Appositive und zusătze können mitsamt ihrem Bezugsausdruck im Satz verschoben werden, die Linksversetzung ist auf die Position vor dem Finitum beschrănt; (c) die Umformungsbeziehungen bzw. die Paraphrasemóglichkeiten: Sie sind bei Linksversetzungen überhaupt nicht gegeben (keine Ropulasatzparaphrase!); (d) die hohe syntaktische Integration der Linksversetzung in den Bezugssatz, die sich u.a. daran zeigt, das die linksversetzte Einheit von einer Negation betroffen werden kann. ${ }^{8}$ Es erscheint demnach als zweckmăBig, die Linksversetzungen aus dem Bereich appositionsverdáchtiger Muster auszuscheiden.

\subsection{Rechtsversetzung}

Die (enge) Rechtsversetzung findet sich z.B. bei Molitor (1979: 118) als Apposition eingestuft, obwohl er spăter (ebd. $216 \mathrm{ff.}$ ) ausdrücklich zwischen Apposition und Right Dislocation unterscheidet. 9 Dieser Typ erscheint allerdings auch in anderen Darstellungen als Beispiel für "Apposition", nur daß in diesen

8 Nach Altmann (1981: 48) führt die hohe Integration "(...) zu dem Schluß, daß der LV-Ausdruck im Vorfeld steht. Wegen des umittelbar folgenden demonstrativen Proelements muB man von doppelter Vorfeldbesetzung ausgehen".

9 Molitor (1979: 216) bemerkt dazu:

"Unter "Right Dislocation" wird eine Operation verstanden, die eine mit einen bestimmten Determinierer versehene NP unter Zuricklassung einer pronominalen Kopie "nach rechts hinter die Grenze ihres urspringlichen Satzes" (...) stellt (...)".

Damit wird angenoumen, das das rechtsversetzte Blement urspringlich in Mittelfeld stand und dort num durch ein Pro-Element vertreten wird. Plausibler erscheint mir Rrifkas Beschreibung (1983: 75), der von einer "nachträglichen Ausbuchstabierung" eines Pronomens spricht, ohne daß eine Tilgungs- und eine Permutationstransformation angesetzt werden. 
nicht thematisiert wird, daB eine von der Apposition distinkte Konstellation "Rechtsversetzung" überhaupt existiert: 10

(10.8) Aber sie wird ein Ende nehmen, (ich meine) diese Nacht. Die beiden Madchen, (ich meine) Inge und Gisela, fahren mit ihren Eltern an die See.

Altmann (1981: $54 \mathrm{f} ., 57 \mathrm{ff.}$ ), der die Rechtsversetzung als "Herausstellung" auffast, die Apposition hingegen nicht, hat wichtige Unterschiede zwischen beiden Mustern herausgearbeitet:

\section{(enge) Rechtsversetzung}

\section{Bezugsausdruck:}

Erkennungsfloskel

Zusătze:

Kasuskongruenz:

stellung:

Paraphrase:

Funktion: korreferente Pro-Form; schwaches Referenzmittel

ich meine

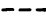

obligatorisch

rechts der Basis oder rechts vom Satz

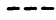

Auflösung unklarer Referenz

\section{Apposition}

NP mit deutlicher

Referenz, (Pron)

übrigens

GP, Sadv, freie ADVB

nicht obligatorisch; APP

of $t$ nominativisch

meist rechts der

Basis

prädikativ: Ropulasatz

$B=$ SBJ,$A=$ PRADIRATIV

Prădikation; Eigen-

schaftszuschreibung

Aufgrund der Ahnlichkeit von Rechtsversetzung und Apposition (ein Satzglied ist Bezugsausdruck; Einschaltungsmarkierung; (mögliche) Adjazenz zum Bezugsausdruck etc.) sowie der Tatsache, daß für diesen Typ eine Einleitungsfloskel (ich meine) charakteristisch ist, mochte ich die Abgrenzungsdebatte Rechtsversetzung versus Apposition auf die Kapitel über "lexenatisch einleitbare Zusătze" verschieben (s. Kap. 12; s. auch Kap. 15).

\subsection{Nachtrag}

Der Nachtrag findet sich als Beispiel für "prăzisierende Apposition" z.B. bei Kusmin und Molitor. 11 Bei beiden wird jedoch nicht explizit von "Nachtrag" als von der Apposition distinktem Muster gesprochen:

10 Erstes Beispiel van Rusmin (1960: 117), das zweite van Schulz/Griesbach (1982: 362); der Bezugsausdruck ist von mir fett markiert, die RV unterstrichen; der zusatz ich meine ist von mir.

$11 \mathrm{zu}$ "Nachtrag" s. Altmann (1981: $70 \mathrm{ff}$.). Die Beispiele (10.9a) aus Kusmin (1960: 109), (10.9b) aus Yolitor (1979: 178); Rundklammerzusátze von mir. 
(10.9a) Die Weltgeschichte hatte ihnen eine Aufgabe gestellt, (und zwar) die größte, die je vergeben wurde.

(10.9b) Ein Auto, und zwar ein großes (Auto), stand vor der Haustür. Er feierte eine Party, und zwar eine Gruppensexparty.

In diesen Beispielen handelt es sich um einen "spezifizierenden Zusatz" zu einem Satzglied des Matrixsatzes. Es sind übrigens nicht nur nachgetragene Attribute möglich, sondern auch nichtattributive NPn, die nicht selten eine Untermenge der vom Basisnomen denotierten Menge bezeichnen. ${ }^{12}$ Man vergleiche ein Beispiel von Altmann (1981: 70; 10.10) mit meinen eigenen:

(10.10) Ich habe mir ein Haus gekauft, (und zwar) das des Bürgermeisters.

(10.11) Ich habe mir ein Haus gekauft, (und zwar) genauer ein Landhaus.

(10.12) Ich habe mir Häuser gekauft, (und zwar) die des Bürgermeisters.

(10.13) Ich habe mir Häuser gekauft, (und zwar) vor allem Landhäuser.

Diese Muster werden später behandelt werden, nămlich bei den "lexematisch einleitbaren Zusătzen" (Kap. 12, 15), da sie - wie (10.11-13) demonstrieren - von bestimmten Ausdrücken eingeleitet werden können. Dort werden sie mit ähnlichen Mustern, wie z.B. dem "Appositionsprototypen" oder der "Rechtsversetzung" verglichen bzw. kontrastiert.

Bei Altmann (1981: $70 \mathrm{ff.}$ ) finden sich unter dem Phänomen Nachtrag nicht ausschließlich basisbezogene Zusätze, sondern auch solche ohne ersichtliches Bezugselement: 13

(10.14) Karl ist weggefabren, (und zwar) allein.

(10.15) Er fuhr die StraBe hinunter, viel zu schnell.

(10.16) (..) wird eine NP aus ihrem ursprünglichen Platz versetzt, und zwar nach links.

Diese nachgetragonen valenzfreien Angaben spezifizieren entweder das Prädikat (Modaladverbiale) oder den Gesamtsatz (z.B. Temporaladverbiale); beide Einheiten könte man folglich als "Bezugselemente" der Nachträge auffassen. Hier offenbart sich ein wesentlicher Unterschied $z$ wischen den Mustern. Die Zusätze in (10.10-13) können ein kategorial gleiches Bezugselement (nämlich NP) haben,

12 Altmann (1981: 70) nennt Adjektiv-, Genitiv-, Präpositional- und Relativsatzattribute. Zudem stellt Altmann (1981: 335) fest: "Bei Attributen im Nachtrag muß die Bezugs-NP nicht getilgt werden, zumindest muB ein ev[tl; W.S.]. vorhandener Artikel erhalten bleiben". Getilgt wird folglich lediglich das Kernnomen, ähnlich wie z.B. bei der Koordination das Auto der Mutter und das des Vaters. Daher scheint es mir günstiger zu sein, hier nicht von nachgetragenen Attributen zu sprechen, sondern von nachgetragenen NPn, deren Kern fakultativ tilgbar ist. Der pure Attributsnachtrag ist nämlich inakzeptabel:

(F10.1) Er ist halt ein Nensch, (und zwar) ein dummer/*dummer.

(F10.2) Ich habe mir ein Haus gekauft, (und zwar) das des Bürgermeisters/*des Bürgenmeisters.

13 Beispiele von Altmann (ebd. 70); nach seinen Ausführungen sind prädikative Attribute, Adjektivadverbiale (= MOD) und freie adverbiale Angaben (= TPMP, KAUS etc.) als nachgetragene Ausdrücke möglich. Ich nenne derartige "Nachträge" vorläufig "nachgetragene Angaben", im Ggs. zu den "nachgetragenen Zusätzen (zu NPn)". 
jene in (10.14-16) haben ein ungleichstufiges "Bezugselement" (nãmlich VP, S), sofern dieser Ausdruck hier noch sinnvoll ist.

Nun erheben sich folgende Fragen: Inwieweit unterscheiden sich beide $\mathrm{Mu}-$ ster? Fallen beide unter eine Grobklasse ("Nachtrag" oder wie auch immer) oder sind zwei verschiedene Muster anzusetzen? Wieviel haben Nachtrăge adverbialer Satzglieder mit Parenthesen (Schaltsătzen) gemeinsam? ${ }^{14}$

Zuerst fallen wohl die Ahnlichkeiten ins Auge: Sowohl "nachgetragene Zusät$z e "$ als auch "nachgetragene Angaben" können optional vom "Funktionslexem" und $z$ war begleitet werden. Beide "Nachträge" können nach dem satzklammerschlieBenden Element stehen - ggf. auch nach Ausklammerungen (Nachfeldbesetzungen), Rechtsversetzungen und Extrapositionen (vgl. Altmann 1981: 71). Beide Konstruktionsmuster lassen eine transformationelle Erklärung über Satzverdoppelung, Tilgung (im 2. Satz) und evtl. Einbettung (des zweiten Satzrestes in den ersten Satz) beschreiben:

(10.17) Sie hat die Haie gefütert. Und zwar hat sie vor allem die Hammerhaie gefüttert.

Sie hat die Haie gefüttert, und zwar vor allem die Hammerhaie. Sie hat die Haie, und zwar vor allem die Hammerhaie, gefüttert.

(10.18) Sie hat die Haie gefütert. Und $z$ war hat sie die Haie gestern gefütert.

Sie hat die Haie gefütert, und zwar gestern.

Sie hat, und zwar gestern, die Haie gefüttert.

Hinsichtlich möglicher lexematischer Erweiterungen treten allerdings deutliche Unterschiede zutage. Hier wird es notwendig, spáteren Untersuchungen vorzugreifen (Kap. 12, 15), bei denen die Erweiterungsmöglichkeiten der "spezifizierenden zusătze" erörtert werden. Während satzgliedbezogene zusătze neben und zwar diverse weitere Lexeme bzw. Floskeln wie vor allem, genauer, z.B. etc. mit sich führen können, ist diese option bei den prädikats- resp. satzbezogenen Nachträgen sehr eingeschrănkt. ${ }^{15}$ Dies ist bedingt durch die verschiedenen Bezugsverhăltnisse. Spezifizierende Zusätze mit gleichrangigem NP-Bezugselement $(10.17,10.19)$ weisen v.a. Menge-Element-Beziehungen auf. Nachgetragene Satzglieder, deren ungleichrangiges Bezugselement ein Satz oder eine VP ist (10.18, 10.20-23), können derartige Beziehungen nicht eingehen:

14 Sie sind nämlich auch in den Satz hinein verschiebbar, wenngleich die Position rechts vom Satz eine Art "Nomalposition" darstellt; vgl. Er fuhr, (und zwar) viel zu schnell, die Straße hinunter.

15 Bei satzgliedbezogenen Zusătzen sind die Hinzufügungsmöglichkeiten unterschiedlich. Bei NP-NPRonstellationen (Die Haie, genauer die Hammerhaie etc.) sind sie äußerst vielfăltig; bei Adj-AdjKonstellationen hingegen bereits eingeschränkter (Die Bemden waren bunt, genauer/ z.B./ ?besanders/ ?ausgenamen/ ??nicht so sehr rot und blau, gewesen). Näheres s. Kap. 12, 15.

Bei prädikats- bzw. satzbezogenen "Nachträgen" ist und zwar generell optional hinzufügbar - genaver/ vor allem/ z.B. etc. nur in speziellen Fällen. 
(10.19) Viele Haie werden vom Menschen gefürchtet, und zwar z.B./ vor allem/ einschlieblich/genauer die Hammer- und Blauhaie.

(10.20) Viele Haie werden vom Menschen gefürchtet, und zwar $z_{\text {.B. }}$ *vor allem/ *einscblieblich/ *genauer auBerordentlich.

(10.21) Viele Haie werden vom Menschen gefürchtet, und zwar z.B./ vor allem/ *einschlieblich/*genauer wegen ihrer Unberechenbarkeit.

(10.22) Pia hat sich einen Jaguar gekauft, und $z$ war *z.B./*vor allem/ * einschlieBlich/*genauer gestern.

(10.23) Pia hat sich einen Jaguar gekauft, und zwar (?)z.B./ (?) vor allem/ * einschlieBlich/*genauer um Kuno zu beeindrucken.

Nachgetragene modale und temporale Adverbiale $(10.20,10.22)$ gestatten keine Erweiterungen durch Funktionslexeme (sie können aber von Gradpartikeln, Satzadverbien und freien Adverbialen begleitet werden). ${ }^{16}$ Die kausale Gruppe $(10.21,10.23)$ kann u.U. von z.B. oder vor allem begleitet werden. Ausgeschlossen sind Hinzufügungen wie einschlieblich und genauer, weil ein gleichrangiges Bezugselement fehlt, hinsichtlich dessen eine Prăzisierung bzw. ein "MengeneinschluB" vorgenommen werden kann.

Die Begleitlexeme nicht-spezifizierender Zusătze (z.B. allgemeiner/ weniger/ ausgenommen/ m.a.W.; vgl. Kap. 12) können nicht mit nachgetragenen Angaben auftreten:

(10.24) Pia hat sich einen Jaguar gekauft, (und zwar) *allgemeiner/ *weni-

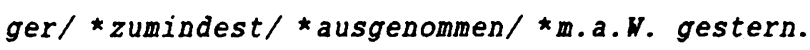

(10.25) Pia hat sich einen Jaguar gekauft, (und zwar) *allgemeiner/*weniger/ ??zumindest/ *ausgenommen/ *m.a.W. um Kuno zu beeindrucken. Wenn man die Liste der "Funktionslexeme" (vgl. Rap. 12), die NP-Zusätze begleiten können, dahingehend prüft, welche von diesen mit nachgetragenen Angaben kombinierbar sind, dann kommt man zu dem Schluß, das lediglich solche vorkommen, die anzeigen, daß ein Beispiel bzw. eine Möglichkeit angegeben wird (10.26) oder eine Hervorhebung folgt (10.27):

(10.26) Wir könnten doch mal die Pia besuchen, (und zwar) z.B. morgen.

(10.27) Pia arbeitet gern, (und zwar) besonders/ hauptsachlich/vor allem/ vornehmlich/ in erster Linie nachts.

Die Erweiterungsmöglichkeiten hängen von der semantischen Beziehung zwischen Bezugselement und zusatz ab. Bei Satzglied-Zusatz-Konstellationen können z.B. diverse Menge-Element-Relationen $z$ wischen beiden Elementen vorherrschen, die jene variantenreiche Erweiterungsoption bedingen. Dies ist bei der Ronstellation Satz/Prädikat-Adverbialnachtrag natürlich ganz anders, denn Menge-Element-Beziehungen treten nicht auf. Auch ein Zusatz, der einen alternativen Ausdruck nennt, tritt nicht auf, da dies nur zwischen verwandten bzw. gleichrangigen Funktionen möglich ist - m.a.W. wenn ein zusatz sich auf ein Satzglied bezieht:

16 Vgl.: Viele Haie werden vom Menschen gefürchtet, und zwar vermutlich sogar außerordentlich. 
(10.28) Pia will die Ledigen schonen, m.a.W. die Unverheirateten.

Pia will die Ledigen morgen schonen, m.a.W. am Weihnachtstag.

(10.29) Pia will die Ledigen schonen, m.a.W. *morgen/ *aus Năchstenliebe. Eine intonatorische/graphematische Abtrennung erfahren beide Muster, und zwar sowohl am Satzende als auch innerhalb eines Satzes $(10.30 ; 10.33)$. Sie weisen also beide das gleiche Markierungsmuster auf. Im Satzinneren kann der spezifizierende zusatz allerdings i.d.R. nur unmittelbar rechts von seinem Bezugselement stehen (10.30). Distanzstellungen sind inakzeptabel oder zumindest von ziemlich fragwürdiger Akzeptabilität (10.31, 10.32; genaueres in Kap. 12); Angabennachtrăge sind stellungsvariabler (10.33):

(10.30) Pia wird die Affen lausen, und zwar (vor allem) die Gibbons. Die Affen, vor allem die Gibbons, wird Pia morgen lausen.

(10.31) ?? Die Affen wird, vor allem die Gibbons, Pia morgen lausen. ?? Die Affen wird Pia, vor allem die Gibbons, morgen lausen. ??Die Affen wird Pia morgen, vor allem die Gibbons, lausen.

(10.32) *Die Gibbons wird, allgemeiner alle Affen, Pia morgen lausen. *Die Gibbons wird Pia, allgemeiner alle Affen, morgen lausen. *Die Gibbons wird Pia morgen, allgemeiner alle Affen, lausen.

(10.33) Pia wird die Affen lausen, und zwar morgen.

Pia wird, und zwar morgen, die Affen lausen.

(?) Pia wird die Affen, und zwar morgen, lausen.

Nun kann die Frage angeschlossen werden, an welchen Positionen die ersichtlich freier verschieb- und einschaltbare Angabe vorzukommen vermag. Ich möchte nămlich überprüfen, ob man es mit einem in den Satz eingeschobenen und somit lediglich permutierten "Nachtrag" oder mit einer Parenthese (in meiner Ausdrucksweise: mit einem Einschub) zu tun hat. 17 Parenthesen (z.B. die Schaltsătze) sind hinsichtlich ihrer stellungsmöglichkeiten sehr variabel. Sie können links bzw. rechts vom Gastsatz oder an den sog. "Parenthesenischen" in diesem auftreten. Zudem kommen sie sogar innerhalb von Wortgruppen (NPn) vor. 18 "Angabennachtrăge" kann man nicht so frei umstellen:

17 Intonatorisch bzw. graphematisch vam Satz abgetrennte Adverbiale werden nicht selten zu den Parenthesen (Prototyp: der Schaltsatz) gerechnet, so z.B. auch bei Altmann (1981: 63 f.). S. auch Kap. 11 in dieser Arbeit.

18 Z.B. Die (ich will mich nicht zu negativ ausdrücken) unterentwickelte Bbflichkeit der Bundesdeutschen wird in Ausland ofters bemängelt. Aber nicht nach dem pränoninalen Attribut: *Die unterentwickelte (ich will mich nicht zu negativ ausdrulcken) Böflichkeit der Bumdesdeutschen wird im Ausland ofters bemăngelt. Vgl. auch: Die (menn sie so mollen) altwodische (*menn sie so mallen) Theorie scheint immer noch aktuell zu sein. Hier unterscheiden sich die "Nachtrăge" nicht von den Parenthesen. Man betrachte die Falle, in denen ein pränominales Satzglied durch eine freie Angabe "spezifiziert" wird:

(F10.3) Seine (und zwar bereits gestern) angereiste (*und zwar bereits gestern) Oma săuft ihm das Bier meg.

(F10.4) Die (und zwar mit Absicht) vernichteten (*und zwar mit Absicht) Beweisstücke waren wichtig gevesen. 
(10.34) Pia (?, und zwar viel zu schne11,/ (?), viel zu schnell,) ist mit ihrem Porsche nach Bause gefahren (, und zwar viel zu schnell/ , viel zu schne11).

(10.35) Pia ist (, und zwar viel zu schnell,/, viel zu schnell,) mit ihrem Porsche (, und zwar viel zu schnell,l, viel zu schnell,) nach Hause gefahren.

(10.36) Pia ist mit ihrem Porsche nach Hause (?, und zwar viel zu schnell,/ ?, viel zu schnell,) gefahren.

(10.37) Unsere Oma (?, und zwar gestern, / (?), gestern,) hat dem Opa das Bier weggesoffen (, und zwar gestern/ gestern).

(10.38) Unsere Oma hat (, und zwar gestern,l, gestern,) dem Opa das Bier weggesoffen.

(10.39) Unsere Oma hat dem Opa (, und zwar gestern,/, gestern,) das Bier ((?), und zwar gestern,/ (?), gestern,) weggesoffen.

(10.40) Pia (?, und zwar um Kuno zu beeindrucken,/, um Kuno zu beeindrukken,) hat sich einen Jaguar gekauft (, und zwar um Kuno zu beeindrucken/, um Kuno zu beeindrucken).

(10.41) Pia hat sich (, und zwar um Kuno zu beeindrucken,/, um Kuno zu beeindrucken,) einen Jaguar (?, und zwar um Kuno zu beeindrucken, / , um Kuno $z u$ beeindrucken,l gekauft.

Kritisch ist insbesondere die Position unmittelbar nach dem präfiniten Satzglied. Diese Position darf wohl (neben den Positionen im Mittelfeld zwischen den Satzgliedern) als typische "Parenthesenische" gelten. 19 Eine parenthetisch eingeschaltete, unbegleitete Angabe, die beim Wegfall der Einschaltungsmarkierung (und ggf. einer Umstellung ins Mittelfeld) eine Satzgliedfunktion ausüben könnte, kann eine Position zwischen Vorfeldelement und Finitum einnehmen $(10.34,10.37,10.40) ;{ }^{20}$ zumindest sind eventuelle Akzeptabilitätsminderungen (10.34, 10.37) nicht kraB. Sobald das nachtragstypische und $z$ war und evtl. noch weitere "Funktionslexeme" (s. Kap. 12) hinzugesetzt werden, entstehen an besagter Position nicht voll akzeptable syntagmen. Hier manifestiert sich ein Unterschied $z$ wischen den dort voll akzeptablen prototypischen Parenthesen (z.B. Schaltsatz) und den mit und zwar eingeleiteten Angaben.

Die Position unmittelbar vor einem klammerschlieBenden Element ist gleichfalls nicht immer voll akzeptabel; diese Akzeptabilitätsminderung nimmt je mehr zu, desto enger das letzte Satzglied (vor Einschub und Klammerschluß) zum

19 Vgl. Altmann (1981: 64 f.), Beringer (1988: 274 f.).

20 Manchmal markiere ich diese Fälle mit "(?)", was lediglich anzeigt, daß mir solche Konstruktionen nicht ganz behagen (Idiosynkrasie). "?" bei den und zwar-Beispielen bedeutet dagegen 'eingeschränkte bzw. fragwürdige Akzeptabilităt' . 
Verb gehörig ist. 21 Dieses Verhalten zeigt sich jedoch auch bei typischen parenthetischen Einheiten:

(10.42) Unsere Oma hat dem Opa (- man glaubt es kaum -) das Bier ((?) - man glaubt es kaum -) weggesoffen.

(10.43) Pia ist (- man glaubt es kaum -) mit dem Rad nach Oslo ((?) - man glaubt es kaum -) gefahren.

Auch im folgenden Kap. 11 zeigt sich diese wahrscheinlich generelle Einschränkung der Akzeptabilitat: Zwischen einer eng ans Verb gebundenen Ergänzung ${ }^{22}$ und dem satzklammerschließenden Prädikatsteil muß zwar eine Parenthesenische angenommen werden, weil ein Einschub dort nicht ungrammatisch wirkt, sie ist jedoch im Vergleich $z u$ den übrigen Parenthesenischen im Satz stark markiert und wirkt - zumindest in gehobenem Standarddeutsch - nicht völlig akzeptabel. ${ }^{23}$ Die Mittelfeldpositionen sind hingegen akzeptabel, ebenso wie bei den typischen Parenthesen.

Ein weiterer Unterschied zwischen Schaltsätzen und den und zwar-begleiteten Angaben besteht in der Stellung links und rechts des "Bezugssatzes"; ein Schaltsatz kann i.d.R. beide Positionen einnehmen:

21 So ist z.B. der Einschub zwischen Akkusativobjekt und Klammerschluß akzeptabler als jener zwischen obl. Direktionaladverbiale und Kammerschluß. Die Stärke der Bindung ans Satzvollverb findet sich expliziert in Heidolph et al. (1981: $703 \mathrm{ff.}$ ).

22 Nach Heidolph et al. (1981: $703 \mathrm{ff}$.) kann man etwa folgende Verbbindungsstärkeskala annehmen ("(" bedeutet 'geringere Verbgebumdenheit als'): SUBJ < DATO < AKKO < DIR.

23 Ich bin mir bewußt, daß "gehobenes Standarddeutsch" expliziert werden muste, was ich jedoch nicht leisten kann. Das intuitive Verständnis dieses Ausdrucks muß genügen. Gemeint ist, daß die Parenthese zwischen stark verbgebundenem Satzglied und Klammerschluß eher umgangssprachlich bzw. der gesprochenen Sprache zugehörig wirkt, während die Besetzung der ubrigen Parenthesenischen eher standardsprachlich anmutet und in geschriebenen Deutsch vorgezogen mürde.

Noch gar nicht angesprochen wurde der Aspekt des Bezugsbereichs einer Parenthese (dazu z.B. Heringer (1988: 277)), der sich rechts von ihr erstreckt:

(F10.5) Pia ist - man glaubt es kaum - zum Kond geflogen.

(F10.6) Pia ist (- man glaubt es kaum -) zum Nond ((?) - man glaubt es kaum -) geradelt. Im ersten Beispiel kann das "Kaum-Glauben" sowohl auf den Gesamtsatz als auch auf den Satzteil zum Mond geflogen. Man kann dies anhand von Kontrastproben belegen (und nicht etwa zu ihren Verwandten in Neuseeland). Im zweiten Beispiel wird kaum geglaubt, daß zum Mond geradelt wurde (auch Gesamtsatzbezug scheint möglich), beim 2. Parenthesevorkanmis wird kaum geglaubt, daß die Fortbewegungsart zum Mond das Radeln ist, insofern ist der Skopus geradelt. Durch entsprechende Akzentverhaltnisse kann der Bezug auf geradelt auch beim 1. Parenthesevorkommis eindeutig markiert werden:

(F10.7) Pia ist - man glaubt es kaum - zum Mond ger a delt (und nicht etwa geflogen).

Es ist also nicht notwendigerweise der Fall, daß der Bezugsbereich von Parenthesen nur ummittelbar rechts von diesen sich erstreckt; intonatorische Markierung kann die Zuordnung über Ronstituenten hinweg mit sichern. Obwohl semantisch betrachtet eine skopusbildende Parenthese vor ihrem Bezugsausdruck zu stehen vermag, ist in Falle der Parenthese unmittelbar vor dem Klammerschluß aus syntaktischen Gründen (Verbenge der letzten Ergänzung) eine mäBige Restriktion festzustellen. 
(10.44) *Und zwar gestern. Unsere Oma soff dem Opa das Bier weg. Man glaubt es kaum. Unsere Oma soff dem Opa das Bier weg.

(10.45) Apropos gestern! Unsere Oma soff dem Opa das Bier weg.

(10.46) Unsere Oma soff dem Opa das Bier weg. (?) Und zwar gestern. Unsere Oma soff dem Opa das Bier weg. Man glaubt es kaum.

Wăhrend die Stellung rechts des Satzes mit Punktabtrennung auch bei der Angabe einigermaßen akzeptabel anmutet $(10.46)$, ist die stellung links vor dem "Bezugssatz" inakzeptabel (10.44). Rechts vom Satz interpretiert man die Angabe wohl als eine Art nachgeliefertes Element, das zum vorherigen Satz gehört, dort aber vergessen wurde. Links von inm dagegen bleibt vermutlich nur die Interpretation, daB eine Abfolge zweier satzwertiger Einheiten vorliegt, ohne daB eine Art "Vorlieferung" eines Elements des Folgesatzes vorläge. Man beachte auch die Akzeptabilitat des freien Themas in (10.45), das als satzwertiger Ausdruck gelten kann.

Insgesamt gesehen ist die "nachgetragene Angabe" stellungsmäßig unfreier als eine typische Parenthese (und freier als ein nachgetragener zusatz). Die Angabe ist somit enger zum Bezugssatz gehörig als ein Schaltsatz und wohl nicht wie dieser als satzwertiger Ausdruck anzusehen.

Die Funktion des "Nachtrags" ist nach Altmann (1981: 71): "er ermöglicht ein mehrgliedriges Rhema". Man betrachte z.B.:

(10.47) ??/pia ist viel zu schn e 11 nach h a use gefahren $\mathrm{ft+} /$. /pia ist nach h a u se gefahren ft+ viel zu schn e $11 \mathrm{ft+}$. Dieses Beispielpaar führt den "Vorteil" der Nachtragskonstruktion vor Augen. Wenn der Sprecher beide Umstănde (Adverbiale) als Rhema ăußern mōchte, kann er dies mit ihrer Hilfe; innerhalb eines Satzes wirken zwei Rhemaakzente kaum akzeptabel. Der Nachtrag erlaubt es, zwei Satzglieder als "neue" bzw. "beachtenswerte" Information zu markieren. ${ }^{24}$ zusătzlich kann als Funktion angesetzt werden, daß das nachgetragene Satzglied den Sachverhalt (das Geschehen), das im Bezugssatz zum Ausdruck kommt, năher zu lokalisieren, zu modifizieren etc. Eine modale, temporale oder kausale spezifikation wird "nachgeschoben", "ergănzt". Auch die satzgliedbezogenen (gleichrangigen) "Nachtrăge" lassen sich funktional so beschreiben.

Die Funktion der Parenthese ist nicht ganz einfach auf einen Nenner zu bringen. Heringer (1988: $276 \mathrm{f}$.) formuliert sie folgenderweise:

"Parenthesen sind Notlōsungen für die lineare Beschränktheit der sprache. Was im Geiste als Kontrapunkt vorhanden ist, muB linear im Satz durch Einschub gebracht werden. Mit den Parenthesen werden sozusagen Nebenhandlungen

24

Genaugenommen ubt der Nachtrag keine syntaktische Funktion (Satzgliedfunktion) in Bezugssatzes aus. Man versuche, die gängigen Satzgliedtests (Topikalisierbarkeit, Erfragbarkeit, Substituierbarkeit) anzurvenden - keiner fällt positiv aus. Das nachgetragene Dlement kännte allerdings als Satzglied in den Bezugssatz integriert werden - eine koglichkeit, die bei Herausstellungskonstruktionen gewhihnlich nicht besteht. Man nehme 2.B. die Vokativische $N$ (nie satzgliedfăhig) oder die Linksversetzung (könnte zwar Satzglied sein, dann aber müßte das Demanstrativm verschwinden). 
zur Haupthandlung des Trägersatzes möglich. (...) Satzförmige Parenthesen kommentieren oft die Haupthandlung, prăzisieren, erläutern, ergănzen; sie führen Nebenthemen, Rückgriffe und Vorgriffe ein, oder sie ziehen ein Fazit (...)".

Dennoch wird selbst bei dieser Grobbestimmung deutlich, daß der funktionale Unterschied zu den und zwar-Phrasen, seien sie "nachgetragen" oder "eingeschoben", erheblich ist.

Was die Weglaßbarkeit betrifft, so sind "nachgetragene" Satzglieder eher entbehrlich als beispielsweise spezifizierende zusătze. Dies wird bedingt durch die unterschiedliche semantische Funktion. Wăhrend NACHTRAGE Hinzufügungen sind, stellen insbesondere spezifizierende Zusätze eine gewisse Modifikation des Bezugssatzgliedes dar und weisen in dieser Hinsicht eine Năhe zu RORREKTUREN auf (vgl. Rap. 13):

(10.48) Ein Mofa, nein: ein Moped brauste um die Ecke.

Ein Fahrzeug, genauer: ein Moped, brauste un die Ecke.

Als eindeutig bestimmendes bzw. differenzierendes Merkmal lảBt sich die Heglabbarkeit jedoch nicht verwenden (vgl, auch Kap. 3, Abs. 8).

Bereichsbildende Sprachausdrücke wie z.B. die Negationspartikel nicht und Gradpartikeln können "nachgetragene Satzglieder" nicht vom Trăgersatz aus affizieren (sondern nur Elemente innerhalb des GASTSATZES). Der bereichsbildende Ausdruck muß "im Nachtrag selbst" stehen, um diesen betreffen zu kōnnen:

(10.49a) Pia ist nicht viel zu schnell nach Hause gefahren, sondern langsam.

(10.49b) Pia ist nicht nach Hause gefahren, und zwar viel zu schne11, sondern ins Maxim gegangen/ * langsam.

(10.50a) Pia ist nach Bause gefahren, und zwar nicht viel zu schne11, sondern langsam.

(10.50b) *Pia ist nicht, und zwar viel zu schnell, nach Hause gefabren, sondern langsam.

(10.51a) Pia hat sogar $g$ e stern den opa besucht.

(10.51b) Pia hat sogar den $O$ pa besucht, und zwar gestern. * Pia hat sogar den Opa besucht, und zwar g e stern.

(10.52a) Pia hat den Opa besucht, und zwar sogar gestern.

(10.52b) ?Pia hat sogar, (und zwar) gestern, den 0 pa besucht. Die Nichtbetreffbarkeit ist unabhangig von der Position des Nachtrags (rechts vom Satz oder in diesen eingeschoben). Satzgliednachtrăge verhalten sich also wie andere HOSPITANTEN (Zusătze) auch, insofern als sie nicht aus dem Gastsatz heraus affizierbar sind. Es bleibt aber darauf hinzuweisen, daß gewisse NP-Zusatze, vor allem solche mit Menge-Element-Beziehungen, durchaus auf anwesende bereichsbildende Operatoren reagieren (vgl. Rap. 12, 15). 
Fazit: Nachgetragene Satzglieder (mit Spezifikationsbeziehung zum Gastsatz oder zur VP bzw. zum hierarchisch obersten Satzvollverb) weisen eine deutliche Ahnlichkeit mit den satzgliedbezogenen "Nachtrăgen" auf. Deshalb wurden beide Phănomene bei Altmann (1981: $70 \mathrm{ff.}$ ) sinnvollerweise einer Großklasse (eben NACHTRAG) zugeordnet. Die Erklärung beider Phänomene als Tilgungsprodukt nach Satzverdoppelung (und evtl. Einbettung in den Gastsatz) spricht allein schon stark zugunsten einer gemeinsamen Funktionsklasse.

Mit Parenthesen, speziell mit Schaltsätzen, haben die nachgetragenen Angaben mehr gemeinsam als die nachgetragenen Satzgliedzusätze, die Parenthesenischen i.d.R. nicht besetzen können und auch funktional nicht über das Konzept der "Nebenhandlung" bzw. des "Nebengedankens" zu erklăren sind. Beides ist bei nachgetragenen (resp. eingeschobenen) Satzgliedern schon naheliegender.

Dennoch bestehen sehr deutliche Unterschiede. Die nachgetragenen Angaben könnten bei entsprechender Umstellung und dem Vegfall der Einschaltungsmarkierung (sowie ggf. der streichung begleitender Elemente wie und zwar etc.) eine syntaktische Funktion im Bezugssatz ausüben (nämlich Adverbial), die Parenthesen - man betrachte etwa die Schaltsatze und die Interjektionen - vermögen dagegen nicht auf diese Weise in ihren "Bezugssatz" integriert zu werden. Sie können dort keine Funktion ausüben. Zudem vermögen die diskutierten Angaben nicht wie Schaltsätze, Interjektionen etc. vor dem "Bezugssatz" zu stehen.

ubrigens ist die Möglichkeit der Integration auch nicht für die satzgliedbezogenen Zusätze gegeben, sie können bei entsprechender Umformung nicht als Satzgliedteil/Attribut fungieren. Wie weiter oben erwăhnt, sollte man bei Fällen wie er ist halt ein Mensch, (und zwar) ein dummer nicht von einem nachgetragenen Attribut sprechen, sondern von einer spezifizierenden NP, deren nominaler Rern tilgbar ist:

(10.53) Die Affen, vor allem die Paviane, begeisterten die Kinder. * Die Affen vor allem die Paviane begeisterten die Kinder.

In solchen Konstellationen liegt eine Art "Doppelsetzung" des betreffenden Satzgliedes vor, ohne daB es tatsăchlich doppelt im Satz vorkäme (der zusatz wird ja mittels des Einschaltungsmusters als außerhalb des Satzes stehend markiert), denn dies würde zu inakzeptablen syntagmen führen. Der "Nachtrag einer Angabe" hat dagegen mit Doppelsetzung o.a. nichts zu tun.

Eine endgültige Einordnung für die und zwar-begleiteten Nachträge, die i.d.R. rechts vom Satz stehen, sich jedoch parentheseartig in den Bezugssatz hinein verschieben lassen, konnte nicht erreicht werden. Ich favorisiere ausgehend von den vorliegenden Testergebnissen im Moment folgende $\mathrm{Zwischenlösung:}$

"Nachgetragene Angaben" und "nachgetragene Zusătze (zu Satzgliedern)" gehören zu den HOSPITANTEN, und zwar in die GroBklasse der ZUSATZE. Bei den spater ausführlich behandelten "spezifizierenden", "prădizierenden" usf. Zusätzen ist dies evident. Bei den Angaben weniger, denn ihr Bezugselement ist ungleichrangig: Satz bzw. Verbalphrase (Prădikat). Zudem kŏnnen sie relativ frei in den Satz eingeschoben werden. Doch von den EINSCHOBEN (Schaltsatz, Interjektion, 
Anrede usw.) unterscheidet sie vor allem, daß sie nach Wegfall des Winschaltungsmusters im Gastsatz eine syntaktische Funktion ausüben könnten (die EINSCHUBE dagegen nicht) und daB sie keine eigene Illokution transportieren (was die Parenthesen/EINSCHUBE tun). Uberdies erscheinen die Angaben nicht wie die EINSCHOBE potentiell auch links vom Satz; in der Parenthesenische zwischen Vorfeld und Finitum ist ihr Auftreten nicht selten mit einer Akzeptabilitäsminderung verbunden.

Nachgetragene und (vor allem) eingeschaltete Angaben werte ich infolge der Testergebnisse als ZUSATZE ZUM SATZ bZw. SATZ-ZUSATZE (z.B. bei potentieller Temporaladverbialfunktion) oder als ZUSATZE ZUR VP bZW. VP-ZUSATZE, wenn potentiell eine Modaladverbialfunktion vorliegt. 


\section{PARENTHESE UND APPOSITION}

Der Begriff "Parenthese" scheint wie der Appositionsbegriff sowohl hinsichtlich seines Umfangs als auch seines Inhalts noch einer befriedigenden Klärung zu bedürfen. Als erste Annăherung diene die Definition in Bußmann (1983: 370):

"In einem komplexen Satz eingefügter selbständiger Ausdruck (Wort, Wortfolge oder Satz), der strukturell unabhăngig ist vom gesamten Satzgefüge

(...). Zu P. [Parenthesen; W.S.] im weiteren Sinne zählt man auch Interjektionen, Anreden und Schaltsătze".

Die Selbständigkeit (s.trukturelle Unabhängigkeit) wird dabei durch das "Einschaltungsmuster" (vgl. Kap. 3, Abs. 2) markiert. Bei der sichtung der Fachliteratur zur Parenthese stößt man noch auf weitere Erscheinungen, die als $\mathrm{Pa}-$ renthesen bezeichnet werden, u.a. mit dem Einschaltungsmuster versehene "Angaben" (Adverbiale; vgl. Kap. 10), Versprecher und Korrekturen. Selbst der Appositionsprototyp wird manchmal der Parenthese zugerechnet (z.B. in Schwyzer 1939; Bayer 1973). Die Vagheiten des Parenthesebegriffs haben m.E. auch damit zu tun, daB nicht immer eindeutig Stellung bezogen wird, ob mit Parenthese eine syntaktische Relation, eine Ronstruktionsart (ähnlich Satzfolge, Phrasenkoordination etc.) oder aber eine pragmatische Erscheinung (im Zusammenhang mit RORREKTUR, NEBENINFORMATION etc.) bezeichnet wird.

Mir erscheint es notwendig, den Begriff Parenthese in seinen unterschiedlichen Fassungen innerhalb einzelner Forschungsansätze vorzustellen, um einen Bereich "parentheseverdăchtiger Muster" zu erhalten. ${ }^{1}$ Dann unternehme ich den Versuch, den Parenthesebereich durch Angabe wesentlicher Eigenschaften solcher Muster zu umreißen sowie mithilfe grammatischer Merkmale intern aufzugliedern, und schließlich herauszuarbeiten, inwiefern lockere Appositionen, ja ZUSATZE im allgemeinen, mit typischen Parenthesen verwandt oder von diesen abzusondern sind. Ich werde im Verlauf dieses Kapitels den Ausdruck Parenthese ablegen (v.a. um traditionellen Implikationen zu entgehen) und die parentheseverdachtigen Muster im Rahmen meines Modells (Kap. 5.5) von HOSPITANTEN in Gastsätzen $z u$ verorten versuchen. Die Rernfrage ist hierbei, ob die zu diskutierenden parentheseverdachtigen Strukturen zum Muster EINSCHUB oder zum Muster ZUSATZ gehoren.

Nicht $z u$ den PARENTHESEN rechnen m.E. die HERAUSSTELLUNGEN, die übrigens auch in der entsprechenden Fachliteratur bisher nicht dazugezählt wurden (zu den Unterschieden vgl. Kap. 10; vgl, auch Altmann 1981).

1 Leider kannten die Ausführungen von Heringer (1988: $274 \mathrm{ff}$.) nur noch peripher berücksichtigt werden, da sie mir zu spát bekannt wurden. 
Schwyzer (1939: 31) beschreibt die Parenthese folgenderweise:

"Vom formal grammatischen Gesichtspunkt ist eine Parenthese ein grammatischer Hauptsatz, der den grammatischen Zusammenhang eines anderen Satzes unterbricht. Der parenthetische Satz kann sich auf ein einzelnes Wort des Gastsatzes beziehen (besonders durch ein Pronomen oder Pronominaladverb); er kann auch eine sprachliche Andeutung des Gedankenverhältnisses enthalten (durch Partikeln und Konjunktionen) (...). Doch ist beides nicht nötig: als ursprünglich ist vielmehr die asyndetische Eingliederung $z u$ betrachten". Diese Bestimmung schränkt er später ein (ebd. 44): "es gibt auch parenthetische (...) Nebensätze". "Visuell kenntlich" würden Parenthesen gemacht durch runde und eckige Klammern, Rommata und Gedankenstriche (ebd. 30). In gesprochener Sprache finde man als Kennzeichen pausen und eine "(...) besondere (im allgemeinen tiefere, doch manchmal auch höhere) stimmlage, die den Einschub mehr oder weniger vom Satz abhebt"; zudem werde die Parenthese schneller als der übrige "Gastsatz" gesprochen (ebd. 32). Formal trete eine Parenthese häufig als Aussagesatz, aber auch als Frage, Befehl oder Wunsch auf (ebd. 32).

Was die stellung der Parenthese betrifft, so komme sie als Prosthothese (Anfangsparenthese), Opisthothese (Endparenthese) - die aufgrund der Endstellung leicht zur Digression geraten kann - und natürlich als (prototypische) Mesothese ("Mittenparenthese") vor (ebd. 33-37). Funktionell bestimmt Schwyzer (1939: 32) die Parenthese als

"(...) eine Mitteilung, die kaum je unbedingt nötig, of aber erwünscht ist, eine nähere Ausführung zu schon Gesagtem oder eine zusätzliche Erklärung; auch ein Ausbruch des Affektes kann in parenthetischer Form auftreten. (...) Die Parenthese ist also gedanklich ein beliebiger Zwischengedanke oder Nebengedanke, der sich in einen vor sich gehenden Gedankenablauf eindrängt".

Interessant ist nun, daB Schwyzer (ebd. 38) neben den im Vordergrund stehenden satzformatigen parenthetischen Einheiten (Haupt- und Nebensatz) auch weitere Elemente mit behandelt; wichtige gemeinsame Merkmale sind dabei das Stellungsverhalten und die Selbständigkeit der Parenthese:

"Der Vokativ zeigt also alle drei stellungen der Parenthese (..) und er darf hier genannt werden, weil er syntaktisch wenigstens ursprünglich kein unselbständiges Wort ist: als Anrede wie als Ruf (...) drückte der Vokativ zunächst einen abgeschlossenen Gedanken aus".

Wie der Vokativ (als "Wortparenthese" im Ggs. zu Satzparenthesen) weisen auch "Vokativpartikeln" (he! hallo!) und Interjektionen die parenthetischen stellungsmerkmale sowie die Eigenständigkeit auf (ebd. 38 f.).

Neben den satz- bzw. außBerungswertigen Vokativen und Interjektionen erwăhnt Schwyer (1939: $39 \mathrm{ff.;}$ Beispiele in (11.1) von Schwyzer) die "echten Wortpa- 
renthesen". 2 Damit lenkt er die Aufmerksamkeit auf ein Muster (11.1b), dessen grammatische Beschreibung bis heute noch aussteht; (11.1a) wăre wohl als Rechtsversetzung (bzw. referenzklärender Zusatz, Kap. 12, 15) zu betrachten:

(11.1a) er (Meyer) ( er (nämlich Meyer).

(11.1b) Isfahan (Heerlager) ( Isfahan (zu deutsch Heerlager). (11.1a, b) erklärt Schwyzer als abgekürzte Satzparenthesen. ${ }^{3}$ Außerdem behandelt Schwyzer die "Satzgliedparenthesen", also Angaben, die durch das Einschaltungsmuster markiert werden:

(11.2) Sie zog ihren Haushalt, ohne Bänglichkeit, ins Enge.

In (11.2) handle es sich um "(...) ursprüngliche satzglieder, die hier (...) in neuern [sic!] Sprachen - wohl erst nach Analogie der eigentlichen Parenthesen - durch die sperrung herausgehoben werden" (ebd. 39).

Auch "Appositionen" ordnet Schwyzer (1939: 41) den parenthetischen Erscheinungen $z u$ :

"In andern [sic!] Fällen ist zwar die Pause gewahrt, aber die satzgeltung viel weiter verschoben und schlieblich der parenthetische Fremdkörper in das Satzganze grammatische eingegliedert worden: eine andere Gestalt der sprachlichen Konzentration [im Original gesperrt; W.S.]".

Zunächst weist er auf die "Satzapposition" (ebd. $41 \mathrm{f}$. ) hin, die z.B. in

(11.3) Er erbielt eine unbeherrschte Zunge, das schimpflichste Gebrechen/ was das schimpflichste Gebrechen ist/ das ist das schimpflichste Gebrechen.

auftritt. Ihr Bezug ist ein ganzer Satz. Danach behandelt er Fälle, die dem "Appositionsprototypen" entsprechen (Zitat ebd. 42):

"Aber auch die gewönliche Apposition, wenigstens die sogenannte selbständige, hat eine parenthetische Romponente: 'Wilhelm, der Eroberer von England' ist gleichwertig mit der relativen Fügung 'Wilhelm, der England erobert hat', aber entwicklungsgeschichtlich würde die Fügung 'Wilhelm - er war Eroberer von England -' entsprechen".

Dabei macht Schwyzer auch auf den (der Kongruenz widersprechenden) Nominativ aufmerksam, der "den ursprünglichen parenthetischen Nominalsatz" (ebd. 43) er-

2 Ich unterscheide Satz (Konstruktion bestehend aus verbalem Valenzträger + Ergänzungen + evtl. Angaben/Partikeln + Illokution) und Außenung (Wortgruppen oder Wörter mit einer Illokution; z.B. Pst! Ballo! Ballo da unten! Meine Damen und Berren! etc.). Man vgl. Engel (1988: 33):

'Nicht die Sprechakte also unterscheiden sich in den verschiedenen Sprachen, wohl aber ihre Ausdrucksformen (...). (...) Texte bestehen also nicht aus sätzen oder anderen sprachlichen Kanstrukten, sondern aus hußerumgen. Sätze sind syntaktische, hußerungen sind kammikative Einheiten (...)".

Eine Anrede wie Meine Damen und Berren! ist äußenungswertig, weil mit ihr eine Illokution (BEGRUSSDN) vollzogen wird. Sie ist insofern auch "satzwertig", als ein verbales Syntagma diese Punktion erfülen könnte (Ich móchte die anwesenden Damen und Herren herzlich begrüßen!) .

3 Schwyzer (1939: 39); vgl. auch ebd.: 'Noch heute kann man in solchen Fällen 'd.h.' oder 'd.i.' usw. brauchen; das Griechische und Lateinische hatten gar keine andere Möglichkeit". 
kennen lasse. Ebenfalls zu den Appositionen rechnen zudem appositive (prädikative) Adjektive, die aus der Kürzung von Kopulasätzen hervorgehen (Schwyzer 1939: 43; sein Beispiel):

(11.4) ein Morgen, rot und golden (ist er/ war er).

Schwyzer hat damit das zu kartierende Terrain abgesteckt, das zwischen zwei Extremen verläuft: voll ausgebauten Schaltsätzen bis hin zur schaltperiode (Digression) und (Satz)Konzentraten wie z.B. der Apposition oder der "Wortparenthese". Die Satzgliedparenthesen sind m.E. schwer einzuordnen und entweder etwa in der Mitte zwischen den Extremen zu suchen oder als eigenständige Erscheinung $z u$ werten. Die folgende "Terrainübersicht" versucht, die parenthetischen Erscheinungen nach Schwyzer zwischen den Extremen zu ordnen, wobei die formale Selbständigkeit als ungefăhres Einordnungsmerkmal angesetzt wird:

(11.5) Schaltperiode (Digression)

Schaltsatz (Hauptsatz)

Schaltsatz (Nebensatz)

(Vokative, Interjektionen)

Satzapposition, (Satzgliedparenthese)

Appositionen

echte Wortparenthesen.

Im folgenden möchte ich untersuchen, ob eine Grenze zwischen Schaltsătzen und Appositionen verläuft und wo diese verortet werden sollte. (Nebenbei: In der letzten umfassenden Appositionsmonographie (Raabe 1979) werden sowohl Schaltsätze als auch NP-Appositive als "Appositionen" gewertet!) Festzuhalten sind die unterschiedlichen Bezugsmöglichkeiten der parenthetischen Elemente: der ganze Satz (z.B. Schaltsatz mit Pronomen, Satzapposition) oder aber ein Satzglied (z.B. Apposition, echte Wortparenthese).

Winkler (1969) untersucht sog. "Einschübe" (er spricht nicht explizit von "Parenthesen", doch dürfte es erlaubt sein, beide Begriffe als gleichwertig zu verstehen), deren intonatorische Merkmale im Vordergrund stehen; diese bestehen in (a) der Unterbrechung des "Spannbogens" des Satzes, (b) der Umlegung des Spannbogens zu einer 'Mulde' (wobei das Sprechtempo zunimmt) und (c) der Wiederaufnahme des Bogens.

Nach dem Einschub setzt der "Aussagebogen" bzgl. Tonhöhe und Tonhöhenverlauf dort an, wo er durch den Einschub unterbrochen wurde. Nicht immer jedoch erscheine der Einschub als 'Mulde', teilweise sind es lediglich tiefere Tonlage, schwächere (zurückgenommene) Sprechweise oder schnelles Sprechtempo, die den Einschub kennzeichnen.

Für meine Analysen wichtig ist die kategoriale Klassifikation der Einschübe bei Winkler (1969: $289 \mathrm{ff} . ;$ Ubersicht S. 290), aus der ich nur hăufigere Muster explizit aufführe und die übrigen unter "Rest" unspezifiziert lasse. Von 179 Einschüben, die ermittelt und untersucht wurden, sind 
- 82 satzformatig: davon sind 34 Schaltsătze, 25 Relativsătze, 13 Adverbialsătze (TEMP, MOD, KAUS) und 10 verbleiben als "Rest".

- 97 nichtsentential: davon sind 37 freie Angaben (LOK, TEMP, MOD), 26 Appositionen, 12 Attribute und 22 bleiben als "Rest".

Dazu einige Beispiele (aus Winkler 1969: 289-294; Einschübe in Klammern; groBgeschriebene Angaben von W.S.):

(11.6) Die Mutter (die ihn kannte; RELATIVSATZ-ATTR) fragte (...).

(11.7) ich muBte (nachdem ich beteuert batte, daB ich diesen Armreif nehmen wollte; TEMP-SATZ) muBte ich die ganze Kollektion noch anprobieren.

(11.8) es konnte Hystaspäs sein (der Grobvater; APPOS) oder Kambyses.

(11.9) (...) lagert sich (im Hals der Pfeife; LOK) eine Mischung aus (...) $a b$.

(11.10) hat jetzt einen Kasten (mit Pulvertüten; ATTR) vor sich stehen. Bei den Einschüben finden sich in Vinklers Untersuchung sehr hăufig "Angaben", relativ häufig Attribute (darunter vor allem "appositive" Relativsätze!), Schaltsätze i.e.S. (ohne mögliche Funktion im "Gastsatz") und auch nicht wenige Appositionen.

Bayer (1973; Zitat S. 71) vertritt einen etwas anderen Parenthesebegriff:

"Untersucht werden nicht nur die sogenannten "Schaltsätze", sondern alle Sequenzen im Bereich eines Satzes, die nicht als dessen Teil beschrieben werden können und auch nicht als "Versprecher" oder sprachliche Fehlplanungen identifizierbar sind. (...) Unter diese Parenthese-Definition fallen vollstăndige sătze nur dann, wenn sie innerhalb [im Orig. gesperrt; W.S.] eines anderen Satzes, des "Trăgersatzes" stehen".

Es ist zu beachten, daB lediglich "eingeschaltete" Elemente zugelassen werden, wodurch z.B. Satzfolgen, Satzanschlüsse (z.B. Digressionen) u.a.m. ausgeschieden werden. Nach Bayer (ebd. 71) rechnen mithin folgende Erscheinungen zur "Parenthese": (a) Schaltsătze, (b) Anredeformen, -floskeln (Sehr geehrte Damen und Berren), (c) absolute Nominative (gemeint sind "Satzappositionen"), (d) Interjektionen, (e) Satzwörter (bitte, danke, nein etc.) und (f) stereotype Floskeln (glaub ich, nicht wahr usw.). Nicht als Parenthesen gewertet werden Einheiten, die als funktionale Teile des Satzes (Satzglieder) analysierbar sind; auch die "weiterführenden Nebensătze" werden aus dem Parenthesebereich ausgegliedert (ebd. 74).

An Bayers Ansatz ist hervorzuheben, daB er eine Abgrenzung gegen sprachliche Fehlplanungen bzw. Versprecher (man könnte hierzu auch Korrekturerscheinungen - vgl. Ortner 1985 - rechnen) vornimmt, auch wenn er diese für nicht unkompliziert hălt (ebd. 72). Als "Versprecher" wăre z.B. das nachstehende Beispiel aus dem Parenthesebereich auszugliedern: 4

4 Mein Beispiel; W.S. Bine explizite Definition von "Versprecher" wird nicht gegeben. Nach Bayer (ebd. 72) "mus die Entscheidung über das Vorliegen eines "Versprechers" bzw. einer regulären Parenthese der Rompetenz des Transkribenten überlassen werden". 
(11.11) Ein Hund, (nein:) eine Katze (?,) jagte das Hühnchen. Bayers Interesse gilt den Funktionen von Parenthesen; er stellt zwei in den Vordergrund (ebd. $79 \mathrm{ff.}$ ): (a) die kontaktbezogene Funktion (Eröffnung, Prüfung und Aufrechterhaltung des Kontaktes innerhalb einer in Kommunikation stehenden Gruppe), vor allem durch Anreden, Satzwörter, Floskeln und Interjektionen; sie vermögen überall im Satz aufzutreten und (b) die Kommentierung der Nachricht bezüglich ihres Inhalts, ihrer Formulierung, ihrer Gliederung oder des in ihr verwendeten Kodes; hier herrschen schaltsätze vor, zudem finden sich "Gliederungssignale" (erstens, zweitens) und Floskeln wie genauer gesagt oder mit anderen Worten. Sie treten bevorzugt inmitten des Satzes auf.

Auch die Parenthesebehandlung von Altmann (1981: $63 \mathrm{ff} ., 160 \mathrm{ff} ., 202 \mathrm{ff}$. , 337) möchte ich kurz besprechen. Interessant ist folgende Aussage (ebd. 63): "Appositionen sind (...) spezielle Typen von Parenthesen, nämlich solche, die sich auf eine Basis im gleichen Satz beziehen und zu dieser syntaktisch und semantisch in bestimmten Beziehungen stehen. Die übrigen Parenthesen unterliegen dieser Einschränkung nicht und sind ungemein vielgestaltig in ihrer Form und Funktion."

Diese Feststellung deutet an, daB es ein allgemeines sprachliches Muster "Parenthese" gibt, das man in Einheiten ohne Bezugselemente und solche mit Bezugselementen, eben Appositionen, gliedern kann. Appositionen können dabei als "satzwertige, prädikative Konstruktionen" (ebd. 336) aufgefaßt werden, wobei Tilgungen verkürzend wirken; damit ist gemeint, daB Appositive systematisch auf (zugrunde liegende) Kopulasätze bezogen werden können. Dieses Merkmal wird auch von mir als besonders wichtig erachtet. Bei parenthesen selbst (z.B. bei Schaltsätzen) werden keine Tilgungen angenommen, da keine Formidentităten vorliegen (ebd. 337). Zu den Parenthesen rechnet Altmann (vgl. ebd. 63 f.):

(a) Satzadverbien: Die alte Generation, natürlich, war noch bereit draufzuzablen.

(b) Satzglieder (aus den Beispielen geht hervor, daß freie Angaben wie Temporal-, Satzadverbiale etc. gemeint sind): Und dieser, nachdem er meine Arbeiten studiert hatte, sicherte mir $z u, d a B(\ldots)$.

(c) Satzstrukturen: Dann kommt die Kontrolle an der Stadtgrenze - "Nehmen sie bitte die Sonnenbrille ab!" - und dann kann es wirklich losgehen.

Vokative und Interjektionen schließt Altinann (1981: 64) aus dem Parenthesebereich aus.

Floskeln und kriteriale Zusātze (wie z.B. übrigens bei Appositionen), die für Parenthesen typisch sein könnten, sind nicht vorhanden (ebd. 64). Topologisch betrachtet sind Parenthesen an bestimmten Satzstellen, den sog. "Parenthesenischen", möglich. Teilweise können sie auch vorfeldfüllend auftreten (ebd. 64). ${ }^{5}$ Letzteres würde eine (nahezu) vollständige Integration des paren-

5 Gemeint ist das Muster (ebd. 64): 
thetischen Elements in den Satzzusammenhang bedeuten; es ist fraglich, ob dies mit dem Einschubcharakter parenthetischer Erscheinungen zu vereinbaren ist.

Bevorzugt treten Parenthesen in assertiven Sprechakten auf, "(..) wesentlich seltener in Fragen, kaum in Imperativen und so gut wie nie in Exklamativen" (Altmann 1981: 161).

Einige wichtige intonatorische Merkmale der Parenthese sind die Einrahmung durch deutliche Pausen, die (Stau)Akzentuierung des Elements unmittelbar vor der Parenthese (wobei darauf hingewiesen wird, das das Bezugselement einer Apposition einen "vergleichsweise deutlich stärkeren Akzent" trage), sowie die "insgesamt "zurückgenommene" Intonation" (ebd. 202 f.). Die Tonmusterselektion wird folgenderweise beschrieben: 6

"Geht die Parenthese der Tonsilbe im umhüllenden Satz voraus oder folgt sie ihr innerhalb des Nachlaufs, so ist der AnschluB an diesen Satz durch einfache progrediente Intonation markiert. (...) Am Satzende ist die Integration in den tief-oder hochtonigen Nachlauf des übrigen Satzes nicht ganz einfach, die Parenthese entwickelt aber keineswegs wie der Nachtrag ein eigenes Tonmuster".

Insgesamt sind nach Altmann (ebd. 337)

"Parenthesen (...) deutlich weniger formal festgelegt als Appositionen. Sie sind wie normale satzförmige Ausdrücke $z u$ beschreiben, die in andere satzförmige Ausdrücke an bestimmten Stellen (...) eingeschoben werden können. (...) Pragmatisch unterscheiden sie sich in nichts von Appositionen".

Sommerfeldt (1984) behandelt die Parenthesen als "Verdichtungserscheinungen im Satzbau". Parenthesencharakteristika sind dabei (ebd. 243 f.): (a) die Art des Einschubs: Hauptsatz; Nebensatz; Wortgruppe; Wort; (b) keine formalgrammatische Verbindung mit dem "Gastsatz"; Verhältnis (unmarkierter) Koordination; (c) Ausgrenzung durch Gedankenstriche, Kommas, Klammern; (d) semantische Uberordnung (11.12) oder Unterordnung (11.13; ebd. 243).

(F11.1) Ich habe mich, was die Spätwirkungen dieser Erfahrung betrifft, doch beträchtlich verschätzt.

(F11.2) Was die Spätwirkungen dieser Erfahrung betrifft, habe ich mich doch beträchtlich verschatzt.

In (F11.2) besetzt die vermeintliche Parenthese das Vorfeld. Ich deute diese Frscheinungen so: Es handelt sich beim was-Satz um ein restriktives Adverbial (Restriktivangabe; Frage: inwiefern/ in welcher Hinsicht? Ersetzung durch insofern; vgl. auch Engel 1988: 225 f.; 281 f.). Im Mittelfeld besteht die typische Ambiguität zwischen Adverbial und "Parenthese" (vgl. Kap. 8.1.1.), im Vorfeld ist der Satz in jeder Hinsicht topikalisiertes Adverbial, vgl. z.B. das Verhalten bei Kontrastnegation und Gradpartikelanwendung:

(F11.3) Was die Spätwirkungen dieser Erfahrung betrifft, habe ich mich nicht beträchtlich verschätzt, sondern was die unmittelbaren Folgen anbelangt l, da habe ich mich beträchtlich verschätzt).

(F11.4) Sogar was die Sp ä trirkungen dieser Erfahrung betrifft, habe ich mich beträchtlich verschätzt (, nicht nur, was die unmittelbaren Folgen anbelangt).

6 Altmann (1981: 202). Zum Begriff Tonsilbe vgl. heidolph u.a. (1981: 855 ff.). 
(11.12) Das Theater - so heiBt es weiter - muB sich was einfallen lassen (...).

(11.13) Ein (...) chronologischer Aufbau erleichtert das Zurechtfinden nur gelegentlich gibt es Vor- und Rückgriffe - (..).

Sommerfeldt (1984: 246) weist auf das Abgrenzungsproblem Apposition vs. Parenthese bei NPn und Nomina hin. Die Kasuskongruenz der Apposition könne nicht mehr als obligatorisches Merkmal angesehen werden. "Es kann aber angenommen werden, daß fehlende Kasuskongruenz ein Zeichen dafür ist, daß es sich um eine Konstruktion handelt, die den Parenthesen nahe steht". Ohne diese Aussage (1984: 246) in ihrem heuristischen Wert anzweifeln zu wollen, muß doch angemerkt werden, daß dieses Kriterium allein zur Abgrenzung von Apposition und Parenthese wohl nicht ausreicht (man denke z.B. an Fälle mit undeutlicher bzw. fehlender Kasusmarkierung).

In Bassarak (1985) wird der Frage nachgegangen, welche Beziehungen zwischen Parenthesen und ihren "Trägersätzen" bestünden, und ob Parenthese + Trăgersatz stets in ein Satzpaar aufeinanderfolgender Sätze auflösbar seien. Interessant dabei ist vor allem die Frage, ob es "(...) semantische Funktionen gibt, die nicht mit Parenthesen, oder solche, die ausschließlich mit Parenthesen vollzogen werden können" (ebd. $368 \mathrm{f}$. ). Bassarak behandelt nur Schaltsätze ohne Konstituentenstatus (im Trägersatz).

Alle Satzfolgen sind nach Bassarak funktionell mit dem Muster 'Parenthese + Trägersatz' variierbar, allein durch Parenthesen vollziehbare Funktionen konnten nicht ermittelt werden (ebd. 369). Dennoch existieren Bedingungen, unter denen einer Satzabfolge das Muster 'Trägersatz + Parenthese' (bedingt oder unbedingt) vorzuziehen ist. Bedingt z.B. in dem Fall (gekürzt, S. 370):

(11.14) Es scheint, als wolle die Mehrzahl unserer wackerer Gelehrten (- Ehre den Ausnahmen! -) dem großen Geheimnis der Liebe vom Schreibtisch her (...) auf die Spur zu kommen. (Ehre den Ausnahmen!) Sie haben keine Zeit dazu, die vier Pforten der Liebe selbst und immer wieder aufzutun.

bei dem die Referenz des Pronomens sie sich verschiebt. Interessanter sind jedoch (Bassarak 1985: 370)

"(..) Fälle von Parenthesen, die aufgrund ihrer referentiellen Beziehung zu einer Konstituerte des Trägersatzes (ihrer Bezugsdomäne) an eine bestimmte syntaktische Position gebunden sind. Diese satzteilbezogenen Parenthesen (...) können daher nicht aus dem Trägersatz herausgelöst und ihm als Nachfolgersatz nachgestellt werden".

(11.15) Bei Beginn der Welt hatten die Menschen vier Arme und vier Beine, wurden aber für ihren Hochmut - denn sie verglichen sich mit den Götern gehälftet und hoffen seitdem, durch Liebe wieder vereint und eins $z u$ werden. (*Denn sie verglichen sich mit den Göttern). 
Um die spezifische Funktion von Paraphrasen zu ermitteln, muB man sich die Frage stellen, warum ein "Sender" eine Konstruktionswahl wie folgt trifft (und nicht die geklammerte Alternative wählt; Beispiel Bassarak 1985: 370):

(11.16) Juno geriet (- sie wuBte sicher, warum -) in Wut und schlug dem armen Experten so heftig ins Gesicht, daß er das Augenlicht verlor. sie wuBte sicher, warum.

Mit Parenthesen würden "Nebenhandlungen vollzogen" (ebd. 373; vgl. Bassarak 1987: 175 ff.), wobei dadurch "(..) nicht nur das zeitliche Nebeneinander zweier Handlungen, sondern auch deren unterschiedliche Wichtigkeit in ikonischer Weise (...)" (ebd. 374) abgebildet würden. Diese "handlungstheoretische Erklărung" ließe sich allerdings auch auf nichtsententiale Einheiten anwenden (ebd. 374). In Bassarak (1987) wird gezeigt, daB Parenthesen eine andere illokutionăre Rolle innehaben können als deren Trägersätze (ebd. 164 ff.); zudem werden mögliche Funktionen von Parenthesen untersucht (ebd. $168 \mathrm{ff.}$ ), von denen ein paar erwähnt seien: Stützung des Verstehens des propositionalen Gehalts des Trägersatzes; Stützung des Verstehens der Trägersatzillokution; text- resp. gesprächsorganisierende Funktionen; Angabe von Erklärungen usw.

Angemerkt sei noch, daß Bassarak (1985: 375) auf Hinweise aus der Indogermanistik aufmerksam macht (und auch auf Schwyzer 1939),

"(..) daß bestimmte subordinierte Sătze und z.T. auch Appositionen und einige Satzglieder historisch jünger sind als asyndetisch verknüpfte sätze und sich möglicherweise durch elliptische Verkürzung als einem sprachlichen Reflex von Operationalisierungen im Denken aus Parenthesen entwickelt haben (...)".

Die nachstehende Obersicht führt die explizit genannten bzw. als Beispiele auffindbaren "parenthetischen Erscheinungen" bei den besprochenen Autoren auf; explizite Erwăhnung wird mit "+", expliziter Ausschluß aus dem Phänomenbereich mit "-" angezeigt; bei Unsicherheit, ob eine Erscheinung dem Parenthesebereich zugeordnet wird oder nicht, erscheint "(+)":

Schwyzer 39 Winkler 69 Bayer 73 Altmann 81 So.feldt 84 Bassarak 85

\begin{tabular}{|c|c|c|c|c|c|}
\hline Hauptsatz & + & + & + & + & + \\
\hline Aussagesatz & + & + & + & + & + \\
\hline Fragesatz & + & & & & \\
\hline Wunschsatz & + & & & & + \\
\hline Nebensatz & + & + & & + & + \\
\hline Relativsatz & & + & & & \\
\hline Adverbialsatz & & + & & $(+)$ & \\
\hline Vokativ & + & & & - & \\
\hline Anrede & & + & + & & \\
\hline Interjektion & + & & + & - & \\
\hline Satzwörter & & & + & & \\
\hline Floskeln & & & + & & \\
\hline
\end{tabular}


Schwyzer 39 Winkler 69 Bayer 73 Altmann 81 So.feldt 84 Bassarak 85

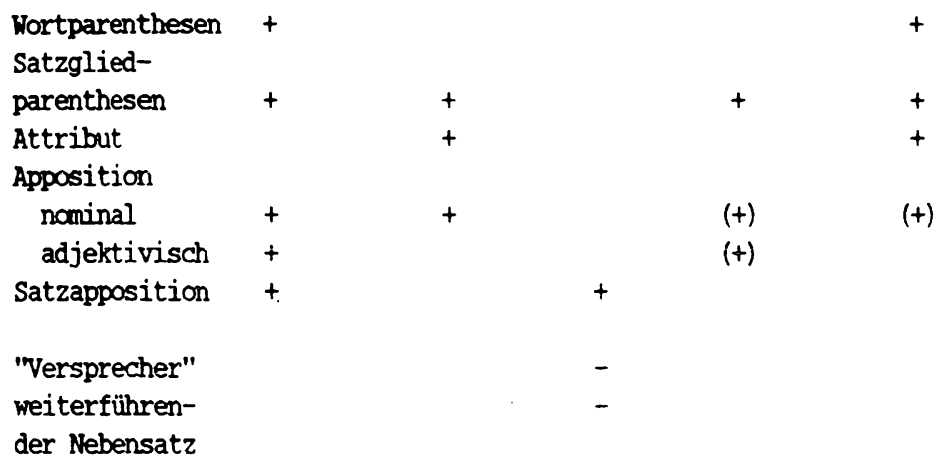

Die tbersicht demonstriert, daß unter dem Begriff Parenthese (als zentral gelten offensichtlich die in allen sechs Arbeiten erwähnten Schaltsätze; auffällig ist zudem die häufige Nennung der eingeschalteten Satzglieder und der "Appositionen") z.T. sehr verschiedenartige Erscheinungen zusammengefaßt werden; ${ }^{7}$ man vgl. z.B. nur Interjektion und Schaltsatz oder Vokativ und Apposition. Das liegt wahrscheinlich u.a. daran, daB man die meisten der mit dem "Einschaltungsmuster" markierten Einheiten als eine Funktion auffassen will. SchlieBlich handelt es sich - in meiner Terminologie - um HOSPITANTEN; doch die interne Gliederung der Muster kommt $z u$ kurz und reflektiert wichtige grammatische Eigenschaften nicht.

Warum muß der Parenthesebereich so ausführlich diskutiert werden? Erstens gibt es Appositionsdefinitionen (z.B. Zemb 1968, 1972, 1978; Raabe 1979), die diverse parenthetische Einheiten mit umfassen; manchmal scheint es so, als ob Parenthese und Apposition nahezu die gleichen Phänomene vereinten. Zweitens sind diverse parenthetische Einheiten den "appositiven Nominalsatelliten" zugerechnet worden; 8 drittens benutzen Parenthesen das typische Muster der "lokkeren Apposition" und können die gleiche Position wie diese einnehmen:

nicht wahr?

meine Damen und Herren

- la $1 a$

$\begin{aligned} \text { (11.17) Pia Müller - } & \text { eine Jirastudentin } \\ & \text { vermutlich } \\ & \text { eine traurige Nachricht }\end{aligned}$

- ist dem Unhold verfalien.

man glaubt es kaum

7 Dies trifft nicht nur für das Deutsche zu; vgl. z.B. Reinhart (1983) und Ziv (1985) für das Englische.

8 So z.B. in v. Polenz (1985: 258 ff.), der Schaltsätze unter die explikativen Naminalattribute einreiht, und in Vater (1986b: 125, 129 f.), der Schaltsätze als appositive Komplemente wertet. 
Eine Gemeinsamkeit der "parentheseverdächtigen" Erscheinungen liegt darin, daß sie nicht direkt dem Satz angehören, daB sie Einschaltungen in einen Gastsatz darstellen, ${ }^{9}$ das sie nicht der Sachverhaltsabbildung und -situierung oder der Gegenstandsidentifizierung dienen wie z.B. die Satzglieder und die Gliedteile, sondern daB sie eher "kommunikativ-pragmatische" resp. text-, kontext- und situationsbezogene Funktionen wahrnehmen (Kontaktaufrechterhaltung und -steuerung z.B. bei Floskeln, Anreden und Vokativen; Kommentare zum Propositionsinhalt z.B. bei Schaltsătzen; Emotionsausdruck z.B. bei Interjektionen u.v.a.).

Nach meiner Terminologie handelt es sich bei den hier behandelten parentheseverdăchtigen Erscheinungen un HOSPITANTEN in Gastsătzen, in Ggs. zu den PARTIZIPANTEN des Trăger- bzw. Matrixsatzes. Im Folgenden versuche ich darzulegen, daß die "parentheseverdăchtigen" Muster in mindestens zwei Großklassen zerfallen:

- die EINSCHOBE (z.B. Schaltsatz, Interjektion, Anrede), die selbst Satzbzw. Xußerungswertigkeit aufweisen und als deren "Bezugselement" der gesamte Satz (z.B. bei "kommentierenden" bzw. "metakommunikativen" Einschüben, vgl. etwa er ist - ich formuliere es im Klartext - ein Schuft) 10 oder aber "Teile der Kommunikationssituation" (bei Anreden, Kontaktsignalen z.B. der Hörer etc.) anzusehen ist. Sie sind sehr stellungsvariabel und erscheinen sowohl zwischen den Satzgliedern (Parenthesenischen) als auch rechts und - mit Einschränkungen - links des Satzes (als Satz- bzw. Xußerungsfolgen);

- die ZUSATZE (z.B. Appositionsprototyp, Rechtsversetzung, Nachtrag), die ein Satzglied als Bezugselement haben (evtl. auch eine Verbalphrase, evtl. auch nur ein Wort, vgl. Kap. 14) oder aber als Satzglied im Gastsatz fungieren könnten. ZUSXTZE sind hinsichtlich ihrer stellungsmöglichkeiten - diese sind hăufig nur mit Bezug auf die Basis des zusatzes formulierbar - deutlich eingeschränkt.

9 ab man 2.B. in Fall

(F11.5) (Meine Damen und Herren!) Pia (- meine Damen und Herren/ jeder meis das -) hat einen Affen als Haustier; (jeder weiß das.)

die links voraufgehende ANREDE bzw. den rechts folgenden Verbzweitsatz, beide auch als Einschaltungen im Satz möglich, als "Grenzfall der PARDMrHIZSE" ansehen soll oder ob man sie dann als syntaktisch wie semantisch selbständige Gebilde betrachtet, ist eine noch offene Frage. Schwyzer (1939: 32 ff.) nimmt an, das ein "Grenzfall" vorliegt. Obwohl ich jetzt keine schlagkräftigen Argumente anführen kann, plädiere ich eher für "Satz- resp. Textteilfolge". Die ANRWDE z.B. kann in der selbständigen Version (links) die "Eröffnung einer Koummikationssequenz" anzeigen, in der parenthetischen Version jedoch nicht. Dort dient sie der "Aufrechterhaltung des Kontakts" (vgl. auch Engel 1988: $60 \mathrm{ff}$. .). Der Verbzweitsatz verhält sich bei parenthetischer Einschaltumg "präsuppositionsartig" und kann scmit nicht cegenstand/Thema des weiteren Textverlaufs werden; als selbständiger Satz vermag er dies durchaus und kann z.B. von Bestreitungen (Kirklich? Nein, das stimmt nicht) betroffen werden (dazu Bartsch 1978; v.a. 8 f.).

10 Angelehnt an ein Beispiel in Antos (1982), S. 127; dort findet sich auch weiteres zu "formulierungskommentierenden Ausdricken". 
Die Eigenschaften der "parentheseverdächtigen Muster" sollen nun beschrieben werden. Die folgenden Ausführungen zielen dabei auf die Eingliederung dieser Muster in die EINSCHUBE und ZUSATZE meines Modells ab, wobei die Unterschiede zwischen beiden, wenn nötig, aufgezeigt werden:

(a) Sie sind intonatorisch bzw. graphematisch markiert ("Einschaltungsmuster") und dies ist ein Indikator dafür, daß diese Einheiten

(b) vom restlichen (Gast)Satz "abgeschirmt" sind; d.h. Negation, Gradpartikeln und sonstige bereichsbildende Ausdrücke können sie aus dem Gastsatz heraus oft nicht affizieren! ${ }^{11}$ Auch eine den Satz (die Proposition) betreffende Frage affiziert sie nicht.

(c) Ihre stellungsmöglichkeiten sind entweder (relativ) unrestringiert oder sie folgen anderen Regeln als die der PARTIZIPANTEN. ${ }^{12}$ Am uneingeschränktesten sind diesbezüglich die EINSCHUBE, die z.T. auch innerhalb von NPn vorkommen können; ${ }^{13}$ die satzbezogenen ZUSATZE ( $z$.B. nachgetragene bzw. eingeschaltete Angaben) sind um einiges unfreier. Für die satzgliedbezogenen ZUSATZE - vor allem die im Zentrum der Analysen stehenden NP-bezogenen ZUSATZE - werden Z.T. starke Stellungsrestriktionen festzustellen sein (vgl. auch Kap. 12, 15). Die Analyse der Stellungsmöglichkeiten wird in diesem Kapitel eine grobe Rolle spielen; daher genügen im Moment pauschale Feststellungen.

(d) Sie sind nicht erfragbar und nehmen an syntaktischen Transformationen, die Satzkonstituenten betreffen, nicht teil; ${ }^{14}$ sollten sie nach Anwendung einer Transformation dennoch beim Bezugselement stehenbleiben, so ist dies nur eine der möglichen optionen und nicht den syntaktischen operationen selbst zuzuschreiben, sondern pragmatischen (stilistischen etc.) Faktoren.

Mc Cawley (1982; v.a. 95 ff.) hat für das Englische demonstriert, das Verbalphrasen sich bei syntaktischen operationen so verhalten, als ob die "parentheticals" gar nicht anwesend wären. Ich will dies anhand eines eigenen Beispiels für das Deutsche zeigen:

(11.19) Pia hat Kuno - er hatte sie getauscht - verziehen, und Hugo tat das auch (, ich meine Kuno verzeihen/ *ich meine Kuno - er hatte sie getäuscht - verzeihen).

Die VP-Pro-Form das (die Rechtsversetzung verdeutlicht das Anaphorisierte) nimmt nur die Elementar-bzw. Gastsatzelemente (die Partizipanten) auf, nicht

11 Bei nanchen NP-ZUSATZWN sind Einschränkungen/Besonderheiten festzustellen; vgl. Kap. 12, 15.

12 Die Stellungsregeln bei Satzgliedern orientieren sich an Faktoren wie kategoriale Füllung des Satzglieds (Pronamen, Voll-NP etc.), Thema-Rhema-Gliedenung, Akzentuierung, Verbgebundenheit etc.

13 Z.B.: Die - wie sie alle wissen/? meine Damen und Herren - exzessiven Orgien müssen aufhören. Man siel.t, daß der Schaltsatzakzeptabel völlig, die Anrede hingegen nicht sehr akzeptabel wirkt.

14 Z.B. PASSIVIERUNG, TOPIKALISIRRUNG, PRONOMINALISIERUNG usf. Man s. auch Schapiro (1977: 14): "Parentheticals and preceding NP do not function as a single constituent under rules which affect NP's". Schapiro fährt fort (1977: 15): "(...) vocatives do not function as a single constituent under rles which affect NP's". 
jedoch den EINSCHUB! Diese Erscheinung gilt im übrigen auch für ZUSATZE (vgl. Kap. 6, 7.A), also wahrscheinlich für alle HOSPITANTEN:

(11.20) Pia hat die Haie, in ihren Augen harmlose Tierchen, ignoriert, und Kuno tat das auch (, ich meine die Haie ignorieren/ *ich meine die Haie, in ihren Augen harmlose Tierchen, ignorieren).

(e) Es sind nur manchmal Umformungsmöglichkeiten gegeben. EINSCHUBE stehen kaum in systematischen Beziehungen zu anderen sprachlichen Mustern (Kopulasatz, Wortgruppe etc.). Wenn überhaupt, dann sind Reduktionen von Schaltsätzen (also EINSCHOBEN) zu ZUSATZEN anzunehmen. ${ }^{15}$ Bei den satzgliedbezogenen ZUSATZEN bestehen ofters, jedoch nicht generell, Umformungsbeziehungen (KopulasatzParaphrase; Satzkopie plus Tilgung).

(f) Sie sind i.d.R. weglaßbar und affizieren den Wahrheitswert des Satzes nicht.

(g) Inwiefern eine Satzgrenzenbestimmtheit vorliegt, ist nicht leicht auszumachen. Zumindest die holophrastischen Einheiten (ANREDE, INTERJEKTION, voKATIV etc.) und wohl auch die meisten Verbzweit-Schaltsätze scheinen Satzgrenzen nicht beachten zu müssen - sie sind selbst als satz- resp. äußerungswertig anzusehen und gehen mit potentiellen Gastsätzen auch Satzfolgen ein. ZUSATZE vermögen keine Satzfolgen $z u$ bilden, da sie wegen ihres Bezugs auf Teile des Gastsatzes (im Grenzfall auf den ganzen Gastsatz?) nicht völlig ausgegliedert werden können.

(h) Die EINSCHUBE, aber auch etliche ZUSATZE, stammen gewöhnlich vom aktuellen Sprecher und müssen deshalb in referierter Rede entsprechend gekennzeichnet werden (Sprecherdivergenz).

(i) Parenthetische Erscheinungen sind weder als Konstituenten des Gastsatzes (wenn überhaupt, dann als dessen Ko-Konstituente unter einem allerobersten S-Symbol) noch als dessen Dependentien anzusehen. ${ }^{16}$ Bei den EINSCHÜBEN ist das eindeutig. EINSCHUBE wie Schaltsätze etc. erfüllen im Gastsatz keine syntaktische Funktion, sondern sind - das ist ein konstruktionaler Aspekt - in diesen eingeschaltet, sind diesem adordiniert (und nicht sub- oder koordiniert).

Bei den satzgliedbezogenen ZUSATZEN kann man immerhin ein evtl. konstituentenartiges (-ăhnliches oder besser: "assoziatives") Verhältnis zum Bezugselement ansetzen. 17 "ZuSATZ" fasse ich als eine syntaktische Funktion (neben Satzglied, Attribut, Partikelfunktion) auf.

Ich gehe nun "top down" vor, indem ich zuerst die grammatisch selbständigsten Einheiten - die EINSCHUBE - behandle, und dann zu den unselbständigeren (überwiegend "gliedassoziierten") Einheiten, den ZUSATZEN, fortschreite.

15 Vgl. 2.B.: Harry Birsch (er gehört der SPD an) hält eine Rede zu Harry Hirsch (CDU) hält eine Rede.

16 Vgl. dazu die Vorschlăge von McCawley (1982), die auf das Deutsche übertragbar sind.

17 Man erinnere sich an meinen Rometenvergleich am Ende von Kap. 6. 
EINSCHOBE sind weder SATZGLIEDER noch SATZGLIEDTEILE und haben auch keine Bezugselemente (Pro-Formen; Satzglieder) wie die HERAUSSTELLUNGEN ( $z$.B. Linksversetzung, Freies Thema) oder die zUSATZE. Sie weisen i.d.R. Satzbezug auf. Ob man darüber hinaus einbedenken muß, daß der "Skopus eines Einschubs" nur Teile des Gastsatzes umfaßt - i.d.R. die rechts von diesem befindlichen - kann hier leider nicht behandelt werden. 18

Mindestens die "kontaktbezogenen Parenthesen" wie ANREDE, KONTAKTPARTIKELN, FORMELN (REDEEROFFNUNG etc.) etc. sind rein hörer-bzw. empfängerbezogene Elemente und selbst satz- resp. äußerungswertig (holophrastisch). Ebenfalls holophrastisch sind die INTERJEKTIONEN. Auch SCHALTSATZE beziehen sich nicht auf (a) den syntaktischen Satz (Prädikat, Satzglieder, Partikelfunktionen), sonst wären sie den satzsyntaktischen operationen resp. den operationalen Verfahren voll zugänglich; (b) den semantischen Satz (das Satzradikal bzw. die Proposition; Satzglieder, Negation etc.), sonst würden sie ja den Wahrheitswert affizieren bzw. einen direkten Beitrag zur Satzproposition leisten; (c) den iliokutionspragmatischen Satz (Modalpartikeln, Satzmodi usw.), weil sie nicht illokutionsbeeinflußend wirken. Viele schaltsatzformen haben überdies eine eigene illokutionäre Rolle. Möglicherweise ist diese Aussage jedoch zu korrigieren, da Schaltsätze evtl. doch illokutionsmodifizierend wirken:

(11.21) Kuno fertigt, und das ist ein Befehl, diese Liste an. Kuno fertigt, ich bitte jedenfalls darum, diese Liste an.

Im folgenden soll nun die äußerst vielgestaltige Menge der EINSCHUBE und ZUSATZE auseinanderdifferenziert werden. Nachdem bereits alle nicht-einschaltungsmarkierten Muster ausgeschieden wurden, wird der vorletzte Aufgliederungsschritt vorgenommen: Aus dem übriggebliebenen Bereich der appositionsund parentheseverdächtigen strukturen sollen die im engeren sinne appositionsverdächtigen Muster aussortiert werden. Ein wichtiges Merkmal stellt dabei das Stellungsverhalten dar, das den Grad der Eigenständigkeit des jeweiligen HOSPITANTEN widerspiegelt: So weisen $z$.B. kommunikations- resp. kontaktbezogene Funktionen wie Anrede, Kontaktsignal (nich' wahr etc.) usf. eine weitgehende stellungsfreiheit auf, denn ihre Verwendung ist ja u.a. auch "extra-

18 Hierzu z.B. Heringer (1988: 277); er zeigt, daß der rechtsbefindliche Skopus einer Partizipial-Parenthese stellumgsabhängig variiert. In (F11.6) umfaßt er alle analytischen Sätze a priori, in (F11.7) nur a priori:

(F11.6) So sind - übertrieben ausgedrückt - alle analytischen Sätze a priori.

(F11.7) So sind alle analytischen Sätze - übertrieben ausgedrickt - a priori.

(F1.8) So sind übertrieben ausgedrückt alle analytischen Sätze a priori.

Derartige "Parenthesen" sind in meinen Augen keine EMNSCHOBE, sondern ZUSATZE, nämlich einschaltungsmarkierte Angaben (Kap. 8.1.1.). Sie könnten bei entsprechender Manipulation in "Bezugssatz" eine syntaktische Funktion ausüben - in (F11.8) z.B. konditionales Adverbial. 
linguistisch" bedingt. ${ }^{19}$ Ebenso sind die Schaltsätze als Kommentare, Nebenhandlungen usw. in ihrer stellung sehr frei.

Wie bereits bemerkt, verhält sich z.B. der "Appositionsprototyp" anders: Seine Position ist relativ zum Bezugselement beschränkt, z.T. ist lediglich unmittelbare Nachstellung möglich. Dies charakterisiert derartige Einschaltungen als ZUSATZE zu bestimmten Satzkonstituenten, die deren Bewegungsfreiheit stark einschränken. Als weitere Differenzierungsmöglichkeiten bieten sich dann vor allem die pragmatischen Funktionen an.

Ich möchte die Stellungsmöglichkeiten der "parentheseverdächtigen" Einheiten mithilfe eines Verbzweit- und eines Verbendsatzes untersuchen. Es ist festzustellen, welche Einheit (Funktion) an welchen Positionen vorzukommen vermag. Die Testsätze werden in einer möglichst unmarkierten Form (hinsichtlich Wortstellung, Intonation etc.) gewählt:

(a) Die Frau hat II gestern IV dem Kind einen Ball VI gegeben(,) VII VIII

(b) daß die Frau gestern III dem Kind IV einen Ball $\mathrm{V}_{\text {II gegeben hat(,) }}^{\text {VI }}$ VII

Erläuterungen: Die Position I in (a) meint, daB die fragliche spracheinheit vor dem Testsatz stehen kann, sei es als erster Teil einer Satzfolge (Er sah gestern eine Frau. Die Frau (...) gegeben) oder, wenn passend, als "einleitendes Element" (Was noch niemand wuBte: Gestern hat (...) gegeben). Die Pos. VII (a) bzw. VI (b) meint, daß die fragliche Einheit, abgetrennt durch Komma (intonatorisch: Pause) oder evtl. andere Zeichen außer Satzpunkt, dem Satz nachfolgen können. Die allerletzte Position (a: VIII; b: VII) sei durch Satzpunkt/Satzpause abgetrennt, so daß wiederum eine Satz- bzw. Außerungsfolge vorliegt.

Zunächst will ich primär kontaktbezogene Einheiten wie VOKATIV, ANREDE, INTERJEKTION etc. prüfen. ${ }^{20}$ VERSPRECHER, die im weitesten sinn auch als kontaktbezogen (präziser: hörerbezogen) aufzufassen sind, bleiben hier außer acht. 21 Korrekturerscheinungen (vgl. hierzu z.B. Rath 1979: 185 ff., Ortner 1985) wie

(11.22) Sie hörten, nein: saben (,) den rosaroten Panther.

(11.23) Es war ein Mofa, [nein:] ein Moped, das den Lärm verursachte.

19 Wenn der Sprecher z.B. erkennt, daß der Hörer unaufmerksam wird, dann wird er eine kontaktverstärkende resp. aufmerksamkeitsheischende Einschaltung vornehmen etc.

20 zu den Spracheinheiten, die primăr "phatische" resp. hörerorientierte Funktionen haben, z.B. beim Aufbau (ANREDE, EDNLETTUNGSFIOSKEL) oder bei der Beendigung (SCHLUSSFLOSKEI, VERABSCFIEDUNG) einer sprachlichen Kormunikation, siehe v. Polenz (1985: 222 ff., v.a. 224). Auch Bayer (1973: 71, $78 \mathrm{ff} ., 101 \mathrm{f} ., 105 \mathrm{f}$.$) .$

21 Eine Abgrenzung der "Versprecher" gegen die PARENTHESEN wird bei Bayer (1973: 72) vorgenoumen. 
werden, obwohl ebenfalls das Einschaltungsmuster "benutzt" wird, erst später berücksichtigt (s. Kap. 12, 16). Molitor (1979: $214 \mathrm{ff.}$ ) grenzt übrigens die "Korrektion" (wie übrigens auch die pure Wiederholung eines Satzteils) gegen die "Apposition" streng ab.

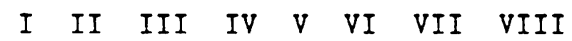

Meine Damen und Herren

(ANREDE)

Frau Huber

(ANREDE)

Lieber Karl

(VOKATIV)

Aua, aha etc.

(INTER JEKTIONEN)

Danke, bitte

(SATZWORTER)

nicht wahr

(FLOSKEL)

glaub ich

(FLOSKEL)

ANREDE, VOKATIV, INTERJEKTION, SATZWORT und FLOSKEL (mit phatischer bzw. kommunikationsbezogener Funktion) sind Einheiten, die offensichtlich kaum stellungsbeschränkungen unterliegen, d.h. sie können vor und nach dem Satz sowie $z$ wischen den Satzgliedern erscheinen. Bei den INTERJEKTIONEN ist zudem vorstellbar, daB sie sogar innerhalb von wortgruppen (NPn) vorkommen, weil ihr "Motiv" auch außersprachlich sein kann (z.B. ein Bienenstich oder eine plötzlich die Sinne affizierende Erscheinung, die die Satzsequenz zu unterbrechen veranlaßt: Sie haben den - au - alten Affen besucht. Sieh doch einmal dort das wunder - ob - schöne Feuerwerk.).22

ANREDEN als Kontaktsignale, die eine "phatische Funktion" haben, sind ebenfalls unrestringiert, weil ihr Auftreten, sofern es sich nicht ohnedies nach "textlinguistischen Mustern" richtet (daB man z.B. die Kommunikation mit einer Anrede eröfnet: Frau Huber, sie wissen ja noch gar nicht (...), u.a. davon

22 Dazu Trabant (1983) und Ehlich (1986). Nach Trabant (ebd. 70) sind für Interjektionen "im Satzinneren bestimmte Positionen ausgeschlossen" (vor allem dann, wenn sie sich auf das im Satz Gesagte beziehen), wobei er vornehmlich die Positionen innerhalb von Satzgliedern anspricht. Seinem Beispiel (Trabants Markierungen!): Am (*ob) Freitag sind viele (*oh) Leute (?oh) abgefahren fügt er das Beispiel Am (Feuerwerk) - oh - Freitag sind viele Leute abgefahren an (kein Bezug auf das im Satz Gesagte!); der Bezug ist ein Geschehen außerhalb des Redezusammenhangs und der Satz ist akzeptabel (ebd. 71). Genau diese "Janusköpfigkeit" spiegelt wesentliche Merkmale der Interjektionen wider. Insofern sind Interjektionen potentiell an relativ vielen Satzstellen moglich, zumindest aber an den Satzgliedgrenzen. 
abhăngt, wann der Sprecher die "Kontaktpflege resp. -auffrischung" für angebracht hält (z.B. bei erkennbarer Unaufmerksamkeit des Hörers). Ahnliche Gründe lassen sich für FLOSKELN der Art nicht wahr, glaub' ich, sagen wir, gell und für VOKATIVE anführen. 23 ubrigens sind vOKATIVE von "Vokativischen NPn" als Herausstellungen klar zu unterscheiden (dazu Altmann 1981: 51 f.).

Die SATZWORTER (Terminus von Bayer 1973: 71; z.B. danke, bitte) sind hinsichtlich ihres Vorkommens augenscheinlich eher an Positionen links und rechts neben dem Satz gebunden, da sie z.T. als sprachliche Reaktionen z.B. auf eine positive Außerung fungieren (vgl. A: Ich habe übrigens ihrem wunsch entsprechen können. B: Danke.). Ob sie innerhalb von Sätzen vorkommen, hängt wohl (wie bei Interjektionen) von "außersprachlichen Faktoren" ab, z.B. wenn während des Aussprechens des Satzes dem Sprecher etwas Gewünschtes zugereicht oder signalisiert wird.

Die untersuchten Einheiten, dies kann ohne weitere Ausführungen wohl geschlossen werden, sind sicher keine Appositionskandidaten. ${ }^{24}$ Insbesondere der Mangel eines Bezugselements, die sehr große Stellungsfreiheit, die Außerungswertigkeit und die (nahezu) fehlende syntaktische Integration in den Gastsatz sowie ihre pragmatischen Funktionen (hörer- bzw. kanalorientiert) schließen diese HOSPITANTEN als appositionsunverdächtig aus. ${ }^{25}$ Ich weise sie zunächst generell den (rein kommunikationsorientierten) EINSCHUBEN zu.

Abschließend sei noch auf den Aufsatz von Burkhardt (1982: 156) hingewiesen, der viele der soeben betrachteten Einheiten als "Gesprächswörter" zusammenfabt und funktional aufgliedert.

Nun will ich mir die typischen Parenthesen, nämlich die Schaltsätze, ansehen. Sie vermögen vor einem Satz vorzukommen:

23 Kormunikationsbezogene Funktionen von Floskeln/Formeln wären z.B. "Verschaffen einer Denkpause", "Anzeige einer hypothetischen Situation" (sagen wir), "dem Hörer anzeigen, daß man noch weiterreden will" etc. Hierzu auch Weiss (1975: $57 \mathrm{ff}$. ).

24 Man vgl. auch die Ausführungen von v. Polenz (1985: $222 \mathrm{ff}$. "Kontakt und Beziehung"). Fr weist (ebd. 223) darauf hin, daß die diesbezüglichen Ausdrucksformen noch nicht genügend in eine grammatische Beschreibung integriert seien:

"Was wir im Folgenden (...) grob unter "Kontakt und Beziehung" exemplarisch behandeln, ist also noch keine wohldefinierte Kategorie, sondern ein vielfältiger Restbereich der Sprachpragmatik (...)".

25 "Große Stellungsfreiheit" ist allerdings als Ceneralisierung unzutreffend; mindestens gewisse "Gliedenmgspartikeln" sind an bestimnte Positionen gebunden - so z.B. die ungangssprachliche Gliederungspartikel ne (mit steigender Intonation; Funktion: 'Bestätigung/Zustimmung heischen', z.T. aber rein rhetorisch) oder die Partikel so (mit fallender Intonation und nachfolgender Satzpause; Funktion: 'Ankmüpfung'):

(F11.9) Pia (??, ne,) hat (??, ne,) den Kuno (??, ne,) doch (??, ne,) verlassen, ne?

(F11.10) So, Pia $(*$, so,) hat $(*$, so, ) also $(*$, so, ) den Kuno $(*$, so, ) verlassen $(*$, so). 
(11.24) Das haben jedenfalls einige vermutet: Die Frau hat gestern dem Kind einen Ball gegeben.

Dann jedoch handelt es sich nicht mehr um "Einschübe", sondern um Satzfolgen. Die Terminologie bedarf kurzer Erläuterung: SATZFOLGE und vor allem asyndetische SATZVERBINDUNG eindeutig auseinanderzuhalten, erscheint schwierig. Wahrscheinlich muß man ohnedies ein "Kontinuum" zwischen zwei voneinander syntaktisch und semantisch unabhängigen sätzen und der Integration eines Satzes in einen anderen (Dependenzverhältnis) annehmen; es wăre in etwa abzustecken mit den Begriffen: SATZFOLGE - asyndetische SATZVERBINDUNG - syndetische SATZVERBINDUNG - ZUSAMMENGEZOGENER SATZ (zu den Termini Helbig/Buscha 1980: 559 ff.). Pragmatik (Sequenzen wie Frage/Antwort etc.), Semantik (inhaltliche Verbindungen zwischen S1 und S2) sowie (Text)Syntax (Vorwärts-, Rückwărtsellipse; Pronominalisierungen, consecutio temporum, Intonation am Ende von S1 etc.) wären $z u$ berücksichtigen. Ich erlaube mir eine gewisse Laxheit in diesem Bereich, da eine exakte Klärung in bezug auf die Parenthese- resp. Appositionsproblematik nicht notwendig scheint.

Zum Schluß: Die Orthographie ist kein sehr zuverlässiges Kriterium, um zwischen Satzverbindung (asyndetisch) und Satzfolge zu differenzieren. Auch die Kriterien für asyndetische Koordination von Helbig/Buscha (1980: 559), "progrediente Intonation" und "semantischer Zusammenhang", reichen vermutlich nicht aus. Die progrediente Intonation als Indikator für die Unabgeschlossenheit eines Satzes kann man jedoch heranziehen, um zwischen der "Komma-Abtrennung" (eher asyndet. Verbindung) und der "Punkt-Abtrennung" (eher satzfolge) zu trennen (Pos. VII/VIII in (a), VI/VII in (b)).

In Făllen wie (11.24) greift die Unterscheidung zwischen Gastsatz und Hospitant nicht mehr, denn wie soll man die Einteilung belegen? Satzsyntaktische Gesichtspunkte wie Einschaltungsmuster, parenthetische stellung etc. treffen ja nicht mehr zu. Andere satzsyntaktische Merkmale wie z.B. die Nicht-Affizierbarkeit durch Negation aus dem jeweils anderen Satz heraus bleiben natürlich bestehen, weil Satzfolgen sich hierin von Satzeinschaltungen nicht unterscheiden.

Falls man über den "Tatbestand" der Satzfolge hinaus zu differenzieren gedächte, müßte man textlinguistisch (Pro-Form beim "Hospitanten") und semantisch-pragmatisch (der erste Satz ist inhaltlich abhängig resp. unselbständig) argumentiexen. Ob man damit etwas gewönne (nămlich einen Unterschied zwischen (11.24) und Satzfolgen wie King Kong saB hungrig im Garten. Die Oma suchte im Keller nach Bananen), sei dahingestellt.

Die stellung rechts neben dem Satz mit Kommabtrennung ruft eine nicht-parenthetische Lesart (asyndetisch koordinativ, evtl. Satzfolge) hervor. Eigen- 
standig und punktgetrennt kommen Schaltsătze durchaus vor, wobei dann eine Satzfolge vorliegt und kein "Einschub". 26

(11.25) Die Frau hat gestern dem Kind einen Ball gegeben, das haben jedenfalls einige vermutet.

(11.26) Die Frau hat (...) gegeben. Das haben jedenfalls einige vermutet. Die eigentliche Parenthese hat im Unterschied zu einer Satzfolge ('aufeinanderfolgende Sachverhalte') die kommunikative Funktion, simultane Begleit-bzw. Nebenhandlungen sprachlich quasi "ikonisch" der Realităt nachzubilden und nicht in eine "künstliche" Nacheinanderfolge zu ordnen (Bassarak 1985).

\section{II III IV $V$ VI VII VIII}

das haben jedenfalls einige vermutet

(VERBZWEIT, AUSSAGE)

gestern war übrigens Mittwoch

( $V-2$ mit Bezug auf TEMP)

war das eigentlich wirklich so?

( $V-1$, FRAGE)

Văre das doch bloß nicht wahr!

( $V-1$, WUNSCH)

Glauben sie das doch endlich!

( $V-1$, AUFFORDERUNG)

Ob das eigentlich wirklich so war?

( $V$-E, FRAGE)

Wenn das doch bloß nicht wahr wäre!

( $V-E$, WUNSCH)

Die kommunikative Funktion (ILLORUTION) des Schaltsatzes spielt offenbar keine wichtige Rolle für die Positionssyntax. Etwas eingeschränkt sind allerdings die Verbendsätze, weil bei deren Vorkommen in Fảlen wie (mit progredienter Intonation!)

(11.27) «Die Frau hat (...) gegeben, ob das eigentlich wirklich so war? *Die Frau hat (...) gegeben, wenn das bloB nicht wahr wäre!

beide Illokutionen in Konflikt geraten und nicht als asyndetisch gereiht aufgefaBt werden kōnnen; eine klarere Abtrennung, z.B. durch deutliche Satzpause (durch Punkt), hebt diesen Ronflikt auf, wobei dann eine Folge zweier auch illokutionār selbständiger sätze vorliegt.

26 Oder man nimmt mit Schwyzer (1939: 34) eine Endparenthese (Opisthothese) an, die "leicht zur Abschweifung oder Digression" werden kann. Schwyzer (ebd. 33) formuliert: "Aber der Begleitgedanke kann auch zuruickgehalten werden, bis der Hauptgedanke sprachlich voll ausgedrückt ist (...)". Man sieht (wie bei der Anfangsparenthese) die zwar extralinguistische (psychologische) Begrumdung, kann aber formal den Zusammenhang zwischen Dazwischenschaltung sorie Vor- und Nachschaltung anerkennen. 
Im übrigen sind alle Schaltsätze obiger Art nicht vorfeldfüllend möglich, weil sie keine syntaktische Funktion im Gastsatz auszữben vermögen. Wenn man wie in (11.28) ein Nachfeld (vgl. hierzu Altmann 1981: $67 \mathrm{ff.;} 166 \mathrm{f.}$ ) in den obigen Sătzen erzeugte,

(11.28) Die Frau hat dem Kind einen Ball gegeben (I) gestern (II). dann könnten die Schaltsătze (wie vermutlich alle EINSCHÖBE) nicht in Position (I) auftreten - dort befindet sich offensichtlich keine Parenthesenische - , sondern lediglich in Position (II) nach dem Nachfeld.

Ich stelle fest, daB Schaltsătze die Merkmale von EINSCHÜEN aufweisen. Die Stellungsmöglichkeiten sind vielfältig, vor allem die mögliche Umformung in Satzfolgen ist ein Rennzeichen von Einschüben. Allerdings muß man durchaus mit Schaltsătzen rechnen, deren stellungsverhalten Einschränkungen erfährt. ${ }^{27}$ Es dürfte aber folgende Minimalcharakteristik auf das Stellungsverhalten von Einschüben zutreffen: Eine (einschaltungsmarkierte) Spracheinheit ist dann als Einschub zu werten, wenn mindestens eine Satz- bzw. Außerungsfolgevariante (also entweder I oder VIII) möglich wäre.

Abschließend sei angemerkt, daß es sich in Fällen wie Wen, wünscht Kuno, soll Pia besuchen? trotz Satzförmigkeit offenbar nicht um EINSCHUB-Erscheinungen handelt. Vielmehr wirken diese Fälle so, als ob der "Gastsatz" eine Valenzstelle des "Einschubverbums" abbände (man vgl. etwa Kuno wünscht, Pia soll ihn besuchen). Die typische Abtrennung mittels Gedankenstrichen ist hierbei inakzeptabel (*Wen - wünscht Kuno - soll Pia besuchen?), "Satzfolgen" sind nicht herstellbar ( $*$ Wen soll Pia besuchen, wüscbt Kuno?) und eine Sprecherdivergenz scheint mir ausgeschlossen zu sein (man vgl. weiter oben die Kriterien (a), (c) und (h)). Weitere Argumente erörtert Grewendorf (1988: 83 ff.)

Nun möchte ich Verbendsătze, die im Gastsatz als Adverbial fungieren könnten, und Verbzweitsätze mit phorischen Bezügen auf Satzglieder des Gastsatzes sowie diverse Relativsatzarten untersuchen:

\section{II III IV $V$ VI VII VIII}

weil Kuno das so wollte

(KAUSALADVERBIALSATZ)

als die Turmuhr drei schlug

(TEMPORALADVERBIALSATZ)

ohne daB ein Grund dazu vorlag

(MODALADVB-SATZ)

$\begin{array}{ccccccc}(-) & + & + & + & + & + & + \\ + & + & + & + & + & + & (+) \\ (-) & + & + & + & + & + & + \\ + & + & + & + & + & + & (+) \\ (-) & + & + & + & + & + & + \\ + & + & + & + & + & + & (+)\end{array}$

27 Man betrachte z.B.:

(F11.11) Pia wird - oder sollte das Ute besser machen? - den Affen futtern.

(F11.12) (ader sollte das Ute besser machen?) Pia wird den Affen füttern. (Oder sollte Ute das besser machen?) 
sie trug übrigens ein rotes Tuch

( $V-2$ mit PERS.PRONOMEN)

die trug übrigens ein rotes Tuch ( $V-2$ mit DEMONSTRATIVPRONOMEN)

es trug ubrigens eine alte Mütze ( $V-2$, mit PERS.PRON)

das trug übrigens eine alte Mütze ( $V-2$, mit DEM.PRON)

er war übrigens sehr dreckig

( $V-2$, mit PERS.PRON)

der war übrigens sehr dreckig

(V-2, mit DEM.PRON)

die ein rotes Tuch trug

(RELATIVSATZ, +RESTRIRTIV)

die übrigens ein rotes Tuch trug

(RELATIVSATZ， -RESTRIKTIV)

das eine alte Mütze trug

(RELATIVSATZ, +RESTRIRTIV)

das übrigens eine alte Mutze trug

(RELATIVSATZ, -RESTRIRTIV)

der sehr dreckig war

(RELATIVSATZ, +RESTRIRTIV)

der übrigens sehr dreckig war

(RELATIVSATZ， -RESTRIRTIV)

was einige Passanten wunderte

(SATZRELATIVSATZ)

was niemand wissen konnte

(SATZRELATIVSATZ)

\begin{tabular}{|c|c|c|c|c|c|c|c|}
\hline$(-)$ & + & + & + & + & $(+)$ & + & + \\
\hline$?$ & + & + & + & $(t)$ & + & + & \\
\hline - & + & + & + & $(+)$ & $(t)$ & $(t)$ & + \\
\hline$?$ & + & + & $(t)$ & $(+)$ & $(+)$ & + & \\
\hline ? & $?$ & $?$ & $?$ & + & $?$ & + & + \\
\hline ? & $?$ & $?$ & + & $?$ & + & + & \\
\hline - & $?$ & $?$ & $?$ & + & $?$ & + & + \\
\hline - & $?$ & $?$ & + & $?$ & + & + & \\
\hline - & $?$ & $?$ & $?$ & $?$ & + & + & + \\
\hline - & $?$ & $?$ & $?$ & + & + & + & \\
\hline - & $?$ & $?$ & $?$ & $?$ & + & + & + \\
\hline - & $?$ & $?$ & $?$ & + & + & + & \\
\hline- & + & - & - & - & - & $(t)$ & - \\
\hline - & + & - & - & - & $(+)$ & - & \\
\hline - & + & - & - & - & - & - & - \\
\hline- & + & - & - & - & - & - & \\
\hline - & - & - & - & + & - & + & - \\
\hline- & - & - & + & - & + & - & \\
\hline - & - & - & - & + & - & - & - \\
\hline - & - & - & + & - & - & - & \\
\hline - & - & - & - & - & + & + & - \\
\hline - & - & - & - & + & + & - & \\
\hline - & - & - & - & - & + & - & - \\
\hline - & - & - & - & + & - & - & \\
\hline$?$ & + & + & + & + & $(+)$ & + & $(t)$ \\
\hline$?$ & + & + & + & $(t)$ & + & $(+)$ & \\
\hline$?$ & + & + & + & + & $(+)$ & + & $(+)$ \\
\hline$?$ & + & + & + & $(t)$ & + & $(t)$ & \\
\hline
\end{tabular}

Bei den einschaltungsmarkierten Angabesătzen muß sehr genau auf die intonatorischen Verháltnisse in der gesprochenen Sprache geachtet werden. Es ist m.E. nicht notwendigerweise der Fall, daB Adverbialsătze als Angaben im Mittelfeld grundsatzlich nur parenthetisch erscheinen können und dann keine syntaktische Funktion im Bezugssatz ausūbten. ${ }^{28}$ Man betrachte:

(11.29) /pia ist nicht $(\mathrm{p}+)$ weil es regnete $(\mathrm{p}+)$ zubause geblieben $\mathrm{p}^{+}$sondern weil es gewitterte $\mathrm{f}++/$.

28 Diese These vertritt Altmann (1981: 63 f., 168, 174). Vgl. auch Rap. 8 . 
$(11.30) * / p i a$ ist nicht $\mathrm{p}+$ weil es regnete $\mathrm{p}+$ zuhause geblieben $\mathrm{p}+$ sondern weil es gewitterte $\mathrm{f}++/$.

(11.31a) /pia ist nicht gekommen $(\mathrm{p}+)$ weil es regnete $\mathrm{ft+} /$.

(11.31b) (?)/pia ist nicht gekommen $\mathrm{ft+}$ weil es regnete $\mathrm{ft+}$.

Die in meinen Augen nichtparenthetische Version der Mittelfeldposition des als Kausaladverbial fungierenden Gliedsatzes ist von eher schwachen (oder gar keinen?) Pausen umgeben, die Intonation ist progredient und eine kontrastierende Negation kann vorgenommen werden (11.29). Der parenthetisch eingefügte Angabesatz wird hingegen von deutlichen Pausen eingerahmt und kann nicht negiert werden (11.30). Nur (11.29) ist äquivalent mit der Satzverfügungsvariante in Beispiel (11.31a), (11.30) wăre gleichwertig mit einer Satzfolgevariante wie in (11.31b), die jedoch hinsichtlich ihrer Akzeptabilităt evtl. nicht unumstritten ist.

Die adverbialen Gliedsätze wurden bereits in Rap. 8.1.1. von ihren eingeschobenen ("appositions- resp. parentheseverdächtigen") Varianten abgegrenzt.

Die nicht als Satzglied fungierenden Angabesätze können nicht links vom Gastsatz erscheinen und mit diesem eine Satzfolge bilden (11.32a), da sie als unselbståndig markiert sind (Subjunktion). Die Variante mit Doppelpunkt (hier erscheint die Trennung etwas schwächer) wirkt zwar nicht välig abweichend, jedoch stark markiert (11.32b); rechts des Bezugssatzes - abgetrennt durch Punkt bzw. Satzpause - erscheint der Angabesatz etwas markiert (11.32c), doch dürfte es sich um eine stilistische Auffälligkeit handeln:

(11.32a) *Als die Turmuhr drei schlug. Die Frau hat (...) gegeben.

(11.32b) ?Als die Turmuhr drei schlug: Die Frau bat (...) gegeben.

(11.32c) (?) Die Frau bat (...). gegeben. Als die Turmuhr drei schlug.

Derartige Muster werte ich trotz ihrer relativ großen stellungsfreiheit als ZUSATZE - und zwar wegen der Möglichkeit, eine syntaktische Funktion ausüben zu können, m.a.W. aufgrund ihrer potentiellen Unselbständigkeit (sie tragen keine eigene Illokution!) bzw. Integrierbarkeit. EINSCHOBE sind dagegen potentiell selbständig und somit imstande, AuBerungsfolgen zu bilden. ${ }^{29}$ Die eingeschalteten Angabesatze stellen folglich ZUSATZE zum Satz dar.

Die eingeschobenen Verbzweitsătze mit Pronomina, die sich auf eine Einheit des Gastsatzes beziehen, unterliegen merklichen stellungsbeschränkungen im Vergleich zu Verbzweit-Schaltsătzen ohne solche Pro-Form-Beziehungen. Die Restriktionen entsprechen wohl den allgemeinen Pronominalisjerungsbedingungen: Kataphorik ist eher inakzeptabel; d.h. diese Sătze stehen im Nachbereich des Bezugselements der Pro-Form. Prinzipiell unterscheiden sich solche Schaltsătze aber sonst nicht von jenen ohne Pro-Form, so daB sie ebenfalls den EINSCHOBEN zugewiesen werden können.

29 Gewiß können diese Ausführungen nur vorlăufig sein. Die EmNSCHOBE erhielten, wenn man sie als eigenständige Außerungen links oder rechts folgen ließe, u.U. einen zu großen kammikativen Eigenwert. Vgl. auch Heringer (1988: 276 f.). 
Die unterschiedlichen Eigenschaften von restriktivem und appositivem Relativsatz - auch im Stellungsverhalten - wurden bereits in Kap. 7. A herausgearbeitet. Der restriktive ist bei beliebiger stellung der Basis sowohl rechtsadjazent als auch (über mehrere Satzglieder hinweg) extraponiert möglich, der appositive kann nur dann extraponiert werden, wenn nur der Klammerschluß Basis und Relativsatz trennt. Der restriktive Relativsatz ist eindeutig Attribut zur Bezugs-NP (vgl. Kap. 7, 7.A). Der nicht-restriktive Relativsatz ist dagegen ein NP-ZUSATZ mit enger Assoziation an seine Basis (ausführlich Kap. 7.A).

Die Satzrelativsätze (die "weiterführenden Nebensätze") sind in ihren Stellungsmöglichkeiten links und rechts des Satzes eingeschränkt, wohl wegen ihrer "subordinativen Markierung" (einleitendes Rel.pron; Verb-End). Dennoch muten sie links vom "Bezugssatz" nicht so abweichend an wie z.B. die etwas weiter oben besprochenen Angabesätze. Die selbständige Hinzufügung rechts vom Bezugssatz ist markiert, kann m.E. allerdings in bestimmten Textsorten (z.B. journalistische Texte) vorkommen. Der Satzrelativsatz bewegt sich bekanntermaBen in der Grauzone zwischen abhängigem Satz (Subordination) und Satzfolge. Seine Einstufung als EINSCHUB oder ZUSATZ (zum Gastsatz) muB offenbleiben; ich tendiere wegen dessen geringer (fehlender?) potentieller selbständigkeit eher zur Wertung als zUSATZ zum Satz.

Nun betrachte ich die Stellungsmöglichkeiten nicht-satzförmiger Angaben, sofern diese parenthetisch markiert sind:

\section{$I$ II III IV $V$ VI VII VIII}

vermutlich

(ADJ, SATZADVERBIAL)

der Gerechtigkeit wegen

(PP, KAUS)

um drei Uhr

(PP, TEMP)

mit theatralischer Geste

(PP, MOD)

hinter einer Ulme

(PP, LOK)

von einem Baum herab

(PP, DIR)

\begin{tabular}{|c|c|c|c|c|c|c|}
\hline - & + & + & + & + & + & ?30 \\
\hline+ & + & + & + & + & $?$ & $(-)$ \\
\hline$(-)$ & + & + & + & + & + & + \\
\hline+ & + & + & + & + & + & $?$ \\
\hline$(-)$ & + & + & + & + & + & + \\
\hline+ & + & + & + & + & + & $?$ \\
\hline$(-)$ & + & + & + & + & + & + \\
\hline+ & + & + & + & + & + & $?$ \\
\hline$(-)$ & + & + & + & + & + & + \\
\hline+ & + & + & + & + & + & $?$ \\
\hline$(-)$ & + & + & + & + & + & + \\
\hline+ & + & + & + & + & + & $?$ \\
\hline
\end{tabular}

Freie Angaben, hier Adverbiale diverser Subklassen, sind also ohne weiteres "parenthetisch" möglich. Die Position links des Satzes

30 Der "Nachtrag" von vermutlich (Pos. VII bzw. VI) wirkt kaum akzeptabel; das Fragezeichen soll jedoch offenhalten, ab manche Subtypen von Satzadverbialen wie z.B. bedaverlicherweise nicht doch in dieser Position akzeptabel wären. Vgl. dazu Altmann (1981: 334). 
(11.33) ??Vermutlich/??Um drei Uhr/ ??Hinter der Ulme: Die Frau hat gestern dem Kind einen Ball gegeben.

kann wohl als inakzeptabel ausgeschieden werden. Die isolierte steliung nach dem "Bezugssatz" ist eher möglich, außer bei Satzadverbialen, die als Sprecherkommentar zur Wahrscheinlichkeit des ausgedrückten Sachverhalts anzusehen sind (emotional wertende Satzadverbiale wirken deutlich annehmbarer):

(11.34) Die Frau hat gestern dem Rind einen Ball gegeben. (?) Hinter der Ulme/ (?) Um drei Uhr/ ??Vermutlich/ (?) Bedauerlicherweise.

Die Bewertung derartiger Konstruktionen ist schwer, denn einerseits sind sie z.B. im journalistischen Bereich nicht selten (zur Erzeugung von Spannung, zur Hervorhebung wichtiger Detailumstände etc.), andererseits finden sie in anderen Textsorten so gut wie nie Anwendung (z.B. in dieser Arbeit).

Entscheidend für die Abtrennbarkeit der Adverbiale ist deren Valenzfreiheit; valenznotwendige Adverbiale können nicht als Einschaltungen markiert werden, weil die dadurch erfolgende Herausnahme aus dem Satz eine ungesättigte valenzstelle zurückläBt, so daB ein unvollständiger und somit inakzeptabler Satz entsteht.

In Position II der Testsătze stelle ich hinsichtlich der Interpretation eine Mehrdeutigkeit fest, wenn man lokale Adverbiale mit den übrigen vergleicht $(11.35)$ :

(11.35) Die Frau, in München/ um drei Uhr/ vermutlich, hat gestern dem Kind einen Ball gegeben.

(11.36) Die Frau, sie war in München/*sie war um drei Uhr/*sie war vermutlich, hat (...) gegeben.

(11.37) Die Frau, und das geschah in München/ um drei Uhr/ vermutlich, hat gestern dem Kind einen Ball gegeben.

Bei Kontaktstellung von NP und "adverbialem Einschub" kann das Lokaladverbial zweifach interpretiert werden: einerseits als (situierende) Prädikation über den Referenten der NP und somit als APPOSITIONSPROTOTYP (später: prädizierender Zusatz), 31 wobei (11.36) die Variante mit Kopulasatzumschreibung bietet und zeigt, daB diese Umformung nur auf die "appositive Variante" anwendbar ist; andererseits kann man in München als eingeschaltete Angabe werten.

(11.37) versucht, die zweite Lesart durch eine und-das-geschab-ADVERBIAL-Umformung zu demonstrieren. ${ }^{32}$ Diese (vielleicht nicht sehr glückliche) Umformung soll eine Eigenschaft freier Angaben veranschaulichen: die situierung des gesamten vom Satz ausgedrückten Sachverhalts.

31 Einheiten wie in München werden selten als "Appositionen" gewertet (Altmann 1981: 59; Engel 1988: 808).

32 U.a. diese Unformung verwendete Renate Bartsch (1972: $22 \mathrm{ff}$.) in ihrer "Adverbialsemantik" zur Subklassifizierung möglicher Adverbiale; in ihrer Ubersicht der Testergebnisse (ebd. 26 f.) kann man herauslesen, daß der Test bei nicht-satzbezogenen (v.a. modalen) Adverbialen negative Ergebnisse liefert. 
Die restlichen eingeschalteten Angaben (oben: temporal und satzmodal; auch die kausalen gehörten dazu) können m.E. nie mit "Appositionen" (ZUSATZEN) verwechselt werden (vgl. 11.36). Engel (1988: 808; daher auch die Beispiele in 11.38, geklammerte Einfügungen von W.S.) allerdings rechnet - meines Wissens als einziger - auch temporale, kausale und konditionale "Angaben" zu den Appositionen:
(11.38)
ein wildes Leben, (*es war) in jenen Jahren,...
seine Schwerhörigkeit, (*sie war) aus verschiedenen Gründen,... ein Ausweichen, (*es war) in diesem Falle,...
(11.39) ein wildes Leben, (es war) eine permanente Alkoholorgie,... seine Schwerhörigkeit, (sie war) eine lastige Behinderung,... ein Ausweichen, (es war) außerordentlich riskant,...

(11.38) veranschaulicht, daB keine kopulativen Satzstrukturen zugrundegelegt werden könnten wie in typischen "Appositionen" (11.39). Ein weiterer (nicht unproblematischer) Unterschied besteht in der Moglichkeit, die eingeschaltete Angabe (potentiell) in den Gastsatz zu integrieren als Satzglied - eine Option, die prototypischen Appositiven verschlossen ist: 33

(11.40) Ein wildes Leben (, in jenen Jahren,) hat (in jenen Jahren) Bukowskis Gesicht gezeichnet.

Ein wildes Leben (, eine permanente Alkoholorgie,) hat (*eine permanente Alkoholorgie) Bukowskis Gesicht gezeichnet.

Abschließend werfe ich noch einen Blick auf die stellungsoptionen von integrierten und "parenthetischen" Angaben bei "normaler Satzbetonung":

(11.41) ??daB zum studium Tarzan nach B a yern gekommen ist. daß, zum Studium (übrigens), Tarzan nach $B$ a yern gekommen ist.

(11.42) *daB mit seinem Freund Kuno nach $B$ o nn fährt. daB - mit seinem Freund (übrigens) - Kuno nach $B$ o nn fährt.

(11.43) *daB ausgeruht und gebräunt Tarzan das $S$ o nnenstudio verlieB. daB, (übrigens) ausgerubt und gebräunt, Tarzan das $S$ o nnenstudio verlieb.

Interessanterweise gelten für die Einschaltversionen nicht die Positionsbeschränkungen, die für satzgliedhafte finale (11.41) und komitative (11.42) Adverbiale sowie prádikative Attribute (als modale Subklasse; 11.43) wirksam sind. ${ }^{34}$ Gerade die Position im subjunktional eingeleiteten Gliedsatz $z$ wischen Subjunktor und erstem Satzglied ist eine stelle, an der nur satzbezogene (temporale, kausale, satzmodale) Adverbiale akzeptabel sind. Parenthetisch sind jedoch an dieser stelle die in Satzgliedversion inakzeptablen Angaben möglich!

33 Ich mogle mich um ein Probleme herum, nämlich um evtl. auftretende Bedeutungsschattierungen zwischen eingeschalteter und integrierter Angabe; genauer kann nicht darauf eingegangen werden.

34 Daher ist zu überlegen, ob die ubliche Verortung finaler Adverbiale in der Großklasse der satzbezogenen Kausaladverbiale uberhaupt zu rechtfertigen ist. 
Die einschaltungsmarkierten Angaben rechne ich zu den ZUSATZEN, vor allem weil sie eine syntaktische Funktion im Gastsatz ausüben könnten und weil sie nicht kommunikativ eigenständig sind; ${ }^{35}$ EINSCHOBE tragen dagegen i.d.R. eine Illokution und üben (auch potentiell) keine syntaktischen Funktionen aus. Die Grammatik dieser Erscheinungen bedarf weiterführender Untersuchungen, die ich leider nicht vornehmen kann.

Obligatorische Funktionen im Satz, also Ergänzungen, sind nicht mit dem "Einschaltungsmuster" versehbar, denn eine valenzstelle ist nicht in Form eines HOSPITANTEN realisierbar; die Einschaltungsmarkierung schirmt das markierte Element u.a. gegen Rektions- oder Valenzforderungen ab:

(11.44) *Dem Kerl gefiel, Pia (SBJ), besonders gut.

* Tarzan hat dem Affen - die Banane (AKKO) - gegeben.

* Die Banane hat Tarzan, dem Affen (DATO), gegeben.

* Sie haben - der Verstorbenen (GENO) - häufig gedacht.

* Die Eifersucht hat ihn, zu dieser Tat (PO), veranlaBt.

* Pia wird morgen - Doktorin der Philosophie (PTIV) - werden.

* Pia hat lange, in Koln (obl. LOK), gewohnt.

*Er bat sich gestern - wie ein Idiot (Obl. MOD) - verhalten.

Natürlich könnten in der gesprochenen sprache derartige strukturen vorkommen; sie sind dann als "Performanzerscheinungen" erklärbar, die unter anderem in Unkonzentriertheit, Schwierigkeit in der Findung des angemessenen (passenden) Ausdrucks begründet sind (ofters treten zudem Zögerungspartikeln auf: /sie hat $\mathrm{p}+$ äh $\mathrm{p}+$ in München ( $\mathrm{p}+$ äh $\mathrm{p}+$ ) gewohnt/.).

Fakultative Ergănzungen, die in bestimmten Kontexten weglaßbar sind, muten zumindest schriftsprachlich ebenfalls inakzeptabel an:

(11.45) ??Pia hat gestern - eine Pizza (AKKO) - gegessen.

?? Pia hat, dem Affen (DATO), eine Banane geklaut.

?? Pia hat tatsáchlich - auf ihrem Vorschlag (PO) - beharrt.

Kurz einzugehen ist an dieser stelle auf die satzformatigen Ergănzungen, die nach Altmann (1981: $168 \mathrm{f.}$ ) nicht parenthetisch im Mittelfeld stehen konnen. Meine (subjektive!) Beurteilung derartiger strukturen ist die folgende:

(11.46) * Sie hat gestern, daB der Abwasch sich türmte (SBJ), gewurmt. *Dieses Vorkommnis hat wieder einmal, daB sich nichts andert (AKRO), bewiesen.

* Sie hat sich, daB er das gesagt hat (GENO/PO), heute erinnert.

(11.47) ?/pia hat immer $(\mathrm{p}+)$ wo es ibr gefiel (LOK) (p+) gewohnt $\mathrm{f}++/$. ?/der brand ist sicher $(\mathrm{p}+)$ weil jemand unachtsam war (KAUS) ( $p+$ ) dort entstanden $\mathrm{f}++/$.

Die hinsichtlich Kasus bzw. Prăposition regierten Ergănzungen (11.46) sind im Mittelfeld gewiB inakzeptabel, seien sie nun satzförmig oder nicht. Bei adver-

35 Vgl. etwas weiter oben die Ausführungen zu den Verbend-Angabesătzen. 
bialen satzförmigen Ergänzungen bin ich mir nicht sicher; unbestreitbar akzeptabel sind allerdings wirklich nur die Varianten ohne Mittelfeldposition (Vorfeld, Extraposition). Vielleicht verläuft zwischen regierten und nichtregierten Ergănzungen - bei Satzformat - eine Akzeptabilitätsgrenze? Uber diese Phänomene sind weitere Untersuchungen wünschenswert, die hier leider unterbleiben müssen.

Nicht uninteressant ist das Verhalten sog. "freier Dative", die sich, wie bei Wegener (1985; v.a. $49 \mathrm{ff.;} 92 \mathrm{ff.}$ ) und bei Eisenberg (1986: $283 \mathrm{ff.}$ ) nachzulesen ist, z.T. als "gar nicht so frei" erweisen; vielmehr verhalten sich etliche Beispiele ähnlich den typischen Dativobjekten.

(11.48a) ??Pia hat heute, der Oma, den Koffer zum Bahnhof getragen.

(11.48b) ??Die Blumen sind gestern - dem Gärtner - verwelkt.

(11.48c) ??Der Arzt operiert, übrigens einem Filmstar, den Blinddarm.

(11.48d) (?) Die Arbeit war - jedenfalls diesem Kerl - zu schwer.

(11.48e) *Der Kerl fällt, dir, noch von der Leiter.

Die z.T. den Angaben zugerechneten "freien Dative" verhalten sich unterschiedlich. 36 Dativus commodi (11.48a) und incommodi (11.48b) sowie der Pertinenzdativ (11.48c) ähneln den Dativobjekten und wirken - wie erwartet, da "Ergānzungen" wie z.B. das Dativobjekt nicht vom Elementarsatz abgeschirmt werden dürfen - in Parenthese gesetzt kaum akzeptabel.

Der Dativus iudicantis (11.48d) scheint mir "parenthetisch" einigermaßen möglich. Ausgerechnet der als Angabe fungierende Dativus ethicus (frei zu beliebigen Verben hinzufügbar usw.) ist parenthetisch völlig ausgeschlossen (11.48e). Das liegt wahrscheinlich an seiner "Modalpartikelhaftigkeit", denn Modalpartikeln können nicht in Parenthese gesetzt werden; ${ }^{37}$ sie könnten als HOSPITANTEN nicht in den Gastsatz hineinwirken, um dort ihre Funktion der illokutiven "Abtönung" wahrzunehmen:

(11.49) Die Männer haben, *eben/*doch/ *mir, kein Verständnis für Frauen. Aufgrund der Skopusabsorbierung (vgl. auch Rap. 8.2) können weder Gradpartikeln noch die Negationspartikel nicht parenthetisch vorkommen:

(11.50) ^Pia glaubt, daB - sogar/ nur/ auch (GP) - Kuno den Geist sah.

*Pia hat, nicht (NEG), dem Kind einen Ball gegeben.

Diese Partikeln, die ja nicht die "normalen" Satzgliedeigenschaften aufweisen, aber andererseits auch nicht als gewönliche Gliedteile - zumindest nicht in jeglicher Vorkommensweise - zu werten sind, können also nicht von ihren Bezugselementen resp. -sătzen "abgeschirmt" werden. 38 Durch die "Einschaltungs-

36 Heidolph u.a. (1981: $207 \mathrm{ff.}$ ) behandeln die "freien Dative" als valenzfreie Adverbiale.

37 Zur "Wodalpartikelhaftigkeit" vgl. Wegener (1985: 51) und Eisenberg (1986: 284).

38 Schulz/Griesbach (1982: 367) werten z.B. die Gradpartikeln als "Rangattribute"; treffend zusarmengefaßt erscheint der Status von (Grad-, Modal) Partikeln in Jung (1982: 120):

"Unter den Elementen des Satzes nehmen die $\mathrm{P}$ a $\mathrm{r} t \mathrm{i} \mathrm{k}$ e $\mathrm{l} \mathrm{n}$ eine Sonderstellung ein. (...) Sie sind nicht satzgliedfähig, d.h., sie können nicht allein vor dem finiten verb im Kernsatz 
markierung" können diese Partikeln nicht den Satz (oder Teile desselben) in ihrem Bezugsbereich (Skopus) haben. Wenn sie ein "Bezugselement" mit sich führen, ist dies natürlich wieder möglich:

(11.51) Es hat - sogar in $M$ ü nchen - gestern wieder geregnet. Sie hat es - (übrigens) nicht in Köln - wiedergefunden.

Es bleiben noch "parenthetische Gliedteilstrukturen" zu untersuchen; ${ }^{39}$ gemeint sind Einheiten, die nach Entfernung des Einschaltungsmusters bzgl. ihrer Bezugselemente als Attribute fungierten. Auf diese Erscheinung wurde ich durch v. Ditfurth (1988: 288 (= 11.52a) u. 220 (=11.52b)) aufmerksam:

(11.52a) Gesteuert aber wird die aus deren Zusammenwirken resultierende Macht von der unsichtbaren Hand des - relativ - freien Spiels wirtschaftlicher Kräfte.

(11.52b) Eine - notgedrungen einseitige - Weigerung, sich an der Fortsetzung des Irrsinns unter diesen Umständen weiter $z u$ beteiligen, würde unserer Sicherheit andererseits auf Jahre hinaus keinen Abbruch tun.

(11.53) In Anlehnung an "Phraseodidaktik" mag "Phraseopragmatik" als ein (zugegeben: schlechter) Arbeitsterminus (...) gelten. 40

Hier finden sich "parenthetisch" ein Attribut zu einem attributiven Adjektiv (11.52a) und eine adnominale Adjektivphrase (11.52b). (11.53) zeigt einen besonders interessanten Fall, da das potentielle Attribut von einem "Funktionslexem" begleitet wird.

Wie verhalten sich nominale Wortgruppen (andere werden vernachlässigt), in denen Attribute zu finden sind, hinsichtlich der Einschaltungsmarkierung?

Erstens: Der Nukleus nimmt für gewöhnlich keine Einschaltungsmarkierung an (11.54a). Erscheinungen dieser Art sind durch Performanzfaktoren wie Unkonzentriertheit, Wortfindungsstörungen etc. zu erklären (11.54b; es können Zögerungspartikeln auftreten). Ein Beleg wie (11.55) legt allerdings nahe, daß Ausnahmen zur oben formulierten "Regel" existieren; ${ }^{41}$ der Kern der interessierenden Nominalgruppe wăre das einschaltungsmarkierte Teile, doch kann der einleitende Artikel ebenfalls auf den Kern des eigentlichen Genitivattributs (Gesellschaftsschichten) bezogen werden, das infolge des Einschaltungsmusters um eigentlichen Kern und den Artikel des Attributs nicht-attributiv erscheint. Die Analyse und Kommentierung solcher Muster stellt eine Forschungsaufgabe dar.

stehen. Sie sind Attributen am nächsten verwandt, können aber in Unterschied zu diesen nicht auf primăre Satzglieder zurückgeführt werden. Außerdem können sich Partikeln auch auf das Verb im Satz beziehen, was bei eigentlichen Attributen nicht der Fall ist".

39 Derartige Muster sind bislang offenbar wenig erforscht. Mur bei Winkler (1969) und Sammerfeldt (1984) fand ich etwas zu solchen Konstruktionen.

40 Aus: Pilz, Klaus D. 1981. Phraseologie. Redensartenforschung. Stuttgart, Metzler. S. 113.

41

Quelle: Goetz, Hans-Werner. 1986. Leben im Mittelalter. München, Beck. S. 80 . 
(11.54a) *Das relativ freie - Spiel - der Kráfte wurde behindert.

(11.54b) /das relativ freie $\mathrm{p}+(a ̈ h \mathrm{p}+)$ spiel $\mathrm{p}+\left(a h^{\mathrm{p}}+\right)$ der kräfte wurde bebindert $\mathrm{f}++/$.

(11.55) Der Franzose Georges Duby stellt (...) fest, daB auf diesem Wege ein groBer Teil der Abgaben (...) wieder an die (unversorgten Teile der) unteren Gesellschaftsschichten zurückfloB.

Zweitens: Obligatorische Nominalkomplemente sind ebenfalls nicht parenthetisch möglich. Es ist einzuräumen, daB die Obligatheit im nominalattributiven Bereich im Ggs. zum verbalen Bereich schwer explizierbar ist, weil viele Nomina auch "absolut" (allein oder nur von einem Determinator begleitet) aufzutreten vermögen. Deshalb werden hier zwei syntagmen angefüht, die auf entsprechende Valenzstrukturen mit "Verb + Ergänzung" bezogen werden können:

(11.56) *Ein Bersteller - von Computern - kann viel Geld verdienen. *Uns ist die Hoffnung - auf bessere Zeiten - schon vergangen.

Drittens: Meine Hypothese lautete vor Oberarbeitung dieser Arbeit, daB Genitivattribute ungeachtet ihres Valenzstatus nie das Einschaltungsmuster anzunehmen vermögen (11.57a). Inzwischen haben sich angesichts der Belege (11.57b) Zweifel daran eingestellt. ${ }^{42}$ offenbar sind "adnominale Genitivangaben" ebenfalls parenthetisch möglich. Möglicherweise ist der Valenzstatus gar nicht das (einzige) einflußnehmende Rriterium. Mir scheint es z.B. denkbar, daB eine obligatorische Einheit in Klammern hinter die Basis gesetzt wird, wenn man den Leser an diese - sie erschien bereits vorher im Text - erinnern möchte (in diesem Fall wäre die Motivation demnach pragmatisch). Bei Ersterwăhnung von "Basis + obligatorischem Gliedteil" darf letzteres allerdings nicht einschaltungsmarkiert vorkommen. Die Untersuchung, inwieweit sich textlinguistische Faktoren wie Vorerwăntheit etc. als steuernd erweisen, muB ich jedoch Nachfolgearbeiten überlassen.

(11.57a) */wir haben über das laster $\mathrm{p}+$ des rauchens $\mathrm{p}+$ diskutiert $\mathrm{ft+} /$. * Sie haben den Bruder - der Mutter - in den Keller geschickt.

(11.57b) Zu den am meisten ausgelegten Bibelbüchern zählte im Mittelalter das Hohelied (der Liebe), (...).

Wieweit solche Dichtungen aber die Wirklichkeit (der festlichen Ausnabmeerscheinungen) des Alltags widerspiegeln, (...).

Viertens: Prăpositionale und adverbiale Attribute sind Z.T. dann (unter hier nicht im Detail analysierbaren Bedingungen) parenthetisch möglich, wenn sie "freie Nominalangaben" darstellen (zu $11.59 \mathrm{vgl}$. Winkler 1969: 291):

(11.58) Wir werden dieses Haus - dort unten/ am Naldrand - kaufen.

(11.59) Er hat einen Kasten (mit Pulvertüten) vor sich stehen.

Die Restriktivităt des Attributes spielt wahrscheinlich eine bedeutende Rolle. Die durch die Abtrennung absorbierten "Gliedteile" (man spricht hier besser nicht von Gliedteilen, sondern ebenfalls von zusătzen) sind nur nicht-restrik-

42 Quelle: Goetz, Hans-Werner. 1986. Leben im Mittelalter. München, Beck. S. 34, 164. 
tiv als Zusatzinformation wie in (11.60a) lesbar. Die restriktive Variante $(11.60 \mathrm{~b})$ ist nicht akzeptabel. Genauere Analysen sind wünschenswert, können hier aber nicht geleistet werden:

(11.60a) Wir werden nicht dieses Haus - am Waldrand - kaufen, sondern jenes Haus - am See - kaufen.

$(11.60 \mathrm{~b})$ *Wir werden nicht dasjenige Haus - am Waldrand - kaufen, sondern das Haus - am See - kaufen.

Bei den Prăpositionen aus/einschlieblich bin ich mir nicht sicher, ob ein "einschaltungsmarkiertes Gliedteil" vorliegt, oder ob es sich um einen spezifizierenden ((und zwar) einschlieblich) oder einen nicht-spezifizierenden $z u-$ satz handelt (man vgl. auch Kap. 12):

(11.61) Die Haie (aus-/einschlieblich der Hammerhaie) näherten sich.

Füftens: Besonders geeignet zur Annahme der Einschaltungsmarkierung sind sowohl vorangestellte Adjektivattribute als auch Modifikatoren dieser Attribute, kurzum: Adjektivphrasen (vgl. 11.52; 11.53).

Stellungsuntersuchungen in diesem Bereich sind nicht vonnöten, da die Regel gilt, daB bei Einschaltungsmarkierung die Position des zuSATzES derjenigen im unmarkierten ATTRIBUT-Fall entspricht. Verschiebungen sind ausgeschlossen, übrigens auch die im gesprochenen (umgangssprachlichen) Deutsch vorkommende Voranstellung vor allem lokaler Adverbial- und Präpositionalattribute: ${ }^{43}$

(11.62) Ich habe dort/ in München die Museen sehr geschätzt.

Ich habe - *dort/*in München - die Museen sehr geschätzt.

Die einschaltungsmarkierten Elemente können nicht mehr als Gliedteile resp. Attribute angesehen werden, weil sie u.a. weder erfragbar (11.63) noch durch nicht oder Gradpartikeln affizierbar sind (11.64):

(11.63) / kuno hat einen kasten $\mathrm{p}+$ mit pulvertüten $\mathrm{p}+$ gefunden $\mathrm{ft+} /$.

A: / was für einen kasten hat kuno gefunden $\mathrm{s}++$ /.

B: ??/ein kasten $\mathrm{p}+$ mit pulvertüten $\mathrm{f}++/$.

(11.64) * Kuno hat nicht einen Kasten - mit Pulvertüten - gefunden, sondern einen Kasten - mit Rnallfröschen.

*/sogar einen kasten p+ mit p u Ivertüten $\mathrm{p}+$ hat kuno gefunden $\mathrm{f}++1$. Ich werte "einschaltungsmarkierte Gliedteile" als ZUSATZE (zu NPn, AdjPn usf.) und gerade der Fall (11.53) mit "Funktionslexem" (vgl.: (...) mag "Phraseopragmatik" als ein (ich gebe es zu: schlechter) Arbeitsterminus (...) gelten) deutet darauf hin, daß hier nicht einfach nur ein Attribut eingeklammert wird. Eventuell sind sie dennoch $z u$ unterscheiden und abzutrennen von Erscheinungen wie beispielsweise dem "Appositionsprototypen", der Rechtsversetzung oder "spezifizierenden zUSATZEN" (vgl. Rap. 12, 15). Sie veisen eher Ahnlichkeiten mit den einschaltungsmarkierten Angaben (s. oben und Rap. 10.3.) auf, da sie auch eine syntaktische Funktion im Gastsatz ausüben könnten, nămlich nach Wegfall des Einschaltungsmusters. Die potentiell attributiven ZUSATZE können

43 Siehe dazu Ulvestad (1974). 
zudem weder auf eine zugrunde liegende prädikative Beziehung (wie der Appositionsprototyp bzw. der "prädizierende Zusatz") noch auf eine mögliche Herleitung aber Satzkopie, Tilgung und ggf. Einbettung (wie die spezifizierenden $z u-$ sātze in Kap. 12, 15) bezogen werden. Insofern unterscheiden sie sich auch von den "abgetrennten" Angaben, die letzteres zulassen.

Die "abgetrennten Gliedteile" (die mit dem bisher Gesagten noch lange nicht ausreichend beschrieben sind) zeigen eine gröBere Verwandtschaft mit beiläufig informierenden ZUSATZEN (übrigens) als mit spezifizierenden (und zwar):

(11.65) Kuno hat einen Kasten - ?? und zwar/ übrigens mit Pulvertüten - gefunden.

(11.66) Eine - ?und zwar/ übrigens notgedrungen einseitige - Weigerung täte uns gut.

(11.67) Das - *und zwar/ ?übrigens relativ - freie Spiel der Kräfte bleibt erbalten.

Nachdem eine beträchtliche Menge sprachlicher Muster als EINSCHÜBE oder ZUSATZE diverser Art ausgeschieden wurden, bleiben nun noch die i.e.s. appositionsverdăchtigen Strukturen übrig. Zunăchst werden nur (im Sinne der Unterscheidungen von Altmann 1981) eindeutige Appositionen berücksichtigt, nicht jedoch Strukturen wie "Rechtsversetzung" oder "Nachtrag". Hier eine Ubersicht über die Stellungsmöglichkeiten (vgl, auch Kap. 6):

\section{II III IV $V$ VI VII VIII}

eine große Brünette

Studentin der Philosophie

ein frecher Bengel

einem frechen Bengel

eine buntbemalte Lederkugel

alt und grauhaarig

jung und frech

auf einer Leiter

\begin{tabular}{|c|c|c|c|c|c|c|c|}
\hline & & - & 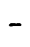 & - & - & - & - \\
\hline & + & - & - & - & - & - & - \\
\hline & + & - & - & - & - & - & - \\
\hline & + & - & - & - & - & - & - \\
\hline & - & - & - & + & - & - & - \\
\hline & - & - & + & - & - & - & - \\
\hline & - & - & - & + & - & $?$ & - \\
\hline & - & - & + & - & $?$ & - & \\
\hline & - & - & - & - & + & $(t)$ & $1-$ \\
\hline & - & - & - & + & $(+)$ & $(-)$ & \\
\hline & + & ? & ? & - & - & - & - \\
\hline & + & $?$ & - & - & - & - & \\
\hline & + & $?$ & $?$ & + & - & - & - \\
\hline & + & $?$ & + & - & - & - & \\
\hline & + & + & + & + & $(+)$ & + & ? \\
\hline & + & + & + & $(+)$ & + & $?$ & \\
\hline
\end{tabular}

Die Appositive, die sich deutlich auf nur eine der möglichen NPn beziehen, folgen dieser i.d.R. unmittelbar. Nur wenn die Basis unmittelbar vor einem 
KlammerschluB steht (z.B. einen Ball), kann das Appositiv evtl. hinter diesen gestellt werden. Distanzstellungen sind deutlich weniger (einem frechen Benge1), häufig auch gar nicht akzeptabel (eine große Brünette; ein frecher Bengel). Sofern die semantischen Bezüge schwanken können (z.B. bei jung und frech, was sowohl auf die Frau als auch auf einem Kind zutreffen könte), ist zur Vermeidung von Bezugsambiguitaten allein die stellung hinter der Bezugs-NP sinnvoll.

Bei ein/einem Bengel bedingt der Unterschied nicht-kongruent/kongruent augenscheinlich, daB die nominativische Version nur unnittelbar rechtsfolgend stehen darf, die kongruente mit allerdings deutlicher Akzeptabilitătsminderung auch nach den Klammerschluß. Die Kongruenz erlaubt dew Empfánger, Bezúge zwischen Einheiten auch über mehrere Ronstituenten hinweg herzustellen. An dieser Stelle kann auch die These entkraftet werden, das "nicht-kongruente Appositiv" sei eigentlich eine Parenthese. 44 Wenngleich Appositive und nicht-satzwertige parenthetische Einschube unzweifelhaft Zusammenhänge aufweisen, so sind doch deren grammatische Eigenschaften verschieden; $\nabla$ gl.:

(11.68a) Pia bat den Udo (, ein/ einen Rocksánger,) in Köln (, *ein/ *einem Rocksänger,) ein Autogramm abgeluchst.

Pia hat dem Udo ein Autogramm (, *ein/ *einem Rocksánger, ) abgeluchst (; *ein/ *einem Rocksánger).

(11.68b) Pia hat dem Udo (- er ist ein Rocksánger -) in Köln (- er ist ein Rocksánger -) ein Autogramm abgeluchst.

Pia hat dem Udo in Köln ein Autogramm (?- er ist ein Rocksánger -) abgeluchst (; er ist ein Rocksánger).

(11.68a) demonstriert die strikte Adjazenz des Appositionsprototypen, die unabhängig von der Kasusmarkierung besteht. (11.68b) zeigt eine entsprechende Parenthese; diese ist verschiebbar und sonit stellungsmảigig unabhangig(er)! Das nimmt auch nicht wunder: schlieblich verhalt sich die Parenthese in (11.68b) eher wie ein selbstandiger Satz (Finitum, Illokution), das Appositiv dagegen eher wie ein Attribut (Adjazenz, z.T. Rongruenz; vgl. auch Kap. 6.22. Konstituenz).

Bei adjektivischen Appositiven kann eine Mehrdeutigkeit auftreten: erstens die Interpretation als sekundare, kopulative Pradikation über einen NP-Referenten (Prototyp) und $z$ weitens die Sicht als eingeschaltetes pradikatives Attribut (somit VP-ZUSATZ oder evtl. SATZ-ZUSATZ):

(11.69) Dann ging Udo, (Udo war) betrunken und mude, nach Bause.

Dann ging Udo, betrunken und müde ['als Udo nach Hause ging, war er betrunken und müde'], nach Bause.

In Appositionsfall ist nur direkte Nachfolgeposition móglich, das eingeschaltete prădikative Attribut kann u.U. verschoben werden. Die Mehrdeutigkeit 10-

44 Nahegelegt z.B. bei Hentschel (1989: 290). 
kaler PPn bei Rechtsadjazenz zu einer NP (vgl. Beispiele 11.35-11.37) wurde bereits angesprochen.

Das Stellungsverhalten der übrigen NP-bezogenen ZUSATZE wird in den Rapiteln 12,13 und 15 abgehandelt. 


\section{ZUSATZE MIT "FUNRTIONSLEXEMEN"}

Nach der Aussonderung aller "enger appositionsverdăchtiger strukturen" (Kap. 7-9) sowie derjenigen, die Beispiele für MEHRFACHE VF-BESETZUNG, HERAUSSTELLUNG, EINSCHUB (traditionell: PARENTHESE) und SATZ-ZUSATZ darstellen (Rap. 911), verbleiben noch die "im engeren sinne appositionsverdächtigen Muster". Das Erkenntnisinteresse konzentriert sich auch weiterhin auf NP-bezogene ZUSATZE.

Ich nehme zunächst eine Aufteilung vor in (A) ZUSATZE, die von sog. "Appositionslexemen" begleitet werden können (12.1a), ja hăufig begleitet werden müssen (12.1b) und (B) (KURZ)ZUSATZE, die gewöhnlich unbegleitet erscheinen; letztere werden in Kap. 13 behandelt. Die begleitenden Lexeme werden hinfort "Funktionslexeme" genannt, da sie anzeigen, welche semantische Funktion (PRAZISIERUNG, BEISPIELNENNUNG etc.) der ZUSATZ ausũbt: ${ }^{1}$

(12.1a) Viele Vögel, (genauer) die Raubvögel, jagt man dort. Manche Haie, (z.B.) Hammer- und Blauhaie, werden gefürchtet.

(12.1b) Manche Raubvögel, ferner die Amseln/*die Amseln, jagt man dort. Die Haie, ausgenommen der Blauhai/ *der Blauhai, waren harmlos.

Gerade die Mōglichkeit, daß Zusãtze von Funktionslexemen begleitet werden können, wird manchmal zu einem wesentlichen Kriterium für das Vorliegen von APPOSITION erhoben. ${ }^{2}$ Doch allein das Vorkommnis eines solchen Lexems beweist noch nicht das Vorliegen eines Falles von ZUSATZ; ${ }^{3}$ es erscheint geboten, weitere grammatische Eigenschaften mit heranzuziehen.

Da auch der "Prototyp" von Funktionslexemen begleitet werden kann, wird er hier wieder aufgegriffen. In diesem Kapitel, wie auch in den folgenden, werden neue Begriffe eingeführt (ZUSATZE diverser Subklasse), um die zur Diskussion stehenden Ronstruktionen klassifikatorisch $z u$ erfassen, und $z$ war unbelastet von traditionellen Begriffssetzungen. Dies heist nicht, daB Begriffe wie "lokkere Apposition", "Rechtsversetzung" etc. obsolet geworden wăren, sondern nur, daß zum zwecke möglichst unvoreingenommener Erfassung darauf verzichtet wurde.

Um zu einer einigermaßen reprăsentativen Datensammlung zu gelangen, wurde versucht, möglichst viele Funktionslexeme zu erfassen. Dabei wurden i.d.R. nur nicht-satzformatige (bzw. verblose) Ausdrücke erfast; Ausdrücke wie ich wie-

1 Altmann (1981), der diese auch zur Analyse einsetzt, nennt sie "Kriteriale Zusătze". Rath (1975: 185 ff., v.a. 200 ff.) bezeichnet sie als "Paraphrasenankündigumgen" bzw. "Rorrektursignale" (ebd. $211 \mathrm{ff}$. ); von "Korrektur- und Bestătigungssignalen" spricht ortner (1985).

2 So z.B. Raabe (1979: $268 \mathrm{ff}$ ), Altmann (1981: $97 \mathrm{ff}$.). Fur das Englische 2.B. Sopher (1971: 409 ff.) und Quirk u.a. (1976: $627 \mathrm{ff.}$.).

3 Man bedenke z.B., daß in einer Folge selbständiger V-2-Hauptsătze ein "Tumktionslexem" auftreten kann:

(F.12.1) Manche Raubvögel jagt man dort; ferner/ übrigens jagt man dort auch Amseln. 
derhole (es): oder oder wie wir uns eigentlich ausdrücken müssen werden weitgehend außer acht gelassen - auch Partizipialphrasen (etwas mehr technisch ausgedrückt usw.) werden nur marginal berücksichtigt.

Erst in späteren Schritten wird überprüt, inwiefern weitere syntaktische Daten mit Teilmengen der Muster in Einklang zu bringen sind. ${ }^{4}$ Es sei kurz angemerkt, daB einige syntaktische Eigenschaften (vgl. die Erörterungen in Kap. 6-11) offenbar grundsătzlich für ZUSATZE gelten dürften, z.B. das "Einschaltungsmuster", die stellungsbeschränkungen relativ zum Basiselement (im Ggs. zu EINSCHOBEN), die Möglichkeit von Paraphrase oder Satzverdoppelungsableitung, das wahrscheinliche Fehlen einer Dependenzrelation und das "Konstituentoidverhältnis" (Verhältnis der Assoziation) von Basis und Zusatz. Gerade weil die Funktionslexeme nicht selten obligatorischer Bestandteil des Zusatzes sind und die bisherige Appositionsforschung m.E. diesen funktionalen Indikatoren zu wenig Beachtung schenkte (und vielleicht zu sehr auf den nur fakultativ um Funktionslexeme erweiterten Prototypen - vgl. Kap. 6 - fixiert war), erwarte ich mir über diesen Zugriff eine fruchtbare Ausgangsposition für Detailanalysen. 5

Zunächst folgt eine alphabetische obersicht über mögliche zur Verfügung stehende Lexeme, die zwar für sich keine Vollständigkeit beanspruchen kann, aber uber bisherige Darstellungen an Umfang hinausgeht. Dabei wird ggf. die Wortartenzugehörigkeit, die syntaktische Funktion und die semantische Leistung angegeben. Die semantische Funktion erachte ich als wichtigen ordnungsfaktor, doch wird dieser später natürlich mit den syntaktischen Eigenschaften der zusatzmuster in Beziehung gesetzt, um zu relevanten Klassen zu gelangen. Bei Lexemen, deren Funktion der eines anderen Lexems ähnelt, wird auf das funktionsverwandte Lexem verwiesen.

Zudem werden öfters veranschaulichende Hinweise gegeben, wie der Zusatz als Satzfolge bzw. verkürzter Satz (bei Tilgung identischer Satzkonstituenten bis auf den zusatz selbst) aussehen könnte. Ich schliebe mich dabei der Ansicht an, daB zumindest ein gröBerer Teil derartiger ZUSATZE über eine sog. "Satzverdoppelungshypothese" erklārbar ist (dazu Altmann 1981: $335 \mathrm{ff}$.). Bei gedoppelten Sätzen würde demnach gleiches Material (Unterstreichung!) getilgt (12.2b) und schlieblich der "Satzrest" in den Gastsatz neben seine Bezugseinheit eingebettet (12.2c). Angesichts von (12.2d) ist evtl. auch eine Satzkonjunktionshypothese zu erwăgen (vgl. 12.2e; fraglich ist, ob diese auf alle Muster zutreffen kann). Besonders hervorzuheben ist die m. E. ungewönliche Stellung des zusatzes in (12.2d), namlich zwischen Kern-NP und Genitivattribut! Zudem demonstriert (12.2f), daB Reduktionen auf dem Konstrukt "Basis + zusatz" operieren können.

4 Obwohl diese Vorgehensweise nicht das Nonplusultra darstellt, wurde auf eine Umarbeitung verzichtet, da begutachtete und veröffentliche Dissertationsfassung sonst zu stark differierten.

5 Eine Ausnahme bildete Altmann (1981), der jedoch aufgrumd seines Forschungsinteresses (die "Herausstellungen") nicht intensiv auf die Aufgliederung des Appositionsmusters eingehen kannte. 
(12.2a) Die Haie waren hungrig. Besonders die Blauhaie waren hungrig.

(12.2b) Die Haie waren hungrig, besonders die Blauhaie.

(12.2c) Die Haie, besonders die Blauhaie, waren hungrig.

(12.2d) (...) wenn nicht die GewiBheit - und das heist die exakte Methode der Mathematik, des näheren der Geometrie, auf die Philosophie ubertragen wird? 6

(12.2e) wenn nicht die GewiBheit der Mathematik (...) übertragen wird und das heiBt wenn nicht die exakte Metbode (...) übertragen wird.

(12.2f) Es erscheint (..) plausibel, daB eine Analyse des Aussehens [eines Nortes], d.h. der Form eines Wortes, oberflächlicher ist (...).7 Wichtig ist auch, daB die ZUSATZE offensichtlich nicht ausschlieblich Nominalphrasen als Bezugselemente (an die sie sich "assoziieren") aufweisen, sondern nicht selten auch an Phrasen anderer Kategorien (Adjektiv-, Verbalphrasen etc.; dazu Kap. 14) herantreten. Die folgenden "assoziierten" HOSPITANTEN bleiben ungeachtet der wechselnden Bezugsphrasen ZUSXTZE, außer wenn sie, . selbst satzförmig, auf ganze Sätze bezogen werden. Dann nämlich bringen Funktionslexeme lediglich die inhaltliche Beziehung zweier Teile einer Satzfolge zum Ausdruck, ohne das sich an der Satzfolge grammatisch etwas ändert. Eine syntaktische Funktion (des Folgesatzes bzgl. des voraufgehenden) wird hier ausgeschlossen.

Zusatz während der Oberarbeitung: Vor allem durch die Aufsătze von ortner (1983; 1985) ist mir - in voller Tragweite allerdings erst wăhrend der Oberarbeitung für die Veröffentlichung - bewußt geworden, daß die Anzahl möglicher Funktionslexeme sehr grob ist und daB eine vielfalt von (inhaltlichen) Funktionen durch sie angezeigt werden kann. Es war nicht mehr durchführbar, bestimmte Muster wie z.B.

(12.3a) Mit einem Satz vollziehen wir eine Sprechhandlung (technisch/ linguistisch gesprochen: eine Illokution).

(12.3b) Dieser faux pas (uberspitzt formuliert: diese Bauchlandung im Fettnäpfchen) war unnötig.

nachträglich zu berücksichtigen. 8 Der "Schaden" dürfte sich jedoch in Grenzen halten, da die Grundfunktionen wie KORREKTUR oder PARAPHRASIEREN (liegt wohl in beiden Fällen oben vor) weitgehend erfaBt sein dürften; eine umfassende funktionale Ausdifferenzierung bleibt eine Forschungsaufgabe.

6 Beleg aus: Küng, Hans. 1978. Existiert Gott? München, Piper. S. 26.

7 Beispiel aus: Schwarze, Christoph \& Dieter Munderlich (Hgg.). 1985. Handbuch der Lexikologie. Königstein/Ts., Athenăum, S. 335. Das Reduzierte füge ich in eckigen Klamern hinzu. zum Begriff "Reduktion" (Tilgung identischer Konstituenten) vgl. Heidolph u.a. (1981: $142 \mathrm{ff}$. .).

8 Vor allem sog. "formulierungskamentierende Ausdrücke" (z.B. Kumo ist ein Betrüger (in Klartext: ein ausgemachter Schuft)/ wären noch zu bericksichtigen gevesen. Hierzu Antos (1982). 
Die Einarbeitung von WIEDERHOLUNG (wörtlicher PARAPHRASIERUNG) und KORRERTUR geschah ebenfalls erst wăhrend der Oberarbeitung (vgl. die Funktionslexeme 19) ja und 28) nochmals zur Wiederholung; 14) genauer, 26) nein und 39) vielmehr zur Korrektur). Daher konnten beide Phänomene, obwohl sie in den Bereich der ZUSATZE hineinspielen (die Wiederholung u.a. im Zusammenhang mit umformulierenden und präzisierenden, die Korrektur u.a. in Verbindung mit präzisierenden Zusätzen), nur stellenweise (aber nicht mehr in jeder Hinsicht) in die Beschreibung integriert werden.

In der begutachteten Fassung der Dissertation wurden WIEDERHOLUNG (und zwar der Fall 12.4a) und RORRERTUR (nämlich der Fall 12.5a) noch aus dem Bereich der ZUSATZE mit einigen unzulänglichen "Argumenten" ausgeschieden. Ich hielt (selbstkonstruierte) Fälle wie $(12.4 \mathrm{a}, 12.5 \mathrm{a})$ zunächst für fragwürdig und war der Ansicht, daB der "Normalfall" von Wiederholung oder Rorrektur aussieht wie in $(12.4 \mathrm{~b}, 12.5 \mathrm{~b})$; da kein vollständiges Einschaltungsmuster vorliegt - es fehlt die rechte Abtrennungsmarkierung -, habe ich derartige Muster ausgesondert. Wăhrend und nach der Lektüre von ortner (1983; 1985) und Rath (1979) sties ich jedoch auf Beispiele von Wiederholung bzw. Korrektur mit vollständigem Einschaltungsmuster (z.B. die Korrektur in 12.5c):

(12.4a) Mit Verstand, ja: mit Verstand, muBt du dies tun.

(12.4b) Mit Verstand, ja: mit Verstand muBt du dies tun.

(12.5a) Ein Mofa, nein: ein Moped, brauste um die Ecke.

(12.5b) Ein Mofa, nein: ein Moped brauste um die Ecke.

Was Krach ist, hören, nein: spüren wir erst danach.

(12.5c) Einmal berücksichtigen die Anhänger (...) nicht genügend - richtiger: überhaupt nicht - den physiologischen Unterschied (...).9

Die VIEDERHOLUNG ist nicht auf den NP-Bereich begrenzt. ${ }^{10}$ Ich war der Meinung, sie stelle ein "Mehrmalssetzen" (Nochmalsetzen) einer Basis und kein Hinzusetzen eines Zusatzes zu einem Basiselement dar, weshalb ich keine syntaktische Relation ansetzen wollte. Eine KORRERTUR sah ich als eine "Neusetzung" einer sprachlichen Einheit (quasi ein "sprachliches Durchstreichen") an; eine Aufteilung in Basis und Zusatz und somit das Postulat einer syntaktischen Relation schien mir fehl am Platz. 11

Vor allem die Ausführungen von Rath (1975) über "Verfahren zur Textkonstitution im Rommunikationsablauf" (ebd. 185 ff.), die ich erst wăhrend der letzten Oberarbeitung zur Renntnis nehmen konnte, legen zusammenhänge nahe (auch

9 Aus: Ortner (1985: 239).

10 Die Wiederholung vird beispielsweise auch van Molitor (1979: 216) aus dem Appositionsbereich ausgeschlossen. Altmann (1981: 52) behandelt die WIFDERHOLUNG als eigenes Muster.

11 Auch Molitor (1979: $214 \mathrm{ff}$.) grenzt Apposition und KORRJXTION voneinander ab. Sein Hauptargument (ebd. 215): "Die Rorrektion erfullt nicht die Prădizierbarkeit der N [= Nomina; H.S.] über einen kanstant gehaltenen cegenstand (...)". Zur Korrektur weiterführend ortner (1985), Glich/Rotschi (1987; v.a. 217 ff.). 
Ortner 1985 deutet auf sie hin). Explizit verweist Rath in Fn. 174 (1975: 210 resp. 243) auf die Verwandtschaft von prăzisierenden bzw. korrigierenden Erweiterungen und Appositionen. Mein Hauptargument, daB nămlich z.B. Rorrekturen ohne vollständige Einschaltungsmarkierung (z.B. ; s. sub verbo nein, Funktionslexem $\mathrm{Nr}$. 26) erscheinen, labt sich angesichts von Beispielen vie (12.5c) nicht aufrechterhalten. Vielmehr ist zwischen einer (primăr pragmatisch und allenfalls sekundăr syntaktisch zu erklärenden) Konstruktionsănderung ohne vollstăndige Einschaltungsmarkierung und einer syntaktifizierten Zusatzkonstruktion mit vollständigem Einschaltungsmuster zu unterscheiden!

Wenn Wiederholungen bzw. Rorrekturen mit Einschaltungsmuster vorliegen, ist ihr Anschluß an die inhaltlichen Funktionen der Zusătze deutlich: nicht-wörtliche Wiederholungen (Paraphrasierungen) können z.B. "umformulierend" wirken, eine Ausdrucksalternative anbieten (12.6a; s. Ordnung der Zusătze, 3.1.) oder aber präzisierend (12.6b). Totale Wiederholung allein (z.B. 12.4a) nimmt wohl eher pragmatische denn semantische Funktionen wahr: Emphase und Intensivierung vor allem. ${ }^{12}$ Korrekturen können voraufgegangene Hortgruppen ganz (TOTALKORREKTUR; KORREKTUR im engeren Sinn, 12.7a) oder teilweise (PRAZISIERUNG, 12.7b) "außer Kraft setzen" (vgl. Rath 1979: 209) und stehen daher mit den spezifizierenden Zusătzen (vgl. Ordnung der Zusătze, 1.) in Zusammenhang.

(12.6a) Pia hat die Ledigen (die Unverheirateten) befragt.

(12.6b) Vom Riff her schwammen Haie (Hammerhaie!) herbei.

(12.7a) Uns haben Haie, nein: Heringe(,) umkreist.

(12.7b) Pia hat Schlangen, genauer: Boas und Pythons, beobachtet.

1) allgemeiner (gesagt) (AdjP (PtGr); KOND), DEPRAZISIERUNG, ERWEITERUNG HIN ZU EINER OBERMENGE.

Die Griechen, allgemeiner die Sudländer, kochen gern mit Knoblauch.

Die Griechen kochen gern mit Knoblauch. Wenn man es allgemeiner nimmt/A11gemeiner gesagt, kochen die Sülander gern mit Knoblauch.

Weitere mógliche Verwendungen:

$A$ ist 20 Meter höher als B, allgemeiner: $A$ ist hober als B.

In Indien verbungern, allgemeiner: sterben, viele Menschen.

12 Vgl. auch Altmann (1981: 52). Zu 'Figuren der Viederholung' s. Lausberg (1982: 80 ff.), Plett (1983: 33 ff.). 
2) also (Konjunktionaladverb), AUFZXHLEN, ANDERSSAGEN. 13

Die Grobkatzen, also Löwen, Tiger und Panther, sind hungrig.

Die Großkatzen sind hungrig. (?) Also sind Löwen, Tiger und Panther hungrig.

Die Großkatzen sind hungrig, also Löwen, Tiger und Panther.

Die Vögel, also unsere gefiederten Freunde, sind von uns bedroht.

Andere Verwendungsweisen:

Welche Prozesse lassen sich auf dem Weg von der Wortmarke zur Bedeutung (PP), also beim lexikalischen Zugriff, beobachten? ${ }^{14}$

Pia hat wieder herumgesumpft, also weder den Haushalt gemacht noch eingekauft (VP).

Zudem vermag also in Fällen wie Meine Meinung ist, daß Deutschunterricht, also daB Historie und Germanistik $z u$ revidieren sind (...) als "Korrektursignal" zu fungieren. 15

3) anders gesagt (PtGr; KOND), ANDERSSAGEN.

Die Raumpflegerin, anders gesagt (/wenn man es anders sagt:) die Putzfrau, wurde zur Chefin befördert.

Eine zusätzliche Anwendungsmöglichkeit wäre beispielsweise:

Kuno ist ledig - anders gesagt: ein Junggeselle - gewesen.

4) ausgenommen/ ausgeschlossen/ ausschlieBlich (Adj/ Prăp; KOND), AUSSCHLUSS EINER TEILMENGE.

Die Vögel, (ausgenommen) die Raubvögel (ausgeschlossen), waren friedlich.

Die Vögel (ausschlieblich der Raubvögel), waren friedlich.

Die Vögel, wenn/ sofern man die $R$. ausnimmt/ ausschließt, waren friedlich. Zusătzlichen Verwendungsweisen muten nicht immer akzeptabel an:

?Dort sterben, ausgenommen: verhungern, viele Menschen.

(?) Die Hemden waren bunt, ausgenommen hellrot und purpur, gewesen.

Bei Fällen mit ausschließlich bin ich nicht sicher, ob ein nicht-spezifizierender Zusatz (ohne mögliche Attributfunktion) vorliegt oder ein mit Einschaltungsmuster markiertes Gliedteil (mit möglicher Attributfunktion; vgl. Kap. 11. Beispiel 11.61). Siehe auch einschlieBlich!

13 Konjunktionaladverbien sind "Satz- bzw. Textteilkonnektoren", die nicht wie koordinierende Konjunktionen platzfest sind, sondern sowohl allein das Vorfeld ausfüllen als auch an bestimnten Mittelfeldpositionen auftreten können. Thre syntaktische Funktion ist nicht immer klar (Adverbial?).

14 Aus: Schwarze/Wunderlich (1985), S. 315.

15 Aus Rath (1979: 213). Dazu ebd. die Aussage:

"Mit dem Korrektursignal "also" wird die Geltung des zuvor geäußerten sprachlichen Elements "Deutschunterricht", zumindest teilweise, außer Kraft gesetzt und durch die Begriffe "Historie und Germanistik" ersetzt. Wenn man das Korrektursignal wegläßt, bleibt der (im ersten Falle korrigierte und daher nur teilweise gulltige) Begriff voll in Geltung (...)". 
5) beispielsweise/ z.B. (Ronjunktionaladverb? Satzadverb? ${ }^{16}$ Ordinative Angabe), 17 BEISPIELNENNUNG, (TEILVEISE) AUFZAHLUNG.

Manche Vögel, beispielsweise/ z.B. Bussarde und Milane, haben es schwer. Manche Vögel haben es schwer, beispielsweise (haben es) Bussarde und Milane (schwer).

Neben rätselhaften Naturerscheinungen und oft als Realität erlebten Träumen sowie Sinnestäuschungen, z.B. der Fata Morgana, wurden sicherlich auch Fiebervisionen (...) für den Menschen zu Quellen mythisch-magischer und religiöser Vorstellungen. 18

Diese Funktion ist nicht $z u$ verwechseln mit derjenigen des "Prototyps" (vgl. auch Abs. 6, 31 dieser Liste):

Manche Vögel, (*manche Vögel sind z.B. Bussarde und Milane), haben es schwer.

Die Anwendung auf nichtnominale Kategorien ist wohl möglich:

(?) Die bunten, z.B. blauen, roten und grünen, Hemden starrten vor Dreck.

In Indien sterben, z.B. verhungern und verdursten, viele Menschen.

6) bekanntlich (Satzadverb), NEBENPRADIKATION (EIGENSCHAFTSZUSCHREIBUNG, KLASSENEINORDNUNG, IDENTIFIKATION etc.).

Bei diesem Muster beachte man, daB eine Satzdoppelungshypothese unannehmbar ist. Auch eine Ableitung aus nachgestelltem Kopulasatz, Tilgung und Einbettung erscheint unzutreffend. Am naheliegendsten ist die Reduktion eines bereits eingebetteten Kopulasatzes (als EINSCHUB).

Die Löwen, (die Löwen/ sie sind) bekanntlich majestätische Großkatzen, sind gefürchtet.

* Die Löwen sind gefürchtet, bekanntlich majestätische Großkatzen (sind gefürchtet).

?? Die Löwen sind gefürchtet, (die Löwen sind) bekanntlich majestätische GroBkatzen.

Weitere Verwendungsweisen sind wohl nicht gegeben:

?? In Indien sterben, bekanntlich verhungern und verdursten, viele Menschen.

16 Werden Bussarde gejagt? Beispielsweise (Antwort Entscheidungsfrage positiv). Es ist beispielsweise der Fall, das Bussarde gejagt werden (Es ist $X$ der Fall-Probe für Satzadverbiale positiv).

17 Zu diesem Begriff Engel (1988: 228 f.); sie setzen Außerungen in Beziehung zu anderen Außerung (steil)en. Man kann sie wohl den SATZADVERBIAL.WN zuordnen (Antwort auf Entscheidungsfrage, Es ist $X$ der Fall-Test).

18 Aus: Winkler, Eike \& Josef Schweikhardt. 1982. Expedition Mensch. Streifzüge durch die Anthropologie. Wien, Heidelberg, Ueberreuter. S. 102. 
7) besonders (GP), RERVORHEBUNG (EINER TEILMENGE), PRAZISIERUNG.

Die Sprachwissenschaft, besonders die moderne Linguistik, hat sich mehr mit solchen (...) strukturen befaBt, (...). 19

Die Sprachwissenschaft hat sich mehr mit solchen (...) strukturen befaBt, (...). Besonders die moderne Linguistik hat sich mehr mit solchen (...) strukturen befaBt, (...).

Die Anwendung auf nichtnominale Kategorien erscheint etwas fragwürdig.

? Bunt, besonders rot und grün, waren die Hemden.

?Bearbeiten, besonders bemalen und bedrucken, will Pia diese Kleider.

8) bzw. (koord. Konj), ALTERNATIVE.

Es wurde Nein, bzw. Milch, getrunken.

Es wurde Wein getrunken, bzw. (es wurde) Milch (getrunken) 20

Auch auf andere Kategorien anwendbar:

Pia soll die Bowle machen (bzw. die Cocktails vorbereiten).

Sie kochten, bzw. grillten, die Würste.

Alle roten, bzw. rotbraunen, Hemden wurden gewaschen.

9) deutlicher (gesagt), s. genauer, präziser.

10) d.h.- (koord. Konj), ANDERSSAGEN, AUFZAHLUNG, PRAZISIERUNG; REFERENZKLARUNG. 21

Die Wüstenschiffe, d.h. die Kamele, waren müde.

Die Wüstenschiffe waren müde, d.h. die Kamele (waren müde).

Alle Vögel, d.h. Raub- und Singvögel, werden dort gejagt.

(...), daß sie - d.h. die Taxifahrerin - dem Wechsel (...) mit Unverständnis gegenüberstand. 22

Zur Verwendung des Konnektors d.h. finden sich auch für nichtnominale Basen Belege zuhauf, was seiner generellen Funktion 'Ankündigung einer Paraphrasierung' zuzuschreiben sein ärfte: ${ }^{23}$

Hiernach werden nicht nur Wörter sensorisch, d.h. graphematisch und phonologisch, als Wortmarken repräsentiert, (...).

19 Aus: Schwarze/hunderlich (1985), S. 7.

20 Man beachte, daß zwei Lesarten möglich sind: 'sowohl Wein als auch Milch' bzw. 'entweder Wein oder Milch'.

$21 \mathrm{zu}$ den semantischen Funktionen von d.h. s. Hindelang (1980).

22 Aus: Hindelang (1980: 127).

23 Zum Begriff 'Konnektor' s. Ortner (1983). Irster Beleg aus: Schwarze/Munderlich (1985), S. 337; zweiter Beleg aus: Süddeutsche Zeitung 16/1990, 20./21.1.90, S. 201; dritter Beleg aus: Glück, Helmut \& Wolfgang Sauer. 1990. Gegenwartsdeutsch. Stuttgart, Metzler. S. 94; letzter Beleg aus: Asimov, Isaac. 1986. Die exakten Geheimnisse unserer Welt. München, Droemer Rnaur. S. 98. 
Wer heute - das heiBt nicht $z$ u Stalins, nicht zu Chruschtschows, nicht $z u$ Breshnews, sondern $z u$ Gorbatschows Zeiten - in Moskau einkauft, (...). Schon während des Krieges, d.h. mit dem Näherrücken der Ostfront, hatte die (...) Regierung (...) Minderheiten (...) "heim ins Reich" geholt.

Je primitiver eine Tierart ist - $d . h$. je weniger diversifiziert und spezialisiert ihre Zellen sind -, desto gröBer ist (...).

11) einschließlich (Präp)/ eingeschlossen (Präp?? Adj? KOMIT?), HERVORHEBUNG EINER TEILMENGE.

Viele Haie, einschließlich des Walhail der Walhai eingeschlossen, sind harmlos.

Viele Haie sind harmlos, einschlieblich des Walhais (sind viele Haie harm10s).

Weitere Anwendung ist wohl möglich.

Im Prinzip müBte das Klonen bei allen Säugetieren möglich sein, die Spezies Mensch eingeschlossen. 24

Zubereiten, einschlieBlich putzen und schneiden, soll Kuno den Salat.

Du sollst bunte, einschlieblich blaue und grüne, Hemden kaufen. Bei Fällen mit einschließlich bin ich nicht sicher, ob ein spezifizierender Zusatz (ohne mögliche Attributfunktion) oder ein mit Einschaltungsmuster markiertes Gliedteil (mit möglicher Attributfunktion; vgl. Kap. 11, Beispiel 11.61 und ausschlieblich) vorliegt. Die nachstehenden Beispiele zeigen eine Attributfunktion und eine zusatzfunktion ohne potentielle Attributfunktion (nach Wegfall der Klammern entstünde ein inakzeptables Syntagma):

Alle Beamten einschließlich der Lehrer (ATTR) bekamen mehr Geld.

Alle Beamten (und $z$ war vermutlich einschlieBlich der Lehrer) (...). Eine Erklärung der Daten muß leider offenbleiben; eine detailliertere Beschreibung der PPn mit aus-/einschlieblich ist wünschenswert.

12) etwa (GP?), BEISPIELNENNUNG. 25

Ein VW 1200, etwa ('(nimm) zum Beispiel') ein Modell von 1952, ist äuBerst robust. 26

Vgl.: Ein VW 1200, (er ist übrigens) etwa ('ungefähr') ein Modell von 1952, ist äuBerst robust.

24 Aus: Asimov (1986: 99).

25 Vgl. Ortner (1983: 101): "Etwa enthält die Anweisung an den Leser: Fasse das im folgenden Gesagte als Exemplifikation zu dem im Satz vor dem Ronnektor etwa Gesagten auf".

26 Beispiel von Raabe (1975: 332); nach Raabe (ebd.) ist die Basis generisch, der Zusatz definit zu lesen. Das Beispiel ist zweifach lesbar: (a) etwa ist eine Gradpartikel, die 'ungefähr' bedeutet, ein W 1200 ist dann nichtgenerisch, sondern quantitativ (' 1 ') aufzufassen; naheliegender ist (b), wobei etwa 'z.B.' bedeutet - dabei ist die Basis generisch zu lesen. 
Auch komplexere Lebewesen, manche Insektenarten etwa, sind in der Lage, (..). 27

Weitere Verwendungsweisen:

In vielen dieser Regionen - etwa in Asien und Afrika - existiert (..) noch immer die Grobfamilie. 28

(?) Die bunten Hemden, etwa grüne, blaue und rote, sind zu teuer.

13) ferner (Adj; ordinative Angabe), ANFUHREN EINER WENIGER BETROFFENEN MENGE. Löwen und Tiger, ferner die Panther, werden gefürchtet.

Lówen und Tiger werden gefürchtet, ferner (werden) die Panther (gefürchtet).

Löwen und Tiger (, ferner die Krokodile,) werden gefürchtet (, ferner die Rrokodile). 29

Weitere Anwendungen sind wahrscheinlich möglich.

Gekocht, ferner gesotten und gegart, wurden die Speisen dort.

(?) Wichtig, ferner eilig, war diese oberweisung gewesen.

14) genauer (Adj; KOND), PRAZISIERUNG, KORREKTUR.

Bisher sind die Worter, genauer die Wortformen, nur als ein noch nicht bedeutungstragender Nahrnehmungsgegenstand betrachtet worden. 30

Darüber hinaus auch $2 . B$. bei:

In jedem Fall setzt die Sprechbandlung ein Ziel, den Entwurf und die Verwirklichung eines Plans (genauer: eines Programms)...voraus. 31

Im Keller, genauer: im Vorratsraum, wimmelte es von Mäusen.

Rote (genauer: rost- und karmesinrote) Farbtöne dominierten das Gemälde.

15) hauptsăchlich (Adj)/ in der Hauptsache (PP), (SADVB), HERVORHEBUNG EINER TEILMENGE (DIE BESONDERS BETROFFEN IST).

Die Vögel, hauptsächlich/ in der Bauptsache die Singvögel, werden gejagt.

Etliche Gewăsser, hauptsáchlich die Binnenseen, sind verschmutzi.

Etliche Gewásser sind verschmutzt, hauptsáchlich/ in der Hauptsache (sind) die Binnenseen (verschmutzt).

Heitere Anwendungen sind wohl meist (einigermaßen) akzeptabel:

(?) Dort sterben - hauptsächlich verhungern und verdursten - viele Menschen. Viele farbige, hauptsächlich rote, grüne und blaue, Hemden verbrannten.

27 Aus: Asimor (1986: 98).

28 Aus: v. Ditfurth (1988: 269).

29 Als 'weniger betroffene Mengen' erscheinen ummittelbar verwandte Teilmengen (Löwen, Tiger, Panther $\varepsilon$ Großkatzen) oder mittelbar verwandte Mengen (Großkatzen, Krokodile $\varepsilon$ Tiere).

30 Aus: Schwarze/hunderlich (1985), S. 274.

31 Aus: Ortner (1985: 249). 
16) ich meine, REFERENZVERDEUTLICHUNG bzw. -KLARUNG, PRAZISIERUNG.

Manche Großkatzen, ich meine Löwen und Tiger, werden gefürchtet.

? Manche Großkatzen werden gefürchtet, ich meine: Löwen und Tiger (werden gef ürchtet) .32

Die Verwendungsbedingungen dieses Ausdrucks sind schwer zu erfassen. Die Akzeptabilität folgender Beispiele ist mir unklar. Hier konfligieren wohl PRAZISIERUNG (genauer) und REFERENZVERDEUTLICHUNG ( $i$ ch meine) miteinander, so daß angenommen werden kann, daß der REFERENZKLARENDE ZUSATZ hier kaum angebracht ist:

Viele bunte Hemden, ich meine/ genauer rote, blaue und grüne, sind verbrannt.

Dort sterben, ich meine/ genauer verhungern und verdursten, viele Menschen. Eindeutig sind dagegen Beispiele mit Pro-Elementen als Bezugseinheiten:

Pia soll das lassen, ich meine/ *genauer ständig Zigaretten rauchen.

Pia kann das doch nicht sein, ich meine/ * genauer nachtragend.

17) in erster Linie (PP; ordinative Angabe); siehe hauptsächlich!

18) insbesondere (GP); siehe besonders!

Der angeführte Beleg ist eigentümlich, weil das Finitum des dab-Satzes im Plural steht, obwohl das subjekt den singular aufweist. ${ }^{33}$ vielleicht wurden Basis und Zusatz als (quasi-)koordiniert angesehen, vielleicht liegt ein Synesis-Phänomen vor?

Es ist außerdem damit $z$ u rechnen, daB die phonologische Aufbereitung des Materials, insbesondere die Auswertung von Redundanzen zur Informationssicherung, von vorneherein in Verflechtung mit dem Lexikonabgleich ablaufen müssen [sic].

19) ja (Konjunktion, Satzäquivalent), 34 wIEDERHOLUNG, ANDERSSAGEN. Pia hat Dracula (ja: Dracula!/ ja: einen Vampir!) geehelicht.

Bei Phrasen anderer Kategorie als NP ebenfalls möglich:

(...) es töne, als stehe sie geradezu an der Rampe, ja, als stehe sie geradezu an der Rampe -.35

32 Die Ableitung via Satzdoppelung erscheint fragwürdig; dazu später (auch Kap. 15) mehr.

33 Aus: Kochendörfer, Günter. 1989. Notation, Modellbildung, Simulation. Tübingen, Narr. S. 173.

34 Zur Einstufung als Konjunktion s. Engel (1988: 743). Helbig/Buscha (1980: 468 ff.) fassen ja, nein, doch, die Interjektionen sowie bitte, danke als Satzäquivalente zusarmen.

Aus: Ortner (1985: 246). 
20) konkret (Adj), BEISPIELNENNUNG, AUFZXHLUNG, PRXZISIERUNG.

Pia kann solche Zustände (konkret: Schlamperei und Unordnung) nicht aussteben.

Man vgl. hierzu beispielsweise, z.B., genauer.

21) korrekt (er) (Adj, KOND), PRAZISIERUNG, KORREKTUR. S. genauer. Und die handelnden Personen - korrekter: die, die handeln könnten - tun auch nichts dagegen. 36

22) (zu)meist; siehe hauptsächlich.

23) m.a.W. (abgekürzte PP; SADVB?), ANDERSSAGEN (AUFZAHLUNG, PRAZISIERUNG) Lowen, Tiger und Panther, m.a.W. viele Großkatzen, werden gefürchtet. Löwen, Tiger und Panther werden gefürchtet, m.a.W. (Nerden) viele GroBkatzen (gefürchtet).

Viele Großkatzen - m.a.W. Löwen, Tiger und Panther - werden gefürchtet. Viele Großkatzen werden gefürchtet, m.a.W. (werden) Löwen, Tiger und Panther (gefürchtet).

Zusatzliche Verwendungsweisen:

Die bunten, m.a.W. blauen, grünen und roten, Hemden verbrannten.

Beide sind untrennbar verbunden, m.a.W.: Sie setzen einander voraus.

24) namentlich (koord. Konj?), AUFZXHLEN, BEISPIELNENNUNG, PRAZISIERUNG.

Die Vögel, namentlich Raub- und Singvögel, werden dort verspeist.

Die Vogel werden dort verspeist, namentlich (werden dort) Raub- und Singvögel (verspeist).

Etliche Haiarten (, namentlich Blauhai und Bammerhai,) werden gefürchtet. (Namentlich Blauhai und Bammerhai werden dort gefürchtet.)

Angewendet auf Bezugselemente nichtnominaler Rategorie, scheint dieser ZUSATZ nicht völlig akzeptabel:

? Wir drücken uns gelegentlich unangemessen aus, namentlich: wir verfehlen das treffende Nort oder wir verwenden es im falschen Augenblick. ? Die bunten, namentlich roten, grünen und blauen, Hemden verbrannten.

25) nămlich (Konjadv), AUFZXHLEN, PRAZISIERUNG.

Jene Großkatzen, nănlich Lówen, Tiger und Panther, werden gefürchtet. 37

36 Aus: Ortner (1985: 250).

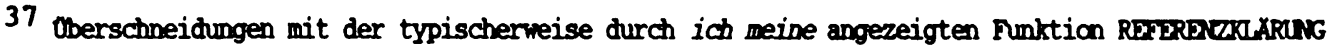
sind nicht auszuschließen: Jene Grabsatzen, nimlich/ ich meine Lömen, Tiger und Panther, werden gefürchtet. Man vgl. dagegen die Variante mit pranaminaler Basis, in der ich meine leicht vorzuziehen ist: Sie, ich meine/ nëmlich Löwen, Tiger und Panther, merden gefürchtet. Binigermaßen deutlich ist der Akzeptabilitătsunterschied bei Distanzstellung: Sie werden gefürchtet, ich meine/ ? nämlich Lömen, Tiger und Panther. 
? Jene Großkatzen werden gefürchtet, (gefürchtet werden) nämlich Löwen, Tiger und Panther.

Nicht zuletzt spielt ein zentraler Begriff der neueren generativen Theorie, nämlich der Begriff der Rektion, auch in Bechs Analysen eine zentrale Rolle. 38

Zusătzliche Vorkommensweisen wären z.B.:

Die Hemden sind bunt, nämlich rot, grün und blau, gewesen.

Mit jenen, nämlich meinen und deinen, Netzen fangen wir Fische.

26) nein (Negationspartikel; Satzäquivalent), KORREKTUR. 39

Frischs Novelle ist die Geschichte nicht einer Nähe, sondern einer Annäherung - nein, eher steter Ferne, ständiger Entfernung.

Was Krach ist, hören, nein: spüren wir erst danach. 40

Wer heute (...) in Moskau einkauft, erhält eine (...) Lektion in sowjetischem, nein: vermutlich eher in russischem Hierarchiedenken. ${ }^{41}$

27) nicht so sehr (NEG + AdvP), ANGABE EINER WENIGER BETROFFENEN TEILMENGE. Die Vögel - die Raubvögel nicht so sehr - werden dort verspeist.

(?) Die Vögel werden dort verspeist, die Raubvögel (werden dort) nicht so sehr (verspeist).

Viele Haie, die Hammerhaie (werden dort) nicht so sehr (gefürchtet), werden dort gefürchtet.

Eine Anwendung uber den NP-Bereich hinaus ist wohl eher auszuschlieBen:

?? Die Hemden waren farbig, nicht so sehr blau und rot, gewesen.

?Auf der Party wurde viel gegessen (nicht so sehr getrunken).

28) nochmals (Adverb)/ noch einmal, WIEDERHOLUNG, ANDERSSAGEN.

Es gibt zu wenig Wohnungen, nochmals: zu wenig Wohnraum(,) in München.

Auch außerhalb des NP-Bereichs anwendbar:

Krepieren, noch einmal: krepieren/ elend verhungern und verdursten, müssen viele Tausend Menschen täglich.

29) obendrein (Adv; ordinative Angabe), ZUSATZLICH ZU NENNENDE MENGE.

Die Vögel, obendrein die Fledermäuse, erhoben sich in die Lüfte.

Die Vogel erhoben sich in die Lüfte, obendrein (erhoben sich) die Fledermäuse (in die Lüfte).

Weitere Verwendung findet sich z.B. bei:

38 Aus: Grewendorf, Günther. 1988. Aspekte der deutschen Syntax. Tubingen, Narr. S. 263.

39 Zu nein als Rorrektursignal s. auch Stickel (1972: 16 f.).

40 Beide Beispiele aus ortner (1985: 241).

41 Aus: Suddeutsche Zeitung 16/1990, 20./21.1.90, S. 210. 
Im Keller, obendrein auf dem Speicher, wimmelte es von Mäusen.

Verärgert, obendrein frustriert, war Kuno an diesem Abend.

30) oder besser, (TREFFENDERE) ALTERNATIVE, PRAZISIERUNG, KORREKTUR.

Die Nazi - oder besser: die einzelnen (...) Nazicliquen - wollten (...) ibren Einfluß sichern. ${ }^{42}$

Die Nazi wollten (...) ihren EinfluB sichern. Oder besser: Die einzelnen (...) Nazicliquen - wollten (...) ihren EinfluB sichern.

Der oder besser-zUSATZ tritt nicht nur bei Nominalphrasen auf:

Wollen wir ein Bier trinken, oder besser, einen Cocktail schlürfen?

Man soll - oder besser: muB - auf Aufrüstung verzichten.

31) präziser (gesagt) (Adj (PtGr); KOND), PRAZISIERUNG, KORREKTUR.

Die Dingwörter, präziser (gesagt) die Nomina, bilden eine Kategorie.

Die Dingwörter bilden eine Kategorie, prăziser (gesagt bilden) die Nomina (eine Kategorie).

Auch auf weitere Kategorien anwendbar. Vgl. genauer.

32) SO auch (Adv + GP), BEISPIEL, HERVORHEBUNG EINER TEILMENGE.

Manche Vögel, so auch Milane und Bussarde, werden dort gejagt.

Manche Vögel werden dort gejagt, so (werden dort) auch Milane und Bussarde (gejagt).

Die Anwendbarkeit über den NP-Bereich hinaus erscheint unklar:

?Das Netter war mies; so stürmte und regnete es auch.

? Die Hemden sind farbig, so auch grün und rot, gewesen.

? Die Würste wurden zubereitet, so auch gegrillt und gebraten.

33) und zwar (Konnektor im VF?), AUFZAHLUNG, PRAZISIERUNG, MARKIERTE VERVOLLSTANDIGUNG. 43

Die Singvógei, und zwar Amsel, Drossel, Fin' und Star, sangen eifrig.

Die Singvögel sangen eifrig, und zwar (sangen) Amsel, Drossel, Fink und Star (eifrig).

Dieser Ausdruck ist sehr vielseitig einsetzbar. Er ist auffäligerweise mit etlichen weiteren Funktionslexemen kombinierbar (dazu etwas spăter); die Anwendung über den NP-Bereich hinaus ist möglich:

42 Aus: Siddeutsche Zeitung 289/1989, 16./17.12.89, S. 130.

43 Dieser Begriff soll zum einen die von Altmann (1981: 71) genannte Nachtragsfumktion "Bildung eines mehrteiligen Rhemas" umreilen, $2 \mathrm{~m}$ anderen soll er darauf hinweisen, das die nachgetragene Binheit für den Bezugssatz wichtig ist, wichtiger jedenfalls, als es z.B. "prádikative Zusătze" (prototypische Appositive) sind. Man nehme z.B. Altmanns Beispiele (ebd. 71), die Nachtragung dieser obligatorischen Funktionen ist für das Satz- und Textverständnis sehr vichtig:

(112.2) Der ist schon wieder befördert warden, zum kammandierenden Ceneral.

(F12.3) Er bat wieder geheurathet. Eine Jfr Obristleutnantin. 
Die Hemden waren farbig, und zwar rot, braun und grün, gewesen. Zubereitet, und zwar gewürzt und gegrillt, wurden die Würste von Pia.

34) überdies (Adv; ordinative Angabe), ZUSATZLICH ZU NENNENDE MENGE.

Die Vögel, überdies die Fledermäuse, flogen herbei.

Die Vögel flogen herbei. überdies (flogen) die Fledermäuse (herbei).

Die Semantik von überdies beschränkt sich nicht nur auf zusätzlich zu berücksichtigende Mengen, sondern kann auf alles, was zusätzlich mit in Betracht gezogen werden kann, angewendet werden:

Die Bemden waren rot, überdies purpur und pink, gewesen.

Arbeiten, überdies lernen und studieren, ist manchmal zuviel.

35) überhaupt; siehe allgemeiner!

36) überwiegend (Sadv, GP?), HERVORHEBUNG EINER TEILMENGE (DIE BESONDERS BETROFFEN IST).

Viele Vogelarten, überwiegend die Raubvögel, werden dort gejagt.

?Viele Vogelarten werden dort gejagt, überwiegend die Raubvögel (werden dort gejagt). $\mathbf{4 4}$

Viele Vogelarten werden dort gejagt, überwiegend (werden dort) die Raubvögel (gejagt).

Offensichtlich ist die Semantik dieses Lexems nicht nur auf (Teil)Mengenhervorhebung beschränkt, sondern es findet allgemein Verwendung zur HERVORREBUNG:

Gekocht, überwiegend gegrillt und gebraten, wurde dort sehr emsig.

Die Hemden waren farbig - überwiegend rot und blau - gewesen.

Das Wetter war schlecht; überwiegend stürmte und regnete es.

37) übrigens (Konjadv; ordinative Angabe?), NEBENPRADIRATION (EIGENSCHAFTSZUSCHREIBUNG, KLASSENEINORDNUNG, IDENTIFIKATION etc.).

Die Löwen, (sie sind) übrigens majestätische Grobkatzen, werden gefürchtet.

*Die Löwen werden gefürchtet, übrigens (werden) majestätische Grobkatzen (gefürchtet).

Eine Satzdoppelung kann man hier wohl nicht ansetzen. Die Anwendungsmöglichkeiten dieses Lexems sind nicht leicht zu erfassen. Es kann über den NP-Bereich hinaus verwendet werden. Ubrigens vermag als Anzeiger einer "Information nebenbei" zu diversen anderen Funktionslexemen zu treten (keineswegs aber zu allen; dazu später mehr):

Dort starben, übrigens genauer: verhungerten und verdursteten, viele Menschen.

Die Bemden waren rot, ubrigens genauer weinrot und ziegelrot, gewesen.

*Das Adjektiv kongruiert, übrigens m.a.W.: stimmt überein, mit dem Nomen.

44 Diese Gradpartikelvariante ist von zweifelhafter Akzeptabilität. 
38) unter anderem (SADVB, GP?), TEILWEISES AUFZAHLEN, HERVORHEBUNG EINER TEILMENGE.

Viele Singvögel, u.a. Amsel, Drossel, Fink und Star, sangen eifrig.

Viele Singvögel sangen eifrig, u.a. (sangen) Amsel, Drossel, Fink und Star (eifrig).

Manche Gewässer waren sehr verschmutzt, u.a. (?die Binnenseen) waren (die Binnenseen) verschmutzt. 45

Dieser Ausdruck ist auch bzgl. anderer Kategorien anwendbar:

Die Hemden waren rot - u.a. weinrot und ziegelrot - gewesen.

Zubereitet, u.a. gewürzt und gegrillt, wurden die würste dort.

39) vielmehr (Konjunktionaladverb), PRAZISIERUNG, KORREKTUR.

Lassen die Leser, vielmehr: eine bedeutsame Minderheit von Lesern, sich nicht mehr beliebig idiotisieren?

Zusătzliche verwendungen (man beachte, daß die Einbettung in den ersten Satz noch erfolgen kann):

Wo steckt der Autor dieses Films, wenn nicht in diesem Kommentar? Oder vielmehr: in der stimme, die diesen Kommentar spricht. 46

40) vor allem (Sadv, GP), HERVORHEBUNG EINER TEILMENGE.

Manche Haiarten, vor allem die Blauhaie, werden gefürchtet.

Manche Haiarten werden gefürchtet, vor allem (die Blauhaie) werden (die Blaubaie) gefürchtet. 47

Zusătzliche Verwendungsmöglichkeiten:

Zubereitet - vor allem gekocht und gegrillt - wurden die Speisen dort.

Diese, vor allem deine und seine, Hemden starren vor Dreck.

Die vor dem Krieg großen deutschsprachigen Minderheiten in Ostmitteleuro-

pa - vor allem in Polen und in der Tschechoslowakei - wurden (...) dezimiert. ${ }^{48}$

41) vornehmlich (GP), siehe vor allem.

42) weniger (MOD), siehe nicht so sehr.

45 Die Gradpartikellesart ist nicht voll akzeptabel.

46 Beide Beispiele zu vielmehr aus: ortner (1985: 249).

47 Hier scheint sowohl die Gradpartikelvariante als auch die Satzadverb-Lesart (vgl. Werden die Blauhaie gefürchtet? $\mathrm{Ja} / \mathrm{Vor}$ allem/ Nein.) annehmbar.

48 Aus: Glück/Sauer (1990: 95). 
43) wie auch (Vergleichs- und Gradpartikel), BEISPIELNENNUNG. vgl. z.B. (...) besaß Drosophila gegenüber den Mendelschen Erbsensträuchern (wie auch gegenüber allen anderen denkbaren Versuchstieren) erhebliche Vorzüge: (...). 49

44) zudem (Konjadv), vgl. ferner, überdies.

45) zumindest (Konjadv; ordinative Angabe), ANGABE DER TEILMENGE, DIE IN JEDEM FALL BETROFFEN IST.

Die Negation, zumindest die logische [Negation], ist eine typische Ad-Formel $(\ldots) .50$

Die Negation ist eine typische Ad-Formel (...). Zumindest ist die logische Negation eine typische Ad-Formel (...).

Der zumindest-Zusatz kann auch in den nachstehenden Beispielen gesetzt werden: Zubereitet, zumindest gekocht und gegrillt, wurden die speisen dort.

(?) Die Jacken waren verschmutzt (zumindest fleckig und speckig) gewesen.

Was kann man über die Funktion derartiger Ronstruktionen, zumal im zusammenhang mit der Möglichkeit, sie überwiegend als verkürzte Satzverbindungen darzustellen, aussagen? Ich greife einige Beispiel heraus:

(12.3) Die Vögel werden dort gejagt; genauer: Die Singvógel werden dort gejagt.

Die Vogel werden dort gejagt, genauer: die singvógel.

Die Vögel, genauer: die Singvögel, werden dort gejagt.

In (12.3) wird der erste Sachverhalt korrigiert (prăzisiert). Er war nicht genau genug. "Mental" abgespeichert wird schlieblich die Information 'Die Singvögel werden dort gejagt'. Aus dieser Reprăsentation ergeben sich dann Konsequenzen für die Fortsetzung des "Textes"; beispielsweise kann jetzt kein Satz folgen wie Die Jagd der Raubvógel erfreut sich dort groBer Beliebtheit (semantische Inkompatibilitát). Trotz der Einschaltungsmarkierung wirkt der zuSATz in die laufende Rede hinein, ohne aber direkt auf den Satz (dessen Wahrheitswert) Einflus zu nehmen.

(12.4) Die Vogel werden dort gejagt. Vor allem die singvogel werden dort gejagt.

Die Vógel werden dort gejagt, vor allem die singvógel.

Die Vögel, vor allem die Singvögel, werden dort gejagt.

Hier wird die Information des ersten Satzes ergånzt. Abgespeichert wird "mental" so etwas wie 'Die vögel werden dort gejagt, wobei die Subklasse der Singvögel davon besonders betroffen ist'.

49 Aus: Asimor (1986: 101).

50 Aus: Schwarze/hunderlich (1985), S. 209. In eckigen Rammern füge ich die getilgte Ronstituente hinzu. 
(12.5) Die Vögel werden dort gejagt, überdies (werden dort) die Fledermäuse (gejagt).

Die Vögel, überdies die Fledermäuse, werden dort gejagt.

Abgespeichert wird hier so etwas wie 'Vögel und auch Fledermäuse werden dort gejagt'. Es wird also die Information über die Jagd der Vögel ergänzt um die Zusatzinformation, daB auch Fledermäuse gejagt werden.

Nun möchte ich eine Zusammenordnung der Funktionen versuchen, die natürlich nur vorläufigen Wert besitzt. (Eine weitere Obersicht findet man in Kap. 16.) Spätere Arbeiten sollten sich mit der weiteren internen Aufgliederung der ZUSATZE auseinandersetzen. Ich ziehe folgende ordnungskriterien heran:

- semantisch-funktionale Gesichtspunkte (Mengenrelationen etc.),

- die Hinzufügbarkeit von und zwar,

- die Hinzufügbarkeit sonstiger eindeutiger Funktionslexeme (ich meine),

- Umformungsbeziehungen (Kopulasătze bei 5.).

Die semantischen Beziehungen $z$ wischen Basis und Zusatz erlauben sowohl relativ klare Abtrennungen zwischen den "GroBklassen" (z.B. zwischen referenzklärenden und spezifizierenden Zusätzen) als auch zwischen den (wichtigsten) subtypen innerhalb der Grobklassen. Die Hinzufügbarkeit von und zwar als erstes, einleitendes Funktionslexem, auch in Verbindung mit weiteren darauffolgenden, gestattet insbesondere die Abtrennung der spezifizierenden Zusătze von den nichtspezifizierenden.

Genannt wird beim jeweiligen ZUSATZ ein Benennungsvorschlag. Zudem gebe ich an, ob die Funktionslexeme obligatorisch oder fakultativ stehen und ob der betreffende Zusatz Einschränkungen hinsichtlich nichtnominaler Bezugselemente aufweist.

Zusatz während der Uberarbeitung: Die generelle fakultative Hinzufügbarkeit von und zwar als Kriterium, das zwischen spezifizierenden und nichtspezifizierenden (sowie Alternantiven nennenden) Zusätzen trennen soll, scheint mir inzwischen nicht mehr so überzeugend. (Man vgl. z.B. die "unerwünschte" Inakzeptabilităt bei folgendem präzisierenden Zusatz: Lassen die Leser, (*und $z$ war) vielmehr: eine bedeutsame Minderheit von Lesern, sich nicht mehr beliebig idiotisieren? Vgl. sub verbo vielmehr, Funktionslexem Nr. 39.) Der Bereich der (nicht)spezifizierenden und Alternativen nennenden zusatze (im folgenden 1. mit 3.) ist möglicherweise nicht mehr aufgrund ausdrucksseitiger (syntaktischer) Merkmale, sondern nur noch aufgrund inhaltlich-funktionaler Kriterien zu gliedern. Zudem scheint mir auch die Binnengliederung der nichtspezifizierenden und der beiden Alternativen nennenden Zusătze nicht mehr völlig stichhaltig zu sein. Ich bleibe dennoch für die Veröffentlichung bei der ursprünglichen Klassifikation, da das Gesamtergebnis der Arbeit durch eine (darstellungstechnisch doch erhebliche) Umarbeitung im Kern unberührt bliebe. 
1. ZUSATZE mit und $z$ War. SPEZIFIZIERENDE ZUSATZE (SPEZ). 51

Basis: Gesamtmenge, Zusatz: Teilmenge/Elemente

1.1. BEISPIELNENNUNG. BEISPIELNENNENDER ZUSATZ (BZ).

Funktionslexeme fakultativ.

(12.6) Manche Haie, [und zwar] beispielsweise/ z.B./ etwa/ konkret:/ namentlich die Hammer- und Blauhaie, werden dort gefürchtet.

1.2. EXPLIZITER TEILMENGENEINSCHLUSS. MENGENEINSCHLIESSENDER ZUSATZ (MZ). Funktionslexeme obligatorisch, sonst Ubergang zur PRAZISIERUNG oder inakzeptables syntagma. 52

(12.7) Viele Haie, [und zwar] einschlieBlich die Hammerhaie, werden dort gefürchtet.

1.3. PRAZISIERUNG. PRAZISIERENDER ZUSATZ (PZ).

Funktionslexeme nicht obligatorisch.

(12.8) Viele Haiarten, [und zwar] deutlicher/genauer/ hauptsächlich/ insbesondere/ konkret:/ korrekt(er):/ (zu)meist/ namentlich/ präziser/vor allem/ vielmehr:/ vornehmlich Hammer- und Blauhaie, sind gefürchtet.

1.4. HERVORHEBUNG. HERVORHEBENDER ZUSATZ (HZ).

Funktionslexeme obligatorisch, sonst PRAZISIERUNGslesart oder Inakzeptabilität. Teilweise bestehen Einschränkungen bei anderen Kategorien als NP.

(12.9) Viele Vogelarten, [und zwar] besonders/ hauptsächlich/in der Hauptsache/ in erster Linie/ insbesondere/ (zu)meist/ überwiegend/ vor allem/ vornehmlich Milane und Bussarde, wurden gejagt.

51 Diese Großklasse könnte man nach Aitmann (1981) auch als "nachtragende Zusätze" resp. "Nachträge" bezeichnen. Altmann (1981: 71) selbst spricht auch von einer Spezifikationsrelation zwischen Bezugselement und Nachtrag.

Gerhild von Schuch verdanke ich den Hinweis auf die problematischen Implikationen des Ausdrucks "spezifizierend (er Zusatz)". "Spezifikation" ist im Rahmen der Kategorialgrammatik die Bezeichnung für die semantische Modifikation eines Operandums (z.B. Nomen) durch einen Operator (z.B. Genitivattribut; Resultat: Mengensubsektion). In Kap. 6 Abs. 21 stelle ich jedoch in Abrede, daß zwischen Basis und Zusatz (Appositiv) eine derartige Relation besteht. Leider ist mir bislang kein alternativer Ausdruck eingefallen. Vielleicht kann eine spätere Arbeit über das Thema damit aufwarten.

52 Viele Haie, die Hamerhaie, werden dort gejagt würde als PRAZISImRUNG aufgefaßt. *Viele Haie, [einschließlich] der Hammerhai, werden dort gejagt ist ungrammatisch. 
2. ZUSATZE ohne und Zwar. NICHTSPEZIFIZIERENDE ZUSATZE (NSPEZ). 53

A) Basis: Menge, Zusatz: Obernenge

2.1. DEPRAZISIERUNG. VERALLGEMEINERNDER ZUSATZ (VZ).

Funktionslexeme nicht obligatorisch.

(12.10) Die Griechen, [*und zwar] allgemeiner/ überhaupt die Südländer, kochen gern mit Knoblauch.

B) Basis: Menge, Zusatz: Teilnenge

2.2. GERINGERE BETROFFENHEIT. ZURUCKNEHMENDER ZUSATZ (ZZ).

Funktionslexeme obligatorisch, sonst PRAZISIERUNG. Z.T. erhebliche Einschrănkungen bei der Anwendung auBerhalb des NP-Bereichs.

(12.11) Viele Vogelarten, [*und zwar] nicht so sehr/weniger die Bussarde, wurden gejagt.

2.3. IN JEDEM FALL/ MINDESTENS BETROFFEN. EINSCHRANKENDER ZUSATZ (EZ). Funktionslexeme obligatorisch, sonst PRAZISIERUNG.

(12.12) Viele Vogelarten, [*und zwar] zumindest die Bussarde, wurden gejagt.

Ein Raubtier, [*und zwar] zumindest ein gefährliches Tier, schlich da berum.

2.4. AUSSCHLUSS. AUSSCHLIESSENDER ZUSATZ (AZ).

Funktionslexeme obligatorisch. Teilweise Einschrănkungen bei Anwendung auf andere Rategorien als NP.

(12.13) Viele Vogelarten, [*und zwar] ausgenommen/ ausgeschlossen die Bussarde, wurden gejagt.

C) Basis: Menge, Zusatz: andere Menge ${ }^{54}$

2.5. ZUSATZLICHE NENNUNG. HINZUFOGENDER ZUSATZ (HFZ).

Funktionslexeme obligatorisch, sonst KORREKTURlesart.

(12.14) Die Vógel, [*und zwarl ferner/ obendrein/ uberdies die Fledermäuse, erhoben sich in die Lüfte.

53 In Abgrenzung zu den spezifizierenden Zusătzen fasse ich die folgenden Subtypen als "nichtspezifizierende Zusătze" zusammen. In Detail malssen allerdings Unstimnigkeiten in Kauf genoumen werden. Der einschränkende Zusatz (2.3.) ist wohl spezifizierend und auch beim ausschließenden Zusatz könnte man dies erwägen. Tatsache ist, daß und zwar nicht hinzugefügt merden kann. Die Gruppe der nichtspezifizierenden Zusătze ist in späteren Untersuchungen genauer unter die Lupe zu nehmen, da sie die inhamogenste darstellt.

54 is existiert in diesen Fălen eine gemeinsame Obermenge, z.B. 'alle fliegenden Tiere' o.ă. 
A) Basis: Ausdruck, Zusatz: alternativer (neist extensionsgleicher) Ausdruck

3.1. ANDERSSAGEN. UMFORMULIERENDER ZUSATZ (UZ).

Funktionslexeme fakultativ.

(12.15) Ihre Existenzformen, anders gesagt/m.a.W. ihre Erscheinungsformen, hat Pia untersucht.

(12.16) Die Ledigen, anders gesagt/ d.h./ ja:/ m.a.N./ (?)nochmals: die Unverheirateten, stellen Pia nach.

B) Basis: Henge, Zusatz: alternative Menge 55

3.2. ALTERNATIVE. ALTERNATIVE ANGEBENDER ZUSATZ (ALZ).

Funktionslexeme (ubberwiegend?) obligatorisch, sonst Hăhe zur RORRERTUR (evtl. auch DEPRAZISIERUNG).

(12.17a) Es wurde Vein, bzw. Milch, getrunken.

(12.17b) Es wurde Nein, bzw./ d.h./ nein(:) Alkohol, getrunken.

Diese Funktion berührt sich evtl. mit der Funktion RORRERTUR.

Als besondere Fålle im Rahmen der Menge-Element-Beziehungen verbleiben die identische WIEDERHOLUNG (Basis: Menge, Zusatz: identische renge; fakultative Funktionslexeme sind z.B. ja, nochmals; s. 12.18a) und die totale RORRERTUR (Basis: "durchgestrichene" Menge $X$, Zusatz: "neugesetzte" Menge $Y$; typisches Funktionslexem ist nein, möglich ist zudem vielmehr; s. 12.18b). In (12.18c) läst sich ein Obergang von der VIEDERHOLUIG zur PRXzISIERUNG beobachten.

(12.18a) Dann kam ein Hai (ja: ein Hai!) angeschwommen.

(12.18b) Dann kam ein Hai, nein: ein Hering(,) angeschwommen.

(12.18c) Dann kam ein Hai ((ja:) ein großer Hai!) angeschwommen.

4. REFERENZKLARUNG. REFERENZKLXRENDE ZUSXTZE (REFZ).

Basis: referenzundeutlicher Ausdruck, Zusatz: referenzklărender Ausdruck

Funktionslexeme fakultativ; das typische Funktionslexem ist ich meine. Teilweise Restriktionen bei Anwendung auf nichtnominale Rategorien.

(12.19) Ich habe sie (, d.h./ ich meine die Haie,) kommen sehen (, die Haie).

Dort - d.h./ ich meine an der bezeichneten Bahnstation - angelangt, erlaubte ich mir, (...). 56

55 Auch hier gibt es eine gemeinsame Obermenge, z.B. 'Getrănke' etc.

56 Beispiel leicht verăndert ubernammen van Hindelang (1980: 127). 
5. NEBENPRADIKATION (ATTRIBUTIVES PRADIKATIV). 57 PRADIZIERENDER ZUSATZ (PRAZ).

Basis: Menge, Zusatz: Bigenschaft, Klasse, Identifikat, Lokalisierung

Funktionslexeme fakultativ. Nur bei NPn anwendbar (evtl. auch als "Satzapposition" möglich, also Basis = Satz, Zusatz = NP).

(12.20) Der Kultusminister von Baden-Württemberg, bekanntlich/ übrigens der Präsident des VFB Stuttgart (Identifikat), beschloB diverse Einsparungen

(12.21) Herr Hirsch, bekanntlich/ übrigens ein Beamter (Klasse)/ faul und arbeitsscheu (Eigenschaft), bereitete den Büroschlaf vor.

(12.22) Das Rathaus, bekanntlich/ übrigens alt und verfallen (Eigenschaft)/ am Marktplatz (Lokalisierung), wird renoviert.

Wenn man nun prüft, welche Lexeme sich nicht nur mit NP-Zusätzen, sondern (bei Bewahrung der Konstruktionssemantik) auch mit AdjP- und PP-Zusätzen (s. Prototyp) verbinden lassen, dann ergibt sich eine klare verteilung:

Nur bekanntlich und übrigens weisen diese Eigenschaft auf und nur diese beiden Lexeme leiten zusătze ein, bei denen Kopulasätze mit sein als Paraphrasen möglich sind und die Annahme von Satzverdoppelungen ausscheidet: 58

(12.23) Die Haie, (sie sind) bekanntlich/ übrigens die Ráuber der Meere/ wild und gefräig, werden gefürchtet.

(12.24) Die Großkatzen, übrigens/ bekanntlich/*also/*z.B./*genauer/ ^m.a.W./ *nämlich/ *präziser/ * und zwar wild und gefräBig, werden gefürchtet.

(12.25) Einige Bauten, übrigens/ (?) bekanntlich/ *also/ *z.B./ *m.a.W./ *nämlich/ *präziser am Marktplatz des Orts, sind renovierungsbedürftig.

Alle anderen Zusätze können nicht mithilfe von Kopulasätzen, die in den Gastsatz eingeschaltet sind, paraphrasiert werden. Eher plausibel sind bei ihnen Satzkopien, bei denen identische Verben, Verbalkomplexe, Verbalphrasen und sonstige Satzglieder bis auf das zusatzlexem und den Kern des zusatzes (die Zusatz-NP etc.; kursiv + unterstrichen) getilgt werden (betrifft die nur fett markierten Elemente):

(12.26) Viele Haie werden sehr gefürchtet. Vor allem die Hammerhaie werden sehr gefürchtet.

Viele Haie werden sehr gefürchtet, vor allem die Hammerhaie.

Viele Haie, vor allem die Hammerhaie, werden sehr gefurchtet.

ob der referenzklärende Zusatz, m.a.W. der von Altmann (1981) als Rechtsversetzung bezeichnete Herausstellungstyp, über Satzkopie plus Tilgungen plus

57 Dieser treffende Terminus stammt von Löbel (1986a: 114).

58 torigens kann als Standardzusatz zum prädizierenden Zusatz (Appositionsprototypen) gelten, bekanntlich ist in seiner Hinzufügbarkeit relativ begrenzt. 
Einschaltung in den Gastsatz erklärbar ist, bleibt vorerst offen. Nach der Tilgung wird das ubriggebliebene in den Satz, i.d.R. unmittelbar nach seiner Bezugskonstituente, eingeschaltet. So könnte jedenfalls eine "transformationelle" Erklärung aussehen. Details habe ich hier nicht geprüft, nicht immer kann die Erklärung via Satzkopie befriedigen. In Betracht zu ziehen ist in manchen Fälen möglicherweise ein zugrundeliegender weiterführender Nebensatz, der reduziert und dann eingeschaltet wird:

(12.27) ? Die Vögel waren friedlich, wobei die Raubvögel ausgenommen waren.

Die Vögel waren friedlich, die Raubvögel ausgenommen.

Die Vögel, die Raubvögel ausgenommen, waren friedlich.

Es făllt auf, daß außer beim Prototypen bei keinem weiteren zusatz die hinzugesetzte NP auch von niedrigerer X-Bar-stufe als N3 (die bei allen statthaft ist; 12.29) sein kann (12.28, 12.30). Der Grund dafür ist augenscheinlich, daB bei bekanntlich und übrigens in der Funktion 'Gleichsetzung/ Einordnung in eine Klasse/ Zuordnung einer Eigenschaft' die Zusatz-NP in Beziehung zum Prădikativ eines Kopulasatzes gesetzt werden kann und dieses Satzglied darf ja als N3, N2, N1 oder N0 erscheinen - bei den übrigen Mustern ist eine solche Beziehung nicht plausibel:

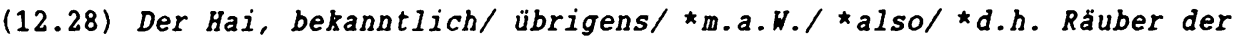
Meere (N2), wird gefürchtet.

(12.29) Der Hai, bekanntlich/ übrigens/ m.a.W./ also/ d.h. der Ráuber der Meere (N3), wird gefürchtet.

(12.30) Manche Vögel, *ausgenommen/*besonders/*genauer/ *ich meine/ *und zwar/ *unter anderem/ *zumindest Bussard (NO), werden dort gejagt. Es sind also bereits erhebliche Unterschiede zu registrieren $z w i s c h e n$ den $Z u-$ sätzen, die von bekanntlich/ übrigens begleitet werden, und allen weiteren!

Wenn man sich die Frage stellt, inwieweit die lexematisch eingeleiteten NPZusătze kombinierbar sind mit übrigens, das prădizierende Zusătze einleiten kann, oder mit dem spezifizierende zusätze einleitenden und zwar, dann ergibt sich die nachstehende Verteilung:

- übrigens: Die Griechen, übrigens/ «und zwar allgemeiner die Südländer, Die Vögel, übrigens/ *und zwar (ausgenommen) die Raubvögel (ausgenommen),

Die Haie, übrigens/ *und zwar bekanntlich die Räuber der Meere, Die Löwen, übrigens/ *und zwar ferner die Tiger,

Die Großkatzen, übrigens/ *und zwar nicht so sehr die Löwen, Die Vögel, (?) übrigens/ *und zwar obendrein/ überdies die Fledermáuse,

Die Vögel, übrigens/ ? und zwar unter anderem Fink und Star, Ubrigens, nicht aber und zwar, kann den zusătzen mit den Funktionen AUSGESCHLOSSENE (TEIL)MENGE, DEPRAZISIERUNG, ZUSATZLICH ZU NENNENDE MENGE, WENIGER BETROFFENE (TEIL)MENGE SOWIE BESONDERS BETROFFENE (TEIL)MENGE vorangestellt werden. Das bedeutet, daB die genannten Funktionen angeschlossen werden, wobei 
übrigens die Beilăufigkeit (Nebensächlichkeit, Nichtnotwendigkeit) des Zusatzes anzeigt und natürlich nicht, daß hier prädizierende Zusătze vorliegen.

- und zwar: Viele Haie, und zwar/ ?übrigens beispielsweise/ z.B. Ratzen- und Walhai,

Ein VW 1200, (?) und zwar/ ??übrigens etwa ein Modell von 1952, Die Großkatzen, und zwar/ *übrigens genauer die Löwen,

Manche Vogel, und $z$ war/ ??übrigens namentlich Finken und stare, Manche Vögel, und zwar/ *übrigens präziser Stare und Finken, Manche Vögel, ?und $z$ war/ *übrigens vielmehr stare und Finken, Und zwar, nicht jedoch übrigens, ist den Funktionen BEISPIEL und PRAZISIERUNG hinzufügbar. Und zwar zeigt (evtl. in Verbindung mit noch prăziseren Funktionslexemen) an, daß eine Spezifizierung erfolgt. Beim wohl eher Korrekturen ankündigenden vielmehr ist die Akzeptabilität von und zwar fraglich.

- beides: Die Großkatzen, und zwar/ ubrigens besonders die Tiger,

Viele Haie, und zwar/ (?) übrigens einschlieblich des Walhais, Manche Haie, und zwar/ übrigens hauptsächlich die Hammerhaie, Manche Haie, und zwar/ übrigens überwiegend Blau- und Tigerhaie, Manche Vögel, und zwar/ ubrigens vor allem die Milane, Beide Ausdrücke sind bei den Funktionen MENGENEINSCHLUSS sowie BESONDERE BETROFFENHEIT EINER (TEIL) MENGE möglich.

- weder-noch: Die Großkatzen, *und zwar/ *ubrigens also Tiger, Lowen etc., desgleichen: anders gesagt; bzw.; d.h.; ich meine; ja; m.a.W.; nămlich; nein; oder besser; so auch; und zwar; ubrigens; vielmehr; zumindest. Dies betrifft die zusätze mit den Funktionen ANDERSSAGEN, ALTERNATIVE, AUFZXRLUNG, KORREKTUR, IN JEDEM FALL BETROFFENE TEILMENGE, REFERENZVERDEUTLICHUNG, NEBENINFORMATION Und NIEDERHOLUNG.

Die Kombinationsrestriktionen sind vornehmlich bedingt durch den Konflikt nicht bzw. kaum miteinander vereinbarer Funktionen. Es ist z.B. nicht möglich, eine PRAZISIERUNG (prảziser/genauer) wie eine BEILAUY'IGE INFORMATION einzuleiten (ubrigens), da die "Relevanzwerte" einander widersprăchen. Allerdings dürften manche Beschrănkungen auch syntaktischer Natur sein (hier gibt es noch einiges zu erforschen), z.B. die Nichtkombinierbarkeit von übrigens und so auch, weil Kompatibilită hier vermutlich keine Rolle spielt; auch als (hervorhebende) Nennung eines Elements (evtl. als Beispiel) ist nämlich durchaus anwendbar. In den entsprechenden Satzdoppelungen zeigen sich die syntaktischen Rombinationsrestriktionen ebenfalls:

(12.31) Manche Săugetiere, übrigens auch/ *so auch der Wal, (...). Manche Săugetiere erreichen enorme GröBen. Ubrigens erreicht auch der Wal enorme Groben.

* Manche Säugetiere erreichen enorme Größen. Obrigens erreicht so auch der Wal enorme Größen. 
Lexematisch einleitbare Zusătze sind z.T. (in Grenzen) miteinander kombinierbar, jedoch müssen einige Regeln beachtet werden. Keinesfalls ist die Kombinierbarkeit in der gleichen Weise so "frei" wie beim prădizierenden Zusatz (vgl. Kap. 6 Abs. 18). Die Regularitäten können hier nur angedeutet werden, da eine umfassende Beispielmenge zu untersuchen wăre, um sie genau zu formulieren. Dies muß leider unterbleiben.

Die Kombination ist als durch Kommata gegliederte Abfolge ofters recht annehmbar. Konjunktorsyndese ist kaum akzeptabel (12.32-12.34). Der Vorzug der Asyndese dürfte darin liegen, daB die semantischen Operationen (Beispiele nennen; Mengenausschlus etc.) nacheinander ablaufen können: zuerst zwischen Basis und erstem Zusatz, dann zwischen dem daraus resultierenden "Produkt" und dem zweiten Zusatz usf. Die Konjunktorsyndese behindert diese "Schritt-fürSchritt"-Folge. Letztlich führen allerdings erst (hier nicht mŏgliche) ausgiebigere Analysen zu deutlichen Regeln.

(12.32) ^Die Großkatzen, also Löwen, Tiger und Panther und m.a.K. die geschmeidigen Jäger, schwărmten wieder aus.

Die Großkatzen, also Löwen, Tiger und Panther, m.a.W. die geschmeidigen Jäger, schwärmten wieder aus.

(12.33) ?Die meisten Haie, einschlieBlich des Walhais und ausgenommen den Blaubai, sind nicht sehr gefährlich.

(?) Die meisten Haie, einschließlich des Walhais, ausgenommen den Blauhai, sind nicht sehr gefährlich.

(12.34) ?Etliche Vogelarten, z.B. die Amsel und vor allem (aber) der Sperling, werden dort gejagt.

(?) Etliche Vogelarten, z.B. die Amsel, vor allem (aber) der Sperling, werden dort gejagt.

Umformulierende Zusătze (und AUFZAHLEN bzw. BEISPIELNENNUNG) sind in engen Grenzen mit sich selbst kombinierbar; i.d.R. konnen der "Basis" maximal zwei Zusătze folgen - weitere Zusătze "überladen" (pragmatisch, evtl. auch semantisch) das Muster "NP + Zusătze". (12.35b) zeigt zudem einen schönen Beleg für die Kombination von ALNZ und SPEZ, also die Kombination auch unterschiedlicher Subklassen:

(12.35a) Die Großkatzen, also Löwen, Tiger und Panther, m.a.W. die geschmeidigen Jăger, (??d.h. unsere eleganten Riesenmiezen,) schwarmten wieder aus.

(12.35b) Und ich hab ihn auch so verstanden, daB er meinte Texte des A11tags, also Dokumente, die nicht literarisch sind, zum Beispiel Reden, politische Reden, Werbetexte, (...). 59

Zusătze, die mit ihrer Basis in einer Element-Menge-Relation stehen, sind gemăs den semantischen (und pragmatischen) Interpretationsregeln für Mengen und

59 Aus: Rath (1979: 195). 
Teilmengen sowie Mengenausschlüsse kombinierbar, wobei (pragmatisch bedingt) wiederum wohl nicht mehr als zwei Zusătze auftreten dürften:

(12.36) Die meisten Haie, einschlieBlich des Walhais, (?? zudem der Katzenhai,l ausgenommen den Blauhai, sind nicht sehr gefährlich. ?? Die meisten Haie, ausgenommen den Blauhai, einschließlich des Walbais, sind nicht sehr gefährlich.

(12.37) Viele Vögel, besonders die Milane, weniger die Bussarde, (??überdies die Fledermáuse,) werden dort gejagt.

??Viele Vögel, weniger die Bussarde, besonders die Milane, werden dort gejagt.

(12.38) Die meisten Haie, ausgenommen den Blauhai, aber einschließlich des Nalhais, sind nicht sehr gefährlich

Viele Vögel, weniger die Bussarde, dafür aber besonders die Milane, werden dort gejagt.

Die akzeptableren Abfolgen sind diejenigen, bei denen zunăchst die besonders betroffene oder explizit eingeschlossene (Teil)Menge genannt und anschließend eine Menge ausgeschlossen oder als weniger betroffen bezeichnet wird. Dennoch kann man durch Hinzufügung von "adversativen" Lexemen (aber; zudem evt1. intensivierende wie dafür) auch in den umgekehrten Fällen völlige Akzeptabilität erreichen.

Die Kombination von verallgemeinerndem Zusatz mit hervorgehobenen Teilmengen ist mäBig bis kaum akzeptabel:

(12.39) ??Die Raubvögel, vor allem die Milane, allgemeiner die Vögel, werden dort gejagt.

(12.40) ? Die Raubvögel, allgemeiner die Vögel, vor allem die Milane, werden dort gejagt.

Die Kombinationsregeln harren noch ihrer gründichen Erarbeitung. Welche Abfolgen statthaft sind, wenn die einzelnen zusatze semantisch kompatibel sind, erfăhrt der Leser in Kap. 15.

Jetzt möchte ich genauer auf die die stellungseigenschaften der verschiedenen einleitbaren Zusătze eingehen (zum prädizierenden Zusatz vgl. Kap. 6, 11). Zunăchst zum Typ bekanntlich/ übrigens die Kónige des Urwalds; die Testsätze:

(12.41) Die Lowen (I) merden (II) heute (III) vam Aussterben (IV) bedroht, (V). daB (I) die Löwen (II) heute (III) vam Aussterben (IV) bedroht merden, (V).

In allen nachfolgenden Stellungstests geht es um die Konstellation mit der Basis in Vorfeld (resp. im Nebensatz als erstes Stellungsglied) und dem Zusatz an den mit den römischen Ziffern markierten Stellen (zu weiteren Stellungsuntersuchungen s. Kap. 15).

Vorab eine generelle Vorbemerkung: Die Beurteilung der Akzeptabilität einer Stellungsoption ist im Folgenden nicht immer leicht. Nicht selten schwankte der Verf. Das Hauptproblem besteht darin, eine deutliche Trennung durchzufuhren zwischen den Funktionsmustern (wohl mit einer gewissen Grammatikalisie- 
rung) und "parole"-Erscheinungen wie z.B. die "spontane Einschiebung einer Information, ganz gleich, an welcher Position des produzierten Syntagmas man angelangt ist". Gerade hier klaffen u.U. die Regeln bzw. Gebrauchsbedingungen, die in geschriebener bzw. gesprochener Sprache wirksam sind, auseinander, so daB einheitliche Aussagen ohnedies eine gewisse Idealisierung darstellen. Daher kann das Nachstehende nur insofern gerechtfertigt werden, als sich wenigstens der Tendenz nach Unterschiede aufweisen lassen - und dies ist m.E. ein Ergebnis, mit dem man im weiteren verlauf argumentieren darf. Trotzdem sind die Resultate mit Vorsicht zu genieBen.

(I) bzw. (II) im Verbend-Satz ist die unmarkierte Normalposition; unmöglich ist (I) im Verbend-Satz. (III) im Verbend-Satz sowie (II) und (III) im Verbzweit-Satz muten m.E. sehr zweifelhaft an, aber vielleicht sind diese optionen nicht ganz unmöglich. Im Einzelfall war die Bewertung äußerst schwierig, der Verf. gehört zu den Deutschsprechern, denen derartige Zusătze im Mittelfeld (bei Distanzstellung zum Bezugselement) generell "verboten" vorkommen; trotzdem erschienen nach lăngerem "Abschmecken" viele Versionen nicht völlig ablehnbar, ja manchmal sogar (einigermaßen) passabel, was eine Konzession v.a. bzgl. gesprochener Varianten sein mag ("nachgeschaltete Information, beinahe egal, wo sie einem einfällt").

Ausgeschlossen ist in jedem Fall die Position (IV); diese Position unmittelbar vor dem Klammerschluß läßt sogar Einschübe (z.B. Schaltsätze, vgl. Kap. 11) nur ungern $z u$. Je unselbstăndiger (d.i. weniger satzformatig) Einschaltungen werden, je enger sie als Zusatz an ihre Basen assoziiert sind, desto eingeschränkter wird ihre positionelle Freiheit und desto unwahrscheinlicher ihre stellung in der "Nische (IV)". Position (V) klingt bei etlichen Typen sehr schlecht, bei wenigen passabel bis gut (zu einigen Gründen hierfür s. Kap. 6).

Um die anderen Beispiele zu prüfen, müssen möglichst ähnliche sătze konstruiert werden (den entsprechenden Verbend-Satz kann man sich denken, er erscheint nicht eigens). In den obigen Rahmen passen aber z.B.

allgemeiner die Graßkatzen, / Die Großkatzen, also die Lömen und Tiger,/ , anders gesagt die Könige der Tiere, / Die Graßkatzen, ausgenommen die Löwen,/ , beispielsweise die Löwen,/ , besonders die Löwen,/ die Löwen, bzw. die Krokodile,/ , d.h. die Könige der Tiere,/ Die Großkatzen, einschließlich die Lömen,/ , ferner die Krokodile,/ Die Groskatzen, genaver Löwen und Tiger,/ , hauptsächlich die Löwen,/ Manche Großkatzen, ich meine Löwen und Tiger,/ Die Löwen (ja: die Löwen!)/Viele Großkatzen, kankret: Löwen und Tiger./Viele Graßkatzen, m.a.W. Lömen und Tiger./ , namentlich Lömen und Tiger,/ , nämlich Löwen und Tiger,/ die Löwen, nein: die Tiger,/ , nicht so sehr die Lömen,/ die Löwen, nochmals: die Lömen,/ , abendrein die Krakodile,/ oder besser viele Graßwildarten,/ , präziser die Löwen und Tiger,/, so auch der Löwe,/ und zwar/ überwiegend Löwen und Tiger, / die Lömen, übrigens die Könige der Tiere,/ die Grabkatzen, unter anderem/ vor allem/ zumindest die Lömen/ die Löwen, vielmehr: die Mehrheit der Löwen. 
bekanntlich/ übrigens

allgemeiner

also

anders gesagt

ausgenommen

beispielsweise

besonders

bzw.

d.h.

einschließlich

ferner

genauer

hauptsăchlich

ich meine

ja

konkret

m.a.w.

namentlich

nănlich

nein

nicht so sehr
+ ?? ?? - ??

- + ?? - ??

+ ?? - - -

- + ?? - -

+ ?? ?? - +

- + ? - +

+ ?? ?? - ??

$-\quad+$ ?? - ??

+ ? ? - +

- + ? - +

+ ?? ?? -

$-\quad+$ ? $-\quad+$

+ ? ? - +

- + ? - +

+ ?? ?? - ??

- + ? - ??

+ ?? ?? - ??

- + ?? - ??

+ ? ? - +

- + ? -+

+ ? ? - +

- + ? -

+ ?? ?? - +

$-\quad+$ ? -+

+ ? ? - +

- + ? -

+ ?? ?? - +

- + ? -4

+ ? ? ? ? +

$-+(+)$ ? +

+ ?? ?? - +

- + ?? -+

+ ?? ?? - ??

$-\quad+$ ?? - ??

+ ? ? $-(+)$

$-\quad+?-(+)$

+ ?? ?? $-(+)$

$-\quad+$ ? $-(+)$

+ ?? ?? - +

$-+(+)-+$

+ ? $\quad$ ? 


\begin{tabular}{|c|c|c|c|c|c|}
\hline & $I$ & II & III & IV & $\mathrm{v}$ \\
\hline & - & + & $?$ & - & $?$ \\
\hline \multirow[t]{2}{*}{ nochmals } & + & $? ?$ & - & - & $(+)$ \\
\hline & - & + & $?$ & - & $(+)$ \\
\hline \multirow[t]{2}{*}{ obendrein/ überdies } & + & ?? & $?$ & - & + \\
\hline & - & + & $?$ & - & + \\
\hline \multirow[t]{2}{*}{ oder besser } & + & $?$ & $?$ & - & $(+)$ \\
\hline & - & + & $?$ & - & $(+)$ \\
\hline \multirow[t]{2}{*}{ prăziser } & + & $?$ & $?$ & - & $(+)$ \\
\hline & - & + & $?$ & - & $(+)$ \\
\hline \multirow{2}{*}{ so auch } & + & $?$ & $?$ & - & $(+)$ \\
\hline & - & + & $?$ & - & $(t)$ \\
\hline \multirow[t]{2}{*}{ und zwar } & + & $?$ & $?$ & - & + \\
\hline & - & + & $?$ & - & + \\
\hline \multirow[t]{2}{*}{ überwiegend } & + & ?? & $?$ & - & $?$ \\
\hline & - & + & $?$ & - & ? \\
\hline \multirow[t]{2}{*}{ unter anderem } & + & $?$ & $?$ & - & + \\
\hline & - & + & $?$ & - & + \\
\hline \multirow[t]{2}{*}{ vielmehr } & + & ?? & $? ?$ & - & + \\
\hline & - & + & $? ?$ & - & + \\
\hline \multirow[t]{2}{*}{ vor allem } & + & $?$ & $?$ & - & + \\
\hline & - & + & $?$ & - & + \\
\hline \multirow[t]{2}{*}{ zumindest } & + & $?$ & $?$ & - & $(t)$ \\
\hline & - & + & $?$ & - & $(t)$ \\
\hline
\end{tabular}

Die Urteile deuten auf eine zweiteilung hin: (a) Weitgehendst auf die unmittelbare Adjazenz rechts der Basis im Vorfeld beschrankt sind der prådizierende Zusatz, aus den Bereich der nichtspezifizierenden zusătze der verallgeneinernde Zusatz, der umformulierende und der Alternativen nennende zusatz. (b) Die spezifizierenden, die meisten nichtspezifizierenden und die referenzklárenden Zusătze (aber auch Korrektur und Wiederholung) zeigen akzeptable stellungen rechts des Satzes; ${ }^{60}$ doch bleiben auch deren Mittelfeldstellungen zweifelhaft. Die Uneinheitlichkeit innerhaib der nichtspezifizierenden zusatze (verallgemeinernder vs. zurücknehmender, einschrănkender, ausschließender, hinzufügender Zusatz) deutet auf eine mógliche Inhomogenitat meiner Klassifikation hin.

Jetzt soll gepruft werden, ob die von Funktionslexemen begleiteten zusătze innerhalb oder außerhalb des Bezugsbereichs von Negationen, Gradpartikeln und Fragen liegen. Es geht um den Nachweis, das in Ggs. zum prådizierenden Zusatz,

60 D.h. in referenzklärender (doch auch in unformulierender) Funtion scheint - il Gos. zu ich meine - nicht am rechten Satzrand plazierbar zu sein. 
der weder durch die Negationspartikel nicht oder Gradpartikeln noch durch Fragen affizierbar ist (vgl. Kap. 6), eine solche "Betroffenheit" bei anderen Zusatztypen u.U. durchaus besteht. Auf ausführliche Analysen zu den möglichen Gründen für Akzeptabilitätsminderungen muß leider verzichtet werden; sie bleiben ein Desiderat. (")" bedeutet 'daraus folgt'.)

(12.42) Viele Vógel, z.B. Milane und Bussarde, wurden dort nicht gejagt > die Milane und Bussarde wurden dort nicht gejagt.

(?) Nicht viele Vögel, z.B. die Milane und Bussarde, wurden dort gejagt, sondern viele Katzen > Nicht die Milane und Bussarde wurden dort gejagt, sondern viele Katzen.

(12.43) Viele Vögel, genauer/ einschließlich/ vor allem die singvögel, wurden nicht gejagt $>$ die Singvögel wurden nicht gejagt.

?? Nicht viele Vögel, genauer/ einschlieblich/ vor allem die singvögel, wurden dort gejagt, sondern viele Katzen

, ??Nicht die Singvögel wurden dort gejagt, sondern viele Katzen.

(12.44) Auch viele $V$ ö gel, einschlieblich/ vor allem/z.B. die Milane und Habichte, wurden dort gejagt

(?) Auch die Milane und Habichte wurden dort gejagt.

Nur/ Sogar viele $V$ o gel, einschlieslich/vor allem/z.B. Milane und Habichte, werden dort gejagt

> *Nur/ ??Sogar Milane und Habichte werden dort gejagt.

(12.45) Auch/ Nur/ Sogar viele $V$ ö gel, genauer die Raubvögel, werden dort gejagt > Auch/ Nur/ Sogar die Raubvógel werden dort gejagt.

(12.46) Ist es der Fall, daß viele Vögel, genauer die singvögel, gejagt werden? > Ist es der Fall, daB die singvógel dort gejagt werden?

(12.47) Verden viele Vögel, einschlieblich/ vor allem/z.B. die Singvögel, dort gejagt? , Werden die Singvogel gejagt?

Die spezifizierenden Zusătze (präzisierender/ hervorhebender/mengeneinschlieBender/ beispielnennender Zusatz) sind von einer nicht-kontrastierenden Trägersatznegatiori affizierbar. Die Verwendung einer kontrastierenden Negaticn wirkt allerdings kaum annehmbar (12.42, 12.43).

Die Anwendung von Gradpartikeln ist nur bei prăzisierenden zusătzen annehmbar, bei den übrigen drei Typen spezifizierender zusătze wirken die Folgerungen nur bei auch akzeptabel (12.44, 12.45). Fragen betreffen auch die Teilmengen, d.h. die Frage nach der Gesamtmenge impliziert die Frage nach der Teilmenge. Obwohl derartige ZUSATZE keine direkten Bestandteile des Satzes sind (sondern HOSPITANTEN), können bestimmte bereichsbildende (bereichsbezogene) Operationen diese betreffen. Dies hat natürlich semantische Gründe: nämlich die Mengen-Teilmengen-Semantik der Basis-Zusatz-Konstellationen.

Ein verallgemeinernder Zusatz kann von einer Satznegation und einer Frage betroffen sein. Bei Kontrastnegation entstehen jedoch inakzeptable syntagmen:

(12.48) Die Griechen, allgemeiner die Südländer, kochen nicht gern ) Die Sudlander kochen nicht gern. 
* Nicht die Griechen, allgemeiner die Südländer, kochen gern, sondern die Eskimos

> *Nicht die Südländer kochen gern, sondern die Eskimos.

(12.49) (?) Auch/ ?? Nur/ ? Sogar die Griechen, allgemeiner die Südländer, kochen nicht gern > (?) Auch/ ?? Nur/ ?Sogar die Südländer kochen gern.

(12.50) Kochen die Griechen, allgemeiner die Südländer, gern?

) Kochen die Südländer gern?

Die Negation erstreckt sich auf die Teilmenge (Griechen), nicht aber auf die hinzugesetzte Obermenge (Südländer). Bei angewendeten Gradpartikeln entstehen je nach der Semantik der einzelnen Gradpartikel unterschiedlich akzeptable Syntagmen; gerade hier müste man sehr ins Detail gehen, was nicht geschehen wird - somit verbleibt das Pauschalurteil, das Gradpartikeln nur sehr beschränkt anwendbar sind.

Bei dem zurücknehmenden Zusatz ist der Zusatz von der Negation weniger betroffen; insofern wirken die Schlüsse in (12.51) kaum akzeptabel:

(12.51) (?) Die Haie, die Sandhaie weniger, werden dort nicht gejagt > * Die Sandhaie werden dort nicht gejagt.

?? Nicht die Haie, die Sandhaie weniger, werden dort gejagt, sondern die Rochen

, ?? Nicht die Sandhaie werden dort gejagt, sondern die Rochen.

(12.52) Auch/ Nur/ Sogar die Haie, die Sandhaie weniger, werden dort gejagt , ? Auch/ *Nur/ ?? Sogar die Sandhaie werden dort gejagt.

(12.53) ? Werden die Haie, die Sandhaie weniger, dort gejagt?

> ?erden die Sandhaie dort gejagt?

? Ist es der Fall, dab die Haie, die Sandhaie weniger, dort gejagt werden?

Bei Gradpartikelanwendung ergeben sich wieder einmal unterschiedlich akzeptable Resultate, je nach Gradpartikelsemantik; von aucb abgesehen scheinen sie nicht sinnvoll anwendbar zu sein. Entscheidungsfragen wirken etwas merkwürdig, scheinen aber nicht inakzeptabel zu sein; man denke sich, das der sender wăhrend der Formulierung der Frage noch einfügen möchte, daß ihn bei der Frage weniger das Gejagtwerden der Sandhaie interessiere als vielmehr die Tatsache, ob Haie prinzipiell gejagt würden.

Beim Typ einschrănkender Zusatz wird der Zusatz von der Satznegation betroffen, nicht jedoch von der Kontrastnegation; diese ist gar nicht anwendbar, weil der AusschluB der Vogelarten keine Zusatzspezifikation dergestalt erlaubt, daB in jedem Fall die Milane in den Sachverhalt involviert waren:

(12.54) Einige Vogelarten, zumindest die Milane, werden dort nicht gejagt > Die Milane werden dort nicht gejagt.

* Nicht einige $V$ o gelarten, zumindest die Milane, werden dort gejagt, sondern einige Haiarten

> Nicht die Milane werden dort gejagt, sondern einige Haiarten. 
(12.55) Auch/ Nur/ Sogar einige Vogelarten, zumindest die Milane, werden dort gejagt , Auch/ ? Nur/ (?) Sogar die Milane werden dort gejagt.

(12.56) Werden einige Vogelarten, zumindest die Milane, dort gejagt?

> Werden die Milane dort gejagt?

Bei Gradpartikeln zeigen sich keine völligen Inakzeptabilităten. Die Frage betrifft ebenfalls den Zusatz.

Die Semantik des Ausnehmens verhindert, daß Negation und Gradpartikeln den Zusatz affizieren:

(12.57) Vogel, ausgenommen die Milane, werden dort nicht gejagt > * Die Milane werden dort nicht gejagt.

Nicht Vogel, ausgenommen die Milane, werden dort gejagt, sondern viele Haie

, * Nicht die Milane werden dort gejagt, sondern viele Haie.

(12.58) Auch/ Nur/ Sogar Vögel, ausgenommen die Milane, werden dort gejagt > *Auch/ *Nur/ * Sogar die Milane werden dort gejagt.

(12.59) Werden viele Vögel, ausgenommen die Milane, dort gejagt?

> * Werden die Milane dort gejagt?

Das Ausnehmen bewirkt z.B. bei der Negation, die ja (zuerst) uber die Gesamtmenge operiert, daß die Milane vom (Nicht) Involviertsein ausgenommen werden, so das kein Schlus dahingehend statthaft ist, das Milane nicht gejagt würden. Natürlich ist auch die zweite Frage nicht impliziert, wenn die Milane per $\mathrm{Zu}-$ satz aus der "in Frage stehenden" Menge ausgeschlossen werden.

Die Satznegation und die Frage betreffen einen hinzufügenden zusatz:

(12.60) Die Vögel, obendrein die Flederwăuse, werden nicht gejagt

> Die Fledermáuse werden nicht gejagt.

?? Nicht die Vögel, obendrein die Fledermăuse, werden gejagt, sondern die Schlangen

, ?? Nicht die Fledermáuse werden gejagt, sonderndie Schlangen.

(12.61) Auch/ ? Nur/ Sogar die Vögel, obendrein die Fledermäuse, werden dort gejagt , Auch' *Nur/ ?Sogar die Fledermäuse werden dort gejagt.

(12.62) Werden die Vögel, obendrein die Fledermäuse, dort gejagt?

> Verden die Fledermáuse dort gejagt?

Die Kontrastnegation ist allerdings nicht annehmbar. Bei den Gradpartikeln entstehen lediglich bei nur starke Einschrănkungen (bei sogar bin ich etwas unsicher).

Der umformulierende zusatz wird von allen bereichsbildenden Operationen affiziert:

(12.63) Ihre Existenzformen, m.a.W. ihre Erscheinungsformen, hat Pia nicht untersucht > Ihre Erscheinungsformen hat Pia nicht untersucht.

(12.64) Nicht ihre Existenzformen, m.a.W. ihre Erscheinungsformen, hat Pia untersucht, sondern ihre Zustandsformen > Nicht ihre Erscheinungsformen hat Pia untersucht, sondern ihre Zustandsformen. 
(12.65) Auch/ Nur/ Sogar ihre Existenzformen, m.a.W. ihre Erscheinungsformen, hat Pia untersucht

> Auch/ Nur/ Sogar ihre Erscheinungsformen hat Pia untersucht.

(12.66) Hat Pia ihre Existenzformen, m.a.W. ihre Erscheinungsformen, untersucht? / Hat Pia ihre Erscheinungsformen untersucht?

Der (verwandte) Alternativen angebende zusatz verhalt so ebenso:

(12.67) Nein, bzw. Milch, wurde nicht getrunken

) Milch wurde nicht getrunken.

Nicht Wein, bzw. Milch, wurde getrunken, sondern Bier

> Nicht Milch wurde getrunken, sondern Bier.

(12.68) Auch/ Nur/ Sogar Wein, bzw. Milch, wurde getrunken

> Auch/Nur/ Sogar Milch wurde getrunken.

(12.69) Nurde Nein, bzw. Milch, getrunken? Vurde Milch getrunken? Wie aufgrund der Korreferenz nicht anders zu erwarten war, sind die Zusătze des Typs referenzklărender zusatz von allen Operationen betroffen:

(12.70) Ich habe sie, (ich meine) die Haie, nicht gejagt

> Ich habe die Haie nicht gejagt.

Ich habe nicht sie, die Haie, gejagt, sondern die Rochen

> Ich habe nicht die Haie gejagt, sondern die Rochen.

(12.71) Auch/ Nur/ Sogar sie, die Haie, habe ich gejagt

> Auch/Nur/ Sogar die Haie habe ich gejagt.

(12.72) Habe ich sie, die Haie, gejagt? Habe ich die Haie gejagt? Die Sonderfälle Totalwiederholung und Totalkorrektur verhalten sich aufgrund ihrer gegenteiligen inhaltlichen Funktion verschieden. Bei der Wiederholung betreffen Folgerungen wegen der identischen Denotate immer sowohl Basis und Zusatz. Bei der Rorrektur ist der Zusatz immer betroffen, weil er statt des "durchgestrichenen", "außer kraft gesetzten" Basisausdrucks den "neugesetzten", "hinfort geltenden" Ausdruck darstellt.

(12.73) Pia kann Haie, nein: Heringe, nicht leiden

) Pia kann Heringe nicht leiden/ «Pia kann Haie nicht leiden.

(12.74) Nicht die Haie, nein: nicht die Heringe(,) will Pia erforschen, sondern die Rochen > Pia will nicht die Heringe erforschen.

(12.75) Auch/ Nur/ Sogar Haie, nein: Heringe(,) wollte Pia erforschen

> Pia wollte auch/ nur/ sogar Heringe erforschen.

(12.76) Wollte Pia Haie, nein: Heringe(,) erforschen? Wollte Pia Heringe erforschen?

Nun noch eine zusammenfassende obersicht, auf deren Vorlăufigkeit hinzuweisen ist. Eingehendere Analysen an einem umfangreicheren Beispielkorpus können diese "Tendenzangaben" - vor allem hinsichtlich der kontrastierenden Negation und im Gradpartikelbereich - evtl. noch etwas korrigieren (oder stützen): 
SatzNeg KNeg Auch Nur Sogar Frage

PRADIZIERENDER ZUSATZ

PRAZISIERENDER ZUSATZ

BEISPIELNENNENDER ZUSATZ

MENGENEINSCHLIESSENDER $U$.

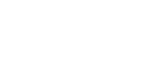

HERVORHEBENDER ZUSATZ

VERALLGEMEINERNDER ZUSATZ

ZURUCKNEHMENDER ZUSATZ

EINSCHRANRENDER ZUSATZ

AUSSCHLIESSENDER ZUSATZ

HINZUFOGENDER ZUSATZ

ALTERNATIVEN NENN. ZUSATZ

REFERENZKLARENDER ZUSATZ

WIEDERHOLUNG

KORREKTUR

Aus der Zusammenfassung ergibt sich, dab

(a) der prädizierende Zusatz ein deutlich "eigen-artiges" Verhalten an den Tag legt, indem er sich als von bereichsbildenden operatoren des Trăgersatzes unabhăngig zeigt.

(b) Alternativen nennende Zusătze, der referenzklärende Zusatz (der sich in dieser Hinsicht völlig konträr zum prädizierenden Zusatz, also zu unserem "Appositionsprototypen", verhält!) sowie Korrektur und Wiederholung allen operationen zugänglich sind. Mit Ausnahme der kontrastierenden Negation reagiert auch der prăzisierende Zusatz auf die durchgeführten Tests.

(c) die Gradpartikeln nur und sogar ausschlieblich bei der eben genannten "Gruppe" anwendbar sind, sonst aber kaum bis gar nicht; nur auch ist relativ unrestringiert in seiner Anwendbarkeit.

(d) die kontrastierende Negation auf nur wenige zusatztypen mit Funktionslexemen angewendet werden kann.

(e) der zurücknehmende und der ausschlieBende Zusatz aufgrund ihrer "ausnehmenden" Semantik von all jenen operationen relativ unbeeinfluBt bleiben.

Heitere Tests und Obersichten über gemeinsame bzw. verschiedene gramatische Eigenschaften der ZUSATZE - unter Berücksichtigung der in Kap. 13 behandelten RURZZUSATZE - finden sich im 15. und 16. Kapitel! 
In diesem Kapitel wird ein sprachliches Muster beschrieben, das meines Wissens bisher noch nie im Rahmen der "Appositionsdiskussion" behandelt wurde, obwohl es stark appositionsverdächtig anmutet, da es rechtsadjazent $z$ u einer nominalen Basis steht und als Einschaltung markiert ist. Ich nenne derartige Zusätze "Kurzzusätze" und verstehe darunter einen Typus, bei dem zu einem Nomen (aber unter Umständen auch zu Wortgruppen anderer Kategorie) ein i.d.R. rundgeklammerter zusatz unmittelbar rechts angefügt wird, der i.d.R. aus nur einem Ausdruck besteht (ein Nomen, Adjektiv, Verb etc.), also gewöhnlich unerweitert (keine Funktionslexeme!) erscheint: z.B. Willy Brandt (SPD). Kurzzusatze sind vornehmlich Erscheinungen der Schriftsprache, kommen allerdings auch in der gesprochenen Sprache vor:

(13.1) / wir haben heute helmut kohl p+ cdu p+ und willy brandt p+ spd p+zu gast $\mathrm{f}++/$.

(13.2) /harry hirsch $\mathrm{p}+$ vierundzwanzig $\mathrm{p}+$ ist der kommende mann $\mathrm{f}++/$. In der gesprochenen sprache zöge man wohl eher explizitere Ausdrücke (determinierte NPs, NPs mit Funktionslexemen, Schaltsătze) vor. ${ }^{1}$ Kurzzusătze sind textsortenspezifisch, sie treten beispielsweise in wissenschaftlichen und journalistischen Texten öfters auf; hingegen dürften sie z.B. in der Belletristik kaum zu finden sein.

In ihrer hăufigsten Realisierungsform (in der von mir angenommenen standardform) treten Kurzzusätze rundgeklammert unmittelbar rechtsadjazent zum Bezugsausdruck (eine Voll-NP) auf, wobei sie nicht durch Zusatzlexeme, Satzadverbien, Gradpartikeln etc. erweitert sind. Diese Zusătze verden i.d.R. nicht von Determinantien begleitet.

Dennoch sind in mehreren Fällen andere Optionen vorhanden: Man kann statt Rundklammern Kommata verwenden (13.3), evtl. Distanzstellungen vornehmen (13.4) und manchmal zusatzlexeme (13.5) und/oder Determinantien (13.6) hinzufügen. Die Frage ist, ob es sich dann immer noch um das gleiche Muster (die gleiche Funktion) handelt, oder ob Ubergänge in andere Typen/Funktionen vorliegen:

(13.3) Helmut Kohl (CDU)/, CDU, griff zur Sahnetorte.

(13.4) Die hat der Prachtbau (100 Millionen) viel gekostet (100 Millionen).

(13.5) James Bond ((uberzeugend:) Timothy Dalton) schlug wieder zu.

(13.6) Die Rechtschreibung (Orthographie/ die Orthographie) ist schwer. Schwyzer (1939: 39) setzt die Kurzzusatze, die er "Wortparenthesen" nennt, in Beziehung zu Satzparenthesen bzw. Schaltsătzen und erklărt sie (historisch)

1 Vgl. Schwyzer (1939: 39) zu den Beispielen er (Meyer)/ Isfahan (Heerlager): "(...) bei lautem Lesen wird man geneigt sein, in solchen Fällen das Schriftbild zu erklären, also z.B. 'er (nănich Meyer)' oder 'Isfahan (zu deutsch Heerlager)' zu lesen. 
als "abgekürzte Satzparenthesen". 2 Somit finden sich auch (sprachgeschichtliche) Hinweise auf eine ("transformationelle") Herleitung bzw. Umformungsbeziehung der Kurzzusätze.

Daß nicht alle die hier in Augenschein genommenen Typen die soeben hervorgehobenen Eigenschaften aufweisen, sei sogleich eingeräumt. Unter anderem liegt das daran, daß die Rundklammerung optional auch für Muster/Funktionen zur Verfügung steht, die nicht zu den Kurzzusătzen zu rechnen sind. 3

In gesprochenem Deutsch, in dem Rurzzusätze gerne erweitert resp. "ausbuchstabiert" werden, wenngleich sie nicht erweitert werden müssen, sind sie mit dem Einschaltungsmuster (pausenumhüllt, progrediente Intonation, "zurückgenommene" Artikulation) versehen - vie alle Hospitanten eines Trăgersatzes.

Was die Kombinierbarkeit mit weiteren Zusătzen betrifft, so stehen die rundgeklammerten Kurzzusătze i.d.R. vor allen übrigen, beispielsweise vor prädizierenden (13.7) und vor spezifizierenden oder umformulierenden zusătzen (13.8) sowie vor Schaltsatzen mit phorischem NP-Bezug (13.9):

(13.7) Harry Hirsch (CDU), (übrigens) Koch aus Passion, (*CDU) trat ein. Immanuel Kant (1724-1804), (bekanntlich) wohnhaft in Königsberg, (*1724-1804) war ein bedeutender Philosoph.

(13.8) Manche Pachyderme (Dickhäuter), besonders die Elefanten, (*Dickhäuter) sind interessant.

James Bond (Sean Connery), anders gesagt 007, (*Sean Connery) löste diesen Fall.

(13.9) Die RNS (Ribonukleinsäure), sie erfüllt wichtige Aufgaben, (*Ribonukleinsäure) wurde geschädigt.

Kurzzusătze teilen mit den prädizierenden zusätzen die Eigenschaft, im Ggs . z.B. zu Attributen von Operationen wie NEGATION (13.10) und GRADPARTIKELANYENDUNG (13.11) oder einer Gliedteilfrage (13.12) aus dem Trägersatz heraus völlig unberührt zu bleiben. Daher ist der Kurzzusatz wohl nicht im Bereich attributiver Funktionen $z u$ verorten:

(13.10) *Nicht Harry Birsch (CDU), sondern Harry Hirsch (SFDi spricht jetzt.

Nicht Harry Hirsch von der CDU, sondern Harry Hirsch von der SPD spricht jetzt.

2 Schwyzer (1939: 39):

"Und bei näherem Zusehen zeigt sich, daß diese angeblichen Wortparenthesen abgekürzte Satzparenthesen sind. Noch heute kann man in solchen Fällen 'd.h.' oder 'd.i.' usw. brauchen; das Griechische und Lateinische hatten gar keine andere Möglichkeit (...). (...) Erst späte griechische Wörterbuicher begnügten sich, das zu erklärende Wort van der Frklärung durch eine Interpunktion zu trennen".

3 Man vgl. z.B. die Kurzzusătze (F13.1) und die rumdgeklammerten prädizierenden Zusătze (F13.2):

(M13.1) Harry Hirsch (SPD) dementierte, das otto Schily (DIE GROWDN) einen Parteiwechsel plane.

(F13.2) Harry Birsch (seit gestern SPD-Sprecher) dementierte, daB Otto Schily (wohl prominentestes Crimen-Aitglied) einen Parteiwechsel plane. 
(13.11) *Sogar Baldur Buhmann (45) kam, nicht nur Baldur Buhmann (39). Sogar der 45-jährige Baldur Buhmann kam, nicht nur der 39-jährige Baldur Buhmann.

(13.12) Welche RNS wird durch Röntgenstrahlen geschädigt? *Die Ribonukleinsäure.

Die "klassischen" Konstituententests wie z.B. Topikalisierung und Substitution (Pro-Form-Bildung) sowie das Verhalten bei Linksversetzung deuten dagegen auf eine Konstituente hin:

(13.13) Harry Hirsch (CDU) bält eine wirre Rede. Harry Hirsch (CDU)/ Er ist ein wirrer Redner.

Harry Hirsch (CDU), der ist ein wirrer Redner.

Beim Versuch, die Konstruktion gesamt zu erfragen, kann der zusatz manchmal (13.16; 13.18), jedoch nicht immer mit völliger Akzeptabilităt hinzugefügt werden; z.T. ist man geneigt, bei der Antwort ausführlichere (erweiterte) $\mathrm{Zu}-$ sätze oder attributive spezifikatoren $z u$ verwenden:

(13.14) Wer machte einen Witz? Helmut Kohl ? (CDU)/ von der CDU. /wer machte einen witz st+/. ?/helmut kohl p+cdu $\mathrm{ft+} /$. / helmut kohl von der cdu $\mathrm{f}++$ /.

(13.15) Wer legt sich bei Geschwindigkeitsbegrenzungen quer? Der ADAC ? 8,8 Millionen Mitglieder// mit seinen 8,8 Millionen Mitgliedern.

(13.16) Wer tagt wieder? Die Spitzenorganisation der Filmwirtschaft (Spio).

(13.17) Wo kennt sich Pia aus? In der Orthographie ? (Rechtschreibung)/ , also in der Rechtschreibung/, zu deutsch Rechtschreibung.

(13.18) Wer wird ihre Frage beantworten? Frau Müller (Apparat 19)/ am Apparat 19.

Wie auch die anderen ZUSATZE (zu den Argumenten s. Kap. 6, 7.A, 12) sehe ich die Kurzzusătze als "phrasenassoziierte Hospitanten" an, die weder im herkömmlichen Sinne Konstituenten dieser Phrase sind noch als deren attributive Modifikatoren fungieren. Ein Dependenzverhältnis des Zusatzes bzgl. der Basis nehme ich nicht an.

KURZZUSATZE zeigen eine ungemein vielgestaltige Ausprăgung. Ich versuche wieder durch eine (allerdings recht unkontrollierte und unsystematische) Beispielsammlung einen Ausgangspunkt für eine ordnung und Klassifikation zu schaffen. Die folgende Sammlung kann keinen Anspruch auf eine annähernde Vollständigkeit erheben. Dennoch dürfte sie ausreichen, um eine Sortierung und Klassifizierung dieser Formen zu erreichen, von der aus weitere, nicht behandelte Typen eingeordnet werden können.

Bei den einzelnen Beispielen erscheinen Paraphrasen (Umschreibungen), welche die jeweilige Funktion der Konstruktion verdeutlichen sollen. GewiB ist die Menge der Beispiele in sich heterogen, so daB einige ausgesondert werden müssen als Beispiele anderer Zusătze. Dies wird im Laufe dieses Kapitels geschehen. 
Die Auflistung der Beispiele ist so aufgebaut, daß zuerst die "prototypischen" Kurzzusätze erscheinen und dann mit ansteigender Beispielnummer die kritischen Fälle folgen (Ab (a9) aufpassen!). Es wird auch angegeben, inwieweit alternative Markierungen neben der Rundklammer (Komma, Gedankenstrich) anwendbar sind sowie ggf. mögliche Erweiterungen des Kurzzusatzes. Zudem werden Stellungsmöglichkeiten des Zusatzes vermerkt: Möglichkeiten der Distanzstellung, der relativen Position zu Attributen oder der Basis nachgestellten Adpositionen, Gradpartikeln und Konjunktionaladverbien. Außerdem wird vermerkt, wenn Kongruenzerscheinungen auftreten. Falls die Option besteht, einen Subtyp auch bei nichtsubstantivischen Basen zu verwenden, wird darauf hingewiesen.

Die Bewertungen der Akzeptabilitäten (vor allem der Markierungsoptionen Komma und Gedankenstrich) fielen mir relativ schwer. Hinzu kommt, daB man im Bereich der (Kurz)Zusätze vielleicht weniger von (Nicht-)Grammatikalität der Strukturen sprechen sollte als von (Un-)Gebräuchlichkeit. ${ }^{4}$ Mit Sicherheit lohnte sich eine spatere gründliche Durcharbeitung dieser Problematik.

(a1) Die DUDEN-Grammatik (1984)/ *, 1984,/ *- 1984 - wurde gründlich überarbeitet.

- Die DUDEN-Grammatik, die 1984 erschien,/ - sie erschien 1984 - (...).

- ANGABE DES ERSCHEINUNGS-, VEROFFENTLICHUNGS- etc. JAHRES

- Erweiterung: keine.

- Stellung: Unmittelbar rechtsadjazent (hinfort: "UR"), evtl. mit Attributen dazwischen. ${ }^{5}$ Distanzstellung ergäbe eine Lesart als eingeschaltete Temporalangabe. Stellung vor Postposition, Konjunktionaladverb, Gradpartikel.

- Kongruenz: keine.

(13.19) Pia hat die DUDEN-Grammatik vermutlich (1984) gelesen.

(13.20) Die DUDEN-Grammatik (1984) jedoch/ sogar (??1984) enthält das.

(13.21) Der DUDEN-Grammatik (1984) zuliebe (??1984) geht er meilenwei

(a2) Aalen (Württemberg)/ ?, Württemberg, / *- Württemberg - ist eine Kreisstadt.

- Aalen in Württemberg; Aalen - es liegt in Württemberg - (...).

- LOKALISIERUNG

- Erweiterung: keine.

4 während man Nicht-Grammatikalităt auf Verletzungen grammatischer Regeln (z.B. Mißachtung von Rektions- oder Valenzbedingungen) beziehen kann, sind Ungebräuchlichkeiten eher auf Pragmatik bzw. Stilistik zu beziehen. Da ich keine befriedigenden Vorschläge zu den verschiedenen Inakzeptabilitätsquellen kenne, milssen die vagen Explikationen hier ausreichen.

5 Vgl.: Die DuDJN-Grammatik (1984) aus Mannheim ((?) 1984) hat sie nicht. 
- Stellung: UR. Kaum nach Postposition, Gradpartikel, Textkonnektor.

- Kongruenz: keine.

(13.22) Er hat Aalen gestern (*Württemberg) besucht (* Württemberg).

(13.23) Aalen (Württemberg) jedoch/ sogar (??Württemberg) hat das.

(13.24) Um Aalens (Württemberg) willen (?Württemberg) soll das geschehen.

(a3) Bundeskanzler Helmut Kohl (CDU)/, CDU,/ ?- CDU - machte einen Hitz. Dann kam Mick Jagger (Rolling Stones)/ *, Rolling Stones,/ ? - Rolling Stones - auf die Bühne.

- Helmut Kohl von der CDU/ - er gehört der CDU an - (...).

(...) Mick Jagger von den Rolling Stones/ - er gehört den Rolling Stones an $-(\ldots)$.

- ANGABE DER ZUGEHORIGKEIT ZU EINER SOZIALEN GRUPPE (PARTEI, BAND etC.)

- Erweiterung: Selten erweitert. Satzadverbien, Gradpartikeln und freie Adverbiale wohl ungebräuchlich. 6

- Stellung: UR; Attribute treten kaum dazwischen. Distanz unmöglich. Möglicherweise nach Postpositionen etc.

- Kongruenz: keine.

(13.25) Man hat Helmut Kohl gestern (*CDU) gehört ( $\left.{ }^{\star} C D U\right)$.

(13.26) Helmut Kohl (CDU) jedoch/ sogar (?CDU) wollte baden gehen ( $\left.{ }^{*} C D U\right)$.

(13.27) Helmut Kohl (CDU) zuliebe (? CDU) gingen sie baden (*CDU).

(a4) Immanuel Kant (1724-1804)/ ?, 1724-1804,/ ?- 1724-1804 - war ein bedeutender Philosoph.

- Immanuel Kant - er lebte von 1724 bis 1804 - (...).

- ANGABE DER LEBENSZEIT EINES INDIVIDUUMS ${ }^{7}$

- Erweiterung: i.d.R. keine; evtl. Satzadverbien (vermutlich 1724-1804).

- Stellung: UR, evtl. mit Attributen dazwischen. 8 Distanzstellung kann bewirken, daB der Zusatz als eingeschaltete Angabe gelesen wird. Position nach Postposition usw. unklar.

- Kongruenz: keine.

(13.28) Damals hat Kant gerne (*1724-1804) unterrichtet (*1724-1804).

(13.29) Immanuel Kant (1724-1804) sogar/ jedoch (?1724-1804) wuBte das.

(13.30) Immanuel Kant (1724-1804) zuliebe (?1724-1804) geschah das.

6 Vgl.: ??Helmut Kohl (seit langem bekanntlich CDU) (...) hält eine Rede.

7 Dieses Muster ist möglicherweise allgemeiner zu fassen als ANGABE ENTES ZETTRAuMS, man vgl.

z.B. Pippins Regierungszeit (751-768), der Dreißigjährige Krieg (1618-1648) usw.

8 Vgl. z.B. Immanuel Kant (1724-1804) aus Königsberg ((?)1724-1804) war (1724-1804 T2MP!) ein bedeutender Philosoph. 
(a5) Danach entschied eine hauchdünne Mehrheit (178 zu 174)/ ??, 178 zu 174,/ ?- 178 zu 174 - zugunsten der kreisrunden sitzordnung (...).9

- (..) eine hauchdünne Mehrheit von 178 zu 174 (Stimmen)/ - sie betrug $178 \mathrm{zu}$ 174 (stimmen) - (...).

- ANGABE EINES ZAHLENVERHALTNISSES

- Erweiterung: eher selten erweitert. Wohl keine Satzadverbien, Gradpartikeln, freie Adverbiale; evtl. übrigens.

- Stellung: Attribute und andere Satzteile können dazwischentreten; jedoch führt Distanzstellung im Mittelfeld zu einer Interpretation als eingeschaltete Angabe (13.31). Nach Postposition, Gradpartikel, Textkonnektor möglich.

- Kongruenz: keine.

(13.31) Danach entschied eine hauchdünne Mehrheit überraschend (178 zu 174) zugunsten der kreisrunden Sitzordnung (178 zu 174).

(13.32) Eine hauchdünne Mehrheit (178 zu 174) jedoch/ sogar (178 zu 174) wurde erzielt (178 zu 174).

(13.33) Einer hauchdünnen Mehrheit (178 zu 174) zuliebe (178 zu 174) tat man das.

(a6) Frau Müller (Apparat 19)/ , Apparat 19,/ ?- Apparat 19 - wird ihre Frage beantworten. 10

- Frau Müller am Apparat 19/ - sie hat den Apparat 19/ sie ist am Apparat 19 erreichbar - (...).

- "LOKALISIERUNG".

- Erweiterung: normalerweise unerweitert. Vielleicht Satzadverbien und Adverbiale (vermutlich/ heute Apparat 19).

- Stellung: UR, evtl. mit Attributen dazwischen. Distanz (am rechten Satzende) evtl. möglich. Wahrscheinlich auch nach Postposition, Gradpartikel, Textkonnektor möglich.

- Kongruenz: keine.

(13.34) Da ist Frau Müller heute (*Apparat 19) zuständig (?Apparat 19).

(13.35) Frau Müller (Apparat 19) jedoch/ sogar ((?) Apparat 19) weiß das.

(13.36) Um Frau Müller (Apparat 19) willen ((?) Apparat 19) geschah das.

9 AUs: DER SPIDCEZ 33/1988, S. 48.

10 Bin ähnliches Beispiel bei Raabe (1975: 326): Frau Grzyb, Apparat 90, enwartet ihren Anruf. Raabe (ebd.) schließt aus solchen Beispielen, "(...) daß es falsch ist, nur parenthetische Kopulasătze als Basis für NP-NP' Konstruktionen anzunehmen". 
(a7) Im vergangenen Herbst hat [er] seine Post-moderne Zentrale bezogen (194 Millionen Mark)/ *, 194 Millionen Mark/ *- 194 Millionen Mark. 11

- (..) Post-moderne Zentrale - sie kostete 100 Millionen Mark - (...).

- ANGABE DER KOSTEN VON ETWAS

- Erweiterung: Gradpartikeln, Satzadverbien, freie Adverbiale (nur/ wabrscheinlich/ bis heute 194 Mill. Mark).

- Stellung: Attribute können dazwischentreten, ebenso Satzglieder, wenn der Zusatz nach dem Klammerschlus erscheint. Position nach Postposition, Gradpartikel und Textkonnektor möglich.

- Kongruenz: keine.

(13.37) Dann hat er seine Post-moderne Zentrale im März (?194 Millionen Mark) beziehen können (194 Millionen Mark).

(13.38) Seine Post-moderne Zentrale (194 Millionen Mark) jedoch (194 Millionen Mark) war funktionell (194 Millionen Mark).

(13.39) Um seiner Post-modernen Zentrale (194 Millionen Mark) willen (194 Millionen Mark) geschah das.

(a8) Der ADAC (8,8 Millionen Mitglieder)/ ??, 8,8 Millionen Mitglieder,/ ?- 8,8 Millionen Mitglieder - erhöht seine Beiträge.

- Der ADAC - er hat 8,8 Millionen Mitglieder - (...).

- ANGABE DER ELEMENTENZAHL EINER MENGE (/ GRUPPE/ KLASSE etc.)

- Erweiterung: Satzadverbien fraglich (?vermutlich); freie Adverbiale (beute); möglicherweise bekanntlich/ übrigens.

- Stellung: Attribute können dazwischentreten; Distanzstellung unwahrscheinlich. Nach Postposition, Gradpartikel, Textkonnektor möglich.

- Kongruenz: keine.

(13.40) Dort hat der ADAC vor kurzem $(\star 8,8$ Millionen Mitglieder) gebaut (*8,8 Millionen Mitglieder).

(13.41) Der ADAC (8,8 Millionen Mitglieder) sogar (8,8 Millionen Mitglieder) stimmte zu.

(a9) [Er] hat [ihm] einen Forderungskatalog ("Nuclear Weapons Requirement study $88^{\prime \prime} /$ / *, "Nuclear Weapons Requirement Study 88 ",/ ??- "Nuclear Weapons Requirement Study 88" - für die Herbstsitzung vorgelegt. 12

- (..) einen Forderungskatalog namens "Nuclear (...) 88"/ - er heiBt "Nuclear (...) $88^{\prime \prime}-(.$.$) .$

- ANGABE DES NAMENS (EINES SCHRIFTSTOCKES etc.)

11 AUs: DER SPIDGET 33/1988, S. 47. EN von mir pronominalisiert.

12 Beispiel aus: DER SPIDGEr 33/1988, S. 14. Eigennamen wurden vom Verf. pronaminalisiert. 
- Erweiterung: keine. 13

- Stellung: Attribute können dazwischentreten; Distanzstellung u.U. am rechten Satzrand möglich. Kann nach Gradpartikeln, Postpositionen und Konjunktionaladverbien erscheinen.

(13.42) Er hat ihm einen Forderungskatalog gestern (?? "Nuclear (..) 88") vorgelegt ("Nuclear (...) 88").

(13.43) Einen Forderungskatalog ("Nuclear (...) 88") sogar/ jedoch ("Nuclear (...) 88") will er nicht.

(13.44) Um des Forderungskatalogs ("Nuclear (...) 88") willen ("Nuclear (...) 88") soll das geschehen.

- Kongruenz: Sofern die Funktion tatsächlich NAMENSANGABE ist, kongruiert der KURZZUSATZ nicht. Obergänge zum ALTERNATIVEN NENNENDEN ZUSATZ bestehen allerdings, wobei dann die Kongruenz realisiert werden muß. (Die in eckige Klammern gesetzten Elemente sollen die Funktionen verdeutlichen.)

(13.45) Das Ende des Romans ([er heiBt] "Der Name der Rose") ist spannend. Das Ende des Romans ([ m.a.W.] "Des Namens der Rose") ist spannend.

(13.46) Der Wirt des Wirtshauses ([es heiBt] "Das lachende Wildschwein") trägt einen Vollbart.

Der Wirt des Wirtshauses ([m.a.W.] "Des lachenden Wildschweins") trägt einen Vollbart.

(a10) James Bond (Sean Connery)/ ??, Sean Connery,/ ??- Sean Connery - verhaute wieder alle Bösewichte.

- James Bond - (er wird) dargestellt/ verkörpert von Sean Connery - (...).

- ANGABE DES SCHAUSPIELERS (EINER FIGUR AUS FILM, THEATER etc.)

- Erweiterung: Seltener erweitert. Evtl. freie Adverbiale (wieder einmal Sean Connery) ${ }^{14}$ evtl. übrigens.

- Stellung: UR; Attribute treten kaum dazwischen. Wohl keine Distanzstellung, aber evtl. nach Postposition, GP, Textkonnektor.

(13.47) Da hat James Bond gestern (*Sean Connery) jeden verhauen (*Sean Connery).

(13.48) James Bond (Sean Connery) jedoch/ sogar (? Sean Connery) kann das (* Sean Connery).

13 Lexeme, die auf ANDERSSAGZN hinweisen, können wohl kaum hinzugefügt werden: Er hat ihm einen Forderungskatalog (??d.h./ ??m.a.W./ ??anders gesagt 'Muklear Weapons Requirement Study 88") vorgelegt.

14 Folgendes Erweiterungsmuster findet sich öfters in Filmbesprechungen: (...) zusammen mit dem amerikanischen Bullengraßmaul Art Ridzik (witzig: James Belushi) wird (...) [aus: IN MavchDN Nr. 39/40, 29.9.-12.10.88, s. 9] oder (...) die junge Amerikanerin Johanna Crass (schnucklig wie immer: Rosanna Arquette) kennenlemen, (...) [aus: DN MrowiPN Nr. 37/38, 15.9.-28.9.88, S. 4] ; hierbei wird in Zusatz eine Meinung des Kritikers uber die schauspielerische Leistung eingebracht, etwa: 'witzig gespielt van James Belushi' oder 'schnucklig wie immer agiert Rosanna Arquette'. 
(13.49) James Bond (Sean Connery) zuliebe (? Sean Connery) geschah das.

- Rongruenz: als KURZZUSATZ keine. Ausnahme: Es liegt ANDERSSAGEN (d.h. ein Alternativen nennender Zusatz) vor:

(13.50) Die neuen Abenteuer James Bonds (m.a.W. Sean Connerys/*m.a.W. Sean Connery) führten ihn nach Jamaica.

(a11) Die Spitzenorganisation der Filmwirtschaft (Spio)/ ??, Spio,/ ??- Spio tagt wieder.

- Die Spitzenorganisation der Filmwirtschaft (kurz/ abgekürzt Spio) (...)/ Die Spitzenorganisation der Filmwirtschaft - ihre Abkürzung heiBt/ lautet "Spio" - (...).

- ANGABE EINER ABKURZUNG

- Erweiterung: normalerweise keine. Möglich wäre (kurz(:) Spio), möglicherweise auch: d.h.l m.a.W.l alsol anders gesagt + Artdf (dann: Alternativen nennender Zusatz).

- Stellung: UR mit evtl. Attributen dazwischen. Distanzstellung unwahrscheinlich. Position nach Postposition usw. unklar.

(13.51) Sicher tagt die Spitzenorganisation der Filmwirtschaft morgen (*Spio) wieder (??Spio).

(13.52) Die Spitzenorganisation der Filmwirtschaft (Spio) sogar/ jedoch (?Spio) tagt wieder.

(13.53) Der Spitzenorganisation der Filmwirtschaft (Spio) zuliebe (?Spio) geschah das.

Dieser KURZZUSATZ kann sich auch an nicht-nominale Basen assoziieren:

(13.54) Die neuhochdeutschen (nhd.) Wörter sind unten aufgelistet.

(13.55) Das ist zur Zeit (z.Z.) weder machbar noch wünschenswert.

- Kongruenz: keine. Bei Alternativen nennenden Zusätzen müssen Basis und Zusatz kongruieren, wobei ein Determinans obligatorisch ist; ohne Determinans ist Kongruenz inakzeptabel. Bei ANGABE EINER ABKURZUNG (also: KURZZUSATZ) tritt vermutlich kein Determinans auf.

(13.56) Die Sitzung des Zentralkomitees (ZK)/ ?? (ZKs)/ (des ZKs) dauerte lange.

(13.57) Die Sitzung des Zentralkomitees (kurz ZK)/*(ZKS)/ ? ((kurz:) des ZKs) dauerte lange.

Die Sitzung des Zentralkomitees * (m.a.W. ZK)/*(ZKs)/ ((m.a.W.) des ZKS) dauerte lange.

(a12) Es wurden 10 Dollar (18 DM)/ *, 18 DM,/ ??- 18 DM - entwendet.

- (..) 10 Dollar - das sind/ entspricht 18 DM - (...).

(...) 10 Dollar - in deutscher Währung: 18 DM - (..).

- ANGABE EINES NECHSELKURS (VERHALTNISS) ES 
- Erweiterung: (wenn ein Alternativen nennender Zusatz vorliegt) also/ ?anders gesagt/ d.i.l ?m.a.w. Evtl. übrigens.

- Stellung: UR; evtl. treten Attribute, Klammerschluß und weitere Satzteile dazwischen. Evt1. nach Postposition, Gradpartikel, Textkonnektor.

- Kongruenz: als Kurzzusatz keine. Bei Alternativen nennenden Zusätzen könnte sie auftreten, falls der Zusatz imstande ist, Flexionsaffixe anzunehmen; dies ist bei währungsangaben jedoch kaum der Fall.

(13.58) Vermutlich wurden 10 Dollar gestern (*18 DM) entwendet (18 DM).

(13.59) 10 Dollar (18 DM) sogar (18 DM) wurden entwendet.

(13.60) Um 10 Dollar (18 DM) willen (18 DM) tat er das.

(a13) Die RNS (Ribonukleinsăure)/ *, Ribonukleinsăure,/ ??- Ribonukleinsäure wird durch Röntgenstrahlen geschädigt.

- Die RNS - das ist die Abkürzung für Ribonukleinsäure - (...).

- ANGABE DES VOLLEN WORTLAUTS; EXPLIKATION EINES KORZELS

- Erweiterung: (nur bei ANDERSSAGEN; "O" bedeutet "Artikellosigkeit") d.i./ d.h./ also + Artdf (d.i.l also diel ?O Ribonukleinsäure).

- Stellung: UR. Distanz inakzeptabel. Kaum nach Postposition usf.

(13.61) Man hat die RNS (Ribonukleinsäure) schon wieder (*Ribonukleinsäure)

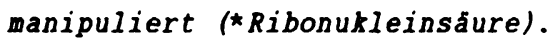

(13.62) Die RNS (Ribonukleinsäure) jedoch (??Ribonukleinsäure) war manipuliert.

(13.63) Um der RNS (Ribonukleinsäure) willen (??Ribonukleinsäure) tat man das.

Zusătzliche Verwendungsweisen sind vorstellbar; sie kommen allerdings seltener vor, da es üblich ist, ein Kürzel nach dem (ersten) Erscheinen des vollen Ausdrucks anzuführen und nicht umgekehrt.

(13.64) Die mhd. (mittelhochdeutschen) Verben kennt sie.

(13.65) Das ist z.Z. (zur Zeit) weder machbar noch wünschenswert.

- Kongruenz: nur falls ein Alternativen nennender zusatz vorliegt.

(13.66) Sie haben des verschrotteten PKHs (Personenkraftwagen) gedacht. Sie haben des verschrotteten PKWs (also des Personenkraftwagens/ *also Personenkraftwagen) gedacht.

(a14) In der Rechtschreibung (Orthographie)/ *, Orthographie, / (?)- Orthographie - kennt Pia sich aus. 15

- In der Rechtschreibung - fachsprachlich heiBt Rechtschreibung "Orthographie" - (...).

15 Man vgl. zur Markierung durch Gedankenstriche den Beleg (...) (in den Sprachatlanten bezeichnet man den Geltungsbereich eines jeden Wortes durch Linien - Isoglossen -) (...) aus: Wendt, Heinz F. 1987. Sprachen. Frankfurt/M., Fischer. S. 6. 
- ANGABE EINES FACHBEGRIFFS bZW. FREMDWORTES

- Erweiterung: (ANDERSSAGEN) d.i./ d.h./ also + Artdf.

- Stellung: UR. Keine Distanzoption. Nach Postp etc. sehr fragwürdig.

(13.67) Da wurde die Rechtschreibung (Orthographie) endlich (*Orthographie) reformiert (*Orthographie).

(13.68) Die Rechtschreibung (Orthographie) sogar (??Orthographie) beherrschte sie.

(13.69) Un der Rechtschreibung (Orthographie) willen (??Orthographie) geschah das.

Diese Funktion ist nicht allein auf den nominalen Bereich beschränkt:

(13.70) Die mehrdeutigen (ambigen) Sätze bereiten Probleme.

(13.71) Subjekt und Finitum stimmen überein (kongruieren) hinsichtlich Person und Numerus.

- Kongruenz: obligatorisch. Es handelt sich folglich um einen Alternativen nennenden Zusatz!

(13.72) Des Vervielfältigens (*Hektographieren/ Hektographierens) wurde sie nicht müde.

(a15) In der Orthographie (Rechtschreibung)/ *, Rechtschreibung,/ ??- Rechtschreibung - kennt Pia sich aus.

- In der Orthographie - das deutsche Wort für Orthographie ist/ heiBt/ lautet "Rechtschreibung" - (...).

- ANGABE DES DEUTSCHEN WORTES

- Erweiterung: (ANDERSSAGEN) d.h./ d.i.l also + Artdf.

- Stellung: UR. Ohne Distanzoption. Nach Postp etc. sehr fragwürdig.

(13.73) Man hat die Orthographie (Rechtschreibung) endlich (*Rechtschreibung) reformiert ( $*$ Rechtschreibung).

(13.74) Die Orthographie (Rechtschreibung) jedoch (??Rechtschreibung) beherrscht sie.

(13.75) Um der Orthographie (Rechtschreibung) willen (??Rechtschreibung) geschab das.

Diese Funktion ist nicht auf den nominalen Bereich beschrănkt:

(13.76) Beide kongruieren (stimmen uberein) binsichtlich Person und Numerus.

(13.77) Die ambigen (mehrdeutigen) Ausdrücke bereiten Probleme.

- Kongruenz: obligatorisch. Somit liegt ein Alternativen nennender zusatz vor.

(13.78) Des Hektographierens (*Vervielfäligen/Vervielfaltigens) wurde sie nicht müde. 
(a16) Markante Größen in diesem ProzeB (...) sind die Existenzformen (Erscheinungsformen)/ ?, Erscheinungsformen,/ ?- Erscheinungsformen - der Sprache $(\ldots) .16$

- (..) die Existenzformen - anders gesagt/ bzw. die Erscheinungsformen (...).

- ANGABE EINES ALTERNATIVEN ODER ZUSATZLICHEN AUSDRUCKS

- Erweiterung: (ALTERNATIVE, ANDERSSAGEN) bzw./ d.h./ d.i./ anders gesagt/ m.a.W.

- Stellung: UR, kaum nach Attributen; wohl nicht mit KlammerschluB dazwischen. 17 Nicht nach Postposition etc.

(13.79) Markante GröBen sind die Existenzformen schon immer (*Erscheinungsformen) gewesen (*Erscheinungsformen).

(13.80) Die Existenzformen (Erscheinungsformen) jedoch (??Erscheinungsformen) kennt sie.

(13.81) Um der Existenzformen (Erscheinungsformen) willen (??Erscheinungsformen) geschah das.

Wiederum kommen auch außerhalb des nominalen Bereichs Verwendungen vor:

(13.82) Die verschobenen (umgestellten) Satzteile verunklaren die Konstruktion.

(13.83) Sie schleuderten (warfen) ihre speere.

- Kongruenz: obligatorisch. Es handelt sich um einen Alternativen nennenden Zusatz.

(13.84) Die Ermittlung des Umfangs (*AusmaB/ AusmaBes) der Katastrophe.

(a17) Andere Völker (Italiener, Spanier, Griechen)/ , Italiener, Spanier, Griechen,/ - Italiener, Spanier, Griechen - wundern sich über die Deutschen.

- Andere Völker - es handelt sich um Italiener, Spanier, Griechen - (...). Andere Völker, nämlich/ und zwar (vor allem/z.B.) die Italiener, (...).

- AUFZAHLUNG (VON ELEMENTEN EINER KLASSE)

- Erweiterung: evtl. Satzadverbien, Gradpartikeln, Adverbiale; evtl. Modalpartikeln (?eben); hier ist wahrscheinlich vieles möglich: (AUFZAHLUNG, BEISPIEL, PRAZISIERUNG, BESONDERE BETROFFENHEIT) besonders/ in der Hauptsache/ insbesondere/ namentlich/námlich/präziser/so auch/ und zwar/ unter anderem/z.B. - Stellung: Attribute können dazwischentreten, evtl. auch ein KlammerschluB, vielleicht auch ein weiteres Satzglied. Nach Postposition, Gradpartikel, Text-. konnektor möglich.

16 Zitiert aus: W. Schmidt. 1984. Geschichte der deutschen Sprache. 5. Aufl. Berlin, S. 22.

17 Markante Groben sind die Existenzformen (Erscheinungsformen) der Sprache ? (Brscheinungsformen) gewesen * (Erscheinungsformen). 
(13.85) Da haben sich andere Völker schon lange (? Italiener, Spanier, Griechen) gewundert (Italiener, Spanier, Griechen).

(13.86) Andere Völker (Italiener, Spanier, Griechen) jedoch (Italiener, Spanier, Griechen) wundern sich.

(13.87) Anderen Völkern (Italiener, Spanier, Griechen) zuliebe (Italiener, Spanier, Griechen) tat man das.

Auch diese Funktion ist über den nominalen Bereich hinaus anzutreffen:

(13.88) Die Hemden waren bunt (rot, grün und blau) gewesen.

(13.89) Dort arbeiteten (hämmerten, sägten, bohrten) die Leute.

- Kongruenz: Bei reinem KURZZUSATZ (13.90; ohne Erweiterung um Determinantien oder Funktionslexeme) keine Kongruenz. Bei erweiterten Nomina erfolgt Kongruenz. Dann liegen spezifizierende (13.91; und zwar/ vor allem/ namentlich) oder Alternativen nennende Zusätze (13.92) vor. Namentlich macht eine unerklarliche Ausnahme, denn die Kongruenz ist optional.

(13.90) Sie musten sich der Haie (Hammerhai, Tigerhai, Blauhai/ *Hammerhais, Tigerhais, Blauhais) erwehren.

(13.91) * Sie muBten sich der Haie ((vor allem/ z.B.) der Hammerhai, der Tigerhai und der Blauhai) erwehren.

(13.92) Die Größe der Reptilien ((??d.h./ ??m.a.W./ namentlich) Schlange, Krokodil, Waran) steht in Brehms Tierleben.

Die GröBe der Reptilien ((d.h./ m.a.W./ namentlich) der Schlange, des Krokodils, des Warans) steht in Brehms Tierleben.

(a18) Baldur Buhmann (45)/, 45,/ ??- 45 - war für diese Rolle zu alt.

- Baldur Buhmann - er ist 45 (Jahre alt) - (...).

Die Möglichkeit der Kopulasatzparaphrase zeigt uns, daß hier gar kein KURZZUSATZ vorliegt, sondern ein Beispiel eines prädizierenden Zusatzes. Dies ist mithilfe eines Konjunktionstests zu erhärten:

(13.93) Reiner Barze1, 63, Ex-Bundestagspräsident, legt (...) Wert auf ein repräsentatives Büro in Bonn. 18

(13.94) Reiner Barzel, 63 und Ex-Bundestagspräsident/Ex-Bundestagspräsident, 63, legt (...).

\section{- ALTERSANGABE}

- Erweiterung: i.d.R. unerweitert. Möglich wären allerdings Gradpartikeln, Satzadverbien, evtl. freie Adverbiale ((vermutlich/auch/seit einem halben Jahr) bereits 45); übrigens.

- Stellung: UR, evtl. mit Attributen dazwischen. Distanzpositionen inakzeptabel. Im Ggs. zu typischen prădizierenden Zusătzen (vgl. Kap. 6) ist die stellung nach Postposition, Konjunktionaladverb etc. kaum annehmbar (s. 13.96/97).

- Kongruenz: keine.

18 Beispiel aus: DER SPIDGZ2, Nr. 34/1987, S. 174. 
(13.95) Da war Baldur Buhmann tatsächlich (*45) zu alt (??45).

(13.96) Baldur Buhmann (45) jedoch (?45) wuBte das.

(13.97) Baldur Buhmann (45) zuliebe (?45) geschah das.

Wie homogen bzw. heterogen sind die Beispiele? Ich versuche eine Zusammenstellung nach Maßgabe der Kriterien. ${ }^{19}$ zuerst stehen die KURZZUSATZE ohne mögliche Funktionsvariation (a1-a8), dann diejenigen, die variieren können; schlieblich stehen markiert durch Unterstreichung jene Zusătze, die wie KURZzUSATZE aussehen, eigentlich aber rundgeklammerte Varianten anderer ZUSATZ-Typen - vgl. Kap. 6, 12 - darstellen (a14-a18):

$$
\text { " - - nur N Brweiterung }
$$

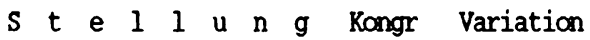 UR ATTR POSTP DIST}

\begin{tabular}{|c|c|c|c|c|c|c|c|c|c|c|}
\hline al & - & - & + & keine & + & $(+)$ & - & - & - & \\
\hline a2 & ? & - & + & keine & + & $?$ & ?? & - & - & \\
\hline a3 & ? & $?$ & + & wohl keine & + & $(-)$ & ? & - & - & \\
\hline a4 & ? & $?$ & - & $(\operatorname{Sadv})$ & + & + & $?$ & - & - & \\
\hline 25 & $? ?$ & $?$ & $?$ & (übrigens) & + & + & + & $(+)$ & - & \\
\hline a6 & + & $?$ & + & (Sadv, ADVB) & + & + & $(t)$ & $?$ & - & \\
\hline a7 & - & - & $?$ & GP, Sadv, ADVB & + & + & + & $(+)$ & - & \\
\hline a8 & ?? & $?$ & $?$ & ADVB, (Sadv, übrigens) & + & + & + & - & - & \\
\hline a9 & - & ?? & + & keine & + & + & + & $(+)$ & - & ANDERSSAGEN \\
\hline alo & ?? & ?? & + & (ADVB), übrigens & + & $(-)$ & ? & - & - & ANDERSSAGEN \\
\hline a11 & ?? & ?? & - & (kurz:/ d.h./ m.a.N.) & + & + & ? & $(-)$ & - & ANDERSSAGEN \\
\hline a12 & - & ?? & + & d.h. (m.a.W./ulbrigens) & + & + & + & $?$ & - & ANDERSSAGTN \\
\hline a13 & - & $? ?$ & $?$ & d.h. & + & - & $(-)$ & - & - & ANDERSSAGZN \\
\hline al4 & - & (?) & - & d.h. & + & - & $(-)$ & - & + & ANDPRSSAGEN \\
\hline a15 & - & $? ?$ & - & d.h. & + & - & $(-)$ & - & + & ANDORSSACNN \\
\hline al6 & $?$ & $?$ & - & bzw./ d.h./ m.a.W. & + & $(-)$ & $(-)$ & - & + & ANDRRSSAGPN \\
\hline al7 & + & + & - & $\begin{array}{l}\text { (Sadv, GP, ADVB), z.B. } \\
\text { besonders, nämlich }\end{array}$ & + & + & + & $?$ & $+/-$ & $\begin{array}{l}\text { PRAZISIMRLIG, } \\
\text { BIISPIII etc. }\end{array}$ \\
\hline a18 & + & ?? & + & $\begin{array}{l}\text { GP, Sadv, ADVB } \\
\text { ubrigens }\end{array}$ & + & $(t)$ & ? & - & - & $\frac{\text { PRXDIZTIRPNDR }}{\text { ZUSATZ }}$ \\
\hline
\end{tabular}

Die KURZZUSATZE und die davon zu trennenden ZUSATZE anderer Typen, die auch rundgeklammert erscheinen können, bieten für Folgeuntersuchungen noch ein weites Feld. Vorläufig darf festgestellt werden, daB es ein eigenständiges Konstruktionsmuster "prototypische KURZZUSATZE" ohne mögliche Funktionsvariation gibt (a1 mit a8) und das variierende rundgeklammerte zusătze existieren, deren Interpretation zwischen KURZZUSATZ und ALTERNATIVE NENNENDER ZUSATZ schwankt

19 Zeichenerklärung: ",," = 'Kommaabtrennung'; "- -" = 'Gedankenstrichabtrennung'; "nur $N^{\prime \prime}=$ 'nur bei nominalen Bezugselementen'; "UR" = 'umittelbar rechtsadjazent'; "ATTR" = 'Attribute dazwischen'; "POSTP" = 'zwischen Bezugselement und Zusatz können Postpositionen, GPn, Konjadv stehen'; "DIST" = 'Distanzstellung'; "Kongr" = 'Kongruenz'; "Variation" = 'mögliche Funktion außer Kurzzusatz bzw. tatsăchliche Funktion (rundgeklammerte Variante eines Zusatzes aus Kap. 12; markiert durch Unterstreichung) '. 
(a9 mit a13); dabei sind unbegleitete, determinantienlose Elemente als KURZZUSATZ, (vor allem um Funktionslexeme) erweiterte und kongruierende Einheiten als ALTERNATIVE NENNENDE ZUSATZE auffaBbar. SchlieBlich sind aus dem Bereich der KURZZUSATZE diejenigen Einheiten auszusondern, die eine rundgeklammerte Variante von ALTERNATIVE NENNENDEN, SPEZIFIZIERENDEN oder PRADIZIERENDEN ZUSATZEN darstellen (a14 mit a18).

Dringend geboten wăre eine systematische Datensammlumg rundgeklammerter Zusătze und - davon ausgehend - sowohl die Beschreibung ihrer grammatischen Eigenschaften als auch deren funktionale Charakterisierung. Es ist durchaus noch manche Differenzierung selbst im Bereich der "typischen KURZZUSATZE" vorstellbar. Weiteres zu den KURZZUSATZEN in Kap. 15. 


\section{ZUSATZE MIT NICHT-SUBSTANTIVISCHEN BASEN}

Im Folgenden geht es um Punkt 3. des Phänomenbereichs (Kap. 2): um Muster mit adjektivischen, verbalen, satzformatigen und anderen Basen. In Kap. 9 wurden lockere Strukturen mit (a) Basis (PP) + Zusatz (PP, Adv), Z.B. am anderen Ufer, hinter den Weiden/ dort, stand das Haus, und (b) Basis (Adv) + Zusatz (PP, Adv), z.B. dort, am anderen Ufer, stand das Haus, rudimentär behandelt; es liegen Beispiele von SPEZIFIZIERENDEN oder ALTERNATIVEN NENNENDEN ZUSATZEN vor. Eine ausführliche Erörterung der Muster muß späteren Untersuchungen vorbehalten bleiben.

Wichtig ist die vorläufige Beantwortung dreier Fragen: Ist die syntaktische Relation ZUSATZ auch zwischen satzformatigen Basen und Hospitanten möglich? Welche nicht-satzformatigen Basis-HOSPITANT-Beziehungen existieren? Welche TYpen von Zusätzen sind möglich? Ich beginne die Erfassung der in Frage kommenden Konstruktionen mit komplexen, satzformatigen Einheiten und schließe mit dem einfachen Muster "Basis (Det) + Zusatz (Det)": 1

\subsection{Basis: (Haupt)Satz, Zusatz: (Haupt)Satz}

(14.1a) $A$ ist $20 \mathrm{~m}$ böher als $B$, oder weniger genau, $A$ ist höher als $B$.

(14.1b) Er kommt, (präziser/ genauer:) er kommt morgen. ${ }^{2}$

(14.1c) Beide sind untrennbar miteinander verbunden, (m.a.W./ d.h./ bzw.l genauer) sie setzen einander voraus.

(14.1d) Rick wird Freigänger; das heiBt/ m.a.W.: Er arbeitet in einem Betrieb (...) und kehrt nach ArbeitsschluB in die strafanstalt zurück.

LEXEME: d.h./ m.a.W. (ANDERSSAGEN); bzw. (ALTERNATIVE); allgemeiner/weniger genau (DEPRAZISIERUNG); $d . h . /$ genauer/ präziser (PRAZISIERUNG).

Hier finden sich Satzverbindungen oder sogar Satzfolgen; dies nehme ich trotz der Kommamarkierung an, die eher auf asyndetische Satzverbindung schlieben läBt. Man beachte, daB intonatorisch der erste Satz i.d.R. fallendes Tonmuster aufweist und eine deutliche (Satz) Pause folgt:

(14.1e) ??/er $k$ o mmt $\mathrm{p}+$ er kommt mo rgen $\mathrm{f}++/$. ler $k \circ$ mmt $\mathrm{ft+}$ er kommt mo rgen $\mathrm{ft+}$.

1 Beispiele überwiegend von Molitor (1979: $185 \mathrm{ff}$.), der sie allesamt als "Apposition" einstuft. Auch bei Raabe (1979; v.a. 289 ff.) finden sich hierher gehörende Appositionsbeispiele (aus dem Französischen). Eingefügte, rundgeklarmerte Funktionslexeme im folgenden von W.S. zur Funktionsverdeutlichung.

2 Ins Deutsche übertragenes Beispiel für Apposition von Molitor (1979: 290). Nach Molitor (ebd. $183 \mathrm{ff.}$ ) fallen derartige "Satzfolgen" ("Hauptsatzreihen") unter das Phänomen Apposition, weil sie als syntaktische Paraphrasen in ein und demselben Kormunikationszusammenhang geäußert würden. 
(14.1f) ??/beide sind untrennbar miteinander verbunden $\mathrm{p}+$ das heiBt $(\mathrm{p}+)$ sie setzen einander voraus $\mathrm{ft+l}$.

/beide sind untrennbar miteinander verbunden $\mathrm{f}++$ das heiBt $(\mathrm{p}+)$ sie setzen einander voraus $\mathrm{f}++/$.

Sie stehen in den angegebenen funktionalen Beziehungen (wie PRAZISIERUNG etc.) zueinander, wobei das jeweilige Verhältnis entweder von einem "Funktionslexem" angezeigt oder, falls dieses fehlt, pragmatisch erschlossen wird. Eine Satzfolge resp. -verbindung sei in Anlehnung an die Kriterien von Helbig/Buscha (1980: 559) beschrieben:

$\begin{array}{lllll}\text { Verknüpfungs- } & \text { Intonation } & \text { Satzzeichen } & \text { semantischer } & \text { Funition } \\ \text { zeichen } & \text { zwischen den } S & \text { zwischen den } S & \text { Zusammenhang } & \text { im and. S }\end{array}$

Satzfolge keine

$\begin{array}{ll}\text { fallend, } & \text { Punkt, } \\ \text { sehr deutliche } & \text { evtl. Konma }\end{array}$

Pause

Satzverbindung

Satzgefüge
Semikolon, Komma keine (Asyndese) progredient, bzw. Konjunktion, Pause Konjadv (Syndese)

(keine) $i^{3}$

Subjunktion, Relpron progredient, Karma schwächere

Pause keiner; vage; durch Weltwissen

durch Weltwissen, kopulativ, adversativ etc.

S2 = Entität, Unstand von S1 ja a

Die Muster in (14.1) haben, wenn man die Kriterien strikt anwendet, widersprüchliche Eigenschaften. Intonatorisch liegt eher die Deutung als Satzfolge nahe, graphematisch (Komma, manchmal semikolon) könnte man auf eine satzverbindung schließen, wobei sogleich auf die "stilistische Varianz" bei schreibzeichen hinzuweisen ist. " Sicherlich entspricht es nicht der sprachwirklichkeit, wenn man das Komma schlicht mit 'mäßiger Abtrennung' (Satzverbindung) und den Punkt mit 'völliger Abtrennung' (Satzfolge) gleichsetzt. Anhand von (14.2) zeigt sich, wie durch den Zusatz von Funktionslexemen aus einer Satzverbindung (14.2b) eine Satzfolge (14.2d) entsteht:

(14.2a) Das Wetter war schlecht, (m.a.w.) es stürmte und schneite.

(14.2b) /das wetter war schlecht $\mathrm{p}+$ es stürmte und schneite $\mathrm{f}++/$.

(14.2c) ?/das wetter war schlecht $\mathrm{p}+$ m.a.w. $(\mathrm{p}+)$ es stürmte und schneite $\mathrm{f}++/$.

(14.2d) /das wetter war schlecht $\mathrm{f}++$ m.a.w. $(\mathrm{p}+)$ es stürmte und schneite $\mathrm{f}+\mathrm{l}$.

3 Z.B. bei Verbzweit-Gliedsätzen wie Pia sagt, Kuno hat jetzt ein Privatkino.

4 Statt des irreführenden Begriffs "Satzzeichen" (nur das Kama kann als solches gelten, da es Hauptsatz (rest) und abhängigen Satz(rest) voneinander trennt) verwendet Engel (1988: 819 ff.) den Terminus "Schreibzeichen" für Korma, Punkt, Semikolon etc. 
Durch den pragmatischen Schluß bzw. die Semantik der Funktionslexeme werden $z w i s c h e n$ den beiden Sätzen der Satzfolge Bezüge hergestellt (zu den möglichen semantischen Bezügen s. Ortner 1983). Dadurch wirkt der zweite Satz z.B. als Prăzisierung des ersten. Anders formuliert: Der Sachverhalt, den der erste Satz zum Ausdruck bringt, wird durch den Sachverhalt des zweiten Satzes präzisiert, indem dieser zusätzliche, zum ersten Sachverhalt passende Information (über Umstände, beteiligte Entitäten etc.) bietet. Das heißt, man schreitet bei der "laufenden" Dekodierung nicht einfach von Satz 1 (Sachverhalt ${ }_{1}$ ) zu Satz $_{2}$ (Sachverhalt ${ }_{2}$ ) fort, sondern korrigiert resp. ergänzt (mental) die dem ersten Satz entnommene Information durch die dem zweiten Satz entnommene.

Diese Satzfolgen könnten (z.T.) auch verkürzt erscheinen, wenn man die Tilgung identischen Sprachmaterials durchführt. Das nachstehende Beispiel demonstriert, daB man zudem in einem Fall eine Art "Satznachtragskonstruktion" bilden kann (14.4; in anderen Fällen geht dies nicht, z.B. 14.6, 14.7):

(14.3) Er kommt, (er kommt) morgen.

(14.4) Er kommt, und zwar kommt er morgen. Er kommt, (und zwar) morgen.

(14.5) Beide sind untrennbar miteinander verbunden, (d.h. sie) setzen einander voraus.

(14.6) ??Beide sind untrennbar miteinander verbunden, und zwar setzen sie einander voraus.

(14.7) ??Rick wird Freigänger, und zwar arbeitet er (...) zurück.

In (14.3) liegt eine (nachtragstypische) Spezifikationsrelation vor, die in den späteren Fällen nicht plausibel in die Beispiele hineininterpretiert werden kann. Bei ANDERSSAGEN beispielsweise will der sprecher ja keinesfalls "spezifizieren", also näher bestimmen, sondern "den gleichen Sachverhalt/die gleiche Sache unter einem etwas anderen Aspekt beleuchten".

Eines kristallisiert sich heraus: Offensichtlich sind die (pragmatischen) Funktionen ANDERSSAGEN, ALTERNATIVE, evtl. auch AUFZAHLUNG (Rick wird Freigänger: $($....)i und (DE) PRAZISIERUNG so aligemeir, daß sie auch zwischer zwei sătzen einer Satzfolge bestehen können. MENGE-ELEMENT-BEZIEHUNGEN sind natürlich bei satzformatigen Einheiten ausgeschlossen, da nur NPn Mengen bzw. Elemente denotieren können. PRAZISIERUNG ist zu verstehen als Angabe eines genaueren Ausdrucks, nicht als Einschränkung einer Menge (bei NPn scheinen Uberschneidungen möglich). Die Möglichkeit, zwei explizite Sätze mit den erwähnten funktionalen Beziehungen zu kürzen (er kommt, morgen.), deutet evtl. darauf hin, daB die subsententialen zusäze in systematischer Beziehung zu expliziten Sătzen stehen.

Das Muster (14.1) selbst fält nicht in den Bereich der HOSPITANTEN, denn es liegt eine Satzfolge vor! (Ein HOSPITANTEN-Fall bestünde, falls der zweite Satz in den ersten eingeschaltet würde.) Die in Kap. 5 entwickelte Begrifflichkeit, daß ein Syntagma oder ein Wort in einem Gastsatz als HOSPITANT (ZUSATZ, EINSCHUB) auftritt, ist auf (14.1) nicht anwendbar. 
14.2. Basis: Verbend-Satz, Zusatz: Verbend-Satz

(14.8a) Es gibt aber Relativsätze, die ambig sind, d.h. [es gibt Relativsätze, J die verschiedene Bedeutung haben. 5

(14.8b) Ein Lautkomplex (...) ist nur dann eine sprachliche AuBerung, wenn er der Kommunikation dient, bzw./ d.h./ m.a.W. [ein Lauthomplex ist nur dann eine sprachliche Außerung,J wenn er sich auf einen Sachverhalt (...) bezieht.

LEXEME: also/ anders gesagt/ d.h./ m.a.W. (ANDERSSAGEN); möglich wären auch (DE) PRAZISIERUNG, ALTERNATIVE; ${ }^{6}$ evtl. AUFZAHLUNG. 7

Ich betrachte mögliche Kürzungen und zusätzlich mögliche Umstellungen:

(14.9) Es gibt aber Relativsätze, die ambig sind, d.h. (es gibt Relativsätze, die) verschiedene Bedeutung haben.

Es gibt aber Relativsätze, die ambig sind ((d.h.) verschiedene Bedeutung haben).

Es gibt aber Relativsätze, die ambig ((d.h.) mehrdeutig) sind.

(14.10) Ein Lautkomplex (...) ist nur dann eine sprachliche AuBerung, wenn er der Kommunikation dient, (wenn er) sich auf einen Sachverhalt (...) bezieht.

Ein Lautkomplex (...) ist nur dann eine sprachliche AuBerung, wenn er der Kommunikation dient ((d.h.) sich auf einen Sachverhalt (...) bezieht).

Ein Lautkomplex (...) ist nur dann eine sprachliche AuBerung, wenn er der Kommunikation (bzw./ d.h./ genauer dem Sachverhaltsbezug) dient.

Hier zeigen sich (optional durch "Funktionslexeme" markierte) Beziehungen wie ANDERSSAGEN, (DE) PRAZISIERUNG etc. bei diversen möglichen Reduktionsstufen u.U. bis hin zu einer Struktur, die wie ein KURZzUSATZ aussieht (14.9; vgl. Kap. 13, Abs. (a15)). Beim Muster 14.2. tritt der zweite Verbend-Satz innerhalb eines Gastsatzes als BOSPITANT auf, und zwar bezogen auf einen Satzteil

5 Einschübe in Klammern von mir. Sie sollen andeuten, inwiefern Tilgungen vorgenammen worden sein könnten, bzw. zu welchen expliziteren Formulienungen Beziehungen bestehen.

6 Vgl.: (F14.1) Es gibt Menschen, die nicht wissen, wer sie sind (genauer: die schizophren sind).

(M14.2) Es gibt Menschen, die schizophren sind (weniger genau: die nicht wissen, wer sie sind).

(F14.3) Es gibt Menschen, die schizophren sind, bzw. die nicht wissen, wer sie sind (ALr TERNATTVE).

7 Die Funktion AUFZXHLung ist möglicherweise aufzulösen in die Funktionen PRAZISImRUnG, BEISPIIIr NIJNuNG und TEIIIING klassen nur als vorläufig und provisorisch aufgefaßt werden; genauere Untersuchungen, vor allem semantische Feinanalysen, sind dringend geboten, können hier aber nicht geleistet werden. 
(auf ein Relativsatzattribut, 14.11a; aber z.B. auch auf adverbiale Gliedsătze: 14.11b). Folglich handelt es sich um einem ZuSATZ (Gastsatz kursiv; Basis unterstrichen; Zusatz fett):

(14.11a) Es gibt Relativsätze, die ambig sind, (d.h.) die verschiedene Bedeutungen haben, weil Eindeutigkeit nicht immer notwendig ist.

(14.11b) Wenn es 12 schlägt, (also/ n.a.W.) wenn die Geisterstunde beginnt, sollte man keine Gruselgeschichten lesen.

In Făllen wie (14.11b) ist infolgedessen kein parenthetischer Einschub des zweiten Konditionalsatzes anzunehmen. Bei gleichen syntaktischen Funktionen und bei möglichem Einschub der bekannten Funktionslexeme liegt die ZUSATZ-Relation vor. Anders sind Fảlle zu werten, bei denen Funktionsdifferenz vorliegt:

(14.12a) Trotzdem (KONZ), sobald er voll von Eindrücken zu Hause anlangte (TEMP), ging eine entmutigende Veränderung in ihm vor.

(14.12b) Obwohl er sich besser fühlte, sobald er voll von Eindrücken $z u$ Hause anlangte, ging eine entmutigende Veränderung in ihm vor. In (14.12b) besteht keine Zusatzrelation; ganz einfach ist dieser Fall jedoch nicht zu klären. Traditionell năhme man an, daB der zweite Adverbial-Gliedsatz eingeschaltet sei (er füllte die Parenthesenische zwischen vorfeldbesetzendem Konzessivsatz und Finitum); in diesem Zusammenhang erinnere ich an meinen Vorschlag in Kap. 11, aufgrund der potentiellen syntaktischen Funktion des "Einschubs" von einem ZUSATZ zu einem Satz (und nicht von EINSCHUB) zu sprechen. Es erscheint allerdings auch nicht abwegig, dem konzessiven Element in (14.12a, b) eine Art "Satzvorlauf-Position" zuzusprechen, da ihm augenscheinlich eine erhebliche Eigenständigkeit zugemessen werden kann, etwa wie in: 8

(14.13) Trotzdem:/ Obwohl er sich besser fühlte: Sobald er (...) anlangte, ging eine entmutigende Veränderung in ihm vor.

Ich kann diesen Fall hier nicht abklären. Festzuhalten bleibt, daB keine zusatzbeziehung existiert.

\subsection{Basis: Infinitivgruppe, Zusatz: Infinitivgruppe}

(14.14) Die Hauptfunktion der Sprache ist, uber die Welt zu sprechen, d.h./ m.a.W.:/ genauer:/ bzw. [die Hauptfunktion der sprache ist,] Informationen über Tatsachen $z u$ geben.

FUNKTIONEN: ANDERSSAGEN, ALTERNATIVE, (DE) PRAZISIERUNG.

Hier darf man zwei aufeinanderfolgende Kopulasätze zugrunde legen, die sich bis auf das differierende Satzglied, die Infinitivgruppe als Prädikativ, gleichen. Die Beziehung zwischen den Infinitivgruppen kann durch ein Funktionsle-

8 Vgl. dazu auch van de Velde (1978: 134). 
xem angezeigt werden. Die zweite Infinitivgruppe vermag als (NICHT)SPEZIFIZIERENDER bZw. als ALTERNATIVEN NENNENNDER ZUSATZ zur ersten zu fungieren.

\subsection{Basis: Verbalphrase, Zusatz: Verbalphrase}

(14.15a) Aber es bleibt doch bestehen, daB wir uns gelegentlich unangemessen ausdrücken, also/ d.h./ m.a.W.:/ bzw./ genauer; [es bleibt doch bestehen, daB wir] das treffende Wort verfehlen.

(14.15b) (...) ist äquivalent mit der Behauptung, daB die entsprechende Klasse erfüllt ist, d.h. [daB die entsprechende Klasse] mindestens ein Element enthält, d.h. [daB die entsprechende Klasse] nicht identisch mit der Nullklasse ist.

(14.15c) DaB der Film "Lysistrata" überhaupt nicht existiert, genauer/ also/ d.h. [daB der Film "Lysistrata"] eine Erfindung ist, (...).

FUNKTIONEN: ANDERSSAGEN, ALTERNATIVE, (DE) PRAZISIERUNG.

Die zweite Verbalphrase (optional mit Funktionslexem) ist ZUSATZ zur ersten.

14.5. Basis: Verb, Zusatz: Verb

(14.16a) In Indien verhungern, allgemeiner: [in Indien] sterben, viele Kinder.

(14.16b) In Indien verhungern viele Kinder. Allgemeiner/Weniger genau/ D.h./ M.a.W.: In Indien sterben viele Kinder.

(14.17a) Das Adjektiv kongruiert (d.h./ m.a.W. stimmt überein) mit dem Substantiv.

(14.17b) Das Adjektiv kongruiert mit dem Substantiv. D.h./ M.a.W.: Das Adjektiv stimmt mit dem Substantiv überein.

(14.18) (...) verzichten wird, genauer gesagt: verzichten muB.

FUNKTIONEN: ANDERSSAGEN, ALTERNATIVE, AUFZAHLUNG, (DE)PRAZISIERUNG.

Wiederum lassen sich Beziehungen zwischen expliziten Satzfolgen und subsententialen Einheiten bis hin zur Wortartenkategorie (V) feststellen, vgl. die $a-$ und b-Varianten. Das zweite Verb fungiert als zuSATZ zum ersten.

14.6. Basis: (attributives) Adjektiv, Zusatz: (attributives) Adjektiv

(14.19) (...) in der abstrakte (also: nicht-lineare) strukturen erzeugt werden.

(14.20a) (..) Kommunikationsakte sind (...) keine stochastischen, d.h. zufälligen, Ereignisse.

(14.20b) Kommunikationsakte sind keine stochastischen Ereignisse. D.h.l M.a.W.: Kommunikationsakte sind keine zufälligen Ereignisse. 
(14.20c) Kommunikationsakte sind keine stochastischen (zufälligen) Ereignisse.

Auch an diesem Beispiel kann sowohl eine Satzfolge (mit identischem Material bis auf das Zusatzelement; 14.20b) als auch eine Reduktion bis hin zum zUSATZ (14.20a) konstruiert werden - hier sogar bis hin zu einer mit dem KURzzUSATZ verwechselbaren Variante (14.20c; vgl. Kap. 13, Abs. (a15)), die allerdings einen Alternativen nennenden Zusatz mit der Funktion ANGABE DES DEUTSCHEN WORTES (FUR EINEN FACHTERMINUS) darstellt.

\subsection{Basis: Prådikativ, Zusatz: Prădikativ}

An ein Prădikativ (hier werden nur Prädikative zum Subjekt betrachtet) kann sich ein ZUSATZ assoziieren, der ebenfalls als Prädikativ fungieren könnte (prädikatives Adjektiv; prädikative NP oder PP):

(14.21a) Die Lexeme sind synonym, [die Lexeme sind] bedeutungsgleich.

(14.21b) Franz ist ledig, d.h. [Franz ist] unverheiratet.

(14.21c) Franz ist ein Junggeselle, also [Franz ist] unverheiratet.

(14.21d) Franz ist ledig, d.h. [Franz ist] ein Junggeselle.

(14.21e) Runo ist von Sinnen - (d.h./ m.a.W.) wahnsinnig - gewesen.

FUNXTIONEN: ANDERSSAGEN, ALTERNATIVE, AUFZABLUNG, 9 (DE) PRAZISIERUNG.

Diese Beispiele führen uns vor Augen, daß der Zusatz - obzwar er öfers mit der Basis kategorial übereinstimmt - nicht immer mit dem Bezugselement in der Kategorie übereinstimmen muß. Es genügt manchmal, daß er die gleiche syntaktische Funktion in entsprechenden selbständigen Sătzen ausüben könnte. Mit anderen Worten: Es darf eine Beziehung zwischen zwei Sătzen angenommen werden, bei denen bis auf das unterschiedliche sprachmaterial in gleicher syntaktischer Funktion Tilgungen angewendet wurden. Man vgl, auch:

(14.22) Franz ist ledig gewesen. D.h. Franz ist ein Junggeselle gewesen. f'ranz ist ledig - d.h. (ein) Junggeselle - gewesen. Franz ist ledig (Junggeselle) gewesen.

\subsection{Basis: Deterninans, Zusatz: Determinans (bzw. Genitiv-MP) 10}

Sogar Determinantien erlauben, daß sich bestimmte ZUSATZE an sie assoziieren, nämlich ALTERNATIVEN NENNENDE, z.T. REFERENZKLARENDE (zumindest bei der Folge Pro-Form + Genitiv-NP) und evtl. SPEZIFIZIERENDE:

9 Z.B.: Die Bemden waren farbig (also/ z.B. pink, rot, grün) gewesen.

10 Die Genitiv-NP kommutiert zwar stellungsmäßig mit Determinantien wie Artikel und Possessivpronomina etc., ich fasse sie aber nicht mit diesen zusammen; Determinantien - der Typ lieb Kindlein mein ist ein Archaismus - kanmen im Ggs. zu Genitiv-NPn nicht nach dem Kernnomen vor. 
(14.23a) Er (...) habe den Bankraub (...) mit seiner, [also/ d.h./ m.a.N./ ?genauer 11 bzw./ ich meine] Krons, Unterstützung ausgefuhrt.

(14.23b) Diese, [also/ bzw./ d.h./ m.a.W./ ?genauer/ ?ich meine] ${ }^{12}$ deine, neue Uhr war wohl teuer.

Auch hier kann ein Zusammenhang mit Satzfolgen gezeigt werden:

(14.24) Diese neue Uhr war wohl teuer. Ich meine/ M.a.W.: Deine neue Uhr war wohl teuer.

Zusammenfassend läßt sich bemerken, daß ein allgemeiner Zusammenhang hergestellt werden konnte zwischen

- Satzfolgen mit oder ohne expliziter Funktionsmarkierung (Es gibt Relativsătze, die ambig sind. (D.h./ M.a.W.:) Es gibt Relativsătze, die mehrdeutig sind.). Satzfolgen sind keine Instantiierungen von "Basis-zUSATZ"-Mustern, weil sich die Konstruktion nicht innerhalb eines komplexen (Gast)Satzes befindet, sondern zwei selbstăndige sătze betrifft.

- subsententialen Kategorien (wie VP, InfGr) nach Anwendung von Tilgung gleichen Materials unter Beibehaltung der verschiedenen Elemente gleicher Kategorie resp. Funktion (Es gibt Relativsätze, die ambig sind, (d.h.) mehrdeutig sind (VP)) und

- Wortartenkategorien (wie Adj, V, Det), die nach solchen Tilgungen als Minimum übrigbleiben. Entweder führen diese auch einen expliziten Funktionsmarker bei sich oder der Hörer muß sich pragmatisch das plausibelste Verháltnis zwischen diesen erschließen (Es gibt Relativsätze die ambig, (also/ d.h.) mehrdeutig, sind.).

Auch zur Grammatik der eben behandelten ZUSATZE bleiben noch etliche Fragen offen. (An manchen Stellen wird in Kap. 15 nochmals kurz auf sie Bezug genommen.) Da die NP-bezogenen ZUSATZE im Mittelpunkt meiner Forschung stehen, kann ich nicht ausfuhrlicher auf sie eingehen.

11 Genaver (PRAZISIFRUWG) erscheint nicht ganz angemessen; der Zusatz bewirkt, daß der Ausdruck "treffender" (deutlicher in seinen referentiellen Bezigen) wird. Fr wird nicht präziser in dem Sinn, daß das Denotat mittels Mengenrestriktion spezifiziert wird.

12 Der Ausdruck ich meine leitet (nach Altmann 1981) einen Ausdruck ein, der die Referenz einer undeutlichen NP, zumeist einer NP-Pro-Form, verdeutlichen soll. Im ersten Beispiel (seiner, Krons,) trifft dies zu; hier im zweiten ist diese neue uhr wegen des deiktischen Demonstrativpronomens referentiell eindeutig, das Possessivpronomen ist nur eine andere Formulienung (ANorRSSAGON). 


\section{ZUSAMMENSCHAU DER NP-BEZOGENEN ZUSATZE}

Im Folgenden werden die grammatischen Eigenschaften jener Muster behandelt, die sich als "im engsten Sinne appositionsverdächtig" herausstellten. Die grammatischen Unterschiede innerhalb der NP-bezogenen Zusätze erweisen sich als nicht geringfügig. Der prädizierende Zusatz (traditionell: "die lockere Apposition") und die übrigen Zusätze sollten nach der folgenden obersicht genauer auseinandergehalten werden können. Ich gehe von sechs Zusatz-Grobklassen aus :

(a) den PRADIZIERENDEN ZUSATZEN (PRAZ; ehedem: "lockere Appositionen");

(b) den REFERENZKLARENDEN ZUSATZEN (REFZ; "Rechtsversetzungen" nach Altmann 1981);

(c) den SPEZIFIZIERENDEN ZUSATZEN (SPEZ; erscheinen bei Altmann 1981 z.T. als "Nachträge");

(d) den NICHT-SPEZIFIZIERENDEN ZUSATZEN (NSPEZ; eine noch problematische "Sammelklasse", die evtl. noch weiter aufzufächern ist);

(e) den ALTERNATIVEN NENNENDEN ZUSATZEN (ALNZ) und

(f) den KURZZUSATZEN (KURZ; "Wortparenthesen" nach Schwyzer 1939: 39). Da sich die Typen (c), (d) und (e) syntaktisch weitgehend gleich verhalten (vgl. Ubersicht am Kapitelende), bestünde die Möglichkeit, sie zu einer GroBklasse zusammenzufassen).

Nur teilweise behandelt werden hier die ebenfalls als NP-ZUSATZE fungierenden "appositiven Relativsätze" (APREL; zu den Merkmalen s. auch Kap. 7.A) und die (NP-)ZUSATZE, die als Attribut fungieren könnten, also einschaltungsmarkierte Gliedteile (EMG) wie z.B. eine - notwendig einseitige - Weigerung (s. Kap. 11). Beide Muster konnten nicht mehr vollständig eingearbeitet werden (und lagen auch nicht im Zentrum meines Forschungsinteresses). Das Beispielmaterial zum Nachweis der jeweiligen Eigenschaften muß knapp gehalten werden (z.T. kann man in der vorigen Kapitelr nachgeschlagen); diese ohnehin beispielreiche Arbeit schwölle sonst unmäBig auf.

\section{Beteiligte Kategorien}

\subsection{Kategorien der Bezugseinheit}

Hier werden auch die nicht-nominalen Rategorien angeführt (vgl. Kap. 14). PRAZ: NP. (Eventuell noch: Satz. ${ }^{1}$ )

1 Die Grammatik der Satzapposition sowie deren Finordnung in eine Grammatik des Deutschen erscheint noch nicht völlig geklärt (vgl. Kap. 6.A). 
REFZ: NPn mit vager Referenz, ${ }^{2}$ insb. Pronomina; auch Pronominaladverbien und Pro-Adverbien ( $d a$, dort etc.) möglich.

SPEZ: NP, Adj, PP, Adv, V, VP, InfGr, V-E-S, [V-2-S]. 3

NSPEZ: NP, Adj, PP, Adv, V, VP, InfGr, V-E-S, [V-2-S].

ALNZ: NP, Adj, PP, Adv, V, VP, InfGr, V-E-S, [V-2-S].

KURZ: Nomen, NP, Kurzwort, Adj, (PP, V). Wohl nicht: Pron, VP, S.

APREL: ausschlieBlich NP.

EMG: NP, Adj, evtl. Adv.

Man verdeutliche sich dies anhand einiger Beispiele für referenzklärende $\mathrm{Zu}-$ sätze (15.1), nichtspezifizierende Zusätze (15.2) und Kurzzusätze, die No (15.3), N3 (15.4) und PP (15.5) zur Basis haben:

(15.1) Das (, ich meine wahnsinnig,) war Mabuse tatsächlich (, ich meine wahnsinnig).

Daran (, an Kunos Tat,) erinnert sich jeder (, an Kunos Tat). Da (, im Silbersee,) lag der Schatz (, im Silbersee).

(15.2) Dort (Adv)/ Im Meer (PP), in allen Gewässern, stank es. Rauchen (V), ferner/ überdies saufen, wollen alle harten Kerle. Zigarren qualmen (VP), zudem/ ferner Bier saufen, wollen sie. DaB Kuno spinnt (V-E-S), ferner daB Hugo säuft, störte Pia nicht.

(15.3) Dickhäuter (Pachyderm) ist ein dreisilbiges Nort.

(15.4) Dickhäuter (Pachyderme) sind z.B. die Elefanten.

(15.5) Mit anderen Worten (M.a.W.) heiBt das (...).

\subsection{Kategoriale Füllung des Zusatzes:}

\subsubsection{Ohne Erweiterungen}

PRAZ:

NO-N3, AdjP, Kopulapartikel, PP, AdvP. 4

REFZ: N3, AdjP, PP, ?Adv, 5 V, VP, V-E-S. 6

2 Man nehme z.B. den Paradefall: Kannst du mir mal das Dingsda (, ich meine das Hygrameter,) 'rüberreichen (, ich meine das Hygrameter). Oder auch: Diese Person (, ich meine deine Schmester,) stürzte Kuno in tiefe Verwirrung (, ich meine deine Schwester). Es sind "vage" (in einigen semantischen Merkmalen nicht spezifizierte) Nomina: Person z.B. bzgl. [+ männlich/ + weiblich].

3 In Kap. 14 (14.1.) habe ich dargelegt, daß zwischen Verbzweitsätzen in Satzfolgen keine ZusatzRelation besteht. Die Möglichkeit, daß der zweite Satz einer Satzfolge ein Funktionslexem mit z.B. spezifizierender Funktion enthalten kann, wird in Klammern hinzugefügt, um die cemeinsamkeit der (nicht-) spezifizierenden und der Alternativen nennenden Zusätze hervorzuheben.

4 zur Erinnerung: Kopulapartikeln sind nur prädikativ auftretende Elemente wie quitt, schade etc.

5 Adverbien sind wahrscheinlich nur teilweise in referenzklärende Zusätze einzubeziehen, und zwar bei adverbalen Basen (nicht bei PPn):

(F15.1) Da, ich meine nebenan,/ dort, ich meine links, ist das, was sie suchen.

(F15.2) (?) In Kunos Zimmer, ich meine nebenan, / ?? In Kunos Zimmer, ich meine dort, findet ihr es. 
SPEZ: $\quad$ N3, AdjP, PP, Adv, V, VP, InfGr, V-E-S, [V-2-S].

NSPEZ: $\quad$ N3, AdjP, PP, Adv, V, VP, InfGr, V-E-S, [V-2-S].

ALNZ: $\quad$ N3, AdjP, PP, Adv, V, VP, InfGr, V-E-S, [V-2-S].

RURZZUSATZ: No, N3, 7 Kurzwort, Zahlausdrücke. 8

APREL: Verbendsatz mit Relativpronomen bzw. -adverb.

EMG: AdjP, Adv (bei Adj-Basen), auch Genitiv-NPn (mögliche Muster sowie deren Bedingungen müssen noch näher erforscht werden!).

Wenn zu einer Pro-Form ein Zusatz unterhalb von N3 bzw. der Kategorien AdjP, PP etc. hinzutritt, dann liegt kein referenzklärender zusatz (Erweiterung ich meine ist inakzeptabel) sondern ein mit übrigens versehbarer prădizierender Zusatz vor:

(15.6) Sie, (übrigens/ *ich meine) Röchin aus Passion/ Iustig und fidel/ in Katmandu, (...).

Andere Zusătze zur Pro-Form sind durchaus statthaft und stellen keine prädizierenden zusătze dar (15.7-15.9). Allerdings ist die Hinzufügung von Kurzzusătzen wohl inakzeptabel (15.10), weil derartige zusätze i.d.R. unmittelbar auf ein autosemantisches Wort Bezug nehmen müssen:

(15.7) Sie, (und zwar) besonders/ einschlieblich/genauer/ vor allem die Hammerhaie, werden gejagt.

(15.8) Sie, allgemeiner alle Fische, werden dort gejagt.

(15.9) Sie, also/ m.a.W. die Schrecken der Meere, werden dort gejagt.

(15.10) Sie (??SPD/??1899-1988) betörte die Mănner.

sie (*Orthographie/ *spio) (...).

sie ((ich meine) die Orthographiel die spio) (...).

(15.10) zeigt anschaulich, wie nahe die Typen beieinander liegen; bereits die Hinzufügung eines Artikels kann die Art des Zusatzes verändern.

Nun noch einige Beispiele zu referenzklărenden Zusatzen; die Distanzvariante wirkt i.d.R. voll akzeptabel, die Kontaktvariante hingegen nicht unbedingt (der Grund hierfür ist mir unklar):

(15.11) Dort (, ich meine aus dem Schrank (PP)/ oben (Adv), ' wâr es feucht (, ich meine auf dem Schrank/ oben).

Das (?, ich meine rauchen (V)/ Bier trinken (VP),) schadet ihr bestimmt (, ich meine rauchen/ Bier trinken).

Das (?, daß Peter spinnt (V-E-S),) störte sie nicht (, daß Peter spinnt, ).

Fazit: Unter dem Aspekt maximaler Kombinationsvariabilităt kann man die spezifizierenden, die nichtspezifizierenden und die Alternativen nennenden Zusătze zusammenfassen. Bei diesen Zusătzen lassen sich nicht nur viele Kategorien

6 Das ist ein vielseitiges Pro-Element: ein ProNkominal, ein Pro-Verbal und ein Pro-Sentential.

7 Siehe z.B.: Die RNS (Ribonukleinsáure; N3/SN), ein Pachyderm (Dickhäuter; NO/CN).

8 Mit "Zahlausdricke" meine ich Zusatz-Binheiten wie: Imanuel Kant (1724-1804) (...). 
gleicher Art miteinander verbinden, sondern in gewissen Grenzen auch ungleiche Kategorien:

(15.12) Kuno behauptet, daB er Extraterristen gesehen habe (V-E-S), genauer: [Kuno behauptet,] mit ihnen gesprochen zu haben (InfGr).

(15.13) Ledig (Adj), überdies ein Ehefeind (NP), ist Opa immer gewesen.

(15.14) Nebenan (Adv), m.a.W. in Kunos Zimmer (PP), herrscht das Chaos. Bei den Kurzzusätzen treten Verbalphrasen und sătze selten als Basen auf: sie sind wohl als idiosynkratisch anzusehen, die zusatzmöglichkeiten sind stark eingeschränkt. 9 Bei den referenzklärenden Zusätzen gibt es nicht allzu viele Basiskategorien, allerdings relativ viele Zusatzkategorien.

Der prädizierende Zusatz weist Nominalphrasen und, wenn man die "Satzapposition" dazurechnen will, auch Sätze als Basis auf, sowie eine genau beschreibbare Menge von Zusatzkategorien, die bei NP-Basis den kategorialen Fü1lungsoptionen für Prädikative in Sătzen mit der Kopula sein (weitgehendst) entsprechen.

\subsubsection{Erweiterungsmöglichkeiten}

In der ersten Zeile werden typische "Erkennungsfloskeln"10 genannt, darunter die Möglichkeiten der Erweiterung um freie Angaben (freie ADVB; v.a. Satzadverbiale und temporale Adverbiale), Partikeln, Ronjunktionaladverbien und subordinierende Konjunktionen; derartige Hinzufügungen signalisieren die "Satzwertigkeit" des zusatzes.

Es ist festzustellen, daB mögliche Erweiterungen um Adverbiale und Gradpartikeln am uneingeschränktesten beim prădizierenden zusatz möglich sind; bei den (nicht-) spezifizierenden Zusätzen sind immer wieder Restriktionen aufweisbar, deren Ursachen hier nicht nachgegangen werden kann. Im Folgenden verden hauptsăchlich Beispiele mit NPn untersucht; daB Erweiterungen auch bei andersformatigen Kategorien möglich sind, wird nur durch vereinzelte Beispiele angedeutet. Inwieweit die Restriktionen bei nicht NP-formatigen Beispielen noch einschneidender sind, kann leider nicht untersucht werden.

9 Vereinzelt könnte man sich (allerdings wohl nur für fremdsprachliche satzformatige Wendungen) Kurzzusătze zu Sătzen vorstellen; zudem kann man natürlich individuell Abkürzungen für Sătze einführen, z.B. als Euphemismus (F15.5). Der Blick in ein x-beliebiges Abkürzungsverzeichnis belegt jedoch, das dies eine idiosynkratische, unsystematische Option darstellt.

(F15.3) Requiescat in pace (R.I.P.).

(F15.4) Save our souls (S.O.S.).

(F15.5) Pia sagte zu Kunibert: "Leck mich am Arsch" (L.m.a.A.).

Vgl. Altmanns Untersuchumgen bzgl. der "kriterialen Zusătze" (1981: 79 ff.). 


\begin{tabular}{|c|c|c|c|c|c|c|}
\hline & $\begin{array}{l}\text { PRAZ } \\
\text { übrigens }\end{array}$ & $\begin{array}{l}\text { SPEZ } \\
\text { und } z \text { war }\end{array}$ & $\begin{array}{l}\text { NSPEZ } \\
--11\end{array}$ & $\begin{array}{c}\text { ALNZ } \\
(m . a . W . / \\
\text { d.h./bzw.) }\end{array}$ & $\begin{array}{c}\text { REFZ } \\
\text { ich meine }\end{array}$ & KURZ \\
\hline freie ADVB & + & + & + & ?? & $\ldots 12$ & --- \\
\hline Gradpt & + & + & + & ?? & --- & --- \\
\hline Modalpt & + & ?? & ?? & ?? & -- & --- \\
\hline Konjadv & + & ?? & ?? & ?? & --- & --- \\
\hline subord. Konj & $(+)$ & --- & --- & --- & --- & --- \\
\hline
\end{tabular}

Bei den Kurzzusätzen bewirken Erweiterungen i.d.R. einen Ubergang in andere Muster (v.a. ALNZ). Bei den spezifizierenden Zusătzen sind Satzadverbien, freie Adverbiale und Gradpartikeln prinzipiell möglich, wobei sich z.T. Kombinationsrestriktionen mit den funktionsanzeigenden Einleitungslexemen ergeben können; ${ }^{13}$ diese resultieren aus den kombinierten wortsemantischen Merkmalen bzw. aus sich widersprechenden "Gebrauchsbedingungen". So kann z.B. das Satzadverb vermutlich nicht zusammen mit dem Funktionslexem genauer vorkommen, weil die Vermutung nicht mit der Präzisierung, die ja auf Tatsachenwissen beruht, vertrăglich ist etc. Die nachstehenden Beispiele zeigen lediglich einen Ausschnitt der (Un) Möglichkeiten:

(15.15) Viele Vögel, (und $z$ war) bedauerlicherweise auch besonders/ einschlieblich/ ? genauer/ (?)z.B. die Habichte, werden dort gejagt. Viele Vögel, sogar besonders/ einschlieBlich/ ?genauer/ ?z.B. die Habichte, werden dort gejagt.

(15.16) Viele Voggel, (und zwar) seit kurzem besonders/(?) einschließlich/ ??genauer/ ??z.B.die Milane, werden dort gejagt.

(15.17) Farbig, bedauerlicherweise besonders/ einschlieblich/?genauer/ ?z.B. rot und grün, waren die Hemden gewesen.

(15.18) Farbig, sogar ?besonders/ einschließlich/?genauer/ (?)z.B. rot und grün, waren die Hemden gewesen.

In engeren Grenzen scheinen auch Moaalpartikeln und Konjunktionaladverbien einfügbar zu sein:

(15.19) ? Viele Vogel, (und zwar) eben doch auch die Milane, (...).

(15.20) Viele Singvögel, allerdings/ (?) trotzdem/ (?) dennoch auch manche Raubvögel, werden dort gejagt.

Kombiniert mit den Funktionslexemen ergeben sich jedoch häufig starke Akzeptabilitätsminderungen, so daß die Hinzufügung nicht im gleichen Maße möglich er-

11 Ubrigens ist meistens hinzufügbar, kann aber nicht als typische Mloskel gelten, weil dadurch lediglich die Beilăufigkeit/Nebensăchlichkeit des Zusatzes ausgedrückt wird.

12 Daß referenzklärende Zusätze ("Rechtsversetzungen") keine Erweiterungsmöglichkeiten aufweisen, hat Altmann (1981: 54, $94 \mathrm{ff}$.$) klar herausgearbeitet und begrindet.$

13 Hierzu Altmann (1981: 71, 102). 
scheint wie beim prädizierenden Zusatz; vielleicht muß man sie sogar als nicht akzeptabel einstufen:

(15.21) Viele Vögel, eben doch ??besonders/ ?einschließlich/ ??genauer/ ??z.B. die Milane, werden dort gejagt.

(15.22) Viele singvögel, allerdings ??besonders/ ?? einschlieblich/ ??genauer/ ??z.B. Amsel, Drossel und Star, werden dort gejagt.

Die Erweiterung durch Subjunktionen dürfte völlig ausgeschlossen sein:

(15.23) Viele Haie, (*obwohl/*weil) besonders/genauer/ einschlieblich/ $z . B$. die Hammerhaie, werden gefürchtet.

Bei nichtspezifizierenden Zusätzen darf man Satzadverbien, Adverbiale und Gradpartikeln hinzusetzen:

(15.24) Manche Haie, vermutlich zumindest/ weniger/ ausgenommen die Ammenhaie, werden dort gejagt.

(15.25) Viele Vögel, allgemeiner/ obendrein/ferner seit kurzem sogar alle flugfähigen Tiere, werden dort gejagt.

(15.26) Putzen, vermutlich ferner/ obendrein schneiden und würzen, soll Kuno die Salate.

Das Hinzusetzen von Modalpartikeln, Konjunktionaladverbien und subordinierenden Konjunktionen führt i.d.R. zu inakzeptablen Resultaten:

(15.27) Manche Haie, (*halt doch/ (?) ja/ *eben; *obwohl/*weil) obendrein/ ferner die Sägefische, werden gefürchtet.

(15.28) Viele Vögel, (*halt doch/ (?) ja/ *eben; *obwohl/*weil) obendrein/ allgemeiner alle fliegenden Tiere, wurden gejagt.

Bei den Alternativen nennenden Zusätzen scheinen die Erweiterungsmöglichkeiten sehr begrenzt zu sein; obzwar ein typisches Funktionslexem nicht existiert, ist die Menge möglicher Funktionslexeme (typischerweise Abkürzungen!) doch begrenzt: anders gesagt/bzw./ d.h./ m.a.W.:

(15.29) Die Könige der Tiere, ?? bedauerlicherweise/ *vermutlich anders gesagt/ m.a.W. die Löwen, (...).

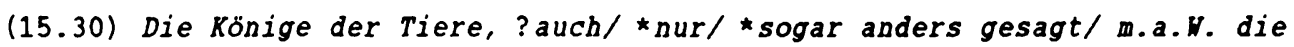
Löwen, (...).

\section{Abtrennung}

Eine graphematische/intonatorische Abtrennung findet sich bei allen Typen. Das Einschaltungsmuster ist ein zentrales Kennzeichen von Zusătzen. Genaueres zu den intonatorischen Eigenschaften der prädizierenden, referenzklärenden und der spezifizierenden Zusătze ist bei Altmann (1981: 183 ff. Zusammenfassung S. 204) nachzulesen. 14

Die Schreibzeichen Komma, Gedankenstrich und Rundklammer sind keinesfalls funktional völlig aquivalent und somit nicht für jedes Muster vőllig frei

14 Gemeint sind die Eigenschaften der "Apposition", der "Rechtsversetzung" und des "Nachtrags". 
wählbar. ${ }^{15}$ Zum einen gibt es einige Fälle, in denen nicht alle drei Zeichen Anwendung finden dürfen (grammatische Restriktion), zum anderen kann man durch die Wahl Nuancen hinsichtlich der stärke des Einschubs zum Ausdruck bringen. Dies liegt wahrscheinlich daran, daß die Rundklammer im Ggs. zu Gedankenstrich und Komma als nicht so stark "abtrennend" bzw. weniger "nachdrücklich" empfunden wird: 16

(15.31) Dieser Kerl (mit dem lustigen Hut) vollführte Kapriolen.

(?) Dieser Kerl - mit dem lustigen Hut - vollführte Kapriolen.

?Dieser Kerl, mit dem lustigen Hut, vollführte Kapriolen.

(15.32) (?) Das ranzige (immerhin war es schon 8 Wochen alt) Fett stank.

?? Das ranzige - immerhin war es schon 8 Wochen alt - Fett stank.

* Das ranzige, immerhin war es schon 8 Wochen alt, Fett stank.

Wenn man z.B. ein Attribut als Zusatz markiert (15.31), geschieht dies am ehesten und eindeutigsten mittels der Rundklammer. Der Gedankenstrich nimmt stärker aus, was zwar nicht zur Inakzeptabilität führt, diesen zusatz jedoch recht stark abtrennt. Die Kommamarkierung führt zu einer gewissen Mehrdeutigkeit, da nun auch eine (zugegebenermaßen etwas fernerliegende) Lesart der PP als hinter das im Vorfeld befindliche subjekt eingeschaltete Instrumentaladverbiale möglich wird. Noch deutlicher werden die Unterschiede bei einem Zusatzversuch innerhalb einer Nominalphrase (15.32): Während der rundgeklammerte Schaltsatz einigermaßen akzeptabel anmutet, wirken Gedankenstriche und Kommamarkierung inakzeptabel.

Unterschiede sind graduell beim Schreibzeichengebrauch festzustellen. Akzeptabilitätsdifferenzen entstehen jedoch nicht notwendigerweise durch syntaktische Abweichungen (Restriktionen), vielmehr sollte man von von angemessener bzw. weniger angemessener Verwendung eines Schreibzeichens sprechen. Sie seien ausschnittweise vorgeführt:

(15.33) Pia ? (Köchin aus Passion)/ ?- Köchin aus Passion -/, Köchin aus Passion, macht heute Karpfen blau.

(i5.34) Sit ? (die piaj/ ?- die Pia -/, die Pia, mag Karpfen blau. Sie mag Karpfen blau * (die Pia)/ ?- die Pial, die Pia.

(15.35) Viele Vögel (einschlieBlich/ vor allem/z.B. die Milane)/ - einschlieblich/ vor allem/z.B. die Milane -/, einschlieblich/ vor allem/z.B. die Milane, werden gejagt.

Viele Vögel werden gejagt (einschlieblich/ vor allem die Milane)/ - einschlieblich/ vor allem die Milane -/, einschlieblich/ vor allem die Milane.

(15.36) Die Haie (obendrein/ferner die Muränen)/ - obendrein/ ferner die Muränen - /, obendrein/ ferner die Muränen, werden gefürchtet.

15 Zum Begriff "Schreibzeichen" vgl. Kap. 14, Fußnote 4.

16 So jedenfalls DUDEN (1972: 372), Berger (1982: 156) und Engel (1988: 838). 
Die Haie werden gefürchtet (obendrein/ ferner die Muränen)/ - obendrein/ ferner die Muränen/, obendrein/ ferner die Muränen.

(15.37) Eine Spielerei (anders gesagt/ m.a.W. eine Laune)/ - anders gesagt/ m.a.W. eine Laune-/, anders gesagt/ m.a.W. eine Laune, ist sein neues Hobby.

(15.38) Eine Spielerei ist sein neues Hobby (anders gesagt/ m.a.W. eine Laune// - anders gesagt/ m.a.W. eine Laune/, anders gesagt/ m.a.W. eine Laune.

(15.39) Harry Hirsch (FDP)/ ??- FDP -/ ?, FDP, hat das gesagt. Immanuel Kant (1724-1804)/??-1724-1804-/*, 1724-1804, lebte in Königsberg.

James Bond (Roger Moore)/ ?? - Roger Moore -/*, Roger Moore, (...). Die Rechtschreibung (Orthographie)/ ?- Orthograpbie -/*, Orthographie, beherrscht sie perfekt.

Die Deutsche Sexpartei (DSP)/ ??-DSP -/*, DSP, wählt Pia.

Nachstehend findet sich eine Zusammenfassung der Tendenzen. Die Markierungen beziehen sich auf die ublichkeit: ${ }^{17}$

\section{Komma Gedankenstrich Rundklammer}

PRAZ:

REFZ (eng):

REFZ (weit) :

SPEZ (eng):

SPEZ (weit):

NSPEZ (eng):

ALNZ (eng):

ALNZ (weit):

Kurzzusatz:

APREL:

EMG :
NSPEZ (weit):

$\begin{array}{ccc}+ & ? & ? \\ + & ? & ? \\ + & ? & - \\ + & + & + \\ + & + & + \\ + & + & + \\ + & + & + \\ + & + & + \\ + & + & + \\ ? / ? ? /- & ? / ? ? /- & + \\ + & + & + \\ - & + & \end{array}$

Einen Block bilden spezifizierende, nichtspezifizierende und Aiternativen nennende Zusätze, bei denen alle drei Schreibzeichen verwendbar sind. Der Kurzzusatz bevorzugt die stets akzeptable Rundklammerung, weist allerdings hinsichtlich der beiden anderen Schreibzeichen schwankungen in der oblichkeit auf (vgl. Ubersicht am Ende von Kap. 13). Diese Bevorzugung deckt sich mit der

17 "-" = 'unüblich'; "??" = 'eher unüblich'; "?" = 'weniger/evtl. üblich'; "(?)" = 'öfters verwendet'. "+" resp. Unmarkiertheit bedeutet, daß eine übliche Verwendung vorliegt. 
oben gemachten Aussage, daß die Rundklammer primăr zur Markierung für Zusätze zu Wörtern (sogar innerhalb von Phrasen) verwendet wird.

Der prädizierende und der referenzklärende Zusatz ähneln sich: das Komma ist am üblichsten; der Gedankenstrich und die Rundklammer sind weniger üblich, die Rundklammer wird beim REFz kaum angewendet.

\section{Stellung}

Die Rontaktstellung ist stets akzeptabel; sie repräsentiert of fenbar die unmarkierte Option. Interessanter sind mögliche Distanzvarianten in einem Verbzweitsatz:

(a) Basis im Vorfeld, Zusatz im Mittelfeld;

(b) Basis im Vorfeld, Zusatz nach KlammerschluB;

(c) Basis im Mittelfeld - Klammerschlus -Zusatz;

(d) Basis im Mittelfeld - Satzglied - KlammerschluB - Zusatz.
(a)
(b)
(c)
(d)

$\begin{array}{lllll}\text { PRAZ: } & \text { ? } 18 & \text { ? }^{19} & \text { ?2 }^{20} & \text { ?? } \\ \text { REFZ: } & (-) & + & + & + \\ \text { SPEZ: } & ?^{21} & + & + & + \\ \text { NSPEZ: } & ? ? & (+) & (+) & (+)\end{array}$

18 Bei Zusatz der Rategorie $\mathbb{N P}$ wohl als stilistische Eigenwilligkeit zu werten; AdjP und PP als Appositive sind in Mittelfeld inakzeptabel oder erhalten eine andere Interpretation.

19 Wie in Rap. 6 bereits erwähnt, hängt die Akzeptabilität von der Rategorie und der Enweiterung des Zusatzes ab (bei NPn; vgl. F15.6). Bei prädizierenden Zusătzen der Rategorien AdjP oder PP bewahrt die Distanzstellung die Lesart nicht (Kopulasatzumschreibung! S. F15.7/8); falls eine andere Lesart (z.B. Nachtrag eines Adverbials) möglich ist, entsteht ein akzeptables Syntagma.

(F15.6) Jener Graf hat alle Fraven betört, ?? Schürzenjäger aus Leidenschaft/ ? ein Schürzenjäger aus Leidenschaft/ (?) vermutlich ein Schürzenjäger aus Leidenschaft/ (?) vermutlich eben doch ein Schürzenjäger aus Leidenschaft.

(15.7) Jener Graf hat alle Fraven betört, *alt und knorrig/ (und zwar) charmant und galant.

(F15.8) Das Rathaus wird bald renoviert, (und zwar) am Marktplatz.

20 Für diese Möglichkeit finden sich manche Beispiele (vgl. Kusmin 1960: 72 ff.), doch systematisch ist diese option nicht. Wiedenm ist die kategoriale Fullung (die N-Bar-Stufe: je höher desto akzeptabler) bzw. die Erweiterung (je mehr Satzadverbien, Gradpartikeln, Floskeln etc. desto selbständiger) ausschlaggebend. AdjP und PP sind aber wiederum nur uninterpretiert (Nachtrag!) akzeptabel.

21 Mur bei einigen Subtypen möglich; selbst dann ist es fraglich, ob ein akzeptabler Ausdruck vorliegt:

(F15.9) ?Viele Vögel sind seit einiger Zeit, ausgenammen/ besonders die Milane, vam Aussterben bedroht. 

(a)
(b)
(c)
(d)

$\begin{array}{lcccc}\text { ALNZ: } & ? ? & (?)^{22} & (?) & (?)^{23} \\ \text { Kurzzusatz: } & ? ? /- & ? /- & +/- & +/- \\ \text { APREL: } & - & - & + & ? ? \\ \text { EMG: } & - & - & - & -\end{array}$

Die stellungsvariabilität ist recht unterschiedlich. Einschaltungsmarkierte Gliedteile haben feste Plätze (wie die attributiven Pendants). Bei den Kurzzusätzen ist Dislokation kaum statthaft. Dabei hat man zwischen den strikt adjazenten (m.E. prototypischen) Kurzzusätzen (die deutsche sexpartei (DSP); Markierungen rechts des Schrägstriches) und den nicht strikt adjazenten Mustern (seine Post-moderne Zentrale (194 Millionen Mark); Markierungen links des Schrägstrichs) zu differenzieren.

Beim prädizierenden Zusatz, der in Form von NP (plus Funktionslexeme etc.) immerhin einige stilistische Variationen gestattet, kommt eine Distanzstellung eher selten vor. Dies liegt vor allem an der möglichen Veränderung der Konstruktion: dem Wechsel zum spezifizierenden Zusatz bei Adjp (dann als modales Adverbial) und PP (dann als lokales Adverbial). Der APREL - mit dem der PRAZ (Kopulaverb!) bisweilen in Verbindung gebracht wurde - kann immerhin über ein klammerschlieBendes Element extraponiert werden.

Die Alternativen nennenden Zusätze scheinen in ihrer stellungsvariabilität nicht ganz so frei zu sein wie die (nicht-) spezifizierenden zusătze; die Urteile sind im einzelnen schwierig und wären durch eingehendere Analysen zu untermauern. Recht variabel sind referenzklärende und spezifizierende zusătze; erstere vielleicht aufgrund ihrer eher pragmatischen Motivation und einer daraus resultierenden lockereren "Syntaktifizierung", letztere evtl. infolge ihrer funktionalen Nähe zu Korrekturen (vgl. Kap. 13).

ZusammengefaBt seien auch die Abfolgebeziehungen der Typen untereinander; sie müssen allerdings noch genauer erforscht werden (z.B. NSPEZ). Sofern sich die zusätze auf die gleiche Basis beziehen, gilt wohl die Grundfolge:

BASIS - (REFZ) - RurZZusatz - PRAZ - SPEZ - ALR - verallgen. Zusatz.

Hierzu ein paar unterstützende Beispiele:

22 Vgl.: (F15.10) Die Existenzformen der Sprache sind (??, anders gesagt die Erscheinungsformen,) von Pia untersucht worden ((?), anders gesagt die Brscheinungsformen).

(F15.11) Ein lediger Mann ist dort umworben worden ((?), anders gesagt/ m.a.W.: ein freier Mann).

$23 \mathrm{Vgl.:}$ (F15.12) Von den Damen ist ein lediger Mann (heftig) ummorben worden ((?), anders gesagt/ m.a.W. ein freier Mann). 
(15.40) Die Deutsche Sexpartei (DSP), ein wüster Haufen/, lustig und fidel, (*DSP) tagt wieder.

(15.41) Die Rechtschreibung (Orthographie), ein Vorschriftendschungel, (*Orthographie) ist kompliziert.

(15.42) Ihre Existenzformen, bislang unerforscht, m.a.W. ihre Zustandsformen, allgemeiner: ihre Erscheinungsformen, müssen erforscht werden.

(15.43) Sie (`DSP), die Deutsche Sexpartei (DSP), ein wüster Haufen, tagt wieder.

Zusatz während der Oberarbeitung: An dieser stelle war eine ausführliche Untersuchung der Abfolgebeziehungen von zusätzen und (a) nicht-restriktiven Relativsätzen, (b) NP-bezogenen Schaltsătzen, (c) Vokativischen NPn (VokNP) am rechten Satzrand, (d) der Extraposition eines Gliedsatzes (EXT GIS) und (e) dem Nachtrag (NT) eines Satzgliedes (Adverbials) geplant. Sie fiel der veröfentlichungsbedingten Kürzung zum Opfer, da sie zur Definition von ZUSATZ sowie zur Subklassifizierung der Zusatz-Muster - dies sind die primären Ziele meiner Arbeit - keinen wesentlichen Beitrag leistet. Eine gesonderte Veröffentlichung wird erwogen.

Nun soll die Stellung der Zusätze relativ zu Postpositionen, nachgestellten Gradpartikeln und Konjunktionaladverbien untersucht werden. Bei dem prădizierenden Zusatz wurde bereits festgestellt, daB nur die Abfolge " $X$ - PRAZ" akzeptabel ist (vgl. Kap. 6).

Die referenzklärenden Zusätze stehen nicht vor Postposition (Rundklammervariante ausgenommen, 15.44a). Die Reihenfolge "PostP - Zusatz" ist nur dann akzeptabel, wenn die Postposition im Zusatz wiederholt wird - die Postposition beim Bezugselement darf dann rückwärtsgetilgt werden. Es muB zwar - wie unsere Beispiele vorführen - nicht unbedingt Inakzeptabilität entstehen, jedoch ändert sich die Konstruktion sowie deren Sinn: der Pia wirkt dann wie ein eingeschalteter dativus commodi.

(15.44a) *Ihr, der Pia, zuliebe kochten sie Hummer.

?? Ihr - der Pia - zuliebe kochten sie Hummer.

? Ihr (der Pia) zuliebe kochten sie Hummer.

(15.44b) *Ihr zuliebe, der Pia, kochten sie Hummer.

* Ihr zuliebe - der Pia - kochten sie Hummer.

* Ihr zuliebe (der Pja) kochten sie Hummer.

(15.44c) Ihr (zuliebe), der Pia zuliebe, kochten sie Hummer.

Ihr zuliebe - (ich meine) der Pia zuliebe - kochten sie Hummer.

Ihr zuliebe (der Pia zuliebe) kochten sie Hummer.

Die Abfolge "REFZ - GP/Konjadv" ist (Rundklammerung evtl. ausgenommen) wieder inakzeptabel. Ohne daB ich Gründe dafür anzugeben vermag, erscheint allerdings die Abweichung beim nachfolgenden Konjunktionaladverb nicht gar so kraB. Die Nachstellung des Zusatzes ist unmarkiert. 
(15.45a) *I hr, der Pia, sogar kochten sie Hummer.

*I hr - der Pia - sogar kochten sie Hummer.

? I hr (der Pia) sogar kochten sie Hummer.

(15.45b) I hr sogar, der Pia, kochten sie Hummer.

I hr sogar - (ich meine) der Pia - kochten sie Hummer.

I hr sogar (der Pia) kochten sie Hummer.

(15.45c) ?? Ihr, der Pia, jedoch kochten sie Hummer.

?? Ihr - der Pia - jedoch kochten sie Hummer.

? Ihr (der Pia) jedoch kochten sie Hummer.

(15.45d) Ihr jedoch, der Pia, kochten sie Hummer.

Ihr jedoch - der Pia - kochten sie Hummer.

Ihr jedoch (der Pia) kochten sie Hummer.

Die spezifizierenden Zusätze lassen sich nicht zwischen Basis und Postposition schieben, außer bei der rundgeklammerten Version, die mir nicht sehr abweichend erscheint, ja evtl. völlig akzeptabel ist. Möglich ist die Position nach der Basispostposition (sie kann auch im Zuge der Rückwärtstilgung gleicher Elemente wegfallen), wobei diese im Zusatz wiederholt werden muß, sonst entsteht ein widersinniges Syntagma $(15.46 \mathrm{~b}$; zuerst verbietet man den Vögeln zuliebe und dann den Vögeln selbst die Jagd). Es nimmt nicht wunder, daß die Postposition zu wiederholen ist, sofern man das Konstrukt "Basis + Zusatz" in Beziehung zu einem verdoppelten Satz setzt, wie (15.46d) demonstriert; dort muß die Postposition ja auch stehen ("0" = "Weglassen des Elements").

(15.46a) *Den Vögeln, genauer den Singvögeln, zuliebe verbot man die Jagd. *Den Vögeln - genauer den Singvögeln - zuliebe verbot man die Jagd. (?) Den Vögeln (genauer den Singvögeln) zuliebe verbot man die Jagd.

$(15.46 \mathrm{~b})$ *Den Vögeln zuliebe, genauer den Singvögeln, verbot man die Jagd.

(15.46c) Den Vögeln (zuliebe), genauer den Singvögeln zuliebe, verbot man die Jagd.

Den Vögeln (zuliebe) - genauer den Singvögeln zuliebe/ $\star_{0}$ - verbot man die Jagd.

Den Vögeln zuliebe (genauer den Singvögeln zuliebe/ $\star 0$ ) verbot man die Jagd.

(15.46d) Den Vögeln zuliebe verbot man die Jagd. Genauer: Man verbot den

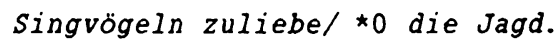

Eine auf das Basisnomen bezogene nachgestellte Gradpartikel verlangt bei den spezifizierenden zusätzen, daß man den zusatz nachfolgen läBt; die sonst inakzeptable Abfolge "Zusatz - GP" ist bei Rundklammerung (einigermaBen) annehmbar. Aus dem Zusatz heraus kann sich eine Gradpartikel nicht auf das Basisnomen beziehen. 
(15.47a) *Manche $V$ ö gel, besonders die Singvögel, sogar jagte man dort. * Manche $V$ ö gel - besonders die singvögel - sogar jagte man dort.

(?) Manche $V$ ö gel (besonders die singvögel) sogar jagte man dort. 24

(15.47b) Manche $V$ ö gel sogar, besonders/ einschließlich/z.B. die Singvögel, jagte man dort.

(15.47c) * Manche $V \ddot{o}$ gel, besonders die Singvögel sogar, jagte man dort. Das Verhalten bzgl. des nachgestellten Konjunktionaladverbs bestätigt die bislang ermittelten Regularitäten: "Zusatz - Konjadv" ist außer bei Rundklammerung wohl inakzeptabel, wenngleich mir auch hier die Abweichung nicht so kraB erscheint wie z.B. bei Postposition und Gradpartikel. Die Folge "Konjadv - Zusatz" ist unmarkiert.

(15.48a) ??Manche Vögel, besonders die Singvögel, jedoch jagte man dort. (?) Manche Vögel (besonders/ einschließlich/z.B. die singvögel) jedoch jagte man dort.

(15.48b) Manche Vögel jedoch, besonders/ einschließlich/genauer/2.B. die Singvögel, jagte man dort.

Die Untersuchung der nichtspezifizierenden Zusätze führt zu erwartbaren Ergebnissen: "X - NSPEZ" ist die akzeptable Reihenfolge, eine Postposition muB im Zusatz wiederholt werden; umgekehrt ist lediglich die Rundklammerversion einigermaBen annehmbar.

(15.49a) *Den Vögeln, weniger den Singvögeln, zuliebe verbot man die Jagd. *Den Singvögeln, allgemeiner den Vögeln, zuliebe verbot man die Jagd.

(?) Den Singvögeln (allgemeiner den Vögeln) zuliebe verbot man die Jagd.

(15.49b) Den Vögeln (zuliebe), weniger den Singvögeln zuliebe, verbot man die Jagd.

Den Singvögeln (zuliebe), allgemeiner den Vögeln zuliebe/ $\star_{0}$, verbot man die Jagd.

(15.49ci * Die $V$ ö gel, weniger die Singuögel, sogar jagte man.

*Die $S$ i ngvögel, allgemeiner die Vögel, sogar jagte man.

(15.49d) Die $V$ ö gel sogar, weniger die singvögel, jagte man.

Die $S$ i nguögel sogar, allgemeiner die Vögel, jagte man.

(15.49e) ??Die Vögel, weniger die Singvögel, jedoch jagte man.

?? Die Singvögel, allgemeiner die Vögel, jedoch jagte man.

(15.49f) Die Vögel jedoch, weniger die Singvögel, jagte man.

Die singvögel jedoch, allgemeiner die Vögel, jagte man.

Der Typ Alternative nennender Zusatz verhält sich im großen und ganzen wie die. vorigen Muster: " $X$ - Zusatz" ist die unmarkierte Folge. Die Postposition muB wiederholt werden.

24 Geschrieben wirkt diese Variante recht akzeptabel, doch es ist fraglich, ob das Symtagma in der gesprochenen Sprache - mit entsprechender Parentheseintonation des Zusatzes und Fokusakzent auf der NP davor - akzeptiert werden kann. 
(15.50a) *Den Ledigen, m.a.W. den Unverheirateten, zuliebe tat man das. (?) Den Ledigen (m.a.W. den Unverheirateten) zuliebe tat man das.

(15.50b) Den Ledigen (zuliebe), m.a.W. den Unverheirateten zuliebe, tat man das.

(15.50c) *Den $L$ e digen, m.a.W. den Unverheirateten, sogar tat man das an. (?) Den $L$ e digen (m.a.W. den Unverheirateten) sogar tat man das an.

(15.50d) Den L e digen sogar, m.a.W. den Unverheirateten, tat man das an.

(15.50e) ??Den Ledigen, m.a.W. den Unverheirateten, jedoch tat man das an. (?) Den Ledigen (m.a.W. den Unverheirateten) jedoch tat man das an.

(15.50f) Den Ledigen jedoch, m.a.W. den Unverheirateten, tat man das an. Die Kurzzusätze weichen in ihren Eigenschaften deutlich ab. Sie stehen strikt beim Bezugselement; Postpositionen, Gradpartikeln und Konjunktionaladverbien folgen nach. Hinzuweisen ist auf von der strikten Adjazenz abweichende Kurzzusătze wie in (15.51d).

(15.51a) Harry Hirsch (CDU) zuliebe (??CDU) wartete man.

Immanuel Kant (1724-1804) zuliebe (??1724-1804) wurde das nicht gedruckt.

Der Deutschen Sexpartei (DSP) zuliebe (??DSP) tat man das.

(15.51b) Harry H i rsch (CDU) sogar (?? CDU) konnte das. Immanuel $K$ a nt (1724-1804) sogar (??1724-1804) wurde gedruckt. Die Deutsche $S$ e xpartei (DSP) sogar (??DSP) druckte Kant.

(15.51c) Harry Hirsch (CDU) jedoch (??CDU) wartete ab. Immanuel Kant (1724-1804) jedoch (??1724-1804) wurde gedruckt. Die Deutsche Sexpartei (DSP) jedoch (??DSP) druckte Kant.

(15.51d) Dem Neubau (? 100 Millionen) zuliebe (100 Millionen) stellte er alle anderen Projekte zurück.

Den $N$ e ubau (?100 Millionen) sogar (100 Millionen) fand man $z u$ teuer.

Den Neubau (?100 Millionen) jedoch (100 Millionen) fand man $z u$ teuer.

Eine Ubersicht der Resultate ergibt das nachstehende Bild:

$\begin{array}{ll}\text { Zusatz steht vor } & \text { Zusatz steht nach } \\ \text { PostP/ GP/ Konjadv } & \text { PostP/ GP/ Konjadv }\end{array}$

$\begin{array}{lll}\text { PRAZ: } & \text { nein, außer evtl. ( ) } & \text { ja } \\ \text { REFZ: } & \text { nein, außer evtl. ( ) } & \text { ja } \\ \text { SPEZ: } & \text { nein, außer evtl. ( ) } & \text { ja } \\ \text { NSPEZ: } & \text { nein, auBer evtl. ( ) } & \text { ja }\end{array}$


Zusatz steht vor

PostP/ GP/ Konjadv
Zusatz steht nach

PostP/ GP/ Konjadv

ALNZ :

nein, außer evtl. ( )

ja

Kurzzusatz: ja

Zu untersuchen bliebe noch, in welchem Umfang und an welchen Stellen ZUSKTZE innerhalb von Phrasen, z.B. NPn, aufzutreten vermögen; bekanntlich ist dies u.a. eine Eigenschaft von gewissen EINSCHUBEN. Ich erinnere an das Beispiel (12.2d): (..) die GewiBheit - und das heiBt die exakte Methode - der Mathematik, (...), in dem ein spezifizierender (oder Alternative nennender?) Zusatz zwischen Kern-NP und Genitivattribut steht. Nachstehend führe ich nur wenige Beispiele für diese Position auf (weitere Analysen wären wünschenswert). Sie zeigen, das prädizierender und referenzklärender zusatz nicht ganz akzeptabel anmuten (15.52a, b), zumindest markiert wirken; vielleicht ist (15.52b) - man spreche die Sequenz einmal aus - aber dennoch möglich. Dagegen sind SPEZ, NSPEZ, ALNZ und KURZ (15.52c-f) evtl. völlig annehmbar. Hier kommt vermutlich zum Vorschein, daß der PRAZ eine Prädikation über die Basis darstellt (ich erinnere an Löbels Begriff des "attributiven Prädikativs"), während SPEZ, NSPEZ und ALNZ eher wie eine Doppelsetzung der Basis wirken (zumindest die spezifizierenden Zusätze haben ohnedies eine Nähe zur KORREKTUR).

(15.52a) ?die GewiBheit - ein Ziel der Menschen - der Mathematik

(15.52b) (?) die - ich meine die Freuden - der Mathematik

(15.52c) die GewiBheit - genauer die exakte Methode - der Mathematik

(15.52d) die GewiBheit - zudem das Vergnügen - der Mathematik

(15.52e) die GewiBheit - m.a.W. die exakte Methode - der Mathematik

(15.52f) das Zentralkommitee $(Z K)$ dieses Landes

\section{Funktionen (auf semantischer und pragmatischer Ebene)}

Bevor die speziellen semantischen Funktionen behandelt werden, seien noch einige allgemeine Hinweise zu den Funktionen von ZUSATZEN vorausgeschickt:

(a) ZUSATZE können, hierin Attributen ähnlich, in einen Satz zusätzliche ("sekundäre") Propositionen resp. Prädikationen einbringen. Somit dienen sie der Propositionsverdichtung, allgemeiner gesprochen: der Informationsverdichtung (Impandierung; vgl. Moskalskaja 1984: $146 \mathrm{ff.}$.). Auch Ortner (1983: 116) weist hierauf hin. Anstatt Propositionen auf mehrere Sätze zu verteilen (worin jedem Propositionsteil, also Prädikaten, Argumenten etc., ein Ausdrucksträger zukommt) werden sie innerhalb eines Satzes zum Ausdruck gebracht, wobei nicht jeder Propositionsteil durch einen Ausdruck repräsentiert wird (dies veran-

25 Typische Kurzzusätze stehen strikt rechtsadjazent, die untypischeren (15.51d) eher nicht. Evtl. muß man in Bereich der Kurzzusätze zwei Submuster annehmen. 
schaulicht 11.53a; aus: Moskalskaja 1984: 151). Die semantischen Relationen müssen aufgrund der Ausdruckskonzentration z.T. erschlossen werden; da jedoch die Zusatz-Muster (mehr oder weniger) stark grammatikalisiert sind (und ggf. Funktionslexeme den Verstehensprozeß unterstützen), fällt die Interpretation i.d.R. nicht allzu schwer.

(11.53a) Am 4. Oktober 1957 leitete Sputnik 1 das Zeitalter der Raumfahrt ein. Sputnik 1 war der erste von Menschenhand geschaffene Himmelskörper > Am 4. Oktober 1957 leitete Sputnik 1, der erste von Menschenhand geschaffene Himmelskörper, das Zeitalter (...) ein.

Die "informationsverdichtende Funktion" wird insbesondere von den prädizierenden, den (nicht)spezifizierenden, den Alternativen nennenden und den Kurzzusätzen (und in geringerem Maße von den Relativsatz-zusätzen und evtl. den referenzklărenden Zusätzen) wahrgenommen.

(b) Vor allem die zusatzmuster, die in Beziehung mit den Funktionen KORREKTUR (Präzisierung, spezifizierung etc.) und PARAPHRASIERUNG (variierende Wiederholung, Umschreibung usf.) stehen, nehmen Funktionen wahr, die man umrißhaft mit Informationsnuancierung resp. -optimierung umschreiben kann, also ein sowohl "darstellungs-" als auch "hörerorientierter" Aspekt. Nicht immer vermögen wir ein Konzept(detail) mit einem Ausdruck vollständig - oder zumindest ausreichend - zu "worten", nicht immer haben wir einen treffenden Ausdruck (ein Lexem) zur Verfügung, so daß wir das "Gemeinte" von vornherein umschreiben werden oder versuchen werden, einen bereits gesetzten Ausdruck, den wir als noch unzulänglich empfinden, nachträglich zu "verbessern". (11.53b) demonstriert einen derartigen Fall: ${ }^{26}$

(11.53b) (...) ich hab ihn auch so verstanden, daB er meinte Texte des A11tags, also Dokumente, die nicht literarisch sind, zum Beispiel Reden, politische Reden, Werbetexte (...).

Doch nicht nur (nicht)spezifizierende und Alternativen nennende zusătze, sondern auch die übrigen Muster (prädizierender Zusatz, Relativsatzzusatz etc.) können der Erhöhung des Textverständnisses (durch In-Erinnerung-Rufen von Vorwissen usf.) dienen; somit sind sie u.a. als strategien für eine "didaktisch" günstige Vermittlung (von Wissen usw.) nutzbar zu machen.

(c) Ein funktionaler Aspekt vor allem der einschaltungsmarkierten Gliedteile, der prădizierenden und der Relativsatzzusătze ist das Phănomen der Informatjonsgewichtung (bzw. -schattierung). Das Einschaltungsmuster kann einerseits einen sprachlichen Ausdruck als "weniger wichtig", "beiläufig" u.ä. markieren, andererseits kann es die Aufmerksamkeit gerade auf den betreffenden Ausdruck lenken und diesen hervorheben (z.B. als Kommentierung durch den Pro-

26 Aus: Rath (1979: 195). Rath (1979: 188 ff.) diskutiert, auch anhand von Textbeispielen, teilweise die hier genannten funktionalen Aspekte von Paraphrasierungen und Korrekturen. 
duzenten wie in $11.53 \mathrm{c}) .27$ Dieser funktionale Aspekt kann hier leider nicht năher untersucht werden.

(11.53c) In Anlehnung an "Phraseodidaktik" mag "Phraseopragmatik" als ein (zugegeben: schlechter) Arbeitsterminus (...) gelten. 28

4.a. PRAZ: (a) Sekundäre (quasi präsupponierte, nicht als Gegenstand weiterer Erörterung angesehene) Eigenschaftszuschreibungen (NP, AdjP), (b) Klasseneinordnungen (NP), (c) Lokalisierungen (PP) usw. Evtl. auch (d) Identitätsaussagen, wobei Ambiguität zwischen prädizierenden und referenzklärenden Zusätzen entstehen kann, vor allem wenn eine unerweiterte NP als Zusatz fungiert; deutlich wird eine Trennung erst durch Hinzufügung entsprechender Funktionslexeme bzw. Erweiterungen (z.B. ADVB; vgl. 11.54a, b). (11.54 c) führt einen Fall vor, bei dem sich die funktional-semantische Nähe bzw. Uberschneidung mit sowohl dem umformulierenden als auch dem präzisierenden zusatz zeigt (man beachte die einerseits obligatorische, andererseits inakzeptable Artikelsetzung):

(15.54a) Der Sprecher der Gruppe, (er ist übrigens zugleich) ihr Leiter, soll bitte herkommen.

(15.54b) Der Sprecher der Gruppe, (ich meine) ihr(en) Leiter, soll bitte herkommen.

(15.54c) Die Experten, genauer: die Fachidioten/m.a.W.: die Fachidioten/ übrigens (*die) Fachidioten, gockelten sich auf.

4.b. REFZ: Verdeutlichung der Referenz eines Pro-Elements bzw. eines Ausdrucks mit unklarer (vager) Referenz. Diese Funktion ist eher "pragmatisch" (Einstellung der Nachricht auf den Hörer bzw. das Hörerverständnis) denn "semantisch" zu begründen. Eine semantisch mengenrelationierende Funktion ist beim REFZ (im Ggs. beispielsweise $z u$ den spezifizierenden Zusătzen) nicht intendiert. Funktionale Ahnlichkeiten weist der REFZ dennoch mit den umformulierenden und den spezifizierenden Zusätzen auf :

(15.54d) Wer heute - das heiBt/ genauer:/ ich meine nicht zu stalins, nicht $z u$ Chruschtschows, nicht zu Breshnews, sondern $z u$ Gorbatschors Zeiten - in Moskau einkauft, (...).

4.C. SPEZ: Bei NP-NP-Konstellationen setzt die Basis eine Gesamtmenge und der Zusatz nennt eine Teilmenge, wobei

(a) die Gesamtmenge (extensional) eingeschränkt (präzisiert) werden kann, so daß das Satzglied (meist Subjekt, objekt) eine "Teilkorrektur" (in Grenzfällen eine "Totalkorrektur") erfährt. Für die Aussage des Restsatzes ist dann auch die Teilmenge relevant - d.h. der zusatz ist semantisch ebenso wichtig wie das eigentliche Satzglied (PZ)! Die Verwandtschaft mit der Korrektur deutet das nachstehende Beispiel an, wobei die Anwesenheit von nein (korrekturin-

27 Psycholinguistische Experimente, die untersuchen, ob das Einschaltungsmuster die Auffäligkeit des markierten Ausdrucks erhöht oder herabsetzt (Behaltensleistung), wären von Interesse.

28 Aus: Pilz, Klaus Dieter. 1981. Phraseologie: Redensartenforschung. Stuttgart, Metzler. S. 113. 
dizierendes Lexem) nur dann akzeptabel wirkt, wenn die Mengen klar distinkt sind (ohne mögliche Durchschnittsmenge):

(15.54e) Sie haben Raubfische, ?nein/ genauer: Haie, beobachtet. Sie haben Raubfische, nein:/ *genauer: Heringe, beobachtet.

(b) aus der Gesamtmenge einzelne Teilmengen resp. Elemente als Beispiele aufgezăhlt werden können (BZ),

(c) Teilmengen bzw. Elemente explizit eingeschlossen werden können - wohl um zu verhindern, daß der Empfänger diese Teilmenge nicht (genügend) berücksichtigt, um diese in Erinnerung zu rufen etc. (MZ) und

(d) Teilmengen/Elemente hervorgehoben werden können, damit ihre besondere Betroffenheit dem Empfänger im Bewußtsein ist etc. (HZ).

Das semantische Konzept von Menge und Element(en) erscheint möglicherweise $z u$ eng gefaBt, wenn man - wie bislang geschehen - vornehmlich Fålle ansieht, in denen wie in den "Tier"-Beispielen auf wohlunterscheidbare objekte referiert wird, und wenn man Abstrakta - überhaupt Ausdrücke, die keine Mengen im engen Sinne denotieren - außer acht läßt. Fälle wie Die Liebe (besonders die Näcbstenliebe) oder Den Kubismus (vor allem Braque und Picasso) schätzt Pia sehr und natürlich die Zusatzbeispiele mit nicht-nominalen Basen (z.B. Zubereitet - vor allem gekocht und gegrillt - hat die Speisen Pia) geben evtl. AnlaB zur Rritik. Man faBte im strengen Sinne wohl weder Die Liebe, Den Kubismus und Zubereitet als Mengen noch die Nächstenliebe, Braque und Picasso sowie gekocht und gegrillt als deren Elemente auf, es sei denn, man operierte mit einem dementsprechend abgewandelten Mengen- bzw. Elementbegriff. Gerade dies mutet m.E. nicht abwegig an, denn Nächstenliebe bezeichnet einen Teilbereich von Liebe (es besteht die Sinnrelation der Hyponymie), Braque und Picasso zwei Vertreter des Kubismus (Kubismus bezeichnet das Umfassendere); schlieblich bezeichnen kochen und grillen zwei Handlungen, die sich - mit weiteren - zu den Handlungen des Zubereitens von Speisen zusammenfassen lassen.

Demnach scheint mir trotz der Problembeispiele das Konzept nicht prinzipiell entkräftet zu sein; vielmehr kommt es mir vor, als sei das problem erstens ein terminologisches bzw. definitorisches (was will man unter Mengen verstehen? etc.) und zweitens eines, das ohnedies genauerer Durcharbeitung bedürte. Da eine "Semantik der Zusätze im Deutschen" im Augenblick meine Kräfte überschreitet und die Arbeit weiter in die Länge zöge, möchte ich bei meiner gewiß noch präzisierungsbedürftigen semantikexplikation bleiben und darauf hinweisen, daß noch Arbeit zu leisten wäre.

4.d. Bei den übrigen Menge-(Teil)Menge-Relationen (den nicht-spezifizierenden Zusătzen) versammeln sich unterschiedliche subfunktionen:

(a) Eine Menge wird hin zu einer hierarchisch höheren obermenge erweitert; hier liegt eine Beziehung vor, die sich zu den spezifizierenden zusätzen konträr verhält (DEPRAZISIERUNG). Semantisch verwandt erscheint hier die Korrektur und evtl. der prăzisierende Zusatz, was besonders das zweite Beispiel (aus: Schwarze/Wunderlich 1985: 49) nahelegt: 
(15.54f) Sie haben dort Haie, allgemeiner:/ ?genauer:/ nein: Raubfische, beobachtet.

Ihr wichtigster Unterschied betrifft die Eigenschaft der Wahrheitsfunktionalität (allgemeiner und genauer: der Distributivität im Sinne von 2.1.2).

(b) Die Basis setzt eine Gesamtmenge und (ba) der Zusatz eine Teilmenge, die als in jedem Fall von dem Sachverhalt betroffen dargestellt wird, in den die Gesamtmenge involviert ist (EZ). Es kann unklar bleiben, ob nun nur die Teilmenge relevant für die semantische Interpretation des Satzes ist, oder ob die Gesamtmenge relevant bleibt. ${ }^{29}$ Im anderen möglichen Fall wird (bb) eine Teilmenge als von dem Sachverhalt weniger betroffen dargestellt, in den die Gesamtmenge involviert ist (2Z, Z.B. Manche Raubvögel, die Bussarde weniger,). Zudem ist es (bc) möglich, daB eine Teilmenge explizit ausgeschlossen wird aus der Betroffenheit von dem Sachverhalt, in den die Gesamtmenge involviert ist (AZ, z.B. Die Raubvögel, ausgenommen die Bussarde,).

(c) Die Basis setzt eine Menge und der Zusatz nennt eine weitere Menge, die auch - evtl. in geringerem MaBe - in den Sachverhalt involviert ist (HFZ). Die Konstruktionsbedeutung weist eine Verwandtschaft mit der koordinativen (additiven) Reihung auf:

(15.54g) Sie haben Vögel (ferner/ zudem Fledermäuse) beobachtet. Sie haben Vögel (ferner/ und Fledermäuse) beobachtet.

4.e. Beim Typ ALNZ bietet der Zusatz einen alternativen Ausdruck zur Basis, der Nuancen (Teilaspekte der Bedeutung der Basis) enthalten, erläutern, hervorheben, verdeutlichen etc. kann, die bei alleiniger Basisnennung (evtl.) nicht präsent wăren (UZ). Basis und Zusatz können in mengeninklusiver (erstes Beispiel: nicht jeder Ledige ist 'nichtvergeben') oder mengenidentischer Beziehung stehen; Berührungspunkte könnten sich mit Wiederholungen und spezifizierenden zusätzen ergeben:

(15.54h) Sie hat die Ledigen, m.a.W. die Nichtvergebenen, gemustert. Sie hat die iedigen, m.a.N. die Unverheirateten, gemustert. Sie hat die Ledigen (ja: die Ledigen!) gemustert. Sie hat die Ledigen, genauer: die Nichtvergebenen, gemustert.

Der Zusatz kann andererseits eine Menge nennen, deren Extension sich nicht mit jener der Basis überlappt (es wurde Nein, bzw. Milch, getrunken); dabei lassen sich Basis und Zusatz ofters einer gemeinsamen Obermenge (Hyponymierelation, z.B. 'Getränke'!) zuordnen (ALZ). Ahnlichkeiten bestehen zur additiven Reihung, zum hinzufügenden zusatz oder zur Korrektur:

29 Vgl.: Viele Vögel, zumindest die Singvögel, werden dort gejagt kann entsprechen Die Singvögel merden dort gejagt und Viele Vögel merden dort gejagt. Bine andere Formulienmgsmöglichkeit, die die Semantik dieser Konstruktion wiedergibt, wäre wohl Viele Vägel werden dort gejagt, aber nur bei den Singuögeln bin ich mir wirklich sicher, daß dies der Fall ist. 
(15.54i) Sie haben Raubvögel, bzw./ ferner/ nein: Amseln und Stare, beobachtet.

Sie haben Raubvögel (bzw./ sowie Amseln und stare) beobachtet.

4.f. Die KURZZUSATZE sind in ihrer vielfalt kaum auf einen funktionalen Nenner $z u$ bringen und stellen ohnedies eine Typ-Klasse dar, die in sich heterogen (und somit wohl noch weiter differenzierbar) ist. Sie bringen "schlaglichtartig" (auf i.d.R. ein Wort konzentriert) Prädikationen unterschiedlichster Art in die Satzaussage ein: der "Verdichtungsgrad" ist maximal. "Typische Rurzzusätze" wie in Willy Brandt (SPD) kann man als konventionalisierte Konzentrate expliziter Aussagen ansehen (z.B. Willy Brandt (er gehört der SPD an)), die sich auf eine Wortgruppe beziehen.

Nichtrestriktive Relativsätze (in meiner Begrifflichkeit: Relativsatzzusătze) sind explizite Prädikationen, welche die NP-Referenten charakterisieren, näher erläutern, beschreiben usf., also nicht restringieren oder identifizieren (vgl. Heidolph u.a. 1981: 827 ff.). Ahnlich können Verbzweitschaltsātze fungieren, die sich phorisch auf eine Wortgruppe des Gastsatzes beziehen:

(15.54j) Diese Leute (die sich wohl für Linguisten halten/ sie halten sich wohl für Linguisten) reden ständig von Rektion.

Einschaltungsmarkierte Gliedteile üben im Ggs. zu deren attributiven Pendants keine restriktive, identifizierende Funktion aus. Sie nennen allerdings wie diese öfters Eigenschaften der Basis-Referenten, nur daB diese lediglich erläuternd, beschreibend etc. fungieren oder aber - vgl. ganz oben (c) - hervorgehoben werden sollen (als Kommentare des "Senders" o.ä.).

\section{Umformungsbeziehungen}

5.a. PRADIZIERENDE ZUSATZE: Kopulasatzparaphrasen; formal könnten sie als nichtrestriktive Relativsätze (15.55a), Verbzweit-Schaltsătze mit anaphorischen Pro-Elementen (rechtsadjazent zur Basis; 15.55b) und Verbzweit-Folgesätze $(15.55 \mathrm{c})$ auftreten:

(15.55a) Kuno hat Pia, die eine Linguistin ist, eingeladen.

(15.55b) Kuno hat Pia - sie ist eine Linguistin - eingeladen.

(15.55c) Kuno hat Pia eingeladen. Sie ist eine Linguistin.

5.b. REFERENZKLARENDE ZUSATZE: Nach Altmann (1981: 337) ist keine plausible Lösung in Sicht; eine Satzverdoppelung wie etwa

(15.56a) Sie (kommt zur Fete), ich meine, die Brigitte (kommt zur Fete). wird abgelehnt. 30 Angesichts des folgenden Beispielpaars scheint eine direkte verdoppelung mit Tilgung identischen Materials bei der Kopie nicht annehmbar;

30 Altmanns Gründe: Grenze zum Nachtrag uberschritten; keine Parenthesenische vor Rechtsversetzungsausdruck; intonatorische Integration in Nachlauf und Nichthinzufibgarkeit von Satzadverbien, Gradpartikeln. 
der zweite Satz müßte eine andere Gliedstellung sowie abweichende Wortformen aufweisen, und dies widerspricht einer direkten Satzkopiehypothese:

$(15.56 \mathrm{~b})$ * (Kuno hat) das (nicht gewollt), ich meine, (Kuno hat) das Auto zu Schrott fahren (nicht gewollt).

(15.56c) (Kuno hat) das (nicht gewollt), ich meine, (Kuno hat) das Auto (nicht) zu schrott fahren (wollen).

Entweder verkompliziert man die Kopiehypothese, indem man allerlei zusatzbedingungen (Zusatztransformationen) einführt, oder man unterläBt derartige Annahmen. Irgendetwas tberzeugendes vermag ich auch nicht anzubieten.

Umformungsbezüge zu KORREKTURSEQUENZEN wirken nicht sonderlich plausibel. In (15.56d) zeigen sich die Unterschiede auf der funktionalen Seite deutlich in den unterschiedlichen Akzeptabilitäten. Während Korrekturen die Basis ganz (nein) oder zumindest teilweise (genauer) außer Kraft setzen, bleibt der Bezugsausdruck des referenzklärenden zusatzes "in Kraft" und wird gleichsam nur "ausgemalt" resp. "konkretisiert".

(15.56d) Kuno hat sie, ich meine die Pial *nein: die Pial ??genauer: die Pia, gestern getroffen.

Verbzweitsätze mit Vollverben (als Schalt- oder Folgesätze) sind wahrscheinlich nicht, zumindest nicht systematisch, in Betracht zu ziehen:

(15.56e) Kuno hat sie (?ich beziehe mich jetzt auf Pia) gestern getroffen. (??Ich beziehe mich jetzt auf Pia.)

5.c. SPEZIFIZIERENDE ZUSATZE: Hier gilt das bei Altmann (1981: $335 \mathrm{f.}$ ) Erarbeitete: Satzverdoppelung, Tilgung gleichen Materials und ggf. Einbettung in den Gastsatz:

(15.57a) Sie hat viele Fische gesehen, und zwar hat sie insbesondere/ einschlieblich/ vor allem/z.B. Haie und Delphine gesehen.

SPEZ lassen sich $z . T$. in Verbindung mit Korrektursequenzen bringen; allerdings kann hierbei von keiner strikten Umformungsbeziehung gesprochen werden (nicht jedes Syntagma der Konstruktion $X$ kann zu einem Syntagma der Konstruktion $Y$ in Beziehung gesetzt werden). 'rotalkorrekcuren (nein) sind nicht als adäquat anzusehen, weil sie die Basis im Gegensatz zu den SPEZ (die Basis "bleibt in Kraft") ganz außer Kraft setzen. Präzisierungen (Teilkorrekturen, genauer) allerdings geben die "Konstruktionsbedeutung" mehr oder minder (allerdings nie völlig) angemessen wieder. Dabei ist genau zu unterscheiden, welcher subtyp umgeformt wird; während ein hervorhebender Zusatz beispielsweise (15.57b) der Teilkorrektur funktional stärker ähnelt, ist die funktionale Differenz beim beispielnennenden Zusatz (15.57c) deutlich größer.

(15.57b) Sie hat viele Fische, vor allem Haie und Rochen/ (?)genauer: Haie und Rochen/ ?nein: Haie und Rochen, beobachten können.

(15.57c) Sie hat viele Fische, z.B. Haie und Rochen/ ?genauer: Haie und Rochen/ ??nein: Haie und Rochen, beobachten können.

Inwieweit Umformungsbeziehungen zu Kurzzusătzen existieren, kann hier nicht geklärt werden. (Ich erinnere daran, daß in Kap. 13 Kurzzusätze deutlich von 
spezifizierenden u.a. unterschieden wurden.) Nach bisherigen Erfahrungen scheint mir diese Option sehr selten zu sein (und evtl. auf die Funktion 'Beispielnennung, Aufzählung von Teilmengen' beschränkt):

(15.57d) Andere Völker (z.B. die Italiener, die Spanier und die Griechen)/ (Italiener, Spanier, Griechen) wundern sich über die Deutschen. Kopulasätze scheinen eher zufällig möglich zu sein, doch gerade bei hervorhebenden Zusätzen sind muten sie akzeptabel an (15.57e); systematische Umformungen, vor allem bei beispielnennenden und einschließenden Zusätzen, dürften generell inakzeptabel sein (15.57f):

(15.57e) Pia hat Haie (, insbesondere/ vor allem Blauhaie,) beobachtet. (Es waren insbesondere/ vor allem Blaubaie.)

(15.57f) Pia hat Haie (, etwa Blau- und Hammerhaie,) studiert. (*Es waren etwa Blau- und Hammerhaie.)

Pia hat die Haie (, einschlieblich die Blauhaie,) verjagt. (*Es waren einschlieblich die Blaubaie.)

5.d. NICHTSPEZIFIZIERENDE ZUSATZE: Hier gilt der gleiche Umformungsweg wie bei den SPEZ (5.c.); man sieht, wie z.B. Satzadverbien und Gradpartikeln, die auf die Zusatz-NP bezogen sind, "mitgenommen" werden (15.58a).

Wenigstens beim Subtyp hinzufügender Zusatz ist eine Beziehung via Satzkonjunktion (dann Tilgung gleichen - unterstrichenen - Materials etc., 15.58b) zu erwägen; allerdings sind z.B. die subtypen einschränkender und ausschließender Zusatz nicht so zu deuten (15.58c):

(15.58a) Sie hat Haie [, ferner vermutlich sogar Delphine,] gesehen, ferner hat sie vermutlich sogar Delphine gesehen.

(15.58b) Die Vögel (, und obendrein die Fledermäuse,) flogen davon. (Und obendrein flogen die Fledermäuse davon.)

$(15.58 \mathrm{c})$ *Viele Vögel, und zumindest die Milane, jagt man dort. *Viele Vögel, und ausgenommen die Milane, jagt man dort.

Beziehungen $z u$ (nicht-totalen) Korrektursequenzen bestehen beim verallgemeinernden Zusatz (15.58d), allerdings nicht im Sinne einer strikten (sondern einer mehr oder minder adäquaten) Umformungsbeziehung. Beim zurücknehmenden und beim ausschlieBenden zusatz besteht keine derartige Beziehung (15.58e).

(15.58d) Die Griechen, allgemeiner: die Südländer/ ?genauer: die Südländer/ ??nein: die Südländer, kochen gern und gut.

(15.58e) Viele Vogelarten, nicht so sehr die Bussarde/ *genauer: die Bus-

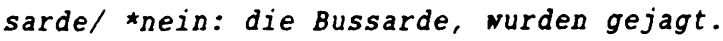

Viele Vogelarten, ausgenommen die Bussarde/ *genauer: die Bussarde/ *nein: die Bussarde, wurden gejagt.

5.e. ALTERNATIVEN NENNENDE ZUSATZE: Am plausibelsten ist wiederum Satzverdoppelung plus Tilgung (und evtl. Einbettung), wobei mir nicht alle Beispiele völlig akzeptabel erscheinen (15.59a; s. auch Abs. 3 dieses Kap.):

(15.59a) Die Damen haben einen ledigen Mann heftig umworben, anders gesagt/ m.a.W., die Damen haben einen freien Mann heftig umworben. 
(?) Die Damen haben einen ledigen Mann heftig umworben, anders gesagt/ m.a.W.: einen freien Mann.

(15.59b) Die Existenzformen der Sprache hat sie untersucht, anders gesagt/ m.a.W. (:) die Erscheinungsformen der Sprache.

Korrektursequenzen sind wiederum kaum (bzw. gar nicht) als systematische Umformungsmöglichkeiten anzusehen, da die Ronstruktionsbedeutungen deutlich differieren; ein umformulierender Zusatz bezeichnet im Unterschied zur nicht-paraphrasierenden Korrektur i.d.R. das gleiche wie seine Basis, nur daB er eine Nuance, einen Aspekt des Bezeichneten (erinnernd, erläuternd, variierend etc.) ins Bewustsein hebt:

(15.59c) Die Existenzformen der Sprache, m.a.W.:/ ?genauer:/ ??nein: die Erscheinungsformen der Sprache, hat sie untersucht.

ALNZ weisen Beziehungen zu Kurzzusătzen auf, deren "Systematizität" hier nicht untersucht werden kann; daher nur zwei Beispiele (vgl. auch Kap. 13):

(15.59d) Die Rechtschreibung (m.a.W.: die Orthographie)/ (Orthographie) beherrscht Pia völlig.

(15.59e) Es wurde Wein (bzw. Bier oder Schnaps)/ (Bier, Schnaps) getrunken. 5.f. KURZZUSATZE: Schwyzer (1939: 39) leitet die "echten Wortparenthesen" von Satzparenthesen ab. Besonders die vielfältigen Funktionen legen es nahe, Umformungen zu Sätzen mit verschiedensten Vollverben anzusetzen, wobei Relativsätze, V-2-Schaltsätze und Satzfolgen in Frage kommen. Man könnte die Kurzzusätze als "konventionalisierte" Reduktionen vollständiger Sătze (bzw. Satzinhalte) auffassen - konventionalisiert deshalb, weil nicht jeder beliebige Satz (inhalt) in einem Kurzzusatz konzentriert (und dann auf eine Basis bezogen) werden kann. Konventionalisiert ist z.B. die Konzentration eines Satzes wie er lebte von 1724 bis 1804 auf den Rurzzusatz (1724-1804), nicht-konventionalisiert ist dagegen die von er kränkelte von 1724 bis $1804 \mathrm{zu}$ *(17241804). Details müssen in zukünftigen Untersuchungen geklärt werden.

(15.60a) Harry Hirsch (SPD)/ (der der SPD angehört) ist der neue Minister. Harry Hirsch iSPD)/ (er gehört der SFD an; ist der nelie Minister. Harry Hirsch ist der neue Minister. Er gehort der SPD an.

(15.60b) Aalen (Württemberg)/(das in Württemberg liegt)/ (es liegt in Wüttemberg) ist eine Kreisstadt. Aalen ist eine Kreisstadt. Es liegt in Württemberg.

(15.60c) Die Deutsche Sexpartei (deren Abkürzung DSP lautet)/(ihre Abkürzung lautet DSP) tagt wieder.

Die Deutsche Sexpartei tagt wieder. Ihre Abkürzung lautet DSP. Nicht immer müssen explizite (Schalt)Sätze angenommen werden; manchmal genügen. satzhafte bzw. satzwertige Ausdrücke wie in Isfahan (Heerlager/zu deutsch: Heerlager) oder die Deutsche Sexpartei, kurz (gesagt): DSP.

Appositive Relativsātze stehen hinsichtlich der Umformungsmöglichkeiten in Beziehungen zu prădizierenden Zusătzen (mit Kopulaverben; vgl. 15.54a), zu Kurz- 
zusätzen (mit Vollverben; vgl. 15.60a-c) und zu NP-bezogenen Gliedteilen mit Einschaltungsmarkierung; in diesem Fall kommt eine Annahme der "älteren" Transformationsgrammatik zum Tragen: daß nämlich Wortgruppenattribute (AdjP, NPgenitiv, PP etc.) auf zugrunde liegende Sätze, unter anderem auf Relativsätze, bezogen wurden $(15.60 d, e)$. Ob alle Fälle dieser Art auf diese Weise umzuformen sind, kann hier nicht geklärt werden. Interessant ist der Fall (15.60f, g), wo in der satzumformung wiederum ein einschaltungsmarkierter Ausdruck erscheint:

(15.60d) Eine - notgedrungen einseitige - Neigerung (...) würde unserer Sicherheit (..) keinen Abbruch tun.

(15.60e) Eine Weigerung - die notgedrungen einseitig ist - würde (...).

(15.60f) In Anlehnung an "Phraseodidaktik" mag "Phraseopragmatik" als ein (zugegeben: schlechter) Arbeitsterminus (...) gelten.

(15.60g) (..) als ein Arbeitsterminus - der - zugegeben - schlecht ist gelten (...).

Zudem stehen appositive Relativsätze, also Relativsatzzusätze, in Beziehung mit Verbzweitschalt- und -folgesätzen. Ob eine uneingeschränkte "Austauschbarkeit" dieser Muster besteht, muß vorerst offenbleiben.

(15.60h) August Stramm (der im 1. Weltkrieg fiel/ er fiel im 1. Weltkrieg) war ein Sprachartist. (Er fiel im 1. Weltkrieg.)

Abschließend noch eine Zusammenschau der (vorläufigen) Ergebnisse:

PRAZ REPZ SPEZ NSPEZ ALNZ RURZ APREZ eingeschalt.

(a) Kopulasatz

- APRET

- V-2-Schaltsatz

- V-2-Folgesatz

(b) Vollverbsatz

- APRET

- V-2-Schaltsatz

- V-2-Folgesatz

(c) Doppelung

(d) KURZ

(e) Korrektursequenz

\section{Kasuskongruenz}

Die nachstehenden Aussagen betreffen nur Muster mit nominalen Basen und nominalen zusätzen.

6.a. PRAZ: ohne Erweiterung statistisch sehr häufig (vgl. Bergenholtz 1985), aber grammatisch außer in Disambiguierungsfällen nicht notwendig; der PRAZ kann fast immer nominativisch erscheinen. Bei erweiterten Nominalphrasen oder $\mathrm{N} 2 / \mathrm{N} 1 / \mathrm{NO}$ ist die Kongruenz unmöglich. 
6.b. REFZ: obligatorisch; die Akkusativrektion von meinen scheint sich nicht bzw. kaum durchsetzen zu können:

(15.61a) Hast du ihm, dem Kuno/ ich meine dem Kuno, die Syntax erklärt? Hast du ihm, *den Kuno/ (?) ich meine den Kuno, die Syntax erklärt?

(15.61b) Warst du dir dessen bewuBt, der/ *die Konsequenzen? Warst du dir dessen bewuBt, ich meine der/?die Konsequenzen? 6.c. SPEZ: Kongruenz ist obligatorisch. Die evtl. in Betracht zu ziehende "neutrale" Nominativmarkierung ist inakzeptabel:

(15.62a) Man hat den Haien, (genauer) den Hammerhaien getrotzt. * Man hat den Haien, (genauer) die Hammerhaie, getrotzt.

(15.62b) Man nahm sich vieler Vögel, (z.B.) der Stare und Finken, an. * Man nahm sich vieler Vögel, (z.B.) die stare und Finken, an.

6.d. NSPEZ: obligatorisch. Es gilt das unter 6.c. Gesagte.

6.e. ALNZ: Die Kongruenz darf als obligatorisch betrachtet werden:

(15.62c) Die Erforschung der Existenzformen, m.a.W. ??die/ der Erscheinungsformen, oblag Pia.

(15.62d) Einem ledigen Mann, m.a.W. einem freien Mann/*m.a.W. ein freier Mann, miBtraut sie.

6.f. Kurzzusatz: Siehe auch Kap. 13. Folgendes ist auseinanderzuhalten:

- Bei den paradigmatischen Typen (wie Harry Hirsch (DSP)) gibt es keine Kongruenzerscheinungen.

- Falls der rundgeklammerte Zusatz als Alternativen nennender Zusatz etc. $z u$ werten ist, tritt Kongruenz ein (vgl. 6.e.).

7. Referenzidentität: wird vernachlässigt (vgl. Kap. 3.8., Kap. 6. Abs. 7).

\section{NeglaBbarkeit}

Im Prinzip sind die Zusätze allesamt entbehrlich, zumindest in syntaktischer Hinsicht. Aus semantischer bzw. pragmatischer sicht man sie allerdings nicht immer ohne weiteres weglassen resp. hinzufügen:

(15.63a) In der DUDEN-Grammatik (1973)/*0 gibt es keinen Abschnitt, der "Der Buchstabe" heiBt.

(15.63b) In Paris (Texas)/ $\star 0$ steht kein Eiffelturm.

(15.63c) *Pia, Köchin aus Passion, haßt das Kochen. Pia, vermeintliche Köchin aus Passion, hast das Kochen.

(15.63d) Viele Haie ( ${ }^{\star}$, besonders die Hammerhaie,) werden dort gefürchtet; die Hammerhaie werden allerdings nicht sehr gefürchtet.

Da es in der Dudengrammatik (1984) einen Abschnitt "Der Buchstabe" gibt, wäre der Satz ohne den Kurzzusatz semantisch abweichend. Paris ohne lokalisierenden Zusatz ware unklar. $(15.63 \mathrm{c})$ ist ohne adverbielle spezifikation semantisch abweichend. In (15.63d) widersprächen sich die Hervorhebung durch den zusatz und die Aussage des Folgesatzes. 
Anwesende Zusätze wirken durchaus in den "laufenden" (Gast)Satz hinein, d.h. von diesen ausgehende "Selektionsrestriktionen" müssen beachtet werden:

(15.64a) Helmut Kohl $O / \star(C D U)$ ist Vorsitzender der DSP.

(15.64b) In Paris 0/ (Frankreich)/*(Texas) steht der Eiffelturm.

$\mathrm{Zu}$ möglichen semantischen bzw. pragmatischen Wechselwirkungen $z$ wischen Zusatz und Gastsatz sind Folgeuntersuchungen wünschenswert.

9. Appositionslexeme: siehe hierzu Abschnitt 1. dieses Kapitels.

10. Appositiv vertritt Basis: wird vernachlässigt (vgl. Kap. 3, 6) .

11. Satzgrenzenbestimmtheit: wird vernachlässigt (s. auch Kap. 3).

\section{Attribution}

Zu nichtattributiven Erweiterungen s. Abs. 1. dieses Kapitels. Hinsichtlich der attributiven Erweiterung zeigt die folgende Ubersicht Tendenzen auf:
PRAZ: relativ frei.
REFZ:
begrenzt. 31
SPEZ: eher begrenzt.
NSPEZ: $\quad$ eher begrenzt.
ALNZ: eher begrenzt.
Kurzzusatz: i.d.R. keine.

Bei (nicht-) spezifizierenden und Alternativen nennenden Zusätzen ist Attribution syntaktisch ohne weiteres möglich, sie wirkt aber (pragmatisch) evtl. etwas übertrieben, der Zusatz erlangt dadurch ein übermäBiges Gewicht.

(15.65a) (?) Viele Haie, (und zwar) vor allem die schrecklichen, blutrünstigen Hammerhaie mit ihren verformten Schnauzen, werden dort gefürchtet.

(15.65b) (?) Viele Haie, ausgenommen die schrecklichen, blutrünstigen Hammerhaie mit ihren verformten Schnauzen, werden dort gejagt.

13. Textnotwendigkeit: wird vernachlässigt.

31 Der Unterschied ist wohl pragmatischer Natur; während beim prädizierenden Zusatz Zusatzinformation verdichtet einem Kern angefügt wird, wird beim referenzklärenden Zusatz lediglich die Referenz eines (vagen) Ausdrucks geklärt. Es mutet etwas merkwürdig an, wenn der referenzklärende Ausdruck viele Attribute enthält:

(F15.13) Ich habe die Pia, eine überaus kluge, äußerst witzige Frau mit einem unglaublichen Charme, heute wieder getroffen.

(F15.14) (?) Ich habe sie, die überaus kluge, äußerst witzige Pia mit einem unglaublichen Charme, heute wieder getraffen. 


\section{Sprecherdivergenz und Sprecherpräsenz}

An Erkenntnis erbringt dieses Kriterium nicht viel (vgl. Kap. 3); zudem erschweren mir nicht ganz klare Einflüsse eine sichere Beurteilung der Beispiele (manche Informanten werten die Fälle anders als ich; die möglichen Abweichungen scheinen nicht rein syntaktisch begründbar, sondern vielmehr pragmatischen Ursprungs $z u$ sein). Immerhin zeigt sich ein gewisses (pragmatisches) Unbehagen (mit "?" markiert) an der direkten Wiedergabe eines prädizierenden (15.66a) bzw. eines referenzklärenden Zusatzes $(15.66 \mathrm{~b})$. Bei dem REFZ ist zudem der Zusatz ich meine (bezogen auf den aktuellen sprecher) inakzeptabel. Der aktuelle Sprecher muß mit sie meint deutlich machen, daß er die Referenz eines Ausdrucks klärt, der vom referierten sprecher stammt. Die Akzeptabilitätsschwankung in Fällen, in denen der REFZ vom referierten sprecher stammt, mögen pragmatisch begründet sein: vielleicht erwartete man, daß diese Hinzufügung in der Wiedergabe der Rede einfach korrigiert wird (der Referierende setzt nur die Voll-NP). Andererseits kann man sich Situationen wie Polizeiverhör etc. vorstellen, die eine exakte und unkorrigierte Wiedergabe erfordern, so daB derartige Fälle manchmal völlig akzeptabel sind.

Ebenfalls eigenartig mutet es an, wenn der aktuelle sprecher den zusatz beim SPEZ (15.66c) außert. Unannehmbar ist es wohl, wenn man wie in (15.66d) einen nicht-spezifizierenden zusatz dem aktuellen sprecher zuschriebe, doch auch hier müssen, sofern dies interessiert, zukünftige Analysen den Gründen auf die spur kommen.

Hinsichtlich ihrer Akzeptabilität schwer $z u$ beurteilen ist die AuBerung eines Kurzzusatzes (15.66f) seitens des aktuellen sprechers innerhalb einer referierten Sequenz; zuerst kam mir das Beispiel inakzeptabel vor, jetzt bin ich mir - infolge einiger positiver Informantenurteile - nicht mehr sicher und bewerte es als relativ akzeptabel. Unter Umständen irritiert auch nur die geschriebene Form, nicht jedoch die gesprochene.

(15.65a) Fia sagte: "Otto", ein Trinker, "hat gerade angerufen". ?Pia sagte: "Otto, ein Trinker, hat gerade angerufen".

Pia sagte, Otto, ein Trinker, habe gerade angerufen.

(15.66b) Pia sagte: "Er", (*ich meine/ sie meint) der otto, "hat gerade angerufen".

?Pia sagte: "Er, der Otto, hat gerade angerufen".

Pia sagte, er, der otto, habe gerade angerufen.

(15.66c) ?Pia sagte: "Die Haie", vor allem die Hammerhaie, "sind häBlich".

Pia sagte: "Die Haie, vor allem die Hammerhaie, sind häBlich".

Pia sagte, die Haie, vor allem die Hammerhaie, seien häblich.

(15.66d) *Pia sagte: "Die Haie", obendrein die Kraken, "sind häBlich".

Pia sagte. "Die Haie, obendrein die Kraken, sind häBlich".

Pia sagte, die Haie, obendrein die Kraken, seien häBlich. 
(15.66e) Pia sagte: "Die Ledigen", m.a.W. die Freien, "stellen ihr nach". Pia sagte: "Die Ledigen, m.a.W. die Freien, stellen ihr nach". Pia sagte, die Ledigen, m.a.W. die Freien, stellten ihr nach.

(15.66f) (?) Pia sagte: "Harry Hirsch" (CDU) "redet als nächster". Pia sagte: "Harry Hirsch (CDU) redet als nächster". Pia sagte, Harry Hirsch (CDU) rede als nachster.

15. Adordinativität: bleibt unberücksichtigt.

16. Tragweite von skopusbildenden Ausdrücken

Siehe hierzu auch Kap. 12, wo sich die Beispiele finden, die zu den nachstehend aufgelisteten Ergebnissen führten. (Beurteilt wird, ob bestimmte Folgerungen gezogen werden können oder nicht.) Beispiele für die Nichtaffizierbarkeit von Kurzzusätzen sind (">" = 'daraus folgt'):

(15.67a) Harry Hirsch (CDU) gewinnt nicht.

> Es ist nicht der Fall, daB Harry Hirsch der CDU angehört.

Kant (1724-1804) lebte nicht in Köln.

> Es ist nicht der Fall, daB Kant von 1724 bis 1804 lebte.

(15.67b) * Nicht Harry Hirsch (CDU) gewinnt, sondern Harry Hirsch (FDP).

* Nicht Kant (1746-1801) lebte in Königsberg, sondern Kant (17241804).

(15.67c) Auch/ Nur/ Sogar Harry Hirsch (CDU) gewann.

> *Auch/ *Nur/ *Sogar Harry Hirsch gehört der CDU an.

*Auch Harry Hirsch (CDU) gewann, nicht nur Harry Hirsch (SPD).

(15.67d) Gewinnt Harry Hirsch (CDU)? > Gehört Harry Hirsch der CDU an? Wie verhält es sich, wenn quantifizierende bereichsbildende Ausdrücke vorkommen (zum Verhalten bzgl. Negation und Gradpartikeln s. Kap. 6, 12)?

(15.67e) Der Kultusminister von Baden-Württemberg, der Präsident des VFB Stuttgart, hat dreimal für das Ministeramt kandidiert

> ??Der Präsident des VFB Stuttgart hat dreimal für das Ministeramt kandidiert. 32

(15.67f) Sie, die Pia, hat dreimal geheiratet

> Die Pia hat dreimal geheiratet.

(15.67g) Manche Haie, z.B. der Tigerhai und der Makohai, greifen mehrmals an > Der Tigerhai und der Makohai greifen mehrmals an.

32 Die Beispiele für prädizierende Zusätze sind heikel und führen u.U. zu nicht geringen logischen Kapriolen. Vielleicht trügt mein Bindruck, daß quantifizierende (Frequenz) Adverbiale sich nicht auf PRAZ erstreckten, denn einigen Informanten kam es durchaus "logisch" vor zu folgern, daß der (jetzige!) VFB-Präsident dreimal fürs Kultusministerant kandidierte; der Satz ist evtl. lediglich "pragmatisch" merkwürdig. Mangels genügender Ausbildung in logischen Formalismen muß ich eine befriedigende $\mathrm{Klärung}$ den entsprechenden Spezialisten überlassen. 
(15.67h) Manche Haie, besonders/ einschließlich die Makohaie, greifen mehrmals an > Die Makohaie greifen mehrmals an.

(15.67i) Die Löwen, allgemeiner die Großkatzen, fressen dreimal täglich > Die Großkatzen fressen dreimal täglich.

(15.67j) Die Großkatzen, weniger die Löwen, fressen dreimal täglich > ? Die Löwen fressen dreimal täglich.

(15.67k) Die GroBkatzen, zumindest die Löwen, fressen dreimal täglich ) Die Löwen fressen dreimal täglich.

(15.671) Die Großkatzen, ausgenommen die Löwen, fressen dreimal täglich > *Die Löwen fressen dreimal täglich.

(15.67m) Die Unverheirateten, m.a.W. die Ledigen, jammern dreimal täglich > Die Ledigen jammern dreimal täglich.

(15.67n) Harry Hirsch (CSU) jammert dreimal täglich $>\star C S U$ jammert dreimal täglich.

Es hängt mit der semantischen Relation zwischen Basis und Zusatz zusammen, ob eine Quantifikation den zusatz (nicht) betrifft. Teilmengen sind, sofern sie nicht ausgenommen oder als weniger betroffen markiert sind, logischerweise betroffen, da ja die Gesamtmenge affiziert ist. Keinesfalls affizierbar sind die Kurzzusätze als Minimalparenthesen (Wortparenthesen) und wahrscheinlich auch die prădizierenden Zusätze, reduzierte Zusatzprädikationen über die Basis-NP (der skopusbildende Ausdruck müBte innerhalb des Zusatzes stehen).

Die Anwendung intensionaler Adjektive führt zu z.T. recht dubios anmutenden Syntagmen. Man ist bei etlichen Konstellationen geneigt, die "Intensionalisierung" auf den zusatz auszudehnen, denn es mutete eigenartig an, z.B. zu assertieren, daß die vermeintlichen Mörder verhaftet wurden, und hinzuzusetzen, daB genauer/z.B. etc. die (tatsächlichen) Raubmörder verhaftet wurden. Dies hat zur Folge, daB man das intensionale Adjektiv auch im Zusatz setzen wird:

(15.68) Die vermeintlichen Mörder, genauer/z.B. die vermeintlichen Raubmörder, wurden verhaftet.

Diese Erscheinung gilt für alle nachstehenden Beispielsatze, die mit "?" markiert sind!

Beim hinzufügenden Zusatz (15.76) bleibt die Auswirkung des Adjektivs auf die Basis-NP begrenzt, der Zusatz müBte durch nochmalige Setzung des Adjektivs "intensionalisiert" werden. Beim referenzklärenden Zusatz wird ebenfalls nur die Basis affiziert. Beim Kurzzusatz ergeben sich eigentümliche Wechselwirkungen: Prinzipiell ist der Kurzzusatz vom Adjektiv nicht erfaßbar, es können jedoch Interpretationsstörungen hervorgerufen werden. Dazu nur ein Beispiel: Wenn man von einem vermeintlichen Rockmusiker (Basis-NP) spricht, meint man, daß er eigentlich gar keiner sei (sondern sich nur als solcher ausgebe o.ä.); wenn man nun aber im Kurzzusatz eine Rockgruppe nennt und ihn damit als ihr angehörig bezeichnet, ergibt sich ein widerspruch: Wie kann ein vermeintlicher Rockmusiker einer Rockgruppe angehören? Logisch (also semantisch) ist das gar nicht einmal auszuschlieben, da sich dieser vermeintliche Rockmusiker mittels 
Vortäuschung falscher Tatsachen in die Gruppe hätte gemogelt haben können. Beim ersten Auftritt müBte es dann zur Enttarnung kommen (er/sie müBte unfähig sein, Rockmusik zu spielen), sonst wäre die Person ja tatsächlich Rockmusiker und vermeintlich eine falsche Kennzeichnung.

(15.69) ?Die vermeintlichen Mörder, genauer die Raubmörder, wurden verhaftet.

(15.70) ?Die vermeintlichen Mörder, z.B. die Raubmörder, wurden verhaftet.

(15.71) ? Die vermeintlichen Mörder, einschlieblich/ vor allem die Raubmörder, wurden verhaftet.

(15.72) ?Die vermeintlichen Mörder, allgemeiner die Schwerverbrecher, wurden verhaftet.

(15.73) ?Die vermeintlichen Mörder, weniger die Raubmörder, wurden verhaftet.

(15.74) ? Die vermeintlichen Mörder, zumindest die Raubmörder, wurden verbaftet.

(15.75) ? Die vermeintlichen Mörder, ausgenommen die Raubmörder, wurden verhaftet.

(15.76) Die vermeintlichen Mörder, obendrein die Diebe, wurden verhaftet.

(15.77) ? Die vermeintlichen Mörder, m.a.W. die Killer, wurden verbaftet.

(15.78) Die vermeintlichen Lebewesen, (ich meine) die Viren, wurden untersucht.

(15.79) Der vermeintliche Vorsitzende (CDU) begann zu reden. Der vermeintliche Rockmusiker (Rolling Stones) wurde interviewt. Die in einigen Punkten (Gradpartikel, Kontrastnegation) noch mit etwas Vorsicht zu genießende Ubersicht über die Ergebnisse zeigt folgendes Bild:

SatzNeg KNeg Auch Nur Sogar Frage Quant Adj

\begin{tabular}{|c|c|c|c|c|c|c|c|}
\hline PRADIZIMRONDER ZUSATZ & - & - & - & - & - & - & - \\
\hline PRAZISIDRENDER ZUSATZ & + & ?? & + & + & + & + & + \\
\hline BEISPIITNDN. ZUSATZ & + & (?) & $(+)$ & - & $? ?$ & + & + \\
\hline $\begin{array}{l}\text { MENGENEETSCHLIFSS. U. } \\
\text { HERVORHIBBDEDER ZUSATZ }\end{array}$ & + & $? ?$ & $(+)$ & - & $? ?$ & + & + \\
\hline VERALLCEMETNIERNDER ZUS. & + & - & $(+)$ & ?? & ? & + & + \\
\hline ZURUCKNIFHMENDER ZUSATZ & - & $(-)$ & $?$ & - & $? ?$ & $?$ & ? \\
\hline EINSCARANKDDNDER ZUSATZ & + & - & + & $?$ & (?) & + & + \\
\hline AUSSCHIIFSSDNDER ZUSATZ & - & - & - & - & - & - & - \\
\hline HINZUFUGENER ZUSATZ & + & ?? & + & - & $?$ & + & + \\
\hline ALTERNATTVE NBNN. ZUSATZ & + & + & + & + & + & + & + \\
\hline REF.KDARDWER ZUSATZ & + & + & + & + & + & + & + \\
\hline KURZZUSATZZE & - & - & - & - & - & - & - \\
\hline
\end{tabular}

Wie der prädizierende Zusatz sperrt sich auch der Kurzzusatz gegen jegliche Beeinflussung mittels operatoren aus dem Gastsatz heraus - überdies der ausschließende Zusatz, der lediglich durch intensionale Adj beeinflußt wird. 
Kurz- und prădizierende Zusătze sind somit paradigmatische Hospitanten im Trăgersatz.

Der ausschließende Zusatz - ein Teil der Basismenge, die in den Sachverhalt involviert ist, wird ausgeschlossen - ist aus semantischen Gründen nicht affizierbar, außer von intensionalen Adjektiven, die über die Gesamtmenge prädizieren und somit implizit auch über die ausgenommene Teilmenge.

Alle übrigen Typen/Funktionen sind mehr oder minder in den satz involviert, wenngleich nicht in vollem sinne wie z.B. ein Satzglied, ein Attribut, eine Partikel etc. Sie stellen, im Ggs. zu prädizierendem Zusatz und Kurzzusatz, allesamt korrekturähnliche Erscheinungen dar: Der Sender setzt ein Satzglied (ich habe das vornehmlich an NPn studiert) und fügt dem eine gewisse Modifikation hinzu. Anders als bei KORREKTUREN wird jedoch das Satzglied nicht "gestrichen" und dann "neu gesetzt", sondern es bleibt erhalten, nur daB es durch den zusatz entweder deutlicher gemacht (REFZ), in seinem referentiellen (extensionalen) Umfang modifiziert (Teilmenge ausgenommen, hervorgehoben, weniger betroffen etc.) oder auch verallgemeinert wird (DEPRAZISIERUNG) usw.

17. Wahrheitswertkonstanz: wird vernachlässigt.

\section{Rekursivität und Koordination}

Das Verhalten hinsichtlich möglicher rekursiver Erzeugung ist nicht bei allen Zusätzen gleich. Die ubersicht zeigt die Tendenzen:

18.a. PRAZ: möglich.

Udo, Hobbykoch, stets hungrig, ein guter Weinkenner, schmatzt.

18.b. REFZ: eher nicht möglich.

??Er, (ich meine) dein Nachbar, (ich meine) dein Freund, schnarcht.

18.c. SPEZ: relativ eingeschränkt; kaum mehr als 2 Zusătze möglich.

Viele Vögel, z.B. die Bussarde, vor allem die Milane, (?weniger die Habichte,) werden dort gejagt.

18.d. NSPEZ: relativ eingeschränkt; kaum mehr als 2 Zusätze möglich.

Manche Haie, zumindest die Hammerhaie, überdies die Kraken, (?ausgenommen die Zitronenhaie), werden gefürchtet.

18.e. ALNZ: ziemlich eingeschränkt; kaum mehr als 1 zusatz möglich.

Die Ledigen, m.a.W. die Unverheirateten (?, anders gesagt die Ehelosen), stellen Kuno nach.

18.f. Kurzzusatz: prinzipiell eher unmöglich, doch manche Muster (die man noch. genauer untersuchen müßte) scheinen akzeptabel zu sein. Nachstehend nur ein kleiner Ausschnitt:

Harry Hirsch * (CDU, 1949-1988)/* (CDU) (1949-1988) spinnt. Jenes $N$ (?) (Nomen, Dingwort)/? (Dingwort, Nomen)/* (Nomen) (Dingwort). Das Nomen (?) (N, Dingwort)/(?) (Dingwort, $N) / \star(N)$ (Dingwort) 
Die Zentrale (?) (8 Millionen, 100 Mitarbeiter)//* (8 Millionen) (100 Mitarbeiter) ist imposant.

In der nachfolgenden Ubersicht zur Koordination wird getestet, welche Einheiten konjunktorsyndetisch (mit und) verbunden werden können. Die Zusammenstellung spiegelt lediglich Tendenzen wieder, genauere und materialreichere Analysen sind vonnöten. Ein paar Beispiele:

(15.80) Sie, *die Brigitte (REFZ) und eine kluge Frau (PRAZ)/*eine kluge Frau (PRAZ) und die Brigitte (REFZ), schnarcht.

(15.81) Sie, schlagfertig wie immer (PRAZ) und Dozentin in Bonn (PRAZ), schnarcht.

(15.82) *Die Haie, vor allem/z.B. die Hammerhaie (SPEZ) und stets gefährlich (PRAZ), umkreisten sie.

(15.83) Sie, die Brigitte (REFZ) und ihre Eltern (REFZ), spinnen.

(15.84) ??Sie, die Haie (REFZ) und einschlieblich/genauer/ vor allem die Hammerhaie (SPEZ), kamen herbei.

(15.85) ?? Die Haie, genauer die Menschenhaie (SPEZ) und einschlieblich die WeiBen Haie (SPEZ), schwammen herbei.

Die Haie, z.B. die Hammerhaie (SPEZ) und vor allem die Blaubaie (SPEZ), schwammen herbei.

(15.86) Die Vögel, genauer die Singvögel (SPEZ) und obendrein die Fledermäuse (NSPEZ), flogen fort.

(15.87) ??Die Haie, ausgenommen den Katzenhai (NSPEZ) und allgemeiner die Fische (NSPEZ), werden dort gejagt.

(15.88) Das Nomen ( $N$ und Dingwort)/ (?) ( $N$, Dingwort) (KURZ) wird groBgeschrieben.

PRAZ REFZ SPEZ NSPEZ ALNZ Kurzzusatz

$\begin{array}{lcccccc}\text { PRAZ: } & (+) & - & - & - & -33 & - \\ \text { REFZ: } & - & (+) & ? ? & ? ? & - & - \\ \text { SPEZ: } & - & (-) & ? ? /+ & ? ? 34 & - & - \\ \text { NSPEZ: } & - & (-) & ? ? & ? ? & - & - \\ \text { ALNZ: } & - & (-) & ? ? & ? ? & ? ? & - \\ \text { Kurzzusatz: } & - & - & - & - & - & -\end{array}$

33 Alternativen nennende Zusätze sind offensichtlich generell von der und-Koordination ausgeschlossen, weil hier keine Referentendiversität vorhanden wäre.

34 Akzeptabel nur beim NSPEZ-TYp ZUSATZZICH BETROFTEN, dort aber systematisch, weil sich die Mengen nicht überschneiden, so daß die in Koordinationskonstruktionen geforderte Referentendiversität der beiden NPs (vgl. Matthews 1981: 225) besteht. 
Ein augenfälliges Ergebnis ist, daB die und-Koordination eher selten (in akzeptabler Weise) realisierbar ist, außer beim prädizierenden zusatz.

Spezifizierende zusätze sind z.T. nicht koordinierbar, weil die beiden Nominalphrasen keine Referentendiversität aufweisen (wie für Konjunkte gefordert), sondern Referenteninklusion; z.T. (15.85) sind sie problemlos koordinierbar (hier müssen noch eingehendere Analysen folgen!).

Auch bei nichtspezifizierenden zusätzen besteht - vom hinzufügenden Zusatz abgesehen - diese Referenteninklusion, welche die Koordination ausschließt. Die Kurzzusätze sind von der Konjunktionsmöglichkeit prinzipiell ausgenommen.

Generell scheint es an der Referentengleichheit (ALNZ) resp. der Referenteninklusion (bei allen Menge-Teilmenge-Konstellationen) zu liegen, daß hier Konjunktion kaum möglich ist. Beim referenzklärenden Zusatz ergeben sich keine Probleme, falls die Konjunkte zwei Referenten(mengen) denotieren, die disjunkt sind (15.83). Beim prädizierenden Zusatz entstehen keine Probleme, weil die Konjunkte zumeist diverse Referenten haben. Eine Ausnahme bilden Koordinationen mit auftretendem Identifikat, sie muten weder voll akzeptabel noch inakzeptabel an:

(15.89) Der Kultusminister von Baden-Württemberg, ?der Präsident des VFB stuttgart und Koch aus Passion,/ (?) Koch aus Passion und der Präsident des VFB Stuttgart, hält eine Ansprache.

19. Pronominalisierung und anaphorische Bezüge: wird vernachlässigt.

\section{Passivierung; syntaktische Operationen}

Es geht um die Frage, ob ZUSATZE bei syntaktischen Operationen, die das Bezugselement betreffen, obligatorisch an dieser operation teilnehmen, oder ob sie davon ausgenommen bleiben können. Anders ausgedrückt: Es geht um die Frage, ob der zusatz unmittelbar beim Bezugselement stehenbleiben muß oder nicht. Im Gegensatz zu den ATTRIBUTEN, die zumeist ihr Bezugseiement begleiten nüssen, sind die Verhältnisse bei den zusätzen unterschiedlich. ${ }^{35}$ Ein Beispiel für Unterschiede im Verhalten (Affizierung durch basisbetreffende syntaktische Operationen) hat man in Kap. 6 Abs. 20 kennengelernt: den elementbezogenen Schaltsatz, der nicht notwendigerweise affiziert wird, und den prädizierenden Zusatz ("Appositionsprototyp"), der affiziert wird.

Wie sich erwarten läBt, sind die diversen Zusätze unterschiedlich frei: je nachdem, ob sie prinzipiell die stellung am Satzende (nach Klammerschluß) einzunehmen vermögen, was z.B. auf den prädizierenden zusatz nicht zutrifft (vgl. Abs. 3 dieses Kapitels). Demnach begleitet der PRAZ i.d.R. sein Bezugselement, ebenso der Kurzzusatz. Der referenzklärende Zusatz, der spezifizierende und

35 Ausnahmen sind z.B. "gefloatete" Adjektivattribute und manche Präpositionalattribute; hierzu z.B. Engel (1988: 627 f.). 
der nichtspezifizierende Zusatz und wahrscheinlich auch die Alternativen nennenden zusätze können hingegen das Bezugselement begleiten oder an der Satzendposition verbleiben. Irgendwelche Mittelfeldstellungen der Zusätze treten hier vermutlich nicht auf. Nachstehend nur einige wenige Beispiele:

(15.90) Pia sucht ibn, den Kuno.

Er (, der Kuno,) wird (??, der Kuno,) von Pia gesucht (, der Kuno).

(15.91) Sie jagten viele Vögel, genauer/z.B. die singvögel.

Viele Vögel (, genauer/ z.B. die Singvögel,) wurden (??, genauer/

$z . B$. die singvögel,) von ibnen (*, genauer/ z.B. die singvögel,)

gejagt (, genauer/z.B. die singvögel).

Auch bei CLEFT/PSEUDOCLEFT, Linksverschachtelung und Satzverschränkung verhalten sich die verschiedenen Zusătze wie bei der Passivierung; wiederum nur einige veranschaulichende Beispiele:

(15.92) Es waren die Haie (, besonders/ einschlieblich/ genauer die Hammerhaie), die sie fürchteten (, besonders/ einschlieblich/ genauer die Hammerhaie).

(15.93) Was sie fürchteten, waren die Haie (, besonders/ einschlieBlich/ genauer die Hammerhaie).

(15.94) Die Vögel (, besonders/ einschließlich/ genauer Eulen und Nachtigallen,) hoffte Pia zu beobachten (, besonders/ einschlieBlich/genaver Eulen und Nachtigallen).

(15.95) Die Haie (, besonders/ einschlieBlich/ genauer die Hammerbaie), glaubten sie, seien gefräBig (, besonders/ einschließlich/genauer die Hammerhaie).

(15.96) Es waren die Existenzformen (, m.a.W. die Erscheinungsformen), die Pia untersuchte (, m.a.W. die Erscheinungsformen).

(15.97) Die Existenzformen, (, m.a.W. die Erscheinungsformen), hoffte Pia zu untersuchen (, m.a.W. die Erscheinungsformen).

(15.98) Die Existenzformen (, m.a.W. die Erscheinungsformen), glaubte sie, untersuchen zu müssen (, m.a.W. die Erscheinungsformen).

(15.99) Sie (, die Haie,) waren es, die sie fürchteten (, die Haie).

(15.100) Sie (, die Haie,) hoffte Pia beobachten zu können (, die Haie). Die ((?), ich meine die Haie), glauben sie, seien gefräBig (, ich meine die Haie).

SPEZ, NSPEZ, ALNZ und REFZ können sowohl das Bezugselement begleiten als auch am rechten Satzrand verbleiben.

\section{Dependenz}

Im Kap. 6 wurden folgende Dependenzmerkmale formuliert: Weglabbarkeit, Rektions- und Valenzbeziehungen, Kongruenz (sie wurde problematisiert) und die Spezifikationsrelation (Strukturierbarkeit in operator und operand; nach Vennemann 1977). 
Beim prädizierenden Zusatz ermittelten wir, daB der Zusatz syntaktisch betrachtet immer weglaßbar ist. Diese Eigenschaft weisen sämtliche Zusätze auf.

Ein Zusatz kann per definitionem nicht ohne Bezugselement auftreten und ist mithin an dieses vorkommensgebunden (gerichtete Konkomitanz), womit ein sehr allgemeines Kriterium für Dependenz erfüllt wäre. Natürlich muß man sich fragen, ob ein so allgemeines Merkmal für das Ansetzen einer Dependenzrelation genügen kann. 36

Das ubernehmen von Kongruenzmerkmalen - zumindest bei einigen Mustern spricht evtl. für Dependenz. 37 Somit wären neben prädizierenden Zusätzen noch referenzklärende, spezifizierende, nichtspezifizierende sowie Alternativen nennende Zusätze als dependent einzustufen - dagegen nicht der prototypische Kurzzusatz. Man kann jedoch erwägen, ob Kongruenz in diesen Fällen "mechanisch" (wie z.B. innerhalb der NP, dann markiert sie offensichtlich Abhängigkeit) oder ob sie "funktional" ist (wie möglicherweise bei unseren Zusätzen) und etwa den gleichen oder einen ähnlichen außersprachlichen Bezug zweier Einheiten markiert. 38 somit wäre sie in den Zusatzbeispielen "funktional" und folglich kein zwingender Indikator für Dependenz.

Valenzbeziehungen spielen im Bereich der Zusätze keine Rolle, weil zusätze nie valenzgefordert sind bzw. nie Valenzstellen ausfüllen können.

Spezifikation (eine operator-Operand-Beziehung) liegt wohl bei keinem unserer Zusatztypen vor, so daB nach MaBgabe dieses Kriteriums Dependenz nicht in Betracht kommt. 39

Außer den sehr allgemeinen Merkmalen Weglaßbarkeit und Vorkommensgebundenheit an ein "Bezugselement" spricht so gut wie nichts für das Vorliegen einer Dependenzrelation, sofern man diese mittels der oben angeführten Merkmale definiert. Dies verträgt sich mit der in Kap. 5 explizierten Modellvorstellung, nach der (speziellere) Dependenzrelationen (Valenz, Rektion etc.) nur im Elementarsatz wirksam sind.

36 Es lassen sich mïhelos Beispiele finden, die die Unangemessenheit der Verabsolutierung des Ronkomitanzkriteriums vor Augen führen. Demach wäre eine koordinierende Konjunktion z.B. von 2 Hauptsätzen dependent, da die Hauptsätze ohne sie, sie aber nicht ohne die Hauptsätze vorkonmen kann. Zwei koordinierte Eigennamen wären aber wohl eher von der Konjumktion dependent, weil sie nicht ohne sie erscheinen dürfen, aber auch die Konjunktion kann nicht allein auftreten (Pia und Kuno kammen/ *Pia Kuno kammen/ *(lnd kammen).

37 Der dependente Ausdruck übernimmt die Kongruenzmerkmale, die dem Regens lexikalisch (z.B. Rektion) oder strukturell (z.B. Subjektsnominativ) zugewiesen werden.

38 Hierzu Ostrowski (1982: 253 ff., 258 ff.).

39 Bei mengensektiven Zusătzen (z.B. die Haie, ausgenommen die Hammerhaie,) ist dies nicht ganz unproblematisch. Die Konstruktionssemantik scheint mir jedoch nicht die gleiche wie bei einer Attribution. 


\section{Ronstituenz}

Nun ist noch zu klären, ob Basis und Zusatz eine Konstituente bilden. Hierzu eine Vorbemerkung: Syntaktische Konstituenz wird operationalisiert durch die bekannten Konstituententests (Topikalisierungstest, Substitutionstest etc.), wobei jeder einzelne Test seine spezifischen Probleme aufweist. So kann offenkundig im Vorfeld mehr als ein Satzglied bzw. eine Konstituente stehen und gesamt ersetzbar sind auch syntagmen, die wohl eher nicht als Konstituenten zählen würden: $\mathbf{4 0}$

(15.101) Gierig (MOD) in die Lungen (DIR) pumpten (VVfin) sie die Luft. Dem Affen (DATO) Likör (AKKO) geben (VVinf) darf (MVfin) nur Pia.

(15.102) Pia will einen Softie ehelichen. Das will Eulalia auch. Möglicherweise ist aber auch der "traditionelle" Konstituentenbegriff zu revidieren und Beispiele wie einen Softie ehelichen sind als Konstituenten anzuerkennen. Wendet man jedoch ein "Bündel" von Tests an, dann wird die Evidenz pro oder contra Konstituenz relativ stark.

Man könnte allerdings auch wie bei der syntaktischen (durchgezogene Linien) und der semantischen Dependenz (der semantischen Konnexion; gepunktete Linie) - z.B. zwischen prädikativem Adjektiv und subjekt - eine syntaktische und eine semantische Konstituenz annehmen. ${ }^{41}$

(15.103)

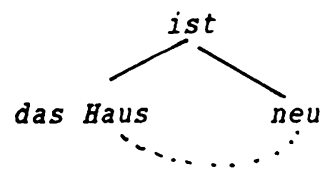

Daraus folgte, daB semantisch zusammengehörige bzw. aufeinander bezogene Aussageteile nicht notwendigerweise eine syntaktische Konstituente bildeten - man denke 2.B. an "gefloatete" NP-Elemente oder an prädikative Attribute. $\mathbf{4 2}$ AuBerdem gälte der UmkehrschluB, daß nicht jede nach Maßgabe der bekannten Ronstituententests syntaktische Ronstituente - man denke z.B. an die "mehrfache Vorfeldbesetzung" oder an ein-bzw. zweistellige VPn im Vorfeld - eine semantische darstellen muB.

Selbstverstandlich bedürte es einer Operationalisierung des Begriffs der semantischen Konstituenz, und die ist nicht einfach zu leisten. Vorläufig muB der vage Vorschlag genügen, semantische Konstituenz als ein Bündel von Merkmalen zu verstehen, die in einer semantischen Reprassentation zusammengefabt bzw. aufeinander bezogen werden müssen (ohne daß sie notwendigerweise als hierarchisch gleichgeordnet $z u$ betrachten wären). Ein anderes Bild: In einer menta-

40 Bei dem Beispiel in (15.102) könnte man allerdings mit Jacobs (1983: $38 \mathrm{ff.}$ ) annehmen, daß hier eine einstellige Verbalphrase als Konstituente figuriert.

41 Beispiel nach Tesnière (1980: 138 f.).

42 Jedenfalls betrăfe dies nicht bestimnte "Oberflăchenstrukturen". 
len Repräsentation von Satzbedeutung, die man sich probeweise als "semantisches Netzwerk" vorstellen kann, in dem zusammengehörige Information nahe beieinander abgespeichert wird, wären natürlich die Zusätze nahe bei ihrer Bezugseinheit abgespeichert, selbst wenn "syntaktisch" keine Berührung/Konstituenz 0. ä. besteht.

Ich wende mich ein letztes Mal der Frage der syntaktischen Konstituenz zu: Den Topikalisierungstest bestehen alle zusatztypen, wobei zu fragen ist, ob nicht eigentlich eine Vorfeldbesetzung (Bezugselement) und eine besetzte Parenthesenische (Zusatz) anzusetzen wären:

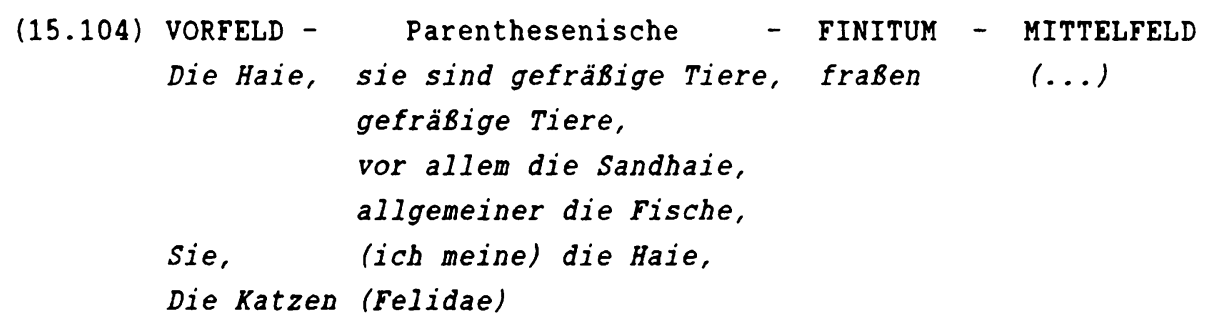

Ebenfalls scheint das Syntagma "Basis plus Zusatz" stets durch eine Pro-Form ersetzbar zu sein:

(15.105) (?) Sie, die Haie,/ die fütterten sie gerne.

Die Haie, besonders/genauer die Sandhaie,/ Sie schwammen herbei. Sie sahen Barry Birsch (SPD)/ ihn.

Wenn man den Fragetest anwendet, dann ergeben sich auch keine markanten Hinweise darauf, daß keine Konstituenz vorliegt:

(15.106) Wen fütterten sie?

(?) Die Haie, gefräBige Tiere (PRAZ).43

(?) Sie, die Haie (REFZ).

Die Haie, vor allem die Sandhaie (SPEZ).

Die Haie, allgemeiner die Fische (NSPEZ).

Die Haie, m.a.W. die Meeresmüllschlucker (ALNZ).

Harry yirsch (SPD). 44

Gliedteilfragen (Attributfragen) kann man nicht anwenden:

(15.107) Welche Haie fütterten sie?

* Die Haie, gefräBige Tiere.

* Die Haie, vor allem die Sandhaie.

* Die Haie, zumindest die Sandhaie.

*Die Haie, m.a.W. die Meeresmüllschlucker.

* Die Haie (Selachoidei).

Die Haie des Barrier-Riffs (GEN.ATTR).

43 Ich erinnere jedoch an die inakzeptablen Fälle, in denen der PRAZ durch AdjP oder PP repräsentiert wird.

44 In der Schriftsprache wohl gängig; wie dies in der gesprochenen Sprache aussähe, bleibt offen. 
(15.108) Welchen Harry Hirsch fütterten sie?

\author{
?? Harry Hirsch (CDU). 45 \\ Den Harry Hirsch mit dem Vollbart (PRAP. ATTR).
}

Bemerkenswert ist die Beobachtung, daß der spezifizierende Zusatz allein manchmal eine korrekte Antwort darstellt (andere zusătze nicht):

(15.109) Welche Haie fütterten sie? *GefräBige Tiere.

Vor allem/Zumindest die Sandhaie.

* Genauer/*Ausgenommen die Sandhaie.

*m.a.W. die Meeresmüllschlucker.

Auch bei einem Koordinationstest verhalten sich die in Frage stehenden Ronstrukte so, als ob Konstituenz vorläge:

(15.110) [Die Haie, gefräBige Meeresräuber,] und [die Rochen, besonders die Stachelrochen,] mag Pia nicht.

(15.111) [Die Ledigen, m.a.W. die Unverheirateten,] und [die Verheirateten, ausgenommen die Bigamisten,] studierte Pia genau.

(15.112) [Harry Hirsch (FDP)] und [Hugo Hummel, ein Windbeutel,] taten sich zusammen.

Dies möge genügen, um $z u$ demonstrieren, daß uns die "klassischen" Konstituententests" hier stets Konstituenz nahelegen, obwohl (zumindest beim Verf.) intuitiv ein gewisses Unbehagen gegen eine solche Beziehung vorhanden ist. Auch die Tatsache, daB die Zusätze bei diversen syntaktischen Operationen ihr Bezugselement begleiten können, spricht für Konstituenz.

Immerhin deutet das Stellungsverhalten einiger Typen an, daB möglicherweise nicht in Rahmen von Teil-Ganzes-strukturen analysiert werden sollte. So können die referenzklärenden, die spezifizierenden, die nichtspezifizierenden und wahrscheinlich auch die Alternativen nennenden zusätze von der Basis disloziert werden resp. nach syntaktischen operationen an der Ausgangsposition verbleiben (Position am rechten Satzrand) - nicht hingegen die kurzzusätze und die prädizierenden zusătze im großen und ganzen wohl auch nicht.

Auch die Feststellung, daB sich außer den Kurzzusätzen alle übrigen zusatztypen - anders als die davorstehenden Attribute - normalerweise hinter Postpositionen, nachgestellten Gradpartikeln und Konjunktionaladverbien befinden (müssen), deutet eventuell auf eine zumindest lockerere zuordnung zum Bezugselement hin als dies bei typischen Ko-Konstituenten wie den Gliedteilen der Fall ist.

Die unterschiedliche BeeinfluBbarkeit resp. Fokussierbarkeit der Zusätze durch Operatoren wie NEGATION, GRADPARTIKEL oder FRAGE (Punkt 16. dieses Ra-

45 Es ist nicht auszuschließen, daß in gesprochener Sprache derartige Rontrastierungsstrategien Verwendung finden. Dennoch kann nur die attributive Kontrastienmg als akzeptabel und stets wählbar gelten (F15.16):

(F15.15) / ich suche nicht den harry hirsch ad u pt sondern den harry hirsch sp $d \mathrm{ftt}$.

(F15.16) / ich suche nicht den harry hirsch von der od u pt sondern den harry hirsch van der sp $d \mathrm{fH}$. 
pitels) deutet u.U. auf einen Unterschied zwischen den Mustern prädizierender Zusatz, Kurzzusatz und ausschließender Zusatz (nicht erfaßbar) sowie allen übrigen (affizierbar) hin. Da hier jedoch sowohl semantische wie auch pragmatische Faktoren eine Rolle spielen, ist für mich nicht zu entscheiden, ob dies als Beweisführung zugunsten oder zuungunsten von Konstituenz zu werten ist.

Die vorläufige Beantwortung der Frage, ob Basis und Zusatz eine Konstituente bilden, läuft auf die Feststellung hinaus, daB sich einerseits deutliche Unterschiede zur "prototypischen Konstituenz" - etwa von Kern und Attribut oder von VP und regierten Ergänzungen - aufweisen lassen, andererseits eine völlige "Beziehungslosigkeit" unangemessen erscheint, so daß ich den Hybridbegriff "Konstituentoid" ansetze (vgl. Kap. 6, 7.A, 7.B) bzw. feststelle, daB sich ein ZUSATZ an ein Element des Gastsatzes "assoziiert", m.a.W. mit ihm eine Assoziationsbildung und keine Konstituentenbildung eingeht.

Jetzt komme ich zur Zusammenschau einer Auswahl m.E. wichtiger Analyseergebnisse, die nochmals die deutliche "Verwandtschaft" spezifizierender, nichtspezifizierender und Alternativen nennender Zusätze (die vielleicht zu einer Großgruppe zusammengefaßt werden können) unterstreichen soll - ebenso wie die Verschiedenheit der restlichen drei Muster. Ebenfalls sei nochmals darauf hingewiesen, daß sich im Bereich der nichtspezifizierenden sowie der Kurzzusätze noch weitere Ausdifferenzierungen erwarten lassen und daß Relativsatzzusätze sowie einschaltungsmarkierte Gliedteile nicht aufgeführt werden (Folgeuntersuchungen sind notwendig):

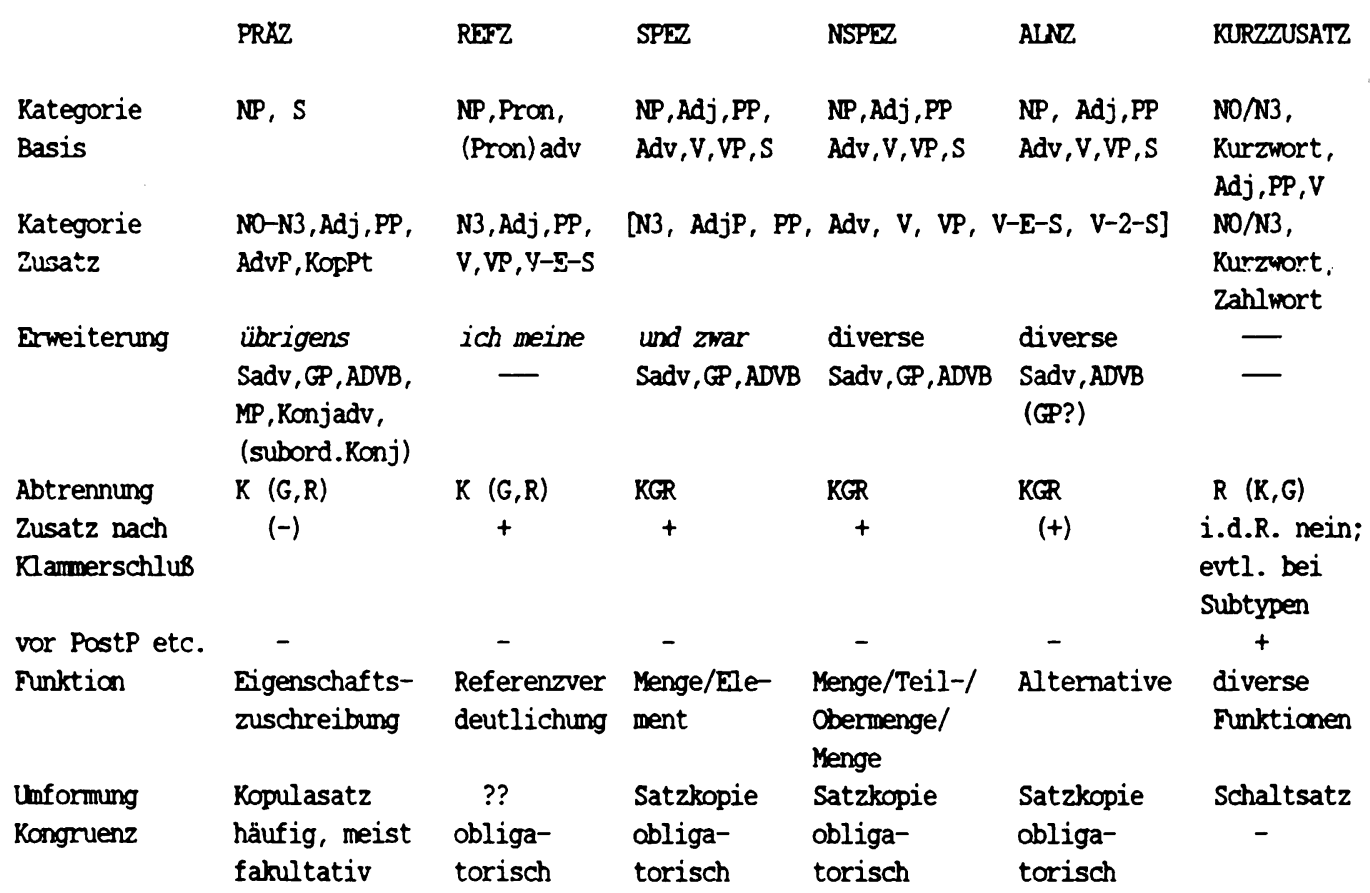


PRAZ

Attribution

affizierbar?

Rekursivität
REFPZ

SPEZ

begrenzt

$+$

- begrenzt

$(t)$

begrenzt
NSPEZ

ALR

begrenzt

begrenzt

kontrovers

begrenzt
KURZZUSATZ

$(-)$

$+\quad-$

sehr begrenzt (-) 
16. ZUSAMMENFASSUNG UND ABSCHLIÉSSENDE OBERSICHTEN

\subsection{Zusamenf assung}

Es folgt zur schnellen Information eine Zusammenfassung wichtiger Arbeitsschritte und -ergebnisse. Aus 16.1. kann man auch die wichtigsten Abgrenzungskriterien (Apposition versus Attribut u.a.m.) ersehen.

In Rapitel 3 wurden grammatische Eigenschaften erörtert, die bei jeweils variierter Bündelung bislang zur Konstitution eines Appositionsbereichs aufgestellt wurden. Es zeigte sich, daB die Menge der in die Diskussion eingebrachten Eigenschaften (insgesamt 17) beträchtlich ist. Dabei stellte sich heraus, daß "bewährte", oft verwendete und z.T. als für Apposition wesentlich angesehene Merkmale heuristisch wenig oder nur bedingt brauchbar sind: so erwiesen sich z.B. Kasuskongruenz, Referenzidentität, Weglaßbarkeit der Apposition oder die Vertretung der Basis durch das Appositiv (und auch seltener verwendete Merkmale wie Satzgrenzenbestimmtheit oder Nahrheitswertkonstanz) als nur bedingt tauglich, um eine konsistente Bestimmung von "Apposition" zu erreichen.

Als wichtig und für die weiteren Analysen bedeutsam gaben sich insbesondere folgende Merkmale zu erkennen: die intonatorische/graphematische Abtrennung des Appositivs (Einschaltungsmarkierung), das stellungsverhalten des Appositivs relativ zur Basis, mögliche Umformungsbeziehungen zwischen Appositionskonstruktionen und anderen Sprachmustern (z.B. satzförmige Paraphrasen), mögliche Begleiter des Appositivs (Appositionslexeme, Satzadverbien etc.) und das Verhalten der Apposition bzgl. skopusbildender Ausdrücke wie Negations- oder Gradpartikeln. Zusätzlich wurden zu berücksichtigende grammatische Eigenschaften wie z.B. die rekursive Erzeugbarkeit von Appositiven etc. und Gesichtspunkte der Dependenz und Konstituenz eingeführt.

In Rapitel 5 wird vorgestellt, wie "Apposition" in verschiedenen Grammatikmodellen behandelt wird. Unterschiedlich ist die 2uordnung "enger" und "lockerer" Syntagmen: Die "engen" sind stark an das Bezugselement gebunden (fakultative Erganzung; restriktiv (X-Bar-Ebene: N2); spezifizierend)., die "iackeron" sehr viel weniger (Angabe; nicht-restriktiv (N3); nicht spezifizierend, sondern pragmatische Funktion). Unter dem Gesichtspunkt der Konstituenz werden "Appositive" ungeachtet des Unterschiedes "eng" versus "locker" i.d.R. als Bestandteile ihrer Bezugseinheiten behandelt. X-Bar-syntaktisch werden "lockere Appositive" als hierarchisch "oberste" Phrasenteile ohne restriktives Potential gewertet, die "engen" Appositive hingegen werden entweder auf unterster Stufe (Vater 1986b) oder auf der Stufe restriktiver Komplemente (Jackendoff 1984) angesiedelt. Dependentiell betrachtet sind "lockere Appositionen" freie Angaben (zum Nomen), "enge Appositionen" $"$ Ergänzungen, da sie nur mit Subklassen von Nomina, nicht jedoch frei mit allen Nomina kombinierbar sind, Kategorialgrammatisch gesehen fallen "lockere Appositionen" nicht unter die Spezifi- 
kationsbeziehung (sie sind also weder Attribut noch Komplement), "enge" sehr wohl (sie sind Adnominale).

Im letzten Abschnitt von Kap. 5 wird ein Modell entworfen, in dessen Rahmen das Appositionsproblem bearbeitet werden soll. Es geht davon aus, das man einen Blenentarsatz (umfaßt Prädikat, Ergånzungen, Angaben, Partikelfunktionen, Gliedteile) annimmt, der um Gáste (Bospitanten) erweitert werden kann. Der Terminus HOSPITANT deutet an, daß die betreffenden Einheiten in geringerem Maße in den "Gastsatz" integriert sind als beispielsweise die Glieder des Elementarsatzes (Aktanten etc.). Die Gãste sind allesamt mit dem Einschaltungsmuster versehen und lassen sich in BIASCHOBE (konstruktionaler Aspekt, kein Bezugselement; z.B. Schaltsatz) und zosxrze (syntaktische Relation, mit Bezugselement; z.B. lockere Apposition) einteilen. Die Găste sind von Rektion, Valenz, Negation, Erfragung etc. aus dem "Gastsatz" heraus nicht erfaßbar. Sie treten kaum in Wechselwirkung mit den Elementarsatzeinheiten. Die ZUSATZE umfassen die i.e.s. appositionsverdăchtigen Einheiten, darüber hinaus aber auch noch mehr (vgl. Kap. 12-15).

In Rapitel 6 wurden wesentliche grammatische Eigenschaften des "Appositionsprototypen" ermittelt. Als bedeutsam erwies sich, daß die Basis derartiger Konstruktionen stets eine Voll-NP ist, wogegen das stets mit dem Einschaltungsmuster versehene Appositiv kategorial von Nominalen beliebiger $\mathrm{X}$-Bar-stufe, von Adjektiv - und Präpositionalphrasen reprảsentiert werden kann. Die typische Appositionsfloskel ist übrigens, mögliche Appositivbegleiter sind Satzadverbien, Gradpartikeln und freie Adverbiale; gezeigt wurden auch seltenere optionen wie begleitende Modalpartikeln, Ronjunktionaladverbien etc. Das Appositiv tritt zumeist rechtsadjazent zur Basis auf, Distanzstellungen wirken entweder stilistisch stark markiert oder führen zum Ronstruktionswechsel. Wenn Permutationsoperationen wie Linksverschachtelung etc. vorgenommen werden, dann unterliegen ihnen i.d.R. Basis und Apposition, nie die Basis allein.

Das Verhältnis des Appositivs zur Basis ist prädizierend, was sich anhand stets möglicher Kopulasatzparaphrasen zeigen läbt. Als semantische AppositivFunktionen sind Eigenschaftszuschreibung, Klasseneinordnung, Gleichsetzung und Lokalisierung (appositive PPn) zu nennen. Menge-Element-Relationen kommen beim Prototypen nicht vor (s. aber Kap. 12, 15). Die Kasuskongruenz des nominalen Appositivs erweist sich als zumeist fakultativ, wenngleich statistisch sehr hăufig. In etlichen Făllen ist die Rongruenz sogar ausgeschlossen (bei Anwesenheit von Appositionslexemen, Gradpartikeln etc. und wenn das Appositiv z.B. von der Rategorie NO, AdjP, PP ist). Referenzidentităt findet man selten vor; sie spielt nur bei den Gleichsetzungen eine Rolle. Die Kriterien Neglabbarkeit des Appositivs, Vertretung der Basis durch das Appositiv, Satzgrenzenbestimmtheit, mögliche Sprecherdivergenz, Attributionsfähigkeit des Appositivs, Textnotwendigkeit, Adordinativität und Wahrheitswertkonstanz erwiesen sich als venig nutzlich (zu allgemein, zu vage etc.). 
Wichtig ist, daB der Prototyp vom Gastsatz aus nie durch bereichsbildende operationen wie Negation, Gradpartikelanwendung etc. erfaBt werden kann! Zudem wurde gezeigt, daß der Prototyp rekursiv erzeugbar ist (mehrere Appositive können koordiniert werden) und daB ein Appositiv eine Passivtransformation mit der Basis (und nicht ohne sie) durchläuft. Appositionen scheinen bzgl. ihres Bezugselements weder in einer gewöhnlichen Dependenz- noch in einer (Ko-) Konstituenzrelation $z u$ stehen. Dies führte $z u$ der Hypothese, daB Appositive in einer besonderen syntaktischen Beziehung der "Assoziation" zu ihrer Basis stehen.

In 6.A wurde die sog. "Satzapposition" behandelt, deren grammatischer Status nicht befriedigend geklärt werden konnte. Gemeinsamkeiten mit dem Prototypen bestehen u.a. in der Hinzufügbarkeit von übrigens, der Umformungsbeziehung zu Kopulasätzen und der Nichtaffizierbarkeit durch Negations- bzw. Gradpartikeln im Bezugssatz. Die Satzapposition kann nicht nur am rechten Satzrand auftreten, sondern auch an den (meisten) Parenthesenischen eines Satzes. Sie wird als Zusatz und nicht als Einschub gewertet, da sie nicht die stellungsmäBige resp. kommunikative Selbständigkeit $z$. B. eines Schaltsatzes aufweist.

In Kapitel 7 nird der Auffassung widersprochen, daB "Apposition" eine Subkategorie der Kategorie Attribut sei. Als Attribute werden Konstruktionen angesehen, deren Basen kategorial durch $N$, Adj und Adv (und evtl. Präp resp. PP) repräsentiert werden, und von denen Satelliten abhängen, die nicht einschaltungsmarkiert sind, mittels einer Gliedteilfrage (welch-? was für ein-?) erfragt werden können, kontrastierend negiert werden und von einer Gradpartikel affiziert werden können, bzgl. ihrer Basen in einem Verhältnis der Dependenz bzw. der Subordination stehen und mit der Basis zusammen eine Konstruktion bilden (sie sind Ko-Konstituente relativ zur Basis). Somit fallen alle einschaltungsmarkierten appositionsverdächtigen Muster aus dem attributiven Bereich heraus!

Als Attribute erweisen sich in Kap. 7: restriktive Relativsätze (1.2.4.), 1 "enge Appositionen" (2.1.-2.7.; sie verhalten sich in vielerlei Hinsicht attributtypisch!), nicht-restriktive Adjektivattribute (4.1.1.), aus NPn gefloatete Elemente (Quantoren, Adjektivattribute; 4.1.3.), der Typus hier am Knie bzw. im Stadion auf der Tribüne (4.2.1.; Adv/PP als Basis plus PP als Attribut!) sowie das Muster eine Chefin wie Pia (5.2.).

In Kap. 7.A wird vorgeführt, daß sich restriktiver und appositiver Relativsatz in wesentlichen grammatischen Eigenschaften unterscheiden: Der appositive Relativsatz (1.2.4.) weist Parentheseintonation auf, ist nicht durch eine Gliedteilfrage erfragbar, ist von Negations- und Gradpartikeln nicht affizierbar, scheint nicht von seiner Basis dependent und eher an sein Bezugselement "assoziiert" als dessen Ko-Konstituente zu sein und steht i.d.R. strikt adjazent bzgl. der Basis, wogegen der restriktive Relativsatz zumeist extraponier-

1 Die Mumern in Kammern verweisen auf den in Kapitel 2 registrierten TYp. 
bar ist. Wahrend der restriktive Relativsatz attributiv fungiert, wird der nicht-restriktive aus dem attributiven Bereich ausgesondert.

Ein Vergleich zwischen dem appositiven Relativsatz, dem "Prototypen" und dem Schaltsatz fuhrt zu der Schlußfolgerung, daß ersterer ebenfalls den Hospitanten zuzuordnen ist, und zwar den NP-Zusătzen (Einschaltungsmuster, unerfragbar, von NegPt/GP nicht affizierbar usf.). Dennoch läbt sich der appositive Rels weder mit dem Prototypen noch mit Schaltsätzen in eins setzen. Zwar kann man den Prototypen u.U. als Reduktionsprodukt nicht-restriktiver Rels mit Kopulaverb ansehen, es treten aber auch Rels mit beliebigen Vollverben auf, die man nicht reduzieren kann, so daß eine prototypische Apposition übrig bleibt. Von typischen Parenthesen unterscheidet sich der Rels u.a. durch seine strikte Adjazenz zur Bezugs-NP. Die Stellungsvariabilităt des Einschubs weist er nicht auf. Der appositive Rels verbleibt als NP-zusatz sui generis (terminologisch: Relativsatz-zusatz) im appositionsverdăchtigen Bereich und wird in den Kap. 15 u. 16 (stellenweise) wieder aufgenommen.

In 7.B wird auf diverse Vorkommnisse von als-Phrasen (5.1.) eingegangen; viele dieser Phrasen können als Adverbiale, manche als Prădikative oder auch Attribute eingestuft werden. Wichtig ist, das nur die NP-adjazente als-Phrase appositionsverdảchtig erscheint; entfernt man solche Phrasen von den Bezugselementen, dann verändert sich die Konstruktion: die als-Phrase wird zum Adverbial.

Es wird gezeigt, daB sich die NP-adjazente als-Phrase nur teilweise attributartig verhät: sie tritt zusammen mit ihrer Basis im vorfeld auf und ist zusammen mit ihr ersetzbar; bereits die gesamte Erfragbarkeit ist schon problematisch, und sicher auszuschließen ist die attributtypische Anwendung einer Gliedteilfrage, die (potentielle) Restriktivität des Satelliten sowie die Affizierbarkeit durch Negations- und Gradpartikeln. Zur Einstufung derartiger Phrasen als zusătze (Appositionen) führen insbesondere die Möglichkeit der Kasuskongruenz und Kopulasatzparaphrase (vgl. Prototyp Kap. 6); ins Feld funren kōnnte man zudem die Nicht-Restriktivităt solcher Phrasen. Entscheidend ist, daB die als-Phrase nicht einschaltungsmarkiert ist; sie könnte allerdings mit dem Einschaltungsmuster versehen werden, aber erst dann wertete ich sie als Hospitant (Zusatz). Oberdies sind als-Phrasen keiner appositionstypischen Erweiterung (Floskel, Satzadverb usf.) făhig. Die NP-adjazente als-Phrase wird schlieblich der attributiven Funktion zugeordnet, wenngleich sie als Muster sui generis an der Peripherie des Attributbereichs anzusiedeln ist. Diese Einordnung bleibt aber problematisch!

In Rapitel 8 werden zum einen die Satzglieder aus dem Appositionsbereich ausgegliedert: nicht parenthetisch markierte Adverbialsătze (im Mittelfeld; 3.6.1.2.), adverbiale als-Phrasen (5.1.) und prădikative Attribute (6.), zum anderen die Partikeln (v.a. nachgestellte Gradpartikeln; 4.1.2.). Satzglieder sind im Unterschied $z u$ den appositionsverdächtigen Hospitanten nicht einschaltungsmarkiert, sie sind mittels Satzgliedfragen (z.B. wo? wann? warum?) er- 
fragbar und können von einer im Gastsatz befindlichen Negation (insonderheit einer kontrastierenden) oder von einer Gradpartikel betroffen werden.

Es stellt sich heraus, daB Adverbialsätze sowohl im Mittelfeld als auch am rechten Satzrand (extraponiert) in der Schriftsprache ambig sein können. In der gesprochenen Sprache markiert dagegen die Intonation den Unterschied $z$ wischen Satzglied (enklitisch; die Adverbialsätze sind kontrastierend negierbar) und Zusatz zum Satz (parenthetisch; keine Kontrastnegation möglich).

Die nicht-satzgliedhaften Partikeln üben einen Einfluß auf Elementarsatzelemente aus (so fokussieren z.B. Gradpartikeln Satzglieder und führen eine quantifizierende bzw. skalierende Interpretation herbei). Sie sind i.d.R. nicht (allein) mit dem Einschaltungsmuster versehbar; daher scheiden auch sie als Appositionskandidaten aus.

In Rapitel 9 werden Fälle "mehrfacher Vorfeldbesetzung" als mögliche appositionsverdächtige Muster diskutiert. Dabei werden enge vorfeldbesetzende Konstruktionen wie oben im Schrank/ im Stadion auf der Tribüne (Typ 4.2.1.), bereits in Kap. 7 als attributive strukturen eingestuft, aus dem appositionsverdächtigen Bereich ausgeschlossen. Dabei ist wichtig, daß beide Konstruktionsteile die gleiche adverbiale Funktion innehaben können (z.B. oben (lokal) im Schrank (lokal)). Sie sind nicht einschaltungsmarkiert, lassen Gliedteilfragen zu und können von Negationen etc. affiziert werden. Analoge lockere Muster (oben, im Schrank, usw.) mit gleichen Adverbialfunktionen (Typ 3.2.) werden zu den ZUSXTZEN gerechnet und später (Kap. 12, 15, 16) wieder aufgenommen.

Echte mehrfache Vorfeldbesetzung (z.B. manchmal tief aus dem Kehlkopf holte er seine stimme; Typ 4.2.2.) liegt bei engen Mustern vor, die keine Einschaltungsmarkierung aufweisen und nur getrennt erfragbar sowie nur getrennt ersetzbar sind; sie können zwar gemeinsam im Vorfeld stehen, sie sind jedoch auch getrennt verschiebbar; beide Adverbiale sind nur jeweils für sich, nicht aber zusammen von Negations-bzw. Gradpartikeln affizierbar und gehören stets unterschiedlichen Adverbialsubklassen an.

Zuletzt kommen Muster zur Sprache, bei denen zwischen Vorfeldelement und Finitum eine satzförmige Einschaltung erfolgt. Handelt es sich um eingefügte Elemente, die potentiell eine Adverbialfunktion erfüllen könnten (also um Typ 3.6.1.2.), werden die Einschaltungen als SATZ-ZUSATZE gewertet. Wenn potentiell keine Funktion erfüllt werden kann - es liegen Schaltsätze vor -, dann wird ein EINSCHUB angenommen (Typ 3.7.; weiteres s. Kap. 11).

Das Kapitel 10 befast sich mit der Abgrenzung von Herausstellungen (nach Altmann 1981). Die Linksversetzung (7.1.) erweist sich als nicht appositionsverdächtig: sie ist nicht vollständig einschaltungsmarkiert, von Partikeln affizierbar, sie steht links (nicht wie in den "verdächtigen Fällen" rechts) vom Bezugsausdruck, ist auf eine feste Position im Satz beschränt (vor dem Vorfeld, in dem der Bezugsausdruck steht) und für Basis + LV sind keinerlei (appositionstypische) Umformungsbeziehungen denkbar. Die Linksversetzung zeigt 
eine hohe syntaktische Integration in den Bezugssatz, moglicherweise besetzen LV und das aufnehmende Pro-Element gemeinsam das Vorfeld.

Die Rechtsversetzung (7.2.) erweist sich als bleibender Appositionskandidat; sie taucht als referenzklárender Zusatz in Kap. 12, 15 wieder auf. Dabei zeigt sich, daB Altmann (1981) diese Funktion mit Recht scharf von der "prototypischen Apposition" (dem prádizierenden Zusatz) abgegrenzt hat.

Kompliziert gestaltet sich der AusschluB beim Nachtrag (7.3.). Nachgetragene Einheiten $z u$ Wortgruppen (primär $z u$ NPn, aber auch PPn etc. Typ: die Haie, und zwar (vor allem) die Hammerhaie) bleiben als "spezifizierende Zusätze" appositionsverdächtig (Weiterbehandlung in Kap. 12, 15).

Nachgetragene freie Angaben könnten nach Wegfall der Einschaltungsmarkierung als Adverbiale im Bezugssatz fungieren. Sie vermögen auch an bestimmten Parenthesenischen im Mittelfeld vorzukommen, nicht jedoch am linken Satzrand und mit fragwürdiger Akzeptabilität zwischen Vorfeld und Finitum, so das sie nicht als EINSCHUB (vormals: Parenthese) einzustufen sind. M.a.W.: Dieser TYp ist stellungsmäBig restringierter als Einschübe und nicht wie diese kommunikativ selbständig. Er ist allerdings nicht von Partikeln affizierbar. Die typische Nachtragseinleitungsfloskel ist und zwar, sonst aber sind derartige Nachtrăge kaum "appositionstypisch" erweiterbar; zudem ist das Bezugselement ungleichrangig (VP bzw. Satz), was bei appositionsverdächtigen Mustern unüblich ist. Als Herleitung können Satzverdoppelungen plus Tilgungen gleichen Materials dienen. Insgesamt erscheint eine Einstufung als EINSCHUB unwahrscheinlich. Die Nachträge dieses Typs werden (wie die parenthetischen Angabesătze) als SATZ-ZUSXTZE gewertet.

In Rapitel 11, in dem zunăchst der Parenthesebegriff anhand einiger Arbeiten diskutiert wird, ergibt sich folgendes Fazit: EIMSCHOBE (3.7.; ehedem: Parenthesen) weisen das Einschaltungsmuster auf, können an allen Parenthesenischen sowie rechts und ofters auch links des Bezugssatzes auftreten (dann bilden sie mit diesem Satzfolgen), sind von Partikeln nie betroffen und nie erfragbar; es existieren keine Umformungsbeziehungen zwischen Einschub und Bezugssatz. Zu den Einschüben zăhlen von den nicht satzformatigen, äußerungswertigen Einheiten: INTERJEKTIONER, ANREDER, VOKATIVE und die SATZWORTER (danke etc.). Ebenfalls als EINSCHUBE sind folgende satzformatige Einheiten anzusehen: Verb-zweit-, Verb-Erst- und Verbend-Schaltsătze, sofern sie nach Fortfall der Einschaltungsmarkierung im Gastsatz keine syntaktische Funktion ausüben konnten.

Verb-End-Sătze, die eine syntaktische Funktion im Gastsatz ausüben könnten, (3.6.1.2.) werden ungeachtet ihrer relativ großen stellungsvariabilität als SATZ-ZUSATZE gewertet; sie gehen keine Satzfolge mit dem Gastsatz ein. Ebenfalls SATZ-ZUSATZE stellen einschaltungsmarkierte, nicht-satzförmige freien Angaben dar (Typ: Pia hat, um drei Uhr, einen Affen geärgert).

Negations-, Grad- und Modalpartikeln sind allein offenbar nie als ZUSATZ oder EINSCKUB möglich. Der Satzrelativsatz (= der weiterführende Nebensatz) 
blieb hinsichtlich seiner Einordnung unklar; er tendiert eher in Richtung SATZ-ZUSATZ, da er nicht über alle stellungseigenschaften eines Einschubs verfügt. Er bildet z.B. keine Satzfolge; der Satzrelativsatz kann keine syntaktische Funktion im Bezugssatz erfüllen usf.

Als Einschaltungen markierte Phrasen, die nach Fortfall des Einschaltungsmusters als Attribute ihrer Bezugselemente fungieren könnten, werden als ZUSATZE (also "Appositionen") zu diesen Phrasen angesehen, allerdings in anderer Weise als z.B. der Appositionsprototyp, der in prädikativer Relation zu seinem Bezugselement steht (Typ: das - relativ - freie Spiel der Kräfte; vgl. Kap. 15). Sie sind weder erfragbar (keine Gliedteilfrage!) noch können sie von Partikeln betroffen werden.

In Rapitel 12 wurde eine möglichst ausführliche Sammlung von "i.e.s. appositionsverdăchtigen Mustern" der Struktur "NP (Basis; z.B. die Haie) + Funktionslexem (z.B. vor allem) + NP (Zusatz; z.B. die Hammerhaie)" angelegt. Die Funktionslexeme wurden hinsichtlich ihrer Kategorie, Funktion und semantischen Leistung untersucht. Fünf deutlich unterscheidbare Großklassen von ZUSATZEN (mit ein paar Submustern) lieBen sich unterscheiden:

(1) spezifizierende (die Haie, vor allem die Hammerhaie,)

(2) nichtspezifizierende (die Haie, obendrein die Barrakudas,)

(3) Alternativen nennende (die Haie, m.a.W. die Räuber der Meere,)

(4) referenzklărende (die, ich meine die Haie,) und

(5) prådizierende Zusătze (die Haie, übrigens eine uralte Tierart,). Den semantisch-funktionalen Unterschieden entsprechen gewisse grammatische Eigenschaften. Nur (5) läBt eine Ropulasatzparaphrase und Zusätze der Kategorie N2, N1, NO, AdjP, PP zu nominalen Basen zu; zudem kann (5) als einzige Klasse mehr als zwei Mal bei einer Basis auftreten. (1) mit (3) (vielleicht auch (4)?) sind eher über eine Satzverdoppelung mit anschließender Tilgung und Einbettung in den Gastsatz zu erklären. Nur (1) kann generell, vor allen spezielleren Funktionslexemen, das Lexem und zwar mit sich führen etc. Außerdem zeigte sich, daß etliche Funktionslexeme auch Zusătze zu Basen nichtnominaler Rategorien (z.B. Putzen, vor allem abstauben und wischen, mag Pia nicht) begleiten bzw. markieren können (dazu năher Kap. 14).

In der bisherigen Forschung zu "i.e.S. appositionsverdächtigen Mustern" wurden die füf ermittelten Muster lange Zeit "in einen (Appositions) Topf geworfen". Nicht alles, was einschaltungsmarkiert rechts von einer NP steht, sollte pauschal der syntaktischen Funktion Apposition zugewiesen werden. Vielmehr sind nach meinen "Ermittlungen" mindestens funf Funktionen innerhalb einer syntaktischen Relation ZUSATZ unterscheidbar (sofern man nicht (1) mit (3) aufgrund ihrer Ahnlichkeiten zusammenfassen wird). Eine sechste Funktion, den Kurzzusatz, demonstriert Kap. 13. Eine siebte stellt der Relativsatz-Zusatz (APREL; 7.A) dar, eine achte das einschaltungsmarkierte Gliedteil (Kap. 11).

In Rapitel 13 wird der sog. KURZzUSATZ - ein i.d.R. unmittelbar rechts von der Basis stehendes, rundgeklammertes, unerweitertes Wort wie z.B. in Willy 
Brandt (SPD) - behandelt. Es wird gezeigt, daß sich mehrerlei hinter diesem Muster verbirgt: sowohl ein eigenständiges (bislang unerforschtes) Muster KURZZUSATZ als auch rundgeklammerte Varianten von Alternativen nennenden, prädizierenden und spezifizierenden zusätzen. Das grammatische Muster KURZZUSATZ ist zur Wahrnehmung relativ vieler semantisch-pragmatischer Funktionen făhig ('Angabe eines Zahlenverhältnisses, eines Erscheinungsjahres, einer Lebenszeitspanne' etc.). Wie alle ZUSATZE legen KURZZUSATZE kein attributartiges Verhalten an den Tag (von Negation usw. nicht erfaßbar; keine Gliedteilfrage etc.); sie verhalten sich wie typische HOSPITANTEN. Die Kurzzusätze bilden die sechste Klasse von NP-bezogenen HOSPITANTEN; die möglichen subfunktionen müssen noch (empirisch) erarbeitet werden.

Das Kapitel 14 befaBte sich mit Einheiten, deren Nertung als ZUSATZE zu Basen ohne nominalen Kern naheliegt. Es stellte sich heraus, das Muster wie Er kommt, (genauer:) er kommt morgen als Satzfolgen (mit semantisch-pragmatischen Bezügen, die über pure Sachverhaltsreihung hinausgehen) einzustufen sind und daB keine ZUSATZ-Beziehung zwischen beiden Teilsătzen einer Satzfolge (selbständige V-2-Hauptsătze!) besteht (Typ 3.6.1.1.). Es wăre widersinnig, eine syntaktische Relation wie z.B. ZUSATZ über die Einzelsatzebene hinaus auszudehnen, da " (...) oberhalb der Grenze des unabhängigen Satzes keine syntaktischen Relationen bestehen" (Lehmann 1983: 339). Beide Teilsătze einer Satzfolge sind formal wie auch funktional selbständig; nach dem ersten Teilsatz ist zudem eine deutliche Satzpause und fallende (keine progrediente) Intonation festzustellen.

An fast alle Wortgruppen unterhalb der Satzebene (VP, AdjP etc.) vermögen sich allerdings zusătze zu assoziieren, jedoch i.d.R. nur ALTERNATIVEN NENNENDE ZUSATZE, ein Teil der SPEZIFIZIERENDEN ZUSATZE und der VERALLGEMEINERNDE ZUSATZ. Bei dem Muster "Basis (Det) + Zusatz (Genitiv-NP)" kann man einen REFERENZKLARENDEN ZUSATZ annehmen. Was niemals vorkommt, sind PRADIZIERENDE ZUSATZE (ehedem: der Appositionsprototyp). Zwar hat die syntaktische Relation ZUSATZ ihre Domäne sicherlich im Bereich nominaler Phrasen, doch müssen andere Phrasen ebenfalls beachtet und in Zukunft noch weiter untersucht werden.

Es konnte ein Zusammenhang zwischen Satzfolgen und subsententialen BasisZusatz-Konstellationen insofern hergestellt werden, als die subsententialen Formen als Reduktionsprodukte von Satzfolgen (mit Einbettung) dargestellt werden können - ein Hinweis auf die Korrektheit der "Satzkopiehypothese". Keine Zusătze scheinen möglich zu Grad-, Modal- und Negationspartikeln, zu Prăpositionen und Konjunktionen, zu Satzadverbien sowie zu Interjektionen.

Das Rapitel 15 bietet eine Zusammenschau über die grammatischen Eigenschaften der wortgruppenbezogenen zusătze, also der fünf einleitbaren zusätze (aus Kap. 12) und der Kurzzusătze (Kap. 13), unter vornehmlicher Berücksichtigung NP-bezogener Einheiten, die in dieser Arbeit von zentralem Interesse waren. Es stellt sich heraus, daß der prädizierende zusatz (entspricht etwa der lockeren Apposition: Udo, Koch aus Passion; nur NP (evtl. auch S) als Bezugselement; 
Paraphrase: Kopulasatz; Erkennungsfloskel: übrigens), der referenzklärende (entspricht etwa der Rechtsversetzung: Er, der Udo; Basen mit vager Referenz; Erkennungsfloskel: ich meine) und der Kurzzusatz (Willy Brandt (SPD); in Rundklammern; Umformungsbeziehung zu gekũrzten Schaltsătzen) als völlig eigenständige Muster anzusehen sind. Zwischen spezifizierenden (die Affen, vor allem die Gibbons), nicht-spezifizierenden (die Gibbons, allgemeiner alle Affen) und Alternativen nennenden Zusătzen (die Ledigen, m.a.W. die Unverheirateten) bestehen viele Gemeinsamkeiten wie z.B. gleiche Bezugselemente, oftmals MengeElement-Semantik und die Erklärung aus Satzdoppelung + Tilgung (+ Einbettung in Gastsatz). Semantisch-funktional erscheinen sie distinkt genug, um als $\mathrm{ZU}-$ SATZ-Submuster zu gelten. Im Bereich der nicht-spezifizierenden Zusätze bleibt die Frage offen, ob diese "Sammelklasse" noch weiter aufzugliedern wăre.

\subsection{Die relevanten distinktiven Merkmale der Hospitanten}

Die Konstitution eines Appositionsbereichs (eines Appositionsbegriffs) hängt davon ab, wie man die grammatischen Eigenschaften sprachlicher Muster relativ zueinander gewichtet und bündelt. Ich führe im folgenden die wesentlichen gramatischen Merkmale auf, die zu meinen Kategorien EINSCHUB (ehedem: Parenthese) und ZUSATZ (ehedem: Apposition) führen. Anhand des Einschaltungsmusters kann der Bereich der Hospitanten von dem der Partizipanten abgetrennt werden. Mit dieser Trennung korrespondieren Unterschiede bzgl. der Erfragbarkeit und der Affizierbarkeit von kontrastierender Negation (nicht $X$, sondern $Y$ ) sowie von Gradpartikeln:

\section{EINSCHALTUNGSMARKIERUNG (vollständig) ? ${ }^{2}$}

keine (bzw. unvollstăndig bei (a5))
(a) P A R T I Z I P A N T E N
(a1) ATTRIBUT
ein Sack mit Papierschnipseln
(a2) SATZGHIBD

Pia hat um 3 Uhr den Hai gefüttert

(a3) PARTTKBZ (FUNKTION)

(a4) MITRFACHE VORPEDDBESEIZUNG

(a5) HIRRAUSSTMTLUNG (speziell LV)

erfragbar (a1, 22, a4)

von NDG/GP affizierbar (a1, a2, a4?) vollständig vorhanden

(b) HOS P I T A T E N

(b1) EINSCHUB

Der Graf ist - so glauben sie mir doch - ein Vampir

(b2) WORTGRUPPEN-ZUSATZ

ein Sack, z.B. ein Müllsack,...

ein Sack (mit Papierschnipseln)...

(b3) SATZ-ZUSATZ

Pia hat - um 3 Uhr - den Hai gefuttert

nie erfragbar

selten affizierbar ${ }^{3}$

2 Vollständig bedeutet, daß es mindestens eine Variante der betreffenden Konstruktion gibt, in der das vermeintliche Appositiv links und rechts markiert ist.

$3 \mathrm{Vgl}$. die einschlägigen tbersichten in Kap. 12, 15. Affizierbar sind generell REFZ, ALNZ, KORREXTUR und WIRDERROLUNG. Nur von GPn betroffen werden manche Subtypen der SPEZ. 
Das wichtigste Merkmal, das im Bereich der HOSPITANTEN die EINSCHUBE von den ZUSATZEN trennt, ist die stellungsfreiheit. EINSCHUBE können wegen ihrer syntaktischen Unabhängigkeit und ihrer kommunikativen Selbständigkeit (sie tragen eine Illokution) potentiell links und rechts des Bezugssatzes und an allen $\mathrm{Pa}-$ renthesenischen auftreten; ZUSATZE sind deutlich stellungsbeschränkt.

\section{STELLUNGSFREIHEIT?}

gering: tendenziell adjazent zum Bezugselement WORTGRUPPDI-ZUSATZ Haie, var allem Blauhaie, (hat Pia gesehen) keine: strikt adjazent EINSCHALTUNGSTARKTIRTE GIIBDTEIIE Haie (mit Riesenmäulern).... (kommunikativ unselbständig) (kommunikativ unselbständig) (keine Illokution) relativ gros: rechts des Satzes oder eingeschaltet ${ }^{4}$ SATZ-ZUSATZ

Pia hat - als gerade die Sonne aufging - den Hai gesehen. maximal: links bzw. rechts des Satzes oder eingeschaltet EINSCHIB

Pia hat - die Sonne ging gerade auf - den Hai gesehen.

EINSCHUB wird nicht als syntaktische Relation aufgefaBt, sondern als konstruktionaler Aspekt ("Einschub in X") im Rahmen etwa von Satzverbindung, -verfügung etc., weil die Ebene des unabhängigen Einzelsatzes überschritten würde. 5 ZUSATZE sind dagegen syntaktisch relational ("Zusatz $z u X "$ ) und an ihre Bezugselemente "assoziiert".

Im Bereich der Zusätze ist vor allem wichtig, ob der Zusatz potentiell eine syntaktische Funktion im Gastsatz erfüllen könnte (nach Wegfall der Einschaltungsmarkierung und ggf. einer Umstellung); dieses Merkmal trennt die in dieser Arbeit zentralen sechs Zusätze von einschaltungsmarkierten Gliedteilen resp. Angaben. Der Relativsatz-Zusatz kann m.E. nach Wegfall der Einschaltungsmarkierung (Wegfall deutlicher Pausen bzw. Ersetzung von Rundklammern oder Gedankenstrichen durch die "neutralen" Kommata) nicht als restriktives Relativsatzattribut fungieren, da seine Bezugs-NPn i.d.R. keiner Restriktion fähig sind (Eigennamen, Pronomen, NPn wie dieser Sack (s.u.) etc.).

\section{POTENTIELLE SYNTAKTISCHE FUNKTION DES ZUSATZES?}

potentielle Adverbialfunktion

SATZ-ZUSATZ

Sie hat (gestern) den hai gesehen. VP-ZUSATZ

Sie hat - laut - nach inm gerufen. pot. Attributsfunktion

ENNSCHALTUAGSM. GHEDTEMII ein Sack (mit Schnipseln)

\author{
keine \\ PRAZ, REZZ., SPEY. \\ NSPEZ, ALNZ, KURZ \\ ein Sack, vor allem ein \\ Kohlensack, \\ REIATTVSATZ-ZUSATZ \\ dieser Sack (der Schnipsel \\ enthält)...
}

4 Wobei die Position zwischen VF-Element und Finitum problematisch sein kann.

5 Vgl. Lehmann (1983: 339 f.). 
Für eine möglichst schnelle Aufgliederung der in dieser Arbeit zentral behandelten Muster kommt man mit der kategorialen Füllung, den Funktionslexemen und den Umformungsbeziehungen (weniger wichtig: die stellungseigenschaften) aus. Die vorgeführte Abgrenzungsstrategie gilt natürlich nur für standardfälle; im Detail wird man immer wieder strittige Beispiele finden, bei denen das Heranziehen vieler Kriterien (dazu v.a. Kap. 15) notwendig wird:

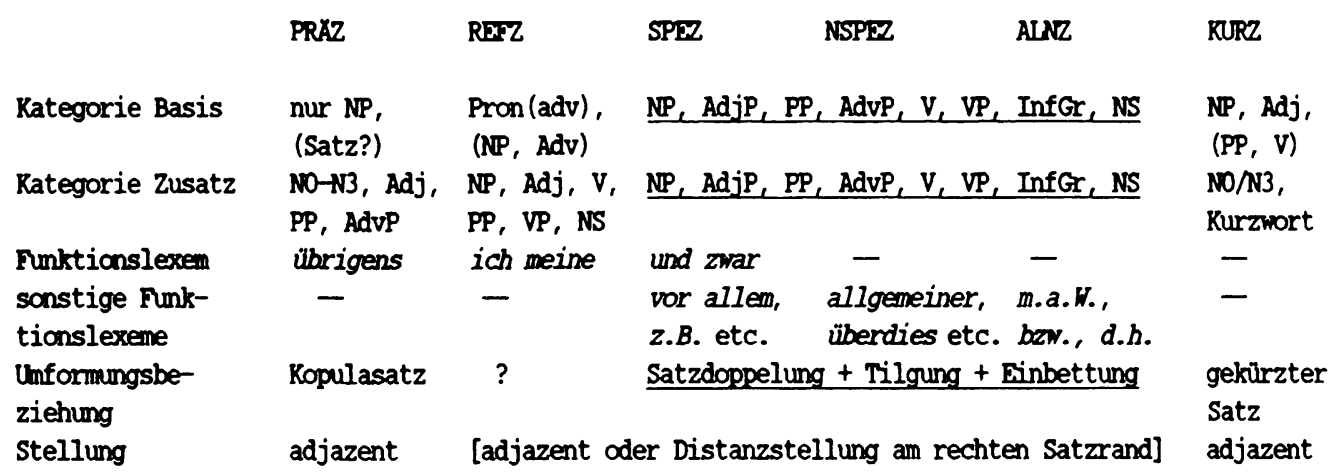

\subsection{Der Bereich der Hospitanten}

\section{1) E I N S H U B}

- traditionell: Parenthese (vor allem 1.1); z.T. aber auch umstritten (insb. 1.2, 1.3)

- konstruktionaler Aspekt: "Binschaltung in X"

- mit dem Einschaltungsmuster versehen

- nicht syntaktisch in den Gastsatz integriert

- keine potentielle syntaktische Funktion in Gastsatz

- nie erfragbar

- von Negationspartikel nicht sowie Gradpartikeln nicht affizierbar

- satz- bzw. äußenmgswertige Einheiten, die illokutives Potential aufweisen

- große Stellungsfreiheit: an allen Parenthesenischen und links bzw. rechts des Bezugssatzes

- Stellung bei nicht-satzformatigen Einheiten (v.a. 1.2) z.T. pragmatisch beschränkt. 6

6 Im Bereich von 1.2) trifft das Merkmal "Stellung links/rechts vom Gastsatz sorie an Parenthesenischen" nicht ohne weiteres $z u$, da z.B. redeeröffnende Partikeln (So, jetzt $(*, s o$,$) kowwen wir$ $(*$, so, zum nächsten Punkt $(*$, so).) pragmatisch (sonst mürden sie ja nichts eröffnen) auf die Position links des Satzes beschrănkt sind, obwohl sie äußernmgswertig sind; es liegt m.E. keine syntaktische Restriktion vor. 


\section{1) Schaltsatz}

- v-2-Schaltsatz

(- das weis doch jeder -)

mit koord. Konjunktion:

(- und/denn das meis jeder $\rightarrow$

- V-E-Schaltsatz 7

(- ab das wohl stimmt? -)

- v-1-Schaltsatz

(- weib das wirklich jeder? -)

mit koord. Konjunktion:

(- oder weist du das nicht? -)

- elliptische Satzstrukturen

(Andern wir - und wie? - den

Lauf der Welt?)
1.2) kanmunikationsbezogene

- Anrede (Liebe Gäste!)

- Vokativ (Dort, liebe Pia, hat...)

- Kontaktumgrenzung (Hallo! Gute Nacht!)

- Kontaktpartikeln (nicht?)

- textgliedernde Ausdrücke (noch etwas/zum anderen)

- Dank, Bitte (Danke!)

- Existimatorische Floskel (meine ich/ glaube ich)
1.3) Interjektionen

diverse Subklassen!

- Dmpfindung (au! oho! pfui!)

- Schallnachahmung

(quak! trara! ticktack)

etc. etc.

eventuell (offenes Problem; vgl. 2.1.2):

- Satzrelativsatzeinschaltung?

(Sie haben - was sie vorher nie

wagten - dem BOB widersprochen)

\section{2) ZUSATZ}

- traditionell: z.T. Apposition (2.2.2), auch Parenthese (2.1) und Attribut (2.2.3)

- relationaler Aspekt: "Zusatz zu X"

- mit dem Einschaltungsmuster versehen

- masßige syntaktische Integration in den Gastsatz (z.B. Stellung, Kongruenz)

- z.T. potentielle syntaktische Funktion in Gastsatz $(2.1,2.2)$

- nie erfragbar

- von Negationspartikel nicht (kontrastierende Negation) sowie Gradpartikeln nur in speziellen Fällen affizierbar

- nicht äußerungswertig (da im Ggs. zu Einschüben kein illokutives Potential; evtl. bilden Relativsatz-Zusätze, vgl. Kap 7.A, hier eine Ausnahme); immerhin gewisse Satzwertigkeit (Annahme reduzierter Sätze, mögliche Begleitung durch freie Angaben usw.)

- Stellungsverhalten: bei (2.1) an Parenthesenischen sowie am rechten Satzrand; bei (2.2.1) strikte Adjazenz; bei (2.2.2) relativ zum Bezugselement zu formulieren (adjazent; evtl. auch am rechten Satzrand); (2.2.3) ist adjazent und eher selten extraponierbar.

7 cemeint sind allerdings nur Verbend-Sătze, die keine syntaktische Funktion im Gastsatz erfullen könnten, denn sonst handelt es sich um SATZ-ZUSXTZE. 


\section{1) SATZ-ZUSATZE}

mit möglicher Adverbial-Funktion

\subsection{1) ENNGESCHALTETE ANGABDN}

Pia hat sich - als es klopfte - im Schrank versteckt.

Pia hat sich, gestern, im Schrank versteckt.

ohne mögliche syntaktische Funktion

\subsection{2) SATZRETATTVSATZEMNSCHALTUNG?}

Sie haben - was sie vorher nie wagten - dem BOB widersprochen.

\section{2) WORTGRUPPEN-ZUSATZE}

\section{mit möglicher Attributfunktion}

2.2.1) EINSCHALTUNGSMARKIFRTE GITBDTETLE Er hat einen Sack (mit Schnipseln) gefunden eine - notwendig einseitige - Weigerung...
2.2.2) WORTGRUPPDNZUSATZE i.e.S.

- PRAZ: Pia, Köchin aus Passion,

- RTrZ: Sie, die Pia,

- SPEZ: die Affen - vor allem die Paviane -

- NSPEZ: die Affen (allgemein alle Primaten)

- ALVZ: die Kamele (m.a.W. die Wüstenschiffe)

- KURZ: Willy Brandt (SPD)

\subsection{3) RELATTVSATZ-ZUSATZ}

Unsere Sonne, ohne die wir nicht existierten,

\subsection{Die Wortgruppenzusātze}

An welche Phrasen können sich welche Zusätze assoziieren? Uber Möglichkeiten und noch zu prüfende Problemfälle informiert die abschließende Ubersicht.

Zeichenerklärung: "+" = 'möglich'; "?" = 'noch zu untersuchen; evtl. Einzelfälle möglich'; "(-)' = 'wohl unmöglich; evtl. Binzelfälle'; "-" = 'auszuschließen'.

$$
\text { NP AdjP AdvP PP } V \text { VP InfGr V-E-S }
$$

\begin{tabular}{|c|c|c|c|c|c|c|c|}
\hline Relativsatzzusatz (APREL) & + & - & - & - & - & - & - \\
\hline prädizierender Zusatz & + & - & - & - & - & - & - \\
\hline Kurzzusatz & + & $?$ & $(-)$ & ? & $(-)$ & - & - \\
\hline einschalt.mark. Gliedteil & + & + & + & - & - & - & - \\
\hline referenzklărender Zusatz & + & ? & + & ? & ? & ? & ? \\
\hline spezifizierender Zusatz & + & + & + & + & + & + & + \\
\hline ichtspezifiz. Zusatz & + & + & + & + & + & + & + \\
\hline ternativen nenn. Zusatz & + & + & + & + & + & + & + \\
\hline
\end{tabular}




\section{B I BLIOGR A PHIB}

Dieses Literaturverzeichnis enthält alle in der vorliegenden Arbeit zitierten bzw. erwähnten Publikationen sowie alle zum Thema Apposition recherchierten Arbeiten, auch wenn sie nicht explizit erwähnt wurden; somit können zukünftige Arbeiten zum gleichen Themenkreis auf meiner Bibliographie aufbauen.

\section{Abkürzungen:}

ĀS American Speech

DSp Deutsche Sprache. Berlin.

FL Foundations of Language. Dordrecht.

FM Le Français Moderne. Paris.

Fol Folia linguistica. The Hague.

IdS Forschungsberichte des Instituts für deutsche Sprache. Tübingen.

几 Jorunal of Linguistics. Cambridge.

LA Linguistische Arbeiten. Tübingen.

LBer Linguistische Berichte. Braunschweig.

LIn Linguistic Inquiry. Cambridge, MA.

Ll 82 La linguistique à la session 1982 de $1^{\prime}$ aggrégation d' allemand. 1982. Journé annuelle des linguistes de $l^{\prime}$ association des germanistes de $l^{\prime}$ enseignement superieur, 12. 12. 1981. (Colloque du centre de recherches germaniques de 1' université Nancy II.). Nancy Univ. II. $189 \mathrm{~S}$.

Mu Muttersprache. Wiesbaden.

RG Reihe Cermanistische Linguistik. Tübingen.

SdG Studien zur deutschen Grammatik. Tübingen.

SdGe Sprache der Gegenwart

SG Studia Granmatica. Berlin.

Sprachw Sprachwissenschaft. Heidelberg.

STL Studien zur theoretischen Linguistik. Munchen.

TBL Tübinger Beiträge zur Linguistik. Tübingen.

ZFSL Zeitschrift für französische Sprache u. Literatur. Wiesbaden.

ZGL Zeitschrift für germanistische Linguistik. Berlin.

ZPhon Zeitschrift für Phonetik, Sprachwissenschaft und Konmunikationsforschung. Berlin.

Abraham, Werner. 1983. Der Dativ im Deutschen. La linguistique à la session 1983 de l'agrégation d'allemand. Journée annuelle des linguistes de l'association des germanistes de l'enseignement superieur, 2-101. (Colloque du centre de recherches germaniques de l'université Nancy II.) Nancy Univ. II. $220 \mathrm{~s}$.

Admoni, Wladimir. ${ }^{3} 1970$ [1966]. Der deutsche Sprachbau. München, Beck. Altmann, Hans. 1976. Die Gradpartikeln im Deutschen. (LA 33.) Tübingen, Niemeyer.

- 1978. Gradpartikelprobleme. (SdG 8.) Tübingen, Narr.

- 1981. Formen der "Herausstellung" im Deutschen. (LA 106.) Tübingen, Niemeyer. 
Andersson, Sven G. \& Sigmund Kvam. 1984. Satzverschränkung im heutigen Deutsch. (SdG 24.) Tübingen, Narr.

Antos, Gerd. 1982. Formulierungskommentierende Ausdrücke. Sprache erkennen und verstehen, hg. v. Klaus Detering u.a., 121-131. (LA 119.) Tübingen, Niemeyer.

Arrive, M. 1962. Discussion: L'apposition. FM 30. 178-183.

- 1964. A propos de la construction 'la ville de Paris': rapports sémantiques et rapports syntaxiques. FM 32. 179-184.

Bänzinger, Andreas. 1970. Kasusabweichungen in der Gegenwartssprache. Freiburg i.d. Schweiz (Diss.).

Bartsch, Angelika. 1982. Zur stellung der Adverbiale in den Werken von Thomas Mann. (Europ. Hochschulschriften, Reihe 1, Deutsche Sprache und Literatur 521.) Frankfurt/M.; Lang.

Bartsch, Renate. 1972. Adverbialsemantik. (Linguistische Forschungen 6.) Frankfurt/M., Athenäum.

- 1978. Satzreihung, Satzgefüge oder Adverbialkonstruktion? Sprache in Gegenwart und Geschichte. FS Heinrich Matthias Heinrichs, hg. v. Dietrich Hartmann u.a., 1-18. Köln, Böhlau.

- \& Jürgen Lenerz \& Veronika Ullmer-Ehrich. 1977. Einführung in die Syntax. Kronberg/Taunus, Scriptor.

- \& Theo Vennemann. 1982. Grundzüge der Sprachtheorie. Tübingen, Niemeyer.

Bassarak, Armin. 1985. Zu den Beziehungen zwischen Parenthesen und ihren Trägersätzen. ZPhon 38, 4. 368-375.

- 1987. Parenthesen als illokutive Handlungen. Motsch (Hg.) 1987. 163-178. Bayer, Xlaus. 1973. Verteilung und Funktion der sogenannten Parenthese in Texten gesprochener sprache. DSp 1. 64-115.

Becker, Rita. 1978. Oberflächenstrukturelle Unterschiede zwischen restriktiven und nichtrestriktiven Relativsătzen im Deutschen. Kölner Linguistische Arbeiten Germanistik 4.

Behaghel, Otto. 1928. Deutsche Syntax. Bd. III. Die Satzgebilde. Heidelberg, Winter.

- 1932. Deutsche Syntax. Bd. IV. Heidelberg, Winter.

Bergenholtz, Henning. 1982. Appositionssyntax im Deutschen. LL 82. 61-104.

- 1985. Kasuskongruenz der Apposition. Beiträge zur Geschichte der deutschen Sprache und Literatur 107, 21-44.

- \& Burkhard Schaeder. 1977. Die Wortarten des Deutschen. Stuttgart, Klett.

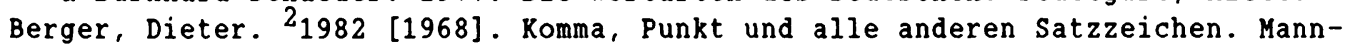
heim u.a., Dudenverlag.

Bergmann, Rolf. 1985. Relativsatz-Probleme in Grammatiken der deutschen Gegenwartssprache. Koller/Moser (Hgg.) 1985. 51-66.

Betten, Anne. 1976. Ellipsen, Anakoluthe und Parenthesen. DSp 3. 207-230.

Bierwisch, Manfred. 1966. Regeln für die Intonation deutscher Sătze. (SG VII.) 99-201. Berlin, Akademie.

Birkenmaier, Willy. 1978. 'Wodkaflasche' und 'Flasche Wodka' auf Russisch. IRAL XVI/3. 219-228.

Bitea, Ioan N. 1977. An Attempt at Defining Apposition in Modern English. Revue Roumaine de Linguistique. Tome XXII/4. 453-477.

Blümel, Rudolf. 1910. Neuhochdeutsche Appositionsgruppen. 1. Teil. Würzburg.

- 1911. Neuhochdeutsche Appositionsgruppen. 2. Teil. Würzburg.

Boas, Hans-Ulrich. 1977. Deep- and Surface-structure Problems of Restrictive and Non-Restrictive Constructions. FoL 11. 39-56. 
Bogacki, Bohdan K. 1973. Types de constructions appositives en Français.

Wroclaw, Zaklad im. Ossolinskich.

Bondy, L. 1963. Discussion: L'apposition. FM 31. 50-54.

Bondzio, Wilhelm. 1967. Untersuchungen zum attributiven Genitiv und zur Nominalgruppe in der deutschen Gegenwartssprache. Berlin (masch. Habil.)

Bonnard, Henri. 1962. Discussion: L'apposition. FM 30. 183-186.

Boudreau, J. 1981. La definition des fonctions 'apposition' et 'épithète' en grammaire traditionelle. Langues et linguistique 7. 155-181.

Bouet, ? ${ }^{1}$. 1963. Discussion: L'apposition (suite). FM 31. 283-287.

Brandt, Margareta. \& Ingemar Persson \& Inger Rosengren \& Lars Ahlander (Hgg.). 1973. Tysk grammatik for universitets bruk. Lund, Gleerup.

Breyer, A. \& K. Bodmann \& S. Bronsert. 1987. Grammatik der rumänischen Sprache der Gegenwart. Leipzig.

Brinker, Klaus. 1972. Konstituentenstrukturgrammatik und operationale Satzgliedanalyse. Frankfurt/M., Athenăum.

- 1977. Modelle und Methoden der strukturalistischen Syntax. Stuttgart, Kohlhammer.

Burkhardt, Armin. 1982. Gesprăchswörter. Konzepte zur Lexikographie, hg. v . Wolfgang Mentrup, 138-171. (RGL 38.) Tübingen, Niemeyer.

Burton-Roberts, Noel. 1975. Nominal Apposition. FL 13. 391-419.

Buscha, Annerose \& Fritz Kempter. ${ }^{1983}$ [1980]. Der Relativsatz. Leipzig, Enzyklopădie.

Bussmann, Hadumod. 1983. Lexikon der Sprachwissenschaft. Stuttgart, Kröner.

Chaurand, Jacques. 1962. Discussion: L'apposition. FM 30. 174-176.

Chevalier, André. 1962. Discussion: Lapposition. FM 30. 177-178.

Chevalier, Jacques C. 1962. Discussion: L'apposition. FM 30. 186-191.

Chomsky, Noam. 1957. Syntactic structures. The Hague, Mouton.

- 1965. Aspects of the Theory of Syntax. Cambridge/Mass., M.I.T. Press.

Clément, Danièle \& Wolf Thümmel. 1975. Grundzüge einer syntax der deutschen standardsprache. Frankfurt/M., Athenaion.

Confais, Jean-Paul. 1985. L'emploi de l'article dans les structures attributives et apparentées (apposition, 'als'...): vue d'ensemble. Nouveaux cahiers d'allemand 3. 95-97.

Delorme, Evelyne \& Ray C. Dougherty. 1972. Appositive NP-Constructions. FL 8. 2-29.

Delplanque, M. 1972. L'apposition. Nature, place, fonction et valeur en français et en allemand. Unveröff. Staatsarbeit $(77 \mathrm{~S}$.$) . Paris-Asnières.$

Dessaintes, Maurice. 1966. L'apposition: une fonction ou un mode de construction asyndétique? Mélanges de grammaire française offerts à M. Maurice Grevisse pour le 30. anniversaire du "Bon Usage", 69-104. Gembloux, Duculot.

Ditfurth, Hoimar von. 1.988. So laßt uns denn ein Apfelbăumchen pflanzen. München, Knaur.

Dittmer, Ernst. 1988. Zur Geschichte des absoluten Akkusativs (Nominativs) im Deutschen. Gedenkschrift für Ingerid Dal, hg. v. John 0. Askedal u.a., 6374. Tübingen, Niemeyer.

Doerfer, Gerhard. 1973. Anatomie der Syntax. (Europ. Hochschulschriften. Reihe 1. 88.) Bern/Frankfurt/M., Lang.

Downing, Bruce T. 1978. Some Universals of Relative Clause Structure. Greenberg (Hg.) 1978. 375-418.

1 Die Publikation gibt nur den Nachnamen, nicht aber den Vornamen an. 
Droop, Helmut G. 1977. Das präpositionale Attribut. (IdS 34.) Tübingen, Narr. Duden. 21972 [1965]. Zweifelsfälle der deutschen Sprache. Bd. 9, bearb. v. Dieter Berger u.a. Mannheim u.a., Dudenverlag.

Duden. ${ }^{4} 1984$ [1959]. Grammatik der deutschen Gegenwartssprache. Bd. 4, hg. u. bearb. v. Günther Drosdowski u.a. Mannheim u.a., Dudenverlag.

Duden. ${ }^{1} 1985$ [1965]. Richtiges und gutes Deutsch. Bd. 9, bearb. v. Dieter Berger, Günther Drosdowski u.a. Mannheim u.a., Dudenverlag.

Dupont, Norbert. 1985. Linguistique du détachement en français. (Sciences pour la communication 12.) Bern u.a., Lang.

Ebert, Karen H. 1973. Functions of relative clauses in reference acts. LBer 23. 1-11.

Ehlich, Konrad. 1986. Interjektionen. (LA 111.) Tübingen, Niemeyer.

Eichbaum, Gali N. 1978. Zum Problem der sogenannten Satzapposition, gebildet von einem Substantiv im Nominativ. Beiträge zu Problemen der Satzglieder, hg. v. Gerhard Helbig, 29-43. Leipzig, Enzyklopädie.

Eisenberg, Peter. 1985. Maß und Zahl. Nach-Chomskysche Linguistik, hg. v. Thomas Ballmer \& Roland Posner, 311-320. Berlin, de Gruyter.

- 1986. Grundriss der deutschen Grammatik. Stuttgart, Metzler.

Emonds, Joseph. 1979. Appositive Relatives Have No Properties. LIn 10, 2. 211243.

Engel, Ulrich. 21982 [1977]. Syntax der deutschen Gegenwartssprache. (Grundlagen der Germanistik 22.) Berlin, E. Schmidt.

- 1986. Die Apposition. Vor-Sätze zu einer neuen deutschen Grammatik, hg. v. Gisela Zifonun, 184-205. (IdS 63.) Tübingen, Narr.

- 1988. Deutsche Grammatik. Heidelberg, Groos.

- \& Pavica Mrazovic (Hgg.). 1986. Kontrastive Grammatik Deutsch-Serbokroatisch. 1. Halbband. München, Sagner.

Engelen, Bernhard. 1975. Untersuchungen zu Satzbauplan und Wortfeld in der geschriebenen deutschen Sprache der Gegenwart. Teilband 1. (Heutiges Deutsch Reihe 1.3.1.) München, Hueber.

- 1984. Einführung in die syntax der deutschen Sprache. Bd. I. Baltmannsweiler, Pädagog. Verl. Burgbücherei Schneider.

- 1986. Einführung in die syntax der deutschen Sprache. Bd. II. Baltmannsweiler, Pädagog. Verl. Burgbücherei Schneider.

Erben, Johannes. 11/97z [1958]. Deutsche Grammatix. Ein Abri3. München, Hueber.

- 1984. Deutsche Syntax. Eine Einführung. Bern, Frankfurt/M., New York, Lang. Faucher, Eugène. 1970. L'apposition en allemand moderne. Etudes de linguistique appliquée [Besançon] 7. 88-96.

Faucher, Eugène. 1982. Le datif "appositif" comme argument pour une définition étroite de $1^{\prime}$ apposition. LL 82. 157-170.

Faucher, Eugène \& R. Metrich. 1982. Comment classer les appositions? LL 82. 136-156.

Fey, Richard. 1912. Neuhochdeutsche Appositionsgruppen. Halle.

Flückiger-studer, Thérèse. 1983. Quantifikation in natürlichen Sprachen. (LA 132.) Tübingen, Niemeyer.

Förster, Uwe. 1972. Neuer Nullkasus? Der Sprachdienst 16, 4. 81-83.

Fries, Norbert. 1988. Präpositionen und Präpositionalphrasen im Deutschen und im Neugriechischen. (LA 208.) Tübingen, Niemeyer.

Galichet, George. 1962. Qu'est-ce que l'apposition? Le Français dans le monde 7. 39-40. 
Gestel, Frank C. van. 1986. X-Bar-Grammar: Attribution and Predication in Dutch. Dordrecht, Foris.

Gippert, Jost. 1981. Zur Dativapposition im Deutschen. Beiträge zur Geschichte der deutschen Sprache und Literatur 103. 31-62.

Glatigny, M. 1966. L'adjectif en apposition. Les langues modernes 34. 264-279.

Greenberg, Joseph H. (Hg.). 1978. Universals of human language. Vol. 4. Syntax. Stanford, stanford UP.

Grewendorf, Günther \& Fritz Hamm \& Wolfgang Sternefeld. 1987. Sprachliches Wissen. Frankfurt a.M., Suhrkamp.

Grewendorf, Günther. 1988. Aspekte der deutschen Syntax. (SdG 33.) Tübingen, Narr.

Gülich, Elisabeth \& Thomas Kotschi. 1987. Reformulierungshandlungen als Mittel der Textkonstitution. Untersuchungen zu französischen Texten aus mündlicher Kommunikation. Motsch (Hg.) 1987. 199-261.

Haberkorn, Didier. 1970. L'apposition en Allemand moderne. These de doctorat. Paris IV.

Haberkorn, Didier. 1982. Apponieren als Handlung. LL 82. 105-116.

Hackel, Werner. 1968. Personeneigennamen als kasusneutrale Apposition. Sprachpflege 6. $113 \mathrm{ff}$.

- 1970. Zum engen appositionellen Syntagma in der deutschen Gegenwartssprache. Jena (masch. Diss.)

- 1972. Zu einem jüngeren Typ des engen appositionellen syntagmas. DaF 9. 341-348.

- 1973. Appositionelle Syntagmen mit gekoppelten Substantiven. DaF 10. 18-22.

- 1975. Betrachtungen zur Kategorie der Apposition. Deutschunterricht (Ost) 28. 43-52.

- 1978. Zur appositionellen Verbindung Substantiv + Personeneigenname in der deutschen Gegenwartssprache. Historizität und gesellschaftliche Bedingtheit der Sprache, hg. v. Franz Bolck. Bd. 2, 97-114. Jena, Universitätsverlag.

- 1986. Zum Verhältnis Eigenname und Apposition. Namenkundliche Informationen [Univ. Leipzig] 49. 1-12.

Haider, Hubert. 1988. Die Struktur der deutschen Nominalphrase. Zeitschrift für Sprachwissenschaft 7.1. 32-59.

Halitsky, David. 1974. Deep-Structure Appositive and Complement NP's. Language 50/3. 446-454.

Harweg, Roland. ${ }^{2} 1979$ [1968]. Pronomina und Textkonstitution. München, Fink.

Haugen, Einar. On Resolving the Close Apposition. AS 28. 165-170.

Hausmann, Franz Joseph. 1982. Horst Raabe: Apposition (Besprechung). Zeitschrift für romanische Philologie 98, Heft 1/2. 434-437.

Havers, W. 1926. Der sogenannte Nominativus pendens. Indogermanische Forschungen $43.207 \mathrm{ff}$.

Heidolph, Karl Erich. 1962. Beziehungen zwischen Kompositum und attributiven Substantivkonstruktionen in der deutschen Gegenwartssprache. Berlin (Diss.)

- 1979. Der Kasus bei Appositionen mit "als" - grammatische Regeln und Oberlegungen zur Vermittlung. DaF 16. 328-331.

- \& Walter Flämig \& Wolfgang Motsch (Hgg.). 1981. Grundzüge einer deutschen Grammatik. Berlin, Akademie.

Helbig, Gerhard. 1972. Zu Problemen des Attributs in der deutschen Gegenwartssprache (1). DaF 9. 332-341.

- 1973. Zu Problemen des Attributs in der deutschen Gegenwartssprache (2). DaF 10. 11-17. 
- 1984a. Die Substantivgruppen mit 'als' und 'wie' im Deutschen. Studien zur deutschen Syntax. Bd. 2, hg. v. Gerhard Helbig, 67-81. Leipzig, Enzyklopädie.

- 1984b. Zu Problemen des Attributs in der deutschen Gegenwartssprache. Studien zur deutschen Syntax. Bd. 2, hg. v. Gerhard Helbig, 132-153. Leipzig, Enzyklopădie.

- 1984c. Was sind Objektsprädikate, Objektsprädikative und prädikative Attribute? DaF 21. 346-350.

- \& Joachim Buscha. ${ }^{6} 1980$ [1972]. Deutsche Grammatik. Leipzig, Enzyklopädie.

Hentschel, Elke. 1986. Funktion und Geschichte deutscher Partikeln. (RGL 63.) Tübingen, Niemeyer.

- 1989. Schwankender Kasusgebrauch im Deutschen: à, je, per, pro. Sprechen und Hören, hg. v. Norbert Reiter, 289-298. (LA 222.) Tübingen, Niemeyer.

Heringer, Hans Jürgen. $2_{1973}$ [1970]. Theorie der deutschen syntax. (Linguistische Reihe 1.) München, Hueber.

- 1988. Lesen lehren lernen: Eine rezeptive Grammatik des Deutschen. Tübingen.

Hiersche, Rolf. 1979. Zur deutschen Satzgliedlehre. Sprachw 4. 233-253.

Hindelang, Götz. 1980. Was heisst das heisst? Perspektive: textintern. Akten des 14. Linguistischen Kolloquiums 1979. Bd. 1., hg. v. Edda Weigand u. Gerhard Tschauder, 123-131. (LA 88.) Tübingen, Niemeyer.

Hockett, Charles F. 1955. Attribution and Apposition. AS 30, 2. 99-102. Höhle, Tilmann. 1982. Explikation für >normale Betonung und >normale Wortstellung<. Satzglieder im Deutschen, hg. v. Werner Abraham, 75-154. (SdG 15.) Tübingen, Narr.

Hollenbach, Barbara E. 1983. Apposition and X-bar rules. Exploring language. 37-58.

Hudson, Richard A. 1987. Zwicky on heads. JL 23. 109-132.

Hujer, 0. \& Karl-Ernst Sommerfeldt. 1964. Zur Behandlung der Apposition im 7. Schuljahr. Deutschunterricht (Ost) 17. 338-345.

Jackendoff, Ray. 1977. X-Syntax: A Study of Phrase Structure. Cambridge/Mass., The MIT Press.

- 1984. On the Phrase "the phrase 'the phrase'". Natural Language and Linguistic Theory 2. 25-37.

Jacobs, Joachim. 1982. Syntax und Semantił. der Negation im Deutschen. (Münchner Studien zur theoretischen Linguistik 2.) München, Fink.

- 1983. Fokus und Skalen. (LA 138.) Tübingen, Niemeyer.

- 1984. Funktionale Satzperspektive und Illokutionssemantik. LBer 91. 25-58. Jung, Walter \& Günter Starke. ${ }^{7} 1982$ [1966]. Grammatik der deutschen Sprache. Leipzig, Bibliograph. Institut.

Kalverkämper, Hartwig. 1978. Textlinguistik der Eigennamen. (Beiträge zur Namensforschung 14.) Stuttgart, klett-Cotta.

Katz, E.: Zur Distribution von Kompositum und Nominalgruppe im Deutschen. Seiler/Lehmann (Hgg.) 1982. 112-129.

Kieffer, Lucien \& Pierre Aron. 1974. Remarques sur l'apposition en allemand. Les langues modernes 68. 539-543.

Klein, Hans-Wilhelm \& Fritz Strohmeyer. 1981. Französische Sprachlehre. Stuttgart, Klett.

Klein, Marten. 1977. Appositionele constructies in het Nederlands. Diss. Univ. Nijmegen. 
- 1979. On the Extraction of Appositive NP's. Utrecht Working Papers in Linguistics 7 . 31-49.

Kniffka, Gabriele. 1986. Zur Distanzstellung von Quantoren und Qualifikatoren im Deutschen. Vater 1986a. 57-82.

Köster, R. 1958. Die Apposition. Sprachpflege 7. 166-167.

Kolde, Gottfried. 1971. Einige Bemerkungen zur Funktion, Syntax und Morphologie der mit 'a $1 \mathrm{~s}$ ' eingeleiteten Nominalphrasen im Deutschen. Mu 81. 182203.

- 1985. Zur Topologie deutscher Substantivgruppen. ZGL 13. 241-277.

Koller, Erwin \& Hans Moser (Hg.). 1985. Studien zur deutschen Grammatik. (Innsbrucker Beiträge zur Kulturwiss.: Germanist. Reihe 25.) Innsbruck, Univ. Institut $f$. Germanistik.

Krifka, Manfred. 1983. Zur semantischen und pragmatischen Motivation syntaktischer Regularitäten. (Studien zur theoretischen Linguistik 5.) München, Fink.

- 1986. Massennomina mit einem Exkurs zu Aktionsarten. Paper des Sonderforschungsbereiches 99, Universität Konstanz.

Kuhn, Wilfried \& Fritz Serzisko. 1982. Eigennamen im Rahmen der Dimension der Apprehension. Seiler/Lehmann (Hgg.) 1982. 277-293.

Kusmin, Iwan. 1960. Die syntaktische Kategorie der Apposition in der deutschen Sprache der Gegenwart. Berlin/Ost (masch. Diss.)

Kvam, Sigmund. 1979. Diskontinuierliche Anordnung von eingebetteten Infinitivphrasen im Deutschen. DSp 7. 313-325.

La linguistique à la session 1982 de $l^{\prime}$ aggrégation d' allemand. 1982. Journée annuelle des linguistes de $l^{\prime}$ association des germanistes de $l^{\prime}$ enseignement supérieur, 12. 12. 1981. (Colloque du centre de recherches germaniques de I' université Nancy II.). Nancy Univ. II. $189 \mathrm{~S}$.

Lange, Klaus-Peter. 1974. Appositive NP-Konstruktionen im Deutschen. DSp 2. 123-132.

Lausberg, Heinrich. 71982. Elemente der literarischen Rhetorik. München, Hueber.

Lee, Donald W. 1952. Close Apposition: an Unresolved Pattern. AS 27. 268-275.

Lee, Duk-Ho. 1979. Aspekte der deutschen Syntax. (Reihe Sprachwissenschaft und Literaturwissenschaft 11.) München, Tudur.

Lehmus, Ursula. 1983. Attribut oder Satzglied? Helsinki.

Lehmann, Christian. 1983. Rektion und syntaktische Relationen. Fol 17. 339378 .

- 1984. Der Relativsatz. (Language universal series 3.) Tübingen, Narr.

Leirbukt, Oddleif. 1978. Uber dativische Apposition bei akkusativischem Bezugswort im Deutschen. LBer 55. 1-17.

Lindgren, Kaj B. 1985. Prolegomena einer Gesprächsgrammatik: Ellipse und Verwandtes. Koller/Moser (Hgg.) 1985. 205-213.

Link, Godehard. 1974. Quantoren-Floating im Deutschen. Syntax und generative Grammatik. Bd. 2, hg. v. Ferenc Kiefer \& David M. Perlmutter, 105-127.

(Linguistische Forschungen 12.) Frankfurt/M., Athenaion.

Ljungerud, Ivar. 1955. Zur Nominalflexion in der deutschen Literatursprache um 1900. Lund, Kopenhagen, Munksgaard.

Löbel, Elisabeth. 1986a. Apposition und Komposition in der Quantifizierung. (LA 166.) Tübingen, Niemeyer.

- 1986b. Apposition in der Quantifizierung. Pragmantax, hg. v. Armin Burkhardt \& Karl-Hermann Körner, 47-59. (LA 171.) Tübingen, Niemeyer. 
- 1988a. Kasuszuweisung und Adjazenzbedingung in der Nominalphrase des Deutschen. Handout (nicht publiziert) zu einem Vortrag vom 18.6.1988 in Passau.

- 1988b. Appositive Nominalphrasen. Linguistik Parisette, hg. v. Heinrich Weber \& Ryszard Zuber, 109-120. (LA 203.) Tübingen, Niemeyer.

Lötscher, Andreas. 1972. Some Problems Concerning Standard German Relative Clauses. The Chicago Which Hunt. Papers from the Relative Clause Festival. April 13, 1972, hg. v. Paul M. Peranteau \& Judith N. Levi \& Gloria C. Phares, 47-58. Chicago.

- 1983. Satzakzent und funktionale Satzperspektive im Deutschen. (LA 127.) Tübingen, Niemeyer.

Lühr, Rosemarie. 1985. Sonderfälle der Vorfeldbesetzung im heutigen Deutsch. DSp 13. 1-23.

Marko, Ernest. 1980. Zum Problem des prädikativen Attributs im Deutschen und im Slowakischen. DaF 17. 96-101.

Matthews, Peter H. 1981. Syntax. Cambridge, Cambridge Univ. Press.

McCawley, James D. 1982. Parentheticals and Discontinuous Constituent Structure. LIn 13, Nr. 1. 91-106.

Molitor, Friedhelm. 1979. Zur Apposition im heutigen Deutsch. Köln (Diss.).

Morgenroth, Ch. 1983. Prädikatives Attribut ${ }_{s}$ und prädikatives Attribut $v$ in der deutschen Gegenwartssprache. DaF 20. 227-230.

Moskalskaja, Olga Ivanova. 1975. Grammatik der deutschen Sprache der Gegenwart. Moskau, Verlag "Hochschule".

- 1984. Textgrammatik. Leipzig, VEB Bibliographisches Institut.

Motsch, Wolfgang. 1965. Untersuchungen zur Apposition im Deutschen. Syntaktische studien. (SG V.) Berlin. 87-132.

- 1987. Satz, Text, sprachliche Handlung. (SG XXV.) Berlin, Akademie.

Mouchet, J.-P. 1962. Discussion: L'apposition (1). FM 30. 172-173.

Müller, Beat Louis. 1985. Der Satz. Definition und sprachtheoretischer status. (RGL 57.) Tübingen, Niemeyer.

Norwood, J. E. 1954. The Loose Appositives in Present-Day English. AS 29. 267271.

Oehrle, Richard T. 1977. Comments on the Paper by Selkirk. Formal Syntax, hg. v. Peter W. Culicover u.a., 317-325. New York u.a., Academic Press.

Ortner, Hanspeter. 1983. Syntaktisch hervorgehobene Konnektoren im Deutschen. DSp 11. 97-121.

- 1985. Hervorgehobene Korrektur- und Bestätigungssignale in Texten geschriebener Sprache. Koller/Moser (Hgg.) 1985. 239-254.

Ostrowski, Manfred. 1982. Zum Konzept der Kongruenz. Seiler/Lehmann (Hgg.) 1982. 252-269.

Pheby, John. 1975. Intonation und Grammatik im Deutschen. (Sammlung AkademieVerlag 19.) Berlin, Akademie.

Pignon, J. 1961. L'apposition. FM 29. 252-257.

- 1962. Discussion: L'apposition. FM 30. 191-192.

Plett, Heinrich F. ${ }^{5} 1983$. Einführung in die rhetorische Textanalyse. Hamburg, Buske.

Polenz, Peter v. 1985. Deutsche Satzsemantik. Berlin, de Gruyter.

Pütz, Herbert. 1982. Objektsprädikate. Satzglieder im Deutschen, hg. v. Werner Abraham, 331-367. (SdG 15.) Tübingen, Narr.

- 1988. Uber Objektsprădikate: Neuere Ableitungen, Bedeutungsvielfalt, Abgrenzung. Gedenkschrift für Ingerid Dal, hg. v. John O. Askedal u.a., 182201. Tübingen, Niemeyer. 
Putnam, Hilary. 1975. The Meaning of Meaning. Mind, Language and Reality. Philosophical papers, Vol. 2, hg. v. Hilary Putnam, 215-271. Cambridge, Mass., Cambridge UP.

Quirk, Randolph \& Sidney Greenbaum \& Geoffrey Leech \& Jan Svartik. 1976. A Grammar of Contemporary English. London, Longman.

Raabe, Horst. 1975. Sind Appositionen mit ihrer Basis referenzidentisch? Sprachsystem und Sprachgebrauch. FS H. Moser, hg. v. Ulrich Engel \& Paul Grebe. Teil 2, 312-339. (SdGe 33.) Düsseldorf, Schwann.

- 1979. Apposition. (TBL 119.) Tübingen, Narr.

Rahmstorf, Gerhard. 1983. Die semantischen Relationen im nominalen Ausdrücken des Deutschen. Mainz (Diss.)

Ramseyer, Rudolf J. 1976. Attributive Zusätze bei Personennamen. Onoma 20. $252-258$.

Rath, Rainer. 1975. Korrektur und Anakoluth im Gesprochenen Deutsch. LBer 37. 1-12.

- 1979. Kommunikationspraxis. Göttingen, Vandenhoeck \& Ruprecht.

Regula, Moritz. 1968. Wesen, Arten und Formen der Apposition und des Attributs. ZFSL 78 . 102-138.

- 1969. Wesen, Form und Anwendungsbereich der Apposition. Eine sprachtheoretische Betrachtung. Mélanges de philologie offerts à Alf Lombard, 164-177. (Etudes romanes de Lund 18.) Lund, Gleerup.

Reinhart, Tanya. 1983. Point of View in Language - The Use of Parentheticals. Essays on Deixis, hg. v. Gisa Rauh, 169-194. (TBL 188.) Tübingen, Narr.

Rioul, René. 1983. Les appositions dans la grammaire française. L'information grammaticale 18. 21-29.

Roey, Jacques van. 1966. Apposition and Coordination in Modern English. Linguistic Research in Belgium, hg. v. Yvan Lebrun, 129-144. Welteren, Universa.

- 1974. A Contrastive Description of English and Dutch Noun Phrases. Paris, Brüssel.

Rohrer, Christian. 1968. Appositive Konstruktionen im Französischen. Word 24. 392-409.

Rosch, Eleanor. 1977. Human Categorization. Studies in Cross-cultural Psychology. Vol. 1., hg. v. N. Warren, 1-49. London u.a.

Rothenberg, Martin. 1970. Encore quelques remarques sur l'apposition en français. Etudes de linguistique appliquée 6. 84-87.

Safir, Kenneth J. 1986. Relativ Clauses in a Theory of Binding and Levels. LIn 17, Nr. 4. 663-689.

Sasina, V.P. 1985. Comparative Function of a Nominal Predicate, Apposition and 'Nominal Joining'. Movoznarstvo 19/3. 59-62.

Schanen, François. 1982. Zum Begriff der Apposition. LL 82. 117-135.

Schapiro, Ben. 1977. English Appositive Structures and Right Movement. (Dissertation Abstracts International 37.) New York (Diss.)

Schäublin, Peter. 1972. Das adnominale Attribut in der deutschen sprache der Gegenwart. (Studia linguistica germanica 5.) Berlin, de Gruyter.

Schenk, A. 1976. Nominale Appositionen mit besonderer Berücksichtigung des Französischen. Bochum (Diss.)

Schönmann, Gerhard. 1959. Der falsche Dativ im Beisatz. Mu 69. 159-161.

Schulz, Dora \& Heinz Griesbach. 11982 [1960]. Grammatik der deutschen Sprache. München, Hueber. 
Schwyzer, Eduard. 1939. Die Parenthese im engern und im weitern Sinn. Abhandlungen der preuBischen Akademie der Wissenschaften. Jahrgang 1939. Philosophisch-historische Klasse. $\mathrm{Nr} .6,3-46$. Berlin.

- 1946. Zur Apposition. Abhandlungen der deutschen Akademie der Wissenschaften zu Berlin. Jahrgang 1945/46. Philosophisch-historische Klasse. Nr. 3, 3-16. Berlin.

Seibicke, Wilfried. 1968. Apposition? Der Sprachdienst 12, 10. 149-151.

- 1969. Apposition? Der Sprachdienst 13. S. 77.

Seiler, Hansjakob. 1960. Relativsatz, Attribut und Apposition. Wiesbaden, Harrassowitz.

- \&Christian Lehmann (Hgg.). 1982. Apprehension: Das sprachliche Erfassen von Gegenständen. Teil 1. (Language Universal Series 1.) Tübingen, Narr.

Selkirk, Elisabeth. 1977. Some Remarks on Noun Phrase Structure. Formal Syntax, hg. v. Peter W. Culicover u.a., 285-316. New York u.a., Academic Press.

Sievers, G. 1969a. Apposition. Der Sprachdienst 13. S. 1 u. 16.

- 1969b. Nochmals Apposition. Der Sprachdienst 13. S. 9 u. $142 \mathrm{f}$.

Smith, John B. 1977. Die Nominalphrase als Prädikativ und als freie Umstandsangabe im Englischen und im Deutschen. Mu 87. 326-336.

Sommerfeldt, Karl-Ernst. 1963. Möglichkeiten und Grenzen der Transformation von Kernsătzen in Nominalgruppen. DaF 3. 175-180.

- 1970. Form und Bedeutung der Attribute beim Substantiv in der deutschen Gegenwartssprache. ZPhon 23. 554-572.

- 1971. Zur Wortstellung in der Gruppe des Substantivs. DaF 8. 13-19.

- 1983. Zu den Nebensätzen ohne Satzgliedwert in der deutschen Sprache der. Gegenwart. ZPhon 36, 4. 413-420.

- 1984. Zu Verdichtungserscheinungen im Satzbau der deutschen Sprache der Gegenwart (unter besonderer Berücksichtigung der Parenthese). ZPhon 37. 242248.

Sopher, H. 1971. Apposition. English Studies 52. 401-412.

Starke, Günter. 1983a. Sätze mit abgesonderten Wortgruppen in der deutschen Sprache der Gegenwart. Sprachpflege 32, Heft 7. 97-100.

- 1983b. Rat und Auskunft. Auf Basis Formaldehyd, in Anlehnung Laborversuche, Versuche November 1982. Sprachpflege 32, Heft 11. 173-174.

- 1985. Eine Reihe Gratulanten - eine Reihe von Gratulanten - die Reihe der Gratulanten. Sprachpflege 34, Heft 3. 29-32.

Stechow, Arnim v. \& Wolfgang Sternefeld. 1987. Bausteine syntaktischen Wissens. Ein Lehrbuch der modernen generativen Grammatik. Wiesbaden, Westdeutscher Verlag.

Steinitz, Renate. 1969. Adverbialsyntax. (SG 10.) Berlin, Akademie-Verlag. Stickel, Gerhard. 1972. 'Ja' und 'nein' als Kontroll- und Korrektursignale.

LBer 17. 12-17.

Strawson, Peter F. 1952. Introduction to Logical Theory. London, Methuen.

Stuurman, Frits. 1983. Appositives and X-bar-Theory. LIn 14. 736-744.

Sugarewa, Tekla. 1974. Adjektivderivate zu Eigennamen. Beiträge zur Geschichte der deutschen Sprache und Literatur (Halle) 94. 199-256.

Sutton, F. W. \& Karl Beilhardt. 1977. Grundzüge der englischen Grammatik. Stuttgart, Klett.

Tamba-Mecz, Irène. 1975. Système de l'identification métaphorique dans la construction appositive. FM 43. 234-255. 
Tamine-Gardes, J. 1984. Introduction à la syntaxe: Les fonctions nominales: les compléments circonstantiels, l'apposition. L'information grammaticale 22. $39-42$.

Tarvainen, Kalevi. 1982. Einführung in die Dependenzgrammatik. (RGL 35.) Tübingen, Niemeyer.

Taylor, Ch. V. 1971. Co-ordinate and Equative Implications in Appositional Relationships. Kivung, Journal of the Linguistic Society of the University of Papua and New Guinea 4. 79-100.

Tesnière, Lucien. ${ }^{2} 1965$ [1959]. Eléments de syntaxe structurale. Paris, Klincksieck.

- 1980. Grundzüge der strukturalen Syntax. Hg. u. übersetzt v. Ulrich Engel. Stuttgart, Klett-Cotta.

Teubert, Wolfgang. 1979. Valenz des Substantivs. Attributive Ergänzungen und Angaben. (SdGe 49.) Düsseldorf, Schwann.

Thim-Mabrey, Christiane. 1985. Satzkonnektoren wie 'allerdings', 'dennoch' und 'übrigens'. Stellungsvarianten im deutschen Aussagesatz. (Regensburger Beiträge zur deutschen Sprach- und Literaturwissenschaft, Reihe B, 28.) Frankfurt/M. , Lang.

Thorne, James Peter. 1988. Non-Restrictice Relative Clauses. On Language. FS Robert P. Stockwell, hg. v. Caroline Duncan-Rose \& Theo Vennemann, 424-436. London/New York, Routledge.

Tilly, Charles. 1963. Discussion: L'apposition. FM 31. 287-291.

Trabant, Jürgen. 1983. Gehören die Interjektionen zur Sprache? Weydt (Hg.) 1983, 69-81.

Twaddell, W. Freeman. 1970. Grammatical Notes: the Auxiliary 'werden'; the Preposition 'als'. FS Detlev W. Schumann, hg. v. Albert R. Schmitt, 383386. München, Delp.

Ulvestad, Bjarne. 1974. Das pränukleare Adverbialattribut bei Nominalen im Deutschen. Gesprochene Sprache, hg. v. H. Moser, 267-282. (SdGe 26.)

Düsseldorf, Schwann.

vater, Heinz. 21979 [1963]. Das System der Artikelformen im gegenwärtigen Deutsch. (LA 78.) Tübingen, Niemeyer.

- 1980. Quantifier floating in German. The Semantics of Determiners, hg. v. Johan v. d. Auwera, 232-249. London, Croom Helm.

- 1985. Einführung in die Nominalphrasensyntax des Deutschen. (KLAGE 10.) Trier, L.A.U.T.

- 1986a. Zur Syntax der Determinantien. (SdG 31.) Tübingen, Narr.

- 1986b. Zur NP-Struktur im Deutschen. Vater 1986a, 123-145.

Velde, Marc van de. 1978. Zur mehrfachen Vorfeldbesetzung im Deutschen. Wortstellung und Bedeutung, hg. v. Maria-Elisabeth Conte u.a., 131-141. (LA 61.) Tübingen, Niemeyer.

Vennemann, Theo. 1977. Konstituenz und Dependenz in einigen neueren Grammatiktheorien. Sprachw 2. 259-301.

- \& Joachim Jacobs. 1982. Sprache und Grammatik. (Ertrăge der Forschung 176.) Darmstadt, Wiss. Buchgesellschaft.

Vildé-Lot, J. 1964. L'apposition dans les grammaires françaises composées par des auteurs soviétiques. FM 32. 101-110.

Voyles, Joseph B. 1983. Ansätze zu einer deutschen Grammatik. Göppingen. Waterman, John T. 1972. The Occurrence of 'als' as Preposition. Monatshefte für deutschen Unterricht, deutsche Sprache und Literatur 64. 132-135. Wegener, Heide. 1985. Der Dativ im heutigen Deutsch. (SdG 28.) Tübingen, Narr. 
Weinrich, Harald. 1982. Textgrammatik der französischen Sprache. Stuttgart, Klett.

Weiss, Andreas. 1975. Syntax spontaner Gespräche. (SdGe 31.) Düsseldorf, Schwann.

Weydt, Harald (Hg.). 1977. Aspekte der Modalpartikeln. Studien zur deutschen Abtönung. (Konzepte der Sprach- und Literaturwissenschaft 23.) Tübingen, Niemeyer.

- 1983. Partikeln und Interaktion. (RGL 44.) Tübingen, Niemeyer.

Wimmer, Rainer. 1973. Der Eigenname im Deutschen. (LA 11.) Tübingen, Niemeyer Winkler, Christian. 1969. Der Einschub. FS Hugo Moser, hg. v. Ulrich Engel u.a., 282-295. Düsseldorf, Schwann.

Winter, Werner. 1966. Vom Genitiv im heutigen Deutsch. Zeitschrift für deutsche Sprache 22. 21-35.

Wittgenstein, Ludwig. 21980 . Philosophische Untersuchungen. Frankfurt/M., Suhrkamp.

Wunderlich, Dieter. 1984. Zur Syntax der Präpositionalphrasen im Deutschen. Zeitschrift für Sprachwissenschaft 3. 65-99.

Wunderlich, Hermann \& H. Reis. ${ }^{3} 1925$ [1892]. Der deutsche Satzbau. Bd. 2. Stuttgart, Cotta.

Zemb, Jean-Marie. 1968. Les structures logiques de la proposition allemande. Paris (Diss.)

- 1972. Satz. Wort. Rede. Freiburg u.a., Herder.

- 1976. Unterordnung, Nebenordnung und Zuordnung. Sprachw 1. 241-262.

- 1978. Vergleichende Grammatik Französisch-Deutsch. Teil 1. (Duden-Sonderreihe. Vergleichende Grammatiken 1) Mannheim u.a., Bibliograph. Institut.

Ziv, Yael. 1973. Why can't appositives be extraposed? Papers in linguistics [Edmonton] 6. 243-256.

- 1985. Parentheticals and Functional Grammar. Syntax and Pragmatics in Functional Grammar, hg. v. A.M. Bolkestein, C. de Groot, J.L. Mackenzie, 181199. (Functional Grammar Series 1.) Dordrecht, Foris.

Zoeppritz, M. 1984. Syntax for German in the User Speciality Languages Syster. (Sprache und Information 9.) Tübingen, Niemeyer.

Zwicky, Arnold M. 1985. Heads. JL 21. 1-29. 